\title{
Distal Ionic Substrate-Catalyst Interactions Enable Long-Range Stereocontrol: Access to Remote Quaternary Stereocenters through a Desymmetrizing Suzuki-Miyaura Reaction
}

Yazhou Lou, Junqiang Wei, Mingfeng Li, Ye Zhu*

Department of Chemistry, Faculty of Science, National University of Singapore, 3 Science Drive 3, Singapore 117543

*Correspondence to: chmzhu@nus.edu.sg

Supporting Information 


\section{Table of Contents}

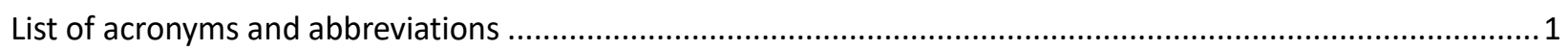

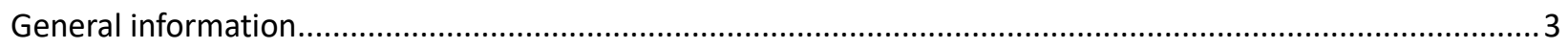

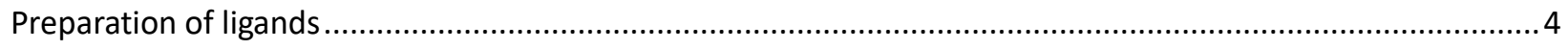

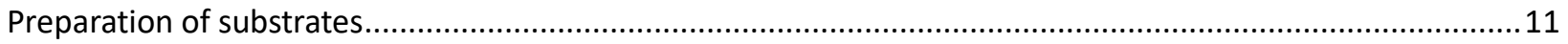

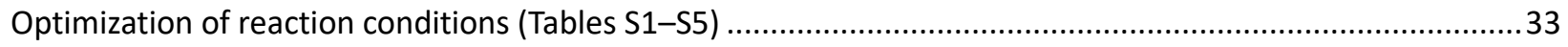

General procedure of catalytic desymmetrization reaction ........................................................................ 38

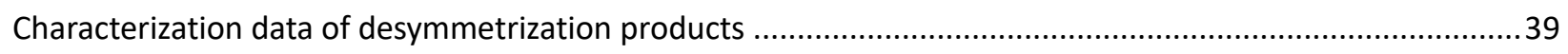

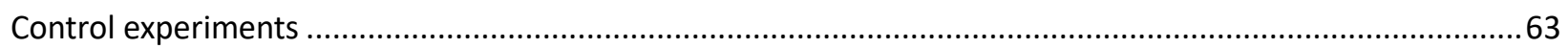

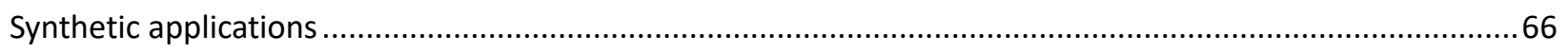

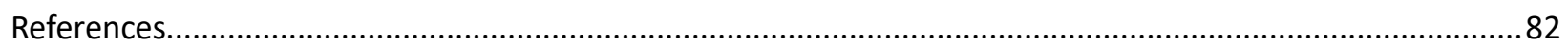

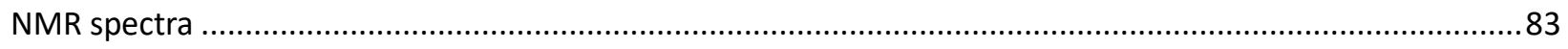

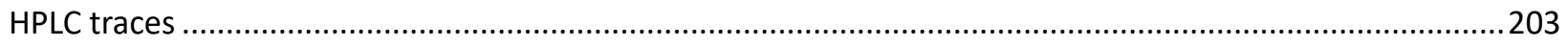

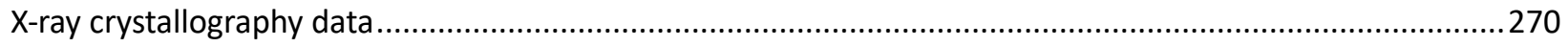




\section{List of acronyms and abbreviations}

\begin{tabular}{|c|c|}
\hline Ac & acetyl \\
\hline $\mathrm{Bu}$ & butyl \\
\hline COD & 1,5-cyclooctadiene \\
\hline Cy & cyclohexyl \\
\hline dan & naphthalene-1,8-diaminato \\
\hline dba & dibenzylideneacetone \\
\hline DCC & dicyclohexylcarbodiimide \\
\hline DCM & dichloromethane \\
\hline DME & 1,2-dimethoxyethane \\
\hline DMF & $\mathrm{N}, \mathrm{N}$-dimethylformamide \\
\hline Et & ethyl \\
\hline HMDS & bis(trimethylsilyl)amide \\
\hline Me & methyl \\
\hline 2-MeTHF & 2-methyltetrahydrofuran \\
\hline NBS & $N$-bromosuccinimide \\
\hline NCS & $N$-chlorosuccinimide \\
\hline $\mathbf{P h}$ & phenyl \\
\hline pin & pinacolato \\
\hline $\operatorname{Pr}$ & propyl \\
\hline RuPhos & 2-dicyclohexylphosphino-2',6'-diisopropoxybiphenyl \\
\hline SPhos & 2-dicyclohexylphosphino-2',6'-dimethoxybiphenyl \\
\hline TBAOH & tetrabutylammonium hydroxide \\
\hline TFA & trifluoroacetic acid \\
\hline THF & tetrahydrofuran \\
\hline
\end{tabular}


Tf

TMS

Tol trifluoromethylsulfonyl

trimethylsilyl

methylphenyl 


\section{General information}

Materials: Commercially available reagents and solvents were used as received. Commercial dry solvents (Aldrich Sure/Seal ${ }^{\mathrm{TM}}$ ) were sparged with nitrogen before used in catalytic reactions. Solvents used for column chromatography were analytical grade.

Methods: Unless otherwise noted, all experiments were set up under an atmosphere of nitrogen in a glovebox or using standard Schlenk techniques. Reactions were monitored by thin layer chromatography (TLC), gas chromatography (GC), or nuclear magnetic resonance (NMR) analysis. Flash column chromatography was performed using Tsingdao silica gel (60, particle size 300-400 mesh). Yields refer to isolated yields after flash column chromatography purification.

Characterization: Products were characterized by means of nuclear magnetic resonance (NMR), mass spectrometry (MS), high performance liquid chromatography (HPLC), and optical rotation. NMR spectra were recorded on a Bruker DPX 400 spectrometer at $300 \mathrm{MHz}$ for ${ }^{1} \mathrm{H} N \mathrm{NMR}, 101 \mathrm{MHz}$ for ${ }^{13} \mathrm{C}$ NMR and $162 \mathrm{MHz}$ for ${ }^{31} \mathrm{P}$ NMR or on a Bruker DPX 500 spectrometer at $500 \mathrm{MHz}$ for ${ }^{1} \mathrm{H}$ NMR, $126 \mathrm{MHz}$ for ${ }^{13} \mathrm{C}$ NMR, $202 \mathrm{MHz}$ for ${ }^{31} \mathrm{P}$ NMR and 471 $\mathrm{MHz}$ for ${ }^{19} \mathrm{~F}$ NMR in $\mathrm{CDCl}_{3}$ with tetramethylsilane as internal standard. Chemical shifts were reported relative to tetramethylsilane $(0 \mathrm{ppm})$ for ${ }^{1} \mathrm{H} \mathrm{NMR}$ and relative to $\mathrm{CDCl}_{3}(77.0 \mathrm{ppm})$ for ${ }^{13} \mathrm{C} \mathrm{NMR} .{ }^{19} \mathrm{~F}$ spectra were calibrated from external standard ( $\left.\mathrm{CFCl}_{3}: 0 \mathrm{ppm}\right) .{ }^{31} \mathrm{P}$ spectra were calibrated from external standard ( 85 wt\% phosphoric acid: 0 ppm). NMR data are reported as: chemical shift (parts per million, $p p m$ ), multiplicity $(s=$ singlet, $d=d o u b l e t, t=t r i p l e t, ~ q$ = quartet, $\mathrm{m}=$ multiplet), coupling constant $(\mathrm{Hz})$, and integration. HPLC analysis was performed on a Shimadzu $i-$ series HPLC system equipped with photodiode array (PDA) detector and Chiralcel and Chiralpak columns $(0.46 \mathrm{~cm} \emptyset \times$ $25 \mathrm{~cm}$ ). The wavelength that is an apex in the spectrum was selected for analysis of the enantiomeric ratio (er) of each compound. Optical rotation $\left([\alpha]_{D}^{\top}\right.$, deg $\left.\bullet \mathrm{cm}^{3} \bullet \mathrm{g}^{-1} \bullet \mathrm{dm}^{-1}\right)$ was measured on a Jasco DIP-1000 Digital Polarimeter at $\lambda=589 \mathrm{~nm}$ in the given solvent at the indicated concentration $(c, \mathrm{~g} / 100 \mathrm{~mL})$ and temperature $\left(\mathrm{T},{ }^{\circ} \mathrm{C}\right) . \mathrm{X}$-ray diffraction was performed on Bruker D8 Venture single crystal X-ray diffractometer. 


\section{Preparation of ligands}

1. Synthetic procedures and characterization data for $(S)-\mathbf{L} \mathbf{4}$

\section{(3'-Bromo-2',6'-diisopropoxy-[1,1'-biphenyl]-2-yl)dicyclohexylphosphane}<smiles>CC(C)COc1c(Br)ccc(OC(C)C)c1-c1ccccc1OC(C)C</smiles>

SI-L1

TFA (27.4 g, $241.1 \mathrm{mmol})$ was added to a solution of RuPhos $(15.0 \mathrm{~g}, 32.1 \mathrm{mmol})$ in $36 \mathrm{~mL}$ DCM, then NBS $(5.72 \mathrm{~g}, 32.1 \mathrm{mmol})$ was added in ten minutes at $0{ }^{\circ} \mathrm{C}$. After addition was completed, the resulting reaction mixture was stirred at this temperature for $30-45 \mathrm{~min}$. The reaction was quenched with ice water and neutralized with $\mathrm{NaHCO}_{3}$ to $\mathrm{pH}$ 9-10.and extracted with DCM three times. The combined organic phases were washed with water and brine, dried over $\mathrm{Na}_{2} \mathrm{SO}_{4}$, and concentrated in vacuo to give the desired product as a white solid which was directly used in the next step without further purification $(17.4 \mathrm{~g}, 99 \%$ yield).

\section{Dicyclohexyl(2',6'-diisopropoxy-3'-methyl-[1,1'-biphenyl]-2-yl)phosphane}<smiles>Cc1ccc(OC(C)C)c(-c2ccccc2P(F)(F)(F)F)c1OC(C)C</smiles>

L1

To SI-L1 (10.9 g, $20.0 \mathrm{mmol}), \mathrm{MeB}(\mathrm{OH})_{2}(3.59 \mathrm{~g}, 60.0 \mathrm{mmol})$, and [Pd(allyl)Cl] 2 (73.1 mg, 0.2 $\mathrm{mmol}$ ) were added $110 \mathrm{~mL}$ dioxane, followed by $2 \mathrm{M} \mathrm{K}_{2} \mathrm{CO}_{3}(8.27 \mathrm{~g}, 60 \mathrm{mmol})$. Then the resulting reaction mixture was warmed to $90^{\circ} \mathrm{C}$ and stirred for $24 \mathrm{~h}$. The reaction was cooled to room temperature and extracted with ethyl acetate $(20 \mathrm{~mL} \times 3)$ for three times. The combined organic phases were washed with brine, dried over $\mathrm{Na}_{2} \mathrm{SO}_{4}$ and concentrated in vacuo. The crude materials were purified by flash column chromatography to give the desired product ( $8.6 \mathrm{~g}, 90 \%$ yield $)$ as a colorless oil.

${ }^{1} \mathrm{H}$ NMR $\left(500 \mathrm{MHz}, \mathrm{CDCl}_{3}\right) \delta 7.56(\mathrm{~d}, J=4.7 \mathrm{~Hz}, 1 \mathrm{H}), 7.31(\mathrm{dd}, J=13.4,9.1 \mathrm{~Hz}, 2 \mathrm{H}), 7.26-7.20(\mathrm{~m}, 1 \mathrm{H}), 7.09(\mathrm{~d}, J$ $=8.3 \mathrm{~Hz}, 1 \mathrm{H}), 6.61(\mathrm{~d}, J=8.4 \mathrm{~Hz}, 1 \mathrm{H}), 4.40-4.30(\mathrm{~m}, 1 \mathrm{H}), 3.78-3.69(\mathrm{~m}, 1 \mathrm{H}), 2.24(\mathrm{~s}, 3 \mathrm{H}), 1.85-1.75(\mathrm{~m}, 2 \mathrm{H}), 1.71-$ $1.54(\mathrm{~m}, 12 \mathrm{H}), 1.32-1.24(\mathrm{~m}, 8 \mathrm{H}), 1.13(\mathrm{dd}, J=6.0,6.0 \mathrm{~Hz}, 6 \mathrm{H}), 0.97(\mathrm{~d}, J=6.1 \mathrm{~Hz}, 3 \mathrm{H}), 0.76(\mathrm{~d}, J=6.1 \mathrm{~Hz}, 3 \mathrm{H}) .{ }^{13} \mathrm{C}$ $\operatorname{NMR}\left(126 \mathrm{MHz}, \mathrm{CDCl}_{3}\right) \delta 154.3,143.7(\mathrm{~d}, J=30.8 \mathrm{~Hz}), 137.0(\mathrm{~d}, J=18.7 \mathrm{~Hz}), 132.1(\mathrm{~d}, J=5.9 \mathrm{~Hz}), 131.9(\mathrm{~d}, J=3.9 \mathrm{~Hz})$, 129.7, 127.1, 125.7, 123.1, 108.1, 74.1, 70.0, 34.8 (d, $J=15.0 \mathrm{~Hz}), 33.1(\mathrm{~d}, J=13.9 \mathrm{~Hz}), 30.7(\mathrm{~d}, J=15.2 \mathrm{~Hz}), 30.2-$ $28.6(\mathrm{~m}), 28.6-27.0(\mathrm{~m}), 26.6(\mathrm{~d}, J=7.6 \mathrm{~Hz}), 22.7,22.4(\mathrm{~d}, J=3.9 \mathrm{~Hz}), 22.1,17.0 .{ }^{31} \mathrm{P} \mathrm{NMR}\left(202 \mathrm{MHz}, \mathrm{CDCl}_{3}\right) \delta-8.91$. 
(3'-Bromo-2',6'-diisopropoxy-5'-methyl-[1,1'-biphenyl]-2-yl)dicyclohexylphosphane

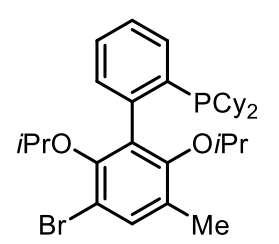

SI-L2

To a solution of $\mathbf{L} 1(8.18 \mathrm{~g}, 17.0 \mathrm{mmol})$ in $20 \mathrm{~mL}$ DCM was added TFA $(14.55 \mathrm{~g}, 127.6 \mathrm{mmol})$ at $0^{\circ} \mathrm{C}$, then NBS $(3.02 \mathrm{~g}, 17.0 \mathrm{mmol})$ was added to the solution portionwise in 5 minutes. After addition was completed, the reaction mixture was stirred for $0.5-1 \mathrm{~h}$ until the reaction was completed. The reaction was quenched with ice water and neutralized with $\mathrm{NaHCO}_{3}$ to $\mathrm{pH}$ 9-10

and extracted with $\mathrm{DCM}(15 \mathrm{~mL} \times 3)$ for three times. The combined organic phases were washed with water and brine, dried over $\mathrm{Na}_{2} \mathrm{SO}_{4}$ and concentrated in vacuo. The crude product was dissolved in toluene and $\mathrm{MeOH}$ was added. The solution was stirred at room temperature for 30 minutes and a white solid precipitated and filtered to give the desired product $(8.2 \mathrm{~g}, 86 \%$ yield) as a white solid which was directly used in next step without further purification.

${ }^{1} \mathrm{H}$ NMR $\left(500 \mathrm{MHz}, \mathrm{CDCl}_{3}\right)$ $\delta$ 7.64-7.62 (m, 1H), 7.39-7.37 (m, 4H), 3.92-3.88 (m, 1H), 3.73-3.69 (m, 1H), $2.25(\mathrm{~s}$, $3 \mathrm{H}), 2.13-2.11(\mathrm{~m}, 2 \mathrm{H}), 1.99-1.97(\mathrm{~m}, 2 \mathrm{H}), 1.80-1.78(\mathrm{~m}, 2 \mathrm{H}), 1.69-1.67(\mathrm{~m}, 4 \mathrm{H}), 1.60-1.58(\mathrm{~m}, 2 \mathrm{H}), 1.35-1.16(\mathrm{~m}$, $8 \mathrm{H}), 1.10-1.06(\mathrm{~m}, 2 \mathrm{H}), 0.94(\mathrm{~d}, J=6.1 \mathrm{~Hz}, 6 \mathrm{H}), 0.87(\mathrm{~d}, J=5.9 \mathrm{~Hz}, 6 \mathrm{H}) .{ }^{13} \mathrm{C} \mathrm{NMR}\left(126 \mathrm{MHz}, \mathrm{CDCl}_{3}\right) \delta 154.1,151.4$, $142.3(\mathrm{~d}, J=32.1 \mathrm{~Hz}), 138.4,133.4,132.6(\mathrm{~d}, J=7.0 \mathrm{~Hz}), 132.2(\mathrm{~d}, J=5.9 \mathrm{~Hz}), 131.7,128.1,127.5,126.9,112.0$, $75.8,74.9,33.8(\mathrm{dd}, J=14.4,5.8 \mathrm{~Hz}), 31.7(\mathrm{dd}, J=19.2,12.5 \mathrm{~Hz}), 27.7(\mathrm{dd}, J=13.0,4.9 \mathrm{~Hz}), 26.5,22.6,22.5(\mathrm{~d}, J=$ 2.7 Hz), 22.4, 16.8. ${ }^{31} \mathrm{P} \mathrm{NMR}\left(202 \mathrm{MHz}, \mathrm{CDCl}_{3}\right)$ 8 -8.01. MS (m/z, ESI): Calcd. for Chemical Formula: $\mathrm{C}_{31} \mathrm{H}_{45} \mathrm{BrO}_{2} \mathrm{P}^{+}[\mathrm{M}+\mathrm{H}]^{+}$: 559.2335, Found: 559.2333 .

\section{(Ss)-(3'-((11-oxidanyl)(p-tolyl)-I3-sulfanyl)-2',6'-diisopropoxy-5'-methyl-[1,1'-biphenyl]-2-}

\section{yl)dicyclohexylphosphane}

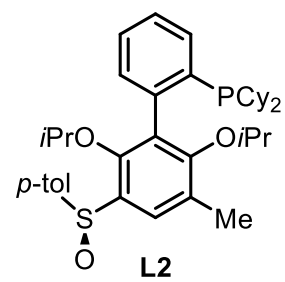

To a solution of SI-L2 (5.59 g, $10.0 \mathrm{mmol})$ in anhydrous $28 \mathrm{~mL}$ THF was added $n$ BuLi (5.5 $\mathrm{mL}, 2.0 \mathrm{~mol} / \mathrm{L}, 11.0 \mathrm{mmol}$ ) as a cyclohexane solution dropwise at $-78^{\circ} \mathrm{C}$. After addition was completed, the reaction mixture was stirred at this temperature for $1 \mathrm{~h}$. Then this reaction solution was transferred to a solution of (-)-menthyl $\left(S_{S}\right)$-p-toluenesulfinate $(3.82 \mathrm{~g}, 13.0 \mathrm{mmol})$

in anhydrous toluene at $0{ }^{\circ} \mathrm{C}$ and the resulting reaction mixture was stirred at room temperature overnight. The reaction was quenched with saturated $\mathrm{NH}_{4} \mathrm{Cl}$ solution and extracted with ethyl acetate for three times. The combined organic phases were washed with brine, dried over $\mathrm{Na}_{2} \mathrm{SO}_{4}$ and concentrated in vacuo. Based on analysis of the NMR 
spectra, the crude material contained two products $(1.1: 1 \mathrm{dr})$. The crude material was purified by flash column chromatography with hexane and ethyl acetate (10:1-3:1) to give the two diastereomers.

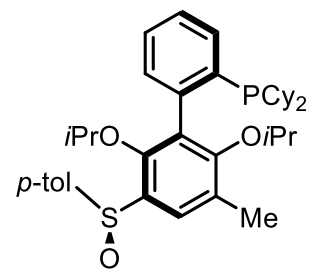

$\left(S_{S}, S\right)-L 2$

(Ss, S)-L2: White solid, $2.0 \mathrm{~g}$, 32\% yield. $[\alpha]_{D^{20}}=-149.0\left(\mathrm{c} 0.005 \mathrm{CHCl}_{3}\right) .{ }^{1} \mathrm{H}$ NMR (500 $\left.\mathrm{MHz}, \mathrm{CDCl}_{3}\right) \delta$ 7.65-7.59 (m, 4H), 7.37-7.36 (m, 3H), 7.26-7.24 (m, 2H), 3.88-3.86 (m, $\left.1 \mathrm{H}\right), 3.84$ $-3.74(\mathrm{~m}, 1 \mathrm{H}), 2.38(\mathrm{~s}, 3 \mathrm{H}), 2.30(\mathrm{~s}, 3 \mathrm{H}), 1.85-1.83(\mathrm{~m}, 3 \mathrm{H}), 1.76-1.71(\mathrm{~m}, 4 \mathrm{H}), 1.68-1.56(\mathrm{~m}$, $4 \mathrm{H}), 1.37-1.32(\mathrm{~m}, 2 \mathrm{H}), 1.18-1.13(\mathrm{~m}, 10 \mathrm{H}), 1.01-0.81(\mathrm{~m}, 11 \mathrm{H}) .{ }^{13} \mathrm{C} \mathrm{NMR}\left(126 \mathrm{MHz}, \mathrm{CDCl}_{3}\right)$ $\delta 157.3,151.3,142.7,142.2,141.9,140.6,138.2(\mathrm{~d}, J=19.8 \mathrm{~Hz}), 132.8,132.4,131.9(\mathrm{~d}$, $J=5.7 \mathrm{~Hz}$ ), $129.7,128.5,127.6,127.1,125.8,125.6,75.6,74.9,34.3$ (d, $J=15.4 \mathrm{~Hz}), 34.0$ (d, J = 15.2 Hz), 31.4 (d, $J=18.6 \mathrm{~Hz}$ ), $30.4(\mathrm{~d}, J=14.3 \mathrm{~Hz}), 29.9(\mathrm{~d}, J=15.5 \mathrm{~Hz}), 28.5(\mathrm{~d}, J=8.9 \mathrm{~Hz}), 27.8(\mathrm{~d}, J=12.8 \mathrm{~Hz}), 27.3(\mathrm{dd}, J=28.1,9.9$ $\mathrm{Hz}$ ), 26.5 (d, J = 10.2 Hz), 22.8, 22.6, 22.4, 22.0, 21.4, 17.4. ${ }^{31} \mathrm{P}$ NMR (202 MHz, CDCl3) $\delta-8.40 . \mathrm{MS}$ (m/z, ESI): Calcd. for Chemical Formula: $\mathrm{C}_{31} \mathrm{H}_{45} \mathrm{O}_{2} \mathrm{P}^{+}[\mathrm{M}+\mathrm{Na}]^{+}:$641.3189, Found: 641.3188 .

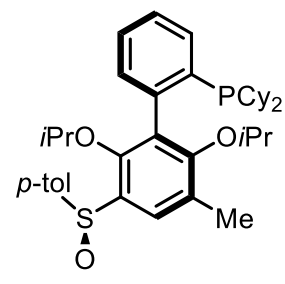

$\left(\mathrm{S}_{s}, R\right)-\mathbf{L} 2$

(Ss, R)-L2 : White solid, $2.3 \mathrm{~g}, 37 \%$ yield. $[\alpha]_{\mathrm{D}}{ }^{20}=-195.0\left(\mathrm{c} 0.005 \mathrm{CHCl}_{3}\right) .{ }^{1} \mathrm{H} \mathrm{NMR}(500 \mathrm{MHz}$, $\left.\mathrm{CDCl}_{3}\right) \delta 7.76(\mathrm{~d}, J=8.8 \mathrm{~Hz}, 1 \mathrm{H}), 7.67(\mathrm{~d}, J=8.2 \mathrm{~Hz}, 2 \mathrm{H}), 7.51(\mathrm{~d}, J=5.6 \mathrm{~Hz}, 1 \mathrm{H}), 7.33(\mathrm{p}, J=6.7$ $\mathrm{Hz}, 2 \mathrm{H}), 7.23(\mathrm{t}, J=6.4 \mathrm{~Hz}, 3 \mathrm{H}), 6.83(\mathrm{~d}, J=8.9 \mathrm{~Hz}, 1 \mathrm{H}), 4.55-4.48(\mathrm{~m}, 1 \mathrm{H}), 3.72-3.64(\mathrm{~m}, 1 \mathrm{H})$, $2.38(\mathrm{~s}, 3 \mathrm{H}), 1.90-1.86(\mathrm{~m}, 2 \mathrm{H}), 1.78-1.39(\mathrm{~m}, 12 \mathrm{H}), 1.25-0.98(\mathrm{~m}, 17 \mathrm{H}), 0.85(\mathrm{~s}, 3 \mathrm{H}) .{ }^{13} \mathrm{C} N M R$ $\left(126 \mathrm{MHz}, \mathrm{CDCl}_{3}\right) \delta 158.6,152.9,143.3,142.1,141.9,140.3,137.0,136.8,132.4,131.5$ (d, $J=5.3 \mathrm{~Hz}), 129.7,129.4,127.6,126.5,126.1(\mathrm{~d}, J=3.4 \mathrm{~Hz}), 124.4,108.0,75.1,69.9,35.3(\mathrm{~d}, J=16.2 \mathrm{~Hz}), 32.8$ (d, $J=14.6 \mathrm{~Hz}), 30.5-29.4(\mathrm{~m}), 28.7(\mathrm{~d}, J=6.4 \mathrm{~Hz}), 27.5(\mathrm{~d}, J=6.4 \mathrm{~Hz}), 27.4,27.1(\mathrm{t}, J=3.4 \mathrm{~Hz}), 26.5(\mathrm{~d}, J=19.4 \mathrm{~Hz})$, 22.7, 22.1, $21.9(\mathrm{~d}, J=14.6 \mathrm{~Hz})$, 21.4. ${ }^{31} \mathrm{P} \mathrm{NMR}\left(202 \mathrm{MHz}, \mathrm{CDCl}_{3}\right) \delta-8.82 . \mathrm{MS}(\mathrm{m} / \mathrm{z}, \mathrm{ESI})$ : Calcd. for Chemical Formula: $\mathrm{C}_{31} \mathrm{H}_{45} \mathrm{O}_{2} \mathrm{P}^{+}[\mathrm{M}+\mathrm{Na}]^{+}:$641.3189, Found: 641.3188 . 


\section{(R)-dicyclohexyl(2',6'-diisopropoxy-3'-methyl-[1,1'-biphenyl]-2-yl)phosphane}

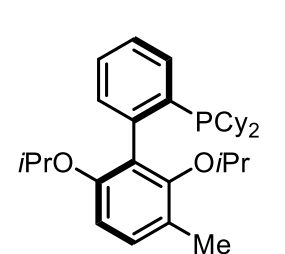

$(R)$-L1

Under $\mathrm{N}_{2}$ atmosphere, $n \mathrm{BuLi}(9.9 \mathrm{~mL}, 2.0 \mathrm{~mol} / \mathrm{L}, 19.8 \mathrm{mmol})$ as a cyclohexane solution was added to $\left(\mathrm{S}_{s}, \mathrm{~S}\right)-\mathbf{L} 2(2.0 \mathrm{~g}, 3.2 \mathrm{mmol})$ in anhydrous $20.0 \mathrm{~mL}$ THF at $-78{ }^{\circ} \mathrm{C}$. Then the resulting reaction mixture was stirred at room temperature for $1-2 \mathrm{~h}$ and $\mathrm{MeOH}(1.0 \mathrm{~mL})$ was added to this reaction mixture at $-78{ }^{\circ} \mathrm{C}$. The reaction was quenched with saturated $\mathrm{NH}_{4} \mathrm{Cl}$ solution and extracted with ethyl acetate $(10 \mathrm{~mL} \times 3)$ three times. The combined organic phases were washed with brine, dried over $\mathrm{Na}_{2} \mathrm{SO}_{4}$, and concentrated in vacuo. The crude materials were purified by flash column chromatography to give the product a colorless oil. $1.5 \mathrm{~g}, 98 \%$ yield. $[\alpha]_{\mathrm{D}}{ }^{20}=-18.36\left(c 0.050 \mathrm{CHCl}_{3}\right)$ for >99.5: 0.5 er. The NMR spectra are consistent with racemic $\mathbf{L} 1$.

HPLC analysis of this compound: Daicel Chiralpak IA, hexane/iso-propanol =95: 5, $1.0 \mathrm{~mL} / \mathrm{min}, \lambda=296 \mathrm{~nm}$, retention time: 8.37 min (major) and $9.31 \mathrm{~min}$ (minor).

\section{(S)-(3'-bromo-2',6'-diisopropoxy-5'-methyl-[1,1'-biphenyl]-2-yl)dicyclohexylphosphane}

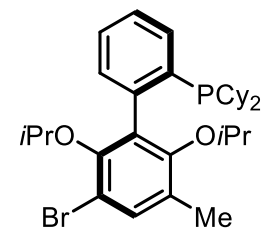

(S)-SI-L2

(R)-L1 (1.5 g, $3.1 \mathrm{mmol})$ was dissolved in $4 \mathrm{~mL} \mathrm{DCM}$ and TFA (2.81 g, $24.6 \mathrm{mmol})$ was added at $0{ }^{\circ} \mathrm{C}$. After stirring for $5 \mathrm{~min}$, NBS $(0.59 \mathrm{~g}, 3.2 \mathrm{mmol})$ was added portion-wise at $0{ }^{\circ} \mathrm{C}$. After addition was completed, the reaction mixture was stirred for $0.5-1 \mathrm{~h}$ until the reaction was completed. The reaction was quenched with ice water and neutralized with $\mathrm{NaHCO}_{3}$ to $\mathrm{pH}$ 9-10 and extracted with DCM $(8 \mathrm{~mL} \times 3)$ for three times. The combined organic phases were washed with water and brine, dried over $\mathrm{Na}_{2} \mathrm{SO}_{4}$, and concentrated in vacuo. The crude product was dissolved in $1.0 \mathrm{~mL}$ toluene and $8.0 \mathrm{~mL} \mathrm{MeOH}$ was added. The solution was stirred at room temperature for 30 minutes and a white solid precipitated and filtrated to give the desired product $1.24 \mathrm{~g}$ in $72 \%$ yield as a white solid which was directly used in the next step without further purification. $[\alpha]_{D}^{20}=+6.00\left(c 0.050 \mathrm{CHCl}_{3}\right)$. The NMR spectra are consistent with racemic SI-L2. 


\section{Diethyl (S)-(2'-(dicyclohexylphosphanyl)-2,6-diisopropoxy-5-methyl-[1,1'-biphenyl]-3-yl)phosphonate}

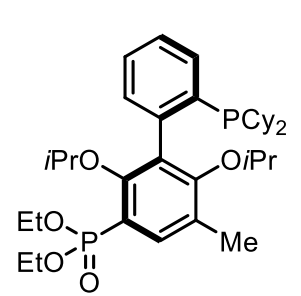

(S)-L3

Under $\mathrm{N}_{2}$ atmosphere, to a solution of (S)-SI-L2 $(1.2 \mathrm{~g}, 2.1 \mathrm{mmol})$ in anhydrous $3.0 \mathrm{~mL}$

THF was added $n$ BuLi $(1.2 \mathrm{~mL}, 2.0 \mathrm{~mol} / \mathrm{L}, 2.35 \mathrm{mmol})$ dropwise as a cyclohexane solution at $78^{\circ} \mathrm{C}$. The reaction mixture was stirred at this temperature for $1 \mathrm{~h}$ and ethyl chlorophosphate (0.44 g, $2.6 \mathrm{mmol}$ ) was added dropwise. The reaction was quenched with saturated $\mathrm{NH}_{4} \mathrm{Cl}$ solution and extracted with ethyl acetate $(8 \mathrm{mLX} 3)$ for three times. The combined organic phases were washed with brine, dried over $\mathrm{Na}_{2} \mathrm{SO}_{4}$ and concentrated in vacuo. The crude materials were purified by flash column chromatography with hexane and ethyl acetate (10:1-3:1) to give the desired products (S)-L3 $1.08 \mathrm{~g}$ in 82\% yield as a colorless oil.

$[\alpha]_{D}^{20}=+22.00\left(c 0.100 \mathrm{CHCl}_{3}\right) .{ }^{1} \mathrm{H}$ NMR $\left(500 \mathrm{MHz}, \mathrm{CDCl}_{3}\right) \delta 7.67-7.61(\mathrm{~m}, 2 \mathrm{H}), 7.34(\mathrm{~d}, J=2.7 \mathrm{~Hz}, 3 \mathrm{H}), 4.24-$ $4.15(\mathrm{~m}, 3 \mathrm{H}), 4.12-4.10(\mathrm{~m}, 1 \mathrm{H}), 3.86-3.76(\mathrm{~m}, 1 \mathrm{H}), 3.73-3.70(\mathrm{~m}, 1 \mathrm{H}), 2.25(\mathrm{~s}, 3 \mathrm{H}), 2.02-1.93(\mathrm{~m}, 2 \mathrm{H}), 1.88-1.84$ (m, 2H), 1.76-1.74 (m, 2H), $1.69-1.60(\mathrm{~m}, 5 \mathrm{H}), 1.48(\mathrm{~d}, J=12.8 \mathrm{~Hz}, 1 \mathrm{H}), 1.38(\mathrm{t}, J=7.0 \mathrm{~Hz}, 3 \mathrm{H}), 1.34(\mathrm{t}, J=7.1 \mathrm{~Hz}$ 3H), 1.26-1.18 (m, 10H), $0.97(\mathrm{dd}, J=5.8,3.0 \mathrm{~Hz}, 6 \mathrm{H}), 0.90(\mathrm{~d}, J=6.1 \mathrm{~Hz}, 3 \mathrm{H}), 0.87(\mathrm{~d}, J=6.1 \mathrm{~Hz}, 3 \mathrm{H}) .{ }^{13} \mathrm{C} \mathrm{NMR}(126$ $\left.\mathrm{MHz}, \mathrm{CDCl}_{3}\right) \delta 158.7,156.8,142.5,138.3(\mathrm{~d}, J=19.4 \mathrm{~Hz}), 135.8(\mathrm{~d}, J=7.6 \mathrm{~Hz}), 132.3,131.9(\mathrm{~d}, J=5.9 \mathrm{~Hz}), 131.0$, $127.6,126.9,126.1(\mathrm{~d}, J=15.3 \mathrm{~Hz}), 117.5,74.8,74.0,61.8(\mathrm{~d}, J=5.7 \mathrm{~Hz}), 61.6(\mathrm{~d}, J=5.3 \mathrm{~Hz}), 34.3(\mathrm{~d}, J=15.4 \mathrm{~Hz})$, $33.9(\mathrm{~d}, J=15.0 \mathrm{~Hz}), 31.3(\mathrm{dd}, J=33.6,17.2 \mathrm{~Hz}), 29.3(\mathrm{~d}, J=12.1 \mathrm{~Hz}), 29.0(\mathrm{~d}, J=11.0 \mathrm{~Hz}), 27.6(\mathrm{dd}, J=14.2,5.4 \mathrm{~Hz})$, $27.3(\mathrm{t}, J=5.4 \mathrm{~Hz}), 26.5(\mathrm{~d}, J=9.2 \mathrm{~Hz}), 22.5(\mathrm{t}, J=12.1 \mathrm{~Hz}), 22.4,21.6,17.1,16.5(\mathrm{dd}, J=11.3,6.9 \mathrm{~Hz}) .{ }^{31} \mathrm{p} \mathrm{NMR}(202$ $\left.\mathrm{MHz}, \mathrm{CDCl}_{3}\right) \delta$ 19.27, -8.29. HRMS (m/z, ESI): Calcd. for Chemical Formula: $\mathrm{C}_{35} \mathrm{H}_{55} \mathrm{O}_{5} \mathrm{P}_{2}{ }^{+}[\mathrm{M}+\mathrm{H}]^{+}: 617.3525$, Found: 617.3531.

\section{Ethyl hydrogen ((S)-2'-(dicyclohexylphosphanyl)-2,6-diisopropoxy-5-methyl-[1,1'-biphenyl]-3-yl)phosphonate}

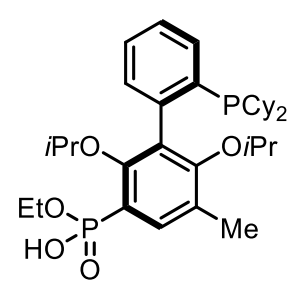

(S)-L4 Under $\mathrm{N}_{2}$ atmosphere, $(S)$ - $\mathbf{L} 3(0.5 \mathrm{~g}, 0.81 \mathrm{mmol})$ was dissolved in $1.0 \mathrm{~mL}$ THF and $2.0 \mathrm{~mL}$ EtOH, followed by addition of $2 \mathrm{M} \mathrm{NaOH}(65 \mathrm{mg}, 1.62 \mathrm{mmol}) .^{[1]}$ The resulting reaction mixture was warmed to $75^{\circ} \mathrm{C}$ and stirred for 3-4 days. After the reaction was completed, removal of the organic phase was performed under reduced pressure and water was added and extracted

with ethyl ether and hexane $(5.0 \mathrm{~mL}, 4: 1 \mathrm{v} / \mathrm{v})$ for three times. The water phase was neutralized with $1 \mathrm{M} \mathrm{HCl}$ to $\mathrm{pH}$ 2-3 and extracted with ethyl acetate $(5 \mathrm{~mL} \times 3)$ for three times. The combined organic phases were washed with 
water and brine, dried over $\mathrm{Na}_{2} \mathrm{SO}_{4}$ and concentrated in vacuo to give the desired ligands without further purification. $0.34 \mathrm{~g}$ in $72 \%$ yield as a white solid.

$[\alpha]_{\mathrm{D}}^{20}=+44.00\left(c 0.010 \mathrm{CHCl}_{3}\right) .{ }^{1} \mathrm{H}$ NMR $\left(500 \mathrm{MHz}, \mathrm{CDCl}_{3}\right) \delta 7.71(\mathrm{~d}, J=15.3 \mathrm{~Hz}, 1 \mathrm{H}), 7.65-7.60(\mathrm{~m}, 1 \mathrm{H}), 7.37$ $(\mathrm{td}, J=6.8,3.5 \mathrm{~Hz}, 3 \mathrm{H}), 6.30(\mathrm{~s}, 1 \mathrm{H}), 4.20-4.09(\mathrm{~m}, 2 \mathrm{H}), 3.82-3.78(\mathrm{~m}, 1 \mathrm{H}), 3.73-3.70(\mathrm{~m}, 1 \mathrm{H}), 2.26(\mathrm{~s}, 3 \mathrm{H}), 1.20-1.18$ $(\mathrm{m}, 2 \mathrm{H}), 1.89(\mathrm{~s}, 1 \mathrm{H}), 1.84-1.58(\mathrm{~m}, 8 \mathrm{H}), 1.50-1.45(\mathrm{~m}, 1 \mathrm{H}), 1.37-1.33(\mathrm{~m}, 3 \mathrm{H}), 1.31-1.14(\mathrm{~m}, 10 \mathrm{H}), 1.00(\mathrm{~d}, J=6.1$ $\mathrm{Hz}, 3 \mathrm{H}), 0.96(\mathrm{~d}, J=6.1 \mathrm{~Hz}, 3 \mathrm{H}), 0.92(\mathrm{~d}, J=6.2 \mathrm{~Hz}, 3 \mathrm{H}), 0.86(\mathrm{~d}, J=6.2 \mathrm{~Hz}, 3 \mathrm{H}) .{ }^{13} \mathrm{C} \mathrm{NMR}(126 \mathrm{MHz}, \mathrm{CDCl}) \delta 158.5$, 156.5 , $142.6,142.4,135.4,132.1(\mathrm{~d}, J=2.6 \mathrm{~Hz}), 132.0(\mathrm{~d}, J=6.5 \mathrm{~Hz}), 130.7,127.7,127.0,126.2(\mathrm{~d}, J=15.7 \mathrm{~Hz})$, 118.3 , 116.8 , $74.9,74.3,61.7(\mathrm{~d}, J=5.7 \mathrm{~Hz}), 33.9(\mathrm{dd}, J=32.6,14.4 \mathrm{~Hz}), 31.8(\mathrm{~d}, J=19.4 \mathrm{~Hz}), 31.0(\mathrm{~d}, J=16.1 \mathrm{~Hz})$, 29.7 , $29.3(\mathrm{~d}, J=12.2 \mathrm{~Hz}), 28.6(\mathrm{~d}, J=8.5 \mathrm{~Hz}), 27.9-26.8(\mathrm{~m}), 26.3(\mathrm{~d}, J=7.8 \mathrm{~Hz}), 22.48(\mathrm{dd}, J=19.9,10.3 \mathrm{~Hz}), 22.2$, $21.5,17.0,16.4$ (d, $J=7.2 \mathrm{~Hz}) .{ }^{31} \mathrm{P}$ NMR $\left(202 \mathrm{MHz}, \mathrm{CDCl}_{3}\right) \delta 20.58,-8.87 . \mathrm{HRMS}$ (m/z, ESI): Calcd. for Chemical Formula: $\mathrm{C}_{33} \mathrm{H}_{51} \mathrm{O}_{5} \mathrm{P}_{2}^{+}[\mathrm{M}+\mathrm{H}]^{+}:$589.3212, Found: 589.3215 .

2. (S)-L5 was prepared starting from SPhos following an analogues route.

\section{Diethyl (S)-(2'-(dicyclohexylphosphanyl)-2,6-dimethoxy-5-methyl-[1,1'-biphenyl]-3-yl)phosphonate}

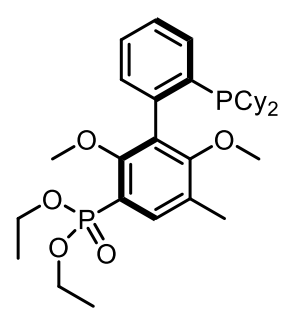

(S)-SI-L3

Prepared from SPhos following an analogous synthetic route. White solid, $[\alpha]_{D}^{20}=+24.00$ (c $0.010 \mathrm{CHCl}_{3}$ ) for 98.5:1.5 er. ${ }^{1} \mathrm{H}$ NMR $\left(500 \mathrm{MHz}, \mathrm{CDCl}_{3}\right) \delta 7.67(\mathrm{~d}, J=14.4 \mathrm{~Hz}, 1 \mathrm{H}), 7.65-7.59$ (m, 1H), $7.39(\mathrm{dd}, J=5.4,3.4 \mathrm{~Hz}, 2 \mathrm{H}), 7.36-7.31(\mathrm{~m}, 1 \mathrm{H}), 4.23-4.09(\mathrm{~m}, 4 \mathrm{H}), 3.45(\mathrm{~s}, 3 \mathrm{H}), 3.33$ $(\mathrm{s}, 3 \mathrm{H}), 2.30(\mathrm{~s}, 3 \mathrm{H}), 1.93-1.81(\mathrm{~m}, 2 \mathrm{H}), 1.79-1.60(\mathrm{~m}, 8 \mathrm{H}), 1.55-1.53(\mathrm{~m}, 2 \mathrm{H}), 1.40-1.32(\mathrm{~m}$, 6H), $1.29-1.00(\mathrm{~m}, 10 \mathrm{H}) .{ }^{13} \mathrm{C} \mathrm{NMR}\left(126 \mathrm{MHz}, \mathrm{CDCl}_{3}\right) \delta 160.9,159.1,141.5(\mathrm{~d}, \mathrm{~J}=32.1 \mathrm{~Hz})$, $137.5(\mathrm{~d}, J=19.9 \mathrm{~Hz}), 136.0(\mathrm{~d}, J=7.8 \mathrm{~Hz}), 132.7,131.5(\mathrm{~d}, J=5.9 \mathrm{~Hz}), 127.7,126.9,126.3(\mathrm{~d}, J=15.4 \mathrm{~Hz}), 117.4$, 115.9, $62.1(\mathrm{~d}, J=5.5 \mathrm{~Hz}), 61.9(\mathrm{~d}, J=4.5 \mathrm{~Hz}), 61.0,60.0,35.3(\mathrm{~d}, J=14.8 \mathrm{~Hz}), 34.4(\mathrm{~d}, J=14.3 \mathrm{~Hz}), 30.6-29.6(\mathrm{~m})$, $28.0-27.1(\mathrm{~m}), 26.5(\mathrm{~d}, J=11.9 \mathrm{~Hz}), 16.5(\mathrm{dd}, J=14.9,8.7 \mathrm{~Hz}) .{ }^{31} \mathrm{P} \mathrm{NMR}\left(202 \mathrm{MHz}, \mathrm{CDCl}_{3}\right) \delta 17.85,-8.62 . \mathrm{HRMS}(\mathrm{m} / \mathrm{z}$ ESI): Calcd. for Chemical Formula: $\mathrm{C}_{31} \mathrm{H}_{47} \mathrm{O}_{5} \mathrm{P}_{2}{ }^{+}[\mathrm{M}+\mathrm{H}]^{+}:$561.2899, Found: 561.2885.

HPLC analysis of this compound: Daicel Chiralpak IH, hexane/iso-propanol $=90: 10,1.0 \mathrm{~mL} / \mathrm{min}, \lambda=272 \mathrm{~nm}$, retention time: $12.27 \mathrm{~min}$ (major) and $11.89 \mathrm{~min}$ (minor). 


\section{Ethyl hydrogen ((S)-2'-(dicyclohexylphosphanyl)-2,6-dimethoxy-5-methyl-[1,1'-biphenyl]-3-yl)phosphonate}

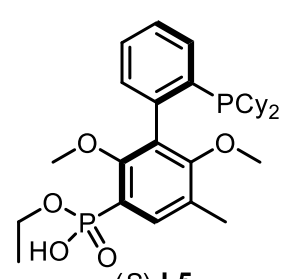

(S)-L5

Prepared from (S)-SI-L3 in 64\% yield. White solid, $[\alpha]_{D}{ }^{20}=+46.00\left(c 0.020 \mathrm{CHCl}_{3}\right) .{ }^{1} \mathrm{H} \mathrm{NMR}$ $\left(500 \mathrm{MHz}, \mathrm{CDCl}_{3}\right) \delta 7.71(\mathrm{~d}, J=14.8 \mathrm{~Hz}, 1 \mathrm{H}), 7.67-7.61(\mathrm{~m}, 1 \mathrm{H}), 7.40(\mathrm{dd}, J=9.2,4.2 \mathrm{~Hz}, 2 \mathrm{H})$, $7.39-7.35(\mathrm{~m}, 1 \mathrm{H}), 5.26(\mathrm{~s}, 1 \mathrm{H}), 4.18-4.10(\mathrm{~m}, 2 \mathrm{H}), 3.43(\mathrm{~s}, 3 \mathrm{H}), 3.35(\mathrm{~s}, 3 \mathrm{H}), 2.29(\mathrm{~s}, 3 \mathrm{H}), 1.88$ $-1.60(\mathrm{~m}, 10 \mathrm{H}), 1.58-1.56(\mathrm{~m}, 2 \mathrm{H}), 1.35(\mathrm{t}, J=7.1 \mathrm{~Hz}, 3 \mathrm{H}), 1.27-1.08(\mathrm{~m}, 10 \mathrm{H}) .{ }^{13} \mathrm{C}$ NMR $(126$

$\left.\mathrm{MHz}, \mathrm{CDCl}_{3}\right) \delta 160.7,159.0,141.5,141.3,135.7(\mathrm{~d}, J=8.0 \mathrm{~Hz}), 132.6,131.7(\mathrm{~d}, J=6.1 \mathrm{~Hz}), 130.6,127.9,127.0,126.3$ (d, $J=15.6 \mathrm{~Hz}), 118.5,116.9,62.0(\mathrm{~d}, J=4.4 \mathrm{~Hz}), 61.1,59.9,35.0(\mathrm{~d}, J=12.5 \mathrm{~Hz}), 34.4(\mathrm{~d}, J=11.9 \mathrm{~Hz}), 30.3(\mathrm{t}, J=12.6$ $\mathrm{Hz}$ ), $29.69(\mathrm{t}, J=12.8 \mathrm{~Hz}), 27.90-27.08(\mathrm{~m}), 26.4(\mathrm{~d}, J=3.6 \mathrm{~Hz}), 16.4(\mathrm{~d}, J=7.8 \mathrm{~Hz}), 16.3 .{ }^{31} \mathrm{P} \mathrm{NMR}\left(202 \mathrm{MHz}, \mathrm{CDCl}_{3}\right)$ $\delta$ 19.56, -7.71. HRMS (m/z, ESI): Calcd. for Chemical Formula: $\mathrm{C}_{29} \mathrm{H}_{43} \mathrm{O}_{5} \mathrm{P}_{2}{ }^{+}[\mathrm{M}+\mathrm{H}]^{+}: 533.2586$, Found: 533.2588 . 


\section{Preparation of substrates}

1. Unless otherwise noted, the general procedure for synthesis of fluorene substrates was described below. ${ }^{[2]}$

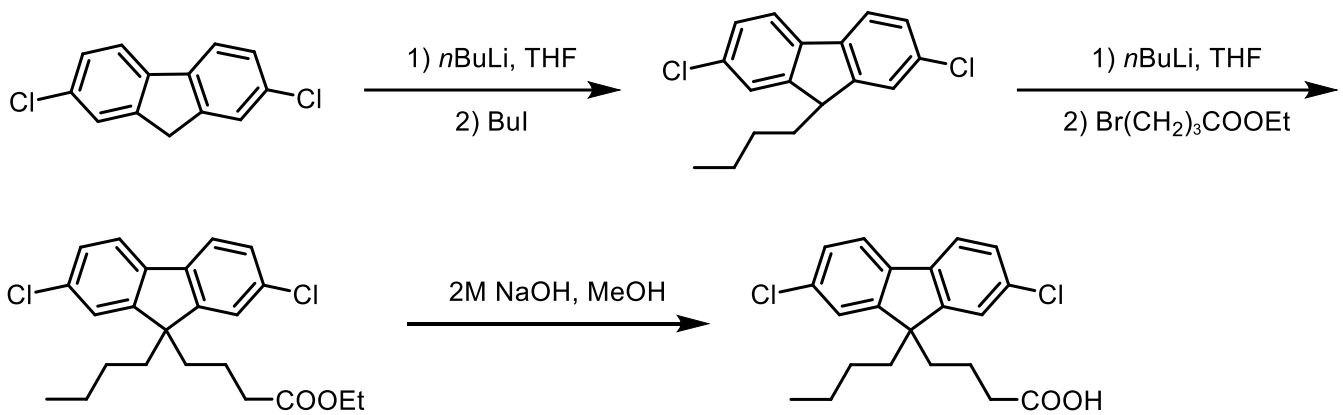

\section{9-butyl-2,7-dichloro-9H-fluorene}

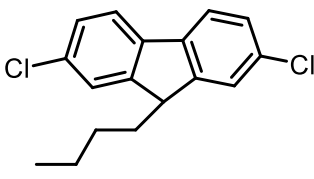

Under $\mathrm{N}_{2}$ atmosphere, 2,7-dichlorofluorene $(6.5 \mathrm{~g}, 27.6 \mathrm{mmol})$ was dissolved in anhydrous $30 \mathrm{~mL}$ THF, then $n \mathrm{BuLi}(14.5 \mathrm{~mL}, 2.0 \mathrm{~mol} / \mathrm{L}, 29.0 \mathrm{mmol})$ as a cyclohexane solution was added dropwise at $-78^{\circ} \mathrm{C}$. After addition was completed, the resulting reaction mixture was stirred for $40 \mathrm{~min}$ at room temperature. 1 -iodobutane $(6.1 \mathrm{~g}, 33.1 \mathrm{mmol})$ was added in one portion at $-78{ }^{\circ} \mathrm{C}$ and stirring at this temperature for $20 \mathrm{~min}$. Then the reaction mixture was warmed to room temperature and stirring for $15 \mathrm{~h}$. The reaction was quenched with saturated $\mathrm{NH}_{4} \mathrm{Cl}$ solution and extracted with ethyl acetate $(20 \times 3 \mathrm{~mL})$ three times. The combined organic phases were washed with brine, dried over $\mathrm{Na}_{2} \mathrm{SO}_{4}$, and concentrated in vacuo. The crude materials were purified by flash column chromatography to give the desired product $7.41 \mathrm{~g}$ in $92 \%$ yield as a white solid.

\section{ethyl 4-(9-butyl-2,7-dichloro-9H-fluoren-9-yl)butanoate (53)}

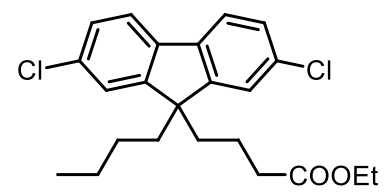

Under $\mathrm{N}_{2}$ atmosphere, 9-butyl-2,7-dichloro-9H-fluorene (2.6 g, $\left.8.9 \mathrm{mmol}\right)$ was dissolved in anhydrous $20 \mathrm{~mL}$ THF and $n$ BuLi $(4.9 \mathrm{~mL}, 2.0 \mathrm{~mol} / \mathrm{L}, 9.8 \mathrm{mmol}$ ) as a cyclohexane solution was added dropwise at $-78^{\circ} \mathrm{C}$. After addition was completed, the reaction mixture was stirring for $30 \mathrm{~min}$ at room temperature and ethyl 4-bromobutanoate (2.09 $\mathrm{g}, 10.7 \mathrm{mmol})$ was 
added in one portion. The resulting reaction mixture was warmed to room temperature and stirred overnight. The reaction was quenched with saturated $\mathrm{NH}_{4} \mathrm{Cl}$ solution and extracted with ethyl acetate $(15 \mathrm{~mL} \times 3)$ three times. The combined organic phases were washed with brine, dried over $\mathrm{Na}_{2} \mathrm{SO}_{4}$, and concentrated in vacuo. The crude materials were purified by flash column chromatography to give the desired product $3.13 \mathrm{~g}$ in $86 \%$ yield as a white solid.

${ }^{1} \mathrm{H}$ NMR $\left(500 \mathrm{MHz}, \mathrm{CDCl}_{3}\right) \delta 7.59(\mathrm{~d}, J=8.0 \mathrm{~Hz}, 2 \mathrm{H}), 7.33(\mathrm{dd}, J=8.0,1.9 \mathrm{~Hz}, 2 \mathrm{H}), 7.31(\mathrm{~d}, J=1.7 \mathrm{~Hz}, 2 \mathrm{H}), 4.06(\mathrm{q}$, $J=7.1 \mathrm{~Hz}, 2 \mathrm{H}), 2.08(\mathrm{t}, J=7.4 \mathrm{~Hz}, 2 \mathrm{H}), 2.03-1.98(\mathrm{~m}, 2 \mathrm{H}), 1.97-1.92(\mathrm{~m}, 2 \mathrm{H}), 1.22(\mathrm{t}, J=7.1 \mathrm{~Hz}, 3 \mathrm{H}), 1.13-1.09(\mathrm{~m}$, $2 \mathrm{H}), 0.99-0.93(\mathrm{~m}, 2 \mathrm{H}), 0.71(\mathrm{t}, J=7.4 \mathrm{~Hz}, 3 \mathrm{H}), 0.62-0.56(\mathrm{~m}, 2 \mathrm{H}) .{ }^{13} \mathrm{C} \mathrm{NMR}\left(126 \mathrm{MHz}, \mathrm{CDCl}_{3}\right) \delta 173.2,151.7,138.6$, 133.3, 127.6, 123.3, 120.9, 60.3, 55.3, 40.1, 39.38, 34.3, 25.7, 22.9, 19.3, 14.2, 13.8. MS (m/z, ESI): Calcd. for Chemical Formula: $\mathrm{C}_{23} \mathrm{H}_{25} \mathrm{Cl}_{2} \mathrm{O}_{2}^{-}[\mathrm{M}+\mathrm{Na}]^{-}: 427.1202$, Found: 427.1201.

\section{4-(9-butyl-2,7-dichloro-9H-fluoren-9-yl)butanoic acid (51)}

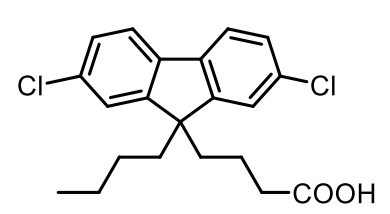

Ethyl 4-(9-butyl-2,7-dichloro-9H-fluoren-9-yl)butanoate (1.5 g, $3.7 \mathrm{mmol})$ was dissolved in $5.0 \mathrm{~mL}$ THF and $5.0 \mathrm{~mL} \mathrm{MeOH}$, and $8 \mathrm{M} \mathrm{NaOH}(0.6 \mathrm{~g}, 14.8 \mathrm{mmol}, 1.8 \mathrm{~mL})$ was added. The reaction mixtures were stirred at room temperature for $2 \mathrm{~h}$ and solvents were removed under reduced pressure. Water was added and the aqueous solution was acidified to $\mathrm{pH} 2-3 \mathrm{with} 1 \mathrm{M}$ $\mathrm{HCl}$ solution. The reaction was extracted with ethyl acetate $(10 \mathrm{~mL} \times 3)$ three times. The combined organic phases were washed with brine, dried over $\mathrm{Na}_{2} \mathrm{SO}_{4}$, and concentrated in vacuo. The crude materials were purified by flash column chromatography to give the desired product. The product was recrystallized in ethyl acetate and hexane to give the desired products $\mathbf{5 1} 0.97 \mathrm{~g}$ in $69 \%$ yield as a white solid.

${ }^{1} \mathrm{H}$ NMR $\left(500 \mathrm{MHz}, \mathrm{CDCl}_{3}\right) \delta 7.59(\mathrm{~d}, J=8.0 \mathrm{~Hz}, 2 \mathrm{H}), 7.33(\mathrm{dd}, J=8.0,1.9 \mathrm{~Hz}, 2 \mathrm{H}), 7.31(\mathrm{~d}, J=1.6 \mathrm{~Hz}, 2 \mathrm{H}), 2.14-$ $2.11(\mathrm{~m}, 2 \mathrm{H}), 2.04-1.99(\mathrm{~m}, 2 \mathrm{H}), 1.97-1.92(\mathrm{~m}, 2 \mathrm{H}), 1.14-1.10(\mathrm{~m}, 2 \mathrm{H}), 0.96-0.92(\mathrm{~m}, 2 \mathrm{H}), 0.71(\mathrm{t}, J=7.4 \mathrm{~Hz}, 3 \mathrm{H})$, $0.62-0.55$ (m, 2H). ${ }^{13} \mathrm{C}$ NMR $\left(126 \mathrm{MHz} \mathrm{CDCl}_{3}\right) \delta 178.4,151.6,138.6,133.4,127.6,123.3,120.9,55.3,40.0,39.3$, 33.8, 25.7, 22.9, 19.1, 13.8. MS (m/z, ESI): Calcd. for Chemical Formula: $\mathrm{C}_{21} \mathrm{H}_{21} \mathrm{Cl}_{2} \mathrm{O}_{2}-[\mathrm{M}-\mathrm{H}]^{-}: 375.0924$, Found: 375.0930 . 


\section{2-(9-butyl-2,7-dichloro-9H-fluoren-9-yl)acetic acid}

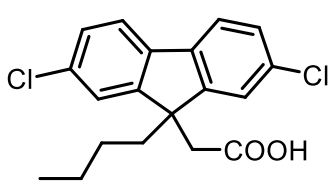

Prepared from 9-butyl-2,7-dichloro-9H-fluorene in two steps, 68\% yield, white solid.

${ }^{1} \mathrm{H}$ NMR $\left(500 \mathrm{MHz}, \mathrm{CDCl}_{3}\right) \delta 7.61-7.56(\mathrm{~m}, 2 \mathrm{H}), 7.38(\mathrm{~d}, J=1.6 \mathrm{~Hz}, 2 \mathrm{H}), 7.35(\mathrm{dd}, J=8.1$, $1.9 \mathrm{~Hz}, 2 \mathrm{H}), 2.90(\mathrm{~s}, 2 \mathrm{H}), 2.07-2.01(\mathrm{~m}, 2 \mathrm{H}), 1.11(\mathrm{dt}, J=14.7,7.4 \mathrm{~Hz}, 2 \mathrm{H}), 0.71(\mathrm{t}, J=7.4$ $\mathrm{Hz}, 3 \mathrm{H}), 0.61-0.54(\mathrm{~m}, 2 \mathrm{H}) .{ }^{13} \mathrm{C} \mathrm{NMR}\left(126 \mathrm{MHz}, \mathrm{CDCl}_{3}\right) \delta 174.5,150.5,138.3,133.3,128.0,123.6,120.9,52.4,43.1$, 38.8, 25.6, 22.7, 13.7. MS (m/z, ESI): Calcd. for Chemical Formula: $\mathrm{C}_{19} \mathrm{H}_{17} \mathrm{Cl}_{2} \mathrm{O}_{2}^{-}[\mathrm{M}-\mathrm{H}]:$ : 347.0611, Found:347.0613.

\section{3-(9-butyl-2,7-dichloro-9H-fluoren-9-yl)propanoic acid}

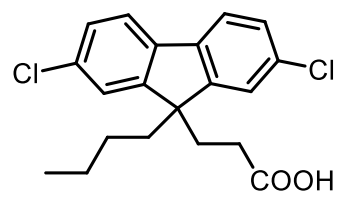

Prepared from 9-butyl-2,7-dichloro-9H-fluorene in two steps, $76 \%$ yield, white solid. ${ }^{1} \mathrm{H}$ $\operatorname{NMR}\left(300 \mathrm{MHz}, \mathrm{CDCl}_{3}\right) \delta 7.62(\mathrm{~d}, J=8.0 \mathrm{~Hz}, 2 \mathrm{H}), 7.42-7.31(\mathrm{~m}, 4 \mathrm{H}), 2.42-2.27(\mathrm{~m}, 2 \mathrm{H})$, $2.05-1.94(\mathrm{~m}, 2 \mathrm{H}), 1.68-1.53(\mathrm{~m}, 2 \mathrm{H}), 1.16-1.12(\mathrm{~m}, 2 \mathrm{H}), 0.73(\mathrm{t}, J=7.4 \mathrm{~Hz}, 3 \mathrm{H}), 0.68-$ $0.48(\mathrm{~m}, 2 \mathrm{H}) .{ }^{13} \mathrm{C} \mathrm{NMR}\left(75 \mathrm{MHz}, \mathrm{CDCl}_{3}\right) \delta 179.2,150.5,138.6,133.5,127.9,123.3,120.9,54.6,39.8,34.2,28.6,25.7$, 22.8, 13.7. MS (m/z, ESI): Calcd. for Chemical Formula: $\mathrm{C}_{20} \mathrm{H}_{19} \mathrm{Cl}_{2} \mathrm{O}_{2}{ }^{-}[\mathrm{M}-\mathrm{H}]: 361.0768$, Found: 361.0761.

\section{(E)-4-(9-butyl-2,7-dichloro-9H-fluoren-9-yl)but-2-enoic acid}

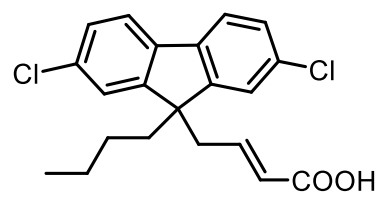

Prepared from 9-butyl-2,7-dichloro-9H-fluorene in two steps, 46\% yield, white solid. ${ }^{1} \mathrm{H}$ NMR $\left(500 \mathrm{MHz}, \mathrm{CDCl}_{3}\right) \delta 7.59(\mathrm{~d}, J=8.0 \mathrm{~Hz}, 2 \mathrm{H}), 7.34(\mathrm{dd}, J=8.1,1.9 \mathrm{~Hz}, 2 \mathrm{H}), 7.32$ $(\mathrm{d}, J=1.6 \mathrm{~Hz}, 2 \mathrm{H}), 6.32(\mathrm{dt}, J=15.3,7.6 \mathrm{~Hz}, 1 \mathrm{H}), 5.59(\mathrm{~d}, J=15.5 \mathrm{~Hz}, 1 \mathrm{H}), 2.83-2.79(\mathrm{~m}$, 2H), $2.03-1.96(\mathrm{~m}, 2 \mathrm{H}), 1.15-1.11(\mathrm{~m}, 2 \mathrm{H}), 0.72(\mathrm{t}, J=7.4 \mathrm{~Hz}, 3 \mathrm{H}), 0.64-0.56(\mathrm{~m}, 2 \mathrm{H}) .{ }^{13} \mathrm{C} \mathrm{NMR}\left(126 \mathrm{MHz}, \mathrm{CDCl}_{3}\right) \delta$ 170.2, 150.5, 146.0, 138.3, 133.5, 128.0, 123.4, 121.1, 54.8, 42.7, 39.1, 25.7, 22.8, 13.7. MS (m/z, ESI): Calcd. for Chemical Formula: $\mathrm{C}_{21} \mathrm{H}_{19} \mathrm{Cl}_{2} \mathrm{O}_{2}{ }_{2}^{-}[\mathrm{M}-\mathrm{H}]^{-}: 373.0768$, Found: 373.0766 .

\section{5-(9-butyl-2,7-dichloro-9H-fluoren-9-yl)pentanoic acid}

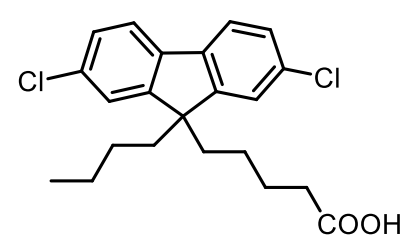

Prepared from 9-butyl-2,7-dichloro-9H-fluorene in two steps, 64\% yield, white solid. ${ }^{1} \mathrm{H}$ NMR $\left(500 \mathrm{MHz}, \mathrm{CDCl}_{3}\right) \delta 7.59(\mathrm{~d}, J=8.0 \mathrm{~Hz}, 2 \mathrm{H}), 7.35-7.29(\mathrm{~m}, 4 \mathrm{H}), 2.18-2.09$ $(\mathrm{m}, 2 \mathrm{H}), 1.99-1.93(\mathrm{~m}, 4 \mathrm{H}), 1.48-1.39(\mathrm{~m}, 2 \mathrm{H}), 1.13-1.09(\mathrm{~m}, 2 \mathrm{H}), 0.71(\mathrm{t}, J=7.4 \mathrm{~Hz}$

3H), $0.68-0.62(\mathrm{~m}, 2 \mathrm{H}), 0.62-0.55(\mathrm{~m}, 2 \mathrm{H}) .{ }^{13} \mathrm{C} \mathrm{NMR}\left(126 \mathrm{MHz}, \mathrm{CDCl}_{3}\right) \delta 179.5,151.9,138.6,133.3,127.5,123.2$, 


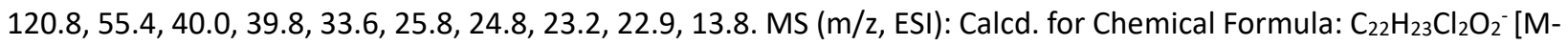
$\mathrm{H}^{-}:$389.1081, Found: 389.1076.

\section{6-(9-butyl-2,7-dichloro-9H-fluoren-9-yl)hexanoic acid}

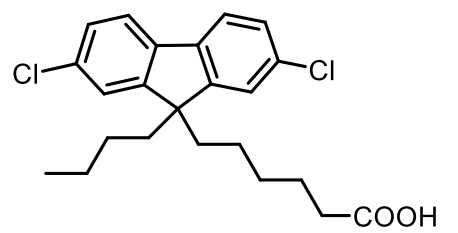

Prepared from 9-butyl-2,7-dichloro-9H-fluorene in two steps, 56\% yield, white solid. ${ }^{1} \mathrm{H}$ NMR $\left(300 \mathrm{MHz}, \mathrm{CDCl}_{3}\right) \delta 7.61(\mathrm{~d}, J=8.0 \mathrm{~Hz}, 2 \mathrm{H}), 7.36-7.30(\mathrm{~m}, 4 \mathrm{H}), 2.20$ $(\mathrm{t}, J=7.4 \mathrm{~Hz}, 2 \mathrm{H}), 2.00-1.94(\mathrm{~m}, 4 \mathrm{H}), 1.51-1.37(\mathrm{~m}, 2 \mathrm{H}), 1.23-1.08(\mathrm{~m}, 4 \mathrm{H}), 0.73$ $(\mathrm{t}, J=7.3 \mathrm{~Hz}, 3 \mathrm{H}), 0.65-0.60(\mathrm{~m}, 4 \mathrm{H}) .{ }^{13} \mathrm{C} \mathrm{NMR}\left(75 \mathrm{MHz}, \mathrm{CDCl}_{3}\right) \delta 179.5,152.0,138.5,133.2,127.3,123.1,120.7,55.4$, 39.9, 33.7, 29.2, 25.7, 24.2, 23.3, 22.9, 13.7. MS (m/z, ESI): Calcd. for Chemical Formula: $\mathrm{C}_{23} \mathrm{H}_{25} \mathrm{Cl}_{2} \mathrm{O}_{2}^{-}[\mathrm{M}-\mathrm{H}]^{-}: 403.1237$, Found: 403.1242 .

\section{7-(9-butyl-2,7-dichloro-9H-fluoren-9-yl)heptanoic acid}

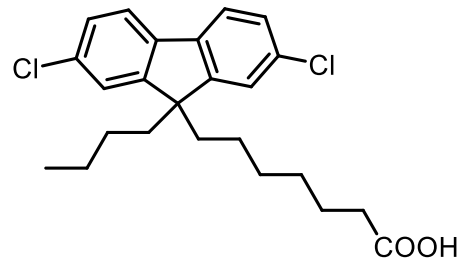

Prepared from 9-butyl-2,7-dichloro-9H-fluorene in two steps, 61\% yield, white solid. ${ }^{1} \mathrm{H}$ NMR $\left(500 \mathrm{MHz}, \mathrm{CDCl}_{3}\right) \delta 7.59(\mathrm{~d}, J=8.0 \mathrm{~Hz}, 2 \mathrm{H}), 7.33(\mathrm{dd}, J=8.0,1.9 \mathrm{~Hz}$, $2 \mathrm{H}), 7.30(\mathrm{~d}, J=1.6 \mathrm{~Hz}, 2 \mathrm{H}), 2.25(\mathrm{t}, J=7.5 \mathrm{~Hz}, 2 \mathrm{H}), 1.97-1.91(\mathrm{~m}, 4 \mathrm{H}), 1.50-$

$1.43(\mathrm{~m}, 2 \mathrm{H}), 1.15-1.07(\mathrm{~m}, 6 \mathrm{H}), 0.71(\mathrm{t}, J=7.4 \mathrm{~Hz}, 3 \mathrm{H}), 0.64-0.56(\mathrm{~m}, 4 \mathrm{H}) .{ }^{13} \mathrm{C}$

NMR $\left(126 \mathrm{MHz}_{\mathrm{CDCl}}\right) \delta 179.4,152.2,138.6,133.2,127.4,123.2,120.8,55.5,40.2,40.0,29.5,28.7,25.8,24.5,23.5$ 22.9, 13.8. $\mathrm{MS}$ (m/z, ESI): Calcd. for Chemical Formula: $\mathrm{C}_{24} \mathrm{H}_{27} \mathrm{Cl}_{2} \mathrm{O}_{2}[\mathrm{M}-\mathrm{H}]$ : 417.1394, Found: 417.1395.

\section{8-(9-butyl-2,7-dichloro-9H-fluoren-9-yl)octanoic acid}

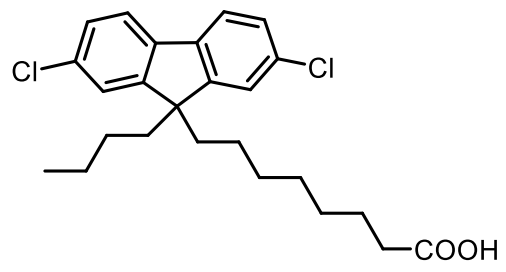

Prepared from 9-butyl-2,7-dichloro-9H-fluorene in two steps, 66\% yield, light green oil. ${ }^{1} \mathrm{H}$ NMR $\left(500 \mathrm{MHz}, \mathrm{CDCl}_{3}\right) \delta 7.62-7.54(\mathrm{~m}, 2 \mathrm{H}), 7.33$ (dd, $J=8.0$, $1.9 \mathrm{~Hz}, 2 \mathrm{H}), 7.30(\mathrm{~d}, J=1.5 \mathrm{~Hz}, 2 \mathrm{H}), 2.28(\mathrm{t}, J=7.5 \mathrm{~Hz}, 2 \mathrm{H}), 1.97-1.90(\mathrm{~m}, 4 \mathrm{H})$, $1.56-1.51(\mathrm{~m}, 2 \mathrm{H}), 1.21-1.15(\mathrm{~m}, 2 \mathrm{H}), 1.13-1.05(\mathrm{~m}, 6 \mathrm{H}), 0.71(\mathrm{t}, J=7.4$

$\mathrm{Hz}, 3 \mathrm{H}), 0.62-0.58(\mathrm{~m}, 4 \mathrm{H}) .{ }^{13} \mathrm{C}$ NMR $\left(126 \mathrm{MHz}, \mathrm{CDCl}_{3}\right) \delta 179.1,152.3,138.6,133.2,127.3,123.2,120.7,55.5,40.2$ 40.1, 33.9, 33.8, 32.7, 28.9, 28.81, 28.82, 28.4, 27.9, 25.8, 24.5, 23.6, 22.9, 13.8. MS (m/z, ESI): Calcd. for Chemical Formula: $\mathrm{C}_{25} \mathrm{H}_{29} \mathrm{Cl}_{2} \mathrm{O}_{2}{ }^{-}[\mathrm{M}-\mathrm{H}]^{-}: 431.1550$, Found: 431.1552 . 
4-(2,7-dichloro-9-methyl-9H-fluoren-9-yl)butanoic acid

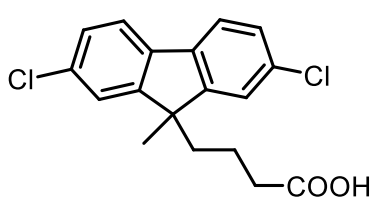

Prepared from 2,7-dichlorofluorene in three steps, $48 \%$ yield, white solid. ${ }^{1} \mathrm{H}$ NMR (500 MHz, $\left.\mathrm{CDCl}_{3}\right) \delta 7.60(\mathrm{~d}, J=7.9 \mathrm{~Hz}, 2 \mathrm{H}), 7.36-7.32(\mathrm{~m}, 4 \mathrm{H}), 2.15(\mathrm{t}, J=7.4 \mathrm{~Hz}, 2 \mathrm{H})$,

152.9, 137.7, 133.4, 127.7, 123.3, 121.1, 51.0, 39.5, 33.7, 26.6, 19.5. MS (m/z, ESI): Calcd. for Chemical Formula: $\mathrm{C}_{18} \mathrm{H}_{15} \mathrm{Cl}_{2} \mathrm{O}_{2}^{-}[\mathrm{M}-\mathrm{H}]^{-}: 333.0455$, Found: 333.0453.

\section{4-(2,7-dichloro-9-octyl-9H-fluoren-9-yl)butanoic acid}

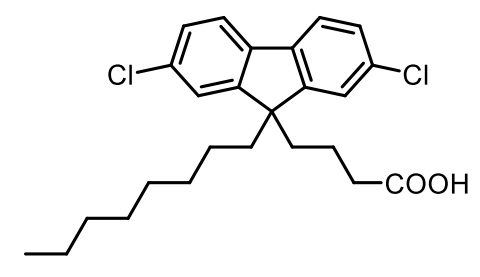

Prepared from 2,7-dichlorofluorene in three steps, 36\% yield, white solid. ${ }^{1} \mathrm{H}$ NMR $\left(500 \mathrm{MHz}, \mathrm{CDCl}_{3}\right) \delta 7.59(\mathrm{~d}, J=8.1 \mathrm{~Hz}, 2 \mathrm{H}), 7.33(\mathrm{dd}, J=8.1,1.9 \mathrm{~Hz}$, $2 \mathrm{H}), 7.30(\mathrm{~d}, J=1.6 \mathrm{~Hz}, 2 \mathrm{H}), 2.12(\mathrm{t}, J=7.4 \mathrm{~Hz}, 2 \mathrm{H}), 2.04-1.99(\mathrm{~m}, 2 \mathrm{H}), 1.96-$ $1.91(\mathrm{~m}, 2 \mathrm{H}), 1.26-1.20(\mathrm{~m}, 2 \mathrm{H}), 1.17-1.04(\mathrm{~m}, 8 \mathrm{H}), 0.96-0.92(\mathrm{~m}, 2 \mathrm{H}), 0.85(\mathrm{t}$, $J=7.2 \mathrm{~Hz}, 3 \mathrm{H}), 0.62-0.58(\mathrm{~m}, 2 \mathrm{H}) .{ }^{13} \mathrm{C} \mathrm{NMR}\left(126 \mathrm{MHz}, \mathrm{CDCl}_{3}\right) \delta 178.2,151.6,138.6,133.4,127.6,123.3,120.9,55.3$, 40.2, 39.3, 33.7, 31.8, 29.8, 29.2, 29.1 23.5, 22.6, 19.1, 14.1. MS (m/z, ESI): Calcd. for Chemical Formula: $\mathrm{C}_{25} \mathrm{H}_{29} \mathrm{Cl}_{2} \mathrm{O}_{2}{ }^{-}$ $[\mathrm{M}-\mathrm{H}]^{-}:$431.1550, Found: 431.1547.

\section{4-(9-benzyl-2,7-dichloro-9H-fluoren-9-yl)butanoic acid}

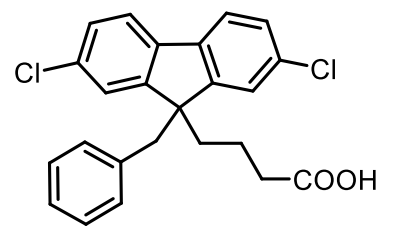

Prepared from 2,7-dichlorofluorene in three steps, $62 \%$ yield, white solid. ${ }^{1} \mathrm{H}$ NMR $\left(500 \mathrm{MHz}, \mathrm{CDCl}_{3}\right) \delta 7.47-7.41(\mathrm{~m}, 2 \mathrm{H}), 7.31-7.26(\mathrm{~m}, 4 \mathrm{H}), 7.09-6.98(\mathrm{~m}, 3 \mathrm{H})$, $6.69-6.62(\mathrm{~m}, 2 \mathrm{H}), 3.14(\mathrm{~s}, 2 \mathrm{H}), 2.22-2.17(\mathrm{~m}, 4 \mathrm{H}), 1.01-0.92(\mathrm{~m}, 2 \mathrm{H}) .{ }^{13} \mathrm{C}$ NMR $(126$

$\left.\mathrm{MHz}, \mathrm{CDCl}_{3}\right) \delta 179.0,150.4,138.5,136.0,133.0,130.2,127.8,127.4,126.3,124.1,120.9,56.1,46.7,37.7,33.8,19.1$. $\mathrm{MS}$ (m/z, ESI): Calcd. for Chemical Formula: $\mathrm{C}_{24} \mathrm{H}_{19} \mathrm{Cl}_{2} \mathrm{O}_{2}{ }^{-}[\mathrm{M}-\mathrm{H}]^{-}:$409.0768, Found: 409.0769. 


\section{4-(2,7-dichloro-9-phenyl-9H-fluoren-9-yl)butanoic acid}

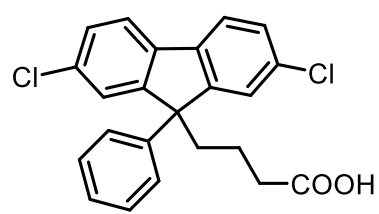

Prepared from 2,7-dichloro-9-phenyl-9H-fluorene ${ }^{[3]}$ in two steps, $42 \%$ yield, white solid. ${ }^{1} \mathrm{H}$ NMR $\left(500 \mathrm{MHz}, \mathrm{CDCl}_{3}\right) \delta 7.66-7.64(\mathrm{~m}, 2 \mathrm{H}), 7.35(\mathrm{dd}, J=8.1,1.9 \mathrm{~Hz}, 2 \mathrm{H})$, $7.28-7.21(m, 3 H), 7.18(d, J=1.6 \mathrm{~Hz}, 2 \mathrm{H}), 7.15-7.11(\mathrm{~m}, 2 \mathrm{H}), 2.63-2.45(\mathrm{~m}, 2 \mathrm{H})$, $2.26(t, J=7.3 \mathrm{~Hz}, 2 \mathrm{H}), 1.11-1.05(\mathrm{~m}, 2 \mathrm{H}) .{ }^{13} \mathrm{C} \mathrm{NMR}\left(126 \mathrm{MHz}, \mathrm{CDCl}_{3}\right) \delta$ 178.3, 152.9, 143.0, 138.2, 133.7, 128.7, 128.0, 127.0, 126.4, 124.7, 121.1, 58.7, 36.7, 33.7, 19.2. MS (m/z, ESI): Calcd. for Chemical Formula: $\mathrm{C}_{23} \mathrm{H}_{17} \mathrm{Cl}_{2} \mathrm{O}_{2}{ }^{-}[\mathrm{M}-\mathrm{H}]^{-}$: 395.0611, Found: 395.0605.

2. Substrates bearing sulfo group were prepared following the procedure shown below. ${ }^{[4]}$

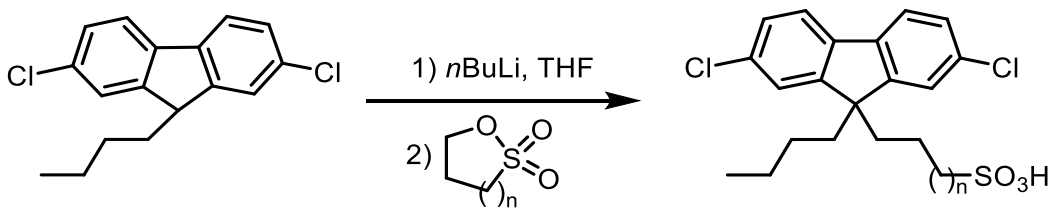

\section{3-(9-butyl-2,7-dichloro-9H-fluoren-9-yl)propane-1-sulfonic acid}

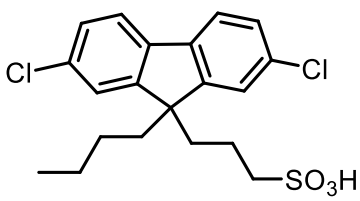

Under $\mathrm{N}_{2}$ atmosphere, 9-butyl-2,7-dichloro-9H-fluorene (0.5 g, $\left.1.7 \mathrm{mmol}\right)$ was dissolved in anhydrous $1.5 \mathrm{~mL}$ THF and $n$ BuLi (0.95 mL, $2.0 \mathrm{~mol} / \mathrm{L}, 1.9 \mathrm{mmol}$ ) as a cyclohexane solution was added dropwise at $-78^{\circ} \mathrm{C}$. After addition was completed, the reaction mixture was stirring for $30 \mathrm{~min}$ at room temperature and ethyl 1,2-oxathiolane 2,2-dioxide (0.25 g, $2.1 \mathrm{mmol})$ was added in one portion. The resulting reaction mixture was warmed to room temperature and stirred overnight. Water was added and the aqueous solution was acidified to $\mathrm{pH} 2-3$ with $1 \mathrm{M} \mathrm{HCl}$ solution and extracted with ethyl acetate $(10 \mathrm{~mL} \times 3)$ three times. The combined organic phases were washed with brine, dried over $\mathrm{Na}_{2} \mathrm{SO}_{4}$, and concentrated in vacuo. The crude materials were purified by flash column chromatography to give the desired product $0.59 \mathrm{~g}$ in $83 \%$ yield as a white solid.

${ }^{1} \mathrm{H} \mathrm{NMR}\left(500 \mathrm{MHz}, \mathrm{CDCl}_{3}\right) \delta 7.49(\mathrm{~d}, J=8.1 \mathrm{~Hz}, 2 \mathrm{H}), 7.35-7.32(\mathrm{~m}, 2 \mathrm{H}), 7.27-7.20(\mathrm{~m}, 2 \mathrm{H}), 2.50-2.48(\mathrm{~m}, 2 \mathrm{H})$, 1.96-1.94 (m, 4H), 1.13-1.07 (m, 4H), $0.72(\mathrm{t}, J=7.3 \mathrm{~Hz}, 3 \mathrm{H}), 0.57-0.53(\mathrm{~m}, 2 \mathrm{H}) .{ }^{13} \mathrm{C} \mathrm{NMR}(126 \mathrm{MHz}, \mathrm{CDCl} 3) \delta 151.7$, $138.5,133.3$, $127.6,123.4,120.9,55.2$, 51.0 , 39.7 , 38.7 , 25.7 , 23.0 , 19.1 , 13.9. MS (m/z, ESI): Calcd. for Chemical Formula: $\mathrm{C}_{20} \mathrm{H}_{21} \mathrm{Cl}_{2} \mathrm{O}_{3} \mathrm{~S}^{-}[\mathrm{M}-\mathrm{H}]^{-}:$: 411.0594, Found: 411.0599 . 


\section{4-(9-butyl-2,7-dichloro-9H-fluoren-9-yl)butane-1-sulfonic acid}

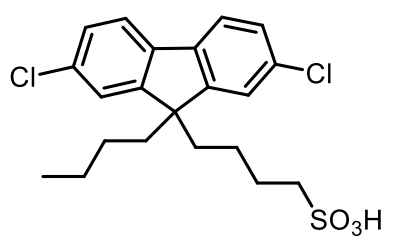

The compound was prepared using a similar procedure to 3-(9-butyl-2,7dichloro-9H-fluoren-9-yl)propane-1-sulfonic acid. $0.63 \mathrm{~g}, 86 \%$ yield, white solid. ${ }^{1} \mathrm{H}$ $\operatorname{NMR}\left(500 \mathrm{MHz}, \mathrm{CDCl}_{3}\right) \delta 7.53(\mathrm{~d}, J=8.0 \mathrm{~Hz}, 2 \mathrm{H}), 7.36-7.24(\mathrm{~m}, 4 \mathrm{H}), 2.52-2.51(\mathrm{~m}, 2 \mathrm{H})$,

1.91-1.87 (m, 4H), 1.43-1.41 (m, 2H), 1.12-1.08 (m, 2H), 0.70 (t, J= 7.3 Hz, 3H), 0.59-0.56 (m, 4H). ${ }^{13} \mathrm{C} \mathrm{NMR}(126 \mathrm{MHz}$, $\left.\mathrm{CDCl}_{3}\right) \delta 151.9,138.6,133.3,127.5,123.3,120.8,55.4,40.1,39.4,25.8,24.2,23.0,22.6,13.9 . \mathrm{MS}$ (m/z, ESI): Calcd. for Chemical Formula: $\mathrm{C}_{21} \mathrm{H}_{23} \mathrm{Cl}_{2} \mathrm{O}_{3} \mathrm{~S}^{-}[\mathrm{M}-\mathrm{H}]^{-}: 425.0750$, Found: 425.0754 .

3. Substrate bearing triflimide was prepared following the synthetic route shown below.
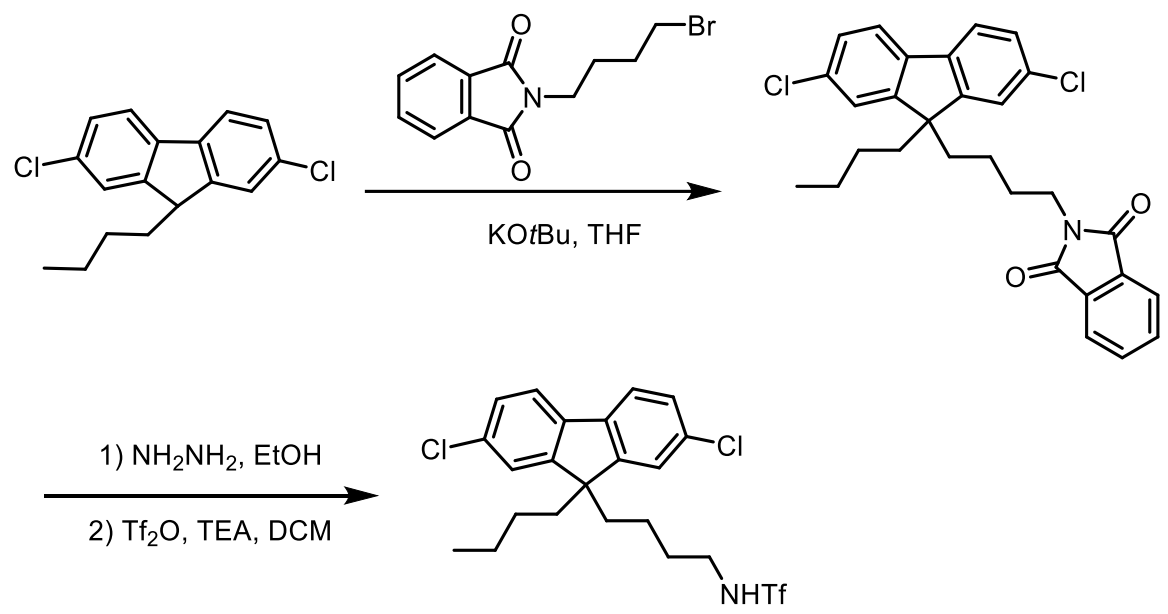

Under $\mathrm{N}_{2}$ atmosphere, 9-butyl-2,7-dichloro-9H-fluorene $(1.0 \mathrm{~g}, 3.43 \mathrm{mmol})$ was dissolved in anhydrous $6 \mathrm{~mL}$ $\operatorname{THF}$ and $t \mathrm{BuOK}(0.36 \mathrm{~g}, 3.8 \mathrm{mmol})$ was added dropwise at $0{ }^{\circ} \mathrm{C}$. After addition was completed, the reaction mixture was stirring for $30 \mathrm{~min}$ at room temperature and 2-(4-bromobutyl)isoindoline-1,3-dione (1.07 g, $3.8 \mathrm{mmol})$ was added in one portion at $0{ }^{\circ} \mathrm{C} .{ }^{[5]}$ The resulting reaction mixture was warmed to room temperature and stirred overnight. The reaction was quenched with saturated $\mathrm{NH}_{4} \mathrm{Cl}$ solution and extracted with ethyl acetate (15 $\left.\mathrm{mL} X 3\right)$ three times. The combined organic phases were washed with brine, dried over $\mathrm{Na}_{2} \mathrm{SO}_{4}$, and concentrated in vacuo. The crude materials were purified by flash column chromatography to give the desired product $1.33 \mathrm{~g}$ in $79 \%$ yield as a white solid. 
The compound prepared from the previous step $(1.33 \mathrm{~g}, 2.7 \mathrm{mmol})$ was dissolved in $8 \mathrm{~mL} E t O H$ and $\mathrm{NH}_{2} \mathrm{NH}_{2}-$ $\mathrm{H}_{2} \mathrm{O}(0.81 \mathrm{~g}, 16.2 \mathrm{mmol})$ was added. The resulting reaction mixture was heated at $70{ }^{\circ} \mathrm{C}$ for $8 \mathrm{~h}$. Then solid was removed and the organic phase was purified by flash column chromatography to give the desired product $0.61 \mathrm{~g}$ in $62 \%$ yield.

At $0{ }^{\circ} \mathrm{C}$, to a mixture of primary amine prepared in the previous step $(0.2 \mathrm{~g}, 0.55 \mathrm{mmol})$ and triethylamine $(0.17$ $\mathrm{g}, 1.66 \mathrm{mmol})$ in anhydrous $1.5 \mathrm{~mL} \mathrm{DCM} . \mathrm{Tf}_{2} \mathrm{O}(0.234 \mathrm{~g}, 0.83 \mathrm{mmol})$ was added dropwise to the reaction mixture. Water was added to quench the reaction. The reaction was extracted with DCM (5 mL X 3) for three times. The combined organic phases were washed with brine, dried over $\mathrm{Na}_{2} \mathrm{SO}_{4}$, and concentrated in vacuo. The crude materials were purified by flash column chromatography to give the desired product. The product was recrystallized in ethyl acetate and hexane to give the desired products $0.170 \mathrm{~g}$ in $62 \%$ yield as a white solid.

\section{N-(4-(9-butyl-2,7-dichloro-9H-fluoren-9-yl)butyl)-1,1,1-trifluoromethanesulfonamide}

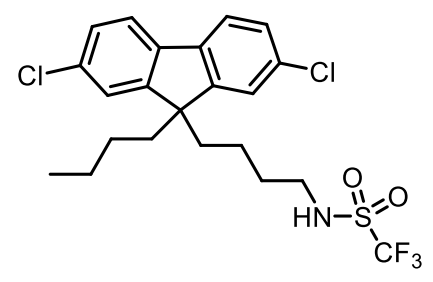

Prepared from 9-butyl-2,7-dichloro-9H-fluorene in four steps, 34\% yield, white solid. ${ }^{1} \mathrm{H}$ NMR $\left(300 \mathrm{MHz}, \mathrm{CDCl}_{3}\right) \delta 7.63(\mathrm{dd}, J=7.9,2.6 \mathrm{~Hz}, 2 \mathrm{H}), 7.39-7.33(\mathrm{~m}, 4 \mathrm{H})$, $4.92(\mathrm{~s}, 1 \mathrm{H}), 3.10-3.06(\mathrm{~m}, 2 \mathrm{H}), 2.10-1.88(\mathrm{~m}, 4 \mathrm{H}), 1.42-1.40(\mathrm{~m}, 2 \mathrm{H}), 1.16-1.14(\mathrm{~m}$, $2 \mathrm{H}), 0.80-0.49(\mathrm{~m}, 7 \mathrm{H}) .{ }^{13} \mathrm{C} \mathrm{NMR}\left(75 \mathrm{MHz}, \mathrm{CDCl}_{3}\right) \delta 151.6,138.6,133.4,127.6,123.1$,

120.9, $119.6(q, J=321.3 \mathrm{~Hz}), 55.3,44.0,39.9,39.5,30.2,25.7,22.8,20.4,13.7 .{ }^{19} \mathrm{~F} \mathrm{NMR}\left(471 \mathrm{MHz}, \mathrm{CDCl}_{3}\right)-77.39$ ppm. MS (m/z, ESI): Calcd. for Chemical Formula: $\mathrm{C}_{22} \mathrm{H}_{23} \mathrm{Cl}_{2} \mathrm{~F}_{3} \mathrm{O}_{2} \mathrm{~S}^{-}[\mathrm{M}-\mathrm{H}]^{-}:$: 492.0784, Found: 492.0784.

4. Preparation of substrate bearing amides.
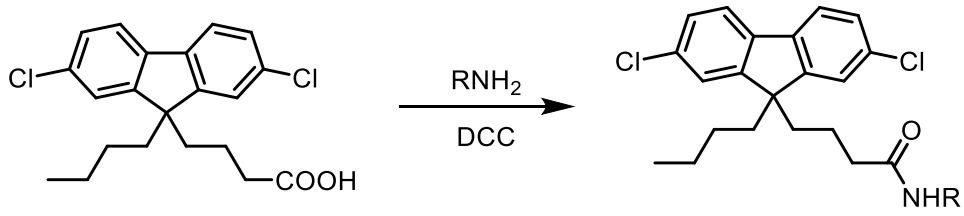


\section{N-butyl-4-(9-butyl-2,7-dichloro-9H-fluoren-9-yl)butanamide}

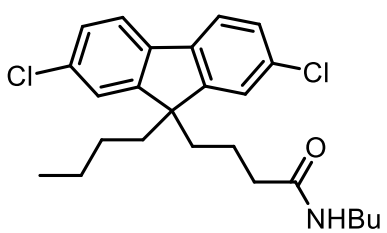

This compound was synthesized by the condensation of 4-(9-butyl-2,7dichloro-9H-fluoren-9-yl)butanoic acid $\mathbf{5 1}$ with $\mathrm{BuNH}_{2}$ using DCC according previous literature, ${ }^{[6]}$ one step, $56 \%$ yield, white solid. ${ }^{1} \mathrm{H} \mathrm{NMR}\left(500 \mathrm{MHz}, \mathrm{CDCl}_{3}\right) \delta 7.59(\mathrm{~d}, J=$ $8.1 \mathrm{~Hz}, 2 \mathrm{H}), 7.33(\mathrm{dd}, J=8.0,1.9 \mathrm{~Hz}, 2 \mathrm{H}), 7.31(\mathrm{~d}, J=1.6 \mathrm{~Hz}, 2 \mathrm{H}), 5.18(\mathrm{~s}, 1 \mathrm{H}), 3.19-$

$3.15(\mathrm{~m}, 2 \mathrm{H}), 2.03-1.98(\mathrm{~m}, 2 \mathrm{H}), 1.96-1.92(\mathrm{~m}, 4 \mathrm{H}), 1.46-1.40(\mathrm{~m}, 2 \mathrm{H}), 1.34-1.29(\mathrm{~m}, 2 \mathrm{H}), 1.13-1.08(\mathrm{~m}, 2 \mathrm{H}), 0.99-$ $0.95(\mathrm{~m}, 2 \mathrm{H}), 0.93-0.88(\mathrm{~m}, 3 \mathrm{H}), 0.70(\mathrm{t}, \mathrm{J}=7.4 \mathrm{~Hz}, 3 \mathrm{H}), 0.59-0.54(\mathrm{~m}, 2 \mathrm{H}) .{ }^{13} \mathrm{C} \mathrm{NMR}\left(126 \mathrm{MHz}, \mathrm{CDCl}_{3}\right) \delta 172.2,151.8$, 138.6, 133.3, 127.6, 123.3, 120.9, 77.3, 77.0, 76.8, 55.3, 40.0, 39.6, 39.2, 36.7, 31.7, 25.7, 22.9, 20.3, 20.1, $13.8,13.7$. MS (m/z, ESI): Calcd. for Chemical Formula: $\mathrm{C}_{25} \mathrm{H}_{30} \mathrm{Cl}_{2} \mathrm{NO}^{-}[\mathrm{M}-\mathrm{H}]^{-}: 430.1710$, Found: 430.1710.

\section{4-(9-butyl-2,7-dichloro-9H-fluoren-9-yl)-N-phenylbutanamide}

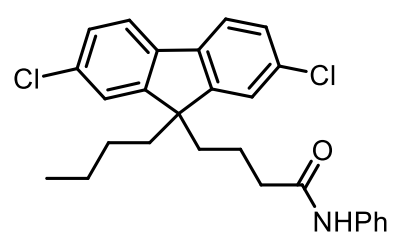

This compound was synthesized by the condensation of 4-(9-butyl-2,7-dichloro9H-fluoren-9-yl)butanoic acid $\mathbf{5 1}$ with $\mathrm{PhNH}_{2}$ using DCC according previous literature. ${ }^{[6]}$ One step, 52\% yield, white solid. ${ }^{1} \mathrm{H}$ NMR $\left(500 \mathrm{MHz}, \mathrm{CDCl}_{3}\right) \delta 7.60(\mathrm{~d}, J=8.1 \mathrm{~Hz}, 2 \mathrm{H})$,

$7.44(\mathrm{~d}, J=7.9 \mathrm{~Hz}, 2 \mathrm{H}), 7.36-7.29(\mathrm{~m}, 6 \mathrm{H}), 7.10(\mathrm{t}, J=7.4 \mathrm{~Hz}, 1 \mathrm{H}), 6.93(\mathrm{~s}, 1 \mathrm{H}), 2.12(\mathrm{t}, J=7.5 \mathrm{~Hz}, 2 \mathrm{H}), 2.09-2.04(\mathrm{~m}$, $2 \mathrm{H}), 1.98-1.94(\mathrm{~m}, 2 \mathrm{H}), 1.13-1.04(\mathrm{~m}, 4 \mathrm{H}), 0.71(\mathrm{t}, J=7.4 \mathrm{~Hz}, 3 \mathrm{H}), 0.62-0.55(\mathrm{~m}, 2 \mathrm{H}) .{ }^{13} \mathrm{C} \mathrm{NMR}(126 \mathrm{MHz}, \mathrm{CDCl})$ $\delta$ 151.7, 138.7, 133.4, 129.0, 127.7, 124.2, 123.3, 120.9, 119.7, 40.0, 39.5, 37.7, 25.7, 22.9, 20.1, 13.8. MS (m/z, ESI): Calcd. for Chemical Formula: $\mathrm{C}_{27} \mathrm{H}_{26} \mathrm{Cl}_{2} \mathrm{NO}^{-}[\mathrm{M}-\mathrm{H}]^{-}:$: 450.1397, Found: 450.1395.

5. Preparation of substrate bearing alcohol.

\section{4-(9-butyl-2,7-dichloro-9H-fluoren-9-yl)butan-1-ol}

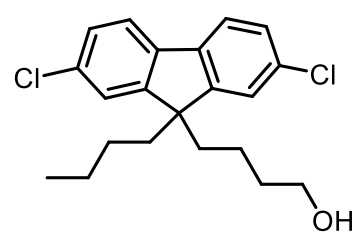

This compound was prepared by reduction of 4-(9-butyl-2,7-dichloro-9H-fluoren9-yl)butanoic acid using lithium triethylborohydride $(1.0 \mathrm{~mol} / \mathrm{L})$ in THF according previous literature. ${ }^{[7]} 92 \%$ yield, white solid. ${ }^{1} \mathrm{H}$ NMR $\left(500 \mathrm{MHz}, \mathrm{CDCl}_{3}\right) \delta 7.59(\mathrm{~d}, J=8.0$ $\mathrm{Hz}, 2 \mathrm{H}), 7.33(\mathrm{dd}, J=8.0,1.9 \mathrm{~Hz}, 2 \mathrm{H}), 7.31(\mathrm{~d}, J=1.8 \mathrm{~Hz}, 2 \mathrm{H}), 3.87(\mathrm{t}, J=6.8 \mathrm{~Hz}, 2 \mathrm{H}), 2.01-1.98(\mathrm{~m}, 2 \mathrm{H}), 1.97-1.93$ $(\mathrm{m}, 2 \mathrm{H}), 1.48-1.37(\mathrm{~m}, 2 \mathrm{H}), 1.14-1.10(\mathrm{~m}, 2 \mathrm{H}), 0.71(\mathrm{t}, J=10.0 \mathrm{~Hz}, 3 \mathrm{H}), 0.69-0.63(\mathrm{~m}, 2 \mathrm{H}), 0.63-0.55(\mathrm{~m}, 2 \mathrm{H}) .{ }^{13} \mathrm{C}$ 
NMR $\left(126 \mathrm{MHz}_{\mathrm{CDCl}}\right) \delta 151.9,138.6,133.3,127.5,123.2,120.8,63.7,55.5,40.0,39.6,28.6,25.8,22.9,20.9,19.9$, 13.8. $\mathrm{MS}$ (m/z, ESI): Calcd. for Chemical Formula: $\mathrm{C}_{21} \mathrm{H}_{23} \mathrm{Cl}_{2} \mathrm{O}^{-}[\mathrm{M}-\mathrm{H}]^{-}: 361.1$, Found: 361.2 .

6. Preparation of substrate bearing cyclohexyl group.

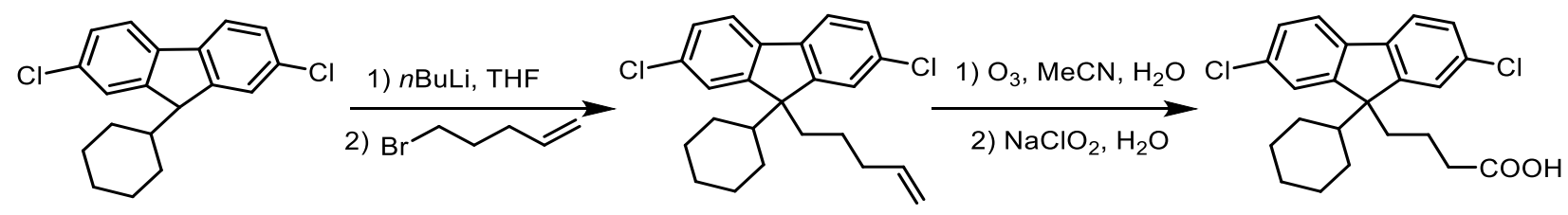

The procedure to prepare 2,7-dichloro-9-cyclohexyl-9H-fluorene is same as the synthesis of 9-butyl-2,7dichloro-9H-fluorene. Under $\mathrm{N}_{2}$ atmosphere, 2,7-dichloro-9-cyclohexyl-9H-fluorene (0.8 g, 2.5 mmol) was dissolved in anhydrous $8 \mathrm{~mL}$ THF and $n B$ BuLi $(1.4 \mathrm{~mL}, 2.8 \mathrm{mmol}, 2.0 \mathrm{mmol} / \mathrm{L})$ was added dropwise at $-78^{\circ} \mathrm{C}$. After addition was completed, the reaction mixture was stirring for $30 \mathrm{~min}$ at room temperature and 5-bromopent-1-ene (0.45 g, 3.0 $\mathrm{mmol}$ ) was added in one portion at $-78^{\circ} \mathrm{C}$ and stirred for $30 \mathrm{~min}$. The resulting reaction mixture was warmed to room temperature and stirred overnight. The reaction was quenched with saturated $\mathrm{NH}_{4} \mathrm{Cl}$ solution and extracted with ethyl acetate $(15 \mathrm{~mL} \times 3)$ three times. The combined organic phases were washed with brine, dried over $\mathrm{Na}_{2} \mathrm{SO}_{4}$, and concentrated in vacuo. The crude materials were purified by flash column chromatography to give the desired product $0.5 \mathrm{~g}$ in $51 \%$ yield as a white solid. (The following steps are performed according to the synthetic route of xanthene substrates.)

\section{4-(2,7-dichloro-9-cyclohexyl-9H-fluoren-9-yl)butanoic acid}

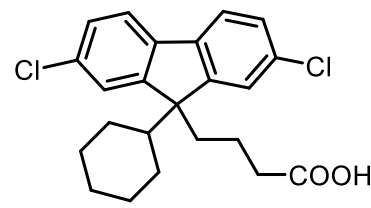

The procedure to this compound is similar to the synthesis of Xanthene-type substrates, starting from 2,7-dichloro-9H-fluorene. Three steps, $18 \%$ yield, white solids.

${ }^{1} \mathrm{H}$ NMR $\left(500 \mathrm{MHz}, \mathrm{CDCl}_{3}\right) \delta 7.57(\mathrm{dd}, J=7.3,1.4 \mathrm{~Hz}, 2 \mathrm{H}), 7.35-7.33(\mathrm{~m}, 3 \mathrm{H}), 7.32(\mathrm{~d}, J$ $=1.9 \mathrm{~Hz}, 1 \mathrm{H}), 2.14(\mathrm{t}, J=7.3 \mathrm{~Hz}, 2 \mathrm{H}), 2.12-2.07(\mathrm{~m}, 2 \mathrm{H}), 1.87-1.83(\mathrm{~m}, 1 \mathrm{H}), 1.67-1.56(\mathrm{~m}, 4 \mathrm{H}), 1.52-1.48(\mathrm{~m}, 2 \mathrm{H})$, $1.21-1.10(\mathrm{~m}, 2 \mathrm{H}), 0.87-0.83(\mathrm{~m}, 2 \mathrm{H}), 0.80-0.76(\mathrm{~m}, 2 \mathrm{H}) .{ }^{13} \mathrm{C} N M R\left(126 \mathrm{MHz}, \mathrm{CDCl}_{3}\right) \delta 177.9,151.3,139.0,133.2$ 127.5, 123.9, 120.6, 58.6, 47.6, 35.6, 33.8, 27.7, 26.8, 26.3, 19.1. MS (m/z, ESI): Calcd. for Chemical Formula: 
$\mathrm{C}_{23} \mathrm{H}_{23} \mathrm{Cl}_{2} \mathrm{O}_{2}^{-}[\mathrm{M}-\mathrm{H}]:: 401.1081$, Found: 401.1082.

7. The general procedure for the synthesis of xanthene substrates is shown below. ${ }^{[8]}$
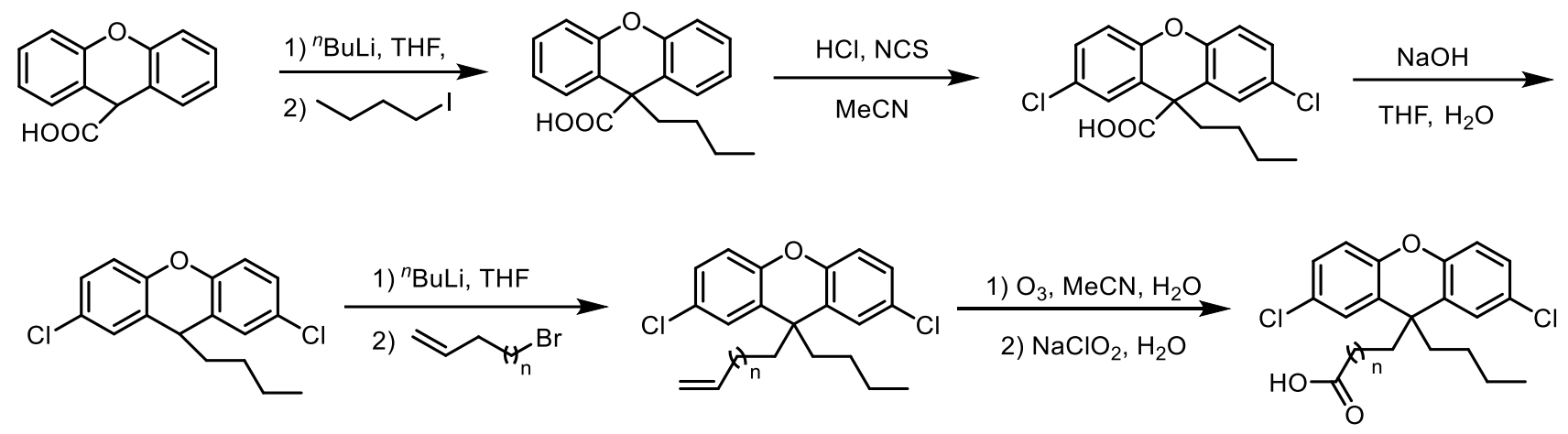

\section{9-butyl-9H-xanthene-9-carboxylic acid}

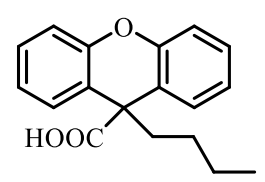

Under $\mathrm{N}_{2}$ atmosphere, $9 \mathrm{H}$-xanthene-9-carboxylic acid $(4.52 \mathrm{~g}, 20 \mathrm{mmol})$ was dissolved in anhydrous THF (100 mL), then $n B u L i(2 \mathrm{M}, 21 \mathrm{~mL}, 42 \mathrm{mmol})$ as a cyclohexane solution was added dropwise at $-78^{\circ} \mathrm{C}$. After addition was completed, the resulting reaction mixture was stirred for $30 \mathrm{~min}$ at room temperature. 1-iodobutane $(4.42 \mathrm{~g}, 24 \mathrm{mmol})$ was added in one portion at $-78^{\circ} \mathrm{C}$ and stirring at this temperature for $20 \mathrm{~min}$. Then the reaction mixture was warmed to room temperature and stirring overnight. The reaction was quenched with saturated $\mathrm{NH}_{4} \mathrm{Cl}$ solution and extracted with ethyl acetate three times. The combined organic phases were washed with brine, dried over $\mathrm{Na}_{2} \mathrm{SO}_{4}$, and concentrated in vacuo. The crude mixture was purified by column chromatography on silica gel (hexane: acetone $=10: 1$ ) to afford the target compound (4.12 $\mathrm{g}$, 73\%) as a white solid.

${ }^{1} \mathrm{H} \mathrm{NMR}\left(500 \mathrm{MHz}, \mathrm{CDCl}_{3}\right) \delta 7.30-7.26(\mathrm{~m}, 2 \mathrm{H}), 7.25-7.23(\mathrm{~m}, 2 \mathrm{H}), 7.11-7.06(\mathrm{~m}, 4 \mathrm{H}), 2.29-2.23(\mathrm{~m}, 2 \mathrm{H})$, $1.12-1.19(\mathrm{~m}, 2 \mathrm{H}), 0.82-0.76(\mathrm{~m}, 2 \mathrm{H}), 0.70(\mathrm{t}, J=7.3 \mathrm{~Hz}, 3 \mathrm{H}) .{ }^{13} \mathrm{C} \mathrm{NMR}\left(126 \mathrm{MHz}, \mathrm{CDCl}_{3}\right) \delta 177.6,150.8,129.0$, $127.3,123.5,121.0,116.9,49.8,40.2,26.1,22.8,13.9$.

\section{9-butyl-2,7-dichloro-9H-xanthene-9-carboxylic acid}




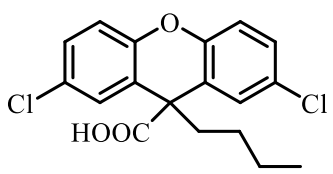

9-butyl-9H-xanthene-9-carboxylic acid (4.12 g, $14.6 \mathrm{mmol}$ ) was dissolved in MeCN $(100 \mathrm{~mL})$, then conc. $\mathrm{HCl}(10 \mathrm{~mL}, 8.3 \mathrm{mmol})$ was added. After that, NCS $(4.31 \mathrm{~g}, 32.1 \mathrm{mmol})$ was added into the reaction mixture at $0{ }^{\circ} \mathrm{C}$. Then the reaction mixture was warmed to room temperature and stirring for $12 \mathrm{~h}$. The reaction was quenched with saturated $\mathrm{NaHCO}_{3}$ solution and extracted with ethyl acetate three times. The combined organic phases were washed with brine, dried over $\mathrm{Na}_{2} \mathrm{SO}_{4}$, and concentrated in vacuo. The crude mixture was purified by column chromatography on silica gel (hexane: acetone = 10: 1$)$ to afford the target compound $(4.77 \mathrm{~g}, 93 \%)$ as a white solid.

${ }^{1} \mathrm{H}$ NMR $\left(400 \mathrm{MHz}, \mathrm{CDCl}_{3}\right) \delta 7.26(\mathrm{~d}, J=2.3 \mathrm{~Hz}, 2 \mathrm{H}), 7.24(\mathrm{~d}, J=1.7 \mathrm{~Hz}, 2 \mathrm{H}), 7.05-7.03(\mathrm{~m}, 1 \mathrm{H}), 7.03-7.01(\mathrm{~m}$, $1 \mathrm{H}), 2.28-2.19(\mathrm{~m}, 2 \mathrm{H}), 1.16-1.21(\mathrm{~m}, 2 \mathrm{H}), 0.88-0.67(\mathrm{~m}, 5 \mathrm{H}) .{ }^{13} \mathrm{C} \mathrm{NMR}\left(126 \mathrm{MHz}, \mathrm{CDCl}_{3}\right) \delta 177.4,149.2,129.5$, $128.6,127.1,121.9,118.4,50.1,39.9,26.2,22.7,13.9$.

\section{9-butyl-2,7-dichloro-9H-xanthene}

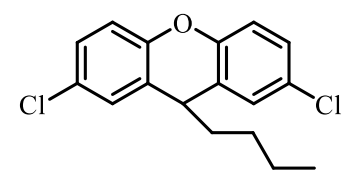

9-butyl-2,7-dichloro-9H-xanthene-9-carboxylic acid (1.97 g, $5.6 \mathrm{mmol})$ was dissolved

in THF- $\mathrm{H}_{2} \mathrm{O}$ co-solvents (8: $1,45 \mathrm{~mL}$ ), then $\mathrm{NaOH}$ (449 mg, $11.2 \mathrm{mmol}$ ) was added. After that, the reaction mixture was heated to $60^{\circ} \mathrm{C}$ and stirring for overnight. The reaction was quenched with $1 \mathrm{~N} \mathrm{HCl}$ solution and extracted with ethyl acetate three times. The combined organic phases were washed with brine, dried over $\mathrm{Na}_{2} \mathrm{SO}_{4}$, and concentrated in vacuo. The crude mixture was purified by column chromatography on silica gel (hexane) to afford the target compound (1.63 g, 95\%) as a white solid.

${ }^{1} \mathrm{H}$ NMR $\left(500 \mathrm{MHz}, \mathrm{CDCl}_{3}\right) \delta 7.17(\mathrm{~d}, J=8.3 \mathrm{~Hz}, 4 \mathrm{H}), 7.02-6.97(\mathrm{~m}, 2 \mathrm{H}), 3.92(\mathrm{t}, J=5.9 \mathrm{~Hz}, 1 \mathrm{H}), 1.74-1.67(\mathrm{~m}$, 2H), $1.26-1.18(\mathrm{~m}, 2 \mathrm{H}), 1.07-1.13(\mathrm{~m}, 2 \mathrm{H}), 0.80(\mathrm{t}, \mathrm{J}=7.3 \mathrm{~Hz}, 3 \mathrm{H}) .{ }^{13} \mathrm{C} \mathrm{NMR}(126 \mathrm{MHz}, \mathrm{CDCl} 3) \delta 150.7,128.3,128.2$, $127.8,126.7,117.8,40.4,39.0,27.4,22.7,14.0$.

\section{9-butyl-2,7-dichloro-9-(pent-3-en-1-yl)-9H-xanthene}

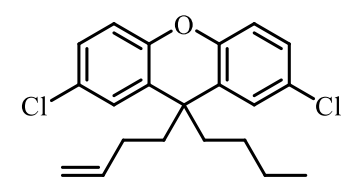

Under $\mathrm{N}_{2}$ atmosphere, 9-butyl-2,7-dichloro-9H-xanthene (1.09 g, $\left.3.6 \mathrm{mmol}\right)$ was dissolved in anhydrous THF (18 mL) and $n B u L i(2 \mathrm{M}, 2.16 \mathrm{~mL}, 4.32 \mathrm{mmol})$ as a cyclohexane solution was added dropwise at $-78^{\circ} \mathrm{C}$. After addition was completed, the reaction mixture was stirring for $30 \mathrm{~min}$ at room temperature and 4-bromopent-1-ene (730 $\mathrm{mg}, 5.4 \mathrm{mmol}$ ) was added dropwise at - 
$78{ }^{\circ} \mathrm{C}$. The resulting reaction mixture was warmed to room temperature and stirred overnight. The reaction was quenched with saturated $\mathrm{NH}_{4} \mathrm{Cl}$ solution and extracted with ethyl acetate three times. The combined organic phases were washed with brine, dried over $\mathrm{Na}_{2} \mathrm{SO}_{4}$, and concentrated in vacuo. The crude mixture was purified by column chromatography on silica gel (hexane) to afford the target compound ( $909 \mathrm{mg}, 70 \%$ ) as a white solid.

${ }^{1} \mathrm{H}$ NMR $\left(400 \mathrm{MHz}, \mathrm{CDCl}_{3}\right) \delta 7.23(\mathrm{~d}, J=2.5 \mathrm{~Hz}, 2 \mathrm{H}), 7.18-7.13(\mathrm{~m}, 2 \mathrm{H}), 6.94(\mathrm{~d}, J=8.7 \mathrm{~Hz}, 2 \mathrm{H}), 5.67-5.54(\mathrm{~m}$, $1 \mathrm{H}), 4.88-4.77(\mathrm{~m}, 2 \mathrm{H}), 2.00-1.94(\mathrm{~m}, 2 \mathrm{H}), 1.91-1.86(\mathrm{~m}, 2 \mathrm{H}), 1.65-1.58(\mathrm{~m}, 2 \mathrm{H}), 1.13-1.18(\mathrm{~m}, 2 \mathrm{H}), 0.87-0.78$ (m, 2H), $0.73(\mathrm{t}, J=7.3 \mathrm{~Hz}, 3 \mathrm{H}) .{ }^{13} \mathrm{C} N M R\left(126 \mathrm{MHz}, \mathrm{CDCl}_{3}\right) \delta 150.0,137.9,128.3,127.8,126.3,126.0,117.6,114.5$, $45.0,44.4,42.7,29.1,26.9,22.8,13.8$.

\section{9-butyl-2,7-dichloro-9-(pent-4-en-1-yl)-9H-xanthene}

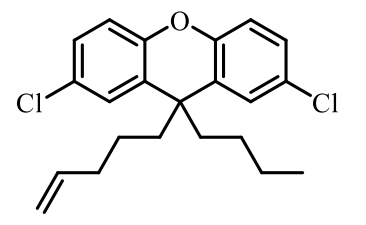

Under $\mathrm{N}_{2}$ atmosphere, 9-butyl-2,7-dichloro-9H-xanthene (1.86 g, $\left.6.06 \mathrm{mmol}\right)$ was dissolved in anhydrous THF (30 mL) and $n B u L i(2 \mathrm{M}, 3.64 \mathrm{~mL}, 7.27 \mathrm{mmol})$ as a cyclohexane solution was added dropwise at $-78^{\circ} \mathrm{C}$. After addition was completed, the reaction mixture

was stirring for $30 \mathrm{~min}$ at room temperature and 5-bromopent-1-ene (1.35 g, 9.09 mmol) was added dropwise at -78 ${ }^{\circ} \mathrm{C}$. The resulting reaction mixture was warmed to room temperature and stirred overnight. The reaction was quenched with saturated $\mathrm{NH}_{4} \mathrm{Cl}$ solution and extracted with ethyl acetate three times. The combined organic phases were washed with brine, dried over $\mathrm{Na}_{2} \mathrm{SO}_{4}$, and concentrated in vacuo. The crude mixture was purified by column chromatography on silica gel (hexane) to afford the target compound ( $1.8 \mathrm{~g}, 79 \%)$.

${ }^{1} \mathrm{H} \mathrm{NMR}\left(500 \mathrm{MHz}, \mathrm{CDCl}_{3}\right) \delta 7.21(\mathrm{~d}, J=2.5 \mathrm{~Hz}, 2 \mathrm{H}), 7.17-7.13(\mathrm{~m}, 2 \mathrm{H}), 6.93(\mathrm{~d}, J=8.7 \mathrm{~Hz}, 2 \mathrm{H}), 5.59(\mathrm{ddt}, J=$ $15.7,11.5,6.6 \mathrm{~Hz}, 1 \mathrm{H}), 4.91-4.85(\mathrm{~m}, 2 \mathrm{H}), 1.91-1.83(\mathrm{~m}, 6 \mathrm{H}), 1.12-1.17(\mathrm{~m}, 2 \mathrm{H}), 0.99-0.91(\mathrm{~m}, 2 \mathrm{H}), 0.85-0.78$ (m, 2H), $0.73(\mathrm{t}, J=7.4 \mathrm{~Hz}, 3 \mathrm{H}) .{ }^{13} \mathrm{C}$ NMR $\left(126 \mathrm{MHz}, \mathrm{CDCl}_{3}\right) \delta 150.1,138.4,128.4,127.9,126.7,126.1,117.7,114.9$ $45.3,44.8,42.9,33.8,27.0,24.1,23.0,14.0$.

\section{9-butyl-2,7-dichloro-9-(pent-5-en-1-yl)-9H-xanthene}




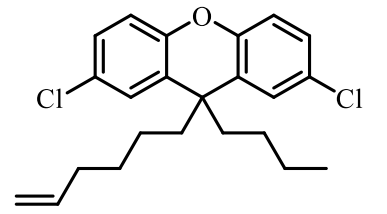

Under $\mathrm{N}_{2}$ atmosphere, 9-butyl-2,7-dichloro-9H-xanthene (1.34 g, $4.4 \mathrm{mmol}$ ) was dissolved in anhydrous THF $(22 \mathrm{~mL})$ and $n$ BuLi $(2 \mathrm{M}, 2.64 \mathrm{~mL}, 5.28 \mathrm{mmol})$ as a cyclohexane solution was added dropwise at $-78^{\circ} \mathrm{C}$. After addition was completed, the reaction mixture was stirring for $30 \mathrm{~min}$ at room temperature and 6-bromopent-1-ene (1.08 $\mathrm{g}, 6.6 \mathrm{mmol})$ was added dropwise at $-78^{\circ} \mathrm{C}$. The resulting reaction mixture was warmed to room temperature and stirred overnight. The reaction was quenched with saturated $\mathrm{NH}_{4} \mathrm{Cl}$ solution and extracted with ethyl acetate three times. The combined organic phases were washed with brine, dried over $\mathrm{Na}_{2} \mathrm{SO}_{4}$, and concentrated in vacuo. The crude mixture was purified by column chromatography on silica gel (hexane) to afford the target compound (1.29 g, $75 \%)$ as a white solid.

${ }^{1} \mathrm{H} \mathrm{NMR}\left(500 \mathrm{MHz}, \mathrm{CDCl}_{3}\right) \delta 7.21(\mathrm{~d}, J=2.5 \mathrm{~Hz}, 2 \mathrm{H}), 7.16(\mathrm{~d}, J=2.5 \mathrm{~Hz}, 1 \mathrm{H}), 7.14(\mathrm{~d}, J=2.5 \mathrm{~Hz}, 1 \mathrm{H}), 6.94(\mathrm{~d}, J=$ 8.7 Hz, 2H), $5.65(\mathrm{ddt}, J=16.9,10.2,6.6 \mathrm{~Hz}, 1 \mathrm{H}), 4.92-4.82(\mathrm{~m}, 2 \mathrm{H}), 1.91-1.83(\mathrm{~m}, 6 \mathrm{H}), 1.26-1.19(\mathrm{~m}, 2 \mathrm{H}), 1.13-$ $1.17(\mathrm{~m}, 2 \mathrm{H}), 0.89-0.79(\mathrm{~m}, 4 \mathrm{H}), 0.73(\mathrm{t}, J=7.4 \mathrm{~Hz}, 3 \mathrm{H}) .{ }^{13} \mathrm{C}$ NMR $\left(126 \mathrm{MHz}, \mathrm{CDCl}_{3}\right) \delta 150.1,138.7,128.3,127.8$ $126.8,126.1,117.7,114.5,45.4,45.2,43.0,33.4,29.0,27.1,24.3,23.0,14.0$.

\section{3-(9-butyl-2,7-dichloro-9H-xanthen-9-yl)propanoic acid}

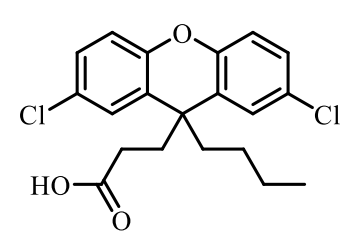

9-(but-3-en-1-yl)-9-butyl-2,7-dichloro-9H-xanthene $(682 \mathrm{mg}, 1.89 \mathrm{mmol})$ was dissolved in $\mathrm{MeCN}-\mathrm{H}_{2} \mathrm{O}$ co-solvents (9: 1, $20 \mathrm{~mL}$ ). The solution was cooled to $0{ }^{\circ} \mathrm{C}$ and sparged with ozone, meanwhile, reaction completeness was confirmed by TLC. An aqueous solution of $\mathrm{NaClO}_{2}(80 \mathrm{wt} \%, 684,7.56 \mathrm{mmol})$ in $\mathrm{H}_{2} \mathrm{O}(8 \mathrm{~mL})$ was added portion-wise to the reaction while maintaining an internal reaction temp $<15^{\circ} \mathrm{C}$. The cold bath was removed from the reaction and the mixture stirred overnight at room temperature. The reaction was quenched with a saturated $\mathrm{Na}_{2} \mathrm{~S}_{2} \mathrm{O}_{3}$ solution at $0{ }^{\circ} \mathrm{C}$ and the reaction mixture was allowed to stir for $4 \mathrm{~h}$ at room temperature, then it was extracted with ethyl acetate three times. The combined organic phases were washed with brine, dried over $\mathrm{Na}_{2} \mathrm{SO}_{4}$, and concentrated in vacuo. The crude mixture was purified by column chromatography on silica gel (hexane: acetone $=10: 1)$ to afford the target compound (444 mg, $62 \%)$ as a white solid.

${ }^{1} \mathrm{H} \mathrm{NMR}\left(500 \mathrm{MHz}, \mathrm{CDCl}_{3}\right) \delta 7.23(\mathrm{~d}, J=2.5 \mathrm{~Hz}, 2 \mathrm{H}), 7.18(\mathrm{~d}, J=2.4 \mathrm{~Hz}, 1 \mathrm{H}), 7.16(\mathrm{~d}, J=2.4 \mathrm{~Hz}, 1 \mathrm{H}), 6.95(\mathrm{~d}, J=$ $8.7 \mathrm{~Hz}, 2 \mathrm{H}), 2.27-2.20(\mathrm{~m}, 2 \mathrm{H}), 1.95-1.88(\mathrm{~m}, 4 \mathrm{H}), 1.13-1.18(\mathrm{~m}, 2 \mathrm{H}), 0.84-0.78(\mathrm{~m}, 2 \mathrm{H}), 0.73(\mathrm{t}, J=7.3 \mathrm{~Hz}, 3 \mathrm{H})$. 
${ }^{13} \mathrm{C} \mathrm{NMR}\left(126 \mathrm{MHz}, \mathrm{CDCl}_{3}\right) \delta 177.7,150.1,128.7,128.4,126.0,125.3,118.0,45.1,42.3,39.6,29.9,27.1,22.9,13.9$. $\operatorname{HRMS}(\mathrm{m} / \mathrm{z}, \mathrm{ESI})$ : Calcd. for Chemical Formula: $\mathrm{C}_{20} \mathrm{H}_{19} \mathrm{Cl}_{2} \mathrm{O}_{3}{ }^{-}[\mathrm{M}-\mathrm{H}]^{-}: 377.0717$, Found: 377.0714 .

\section{4-(9-butyl-2,7-dichloro-9H-xanthen-9-yl)butanoic acid}

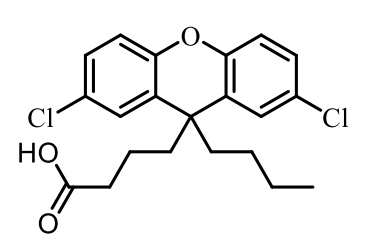

9-butyl-2,7-dichloro-9-(pent-4-en-1-yl)-9H-xanthene (1.8 g, $4.8 \mathrm{mmol})$ was dissolved in $\mathrm{MeCN}-\mathrm{H}_{2} \mathrm{O}$ co-solvents $(9: 1,50 \mathrm{~mL})$.The solution was cooled to $0{ }^{\circ} \mathrm{C}$ and sparged with ozone, meanwhile, reaction completeness was confirmed by TLC. An aqueous solution of $\mathrm{NaClO}_{2}$ (80 wt\%, $1.74 \mathrm{~g}, 19.2 \mathrm{mmol}, 4$ eq.) in $\mathrm{H}_{2} \mathrm{O}(20 \mathrm{~mL}$ ) was added portion-wise to the reaction while maintaining an internal reaction temp $<15^{\circ} \mathrm{C}$. The cold bath was removed from the reaction and the mixture stirred overnight at room temperature. The reaction was quenched with a saturated $\mathrm{Na}_{2} \mathrm{~S}_{2} \mathrm{O}_{3}$ solution at $0{ }^{\circ} \mathrm{C}$ and the reaction mixture was allowed to stir for $2 \mathrm{~h}$ at room temperature, then it was extracted with ethyl acetate three times. The combined organic phases were washed with brine, dried over $\mathrm{Na}_{2} \mathrm{SO}_{4}$, and concentrated in vacuo. The crude mixture was purified by column chromatography on silica gel (hexane: acetone $=10: 1)$ to afford the target compound $(1.23 \mathrm{~g}$, $65 \%)$ as a white solid.

${ }^{1} \mathrm{H}$ NMR $\left(500 \mathrm{MHz}, \mathrm{CDCl}_{3}\right) \delta 7.22(\mathrm{~d}, J=2.5 \mathrm{~Hz}, 2 \mathrm{H}), 7.16(\mathrm{dd}, J=8.7,2.5 \mathrm{~Hz}, 2 \mathrm{H}), 6.94(\mathrm{~d}, J=8.7 \mathrm{~Hz}, 2 \mathrm{H}), 2.20(\mathrm{t}$, $J=7.3 \mathrm{~Hz}, 2 \mathrm{H}), 1.96-1.92(\mathrm{~m}, 2 \mathrm{H}), 1.87-1.83(\mathrm{~m}, 2 \mathrm{H}), 1.23-1.17(\mathrm{~m}, 2 \mathrm{H}), 1.16-1.11(\mathrm{~m}, 2 \mathrm{H}), 0.84-0.78(\mathrm{~m}, 2 \mathrm{H})$, $0.72(\mathrm{t}, J=7.4 \mathrm{~Hz}, 3 \mathrm{H}) .{ }^{13} \mathrm{C}$ NMR $\left(126 \mathrm{MHz}, \mathrm{CDCl}_{3}\right) \delta 177.3,150.1,128.5,128.1,126.2,126.1,117.9,45.5,44.2,42.8$, 33.5, 27.0, 22.9, 20.2, 14.0. HRMS (m/z, ESI): Calcd. for Chemical Formula: $\mathrm{C}_{21} \mathrm{H}_{21} \mathrm{Cl}_{2} \mathrm{O}_{3}{ }^{-}[\mathrm{M}-\mathrm{H}]^{-}:$: 391.0873, Found: 391.0871.

\section{5-(9-butyl-2,7-dichloro-9H-xanthen-9-yl)pentanoic acid}

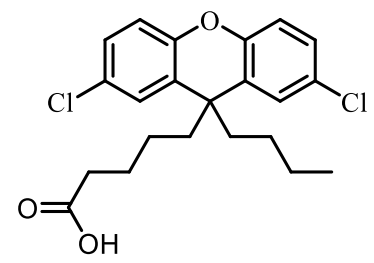

9-butyl-2,7-dichloro-9-(hex-5-en-1-yl)-9H-xanthene (1.29 g, $3.32 \mathrm{mmol})$ was dissolved in $\mathrm{MeCN}-\mathrm{H}_{2} \mathrm{O}$ co-solvents (9: 1, $35 \mathrm{~mL}$ ). The solution was cooled to $0{ }^{\circ} \mathrm{C}$ and sparged with ozone, meanwhile, reaction completeness was confirmed by TLC. An aqueous solution of $\mathrm{NaClO}_{2}$ (80 wt\%, $1.2 \mathrm{~g}, 13.28 \mathrm{mmol}$ ) in $\mathrm{H}_{2} \mathrm{O}(14 \mathrm{~mL}$ ) was added

portion-wise to the reaction while maintaining an internal reaction temp $<15^{\circ} \mathrm{C}$. The cold bath was removed from the reaction and the mixture stirred overnight at room temperature. The reaction was quenched with a saturated 
$\mathrm{Na}_{2} \mathrm{~S}_{2} \mathrm{O}_{3}$ solution at $0{ }^{\circ} \mathrm{C}$ and the reaction mixture was allowed to stir for $2 \mathrm{~h}$ at room temperature, then it was extracted with ethyl acetate three times. The combined organic phases were washed with brine, dried over $\mathrm{Na}_{2} \mathrm{SO}_{4}$, and concentrated in vacuo. The crude mixture was purified by column chromatography on silica gel (hexane: acetone = 10: 1 ) to afford the target compound ( $865 \mathrm{mg}, 64 \%)$ as a white solid.

${ }^{1} \mathrm{H}$ NMR $\left(500 \mathrm{MHz}, \mathrm{CDCl}_{3}\right) \delta 7.20(\mathrm{~d}, J=2.5 \mathrm{~Hz}, 2 \mathrm{H}), 7.17-7.13(\mathrm{~m}, 2 \mathrm{H}), 6.93(\mathrm{dd}, J=8.7,1.0 \mathrm{~Hz}, 2 \mathrm{H}), 2.17(\mathrm{t}, J=$ $7.7 \mathrm{~Hz}, 2 \mathrm{H}), 1.90-1.84(\mathrm{~m}, 4 \mathrm{H}), 1.51-1.44(\mathrm{~m}, 2 \mathrm{H}), 1.12-1.17(\mathrm{~m}, 2 \mathrm{H}), 0.92-0.86(\mathrm{~m}, 2 \mathrm{H}), 0.84-0.77(\mathrm{~m}, 2 \mathrm{H})$, $0.73(\mathrm{t}, J=7.3 \mathrm{~Hz}, 3 \mathrm{H}) .{ }^{13} \mathrm{C} \mathrm{NMR}\left(126 \mathrm{MHz}, \mathrm{CDCl}_{3}\right) \delta 178.2,150.1,128.4,128.0,126.5,126.0,117.8,45.3,45.0,42.9$, 33.6, 27.0, 24.9, 24.4, 23.0, 14.0. $\mathrm{HRMS}$ (m/z, ESI): Calcd. for Chemical Formula: $\mathrm{C}_{22} \mathrm{H}_{23} \mathrm{Cl}_{2} \mathrm{O}_{3}{ }^{-}[\mathrm{M}-\mathrm{H}]^{-}:$: 405.1030, Found: 405.1028.

8. Substrates bearing extra distant chloroarenes were prepared following the scheme shown below.

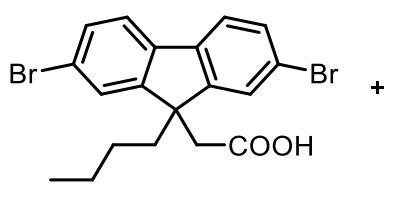<smiles>[R]c1ccc(Cl)c(OCOCCO[Na])c1</smiles>

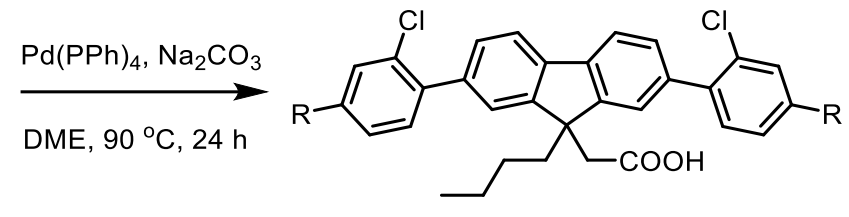

\section{2-(2,7-dibromo-9-butyl-9H-fluoren-9-yl)acetic acid}

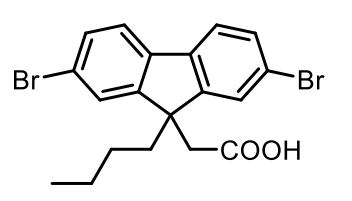

Prepared from 2,7-dibromofluorene in three steps, $52 \%$ yield, white solid. ${ }^{1} \mathrm{H}$ NMR $\left(500 \mathrm{MHz}, \mathrm{CDCl}_{3}\right) \delta 7.57-7.45(\mathrm{~m}, 6 \mathrm{H}), 2.90(\mathrm{~s}, 2 \mathrm{H}), 2.06-1.99(\mathrm{~m}, 2 \mathrm{H}), 1.12(\mathrm{dt}, J=14.7$, $7.4 \mathrm{~Hz}, 2 \mathrm{H}), 0.71(\mathrm{t}, J=7.4 \mathrm{~Hz}, 3 \mathrm{H}), 0.62-0.58(\mathrm{~m}, 2 \mathrm{H}) .{ }^{13} \mathrm{C} \mathrm{NMR}\left(126 \mathrm{MHz}, \mathrm{CDCl}_{3}\right) \delta 174.5$, 150.7, 138.8, 130.9, 126.5, 121.5, 121.4, 52.4, 43.1, 38.8, 25.6, 22.7, 13.8. MS (m/z, ESI): Calcd. for Chemical Formula: $\mathrm{C}_{19} \mathrm{H}_{17} \mathrm{Br}_{2} \mathrm{O}_{2}^{-}[\mathrm{M}-\mathrm{H}]^{-}:$434.9601, Found: 434.9598.

2-(9-butyl-2,7-bis(2-chlorophenyl)-9H-fluoren-9-yl)acetic acid 


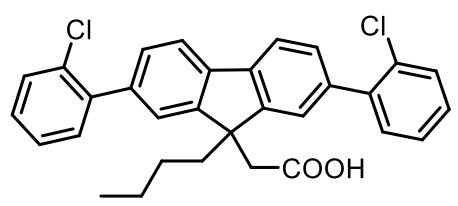

Under $\mathrm{N}_{2}$ atmosphere, to a mixture of 2-(2,7-dibromo-9-butyl-9H-fluoren-9yl)acetic acid (0.4 g, $0.9 \mathrm{mmol})$, (4-chlorophenyl)boronic acid (0.31 g, $2.0 \mathrm{mmol})$ and $\mathrm{Pd}\left(\mathrm{PPh}_{3}\right)_{4}$ were added $2.8 \mathrm{~mL}$ DME and followed by $2 \mathrm{M} \mathrm{Na}_{2} \mathrm{CO}_{3} .{ }^{[9]}$ The resulting reaction mixture was heated to $90{ }^{\circ} \mathrm{C}$ and stirred for $24 \mathrm{~h}$. Water was added and the aqueous solution was acidified to $\mathrm{pH}$ 2-3 with $1 \mathrm{M} \mathrm{HCl}$ solution and extracted with ethyl acetate $(10 \mathrm{~mL} \times 3)$ three times. The combined organic phases were washed with brine, dried over $\mathrm{Na}_{2} \mathrm{SO}_{4}$, and concentrated in vacuo. The crude materials were purified by flash column chromatography to give the desired product $0.42 \mathrm{~g}$ in $91 \%$ yield as a light white solid.

${ }^{1} \mathrm{H}$ NMR $\left(500 \mathrm{MHz}, \mathrm{CDCl}_{3}\right) \delta 7.80(\mathrm{~d}, J=7.8 \mathrm{~Hz}, 2 \mathrm{H}), 7.58(\mathrm{~d}, J=1.1 \mathrm{~Hz}, 2 \mathrm{H}), 7.51(\mathrm{dd}, J=7.8,1.3 \mathrm{~Hz}, 2 \mathrm{H}), 7.46-$ $7.41(\mathrm{~m}, 4 \mathrm{H}), 7.37-7.30(\mathrm{~m}, 4 \mathrm{H}), 2.96(\mathrm{~s}, 2 \mathrm{H}), 2.23-2.17(\mathrm{~m}, 2 \mathrm{H}), 1.16-1.11(\mathrm{~m}, 2 \mathrm{H}), 0.78-0.70(\mathrm{~m}, 5 \mathrm{H}) .{ }^{13} \mathrm{C} \mathrm{NMR}$ $\left(126 \mathrm{MHz}, \mathrm{CDCl}_{3}\right) \delta 174.3,149.0,140.7,139.7,138.4,132.6,131.5,130.1,128.9,128.5,126.9,124.7,119.7,52.4$ 43.4, 38.4, 25.9, 22.8, 13.8. MS (m/z, ESI): Calcd. for Chemical Formula: $\mathrm{C}_{31} \mathrm{H}_{25} \mathrm{Cl}_{2} \mathrm{O}_{2}^{-}$[M-H] : 499.1237, Found: 499.1232.

\section{2-(9-butyl-2,7-bis(2-chloro-4-methoxyphenyl)-9H-fluoren-9-yl)acetic acid}

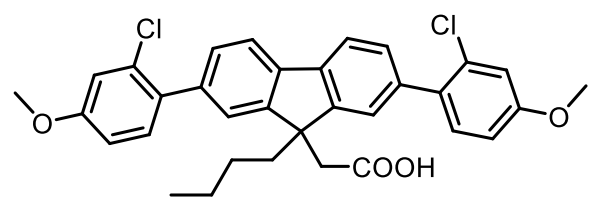

This compound was prepared using a similar method to 2-(9-butyl2,7-bis(2-chlorophenyl)-9H-fluoren-9-yl)acetic acid. 91\% yield, white solid. ${ }^{1} \mathrm{H}$ NMR $\left(500 \mathrm{MHz}, \mathrm{CDCl}_{3}\right) \delta 7.77(\mathrm{~d}, J=7.8 \mathrm{~Hz}, 2 \mathrm{H}), 7.54(\mathrm{~d}, J=0.9$

$\mathrm{Hz}, 2 \mathrm{H}), 7.42(\mathrm{dd}, J=7.8,1.5 \mathrm{~Hz}, 2 \mathrm{H}), 7.34(\mathrm{~d}, J=8.5 \mathrm{~Hz}, 2 \mathrm{H}), 7.05(\mathrm{~d}, J=2.6 \mathrm{~Hz}, 2 \mathrm{H}), 6.90(\mathrm{dd}, J=8.5,2.6 \mathrm{~Hz}, 2 \mathrm{H})$, $3.86(\mathrm{~s}, 6 \mathrm{H}), 2.93(\mathrm{~s}, 2 \mathrm{H}), 2.24-2.15(\mathrm{~m}, 2 \mathrm{H}), 1.16-1.11(\mathrm{~m}, 2 \mathrm{H}), 0.79-0.69(\mathrm{~m}, 5 \mathrm{H}) .{ }^{13} \mathrm{C} \mathrm{NMR}(126 \mathrm{MHz}, \mathrm{CDCl} 3) \delta$ $159.3,149.0,139.4,138.1,133.2,133.1,132.0,129.0,124.8,119.6,115.2,113.1,55.6,52.3,43.6,38.3,25.9,22.9$, 13.8. MS (m/z, ESI): Calcd. for Chemical Formula: $\mathrm{C}_{33} \mathrm{H}_{29} \mathrm{Cl}_{2} \mathrm{O}_{4}{ }^{-}[\mathrm{M}-\mathrm{H}]^{-}: 559.1448$, Found: 559.1444.

9. Substrates bearing heteroatom adjacent to C9. 

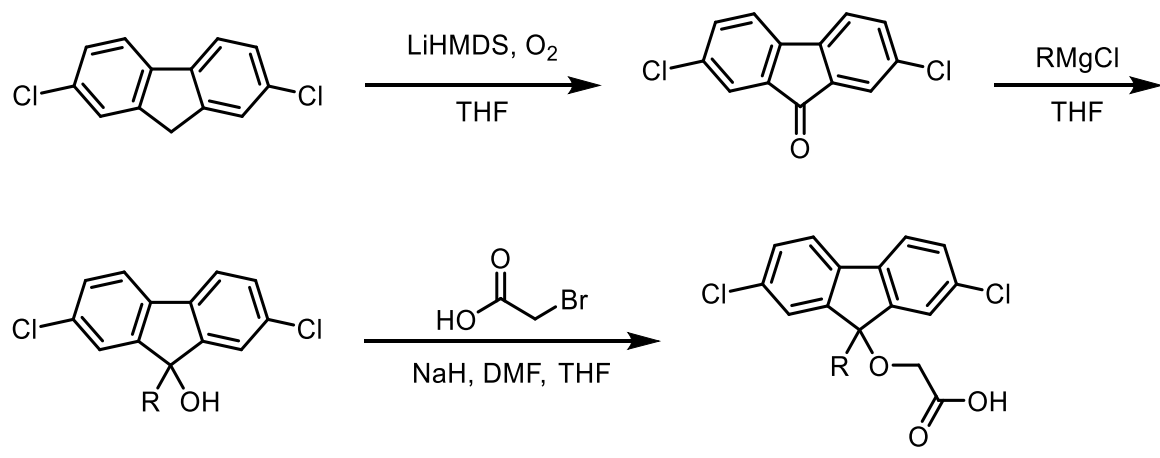

\section{2,7-dichloro-9H-fluoren-9-one}

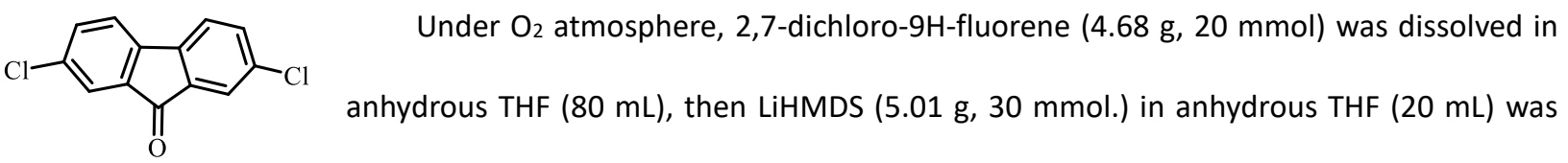
added dropwise at $0{ }^{\circ} \mathrm{C} \cdot{ }^{[10]} \mathrm{And}$, the resulting reaction mixture was stirred for $30 \mathrm{~min}$ at the same temperature. After that, the reaction mixture was allowed to warm to $60^{\circ} \mathrm{C}$ and stirring at this temperature overnight. Then the reaction mixture was warmed to room temperature and quenched with saturated $\mathrm{NH}_{4} \mathrm{Cl}$ solution and extracted with ethyl acetate three times. The combined organic phases were washed with brine, dried over $\mathrm{Na}_{2} \mathrm{SO}_{4}$, and concentrated in vacuo. The crude mixture was purified by column chromatography on silica gel (hexane: ethyl acetate $=20: 1$ ) to afford the target compound ( $3.98 \mathrm{~g}, 80 \%$ yield) as a yellow solid.

${ }^{1} \mathrm{H}$ NMR $\left(500 \mathrm{MHz}, \mathrm{CDCl}_{3}\right) \delta 7.63(\mathrm{~d}, J=1.8 \mathrm{~Hz}, 2 \mathrm{H}), 7.47(\mathrm{dd}, J=7.9,1.9 \mathrm{~Hz}, 2 \mathrm{H}), 7.45-7.43(\mathrm{~m}, 2 \mathrm{H})$.

\section{2,7-dichloro-9-methyl-9H-fluoren-9-ol}

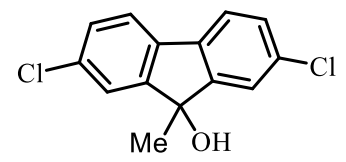

Under $\mathrm{N}_{2}$ atmosphere, 2,7-dichloro-9H-fluoren-9-one (2.12 g, $\left.8.5 \mathrm{mmol}\right)$ was dissolved in anhydrous THF (50 mL), then $\mathrm{MeMgCl}(2 \mathrm{M}, 12.75 \mathrm{~mL}, 25.5 \mathrm{mmol}$.) was added dropwise at $0^{\circ} \mathrm{C}$. And, the resulting reaction mixture was stirred for $30 \mathrm{~min}$ at the same temperature. After that, the reaction mixture was allowed to warm to room temperature and stirring at this temperature for $12 \mathrm{~h}$. Then the reaction mixture was quenched with saturated $\mathrm{NH}_{4} \mathrm{Cl}$ solution and extracted with ethyl acetate three times. The combined organic phases were washed with brine, dried over $\mathrm{Na}_{2} \mathrm{SO}_{4}$, and concentrated in vacuo. The crude mixture was purified by column chromatography on silica gel (hexane: ethyl acetate $=15: 1$ ) to afford the target compound (1.85 g, 82\% yield) as a yellow solid.

${ }^{1} \mathrm{H}$ NMR $\left(500 \mathrm{MHz}, \mathrm{CDCl}_{3}\right) \delta 7.53-7.48(\mathrm{~m}, 4 \mathrm{H}), 7.34(\mathrm{dd}, J=8.1,2.0 \mathrm{~Hz}, 2 \mathrm{H}), 1.70(\mathrm{~s}, 3 \mathrm{H}) .{ }^{13} \mathrm{C} \mathrm{NMR}(126 \mathrm{MHz}$ 


\section{9-butyl-2,7-dichloro-9H-fluoren-9-ol}

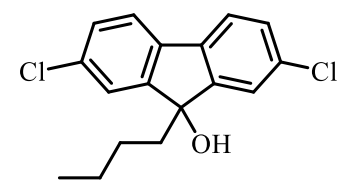

Under $\mathrm{N}_{2}$ atmosphere, 2,7-dichloro-9H-fluoren-9-one (2.12 g, $\left.8.5 \mathrm{mmol}\right)$ was

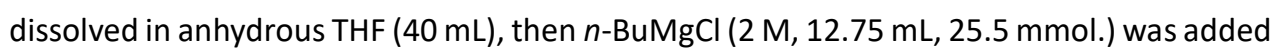
dropwise at $0{ }^{\circ} \mathrm{C}$. And, the resulting reaction mixture was stirred for $30 \mathrm{~min}$ at the same temperature. After that, the reaction mixture was allowed to warm to room temperature and stirring at this temperature for $12 \mathrm{~h}$. Then the reaction mixture was quenched with saturated $\mathrm{NH}_{4} \mathrm{Cl}$ solution and extracted with ethyl acetate three times. The combined organic phases were washed with brine, dried over $\mathrm{Na}_{2} \mathrm{SO}_{4}$, and concentrated in vacuo. The crude mixture was purified by column chromatography on silica gel (hexane: ethyl acetate = 15: 1 ) to afford the target compound $(1.85 \mathrm{~g}, 71 \%)$ as a yellow solid.

${ }^{1} \mathrm{H}$ NMR $\left(500 \mathrm{MHz}, \mathrm{CDCl}_{3}\right) \delta 7.51-7.45(\mathrm{~m}, 4 \mathrm{H}), 7.35-7.31(\mathrm{~m}, 2 \mathrm{H}), 2.13-2.03(\mathrm{~m}, 3 \mathrm{H}), 1.24-1.15(\mathrm{~m}, 2 \mathrm{H})$, $0.89-0.81(\mathrm{~m}, 2 \mathrm{H}), 0.77(\mathrm{t}, J=7.4 \mathrm{~Hz}, 3 \mathrm{H}) .{ }^{13} \mathrm{CNMR}\left(126 \mathrm{MHz}, \mathrm{CDCl}_{3}\right) \delta 150.6,137.2,134.2,129.4,124.3,121.2,82.5$ $39.4,26.0,22.9,13.9$.

\section{2-((2,7-dichloro-9-methyl-9H-fluoren-9-yl)oxy)acetic acid}

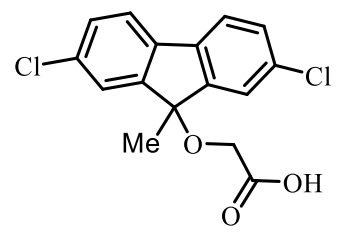

Under $\mathrm{N}_{2}$ atmosphere, $\mathrm{NaH}$ (60\% dispersion in mineral oil, $360 \mathrm{mg}, 9 \mathrm{mmol}$ ) was suspended in anhydrous THF $(10 \mathrm{~mL})$ and the mixture was allowed to cool to $0{ }^{\circ} \mathrm{C}$. Next, methyl bromoacetate $(626 \mathrm{mg}, 4.5 \mathrm{mmol})$ in anhydrous THF $(2 \mathrm{~mL})$ was added dropwise into the reaction mixture at $0{ }^{\circ} \mathrm{C}$, and the solution was allowed to warm to room temperature and stir for $1 \mathrm{~h}$. 2,7 dichloro-9-methyl-9H-fluoren-9-ol (921 mg, $3 \mathrm{mmol}$ ) anhydrous DMF (3 mL) was added dropwise at $0{ }^{\circ} \mathrm{C} \cdot{ }^{[11]} \mathrm{And}$, the resulting reaction mixture was stirred for $30 \mathrm{~min}$ at the same temperature. After that, the reaction mixture was allowed to warm to room temperature and stirring at this temperature overnight. Then the reaction mixture was quenched with saturated $\mathrm{NH}_{4} \mathrm{Cl}$ solution and extracted with ethyl acetate three times. The combined organic phases were washed with brine, dried over $\mathrm{Na}_{2} \mathrm{SO}_{4}$, and concentrated in vacuo. The crude mixture was purified by column chromatography on silica gel (hexane: acetone $=5: 1$ ) to afford the target compound (700 mg, $62 \%)$ as a yellow solid.

${ }^{1} \mathrm{H}$ NMR $\left(500 \mathrm{MHz}, \mathrm{CDCl}_{3}\right) \delta 7.54(\mathrm{dd}, J=8.1,0.6 \mathrm{~Hz}, 2 \mathrm{H}), 7.46(\mathrm{dd}, J=2.0,0.5 \mathrm{~Hz}, 2 \mathrm{H}), 7.39(\mathrm{dd}, J=8.1,1.9 \mathrm{~Hz}$, 
2H), 3.47 (s, 2H), $1.76(\mathrm{~s}, 3 \mathrm{H}) .{ }^{13} \mathrm{C}$ NMR $\left(126 \mathrm{MHz}, \mathrm{CDCl}_{3}\right) \delta 173.2,146.9,137.5,134.6,130.2,124.6,121.5,85.2,61.8$, 26.1. HRMS (m/z, ESI): Calcd. for Chemical Formula: $\mathrm{C}_{16} \mathrm{H}_{11} \mathrm{Cl}_{2} \mathrm{O}_{3}{ }^{-}[\mathrm{M}-\mathrm{H}]^{-}: 321.0091$, Found: 321.0088.

\section{2-((9-butyl-2,7-dichloro-9H-fluoren-9-yl)oxy)acetic acid}

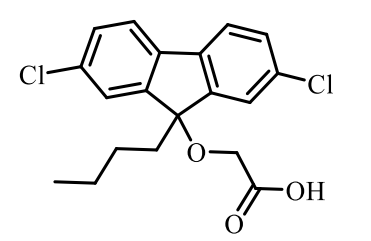

Under $\mathrm{N}_{2}$ atmosphere, $\mathrm{NaH}$ (60\% dispersion in mineral oil, $72 \mathrm{mg}, 3$ equiv) was suspended in anhydrous THF (5 mL) and the mixture was allowed to cool to $0{ }^{\circ} \mathrm{C}$. Next, methyl bromoacetate $(209 \mathrm{mg}, 1.5 \mathrm{mmol})$ in anhydrous THF $(2 \mathrm{~mL})$ was added dropwise into the reaction mixture at $0^{\circ} \mathrm{C}$, and the solution was allowed to warm to room temperature and stir for $1 \mathrm{~h}$. 9butyl-2,7-dichloro-9H-fluoren-9-ol $(307 \mathrm{mg}, 1 \mathrm{mmol})$ anhydrous DMF $(1.5 \mathrm{~mL})$ was added dropwise at $0{ }^{\circ} \mathrm{C}$. And, the resulting reaction mixture was stirred for $30 \mathrm{~min}$ at the same temperature. After that, the reaction mixture was allowed to warm to room temperature and stirring at this temperature overnight. Then the reaction mixture was quenched with saturated $\mathrm{NH}_{4} \mathrm{Cl}$ solution and extracted with ethyl acetate three times. The combined organic phases were washed with brine, dried over $\mathrm{Na}_{2} \mathrm{SO}_{4}$, and concentrated in vacuo. The crude mixture was purified by column chromatography on silica gel (hexane: acetone $=5: 1$ ) to afford the target compound $(281 \mathrm{mg}, 77 \%)$ as a yellow solid.

${ }^{1} \mathrm{H}$ NMR $\left(500 \mathrm{MHz}, \mathrm{CDCl}_{3}\right) \delta 7.57-7.51(\mathrm{~m}, 2 \mathrm{H}), 7.46-7.35(\mathrm{~m}, 4 \mathrm{H}), 3.48(\mathrm{~s}, 2 \mathrm{H}), 2.24-2.12(\mathrm{~m}, 2 \mathrm{H}), 1.19-$ $1.16(\mathrm{~m}, 2 \mathrm{H}), 0.86-0.68(\mathrm{~m}, 5 \mathrm{H}) .{ }^{13} \mathrm{C} N M R\left(126 \mathrm{MHz}, \mathrm{CDCl}_{3}\right) \delta 172.6,145.9,138.3,134.6,130.2,124.7,121.4,88.6$ 61.6, 39.1, 25.5, 22.8, 13.9. HRMS (m/z, ESI): Calcd. for Chemical Formula: $\mathrm{C}_{19} \mathrm{H}_{17} \mathrm{Cl}_{2} \mathrm{O}_{3}{ }^{-}[\mathrm{M}-\mathrm{H}]^{-}:$: 363.0560, Found: 363.0563.

10. Substrate for synthetic applications.

\section{4-(9-((1,3-dioxolan-2-yl)methyl)-2,7-dichloro-9H-fluoren-9-yl)butanoic acid (57)}


The substrate was prepared following the general procedure for synthesis of fluorene substrates described in Part 1.<smiles>Clc1ccc2c(c1)Cc1cc(Cl)ccc1-2</smiles>
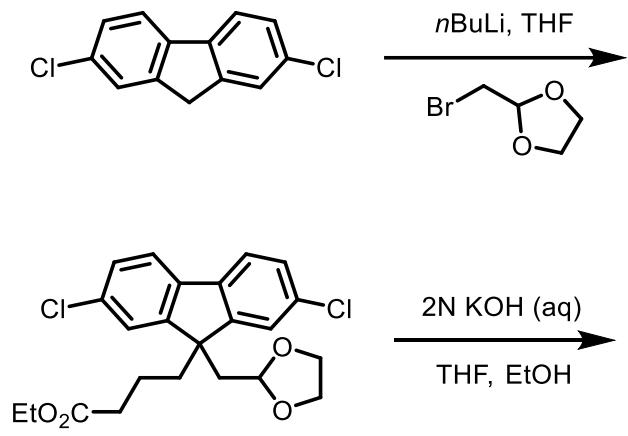

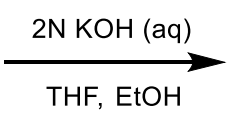

THF, EtOH
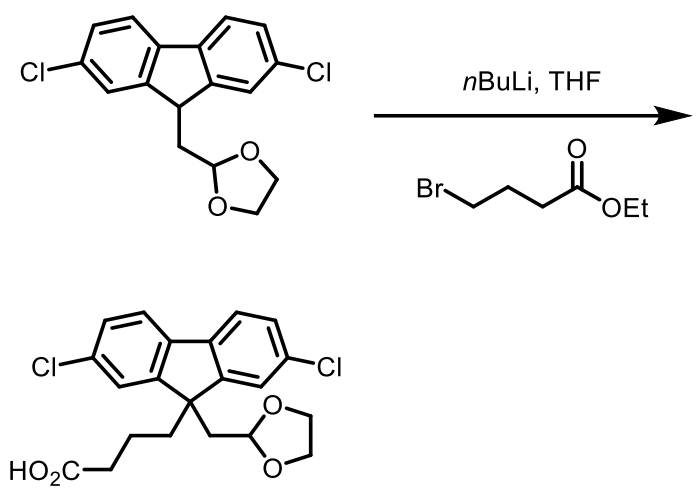

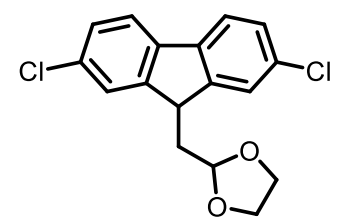

Under $\mathrm{N}_{2}$ atmosphere, 2,7-dichloro-9H-fluorene (1.7 g, $5 \mathrm{mmol}$ ) was dissolved in anhydrous THF $(20 \mathrm{~mL})$. A solution of $n B$ BuLi in cyclohexane $(2 \mathrm{M}, 3.0 \mathrm{~mL}, 6.0 \mathrm{mmol})$ was added dropwise at $-78{ }^{\circ} \mathrm{C}$. Then, the reaction mixture was stirring for $30 \mathrm{~min}$ at room temperature, and cooled back to $-78^{\circ} \mathrm{C}$. 2-(bromomethyl)-1,3-dioxolane $(1.09 \mathrm{~g}, 6.5 \mathrm{mmol})$ was added dropwise at $-78{ }^{\circ} \mathrm{C}$. The resulting reaction mixture was warmed to room temperature and stirred overnight. The reaction was quenched with saturated $\mathrm{NH}_{4} \mathrm{Cl}$ solution and extracted with ethyl acetate three times. The combined organic phases were washed with brine, dried over $\mathrm{Na}_{2} \mathrm{SO}_{4}$, and concentrated in vacuo. The crude product was purified by column chromatography on silica gel (hexane) to afford a light-yellow solid (788 mg, 49\%).

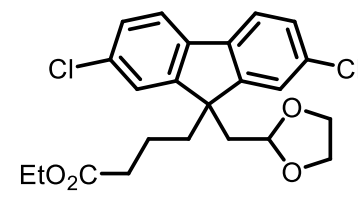

Under $\mathrm{N}_{2}$ atmosphere, 2-((2,7-dichloro-9H-fluoren-9-yl)methyl)-1,3-dioxolane (788 $\mathrm{mg}, 2.46 \mathrm{mmol})$ was dissolved in anhydrous THF $(10 \mathrm{~mL})$. A solution of $n$ BuLi in cyclohexane $(2 \mathrm{M}, 1.48 \mathrm{~mL}, 2.95 \mathrm{mmol})$ was added dropwise at $-78{ }^{\circ} \mathrm{C}$. Then, the reaction mixture was stirring for $30 \mathrm{~min}$ at room temperature, and cooled back to $-78{ }^{\circ} \mathrm{C}$. Ethyl 4 -bromobutyrate $(600 \mathrm{mg}$, $3.08 \mathrm{mmol}$ ) was added dropwise at $-78^{\circ} \mathrm{C}$. The resulting reaction mixture was warmed to room temperature and stirred overnight. The reaction was quenched with saturated $\mathrm{NH}_{4} \mathrm{Cl}$ solution and extracted with ethyl acetate three times. The combined organic phases were washed with brine, dried over $\mathrm{Na}_{2} \mathrm{SO}_{4}$, and concentrated in vacuo. The crude product was purified by column chromatography on silica gel (hexane : acetone $=100: 1$ ) to afford a lightyellow solid (448 $\mathrm{mg}, 46 \%)$. 


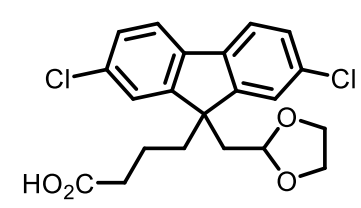

Ethyl 4-(9-((1,3-dioxolan-2-yl)methyl)-2,7-dichloro-9H-fluoren-9-yl)butanoate (1.0 g, $2.3 \mathrm{mmol}$ ) was dissolved in $3.0 \mathrm{~mL}$ THF and $3.0 \mathrm{~mL} \mathrm{EtOH}$, and $2 \mathrm{M} \mathrm{KOH}(11.5 \mathrm{mmol}, 23$ $\mathrm{mL}$ ) was added. The reaction mixtures were stirred at reflux overnight and solvents were removed under reduced pressure. Water was added and the aqueous solution was acidified to $\mathrm{pH} 2-3$ with $1 \mathrm{M} \mathrm{HCl}$ solution. The reaction was extracted with ethyl acetate $(10 \mathrm{~mL} \times 3)$ three times. The combined organic phases were washed with brine, dried over $\mathrm{Na}_{2} \mathrm{SO}_{4}$, and concentrated in vacuo. The crude materials were purified by flash column chromatography (hexane $:$ acetone $=5: 1)$ to give the desired product $(0.85 \mathrm{~g}, 91 \%)$ yield as a white solid. ${ }^{1} \mathrm{H}$ NMR $\left(500 \mathrm{MHz}, \mathrm{CDCl}_{3}\right) \delta 7.57(\mathrm{~d}, J=8.1 \mathrm{~Hz}, 2 \mathrm{H}), 7.35(\mathrm{~d}, J=1.9 \mathrm{~Hz}, 2 \mathrm{H}), 7.32(\mathrm{dd}, J=8.1,1.8 \mathrm{~Hz}, 2 \mathrm{H}), 4.27(\mathrm{t}, J=4.8 \mathrm{~Hz}$, $1 \mathrm{H}), 3.76-3.70(\mathrm{~m}, 2 \mathrm{H}), 3.59-3.50(\mathrm{~m}, 2 \mathrm{H}), 2.29(\mathrm{~d}, \mathrm{~J}=4.8 \mathrm{~Hz}, 2 \mathrm{H}), 2.11(\mathrm{t}, \mathrm{J}=7.4 \mathrm{~Hz}, 2 \mathrm{H}), 2.07-2.00(\mathrm{~m}, 2 \mathrm{H}), 0.92$ -0.85 (m, 2H). ${ }^{13} \mathrm{C}$ NMR $\left(101 \mathrm{MHz}, \mathrm{CDCl}_{3}\right) \delta$ 177.0, 150.7, 138.4, 133.4, 128.0, 124.1, 121.1, 102.0, 64.6, 52.8, 43.7, 33.5, 18.8. $\mathrm{MS}$ (m/z, ESI): Calcd. for Chemical Formula: $\mathrm{C}_{21} \mathrm{H}_{19} \mathrm{Cl}_{2} \mathrm{O}_{4}^{-}[\mathrm{M}-\mathrm{H}]^{-}: 405.0666$, Found: 405.0667. 


\section{Optimization of reaction conditions (Tables S1-S5)}

Table S1. Pd sources ${ }^{\text {a }}$
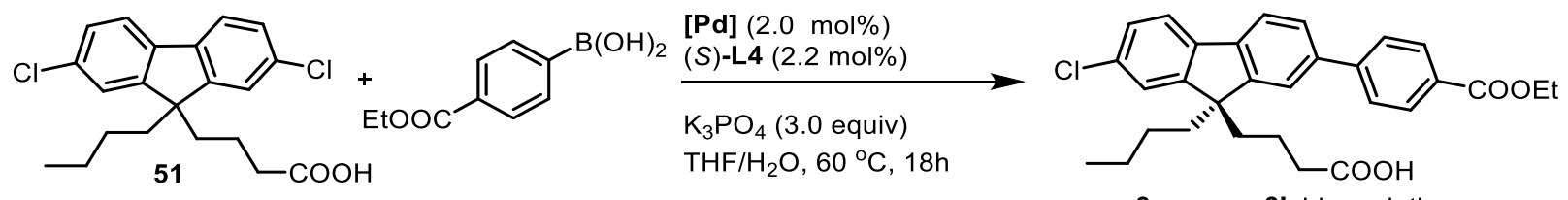

3: mono-; 3': bis-arylation

\begin{tabular}{llllll}
\hline entry & Pd source & $\mathbf{5 1}(\%)^{\mathrm{b}}$ & $\mathbf{3}(\%)^{\mathrm{b}}$ & $\mathbf{3}(\mathrm{er})^{\mathrm{c}}$ & $\mathbf{3}^{\mathbf{\prime}}(\%)^{\mathrm{b}}$ \\
\hline 1 & {$[\mathrm{Pd}(\mathrm{allyl}) \mathrm{Cl}]_{2}$} & 9 & 76 & $93: 7$ & 15 \\
2 & $\mathrm{Pd}\left(\mathrm{CH}_{2} \mathrm{TMS}\right)_{2}(\mathrm{COD})$ & 8 & 75 & $94: 6$ & 17 \\
3 & $\mathrm{Pd}(\mathrm{OAc})_{2}$ & 5 & 79 & $77: 23$ & 16 \\
4 & $\mathrm{Pd}_{2}(\mathrm{dba})_{3}$ & 4 & 79 & $94: 6$ & 17 \\
5 & {$\left[\mathrm{Pd}(2-\text { Butenyl)Cl }]_{2}\right.$} & 6 & 78 & $83: 17$ & 16
\end{tabular}

[a] Unless otherwise stated, the reactions were performed with substrate $(0.1 \mathrm{mmol})$, aryl boronic acid $(0.1 \mathrm{mmol})$,

[Pd] (2.0 mol\%), (S)-L4 (2.2 mol\%), $\mathrm{K}_{3} \mathrm{PO}_{4}(0.3 \mathrm{mmol})$ in $0.95 \mathrm{~mL} \mathrm{THF}$ and $0.05 \mathrm{~mL} \mathrm{H} \mathrm{H}_{2}$ at $60{ }^{\circ} \mathrm{C}$ for $18 \mathrm{~h}$. [b] The ratio of starting material, mono and bis were determined by their corresponding ${ }^{1} \mathrm{H}$ NMR of crude reaction mixture. [c] Determined using chiral chromatography. 
Table S2. Solvents ${ }^{a}$
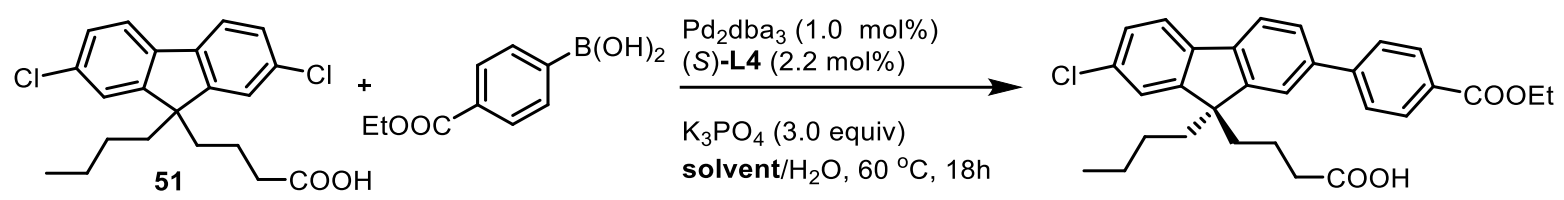

3: mono-; 3': bis-arylation

\begin{tabular}{llllll}
\hline entry & solvent & $\mathbf{5 1}(\%)^{\mathrm{b}}$ & $\mathbf{3}(\%)^{\mathrm{b}}$ & $\mathbf{3}(\mathrm{er})^{\mathrm{c}}$ & $\mathbf{3}^{\prime}(\%)^{\mathrm{b}}$ \\
\hline 1 & THF & 4 & 79 & $94: 6$ & 17 \\
2 & Ethyl acetate & 13 & 70 & $94: 6$ & 17 \\
3 & Dimethylformamide & 33 & 52 & $66: 34$ & 17 \\
4 & Dimethoxyethane & 13 & 68 & $93: 7$ & 19 \\
5 & Toluene & 18 & 64 & $91: 9$ & 18 \\
6 & 2-MeTHF & 14 & 73 & $94: 6$ & 13
\end{tabular}

[a] Unless otherwise stated, the reactions were performed with $51(0.1 \mathrm{mmol})$, aryl boronic acid $(0.1 \mathrm{mmol})$, $\mathrm{Pd}_{2}(\mathrm{dba})_{3}(1.0 \mathrm{~mol} \%),(\mathrm{S})-\mathrm{L} 4$ (2.2 mol\%), $\mathrm{K}_{3} \mathrm{PO}_{4}(0.3 \mathrm{mmol})$ in $0.95 \mathrm{~mL} \mathrm{THF}$ and $0.05 \mathrm{~mL} \mathrm{H} \mathrm{O}_{2}$ at $60{ }^{\circ} \mathrm{C}$ for $18 \mathrm{~h}$. [b] The ratio of starting material, mono and bis were determined by their corresponding ${ }^{1} \mathrm{H}$ NMR of crude reaction mixture. [c] Determined using chiral chromatography. 
Table S3. Metal to ligand ratio, catalyst loading, temperature, quantity of base and boronic acid ${ }^{\text {a }}$
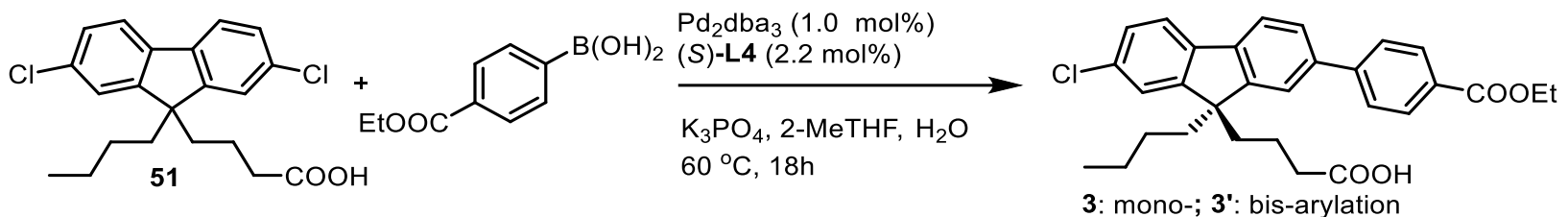

\begin{tabular}{|c|c|c|c|c|c|}
\hline entry & condition changes & $51(\%)^{b}$ & $3(\%)^{b}$ & $3(e r)^{c}$ & $3^{\prime}(\%)^{b}$ \\
\hline 1 & -- & 14 & 73 & $94: 6$ & 13 \\
\hline 2 & $45^{\circ} \mathrm{C}$ & $>90 \%$ & $<10 \%$ & n.d. & n.d. \\
\hline 3 & $75^{\circ} \mathrm{C}$ & 12 & 73 & $93: 7$ & 15 \\
\hline 4 & $1.0 \mathrm{~mol}^{2} \mathrm{Pd}_{2} \mathrm{bda}_{3}, 4.4 \mathrm{~mol} \%(S)-\mathrm{L} 4$ & 10 & 73 & $93.5: 6.5$ & 17 \\
\hline 5 & $2.0 \mathrm{~mol}^{2} \mathrm{Pd}_{2} \mathrm{bda}_{3}, 4.4 \mathrm{~mol} \%(S)-\mathrm{L} 4$ & 9 & 73 & 93.5:6.5 & 18 \\
\hline 6 & 10 equiv $\mathrm{K}_{3} \mathrm{PO}_{4}, 0.165 \mathrm{~mL} \mathrm{H}{ }_{2} \mathrm{O}$ & 8 & 74 & 93.5:6.5 & 18 \\
\hline \multirow[t]{2}{*}{7} & 1.1 equiv aryl boronic acid, 10 eq $\mathrm{K}_{3} \mathrm{PO}_{4}$, & 7 & 74 & $95: 5$ & 19 \\
\hline & $0.165 \mathrm{~mL} \mathrm{H} \mathrm{H}_{2}$ & & & & \\
\hline \multirow[t]{2}{*}{8} & 1.2 equiv aryl boronic acid, 10 eq $\mathrm{K}_{3} \mathrm{PO}_{4}$ & 6 & 74 & $96: 4$ & 20 \\
\hline & $0.165 \mathrm{~mL} \mathrm{H} 2 \mathrm{O}$ & & & & \\
\hline
\end{tabular}

[a] Unless otherwise stated, the reactions were performed with $\mathbf{5 1}(0.1 \mathrm{mmol})$, aryl boronic acid $(0.1 \mathrm{mmol})$, $\mathrm{Pd}_{2}(\mathrm{dba})_{3}(1.0 \mathrm{~mol} \%),(S)-\mathrm{L} 4(2.2 \mathrm{~mol} \%), \mathrm{K}_{3} \mathrm{PO}_{4}(0.3 \mathrm{mmol})$ in $0.95 \mathrm{~mL} \mathrm{THF}$ and $0.05 \mathrm{~mL} \mathrm{H} \mathrm{O}_{2}$ at $60{ }^{\circ} \mathrm{C}$ for $18 \mathrm{~h}$. [b] The ratio of starting material, mono and bis were determined by their corresponding ${ }^{1} \mathrm{H}$ NMR of crude reaction mixture. [c] Determined using chiral chromatography. 
Table S4. Concentration ${ }^{\mathrm{a}}$

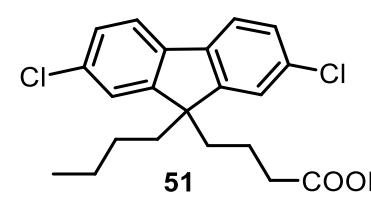

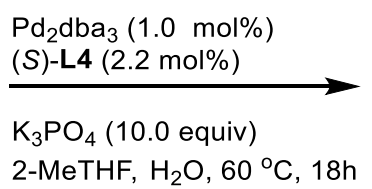

2-MeTHF, $\mathrm{H}_{2} \mathrm{O}, 60^{\circ} \mathrm{C}, 18 \mathrm{~h}$

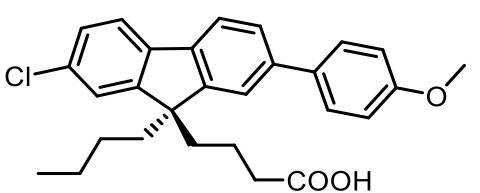

20: mono-; 20': bis-arylation

\begin{tabular}{|c|c|c|c|c|c|}
\hline entry & Condition changes & $51(\%)^{b}$ & $20(\%)^{b}$ & $20(\mathrm{er})^{\mathrm{c}}$ & $20^{\prime}(\%)^{b}$ \\
\hline 1 & -- & 10 & 72 & $94: 6$ & 18 \\
\hline 2 & $0.5 \mathrm{~mL}$ 2-MeTHF & 7 & 75 & $96: 4$ & 18 \\
\hline 3 & $2.0 \mathrm{~mL}$ 2-MeTHF & 6 & 80 & $96: 4$ & 14 \\
\hline 4 & $6.0 \mathrm{~mL}$ 2-MeTHF & 8 & 80 & $97: 3$ & 12 \\
\hline 5 & $6.0 \mathrm{~mL}$ 2-MeTHF, $1.0 \mathrm{~mL} \mathrm{H} \mathrm{H}_{2}$ & 5 & 69 & $95: 5$ & 26 \\
\hline
\end{tabular}

[a] Unless otherwise stated, the reactions were performed with $51(0.1 \mathrm{mmol})$, arylboronic acid $(0.12 \mathrm{mmol})$, $\mathrm{Pd}_{2}(\mathrm{dba})_{3}(1.0 \mathrm{~mol} \%),(\mathrm{S})-\mathrm{L} 4(2.2 \mathrm{~mol} \%), \mathrm{K}_{3} \mathrm{PO}_{4}(1.0 \mathrm{mmol})$ in $0.95 \mathrm{~mL}$ 2-MeTHF and $0.165 \mathrm{~mL} \mathrm{H} \mathrm{O}$ at $60{ }^{\circ} \mathrm{C}$ for $18 \mathrm{~h}$. [b] The ratio of starting material, mono and bis were determined by their corresponding ${ }^{1} \mathrm{H}$ NMR of crude reaction mixture. [c] Determined using chiral chromatography. 
Table S5. Bases ${ }^{a}$
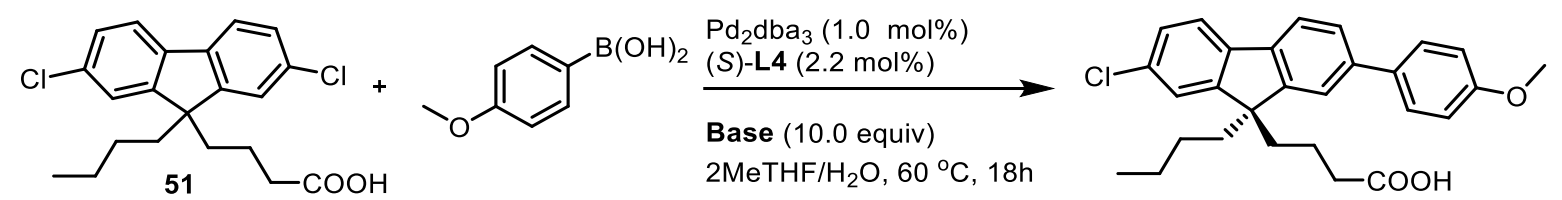

20: mono-; 20': bis-arylation

\begin{tabular}{llllll}
\hline entry & base & $\mathbf{5 1}(\%)^{\mathrm{b}}$ & $\mathbf{2 0}(\%)^{\mathrm{b}}$ & $\mathbf{2 0}(\mathrm{er})^{\mathrm{c}}$ & $\mathbf{2 0}^{\prime}(\%)^{\mathrm{b}}$ \\
\hline 1 & $\mathrm{~K}_{3} \mathrm{PO}_{4}$ & 6 & 80 & $96: 4$ & 14 \\
2 & $\mathrm{Li}_{2} \mathrm{CO}_{3}$ & 72 & 26 & $75: 25$ & 2 \\
3 & $\mathrm{Na}_{2} \mathrm{CO}_{3}$ & 13 & 71 & $96: 4$ & 16 \\
4 & $\mathrm{~K}_{2} \mathrm{CO}_{3}$ & 10 & 73 & $96: 4$ & 18 \\
5 & $\mathrm{Cs}_{2} \mathrm{CO}_{3}$ & 9 & 70 & $96: 4$ & 21 \\
6 & $\mathrm{LiOH}_{7}$ & 61 & 36 & $86: 13$ & 3 \\
8 & $\mathrm{NaOH}$ & 7 & 74 & $97: 3$ & 19 \\
9 & $\mathrm{KOH}$ & 7 & 73 & $97: 3$ & 20 \\
10 & $\mathrm{CSOH}$ & 6 & 70 & $97: 3$ & 24 \\
\hline
\end{tabular}

[a] Unless otherwise stated, the reactions were performed with $51(0.1 \mathrm{mmol})$, arylboronic acid $(0.12 \mathrm{mmol})$, $\mathrm{Pd}_{2}(\mathrm{dba})_{3}(1.0 \mathrm{~mol} \%),(\mathrm{S})-\mathrm{L} 4(2.2 \mathrm{~mol} \%), \mathrm{K}_{3} \mathrm{PO}_{4}(1.0 \mathrm{mmol})$ in $2.0 \mathrm{~mL}$ 2-MeTHF and $0.165 \mathrm{~mL} \mathrm{H} \mathrm{O} \mathrm{O}$ at $60{ }^{\circ} \mathrm{C}$ for $18 \mathrm{~h}$. [b] The ratio of starting material, mono and bis were determined by their corresponding ${ }^{1} \mathrm{H}$ NMR of crude reaction mixture. [c] Determined using chiral chromatography. 


\section{General procedure of catalytic desymmetrization reaction}

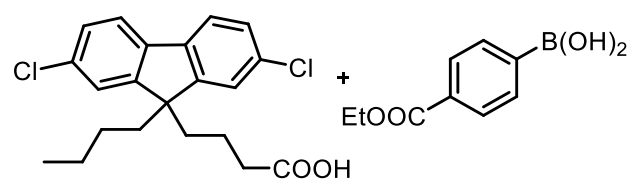

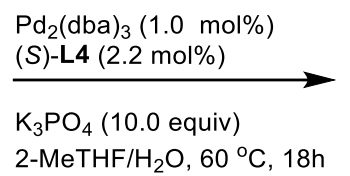

Under $\mathrm{N}_{2}$ atmosphere, to a mixture of $\mathrm{Pd}_{2} \mathrm{dba}_{3}(2.3 \mathrm{mg}, 0.0025 \mathrm{mmol})$ and $(\mathrm{S})-\mathrm{L} 4(3.2 \mathrm{mg}, 0.0055 \mathrm{mmol})$ was added $0.5 \mathrm{~mL} \mathrm{2-MeTHF,} \mathrm{and} \mathrm{the} \mathrm{mixture} \mathrm{was} \mathrm{stirred} \mathrm{at} \mathrm{room} \mathrm{temperature} \mathrm{for} 20 \mathrm{~min}$. The resulting metal-ligand complex solution was added to a reaction flask containing $4.5 \mathrm{~mL}$ 2-MeTHF solution of 4-(9-butyl-2,7-dichloro-9Hfluoren-9-yl)butanoic acid $(94.4 \mathrm{mg}, 0.25 \mathrm{mmol}$ ) and (4-(ethoxycarbonyl)phenyl)boronic acid $(58.2 \mathrm{mg}, 0.3 \mathrm{mmol})$, and $\mathrm{K}_{3} \mathrm{PO}_{4}(530.7 \mathrm{mg}, 2.5 \mathrm{mmol})$, followed by addition of $0.41 \mathrm{~mL} \mathrm{H} 2 \mathrm{O}$. Then the resulting reaction mixture was stirred at $60^{\circ} \mathrm{C}$ for $18 \mathrm{~h}$. The reaction was then quenched with water, neutralized to $\mathrm{pH}$ 3-5 with $1 \mathrm{M} \mathrm{HCl}$ and extracted with ethyl acetate $(4 \mathrm{~mL})$ for three times. The combined organic phases were washed with brine, dried over $\mathrm{Na}_{2} \mathrm{SO}_{4}$ and concentrated in vacuo. The crude materials were purified by flash column chromatography with hexane and ethyl acetate as the eluents to give the desired products. 


\section{Characterization data of desymmetrization products}

(R)-ethyl 4-(9-butyl-7-chloro-9-(4-((trifluoromethyl)sulfonamido)butyl)-9H-fluoren-2-yl)benzoate (1)

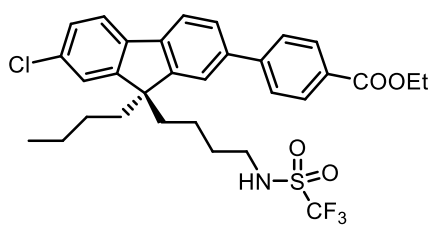

The reaction was carried out under conditions in Table 1.

White solid, $26.8 \mathrm{mg}, 44 \%$ yield, $[\alpha]_{\mathrm{D}}{ }^{20}=+1.67\left(\mathrm{c} 0.100 \mathrm{CHCl}_{3}\right)$ for $73: 27$ er. ${ }^{1} \mathrm{H}$ $\operatorname{NMR}\left(500 \mathrm{MHz}, \mathrm{CDCl}_{3}\right) \delta 8.23-8.07(\mathrm{~m}, 2 \mathrm{H}), 7.75(\mathrm{ddd}, J=9.0,8.5,4.8 \mathrm{~Hz}, 3 \mathrm{H})$, $7.69-7.63(\mathrm{~m}, 2 \mathrm{H}), 7.58(\mathrm{~d}, J=1.2 \mathrm{~Hz}, 1 \mathrm{H}), 7.37(\mathrm{dd}, J=8.0,1.9 \mathrm{~Hz}, 1 \mathrm{H}), 7.35(\mathrm{~d}, J=1.7 \mathrm{~Hz}, 1 \mathrm{H}), 4.65(\mathrm{~s}, 1 \mathrm{H}), 4.44(\mathrm{q}$, $J=7.1 \mathrm{~Hz}, 2 \mathrm{H}), 3.09-3.06(\mathrm{~m}, 2 \mathrm{H}), 2.14-1.97(\mathrm{~m}, 4 \mathrm{H}), 1.47-1.37(\mathrm{~m}, 5 \mathrm{H}), 1.14-1.10(\mathrm{~m}, 2 \mathrm{H}), 0.76-0.59(\mathrm{~m}, 7 \mathrm{H}) .{ }^{13} \mathrm{C}$ NMR $\left(126 \mathrm{MHz} \mathrm{CDCl}_{3}\right) \delta 166.6,152.2,150.6,145.5,140.2,139.5,139.1,133.4,130.1,129.3,127.6,127.0,126.7$ 123.2, 121.4, 121.1, 120.3, $119.6(q, J=321.4 \mathrm{~Hz}), 61.1,55.3,44.0,40.1,39.7,30.5,25.8,22.9,20.6,14.4,13.8 .{ }^{19} \mathrm{~F}$ NMR (471 MHz, $\mathrm{CDCl}_{3}$ ) $\delta$-77.40. HRMS (m/z, ESI): Calcd. for Chemical Formula: $\mathrm{C}_{31} \mathrm{H}_{32} \mathrm{ClF}_{3} \mathrm{NO}_{4} \mathrm{~S}^{-}[\mathrm{M}-\mathrm{H}]^{-}: 606.1698$, Found: 606.1700. HPLC analysis of the reaction product: Daicel Chiralpak IB, hexane/iso-propanol = $99: 1,1.0 \mathrm{~mL} / \mathrm{min}$, $\lambda=321 \mathrm{~nm}$, retention time: $24.47 \mathrm{~min}$ (major) and $27.09 \mathrm{~min}$ (minor).

\section{(R)-ethyl 4-(9-butyl-7-chloro-9-(4-hydroxybutyl)-9H-fluoren-2-yl)benzoate (4)}

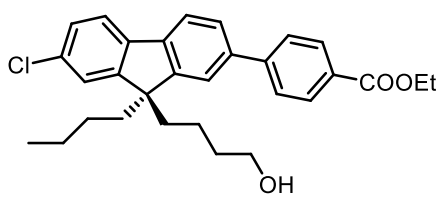

The reaction was carried out under conditions in Table 1.

White solid, $51.0 \mathrm{mg}, 43 \%$ yield, $[\alpha]_{\mathrm{D}}{ }^{20}=+1.10\left(\mathrm{c} 0.100 \mathrm{CHCl}_{3}\right)$ for $56: 44$ er. ${ }^{1} \mathrm{H}$ $\operatorname{NMR}\left(500 \mathrm{MHz}, \mathrm{CDCl}_{3}\right) \delta 8.16(\mathrm{~d}, J=8.5 \mathrm{~Hz}, 2 \mathrm{H}), 7.77-7.73(\mathrm{~m}, 3 \mathrm{H}), 7.69-7.62(\mathrm{~m}$,

2H), $7.58(\mathrm{~d}, J=0.9 \mathrm{~Hz}, 1 \mathrm{H}), 7.40-7.32(\mathrm{~m}, 2 \mathrm{H}), 4.44(\mathrm{q}, J=7.1 \mathrm{~Hz}, 2 \mathrm{H}), 3.88(\mathrm{t}, J=6.8 \mathrm{~Hz}, 2 \mathrm{H}), 2.11-1.99(\mathrm{~m}, 4 \mathrm{H})$, $1.92(\mathrm{~s}, 1 \mathrm{H}), 1.47-1.42(\mathrm{~m}, 5 \mathrm{H}), 1.15-1.11(\mathrm{~m}, 2 \mathrm{H}), 0.76-0.62(\mathrm{~m}, 7 \mathrm{H}) .{ }^{13} \mathrm{C} \mathrm{NMR}(126 \mathrm{MHz}, \mathrm{CDCl} 3) \delta 171.1,166.5$ $152.5,150.9,145.7,140.2,139.3,139.1,133.3,130.1,129.2,127.4,127.0,126.6,123.3,121.5,121.0,120.3,63.8$ 61.0, 55.4, 40.1, 39.7, 28.6, 25.9, 23.0, 20.9, 20.0, 14.4, 13.8. MS (m/z, ESI): Calcd. for Chemical Formula: $\mathrm{C}_{30} \mathrm{H}_{33} \mathrm{ClO}_{3} \mathrm{Na}^{+}[\mathrm{M}+\mathrm{Na}]^{+}:$499.2, Found: 499.3. HPLC analysis of the reaction product: Daicel Chiralpak IA, hexane/isopropanol $=99: 1,1.0 \mathrm{~mL} / \mathrm{min}, \lambda=324 \mathrm{~nm}$, retention time: $7.04 \mathrm{~min}$ (major) and $7.74 \mathrm{~min}$ (minor). 


\section{(R)-ethyl 4-(9-butyl-9-(4-(butylamino)-4-oxobutyl)-7-chloro-9H-fluoren-2-yl)benzoate (5)}

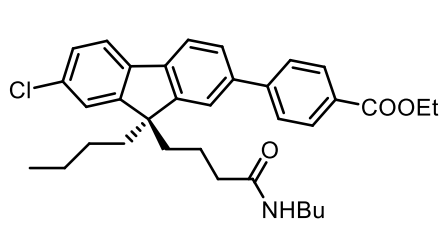

The reaction was carried out under conditions in Table 1.

White solid, $54.3 \mathrm{mg}, 40 \%$ yield, $[\alpha]_{\mathrm{D}}{ }^{20}=-1.80\left(\mathrm{c} 0.040 \mathrm{CHCl}_{3}\right)$ for $63: 37$ er. ${ }^{1} \mathrm{H}$ $\operatorname{NMR}\left(500 \mathrm{MHz}, \mathrm{CDCl}_{3}\right) \delta 8.18-8.13(\mathrm{~m}, 2 \mathrm{H}), 7.78-7.71(\mathrm{~m}, 3 \mathrm{H}), 7.68-7.62(\mathrm{~m}$, 2H), $7.58(\mathrm{~d}, J=1.3 \mathrm{~Hz}, 1 \mathrm{H}), 7.36(\mathrm{dd}, J=7.6,1.5 \mathrm{~Hz}, 2 \mathrm{H}), 5.15(\mathrm{~s}, 1 \mathrm{H}), 4.44(\mathrm{q}, J=7.1 \mathrm{~Hz}, 2 \mathrm{H}), 3.18-3.11(\mathrm{~m}, 2 \mathrm{H})$, $2.11-1.98(\mathrm{~m}, 4 \mathrm{H}), 1.94(\mathrm{t}, J=7.5 \mathrm{~Hz}, 2 \mathrm{H}), 1.45(\mathrm{t}, J=7.1 \mathrm{~Hz}, 3 \mathrm{H}), 1.41-1.36(\mathrm{~m}, 2 \mathrm{H}), 1.14-1.10(\mathrm{~m}, 2 \mathrm{H}), 1.04-1.01$ $(\mathrm{m}, 2 \mathrm{H}), 0.93-0.86(\mathrm{~m}, 3 \mathrm{H}), 0.71(\mathrm{t}, J=7.3 \mathrm{~Hz}, 3 \mathrm{H}), 0.67-0.61(\mathrm{~m}, 2 \mathrm{H}) .{ }^{13} \mathrm{C} \mathrm{NMR}\left(126 \mathrm{MHz}, \mathrm{CDCl}_{3}\right) \delta 172.3,166.6$, $152.4,150.7,140.2,139.4,139.1,130.1,129.2,127.5,127.1,126.6,123.3,121.6,121.1,120.3,76.9,61.0,55.3,40.1$, 39.7, 39.1, 36.8, 31.7, 25.9, 22.9, 20.4, 20.0, 14.4, 13.8, 13.7. HRMS (m/z, ESI): Calcd. for Chemical Formula: $\mathrm{C}_{34} \mathrm{H}_{39} \mathrm{ClNO}_{3}^{-}[\mathrm{M}-\mathrm{H}]^{-}:$544.2633, Found: 544.2624. HPLC analysis of the reaction product: Daicel Chiralpak IA, hexane/iso-propanol = 96: 4, $1.0 \mathrm{~mL} / \mathrm{min}, \lambda=330 \mathrm{~nm}$, retention time: $19.73 \mathrm{~min}$ (minor) and $20.89 \mathrm{~min}$ (major).

\section{(R)-ethyl 4-(9-butyl-7-chloro-9-(4-oxo-4-(phenylamino)butyl)-9H-fluoren-2-yl)benzoate (6)}

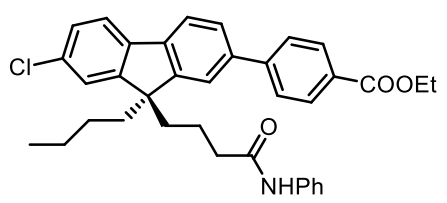

The reaction was carried out under conditions in Table 1.

White solid, $53.3 \mathrm{mg}, 38 \%$ yield, $[\alpha]_{\mathrm{D}}{ }^{20}=-4.00\left(\mathrm{c} 0.100 \mathrm{CHCl}_{3}\right)$ for $60: 40$ er. ${ }^{1} \mathrm{H}$ $\operatorname{NMR}\left(500 \mathrm{MHz}, \mathrm{CDCl}_{3}\right) \delta 8.18-8.09(\mathrm{~m}, 2 \mathrm{H}), 7.77(\mathrm{~d}, J=7.9 \mathrm{~Hz}, 1 \mathrm{H}), 7.75-7.70$

(m, 2H), $7.69-7.62(\mathrm{~m}, 2 \mathrm{H}), 7.60(\mathrm{~s}, 1 \mathrm{H}), 7.40(\mathrm{~d}, J=7.9 \mathrm{~Hz}, 2 \mathrm{H}), 7.36(\mathrm{dd}, J=6.1,2.3 \mathrm{~Hz}, 2 \mathrm{H}), 7.26(\mathrm{~d}, J=8.3 \mathrm{~Hz}, 2 \mathrm{H})$, $7.08(t, J=7.4 \mathrm{~Hz}, 1 \mathrm{H}), 6.92(\mathrm{~s}, 1 \mathrm{H}), 4.44(\mathrm{q}, J=7.1 \mathrm{~Hz}, 2 \mathrm{H}), 2.14-2.11(\mathrm{~m}, 4 \mathrm{H}), 2.06-1.96(\mathrm{~m}, 2 \mathrm{H}), 1.46(\mathrm{t}, J=7.1 \mathrm{~Hz}$, $3 \mathrm{H}), 1.13-1.00(\mathrm{~m}, 4 \mathrm{H}), 0.73-0.69(\mathrm{~m}, 3 \mathrm{H}), 0.67-0.64(\mathrm{~m}, 2 \mathrm{H}) .{ }^{13} \mathrm{C} \mathrm{NMR}\left(126 \mathrm{MHz}, \mathrm{CDCl}_{3}\right) \delta 170.6,166.6,152.3,150.6$, $145.6,140.2,139.4,139.2,137.7,133.4,130.1,129.2,129.0,127.6,127.1,126.7,124.2,123.3,121.6,121.1,120.3$ 119.6, 61.0, 55.3, 40.1, 39.5, 37.7, 25.8, 22.9, 20.2, 14.4, 13.8. HRMS (m/z, ESI): Calcd. for Chemical Formula: $\mathrm{C}_{36} \mathrm{H}_{35} \mathrm{ClNO}_{3}^{-}[\mathrm{M}-\mathrm{H}]^{-}:$564.2311, Found: 564.2308. HPLC analysis of the reaction product: Daicel Chiralpak IA, hexane/iso-propanol = 96: 4, $1.0 \mathrm{~mL} / \mathrm{min}, \lambda=329 \mathrm{~nm}$, retention time: $22.32 \mathrm{~min}$ (minor) and $35.64 \mathrm{~min}$ (major). 


\section{(R)-2-(9-butyl-2-chloro-7-(4-(ethoxycarbonyl)phenyl)-9H-fluoren-9-yl)acetic acid (7)}

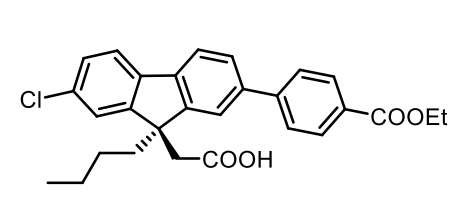

White solid, $48.3 \mathrm{mg}, 42 \%$ yield, $[\alpha]_{\mathrm{D}}^{20}=-0.30\left(\mathrm{c} 0.100 \mathrm{CHCl}_{3}\right)$ for 82.5:17.5 er.

${ }^{1} \mathrm{H}$ NMR $\left(500 \mathrm{MHz}, \mathrm{CDCl}_{3}\right) \delta 8.16-8.11(\mathrm{~m}, 2 \mathrm{H}), 7.75(\mathrm{dd}, J=7.9,0.4 \mathrm{~Hz}, 1 \mathrm{H}), 7.72$ $-7.68(\mathrm{~m}, 2 \mathrm{H}), 7.68-7.62(\mathrm{~m}, 3 \mathrm{H}), 7.44(\mathrm{~d}, J=1.8 \mathrm{~Hz}, 1 \mathrm{H}), 7.37(\mathrm{dd}, J=8.1,1.9 \mathrm{~Hz}$,

$1 \mathrm{H}), 4.44(\mathrm{q}, J=7.1 \mathrm{~Hz}, 2 \mathrm{H}), 3.02-2.92(\mathrm{~m}, 2 \mathrm{H}), 2.15-2.11(\mathrm{~m}, 2 \mathrm{H}), 1.45(\mathrm{t}, J=7.1 \mathrm{~Hz}, 3 \mathrm{H}), 1.16-1.12(\mathrm{~m}, 2 \mathrm{H}), 0.71(\mathrm{t}$, $J=7.4 \mathrm{~Hz}, 3 \mathrm{H}), 0.68-0.60(\mathrm{~m}, 2 \mathrm{H}) .{ }^{13} \mathrm{CNMR}\left(126 \mathrm{MHz}, \mathrm{CDCl}_{3}\right) \delta 174.1,166.6,151.2,149.5,145.5,139.8,139.4,138.8$, 133.3, 130.1, 129.2, 128.0, 127.1, 127.0, 123.7, 122.0, 121.1, 120.4, 61.0, 52.4, 43.3, 38.8, 25.7, 22.8, 14.4, 13.8. HRMS (m/z, ESI): Calcd. for Chemical Formula: $\mathrm{C}_{28} \mathrm{H}_{26} \mathrm{ClO}_{4}{ }^{-}[\mathrm{M}-\mathrm{H}]^{-:}$461.1525, Found: 461.1530. HPLC analysis of the reaction product: Daicel Chiralpak IA, hexane/iso-propanol $=90: 10,1.0 \mathrm{~mL} / \mathrm{min}, \lambda=331 \mathrm{~nm}$, retention time: 6.16 $\min$ (major) and $10.84 \min$ (minor).

\section{(R)-3-(9-butyl-2-chloro-7-(4-(ethoxycarbonyl)phenyl)-9H-fluoren-9-yl)propanoic acid (8)}

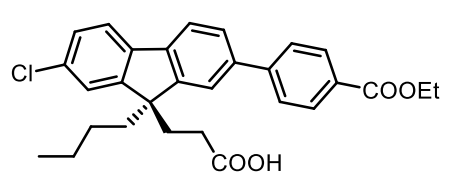

White solid, $66.3 \mathrm{mg}, 56 \%$ yield, $[\alpha]_{D}{ }^{20}=+5.30\left(c 0.100 \mathrm{CHCl}_{3}\right)$ for $90.5: 9.5$ er. ${ }^{1} \mathrm{H}$ NMR $\left(500 \mathrm{MHz}, \mathrm{CDCl}_{3}\right) \delta 8.17-8.12(\mathrm{~m}, 2 \mathrm{H}), 7.76(\mathrm{~d}, \mathrm{~J}=7.9 \mathrm{~Hz}, 1 \mathrm{H}), 7.73$ $-7.69(\mathrm{~m}, 2 \mathrm{H}), 7.67(\mathrm{~d}, J=8.0 \mathrm{~Hz}, 1 \mathrm{H}), 7.64(\mathrm{dd}, J=7.9,1.6 \mathrm{~Hz}, 1 \mathrm{H}), 7.58(\mathrm{~d}, J=$ $1.3 \mathrm{~Hz}, 1 \mathrm{H}), 7.39-7.32(\mathrm{~m}, 2 \mathrm{H}), 4.44(\mathrm{q}, J=7.1 \mathrm{~Hz}, 2 \mathrm{H}), 2.45-2.32(\mathrm{~m}, 2 \mathrm{H}), 2.08-1.99(\mathrm{~m}, 2 \mathrm{H}), 1.62(\mathrm{t}, J=8.1 \mathrm{~Hz}$, 2H), $1.45(\mathrm{t}, J=7.1 \mathrm{~Hz}, 3 \mathrm{H}), 1.15-1.10(\mathrm{~m}, 2 \mathrm{H}), 0.73-0.63(\mathrm{~m}, 5 \mathrm{H}) .{ }^{13} \mathrm{C} \mathrm{NMR}\left(126 \mathrm{MHz}, \mathrm{CDCl}_{3}\right) \delta 166.5,151.2,149.6$, $145.4,140.2,139.6,139.2,133.5,130.1,127.9,127.0,127.0,123.4,121.6,121.2,120.4,61.1,54.6,40.0,34.4,28.6$, 25.9, 22.9, 14.4, 13.8. HRMS (m/z, ESI): Calcd. for Chemical Formula: $\mathrm{C}_{29} \mathrm{H}_{28} \mathrm{ClO}_{4}{ }^{-}[\mathrm{M}-\mathrm{H}]^{-}: 475.1682$, Found: 475.1678. HPLC analysis of the reaction product: Daicel Chiralpak IA, hexane/iso-propanol $=90: 10,1.0 \mathrm{~mL} / \mathrm{min}, \lambda=320 \mathrm{~nm}$, retention time: $5.58 \mathrm{~min}$ (major) and $6.17 \mathrm{~min}$ (minor).

\section{(R)-4-(9-butyl-2-chloro-7-(4-(ethoxycarbonyl)phenyl)-9H-fluoren-9-yl)butanoic acid (3)}

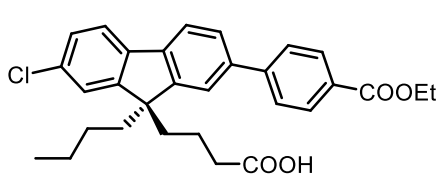

White solid, $82.4 \mathrm{mg}, 67 \%$ yield, $[\alpha]_{\mathrm{D}}{ }^{20}=+1.20\left(\mathrm{c} 0.080 \mathrm{CHCl}_{3}\right)$ for $96: 4$ er. ${ }^{1} \mathrm{H}$ $\operatorname{NMR}\left(500 \mathrm{MHz}, \mathrm{CDCl}_{3}\right) \delta 8.15(\mathrm{~d}, J=8.2 \mathrm{~Hz}, 2 \mathrm{H}), 7.76(\mathrm{~d}, J=7.9 \mathrm{~Hz}, 1 \mathrm{H}), 7.73(\mathrm{~d}, J$ $=8.2 \mathrm{~Hz}, 2 \mathrm{H}), 7.67(\mathrm{~d}, J=8.7 \mathrm{~Hz}, 1 \mathrm{H}), 7.63(\mathrm{dd}, J=7.9,1.3 \mathrm{~Hz}, 1 \mathrm{H}), 7.58(\mathrm{~d}, J=1.5$

$\mathrm{Hz}, 1 \mathrm{H}), 7.36(\mathrm{dd}, J=6.9,1.6 \mathrm{~Hz}, 2 \mathrm{H}), 4.44(\mathrm{q}, J=7.1 \mathrm{~Hz}, 2 \mathrm{H}), 2.16-1.99(\mathrm{~m}, 6 \mathrm{H}), 1.44(\mathrm{t}, J=7.2 \mathrm{~Hz}, 3 \mathrm{H}), 1.14-1.09$ 
$(\mathrm{m}, 2 \mathrm{H}), 1.02-0.98(\mathrm{~m}, 2 \mathrm{H}), 0.71(\mathrm{t}, J=7.3 \mathrm{~Hz}, 3 \mathrm{H}), 0.64(\mathrm{t}, J=8.0 \mathrm{~Hz}, 2 \mathrm{H}) .{ }^{13} \mathrm{C} \mathrm{NMR}\left(126 \mathrm{MHz}, \mathrm{CDCl}_{3}\right) \delta 166.6,152.2$ $150.6,145.7,140.1,139.4,139.1,133.3,130.1,127.6,127.1,126.7,123.3,121.6,121.1,120.3,61.0,55.2,40.2,39.4$ 33.5, 25.8, 22.9, 19.1, 14.4, 13.8. HRMS (m/z, ESI): Calcd. for Chemical Formula: $\mathrm{C}_{30} \mathrm{H}_{30} \mathrm{ClO}_{4}^{-}\left[\mathrm{M}^{-} \mathrm{H}^{-}:\right.$: 489.1837, Found: 489.1839. HPLC analysis of the reaction product: Daicel Chiralpak IA, hexane/iso-propanol $=90: 10,1.0 \mathrm{~mL} / \mathrm{min}, \lambda=$ $272 \mathrm{~nm}$, retention time: $4.87 \mathrm{~min}$ (major) and $6.05 \mathrm{~min}$ (minor).

\section{(R)-5-(9-butyl-2-chloro-7-(4-(ethoxycarbonyl)phenyl)-9H-fluoren-9-yl)pentanoic acid (9)}

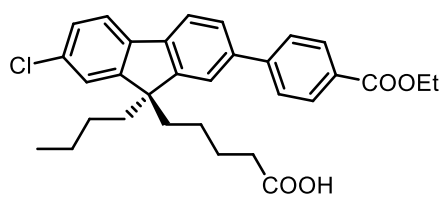

White solid, $75.9 \mathrm{mg}, 60 \%$ yield, $[\alpha]_{\mathrm{D}}^{20}=+12.4\left(\right.$ c $\left.0.100 \mathrm{CHCl}_{3}\right)$ for 95.5:4.5 er.

${ }^{1} \mathrm{H}$ NMR $\left(300 \mathrm{MHz}, \mathrm{CDCl}_{3}\right) \delta 8.17(\mathrm{~d}, \mathrm{~J}=8.3 \mathrm{~Hz}, 2 \mathrm{H}), 7.77-7.74(\mathrm{~m}, 1 \mathrm{H}), 7.74-7.72$ $(\mathrm{m}, 2 \mathrm{H}), 7.67-7.62(\mathrm{~m}, 2 \mathrm{H}), 7.5(\mathrm{~s}, 1 \mathrm{H}), 7.37(\mathrm{dd}, J=6.5,1.8 \mathrm{~Hz}, 2 \mathrm{H}), 4.45(\mathrm{q}, J=7.1$

$\mathrm{Hz}, 2 \mathrm{H}), 2.15-2.00(\mathrm{~m}, 6 \mathrm{H}), 1.51-1.36(\mathrm{~m}, 5 \mathrm{H}), 1.15-1.12(\mathrm{~m}, 2 \mathrm{H}), 0.94-0.54(\mathrm{~m}, 7 \mathrm{H}) .{ }^{13} \mathrm{C} \mathrm{NMR}(75 \mathrm{MHz}, \mathrm{CDCl} 3) \delta$ $178.6,166.6,152.5,150.9,145.7,140.2,139.3,139.1,133.3,130.1,129.2,127.4,127.0,126.6,123.3,121.5,120.9$, 120.2, 61.0, 55.3, 40.1, 39.9, 33.5, 33.4, 25.9, 24.9, 23.3, 23.0, 14.4, 13.8. HRMS (m/z, ESI): Calcd. for Chemical Formula: $\mathrm{C}_{31} \mathrm{H}_{32} \mathrm{ClO}_{4}^{-}[\mathrm{M}-\mathrm{H}]^{-}:$503.1995, Found: 503.1993. HPLC analysis of the reaction product: Daicel Chiralpak IA, hexane/iso-propanol = 90: 10, $1.0 \mathrm{~mL} / \mathrm{min}, \lambda=320 \mathrm{~nm}$, retention time: $14.54 \mathrm{~min}$ (major) and $16.07 \mathrm{~min}$ (minor).

\section{(R)-6-(9-butyl-2-chloro-7-(4-(ethoxycarbonyl)phenyl)-9H-fluoren-9-yl)hexanoic acid (10)}

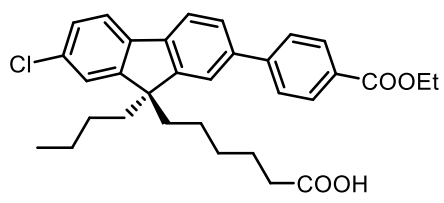

White solid, $81.5 \mathrm{mg}, 63 \%$ yield, $[\alpha]_{\mathrm{D}}^{20}=+6.90\left(\right.$ c $\left.0.100 \mathrm{CHCl}_{3}\right)$ for 93.5:6.5 er.

${ }^{1} \mathrm{H}$ NMR $\left(500 \mathrm{MHz}, \mathrm{CDCl}_{3}\right) \delta 8.16(\mathrm{~d}, J=8.3 \mathrm{~Hz}, 2 \mathrm{H}), 7.75(\mathrm{dd}, J=10.7,8.2 \mathrm{~Hz}, 3 \mathrm{H})$, $7.68-7.61(m, 2 H), 7.57(s, 1 H), 7.38-7.31(m, 2 H), 4.44(q, J=7.1 \mathrm{~Hz}, 2 H), 2.17$

$(\mathrm{t}, J=7.5 \mathrm{~Hz}, 2 \mathrm{H}), 2.04-2.00(\mathrm{~m}, 4 \mathrm{H}), 1.47-1.39(\mathrm{~m}, 5 \mathrm{H}), 1.18-1.08(\mathrm{~m}, 4 \mathrm{H}), 0.74-0.62(\mathrm{~m}, 7 \mathrm{H}) .{ }^{13} \mathrm{C} \mathrm{NMR}(126 \mathrm{MHz}$ $\left.\mathrm{CDCl}_{3}\right) \delta 178.4,166.6,152.7,151.1,145.7,140.2,139.3,139.1,133.2,130.1,129.2,127.4,127.0,126.5,123.3,121.5$ $120.9,120.2,61.0,55.4,40.1,33.6,29.7,29.3,25.9,24.3,23.4,23.0,14.4,13.8 . ~ H R M S$ (m/z, ESI): Calcd. for Chemical Formula: $\mathrm{C}_{32} \mathrm{H}_{34} \mathrm{ClO}_{4}{ }^{-}[\mathrm{M}-\mathrm{H}]^{-}:$517.2151, Found: 517.2150. HPLC analysis of the reaction product: Daicel Chiralpak IA, hexane/iso-propanol = 98: $2,1.0 \mathrm{~mL} / \mathrm{min}, \lambda=324 \mathrm{~nm}$, retention time: $13.09 \mathrm{~min}$ (minor) and $15.24 \mathrm{~min}$ (major). 
(R)-7-(9-butyl-2-chloro-7-(4-(ethoxycarbonyl)phenyl)-9H-fluoren-9-yl)heptanoic acid (11)

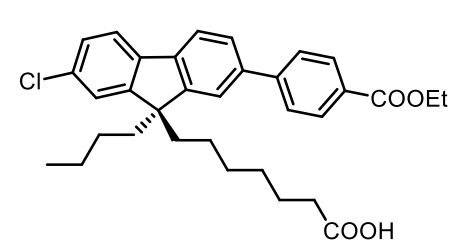

White solid, $72.6 \mathrm{mg}, 54 \%$ yield, $[\alpha]_{D}^{20}=+9.30\left(\right.$ c $\left.0.100 \mathrm{CHCl}_{3}\right)$ for 89:11 er.

${ }^{1} \mathrm{H} \mathrm{NMR}\left(500 \mathrm{MHz}, \mathrm{CDCl}_{3}\right) \delta 8.16(\mathrm{~d}, J=8.3 \mathrm{~Hz}, 2 \mathrm{H}), 7.75(\mathrm{t}, J=8.6 \mathrm{~Hz}, 3 \mathrm{H}), 7.69-$ $7.60(\mathrm{~m}, 2 \mathrm{H}), 7.57(\mathrm{~d}, J=1.1 \mathrm{~Hz}, 1 \mathrm{H}), 7.35(\mathrm{dd}, J=6.4,1.9 \mathrm{~Hz}, 2 \mathrm{H}), 4.44(\mathrm{q}, J=7.1$

$\mathrm{Hz}, 2 \mathrm{H}), 2.23(\mathrm{t}, J=7.5 \mathrm{~Hz}, 2 \mathrm{H}), 2.06-1.96(\mathrm{~m}, 4 \mathrm{H}), 1.49-1.42(\mathrm{~m}, 5 \mathrm{H}), 1.16-1.07(\mathrm{~m}, 6 \mathrm{H}), 0.73-0.60(\mathrm{~m}, 7 \mathrm{H}) .{ }^{13} \mathrm{C}$ NMR $\left(126 \mathrm{MHz}, \mathrm{CDCl}_{3}\right) \delta$ 178.6, 166.6, 152.8, 151.2, 145.7, 140.2, 139.2, 139.1, 133.2, 130.1, 129.2, 127.3, 127.0, 126.5, 123.3, 121.5, 120.9, 120.2, 64.7, 61.0, 55.5, 40.2, 40.2, 33.7, 29.5, 28.7, 25.9, 24.5, 23.6, 23.0, 14.4, 13.8. HRMS (m/z, ESI): Calcd. for Chemical Formula: $\mathrm{C}_{33} \mathrm{H}_{36} \mathrm{ClO}_{4}{ }^{-}[\mathrm{M}-\mathrm{H}]^{-}: 531.2308$, Found: 531.2303. HPLC analysis of the reaction product: Daicel Chiralpak IA, hexane/iso-propanol $=98: 2,1.0 \mathrm{~mL} / \mathrm{min}, \lambda=319 \mathrm{~nm}$, retention time: 12.50 $\min$ (minor) and $14.31 \mathrm{~min}$ (major).

(R)-8-(9-butyl-2-chloro-7-(4-(ethoxycarbonyl)phenyl)-9H-fluoren-9-yl)octanoic acid (12)

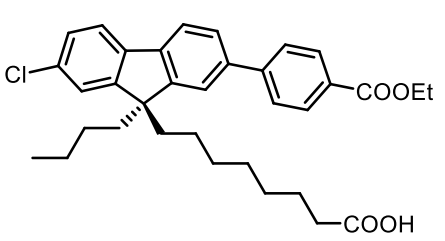

White solid, $43.6 \mathrm{mg}, 32 \%$ yield, $[\alpha]_{\mathrm{D}}{ }^{20}=+2.40\left(\mathrm{c} 0.080 \mathrm{CHCl}_{3}\right)$ for $86.5: 13.5 \mathrm{er}$. ${ }^{1} \mathrm{H}$ NMR $\left(500 \mathrm{MHz}, \mathrm{CDCl}_{3}\right) \delta 8.18-8.13(\mathrm{~m}, 2 \mathrm{H}), 7.78-7.71(\mathrm{~m}, 3 \mathrm{H}), 7.68-7.61$ $(\mathrm{m}, 2 \mathrm{H}), 7.57(\mathrm{~d}, J=1.2 \mathrm{~Hz}, 1 \mathrm{H}), 7.35(\mathrm{dd}, J=6.6,1.9 \mathrm{~Hz}, 2 \mathrm{H}), 4.44(\mathrm{q}, J=7.1 \mathrm{~Hz}, 2 \mathrm{H})$,

$2.26(\mathrm{t}, J=7.5 \mathrm{~Hz}, 2 \mathrm{H}), 2.05-1.97(\mathrm{~m}, 4 \mathrm{H}), 1.54-1.49(\mathrm{~m}, 2 \mathrm{H}), 1.45(\mathrm{t}, J=8.8 \mathrm{~Hz}, 3 \mathrm{H}), 1.19-1.08(\mathrm{~m}, 8 \mathrm{H}), 0.71(\mathrm{t}, J=$ $7.4 \mathrm{~Hz}, 3 \mathrm{H}), 0.69-0.62(\mathrm{~m}, 4 \mathrm{H}) .{ }^{13} \mathrm{C} N M R\left(126 \mathrm{MHz}, \mathrm{CDCl}_{3}\right) \delta$ 166.6, 152.9, 151.2, 145.8, 140.2, 139.2, 139.1, 133.2, $130.1,129.2,127.3,127.0,126.4,123.3,121.5,120.9,120.2,61.0,55.5,40.2,40.1,33.6,28.9,28.8,25.9,24.5,23.7$, 23.0, 14.4, 13.8. HRMS (m/z, ESI): Calcd. for Chemical Formula: $\left.\mathrm{C}_{34} \mathrm{H}_{38} \mathrm{ClO}_{4}{ }^{-}[\mathrm{M}-\mathrm{H}]\right]^{-}: 545.2464$, Found: 545.2465. HPLC analysis of the reaction product: Daicel Chiralpak IH, hexane/iso-propanol $=97: 3,1.0 \mathrm{~mL} / \mathrm{min}, \lambda=336 \mathrm{~nm}$, retention time: $12.00 \mathrm{~min}$ (major) and $14.12 \mathrm{~min}$ (minor).

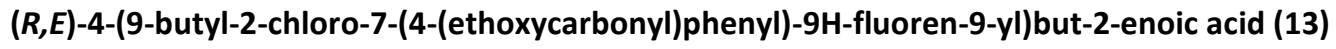

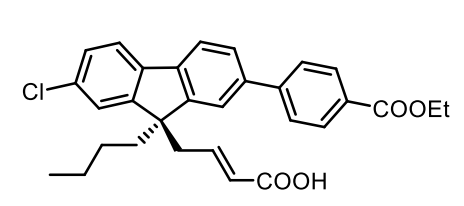

White solid, $70.7 \mathrm{mg}, 58 \%$ yield, $[\alpha]_{\mathrm{D}}{ }^{20}=+57.80\left(\right.$ c $\left.0.100 \mathrm{CHCl}_{3}\right)$ for $91.5: 8.5$ er. ${ }^{1} \mathrm{H}$ NMR $\left(500 \mathrm{MHz}, \mathrm{CDCl}_{3}\right) \delta 8.15(\mathrm{dd}, J=6.5,4.8 \mathrm{~Hz}, 2 \mathrm{H}), 7.76(\mathrm{~d}, J=7.8 \mathrm{~Hz}$, 1H), $7.73-7.69(m, 2 H), 7.68-7.63(m, 2 H), 7.57(d, J=1.1 \mathrm{~Hz}, 1 \mathrm{H}), 7.39-7.34$

$(\mathrm{m}, 2 \mathrm{H}), 6.44(\mathrm{dt}, J=15.3,7.5 \mathrm{~Hz}, 1 \mathrm{H}), 5.63(\mathrm{~d}, J=15.5 \mathrm{~Hz}, 1 \mathrm{H}), 4.44(\mathrm{q}, J=7.1 \mathrm{~Hz}, 2 \mathrm{H}), 2.89-2.85(\mathrm{~m}, 2 \mathrm{H}), 2.12-2.03$ 
$(\mathrm{m}, 2 \mathrm{H}), 1.45(\mathrm{t}, J=7.1 \mathrm{~Hz}, 3 \mathrm{H}), 1.14-1.11(\mathrm{~m}, 2 \mathrm{H}), 0.72(\mathrm{t}, J=10.0 \mathrm{~Hz}, 3 \mathrm{H}), 0.69-0.62(\mathrm{~m}, 2 \mathrm{H}) .{ }^{13} \mathrm{C} \mathrm{NMR}(126 \mathrm{MHz}$ $\left.\mathrm{CDCl}_{3}\right) \delta 170.0,166.5,151.1,149.4,146.5,145.5,139.8,139.5,138.8,133.4,130.1,129.3,128.0,127.1,127.1,123.4$ 123.2, 121.8, 121.3, 120.6, 61.0, 54.7, 42.8, 39.1, 25.8, 22.9, 14.4, 13.8. HRMS (m/z, ESI): Calcd. for Chemical Formula: $\mathrm{C}_{30} \mathrm{H}_{28} \mathrm{ClO}_{4}^{-}[\mathrm{M}-\mathrm{H}]^{-}:$487.1682, Found: 487.1676. HPLC analysis of the reaction product: Daicel Chiralpak IH, hexane/iso-propanol = 95: 5, $1.0 \mathrm{~mL} / \mathrm{min}, \lambda=339 \mathrm{~nm}$, retention time: $10.05 \mathrm{~min}$ (minor) and $12.69 \mathrm{~min}$ (major).

(R)-diethyl 4,4'-(9-butyl-9-(3-(ethoxysulfonyl)propyl)-9H-fluorene-2,7-diyl)dibenzoate (2)

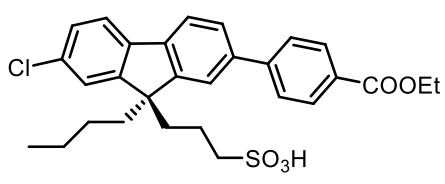

After the reaction was completed, the reaction mixture was neutralized with $1 \mathrm{M} \mathrm{HCl}$ to $\mathrm{pH} 2-3$, extracted with ethyl acetate, dried with $\mathrm{Na}_{2} \mathrm{SO}_{4}$, the organic phase was removed under reduced pressure.

The product was then converted to the corresponding ethyl sulfonate using triethyl orthoacetate ${ }^{[12]}$ for isolation, characterization, and HPLC analysis. White solid, $90.5 \mathrm{mg}, 65 \%$ yield, $[\alpha]_{\mathrm{D}}{ }^{20}=+9.667\left(\mathrm{c} 0.030 \mathrm{CHCl}_{3}\right)$ for $95: 5$ er. ${ }^{1} \mathrm{H}$ $\operatorname{NMR}\left(500 \mathrm{MHz}, \mathrm{CDCl}_{3}\right) \delta 8.19-8.13(\mathrm{~m}, 2 \mathrm{H}), 7.77(\mathrm{~d}, J=7.9 \mathrm{~Hz}, 1 \mathrm{H}), 7.75-7.71(\mathrm{~m}, 2 \mathrm{H}), 7.70-7.63(\mathrm{~m}, 2 \mathrm{H}), 7.59$ $(\mathrm{d}, J=1.2 \mathrm{~Hz}, 1 \mathrm{H}), 7.40-7.34(\mathrm{~m}, 2 \mathrm{H}), 4.44(\mathrm{q}, J=7.1 \mathrm{~Hz}, 2 \mathrm{H}), 4.13-4.05(\mathrm{~m}, 2 \mathrm{H}), 2.88-2.81(\mathrm{~m}, 2 \mathrm{H}), 2.30-2.18$ (m, 2H), 2.06-2.03 (m, 2H), $1.45(\mathrm{t}, J=7.1 \mathrm{~Hz}, 3 \mathrm{H}), 1.25-1.20(\mathrm{~m}, 5 \mathrm{H}), 1.15-1.12(\mathrm{~m}, 2 \mathrm{H}), 0.71(\mathrm{t}, J=7.4 \mathrm{~Hz}, 3 \mathrm{H}), 0.67-$ $0.63(\mathrm{~m}, 2 \mathrm{H}) .{ }^{13} \mathrm{CNMR}\left(126 \mathrm{MHz}, \mathrm{CDCl}_{3}\right) \delta 151.5,149.9,145.5,139.6,139.1,139.1,133.5,130.2,127.8,127.1,127.0$, 123.2, 121.5, 121.2, 120.5, 66.0, 61.1, 55.1, 50.4, 40.1, 38.3, 25.8, 22.9, 18.4, 15.0, 14.4, 13.8. HRMS (m/z, ESI): Calcd. for Chemical Formula: $\mathrm{C}_{31} \mathrm{H}_{36} \mathrm{ClO}_{5} \mathrm{SNa}^{+}[\mathrm{M}+\mathrm{Na}]^{+}: 577.1786$, Found: 577.1779. HPLC analysis of the esterification product: Daicel Chiralpak IA, hexane/iso-propanol $=97: 3,1.0 \mathrm{~mL} / \mathrm{min}, \lambda=322 \mathrm{~nm}$, retention time: 14.97 min (major) and $17.49 \mathrm{~min}$ (minor).

\section{Ethyl (R)-4-(9-butyl-2-chloro-7-(4-methoxyphenyl)-9H-fluoren-9-yl)butane-1-sulfonate (14)}

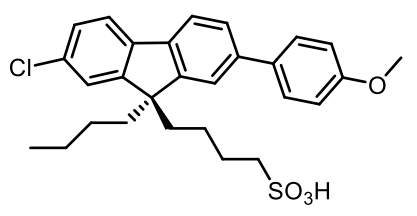

After the reaction was completed, the reaction mixture was neutralized with 1

$\mathrm{M} \mathrm{HCl}$ to $\mathrm{pH} 2-3$, extracted with ethyl acetate, dried with $\mathrm{Na}_{2} \mathrm{SO}_{4}$, the organic phase was removed under reduced pressure.

The product was then converted to the corresponding ethyl sulfonate using triethyl orthoacetate ${ }^{[12]}$ for isolation, characterization, and HPLC analysis. White solid, $89.0 \mathrm{mg}, 68 \%$ yield, $[\alpha]_{D}^{20}=+14.00\left(c 0.020 \mathrm{CHCl}_{3}\right)$ for $94.5: 5.5 \mathrm{er}$. 
${ }^{1} \mathrm{H}$ NMR $\left(500 \mathrm{MHz}, \mathrm{CDCl}_{3}\right) \delta 7.72(\mathrm{~d}, J=7.7 \mathrm{~Hz}, 1 \mathrm{H}), 7.64(\mathrm{~d}, J=8.0 \mathrm{~Hz}, 1 \mathrm{H}), 7.63-7.59(\mathrm{~m}, 2 \mathrm{H}), 7.57(\mathrm{dd}, J=7.9,1.7$ $\mathrm{Hz}, 1 \mathrm{H}), 7.49(\mathrm{~d}, J=1.2 \mathrm{~Hz}, 1 \mathrm{H}), 7.35(\mathrm{dd}, J=8.0,1.9 \mathrm{~Hz}, 1 \mathrm{H}), 7.32(\mathrm{~d}, J=1.6 \mathrm{~Hz}, 1 \mathrm{H}), 7.06-7.01(\mathrm{~m}, 2 \mathrm{H}), 4.17(\mathrm{q}, J=$ $7.1 \mathrm{~Hz}, 2 \mathrm{H}), 3.90(\mathrm{~s}, 3 \mathrm{H}), 2.87-2.83(\mathrm{~m}, 2 \mathrm{H}), 2.09-2.00(\mathrm{~m}, 4 \mathrm{H}), 1.69-1.62(\mathrm{~m}, 2 \mathrm{H}), 1.32(\mathrm{t}, J=7.1 \mathrm{~Hz}, 3 \mathrm{H}), 1.14-1.08$ (m, 2H), $0.80-0.64(\mathrm{~m}, 7 \mathrm{H}) .{ }^{13} \mathrm{C}$ NMR $\left(126 \mathrm{MHz}, \mathrm{CDCl}_{3}\right) \delta 159.3,152.0,150.3,140.4,139.5,138.7,133.8,132.9$ $128.2,127.5,126.0,123.1,120.9,120.7,120.1,114.3,65.8,55.4,55.1,50.2,40.2,39.8,25.8,23.7,23.0,22.6,15.1$, 13.8. MS (m/z, ESI): Calcd. for Chemical Formula: $\mathrm{C}_{30} \mathrm{H}_{34} \mathrm{ClO}_{4} \mathrm{~S}^{-}[\mathrm{M}-\mathrm{H}]^{-}:$497.1559, Found: 497.1568. HPLC analysis of the esterification product: Daicel Chiralpak IA, hexane/iso-propanol $=97: 3,1.0 \mathrm{~mL} / \mathrm{min}, \lambda=325 \mathrm{~nm}$, retention time: $8.07 \mathrm{~min}$ (minor) and $9.58 \mathrm{~min}$ (major).

\section{(R)-4-(2-chloro-7-(4-(ethoxycarbonyl)phenyl)-9-methyl-9H-fluoren-9-yl)butanoic acid (15)}

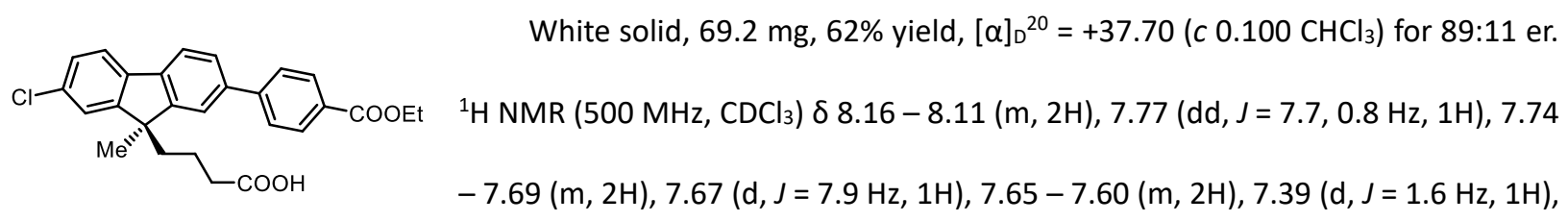

$7.36(\mathrm{dd}, J=8.0,1.9 \mathrm{~Hz}, 1 \mathrm{H}), 4.43(\mathrm{q}, J=7.1 \mathrm{~Hz}, 2 \mathrm{H}), 2.17-2.06(\mathrm{~m}, 4 \mathrm{H}), 1.53(\mathrm{~s}, 3 \mathrm{H}), 1.44(\mathrm{t}, J=7.1 \mathrm{~Hz}, 3 \mathrm{H}), 1.07-$ $1.03(\mathrm{~m}, 2 \mathrm{H}) .{ }^{13} \mathrm{C} N M R\left(126 \mathrm{MHz}, \mathrm{CDCl}_{3}\right) \delta 177.8,166.6,153.5,152.0,145.6,139.5,139.2,138.1,133.4,130.1,129.2$ 127.6, 127.0, 126.8, 123.3, 121.6, 121.2, 120.5, 61.0, 51.0, 39.6, 33.6, 26.8, 19.6, 14.4. MS (m/z, ESI): Calcd. for Chemical Formula: $\mathrm{C}_{27} \mathrm{H}_{24} \mathrm{ClO}_{4}^{-}[\mathrm{M}-\mathrm{H}]^{-}:$447.1369, Found: 447.1364. HPLC analysis of the reaction product: Daicel Chiralpak IA, hexane/iso-propanol = 90: 10, $1.0 \mathrm{~mL} / \mathrm{min}, \lambda=315 \mathrm{~nm}$, retention time: 6.12 min (major) and $9.40 \mathrm{~min}$ (minor).

\section{(R)-4-(2-chloro-7-(4-(ethoxycarbonyl)phenyl)-9-octyl-9H-fluoren-9-yl)butanoic acid (16)}

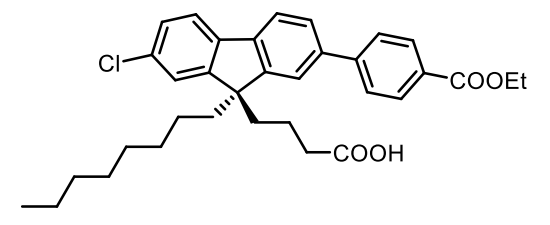

White solid, $96.8 \mathrm{mg}, 71 \%$ yield, $[\alpha]_{D}{ }^{20}=-4.60\left(c 0.100 \mathrm{CHCl}_{3}\right.$ ) for 94.5:5.5 er. ${ }^{1} \mathrm{H}$ NMR (500 MHz, $\left.\mathrm{CDCl}_{3}\right) \delta 8.16-8.11(\mathrm{~m}, 2 \mathrm{H}), 7.75(\mathrm{~d}, J=7.9$ $\mathrm{Hz}, 1 \mathrm{H}), 7.74-7.70(\mathrm{~m}, 2 \mathrm{H}), 7.66(\mathrm{~d}, J=8.7 \mathrm{~Hz}, 1 \mathrm{H}), 7.62(\mathrm{dd}, J=7.9,1.6 \mathrm{~Hz}$

1H), $7.57(\mathrm{~d}, J=1.2 \mathrm{~Hz}, 1 \mathrm{H}), 7.35(\mathrm{dd}, J=6.7,1.9 \mathrm{~Hz}, 2 \mathrm{H}), 4.43(\mathrm{q}, J=7.1 \mathrm{~Hz}, 2 \mathrm{H}), 2.14(\mathrm{t}, J=7.3 \mathrm{~Hz}, 2 \mathrm{H}), 2.10-1.93$ $(\mathrm{m}, 4 \mathrm{H}), 1.44(\mathrm{t}, J=7.1 \mathrm{~Hz}, 3 \mathrm{H}), 1.24-1.18(\mathrm{~m}, 2 \mathrm{H}), 1.15-0.96(\mathrm{~m}, 10 \mathrm{H}), 0.82(\mathrm{t}, J=7.2 \mathrm{~Hz}, 3 \mathrm{H}), 0.70-0.63(\mathrm{~m}, 2 \mathrm{H})$. ${ }^{13} \mathrm{C}$ NMR $\left(126 \mathrm{MHz}_{\mathrm{CDCl}}\right)$ $\delta 178.0,166.6,152.2,150.6,145.7,140.1,139.4,139.1,133.3,130.1,129.2,127.5,127.0$ 
$126.7,123.3,121.6,121.0,120.3,61.0,55.3,40.3,39.3,33.7,31.7,29.8,29.1_{1}, 29.12,23.6,22.6,19.1,14.4,14.1$. HRMS (m/z, ESI): Calcd. for Chemical Formula: $\mathrm{C}_{34} \mathrm{H}_{38} \mathrm{ClO}_{4}{ }^{-}[\mathrm{M}-\mathrm{H}]^{-}: 545.2464$, Found: 545.2461 . HPLC analysis of the reaction product: Daicel Chiralpak IA, hexane/iso-propanol $=90: 10,1.0 \mathrm{~mL} / \mathrm{min}, \lambda=330 \mathrm{~nm}$, retention time: 4.33 $\min$ (major) and $5.11 \mathrm{~min}$ (minor).

\section{(S)-4-(9-benzyl-2-chloro-7-(4-(ethoxycarbonyl)phenyl)-9H-fluoren-9-yl)butanoic acid (17)}

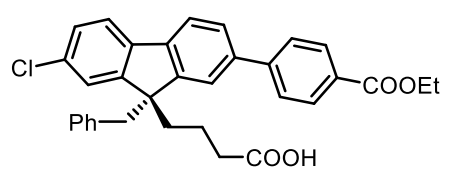

White solid, $86.4 \mathrm{mg}, 66 \%$ yield, $[\alpha]_{\mathrm{D}}^{20}=-56.70\left(\mathrm{c} 0.100 \mathrm{CHCl}_{3}\right)$ for $92.5: 7.5$ er. ${ }^{1} \mathrm{H}$ NMR $\left(500 \mathrm{MHz}, \mathrm{CDCl}_{3}\right) \delta 8.17-8.10(\mathrm{~m}, 2 \mathrm{H}), 7.70-7.65(\mathrm{~m}, 2 \mathrm{H}), 7.63(\mathrm{~d}$, $J=7.7 \mathrm{~Hz}, 1 \mathrm{H}), 7.59(\mathrm{dd}, J=7.9,1.6 \mathrm{~Hz}, 1 \mathrm{H}), 7.54(\mathrm{dd}, J=7.5,1.1 \mathrm{~Hz}, 1 \mathrm{H}), 7.44$ $(\mathrm{d}, J=1.2 \mathrm{~Hz}, 1 \mathrm{H}), 7.32(\mathrm{dd}, J=7.9,1.5 \mathrm{~Hz}, 2 \mathrm{H}), 7.14-7.08(\mathrm{~m}, 1 \mathrm{H}), 7.08-7.02(\mathrm{~m}, 2 \mathrm{H}), 6.77-6.69(\mathrm{~m}, 2 \mathrm{H}), 4.44(\mathrm{q}$ $J=7.1 \mathrm{~Hz}, 2 \mathrm{H}), 3.16(\mathrm{AB}, 2 \mathrm{H}), 2.32-2.20(\mathrm{~m}, 2 \mathrm{H}), 2.16(\mathrm{t}, J=7.3 \mathrm{~Hz}, 2 \mathrm{H}), 1.45(\mathrm{t}, J=7.1 \mathrm{~Hz}, 3 \mathrm{H}), 1.03-0.95(\mathrm{~m}, 2 \mathrm{H})$. ${ }^{13} \mathrm{C}$ NMR $\left(126 \mathrm{MHz}, \mathrm{CDCl}_{3}\right) \delta 177.8,166.6,151.3,149.3,145.6,139.9,138.8,136.6,133.0,130.4,130.1,129.1,127.7$ 127.4, 127.0, 126.8, 126.3, 124.1, 122.7, 121.1, 120.3, , 61.0, 55.9, 46.9, 37.5, 33.6, 19.2, 14.4. HRMS (m/z, ESI): Calcd. for Chemical Formula: $\mathrm{C}_{33} \mathrm{H}_{28} \mathrm{ClO}_{4}^{-}[\mathrm{M}-\mathrm{H}]^{-}:$523.1682, Found: 523.1677. HPLC analysis of the reaction product: Daicel Chiralpak IA, hexane/iso-propanol = 90: 10, $1.0 \mathrm{~mL} / \mathrm{min}, \lambda=330 \mathrm{~nm}$, retention time: 6.76 min (major) and $15.04 \mathrm{~min}$ (minor).

\section{(S)-4-(2-chloro-9-cyclohexyl-7-(4-(ethoxycarbonyl)phenyl)-9H-fluoren-9-yl)butanoic acid (18)}

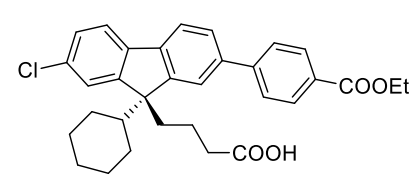

White solid, $79.6 \mathrm{mg}, 61 \%$ yield, $[\alpha]_{\mathrm{D}}{ }^{20}=-5.80\left(\mathrm{c} 0.100 \mathrm{CHCl}_{3}\right)$ for $91: 9 \mathrm{er} .{ }^{1} \mathrm{H}$ $\operatorname{NMR}\left(500 \mathrm{MHz}, \mathrm{CDCl}_{3}\right) \delta 8.16-8.12(\mathrm{~m}, 2 \mathrm{H}), 7.74-7.71(\mathrm{~m}, 3 \mathrm{H}), 7.66-7.59(\mathrm{~m}, 3 \mathrm{H})$, $7.38-7.33(\mathrm{~m}, 2 \mathrm{H}), 4.43(\mathrm{q}, J=7.1 \mathrm{~Hz}, 2 \mathrm{H}), 2.25-2.10(\mathrm{~m}, 4 \mathrm{H}), 1.94-1.87(\mathrm{~m}, 1 \mathrm{H})$, $1.68-1.51(\mathrm{~m}, 6 \mathrm{H}), 1.44(\mathrm{t}, J=7.1 \mathrm{~Hz}, 3 \mathrm{H}), 1.19-1.11(\mathrm{~m}, 2 \mathrm{H}), 1.00-0.84(\mathrm{~m}, 4 \mathrm{H}) .{ }^{13} \mathrm{C} \mathrm{NMR}\left(126 \mathrm{MHz}, \mathrm{CDCl}_{3}\right) \delta$ $177.4,166.6,151.9,150.3,145.7,140.5,139.5,139.1,133.1,130.1,129.1,127.4,127.1,126.6,123.9,122.3,120.8$, 120.0, 61.0, 58.6, 47.7, 35.6, 33.7, 27.9, 27.8, 26.9, 26.4, 19.2, 14.4. HRMS (m/z, ESI): Calcd. for Chemical Formula: $\mathrm{C}_{32} \mathrm{H}_{32} \mathrm{ClO}_{4}^{-}[\mathrm{M}-\mathrm{H}]^{-}:$515.1995, Found: 515.1989. HPLC analysis of the reaction product: Daicel Chiralpak IA, hexane/iso-propanol = 95: 5, $1.0 \mathrm{~mL} / \mathrm{min}, \lambda=321 \mathrm{~nm}$, retention time: $7.63 \mathrm{~min}$ (major) and $9.39 \mathrm{~min}$ (minor). 


\section{(S)-4-(2-chloro-7-(4-(ethoxycarbonyl)phenyl)-9-phenyl-9H-fluoren-9-yl)butanoic acid (19)}

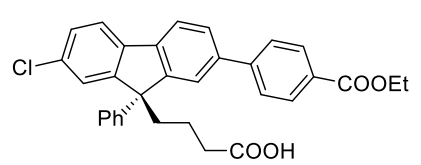

White solid, $80.1 \mathrm{mg}, 63 \%$ yield, $[\alpha]_{\mathrm{D}}{ }^{20}=-55.20\left(\right.$ c $\left.0.100 \mathrm{CHCl}_{3}\right)$ for $94.5: 5.5 \mathrm{er}$.

${ }^{1} \mathrm{H} \mathrm{NMR}\left(500 \mathrm{MHz}, \mathrm{CDCl}_{3}\right) \delta 8.08(\mathrm{~d}, J=8.5 \mathrm{~Hz}, 2 \mathrm{H}), 7.81(\mathrm{~d}, J=7.9 \mathrm{~Hz}, 1 \mathrm{H}), 7.72(\mathrm{~d}, J$ $=8.1 \mathrm{~Hz}, 1 \mathrm{H}), 7.66-7.61(\mathrm{~m}, 3 \mathrm{H}), 7.46(\mathrm{~d}, J=1.2 \mathrm{~Hz}, 1 \mathrm{H}), 7.36(\mathrm{dd}, J=8.1,1.9 \mathrm{~Hz}, 1 \mathrm{H}), 7.27-7.18(\mathrm{~m}, 6 \mathrm{H}), 4.41(\mathrm{q}, J$ $=7.1 \mathrm{~Hz}, 2 \mathrm{H}), 2.63-2.53(\mathrm{~m}, 2 \mathrm{H}), 2.26(\mathrm{t}, J=7.2 \mathrm{~Hz}, 2 \mathrm{H}), 1.42(\mathrm{t}, J=7.1 \mathrm{~Hz}, 3 \mathrm{H}), 1.17-1.10(\mathrm{~m}, 2 \mathrm{H}) .{ }^{13} \mathrm{C} N M R(126$ $\left.\mathrm{MHz}, \mathrm{CDCl}_{3}\right) \delta 178.7,166.5,153.5,151.9,145.4,143.6,139.8,138.7,133.7,130.0,129.2,128.6,127.9,127.0,126.9$, 126.5, 124.7, 123.1, 121.2, 120.5, 61.0, 58.8, 36.9, 33.8, 19.2, 14.4. HRMS (m/z, ESI): Calcd. for Chemical Formula: $\mathrm{C}_{32} \mathrm{H}_{26} \mathrm{ClO}_{4}{ }^{-}[\mathrm{M}-\mathrm{H}]^{-}: 509.1525$, Found: 509.1522. HPLC analysis of the reaction product: Daicel Chiralpak IA, hexane/iso-propanol = 95: 5, $1.0 \mathrm{~mL} / \mathrm{min}, \lambda=340 \mathrm{~nm}$, retention time: $12.33 \mathrm{~min}$ (major) and $17.25 \mathrm{~min}$ (minor).

\section{(R)-4-(9-butyl-2-chloro-7-(4-methoxyphenyl)-9H-fluoren-9-yl)butanoic acid (20)}

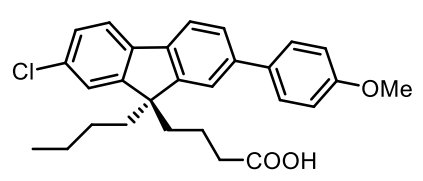

White solid, $76.2 \mathrm{mg}, 68 \%$ yield, $[\alpha]_{D}{ }^{20}=+5.00\left(c 0.100 \mathrm{CHCl}_{3}\right)$ for $96: 4$ er. ${ }^{1} \mathrm{H}$ $\operatorname{NMR}\left(500 \mathrm{MHz}, \mathrm{CDCl}_{3}\right) \delta 7.71(\mathrm{~d}, J=7.9 \mathrm{~Hz}, 1 \mathrm{H}), 7.63(\mathrm{~d}, J=8.7 \mathrm{~Hz}, 1 \mathrm{H}), 7.62-7.58$ (m, 2H), 7.55 (dd, $J=7.9,1.7 \mathrm{~Hz}, 1 \mathrm{H}), 7.50(\mathrm{~d}, J=1.2 \mathrm{~Hz}, 1 \mathrm{H}), 7.34$ (dd, $J=6.6,1.9 \mathrm{~Hz}$, 2H), $7.04-7.00(\mathrm{~m}, 2 \mathrm{H}), 3.89(\mathrm{~s}, 3 \mathrm{H}), 2.12(\mathrm{t}, J=7.4 \mathrm{~Hz}, 2 \mathrm{H}), 2.08-1.93(\mathrm{~m}, 4 \mathrm{H}), 1.11(\mathrm{dd}, J=14.8,7.4 \mathrm{~Hz}, 2 \mathrm{H}), 1.05$ $-0.98(\mathrm{~m}, 2 \mathrm{H}), 0.70(\mathrm{t}, J=7.4 \mathrm{~Hz}, 3 \mathrm{H}), 0.68-0.61(\mathrm{~m}, 2 \mathrm{H}) .{ }^{13} \mathrm{C} \mathrm{NMR}\left(126 \mathrm{MHz}, \mathrm{CDCl}_{3}\right) \delta 177.3,159.2,152.0,150.4$, $140.3,139.5,138.7,133.9,132.8,128.2,127.4,126.0,123.2,121.1,120.7,120.1,114.3,55.4,55.1,40.2,39.5,33.7$, 25.8, 23.0, 19.2, 13.8. HRMS (m/z, ESI): Calcd. for Chemical Formula: $\mathrm{C}_{28} \mathrm{H}_{28} \mathrm{ClO}_{3}{ }^{-}[\mathrm{M}-\mathrm{H}]$ ]: 447.1732, Found: 447.1727. HPLC analysis of the reaction product: Daicel Chiralpak IH, hexane/iso-propanol $=99: 1,1.0 \mathrm{~mL} / \mathrm{min}, \lambda=327 \mathrm{~nm}$, retention time: $19.36 \mathrm{~min}$ (minor) and $21.70 \mathrm{~min}$ (major).

\section{(R)-4-(9-butyl-2-chloro-7-(4-(diphenylamino)phenyl)-9H-fluoren-9-yl)butanoic acid (21)}

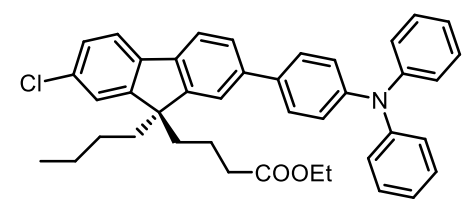

After the reaction was completed, the reaction mixture was neutralized with $1 \mathrm{M} \mathrm{HCl}$ to $\mathrm{pH} \mathrm{2-3} \mathrm{and} \mathrm{the} \mathrm{crude} \mathrm{product} \mathrm{was} \mathrm{dissolved} \mathrm{in} \mathrm{EtOH}$, then $10 \mu \mathrm{L}$ conc. $\mathrm{H}_{2} \mathrm{SO}_{4}$ was added and the reaction mixture was stirred at $65{ }^{\circ} \mathrm{C}$ for $10 \mathrm{~h}$.

The esterification product was obtained as a white solid, $111.8 \mathrm{mg}, 73 \%$ yield, $[\alpha]_{D}^{20}=+5.10\left(c 0.100 \mathrm{CHCl}_{3}\right)$ for 95.5:4.5 er. ${ }^{1} \mathrm{H}$ NMR $\left(500 \mathrm{MHz}, \mathrm{CDCl}_{3}\right) \delta 7.73(\mathrm{~d}, J=7.9 \mathrm{~Hz}, 1 \mathrm{H}), 7.65(\mathrm{~d}, J=8.6 \mathrm{~Hz}, 1 \mathrm{H}), 7.62-7.52(\mathrm{~m}, 4 \mathrm{H}), 7.39-$ 
$7.28(\mathrm{~m}, 6 \mathrm{H}), 7.25-7.15(\mathrm{~m}, 6 \mathrm{H}), 7.08(\mathrm{dd}, J=10.6,4.0 \mathrm{~Hz}, 2 \mathrm{H}), 4.12(\mathrm{q}, J=7.1 \mathrm{~Hz}, 2 \mathrm{H}), 2.12-2.00(\mathrm{~m}, 6 \mathrm{H}), 1.24-$ $1.17(\mathrm{~m}, 3 \mathrm{H}), 1.13-1.12(\mathrm{~m}, 2 \mathrm{H}), 1.05-1.02(\mathrm{~m}, 2 \mathrm{H}), 0.73-0.65(\mathrm{~m}, 5 \mathrm{H}) .{ }^{13} \mathrm{C} \mathrm{NMR}\left(126 \mathrm{MHz}, \mathrm{CDCl}_{3}\right) \delta 173.3,152.2,150.5$ $147.7,147.3,140.0,139.5,138.9,135.3,132.8,129.3,127.8,127.4,125.9,124.4,124.0,123.2,123.0,120.9,120.8$, 120.2, 60.2, 55.2, 40.2, 39.6, 34.4, 25.9, 23.0, 19.5, 14.2, 13.8. HRMS (m/z, ESI): Calcd. for Chemical Formula: $\mathrm{C}_{41} \mathrm{H}_{41} \mathrm{ClNO}_{2}{ }^{+}[\mathrm{M}+\mathrm{H}]^{+}:$614.2767, Found: 614.2782.

A sample of the ester was hydrolyzed to provide the parent carboxylic acid for the HPLC analysis of the reaction product: Daicel Chiralpak IA, hexane/iso-propanol $=99.4: 0.6,1.0 \mathrm{~mL} / \mathrm{min}, \lambda=348 \mathrm{~nm}$, retention time: $9.98 \mathrm{~min}$ (major) and $10.26 \mathrm{~min}$ (minor).

\section{(R)-4-(9-butyl-2-chloro-7-(4-nitrophenyl)-9H-fluoren-9-yl)butanoic acid (22)}

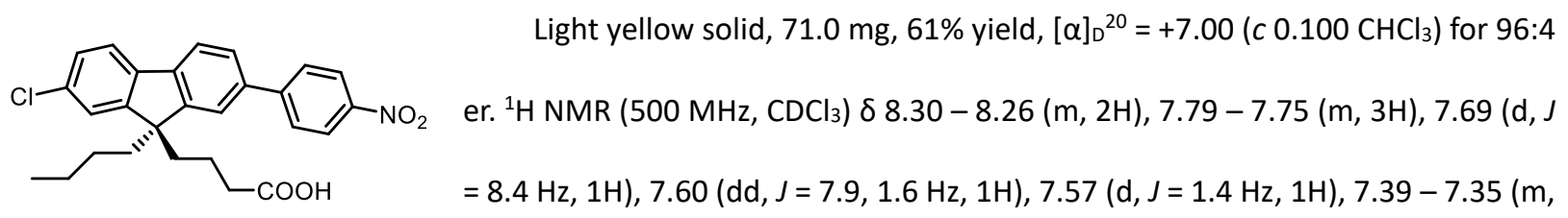

$2 \mathrm{H}), 2.17-1.99(\mathrm{~m}, 6 \mathrm{H}), 1.13-1.09(\mathrm{~m}, 2 \mathrm{H}), 1.01-1.97(\mathrm{~m}, 2 \mathrm{H}), 0.70(\mathrm{t}, J=7.3 \mathrm{~Hz}, 3 \mathrm{H}), 0.67-0.61(\mathrm{~m}, 2 \mathrm{H}) .{ }^{13} \mathrm{C} \mathrm{NMR}$ $\left(126 \mathrm{MHz}_{1} \mathrm{CDCl}_{3}\right) \delta 179.0,152.1,150.8,147.7,146.9,141.0,138.8,137.9,133.7,127.8,127.7,126.8,124.1,123.3$, 121.7, 121.3, 120.5, 76.8, 55.3, 40.3, 39.2, 33.7, 25.8, 22.9, 19.0, 13.8. HRMS (m/z, ESI): Calcd. for Chemical Formula: $\mathrm{C}_{27} \mathrm{H}_{25} \mathrm{ClNO}_{4}^{-}[\mathrm{M}-\mathrm{H}]^{-}:$462.1478, Found: 462.1474. HPLC analysis of the reaction product: Daicel Chiralpak IA, hexane/iso-propanol = 95: 5, $1.0 \mathrm{~mL} / \mathrm{min}, \lambda=340 \mathrm{~nm}$, retention time: $8.67 \mathrm{~min}$ (major) and 9.84 min (minor).

\section{(R)-4-(2-(3-(benzyloxy)phenyl)-9-butyl-7-chloro-9H-fluoren-9-yl)butanoic acid (23)}

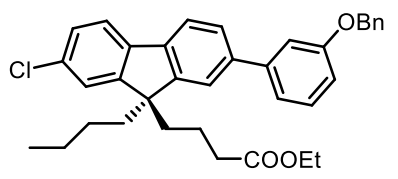

After the reaction was completed, the reaction mixture was neutralized with $1 \mathrm{M}$ $\mathrm{HCl}$ to $\mathrm{pH} \mathrm{2-3}$ and the crude product was dissolved in $\mathrm{EtOH}$, then $10 \mu \mathrm{L}$ conc. $\mathrm{H}_{2} \mathrm{SO}_{4}$ was added and the reaction mixture was stirred at $65^{\circ} \mathrm{C}$ for $10 \mathrm{~h}$. The esterification product was obtained as a white solid, $85.6 \mathrm{mg}, 62 \%$ yield, $[\alpha]_{\mathrm{D}}{ }^{20}=+3.60\left(c 0.100 \mathrm{CHCl}_{3}\right)$ for $97.5: 2.5$ er. ${ }^{1} \mathrm{H} \mathrm{NMR}(500 \mathrm{MHz}, \mathrm{CDCl}) \delta 7.74(\mathrm{~d}, J=7.9 \mathrm{~Hz}$, 1H), $7.68-7.64(m, 1 H), 7.60(d, J=7.9 \mathrm{~Hz}, 1 \mathrm{H}), 7.56-7.49(\mathrm{~m}, 3 \mathrm{H}), 7.46-7.30(\mathrm{~m}, 8 \mathrm{H}), 7.02(\mathrm{dd}, J=8.2,1.8 \mathrm{~Hz}, 1 \mathrm{H})$, $5.19(\mathrm{~s}, 2 \mathrm{H}), 4.10-3.98(\mathrm{~m}, 2 \mathrm{H}), 2.14-1.98(\mathrm{~m}, 6 \mathrm{H}), 1.19(\mathrm{t}, J=7.1 \mathrm{~Hz}, 3 \mathrm{H}), 1.14-1.10(\mathrm{~m}, 2 \mathrm{H}), 1.07-0.98(\mathrm{~m}, 2 \mathrm{H})$, $0.72(\mathrm{t}, J=7.3 \mathrm{~Hz}, 3 \mathrm{H}), 0.68-0.65(\mathrm{~m}, 2 \mathrm{H}) .{ }^{13} \mathrm{C} \mathrm{NMR}\left(126 \mathrm{MHz}, \mathrm{CDCl}_{3}\right) \delta$ 173.3, 159.2, 152.3, 150.5, 142.9, 140.3, 139.5, 
$139.4,137.0,133.0,129.9,128.7,128.1,127.6,127.4,126.4,123.3,121.6,120.9,120.1,120.0,114.2,113.3,70.2$ 60.2, 55.2, 40.2, 39.5, 34.3, 25.9, 23.0, 19.5, 14.2, 13.8. HRMS (m/z, ESI): Calcd. for Chemical Formula: $\mathrm{C}_{36} \mathrm{H}_{37} \mathrm{CINaO}_{3}{ }^{+}$ $[\mathrm{M}+\mathrm{Na}]^{+}:$575.2323, Found: 575.2322. HPLC analysis: Daicel Chiralpak IB, hexane/iso-propanol = $99: 1,1.0 \mathrm{~mL} / \mathrm{min}$, $\lambda=318 \mathrm{~nm}$, retention time: $7.00 \mathrm{~min}$ (minor) and $8.29 \mathrm{~min}$ (major).

\section{(R)-4-(9-butyl-2-chloro-7-(3-(ethoxycarbonyl)phenyl)-9H-fluoren-9-yl)butanoic acid (24)}

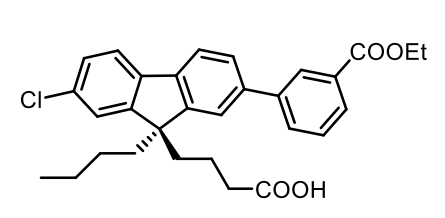

White solid, $75.7 \mathrm{mg}, 62 \%$ yield, $[\alpha]_{D}{ }^{20}=-9.60\left(c^{0} 0.100 \mathrm{CHCl}_{3}\right)$ for $96: 4$ er. ${ }^{1} \mathrm{H}$ $\operatorname{NMR}\left(500 \mathrm{MHz}, \mathrm{CDCl}_{3}\right) \delta 8.34(\mathrm{t}, J=1.6 \mathrm{~Hz}, 1 \mathrm{H}), 8.08-8.02(\mathrm{~m}, 1 \mathrm{H}), 7.85$ (ddd, $J=$ 7.7, 1.9, $1.2 \mathrm{~Hz}, 1 \mathrm{H}), 7.76(\mathrm{~d}, J=7.9 \mathrm{~Hz}, 1 \mathrm{H}), 7.64$ (ddd, $J=9.5,7.5,1.6 \mathrm{~Hz}, 2 \mathrm{H}), 7.58$ $-7.51(\mathrm{~m}, 2 \mathrm{H}), 7.35(\mathrm{dd}, J=7.4,1.7 \mathrm{~Hz}, 2 \mathrm{H}), 4.45(\mathrm{q}, J=7.1 \mathrm{~Hz}, 2 \mathrm{H}), 2.15-1.97(\mathrm{~m}, 6 \mathrm{H}), 1.45(\mathrm{t}, J=7.1 \mathrm{~Hz}, 3 \mathrm{H}), 1.12-$ $1.07(\mathrm{~m}, 2 \mathrm{H}), 1.03-0.96(\mathrm{~m}, 2 \mathrm{H}), 0.71(\mathrm{t}, J=7.4 \mathrm{~Hz}, 3 \mathrm{H}), 0.66-0.62(\mathrm{~m}, 2 \mathrm{H}) .{ }^{13} \mathrm{C} \mathrm{NMR}\left(126 \mathrm{MHz}, \mathrm{CDCl}_{3}\right) \delta 177.7,166.8$, $152.1,150.5,141.7,139.7,139.6,139.2,133.2,131.6,131.0,128.9,128.3,128.3,127.5,126.5,123.3,121.6,121.0$, 120.3, 61.2, 55.2, 40.2, 39.4, 33.7, 25.8, 22.9, 19.2, 14.4, 13.8. HRMS (m/z, ESI): Calcd. for Chemical Formula: $\mathrm{C}_{30} \mathrm{H}_{30} \mathrm{ClO}_{4}{ }^{-}[\mathrm{M}-\mathrm{H}]:$ : 489.1838, Found: 489.1836. HPLC analysis of the reaction product: Daicel Chiralpak $\mathrm{IH}$, hexane/iso-propanol = 95: 5, $1.0 \mathrm{~mL} / \mathrm{min}, \lambda=316 \mathrm{~nm}$, retention time: $6.59 \mathrm{~min}$ (minor) and $7.19 \mathrm{~min}$ (major).

\section{(R)-4-(9-butyl-2-chloro-7-(2-methoxyphenyl)-9H-fluoren-9-yl)butanoic acid (25)}

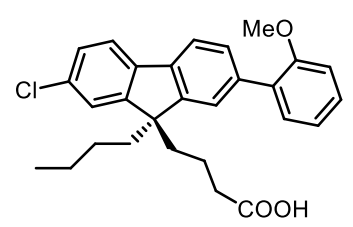

White solid, $76.0 \mathrm{mg}, 68 \%$ yield, $[\alpha]_{D}{ }^{20}=+140.0\left(\mathrm{c} 0.005 \mathrm{CHCl}_{3}\right)$ for $96.5: 3.5 \mathrm{er} .{ }^{1} \mathrm{H}$ $\operatorname{NMR}\left(500 \mathrm{MHz}, \mathrm{CDCl}_{3}\right) \delta 7.74-7.69(\mathrm{~m}, 1 \mathrm{H}), 7.64(\mathrm{~d}, J=7.6 \mathrm{~Hz}, 1 \mathrm{H}), 7.54$ (dd, $J=4.0,2.3$ $\mathrm{Hz}, 2 \mathrm{H}), 7.41(\mathrm{~d}, J=7.5 \mathrm{~Hz}, 1 \mathrm{H}), 7.38-7.31(\mathrm{~m}, 3 \mathrm{H}), 7.08(\mathrm{t}, J=7.5 \mathrm{~Hz}, 1 \mathrm{H}), 7.03(\mathrm{~d}, J=8.2$

$\mathrm{Hz}, 1 \mathrm{H}), 3.83(\mathrm{~s}, 3 \mathrm{H}), 2.14-1.92(\mathrm{~m}, 6 \mathrm{H}), 1.18-1.04(\mathrm{~m}, 6 \mathrm{H}), 0.75-0.71(\mathrm{~m}, 5 \mathrm{H}) .{ }^{13} \mathrm{C} \mathrm{NMR}\left(126 \mathrm{MHz}, \mathrm{CDCl}_{3}\right) \delta 178.6$, $156.6,152.4,149.3,139.6,138.7,137.8,132.8,130.9,130.9,128.6,128.6,127.3,124.4,123.3,121.0,120.7,119.4$, 111.5, 55.7, 40.0, 39.4, 34.2, 25.9, 23.0, 19.4, 13.8. HRMS (m/z, ESI): Calcd. for Chemical Formula: $\mathrm{C}_{28} \mathrm{H}_{29} \mathrm{ClNaO}_{3}{ }^{+}$ $[\mathrm{M}+\mathrm{Na}]^{+}:$471.1697, Found: 471.1695. HPLC analysis of the reaction product: Daicel Chiralpak IA, hexane/isopropanol = 99: $1,1.0 \mathrm{~mL} / \mathrm{min}, \lambda=324 \mathrm{~nm}$, retention time: $13.88 \mathrm{~min}$ (major) and $15.32 \mathrm{~min}$ (minor). 


\section{Ethyl (R)-4-(9-butyl-2-chloro-7-(2,5-dimethylphenyl)-9H-fluoren-9-yl)butanoate (26)}

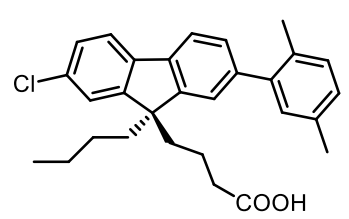

White solid, $64.6 \mathrm{mg}, 58 \%$ yield, $[\alpha]_{D}{ }^{20}=-1.30\left(\right.$ c $\left.0.100 \mathrm{CHCl}_{3}\right)$ for $95: 5$ er. ${ }^{1} \mathrm{H}$ NMR $\left(500 \mathrm{MHz}, \mathrm{CDCl}_{3}\right) \delta 7.71(\mathrm{~d}, J=7.7 \mathrm{~Hz}, 1 \mathrm{H}), 7.65(\mathrm{~d}, J=7.5 \mathrm{~Hz}, 1 \mathrm{H}), 7.33(\mathrm{dd}, J=15.0,7.6$ $\mathrm{Hz}, 3 \mathrm{H}), 7.20(\mathrm{~d}, J=7.7 \mathrm{~Hz}, 1 \mathrm{H}), 7.15-7.08(\mathrm{~m}, 2 \mathrm{H}), 2.39(\mathrm{~s}, 3 \mathrm{H}), 2.26(\mathrm{~s}, 3 \mathrm{H}), 2.13-2.08$ (m, $2 \mathrm{H}), 2.06-2.02(\mathrm{~m}, 2 \mathrm{H}), 2.00-1.94(\mathrm{~m}, 2 \mathrm{H}), 1.13-1.08(\mathrm{~m}, 2 \mathrm{H}), 1.06-0.97(\mathrm{~m}, 2 \mathrm{H}), 0.74-0.62(\mathrm{~m}, 5 \mathrm{H}) .{ }^{13} \mathrm{C} \mathrm{NMR}$ (126 MHz, $\left.\mathrm{CDCl}_{3}\right) \delta$ 152.0, 149.5, 141.9, 141.4, 139.6, 138.6, 135.3, 132.9, 132.2, 130.5, 130.4, 128.3, 128.0, 127.4, 123.8, 123.2, 120.7, 119.5, 55.1, 40.1, 39.5, 33.8, 25.9, 22.9, 20.9, 20.0, 19.2, 13.8. HRMS (m/z, ESI): Calcd. for Chemical Formula: $\mathrm{C}_{29} \mathrm{H}_{30} \mathrm{ClO}_{2}{ }^{-}[\mathrm{M}-\mathrm{H}]=$ : 445.1940, Found: 445.1939. HPLC analysis of the reaction product: Daicel Chiralpak IA, hexane/iso-propanol = 99: 1, $1.0 \mathrm{~mL} / \mathrm{min}, \lambda=320 \mathrm{~nm}$, retention time: $9.86 \mathrm{~min}$ (major) and $11.75 \mathrm{~min}$ (minor).

\section{Ethyl (R)-4-(9-butyl-2-chloro-7-(2,4-difluorophenyl)-9H-fluoren-9-yl)butanoate (27)}

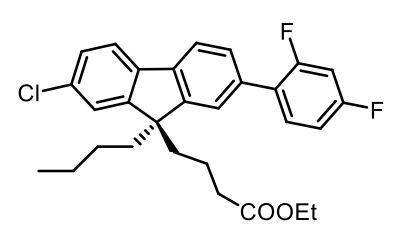

After the reaction was completed, the reaction mixture was neutralized with $1 \mathrm{M}$ $\mathrm{HCl}$ to $\mathrm{pH} 2-3$ and the crude product was dissolved in $\mathrm{EtOH}$, then $10 \mu \mathrm{L}$ conc. $\mathrm{H}_{2} \mathrm{SO}_{4}$ was added and the reaction mixture was stirred at $65^{\circ} \mathrm{C}$ for $10 \mathrm{~h}$. The esterification product was obtained as a white solid, $68.6 \mathrm{mg}, 57 \%$ yield, $[\alpha]_{\mathrm{D}}{ }^{20}=-0.40\left(c 0.100 \mathrm{CHCl}_{3}\right)$ for $93: 7$ er. ${ }^{1} \mathrm{H} \mathrm{NMR}(500 \mathrm{MHz}, \mathrm{CDCl} 3)$ $\delta 7.74(\mathrm{dd}, J=7.9,0.5 \mathrm{~Hz}, 1 \mathrm{H}), 7.67-7.63(\mathrm{~m}, 1 \mathrm{H}), 7.52-7.46(\mathrm{~m}, 3 \mathrm{H}), 7.35(\mathrm{dd}, J=6.6,1.9 \mathrm{~Hz}, 2 \mathrm{H}), 7.03-6.92(\mathrm{~m}$, $2 \mathrm{H}), 4.09-4.00(\mathrm{~m}, 2 \mathrm{H}), 2.10-1.96(\mathrm{~m}, 6 \mathrm{H}), 1.19(\mathrm{t}, J=7.1 \mathrm{~Hz}, 3 \mathrm{H}), 1.1-1.09(\mathrm{~m}, 2 \mathrm{H}), 1.06-0.98(\mathrm{~m}, 2 \mathrm{H}), 0.72(\mathrm{t}, J=$ $7.4 \mathrm{~Hz}, 3 \mathrm{H}), 0.69-0.62(\mathrm{~m}, 2 \mathrm{H}) .{ }^{13} \mathrm{C}$ NMR $\left(126 \mathrm{MHz}, \mathrm{CDCl}_{3}\right) \delta 173.3,152.3,150.1,139.6,139.2,134.1,133.2,131.5$ (dd, $J=9.4,4.9 \mathrm{~Hz}), 128.1(\mathrm{~d}, J=2.3 \mathrm{~Hz}), 127.5,123.5(\mathrm{~d}, J=2.8 \mathrm{~Hz}), 123.3,121.0,119.9,111.6(\mathrm{dd}, J=21.1,3.5 \mathrm{~Hz})$, $104.4(\mathrm{t}, \mathrm{J}=25.9 \mathrm{~Hz}), 104.2,104.2,60.2,55.2,40.0,39.5,34.3,25.8,22.9,19.5,14.2,13.8 .{ }^{19} \mathrm{~F} \mathrm{NMR}\left(471 \mathrm{MHz}, \mathrm{CDCl}_{3}\right)$ $\delta-111.53(\mathrm{~d}, J=7.3 \mathrm{~Hz}),-113.09(\mathrm{~d}, J=7.3 \mathrm{~Hz})$. HRMS (m/z, ESI): Calcd. for Chemical Formula: $\mathrm{C}_{29} \mathrm{H}_{29} \mathrm{ClF}_{2} \mathrm{NaO}_{2}{ }^{+}$ $[\mathrm{M}+\mathrm{Na}]^{+}:$505.1716, Found: 505.1708.

A sample of the ester was hydrolyzed to provide the parent carboxylic acid for the HPLC analysis of the reaction product: Daicel Chiralpak IA, hexane/iso-propanol = 99: 1, $1.0 \mathrm{~mL} / \mathrm{min}, \lambda=317 \mathrm{~nm}$, retention time: $12.33 \mathrm{~min}$ (major) and $13.17 \mathrm{~min}$ (minor). 


\section{Ethyl (R)-4-(9-butyl-2-chloro-7-(naphthalen-2-yl)-9H-fluoren-9-yl)butanoate (28)}

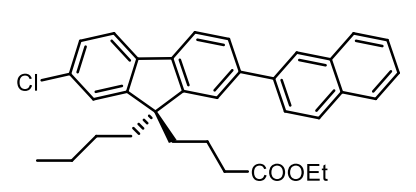

After the reaction was completed, the reaction mixture was neutralized with 1

$\mathrm{M} \mathrm{HCl}$ to $\mathrm{pH} 2-3$ and the crude product was dissolved in $\mathrm{EtOH}$, then $10 \mu \mathrm{L}$ conc. $\mathrm{H}_{2} \mathrm{SO}_{4}$ was added and the reaction mixture was stirred at $65^{\circ} \mathrm{C}$ for $10 \mathrm{~h}$. The esterification product was obtained as a white solid, $86.5 \mathrm{mg}, 70 \%$ yield, $[\alpha]_{\mathrm{D}}^{20}=+7.60$ (c 0.100

$\mathrm{CHCl}_{3}$ ) for 94:6 er. ${ }^{1} \mathrm{H} \mathrm{NMR}\left(500 \mathrm{MHz}, \mathrm{CDCl}_{3}\right) \delta 8.13(\mathrm{~d}, J=1.3 \mathrm{~Hz}, 1 \mathrm{H}), 7.96(\mathrm{t}, J=7.1 \mathrm{~Hz}, 2 \mathrm{H}), 7.91(\mathrm{~d}, J=7.8 \mathrm{~Hz}, 1 \mathrm{H})$, $7.84(\mathrm{dd}, J=8.5,1.8 \mathrm{~Hz}, 1 \mathrm{H}), 7.79(\mathrm{~d}, J=7.9 \mathrm{~Hz}, 1 \mathrm{H}), 7.74(\mathrm{dd}, J=7.9,1.6 \mathrm{~Hz}, 1 \mathrm{H}), 7.70-7.66(\mathrm{~m}, 2 \mathrm{H}), 7.58-7.49(\mathrm{~m}$, 2H), 7.37-7.36 (m, 2H), $4.05(\mathrm{q}, J=7.1 \mathrm{~Hz}, 2 \mathrm{H}), 2.17-1.98(\mathrm{~m}, 6 \mathrm{H}), 1.19(\mathrm{t}, J=7.1 \mathrm{~Hz}, 3 \mathrm{H}), 1.15-1.13(\mathrm{~m}, 2 \mathrm{H}), 1.09-$ $1.00(\mathrm{~m}, 2 \mathrm{H}), 0.76-0.65(\mathrm{~m}, 5 \mathrm{H}) .{ }^{13} \mathrm{C} \mathrm{NMR}\left(126 \mathrm{MHz}, \mathrm{CDCl}_{3}\right) \delta 173.3,152.3,150.6,140.5,139.4,138.7,133.7,133.0$, $132.7,128.5,128.2,127.7,127.4,126.7,126.4,126.0,125.8,125.7,123.3,121.8,120.9,120.3,60.2,55.3,40.2,39.6$, 34.4, 25.9, 23.0, 19.5, 14.2, 13.8. HRMS (m/z, ESI): Calcd. for Chemical Formula: $\mathrm{C}_{33} \mathrm{H}_{33} \mathrm{ClNaO}_{2}{ }^{+}[\mathrm{M}+\mathrm{Na}]^{+}: 519.2061$, Found: 519.2062 .

A sample of the ester was hydrolyzed to provide the parent carboxylic acid for HPLC analysis of the initial reaction product: Daicel Chiralpak IA, hexane/iso-propanol $=97: 3,1.0 \mathrm{~mL} / \mathrm{min}, \lambda=321 \mathrm{~nm}$, retention time: $7.63 \mathrm{~min}$ (major) and $8.47 \mathrm{~min}$ (minor).

\section{Ethyl (R)-4-(9-butyl-2-chloro-7-(dibenzo[b,d]furan-4-yl)-9H-fluoren-9-yl)butanoate (29)}

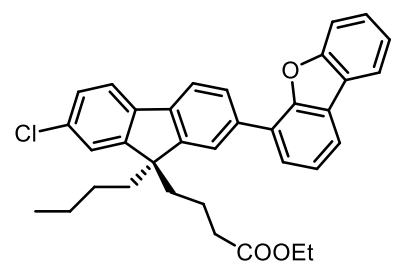

After the reaction was completed, the reaction mixture was neutralized with $1 \mathrm{M} \mathrm{HCl}$ to $\mathrm{pH} 2-3$ and the crude product was dissolved in $\mathrm{EtOH}$, then $10 \mu \mathrm{L}$ conc. $\mathrm{H}_{2} \mathrm{SO}_{4}$ was added and the reaction mixture was stirred at $65{ }^{\circ} \mathrm{C}$ for $10 \mathrm{~h}$. The esterification product was obtained as a white solid, $83.0 \mathrm{mg}, 62 \%$ yield, $[\alpha]_{D^{20}}=$ $+16.00\left(\mathrm{c} 0.100 \mathrm{CHCl}_{3}\right)$ for $96: 4$ er. ${ }^{1} \mathrm{H} \mathrm{NMR}\left(500 \mathrm{MHz}, \mathrm{CDCl}_{3}\right) \delta 8.05-8.01(\mathrm{~m}, 1 \mathrm{H}), 8.00-7.93(\mathrm{~m}, 2 \mathrm{H}), 7.90(\mathrm{~s}, 1 \mathrm{H})$, $7.85(\mathrm{~d}, J=7.9 \mathrm{~Hz}, 1 \mathrm{H}), 7.72-7.68(\mathrm{~m}, 2 \mathrm{H}), 7.64(\mathrm{~d}, J=8.2 \mathrm{~Hz}, 1 \mathrm{H}), 7.50(\mathrm{dt}, J=15.2,7.4 \mathrm{~Hz}, 2 \mathrm{H}), 7.43-7.35(\mathrm{~m}, 3 \mathrm{H})$, $4.05(q, J=7.1 \mathrm{~Hz}, 2 \mathrm{H}), 2.17-1.99(\mathrm{~m}, 6 \mathrm{H}), 1.19-1.12(\mathrm{~m}, 7 \mathrm{H}), 0.78-0.75(\mathrm{~m}, 5 \mathrm{H}) .{ }^{13} \mathrm{C} \mathrm{NMR}\left(126 \mathrm{MHz}, \mathrm{CDCl}_{3}\right) \delta 173.3$, $156.2,153.4,152.5,150.2,139.7,139.4,135.6,133.1,128.0,127.5,127.3,126.8,126.2,125.0,124.2,123.4,123.3_{1}$, $123.32,122.8,121.0,120.7,120.0,119.7,111.9,60.2,55.2,40.1,39.5,34.5,25.9,23.0,19.6,14.2,13.8$. HRMS (m/z, ESI): Calcd. for Chemical Formula: $\mathrm{C}_{35} \mathrm{H}_{33} \mathrm{ClNaO}_{3}{ }^{+}[\mathrm{M}+\mathrm{Na}]^{+}: 559.2010$, Found: 559.2004. 
A sample of the ester was hydrolyzed to provide the parent carboxylic acid for the HPLC analysis of the reaction product: Daicel Chiralpak IA, hexane/iso-propanol = 97: 3, $1.0 \mathrm{~mL} / \mathrm{min}, \lambda=324 \mathrm{~nm}$, retention time: $7.59 \mathrm{~min}$ (major) and $8.18 \mathrm{~min}$ (minor).

\section{Ethyl (R)-4-(9-butyl-7-chloro-9',9'-dimethyl-9H,9'H-[2,2'-bifluoren]-9-yl)butanoate (30)}

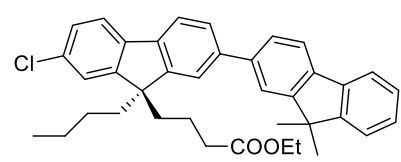

White solid, $91.5 \mathrm{mg}, 65 \%$ yield, $[\alpha]_{\mathrm{D}}{ }^{20}=+3.60\left(c 0.100 \mathrm{CHCl}_{3}\right)$ for $98: 2$ er. ${ }^{1} \mathrm{H}$

$\operatorname{NMR}\left(500 \mathrm{MHz}, \mathrm{CDCl}_{3}\right) \delta 7.83(\mathrm{~d}, J=7.7 \mathrm{~Hz}, 1 \mathrm{H}), 7.81-7.78(\mathrm{~m}, 1 \mathrm{H}), 7.76(\mathrm{~d}, J=7.8$ $\mathrm{Hz}, 1 \mathrm{H}), 7.73(\mathrm{~d}, J=1.2 \mathrm{~Hz}, 1 \mathrm{H}), 7.69-7.65(\mathrm{~m}, 3 \mathrm{H}), 7.61(\mathrm{~d}, J=1.2 \mathrm{~Hz}, 1 \mathrm{H}), 7.51-7.48(\mathrm{~m}, 1 \mathrm{H}), 7.42-7.34(\mathrm{~m}, 4 \mathrm{H})$, $4.09-4.02(\mathrm{~m}, 2 \mathrm{H}), 2.15-2.00(\mathrm{~m}, 6 \mathrm{H}), 1.60(\mathrm{~s}, 6 \mathrm{H}), 1.19(\mathrm{t}, J=7.1 \mathrm{~Hz}, 3 \mathrm{H}), 1.16-1.11(\mathrm{~m}, 2 \mathrm{H}), 1.07-1.01(\mathrm{~m}, 2 \mathrm{H})$, $0.74-0.65$ (m, 5H). ${ }^{13} \mathrm{C}$ NMR $\left(126 \mathrm{MHz}, \mathrm{CDCl}_{3}\right) \delta 173.3,154.3,153.9,152.3,150.5,141.0,140.6,139.5,139.2,138.9$ 138.5, 132.9, 127.4, 127.3, 127.1, 126.5, 126.3, 123.3, 122.6, 121.5, 121.4, 120.8, 120.3, 120.1, 120.1, 60.2, 55.2, 47.0, 40.3, 39.6, 34.4, 27.3, 25.9, 23.0, 19.5, 14.2, 13.8. HRMS (m/z, ESI): Calcd. for Chemical Formula: $\mathrm{C}_{38} \mathrm{H}_{39} \mathrm{CINaO}_{2}{ }^{+}$ $[\mathrm{M}+\mathrm{Na}]^{+}:$585.2531, Found: 585.2525.

A sample of the ester was hydrolyzed to provide the parent carboxylic acid for the HPLC analysis of the reaction product: Daicel Chiralpak IA, hexane/iso-propanol =99: 1, $1.0 \mathrm{~mL} / \mathrm{min}, \lambda=330 \mathrm{~nm}$, retention time: $22.16 \mathrm{~min}$ (major) and $24.66 \mathrm{~min}$ (minor).

\section{Ethyl (R)-4-(9-butyl-2-chloro-7-(dibenzo[b,d]thiophen-4-yl)-9H-fluoren-9-yl)butanoate (31)}

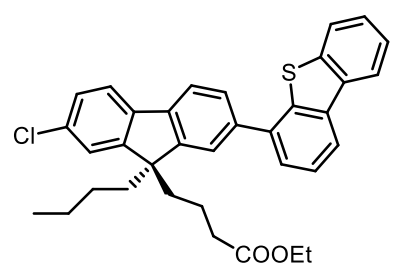

After the reaction was completed, the reaction mixture was neutralized with 1 $\mathrm{M} \mathrm{HCl}$ to $\mathrm{pH} 2-3$ and the crude product was dissolved in $\mathrm{EtOH}$, then $10 \mu \mathrm{L}$ conc. $\mathrm{H}_{2} \mathrm{SO}_{4}$ was added and the reaction mixture was stirred at $65^{\circ} \mathrm{C}$ for $10 \mathrm{~h}$. The esterification product was obtained as a white solid, $96.4 \mathrm{mg}, 70 \%$ yield, $[\alpha]_{\mathrm{D}}^{20}=+20.50$ (c 0.100

$\left.\mathrm{CHCl}_{3}\right)$ for $96: 4$ er. ${ }^{1} \mathrm{H} \mathrm{NMR}\left(500 \mathrm{MHz}, \mathrm{CDCl}_{3}\right) \delta 8.25-8.17(\mathrm{~m}, 2 \mathrm{H}), 7.87(\mathrm{dd}, J=5.7,3.3 \mathrm{~Hz}, 1 \mathrm{H}), 7.83(\mathrm{~d}, J=7.8 \mathrm{~Hz}$, $1 \mathrm{H}), 7.79(\mathrm{~s}, 1 \mathrm{H}), 7.74-7.67(\mathrm{~m}, 2 \mathrm{H}), 7.64-7.58(\mathrm{~m}, 2 \mathrm{H}), 7.54-7.47(\mathrm{~m}, 2 \mathrm{H}), 7.40-7.35(\mathrm{~m}, 2 \mathrm{H}), 4.06(\mathrm{q}, J=7.1 \mathrm{~Hz}$ $2 \mathrm{H}), 2.13-2.07(\mathrm{~m}, 6 \mathrm{H}), 1.21-1.08(\mathrm{~m}, 7 \mathrm{H}), 0.80-0.70(\mathrm{~m}, 5 \mathrm{H}) .{ }^{13} \mathrm{C} \mathrm{NMR}\left(126 \mathrm{MHz}, \mathrm{CDCl}_{3}\right) \delta 173.3,152.4,150.4$, $139.9,139.8,139.6,139.3,138.8,137.3,136.3,135.8,133.2,127.6,127.5,126.9,125.2,124.4,123.3,122.8,122.6$ 121.8, 121.0, 120.5, 120.3, 60.2, 55.3, 40.1, 39.5, 34.5, 25.9, 23.0, 19.6, 14.2, 13.9. HRMS (m/z, ESI): Calcd. for 
Chemical Formula: $\mathrm{C}_{35} \mathrm{H}_{33} \mathrm{ClNaO}_{3} \mathrm{~S}[\mathrm{M}+\mathrm{Na}]^{+}:$575.1782, Found: 575.1781.

A sample of the ester was hydrolyzed to provide the parent carboxylic acid for the HPLC analysis of the reaction product: Daicel Chiralpak IA, hexane/iso-propanol $=97: 3,1.0 \mathrm{~mL} / \mathrm{min}, \lambda=320 \mathrm{~nm}$, retention time: $8.37 \mathrm{~min}$ (major) and $9.64 \mathrm{~min}$ (minor).

\section{Ethyl (R)-4-(9-butyl-2-chloro-7-(thianthren-1-yl)-9H-fluoren-9-yl)butanoate (32)}

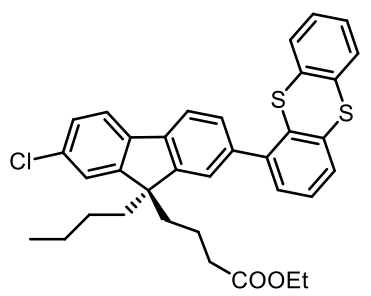

After the reaction was completed, the reaction mixture was neutralized with $1 \mathrm{M}$ $\mathrm{HCl}$ to $\mathrm{pH} 2-3$ and the crude product was dissolved in $\mathrm{EtOH}$, then $10 \mu \mathrm{L}$ conc. $\mathrm{H}_{2} \mathrm{SO}_{4}$ was added and the reaction mixture was stirred at $65^{\circ} \mathrm{C}$ for $10 \mathrm{~h}$. The esterification product was obtained as a white solid, $89.0 \mathrm{mg}, 61 \%$ yield, $[\alpha]_{\mathrm{D}}^{20}=+7.80\left(\mathrm{c} 0.100 \mathrm{CHCl}_{3}\right)$ for

94.5:5.5 er. ${ }^{1} \mathrm{H}$ NMR (500 MHz, $\left.\mathrm{CDCl}_{3}\right) \delta 7.80-7.77(\mathrm{~m}, 1 \mathrm{H}), 7.71-7.67(\mathrm{~m}, 1 \mathrm{H}), 7.56(\mathrm{dd}, J=7.6,1.5 \mathrm{~Hz}, 1 \mathrm{H}), 7.53$ (dd, $J=7.7,1.2 \mathrm{~Hz}, 1 \mathrm{H}), 7.42(\mathrm{~d}, J=1.1 \mathrm{~Hz}, 1 \mathrm{H}), 7.41-7.36(\mathrm{~m}, 4 \mathrm{H}), 7.35-7.32(\mathrm{~m}, 2 \mathrm{H}), 7.26(\mathrm{td}, J=7.5,1.4 \mathrm{~Hz}, 1 \mathrm{H})$, $7.19(\mathrm{td}, J=7.5,1.4 \mathrm{~Hz}, 1 \mathrm{H}), 4.05(\mathrm{q}, J=7.1 \mathrm{~Hz}, 2 \mathrm{H}), 2.14-2.00(\mathrm{~m}, 6 \mathrm{H}), 1.20-1.05(\mathrm{~m}, 7 \mathrm{H}), 0.81-0.69(\mathrm{~m}, 5 \mathrm{H}) .{ }^{13} \mathrm{C}$ NMR $\left(126 \mathrm{MHz} \mathrm{CDCl}_{3}\right) \delta 173.3,152.4,149.6,142.8,139.7,139.4,139.3,136.4,136.1,135.4,135.2,133.2,129.2$ $128.8,128.6,128.3,128.3,127.8,127.6,127.5,127.1,124.4,123.3,121.0,119.7,60.2,55.2,40.1,39.6,34.5,25.9$, 23.1, 19.6, 14.2, 13.9. HRMS (m/z, ESI): Calcd. for Chemical Formula: $\mathrm{C}_{35} \mathrm{H}_{34} \mathrm{ClO}_{2} \mathrm{~S}_{2}{ }^{+}[\mathrm{M}+\mathrm{H}]^{+}:$585.5275, Found: 585.5309.

A sample of the ester was hydrolyzed to provide the parent carboxylic acid for the HPLC analysis of the reaction product: Daicel Chiralpak IA, hexane/iso-propanol =97: 3, $1.0 \mathrm{~mL} / \mathrm{min}, \lambda=320 \mathrm{~nm}$, retention time: $7.08 \mathrm{~min}$ (major) and $8.06 \mathrm{~min}$ (minor).

\section{Ethyl (R)-4-(9-butyl-2-chloro-7-(9-phenyl-9H-carbazol-2-yl)-9H-fluoren-9-yl)butanoate (33)}

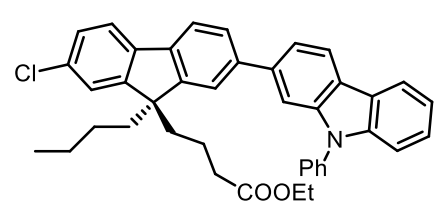

After the reaction was completed, the reaction mixture was neutralized with $1 \mathrm{M} \mathrm{HCl}$ to $\mathrm{pH} \mathrm{2-3} \mathrm{and} \mathrm{the} \mathrm{crude} \mathrm{product} \mathrm{was} \mathrm{dissolved} \mathrm{in} \mathrm{EtOH}$, then $10 \mu \mathrm{L}$ conc. $\mathrm{H}_{2} \mathrm{SO}_{4}$ was added and the reaction mixture was stirred at $65{ }^{\circ} \mathrm{C}$ for $10 \mathrm{~h}$. The esterification product was obtained as a white solid, $97.5 \mathrm{mg}, 64 \%$ yield, $[\alpha]_{D}{ }^{20}=$

+4.6 (c $\left.0.100 \mathrm{CHCl}_{3}\right)$ for 92.5:7.5 er. ${ }^{1} \mathrm{H} \mathrm{NMR}\left(500 \mathrm{MHz}, \mathrm{CDCl}_{3}\right) \delta 8.47(\mathrm{~s}, 1 \mathrm{H}), 8.29(\mathrm{~d}, J=7.7 \mathrm{~Hz}, 1 \mathrm{H}), 7.81-7.73(\mathrm{~m}$, 
3H), $7.72-7.63(\mathrm{~m}, 6 \mathrm{H}), 7.53(\mathrm{dd}, J=9.2,4.6 \mathrm{~Hz}, 2 \mathrm{H}), 7.48(\mathrm{~d}, J=3.7 \mathrm{~Hz}, 2 \mathrm{H}), 7.38-7.36(\mathrm{~m}, 3 \mathrm{H}), 4.08(\mathrm{q}, J=7.1 \mathrm{~Hz}$, $2 \mathrm{H}), 2.20-2.01(\mathrm{~m}, 6 \mathrm{H}), 1.23-1.07(\mathrm{~m}, 7 \mathrm{H}), 0.76-0.73(\mathrm{~m}, 5 \mathrm{H}) .{ }^{13} \mathrm{C} N M R\left(126 \mathrm{MHz}, \mathrm{CDCl}_{3}\right) \delta 173.3,152.3,150.6$, $141.5,140.4,139.6,138.6,137.7,133.7,132.8,130.0,127.6,127.6,127.4,127.1,126.6,126.2,125.6,124.0,123.5$, $123.3,121.7,120.8,120.5,120.2,120.1,118.9,110.1,110.0,60.2,55.2,40.3,39.6,34.4,25.9,23.0,19.5,14.2,13.9$. HRMS (m/z, ESI): Calcd. for Chemical Formula: $\mathrm{C}_{41} \mathrm{H}_{38} \mathrm{CINNaO}_{2}{ }^{+}[\mathrm{M}+\mathrm{Na}]^{+}:$634.2483, Found: 634.2478.

A sample of the ester was hydrolyzed to provide the parent carboxylic acid for the HPLC analysis of the reaction product: Daicel Chiralpak IA, hexane/iso-propanol =97: 3, $1.0 \mathrm{~mL} / \mathrm{min}, \lambda=330 \mathrm{~nm}$, retention time: $10.78 \mathrm{~min}$ (major) and $11.99 \min$ (minor).

\title{
(R)-4-(9-butyl-2-chloro-7-(4-methoxyphenyl)-9H-xanthen-9-yl)butanoic acid (34)
}

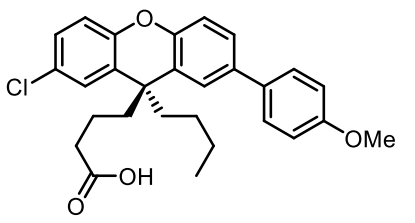

\author{
White solid, $64.2 \mathrm{mg}, 55 \%$ yield, $[\alpha]_{\mathrm{D}}{ }^{20}=-3.80\left(c 0.50 \mathrm{CHCl}_{3}\right)$ for $95.5: 4.5$ er. ${ }^{1} \mathrm{H}$ \\ $\operatorname{NMR}\left(500 \mathrm{MHz}, \mathrm{CDCl}_{3}\right) \delta 7.50-7.46(\mathrm{~m}, 2 \mathrm{H}), 7.41(\mathrm{~d}, J=2.2 \mathrm{~Hz}, 1 \mathrm{H}), 7.37(\mathrm{dd}, J=8.4$, \\ $2.2 \mathrm{~Hz}, 1 \mathrm{H}), 7.25(\mathrm{~d}, J=2.5 \mathrm{~Hz}, 1 \mathrm{H}), 7.16(\mathrm{dd}, J=8.7,2.4 \mathrm{~Hz}, 1 \mathrm{H}), 7.04(\mathrm{~d}, J=8.4 \mathrm{~Hz}$,
} 1H), $6.99-6.95(\mathrm{~m}, 3 \mathrm{H}), 3.85(\mathrm{~s}, 3 \mathrm{H}), 2.19(\mathrm{td}, J=7.2,2.0 \mathrm{~Hz}, 2 \mathrm{H}), 2.12-2.06(\mathrm{~m}, 1 \mathrm{H}), 1.99-1.93(\mathrm{~m}, 2 \mathrm{H}), 1.90-$ $1.84(\mathrm{~m}, 1 \mathrm{H}), 1.31-1.21(\mathrm{~m}, 2 \mathrm{H}), 1.15-1.11(\mathrm{~m}, 2 \mathrm{H}), 0.90-0.82(\mathrm{~m}, 2 \mathrm{H}), 0.71(\mathrm{t}, J=7.3 \mathrm{~Hz}, 3 \mathrm{H}) .{ }^{13} \mathrm{C} \mathrm{NMR}(126 \mathrm{MHz}$ $\left.\mathrm{CDCl}_{3}\right) \delta 178.1,159.1,150.6,150.5,136.4,133.6,128.1,128.0,127.8,126.8,126.4,126.2,124.4,117.9,116.6,114.4$ 55.5, 45.6, 44.1, 42.7, 33.7, 27.0, 23.0, 20.2, 14.0. HRMS (m/z, ESI): Calcd. for Chemical Formula $\mathrm{C}_{28} \mathrm{H}_{28} \mathrm{ClO}_{4}{ }^{-}[\mathrm{M}-$ $\mathrm{H}^{-}$:463.1682, Found: 463.1668. HPLC analysis of the reaction product: Daicel Chiralpak IA, hexane/iso-propanol = 95: 5, $1.0 \mathrm{~mL} / \mathrm{min}, \lambda=273 \mathrm{~nm}$, retention time: $5.84 \mathrm{~min}$ (major) and $8.55 \mathrm{~min}$ (minor).

\section{(S)-4-(9-butyl-2-chloro-7-(4-methoxyphenyl)-9H-xanthen-9-yl)butanoic acid (ent-34)}

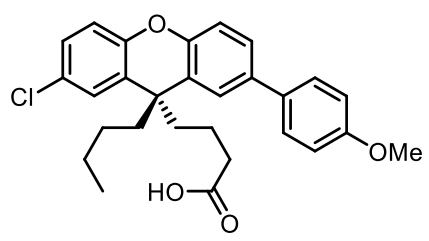

The reaction was carried out using $(R)$-L4 as the ligand. White solid, $61.0 \mathrm{mg}$, $53 \%$ yield, $[\alpha]_{D}{ }^{20}=+5.83\left(c 1.00 \mathrm{CHCl}_{3}\right)$ for $4: 96$ er. HPLC analysis of the reaction product: Daicel Chiralpak IA, hexane/iso-propanol = 95: 5, $1.0 \mathrm{~mL} / \mathrm{min}, \lambda=273 \mathrm{~nm}$, retention time: $7.87 \mathrm{~min}$ (major) and $5.87 \mathrm{~min}$ (minor). 
(R)-5-(9-butyl-2-chloro-7-(4-methoxyphenyl)-9H-xanthen-9-yl)pentanoic acid (35)

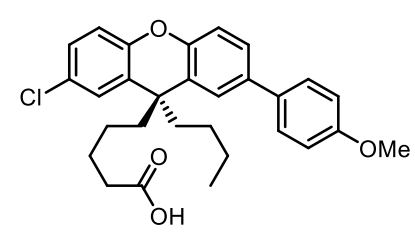

White solid, $50 \mathrm{mg}, 42 \%$ yield, $[\alpha]_{\mathrm{D}^{20}}=-10.00\left(\mathrm{c} 0.040 \mathrm{CHCl}_{3}\right)$ for $93: 7$ er. ${ }^{1} \mathrm{H}$ $\operatorname{NMR}\left(500 \mathrm{MHz}, \mathrm{CDCl}_{3}\right) \delta 7.48(\mathrm{dt}, J=8.7,2.2 \mathrm{~Hz}, 2 \mathrm{H}), 7.43-7.34(\mathrm{~m}, 2 \mathrm{H}), 7.25-$ $7.22(\mathrm{~m}, 1 \mathrm{H}), 7.15(\mathrm{dd}, J=8.7,2.1 \mathrm{~Hz}, 1 \mathrm{H}), 7.06-7.02(\mathrm{~m}, 1 \mathrm{H}), 7.01-6.94(\mathrm{~m}, 3 \mathrm{H})$, $3.85(\mathrm{~s}, 3 \mathrm{H}), 2.14(\mathrm{t}, J=7.6 \mathrm{~Hz}, 2 \mathrm{H}), 2.01-1.86(\mathrm{~m}, 4 \mathrm{H}), 1.47(\mathrm{q}, J=7.8 \mathrm{~Hz}, 2 \mathrm{H}), 1.17-1.12(\mathrm{~m}, 2 \mathrm{H}), 0.96-0.83(\mathrm{~m}$, 4H), $0.72(\mathrm{t}, J=7.4 \mathrm{~Hz}, 3 \mathrm{H}) .{ }^{13} \mathrm{C} N M R\left(126 \mathrm{MHz}, \mathrm{CDCl}_{3}\right) \delta 178.4,159.1,150.6,150.5,136.3,133.6,128.1,128.0,127.7$, 127.2, 126.2, 126.1, 124.7, 124.3, 117.8, 116.6, 114.4, 55.5, 45.3, 45.1, 42.8, 33.6, 27.1, 25.0, 24.5, 23.0, 14.0. HRMS (m/z, ESI): Calcd. for Chemical Formula $\mathrm{C}_{29} \mathrm{H}_{30} \mathrm{ClO}_{4}{ }^{-}[\mathrm{M}-\mathrm{H}]^{-}: 477.1838$, Found: 477.1841. HPLC analysis of the reaction product: Daicel Chiralpak IA, hexane/iso-propanol = 99: 1, $1.0 \mathrm{~mL} / \mathrm{min}, \lambda=273 \mathrm{~nm}$, retention time: 22.29 min (major) and $20.59 \mathrm{~min}$ (minor).

\section{(R)-4-(9-butyl-2-chloro-7-(4-(diphenylamino)phenyl)-9H-xanthen-9-yl)butanoic acid (36)}

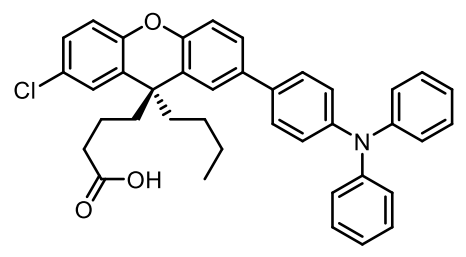

White solid, $95 \mathrm{mg}, 63 \%$ yield, $[\alpha]_{\mathrm{D}}{ }^{20}=+2.25\left(\mathrm{c} 0.400 \mathrm{CHCl}_{3}\right)$ for $97: 3$ er. ${ }^{1} \mathrm{H}$ $\operatorname{NMR}\left(500 \mathrm{MHz}, \mathrm{CDCl}_{3}\right) \delta 7.48-7.42(\mathrm{~m}, 3 \mathrm{H}), 7.40(\mathrm{dd}, J=8.4,2.2 \mathrm{~Hz}, 1 \mathrm{H}), 7.31-$ $7.22(\mathrm{~m}, 6 \mathrm{H}), 7.14(\mathrm{td}, J=9.9,8.6,5.4 \mathrm{~Hz}, 6 \mathrm{H}), 7.06-6.99(\mathrm{~m}, 3 \mathrm{H}), 6.97(\mathrm{~d}, J=8.6$ $H z, 1 H), 2.16-2.06(m, 3 H), 1.98-1.81(m, 3 H), 1.25-1.09(m, 4 H), 0.90-0.84$ (m, 2H), $0.71(\mathrm{t}, J=7.3 \mathrm{~Hz}, 3 \mathrm{H}) .{ }^{13} \mathrm{C}$ NMR $\left(126 \mathrm{MHz}, \mathrm{CDCl}_{3}\right) \delta 178.1,150.7,150.5,147.8,147.1,136.2,134.9,129.4$, $128.1,127.9,127.6,126.8,126.3,126.1,124.5,124.3,124.2,123.0,117.9,116.7,45.8,44.0,42.7,33.6,27.0,23.0$, 20.2, 14.0. HRMS (m/z, ESI): Calcd. for Chemical Formula $\mathrm{C}_{39} \mathrm{H}_{35} \mathrm{ClNO}_{3}{ }^{-}[\mathrm{M}-\mathrm{H}]^{-}: 600.2311$, Found: 600.2312. HPLC analysis of the reaction product: Daicel Chiralpak IA, hexane/iso-propanol $=97: 3,1.0 \mathrm{~mL} / \mathrm{min}, \lambda=321 \mathrm{~nm}$, retention time: $5.83 \mathrm{~min}$ (major) and $6.30 \mathrm{~min}$ (minor).

\section{(R)-4-(9-butyl-2-chloro-7-(4-nitrophenyl)-9H-xanthen-9-yl)butanoic acid (37)}

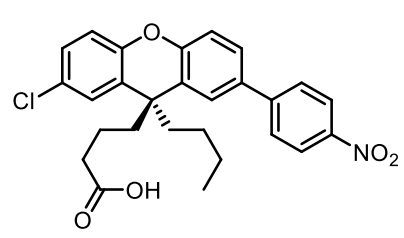

Yellow solid, $84 \mathrm{mg}, 70 \%$ yield, $[\alpha]_{\mathrm{D}}{ }^{20}=-7.00\left(\mathrm{c} 0.100 \mathrm{CHCl}_{3}\right)$ for $96: 4 \mathrm{er} .{ }^{1} \mathrm{H} \mathrm{NMR}$ $\left(500 \mathrm{MHz}, \mathrm{CDCl}_{3}\right) \delta 8.24(\mathrm{~d}, J=8.5 \mathrm{~Hz}, 2 \mathrm{H}), 7.68(\mathrm{~d}, J=8.7 \mathrm{~Hz}, 2 \mathrm{H}), 7.53(\mathrm{~d}, J=2.2 \mathrm{~Hz}$,

1H), $7.44(\mathrm{dd}, J=8.5,2.1 \mathrm{~Hz}, 1 \mathrm{H}), 7.28-7.25(\mathrm{~m}, 1 \mathrm{H}), 7.18(\mathrm{dd}, J=8.7,2.4 \mathrm{~Hz}, 1 \mathrm{H})$,

$7.11(\mathrm{~d}, J=8.4 \mathrm{~Hz}, 1 \mathrm{H}), 7.00(\mathrm{~d}, J=8.7 \mathrm{~Hz}, 1 \mathrm{H}), 2.32-2.13(\mathrm{~m}, 3 \mathrm{H}), 2.05-1.96(\mathrm{~m}, 1 \mathrm{H}), 1.91-1.89(\mathrm{~m}, 2 \mathrm{H}), 1.33(\mathrm{~d}$, 
$J=14.2 \mathrm{~Hz}, 2 \mathrm{H}), 1.13(\mathrm{dd}, J=7.3,3.1 \mathrm{~Hz}, 2 \mathrm{H}), 0.88-0.84(\mathrm{~m}, 2 \mathrm{H}), 0.71(\mathrm{t}, J=7.3 \mathrm{~Hz}, 3 \mathrm{H}) .{ }^{13} \mathrm{C} \mathrm{NMR}\left(126 \mathrm{MHz}, \mathrm{CDCl}_{3}\right)$ $\delta 179.2,152.3,150.2,147.2,146.9,134.1,128.6,128.1,127.5,127.0,126.6,126.1,125.6,125.2,124.3,118.0,117.2$ 46.2, 43.6, 42.8, 33.6, 26.9, 22.9, 20.1, 13.9. HRMS (m/z, ESI): Calcd. for Chemical Formula $\mathrm{C}_{27} \mathrm{H}_{25} \mathrm{CINO}_{5}{ }^{-}[\mathrm{M}-\mathrm{H}]$ ]: 478.1427, Found: 478.1432. HPLC analysis of the reaction product: Daicel Chiralpak IA, hexane/iso-propanol = 95: 5, $1.0 \mathrm{~mL} / \mathrm{min}, \lambda=332 \mathrm{~nm}$, retention time: $8.38 \mathrm{~min}$ (major) and $16.58 \mathrm{~min}$ (minor).

(R)-5-(9-butyl-2-chloro-7-(4-nitrophenyl)-9H-xanthen-9-yl)pentanoic acid (38)

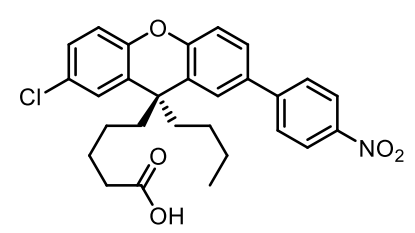

Yellow solid, $62 \mathrm{mg}, 50 \%$ yield, $[\alpha]_{\mathrm{D}}{ }^{20}=-1.00\left(\mathrm{c} 0.100 \mathrm{CHCl}_{3}\right)$ for $95: 5$ er. ${ }^{1} \mathrm{H}$ NMR $\left(500 \mathrm{MHz}, \mathrm{CDCl}_{3}\right) \delta 8.30(\mathrm{~d}, J=8.7 \mathrm{~Hz}, 2 \mathrm{H}), 7.70(\mathrm{~d}, J=8.7 \mathrm{~Hz}, 2 \mathrm{H}), 7.51-7.45(\mathrm{~m}$,

2H), $7.25(\mathrm{~s}, 1 \mathrm{H}), 7.18(\mathrm{dd}, J=8.6,2.4 \mathrm{~Hz}, 1 \mathrm{H}), 7.11(\mathrm{~d}, J=8.4 \mathrm{~Hz}, 1 \mathrm{H}), 6.99(\mathrm{~d}, J=8.6$

$\mathrm{Hz}, 1 \mathrm{H}), 2.16(\mathrm{td}, J=7.6,5.1 \mathrm{~Hz}, 2 \mathrm{H}), 2.04-1.93(\mathrm{~m}, 4 \mathrm{H}), 1.51-1.47(\mathrm{~m}, 2 \mathrm{H}), 1.18-1.13(\mathrm{~m}, 2 \mathrm{H}), 0.98-0.92(\mathrm{~m}$, $2 \mathrm{H}), 0.88-0.85(\mathrm{~m}, 2 \mathrm{H}), 0.73(\mathrm{t}, J=7.3 \mathrm{~Hz}, 3 \mathrm{H}) .{ }^{13} \mathrm{C} \mathrm{NMR}\left(126 \mathrm{MHz}, \mathrm{CDCl}_{3}\right) \delta 176.6,152.3,150.1,147.3,147.0,134.2$, $130.9,128.6,128.0,127.5,127.0,126.9,126.7,126.1,125.6,125.3,124.4,117.9,117.3,45.5,45.0,42.8,33.2$, 27.1, 24.9, 24.5, 23.0, 14.0. HRMS (m/z, ESI): Calcd. for Chemical Formula $\mathrm{C}_{28} \mathrm{H}_{27} \mathrm{CINO}_{5}{ }^{-}[\mathrm{M}-\mathrm{H}]$ ]: 492.1583, Found: 492.1587. HPLC analysis of the reaction product: Daicel Chiralpak IA, hexane/iso-propanol $=99: 1,1.0 \mathrm{~mL} / \mathrm{min}, \lambda=331 \mathrm{~nm}$, retention time: $45.37 \mathrm{~min}$ (major) and $49.80 \mathrm{~min}$ (minor).

\section{(R)-4-(9-butyl-2-chloro-7-(4-cyanophenyl)-9H-xanthen-9-yl)butanoic acid (39)}

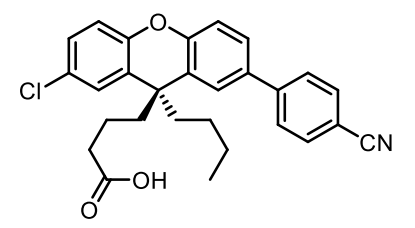

White solid, $70 \mathrm{mg}, 61 \%$ yield, $[\alpha]_{\mathrm{D}}{ }^{20}=-5.00\left(\mathrm{c} 0.200 \mathrm{CHCl}_{3}\right)$ for $97: 3$ er. ${ }^{1} \mathrm{H} \mathrm{NMR}$ (500 MHz, $\left.\mathrm{CDCl}_{3}\right) \delta 7.72-7.68(\mathrm{~m}, 2 \mathrm{H}), 7.67-7.63(\mathrm{~m}, 2 \mathrm{H}), 7.49(\mathrm{~d}, J=2.2 \mathrm{~Hz}, 1 \mathrm{H})$,

$7.44(\mathrm{dd}, J=8.5,2.2 \mathrm{~Hz}, 1 \mathrm{H}), 7.27(\mathrm{~s}, 1 \mathrm{H}), 7.18(\mathrm{dd}, J=8.6,2.4 \mathrm{~Hz}, 1 \mathrm{H}), 7.11(\mathrm{~d}, J=8.5$ $\mathrm{Hz}, 1 \mathrm{H}), 6.99(\mathrm{~d}, J=8.7 \mathrm{~Hz}, 1 \mathrm{H}), 2.27-2.21(\mathrm{~m}, 2 \mathrm{H}), 2.18-2.10(\mathrm{~m}, 1 \mathrm{H}), 2.02-1.85(\mathrm{~m}, 3 \mathrm{H}), 1.30-1.20(\mathrm{~m}, 2 \mathrm{H}), 1.15$ - $1.09(\mathrm{~m}, 2 \mathrm{H}), 0.89-0.81(\mathrm{~m}, 2 \mathrm{H}), 0.71(\mathrm{t}, \mathrm{J}=7.3 \mathrm{~Hz}, 3 \mathrm{H}) .{ }^{13} \mathrm{C} \mathrm{NMR}\left(126 \mathrm{MHz}, \mathrm{CDCl}_{3}\right) \delta 178.9,152.0,150.2,145.3$, $134.6,132.8,128.5,128.0,127.5,126.8,126.6,126.1,125.3,125.1,119.2,117.9,117.2,110.6,46.0,43.8,42.7,33.6$, 26.9, 22.9, 20.1, 13.9. HRMS (m/z, ESI): Calcd. for Chemical Formula $\mathrm{C}_{28} \mathrm{H}_{25} \mathrm{ClNO}_{3}{ }^{-}[\mathrm{M}-\mathrm{H}]^{-}: 458.1528$, Found: 458.1531. HPLC analysis of the reaction product: Daicel Chiralpak IA, hexane/iso-propanol $=95: 5,1.0 \mathrm{~mL} / \mathrm{min}, \lambda=319 \mathrm{~nm}$, retention time: $8.05 \mathrm{~min}$ (major) and $10.89 \mathrm{~min}$ (minor). 


\section{(R)-4-(9-butyl-2-chloro-7-(6-methoxypyridin-3-yl)-9H-xanthen-9-yl)butanoic acid (40)}

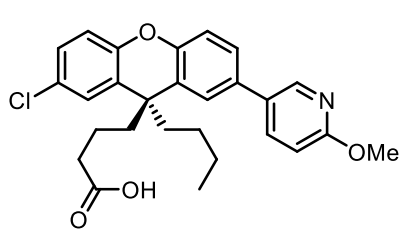

White solid, $63 \mathrm{mg}, 54 \%$ yield, $[\alpha]_{\mathrm{D}}{ }^{20}=-13.00\left(\mathrm{c} 0.100 \mathrm{CHCl}_{3}\right)$ for $95: 5$ er. ${ }^{1} \mathrm{H} \mathrm{NMR}$ $\left(500 \mathrm{MHz}, \mathrm{CDCl}_{3}\right) \delta 8.37(\mathrm{~d}, J=2.5 \mathrm{~Hz}, 1 \mathrm{H}), 7.77(\mathrm{dd}, J=8.6,2.5 \mathrm{~Hz}, 1 \mathrm{H}), 7.39(\mathrm{~d}, J=$ $2.2 \mathrm{~Hz}, 1 \mathrm{H}), 7.34(\mathrm{dd}, J=8.5,2.2 \mathrm{~Hz}, 1 \mathrm{H}), 7.25(\mathrm{~d}, J=2.4 \mathrm{~Hz}, 1 \mathrm{H}), 7.16(\mathrm{dd}, J=8.7,2.5$ $\mathrm{Hz}, 1 \mathrm{H}), 7.07(\mathrm{~d}, J=8.4 \mathrm{~Hz}, 1 \mathrm{H}), 6.97(\mathrm{~d}, J=8.6 \mathrm{~Hz}, 1 \mathrm{H}), 6.80(\mathrm{~d}, J=8.5 \mathrm{~Hz}, 1 \mathrm{H}), 3.97(\mathrm{~s}, 3 \mathrm{H}), 2.25-2.16(\mathrm{~m}, 2 \mathrm{H}), 2.12$ $-2.04(\mathrm{~m}, 1 \mathrm{H}), 2.01-1.83(\mathrm{~m}, 3 \mathrm{H}), 1.30-1.21(\mathrm{~m}, 2 \mathrm{H}), 1.15-1.10(\mathrm{~m}, 2 \mathrm{H}), 0.86(\mathrm{q}, J=8.4,8.0 \mathrm{~Hz}, 2 \mathrm{H}), 0.71(\mathrm{t}, J=$ $7.3 \mathrm{~Hz}, 3 \mathrm{H}) .{ }^{13} \mathrm{C}$ NMR $\left(101 \mathrm{MHz}, \mathrm{CDCl}_{3}\right) \delta 178.2,163.5,151.1,150.4,144.7,137.7,133.3,130.1,128.3,127.9,126.8$, 126.3, 126.1, 124.9, 124.6, 117.9, 117.0, 110.9, 53.9, 45.7, 44.0, 42.7, 33.8, 27.0, 23.0, 20.3, 14.0. HRMS (m/z, ESI): Calcd. for Chemical Formula $\mathrm{C}_{27} \mathrm{H}_{27} \mathrm{ClNO}_{4}{ }^{-}[\mathrm{M}-\mathrm{H}]^{-}: 464.1634$, Found: 464.1633 . HPLC analysis of the reaction product: Daicel Chiralpak IA, hexane/iso-propanol = 99: 1, $1.0 \mathrm{~mL} / \mathrm{min}, \lambda=271 \mathrm{~nm}$, retention time: $22.07 \mathrm{~min}$ (major) and 24.07 $\min (\operatorname{minor})$.

\section{(R)-5-(9-butyl-2-chloro-7-(6-methoxypyridin-3-yl)-9H-xanthen-9-yl)pentanoic acid (41)}

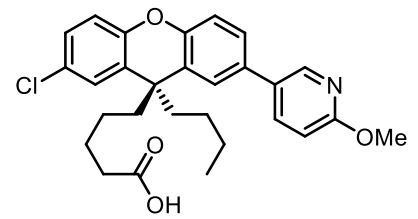

White solid, $70 \mathrm{mg}, 58 \%$ yield, $[\alpha]_{\mathrm{D}^{20}}=-7.00\left(\mathrm{c} 0.100 \mathrm{CHCl}_{3}\right)$ for $94.5: 5.5$ er. ${ }^{1} \mathrm{H}$ $\operatorname{NMR}\left(400 \mathrm{MHz}, \mathrm{CDCl}_{3}\right) \delta 8.36(\mathrm{~d}, J=2.5 \mathrm{~Hz}, 1 \mathrm{H}), 7.76(\mathrm{dd}, J=8.6,2.5 \mathrm{~Hz}, 1 \mathrm{H}), 7.37$ $(\mathrm{d}, J=2.2 \mathrm{~Hz}, 1 \mathrm{H}), 7.34(\mathrm{dd}, J=8.4,2.2 \mathrm{~Hz}, 1 \mathrm{H}), 7.24(\mathrm{~d}, J=2.5 \mathrm{~Hz}, 1 \mathrm{H}), 7.16$ (dd, $J=$

8.6, $2.5 \mathrm{~Hz}, 1 \mathrm{H}), 7.06(\mathrm{~d}, J=8.4 \mathrm{~Hz}, 1 \mathrm{H}), 6.96(\mathrm{~d}, J=8.7 \mathrm{~Hz}, 1 \mathrm{H}), 6.83(\mathrm{~d}, J=8.6 \mathrm{~Hz}, 1 \mathrm{H}), 3.98(\mathrm{~s}, 3 \mathrm{H}), 2.15$ (dd, $J=8.4$, $6.8 \mathrm{~Hz}, 2 \mathrm{H}), 2.03-1.85(\mathrm{~m}, 4 \mathrm{H}), 1.50-1.42(\mathrm{~m}, 2 \mathrm{H}), 1.17-1.11(\mathrm{~m}, 2 \mathrm{H}), 0.96-0.90(\mathrm{~m}, 2 \mathrm{H}), 0.88-0.83(\mathrm{~m}, 2 \mathrm{H})$, $0.72(\mathrm{t}, J=7.3 \mathrm{~Hz}, 3 \mathrm{H}) .{ }^{13} \mathrm{C}$ NMR $\left(126 \mathrm{MHz}, \mathrm{CDCl}_{3}\right) \delta 178.3,163.5,151.1,150.4,144.6,137.7,133.2,130.1,128.2$ $127.8,127.1,126.3,126.1,125.2,124.4,117.8,116.9,111.0,53.9,45.4,44.9,42.8,33.6,27.1,24.9,24.4,23.0,14.0$. HRMS (m/z, ESI): Calcd. for Chemical Formula $\mathrm{C}_{28} \mathrm{H}_{29} \mathrm{ClNO}_{4}{ }^{-}[\mathrm{M}-\mathrm{H}]^{-}: 478.1791$, Found: 478.1800. HPLC analysis of the reaction product: Daicel Chiralpak IA, hexane/iso-propanol $=99: 1,1.0 \mathrm{~mL} / \mathrm{min}, \lambda=272 \mathrm{~nm}$, retention time:23.35 min (major) and $20.51 \mathrm{~min}$ (minor). 
(R)-4-(9-butyl-2-chloro-7-(thiophen-3-yl)-9H-xanthen-9-yl)butanoic acid (42)

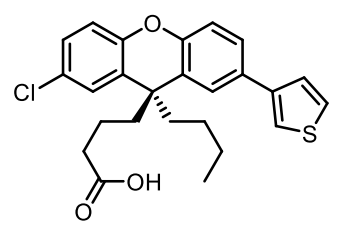

White solid, $60 \mathrm{mg}, 55 \%$ yield, $[\alpha]_{\mathrm{D}^{20}}=-0.40\left(\mathrm{c} 1.000 \mathrm{CHCl}_{3}\right)$ for $95: 5$ er. ${ }^{1} \mathrm{H} \mathrm{NMR}$

$\left(500 \mathrm{MHz}, \mathrm{CDCl}_{3}\right) \delta 7.48(\mathrm{~d}, J=2.1 \mathrm{~Hz}, 1 \mathrm{H}), 7.43(\mathrm{dd}, J=8.4,2.1 \mathrm{~Hz}, 1 \mathrm{H}), 7.38(\mathrm{t}, J=2.1$ $\mathrm{Hz}, 1 \mathrm{H}), 7.35(\mathrm{~d}, J=2.2 \mathrm{~Hz}, 2 \mathrm{H}), 7.25(\mathrm{~d}, J=2.4 \mathrm{~Hz}, 1 \mathrm{H}), 7.16(\mathrm{dd}, J=8.7,2.4 \mathrm{~Hz}, 1 \mathrm{H}), 7.03$ $(\mathrm{d}, J=8.5 \mathrm{~Hz}, 1 \mathrm{H}), 6.97(\mathrm{~d}, J=8.6 \mathrm{~Hz}, 1 \mathrm{H}), 2.20(\mathrm{td}, J=7.2,4.7 \mathrm{~Hz}, 2 \mathrm{H}), 2.14-2.05(\mathrm{~m}, 1 \mathrm{H}), 1.97-1.93(\mathrm{~m}, 2 \mathrm{H}), 1.89-$ $1.82(\mathrm{~m}, 1 \mathrm{H}), 1.31-1.20(\mathrm{~m}, 2 \mathrm{H}), 1.14-1.11(\mathrm{~m}, 2 \mathrm{H}), 0.90-0.82(\mathrm{~m}, 2 \mathrm{H}), 0.71(\mathrm{t}, J=7.3 \mathrm{~Hz}, 3 \mathrm{H}) .{ }^{13} \mathrm{C} \mathrm{NMR}(126 \mathrm{MHz}$, $\left.\mathrm{CDCl}_{3}\right) \delta 179.0,150.8,150.4,142.1,131.6,128.2,128.1,127.9,126.8,126.4,126.4,126.2,126.1,124.5,124.2,119.7$ 117.9, 116.7, 45.9, 43.9, 42.7, 33.8, 27.0, 23.0, 20.2, 14.0. HRMS (m/z, ESI): Calcd. for Chemical Formula $\mathrm{C}_{25} \mathrm{H}_{24} \mathrm{ClO}_{3} \mathrm{~S}^{-}$ [M-H]": 439.1140, Found: 439.1148. HPLC analysis of the reaction product: Daicel Chiralpak IA, hexane/iso-propanol = 99: $1,1.0 \mathrm{~mL} / \mathrm{min}, \lambda=272 \mathrm{~nm}$, retention time: $16.93 \mathrm{~min}$ (major) and $18.82 \mathrm{~min}$ (minor).

\section{(R)-3-(9-butyl-2-chloro-7-(dibenzo[b,d]thiophen-4-yl)-9H-xanthen-9-yl)propanoic acid (43)}

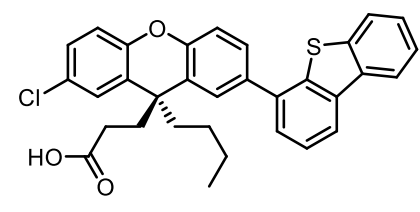

White solid, $70 \mathrm{mg}, 53 \%$ yield, $[\alpha]_{\mathrm{D}}{ }^{20}=+85.00\left(\mathrm{c} 0.040 \mathrm{CHCl}_{3}\right)$ for $96: 4$ er. ${ }^{1} \mathrm{H}$ $\operatorname{NMR}\left(500 \mathrm{MHz}, \mathrm{CDCl}_{3}\right) \delta 8.18-8.13(\mathrm{~m}, 2 \mathrm{H}), 7.81-7.77(\mathrm{~m}, 1 \mathrm{H}), 7.75(\mathrm{~d}, \mathrm{~J}=2.0 \mathrm{~Hz}$, 1H), $7.57-7.50(\mathrm{~m}, 2 \mathrm{H}), 7.49-7.41(\mathrm{~m}, 3 \mathrm{H}), 7.29(\mathrm{~d}, J=2.3 \mathrm{~Hz}, 1 \mathrm{H}), 7.20$ (dd, $J=$ 8.7, $2.4 \mathrm{~Hz}, 1 \mathrm{H}), 7.16(\mathrm{dd}, J=8.4,1.6 \mathrm{~Hz}, 1 \mathrm{H}), 7.02(\mathrm{dd}, J=8.7,1.6 \mathrm{~Hz}, 1 \mathrm{H}), 2.39-$ $2.24(\mathrm{~m}, 2 \mathrm{H}), 2.10-1.95(\mathrm{~m}, 4 \mathrm{H}), 1.21(\mathrm{dd}, J=8.4,6.4 \mathrm{~Hz}, 2 \mathrm{H}), 1.04-0.92(\mathrm{~m}, 2 \mathrm{H}), 0.78(\mathrm{t}, J=7.4 \mathrm{~Hz}, 3 \mathrm{H}) .{ }^{13} \mathrm{C} \mathrm{NMR}$ $\left(126 \mathrm{MHz}, \mathrm{CDCl}_{3}\right) \delta 178.5,151.2,150.4,139.6,138.8,136.5,136.2,135.9,128.6,128.4,128.3,127.0,126.6,126.1_{1}$, $126.12,126.0,125.3,124.6,123.7,122.8,121.9,120.6,118.1,117.1,45.2,42.2,39.8,30.2,27.2$, 23.1, 14.1. HRMS

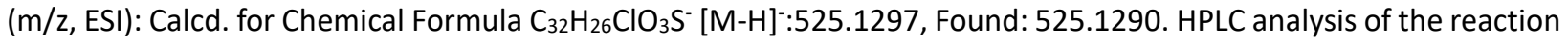
product: Daicel Chiralpak IA, hexane/iso-propanol = 99: 1, $1.0 \mathrm{~mL} / \mathrm{min}, \lambda=332 \mathrm{~nm}$, retention time: 27.84 min (major) and $36.43 \mathrm{~min}$ (minor). 


\section{(R)-4-(9-butyl-2-chloro-7-(dibenzo[b,d]thiophen-4-yl)-9H-xanthen-9-yl)butanoic acid (44)}

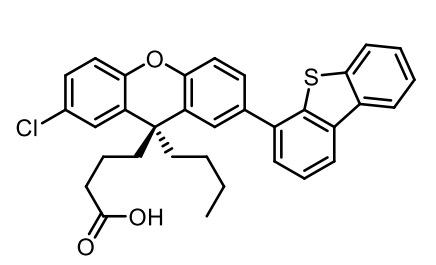

White solid, $95 \mathrm{mg}, 70 \%$ yield, $[\alpha]_{\mathrm{D}}{ }^{20}=-14.00\left(\mathrm{c} 0.100 \mathrm{CHCl}_{3}\right)$ for $97.5: 2.5 \mathrm{er}^{1}{ }^{1} \mathrm{H}$ NMR $\left(500 \mathrm{MHz}, \mathrm{CDCl}_{3}\right) \delta 8.20-8.16(\mathrm{~m}, 1 \mathrm{H}), 8.14(\mathrm{dd}, J=7.8,1.2 \mathrm{~Hz}, 1 \mathrm{H}), 7.84-$ $7.80(\mathrm{~m}, 1 \mathrm{H}), 7.73(\mathrm{~d}, J=2.2 \mathrm{~Hz}, 1 \mathrm{H}), 7.55(\mathrm{t}, J=7.6 \mathrm{~Hz}, 1 \mathrm{H}), 7.53-7.48(\mathrm{~m}, 2 \mathrm{H}), 7.47$ $-7.41(m, 2 H), 7.27(d, J=2.5 \mathrm{~Hz}, 1 \mathrm{H}), 7.18(\mathrm{dd}, J=8.7,2.5 \mathrm{~Hz}, 1 \mathrm{H}), 7.15(\mathrm{~d}, J=8.4$ $\mathrm{Hz}, 1 \mathrm{H}), 7.01(\mathrm{~d}, J=8.7 \mathrm{~Hz}, 1 \mathrm{H}), 2.22(\mathrm{t}, J=7.4 \mathrm{~Hz}, 2 \mathrm{H}), 2.08-1.95(\mathrm{~m}, 3 \mathrm{H}), 1.92-1.86(\mathrm{~m}, 1 \mathrm{H}), 1.39-1.27(\mathrm{~m}, 2 \mathrm{H})$, $1.19(\mathrm{p}, J=7.4 \mathrm{~Hz}, 2 \mathrm{H}), 1.02-0.96(\mathrm{~m}, 1 \mathrm{H}), 0.93-0.86(\mathrm{~m}, 1 \mathrm{H}), 0.77(\mathrm{t}, J=7.4 \mathrm{~Hz}, 3 \mathrm{H}) .{ }^{13} \mathrm{C} \mathrm{NMR}\left(126 \mathrm{MHz}, \mathrm{CDCl}_{3}\right) \delta$ $178.1,151.3,150.4,139.6,138.7,136.7,136.5,136.0,135.9,128.3,128.1,127.9,127.0,126.9,126.7,126.2,126.1$, 125.3, 124.6, 124.5, 122.8, 121.9, 120.5, 117.9, 116.9, 45.6, 44.4, 42.8, 33.9, 27.1, 23.1, 20.4, 14.1. HRMS (m/z, ESI): Calcd. for Chemical Formula $\mathrm{C}_{33} \mathrm{H}_{28} \mathrm{ClO}_{3} \mathrm{~S}^{-}[\mathrm{M}-\mathrm{H}]^{-}: 539.1453$, Found: 539.1443.

HPLC analysis of the reaction product: Daicel Chiralpak IA, hexane/iso-propanol $=95: 5,1.0 \mathrm{~mL} / \mathrm{min}, \lambda=332 \mathrm{~nm}$, retention time: $5.63 \mathrm{~min}$ (major) and $6.22 \mathrm{~min}$ (minor).

\section{(R)-5-(9-butyl-2-chloro-7-(dibenzo[b,d]thiophen-4-yl)-9H-xanthen-9-yl)pentanoic acid (45)}

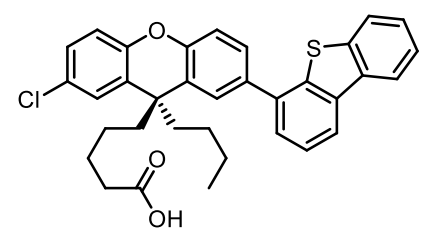

White solid, $93 \mathrm{mg}, 67 \%$ yield, $[\alpha]_{\mathrm{D}}^{20}=-16.00\left(\mathrm{c} 0.100 \mathrm{CHCl}_{3}\right)$ for $95.5: 4.5 \mathrm{er}$. ${ }^{1} \mathrm{H} \mathrm{NMR}\left(400 \mathrm{MHz}, \mathrm{CDCl}_{3}\right) \delta 8.20-8.13(\mathrm{~m}, 2 \mathrm{H}), 7.85-7.80(\mathrm{~m}, 1 \mathrm{H}), 7.74(\mathrm{~d}, J=2.2$ $\mathrm{Hz}, 1 \mathrm{H}), 7.57(\mathrm{t}, J=7.6 \mathrm{~Hz}, 1 \mathrm{H}), 7.51-7.44(\mathrm{~m}, 4 \mathrm{H}), 7.27(\mathrm{~d}, J=2.5 \mathrm{~Hz}, 1 \mathrm{H}), 7.20-$

$7.17(\mathrm{~m}, 1 \mathrm{H}), 7.14(\mathrm{~d}, J=8.4 \mathrm{~Hz}, 1 \mathrm{H}), 7.00(\mathrm{~d}, J=8.7 \mathrm{~Hz}, 1 \mathrm{H}), 2.23-2.16(\mathrm{~m}, 2 \mathrm{H}), 2.03-1.86(\mathrm{~m}, 4 \mathrm{H}), 1.57-1.49(\mathrm{~m}$, 2H), $1.21(\mathrm{~d}, J=7.4 \mathrm{~Hz}, 2 \mathrm{H}), 1.03-0.85(\mathrm{~m}, 4 \mathrm{H}), 0.78(\mathrm{t}, J=7.3 \mathrm{~Hz}, 3 \mathrm{H}) .{ }^{13} \mathrm{C}$ NMR $\left(126 \mathrm{MHz}, \mathrm{CDCl}_{3}\right) \delta 178.9,151.2$, $150.4,139.6,138.8,136.7,136.5,136.0,135.9,128.3,128.0,127.8,127.2,127.0,126.6,126.11,126.12,125.3,124.8$, 124.6, 122.8, 121.9, 120.5, 117.8, 116.9, 45.4, 45.2, 42.8, 33.8, 27.2, 25.1, 24.6, 23.1, 14.1. HRMS (m/z, ESI): Calcd. for Chemical Formula $\mathrm{C}_{34} \mathrm{H}_{30} \mathrm{ClO}_{3} \mathrm{~S}^{-}[\mathrm{M}-\mathrm{H}]: 553.1610$, Found: 553.1599. HPLC analysis of the reaction product: Daicel Chiralpak IA, hexane/iso-propanol = 99: 1, $1.0 \mathrm{~mL} / \mathrm{min}, \lambda=332 \mathrm{~nm}$, retention time: $21.52 \mathrm{~min}$ (major) and $20.12 \mathrm{~min}$ (minor). 
(R)-4-(9-butyl-2-chloro-7-(thianthren-1-yl)-9H-xanthen-9-yl)butanoic acid (46)

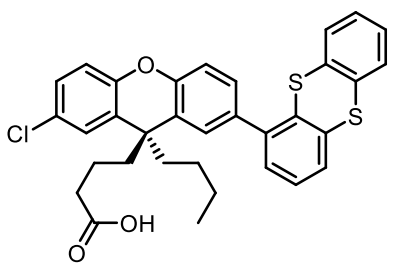

White solid, $76 \mathrm{mg}, 53 \%$ yield, $[\alpha]_{\mathrm{D}}{ }^{20}=+23.00\left(\mathrm{c} 0.100 \mathrm{CHCl}_{3}\right)$ for $97: 3$ er. ${ }^{1} \mathrm{H} \mathrm{NMR}$ $\left(500 \mathrm{MHz}, \mathrm{CDCl}_{3}\right) \delta 7.51(\mathrm{dd}, J=7.3,1.8 \mathrm{~Hz}, 1 \mathrm{H}), 7.48(\mathrm{dd}, J=7.8,1.4 \mathrm{~Hz}, 1 \mathrm{H}), 7.31$ (dd, $J=6.9,2.0 \mathrm{~Hz}, 1 \mathrm{H}), 7.29-7.25(\mathrm{~m}, 4 \mathrm{H}), 7.23-7.17(\mathrm{~m}, 3 \mathrm{H}), 7.13(\mathrm{td}, J=7.5,1.4 \mathrm{~Hz}, 1 \mathrm{H})$, $7.10(\mathrm{~d}, J=8.3 \mathrm{~Hz}, 1 \mathrm{H}), 7.01(\mathrm{~d}, J=8.7 \mathrm{~Hz}, 1 \mathrm{H}), 2.21(\mathrm{t}, J=7.5 \mathrm{~Hz}, 2 \mathrm{H}), 2.09-1.85(\mathrm{~m}$, $4 \mathrm{H}), 1.43-1.33(\mathrm{~m}, 1 \mathrm{H}), 1.29(\mathrm{~d}, J=4.7 \mathrm{~Hz}, 1 \mathrm{H}), 1.18(\mathrm{p}, J=7.3 \mathrm{~Hz}, 2 \mathrm{H}), 1.03-0.98(\mathrm{~m}, 1 \mathrm{H}), 0.93-0.87(\mathrm{~m}, 1 \mathrm{H}), 0.76$ $(\mathrm{t}, J=7.3 \mathrm{~Hz}, 3 \mathrm{H}) .{ }^{13} \mathrm{C}$ NMR $\left(126 \mathrm{MHz}, \mathrm{CDCl}_{3}\right) \delta 178.9,151.1,150.4,142.1,136.5,136.0,135.4,135.3,129.2,128.8$, $128.7,128.4,128.3,127.9,127.7,127.2,126.8,126.2,123.7,117.9,116.3,45.7,44.4,42.6,34.0,27.1,23.0,20.4$, 14.1. HRMS (m/z, ESI): Calcd. for Chemical Formula $\mathrm{C}_{33} \mathrm{H}_{28} \mathrm{ClO}_{3} \mathrm{~S}^{-}[\mathrm{M}-\mathrm{H}]^{-}: 571.1174$, Found: 571.1166. HPLC analysis of the reaction product: Daicel Chiralpak IA, hexane/iso-propanol $=99: 1,1.0 \mathrm{~mL} / \mathrm{min}, \lambda=262 \mathrm{~nm}$, retention time: $18.98 \mathrm{~min}$ (major) and $21.52 \mathrm{~min}$ (minor).

Ethyl (R)-2'-(9-butyl-7-(2-chlorophenyl)-9-(2-ethoxy-2-oxoethyl)-9H-fluoren-2-yl)-[1,1'-biphenyl]-4-carboxylate (47)

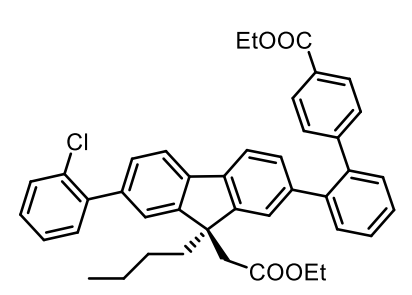

The reaction mixture was neutralized with $1 \mathrm{M} \mathrm{HCl}$ to $\mathrm{pH} \mathrm{2-3} \mathrm{and} \mathrm{the} \mathrm{crude}$ product was dissolved in $\mathrm{EtOH}$, then $10 \mu \mathrm{L}$ conc. $\mathrm{H}_{2} \mathrm{SO}_{4}$ was added and the reaction mixture was stirred at $65^{\circ} \mathrm{C}$ for $10 \mathrm{~h}$. The esterification product was obtained as a white solid, $84.6 \mathrm{mg}, 53 \%$ yield, $[\alpha]_{\mathrm{D}}{ }^{20}=-12.20\left(c 0.100 \mathrm{CHCl}_{3}\right)$ for $85.5: 14.5$ er. ${ }^{1} \mathrm{H}$ NMR (500

$\left.\mathrm{MHz}, \mathrm{CDCl}_{3}\right) \delta 7.90(\mathrm{~d}, J=8.5 \mathrm{~Hz}, 2 \mathrm{H}), 7.75-7.71(\mathrm{~m}, 1 \mathrm{H}), 7.65(\mathrm{~d}, J=7.8 \mathrm{~Hz}, 1 \mathrm{H}), 7.58-7.54(\mathrm{~m}, 1 \mathrm{H}), 7.48(\mathrm{ddd}, J=$ 6.8, 4.9, 1.2 Hz, 4H), $7.42(\mathrm{dt}, J=7.5,1.6 \mathrm{~Hz}, 2 \mathrm{H}), 7.35(\mathrm{td}, J=7.4,1.4 \mathrm{~Hz}, 1 \mathrm{H}), 7.31-7.29(\mathrm{~m}, 2 \mathrm{H}), 7.25(\mathrm{dd}, J=7.8$, $1.6 \mathrm{~Hz}, 1 \mathrm{H}), 7.13(\mathrm{~d}, J=1.1 \mathrm{~Hz}, 1 \mathrm{H}), 4.36-4.31(\mathrm{~m}, 2 \mathrm{H}), 3.77(\mathrm{q}, J=7.1 \mathrm{~Hz}, 2 \mathrm{H}), 2.77(\mathrm{br}, 2 \mathrm{H}), 2.02-1.94(\mathrm{~m}, 1 \mathrm{H})$, 1.80-1.76 (m, 1H), $1.36(\mathrm{t}, J=7.1 \mathrm{~Hz}, 3 \mathrm{H}), 1.02-0.98(\mathrm{~m}, 2 \mathrm{H}), 0.85(\mathrm{t}, J=7.1 \mathrm{~Hz}, 3 \mathrm{H}), 0.69(\mathrm{t}, J=7.1 \mathrm{~Hz}, 3 \mathrm{H}), 0.59-0.54$ (m, 2H). ${ }^{13} \mathrm{CNMR}\left(126 \mathrm{MHz}, \mathrm{CDCl}_{3}\right) \delta 170.2,166.4,149.4,149.0,146.5,141.0,140.8,140.2,139.9,139.6,139.0$, $138.1,131.4,130.8,130.5,130.0,129.9,129.2,129.0,128.7,128.5,128.4,128.1,127.6,126.8,125.1,124.7,119.7$, $119.4,60.9,59.9,52.5,44.0,38.9,25.8,22.7,14.3,13.81,13.82 . \mathrm{MS}$ (m/z, ESI): Calcd. for Chemical Formula: $\mathrm{C}_{42} \mathrm{H}_{39} \mathrm{ClNaO}_{4}{ }^{+}[\mathrm{M}+\mathrm{Na}]^{+}: 665.2429$, Found: 665.2423 . HPLC analysis of the reaction product: Daicel Chiralpak IA, hexane/iso-propanol = 95: 5, $1.0 \mathrm{~mL} / \mathrm{min}, \lambda=330 \mathrm{~nm}$, retention time: $8.06 \mathrm{~min}$ (major) and $8.89 \mathrm{~min}$ (minor). 
Ethyl (R)-2'-(9-butyl-7-(2-chloro-4-methoxyphenyl)-9-(2-ethoxy-2-oxoethyl)-9H-fluoren-2-yl)-5'-methoxy-[1,1'biphenyl]-4-carboxylate (48)

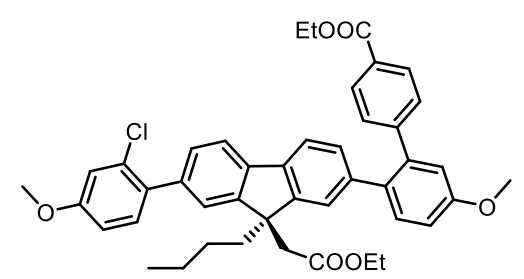

After the reaction was completed, the reaction mixture was neutralized with $1 \mathrm{M} \mathrm{HCl}$ to $\mathrm{pH} 2-3$ and the crude product was dissolved in $\mathrm{EtOH}$, then $10 \mu \mathrm{L}$ conc. $\mathrm{H}_{2} \mathrm{SO}_{4}$ was added and the reaction mixture was stirred at $65^{\circ} \mathrm{C}$ for $10 \mathrm{~h}$. The esterification product was obtained as a white solid, $97.9 \mathrm{mg}, 56 \%$ yield, $[\alpha]_{D}{ }^{20}=-35.10\left(c 0.100 \mathrm{CHCl}_{3}\right)$ for $89.5: 10.5$ er. ${ }^{1} \mathrm{H} \mathrm{NMR}\left(500 \mathrm{MHz}, \mathrm{CDCl}_{3}\right) \delta 7.93-7.86(\mathrm{~m}, 2 \mathrm{H}), 7.69(\mathrm{~d}, J=7.8$ $\mathrm{Hz}, 1 \mathrm{H}), 7.61(\mathrm{~d}, J=7.8 \mathrm{~Hz}, 1 \mathrm{H}), 7.48(\mathrm{~d}, J=8.4 \mathrm{~Hz}, 1 \mathrm{H}), 7.44(\mathrm{~d}, J=1.1 \mathrm{~Hz}, 1 \mathrm{H}), 7.38(\mathrm{dd}, J=7.8,1.5 \mathrm{~Hz}, 1 \mathrm{H}), 7.32(\mathrm{~d}$, $J=8.5 \mathrm{~Hz}, 1 \mathrm{H}), 7.31-7.28(\mathrm{~m}, 2 \mathrm{H}), 7.20(\mathrm{dd}, J=7.8,1.5 \mathrm{~Hz}, 1 \mathrm{H}), 7.08-7.02(\mathrm{~m}, 3 \mathrm{H}), 7.00(\mathrm{~d}, J=2.7 \mathrm{~Hz}, 1 \mathrm{H}), 6.91(\mathrm{dd}$, $J=8.5,2.6 \mathrm{~Hz}, 1 \mathrm{H}), 4.37-4.30(\mathrm{~m}, 2 \mathrm{H}), 3.92(\mathrm{~s}, 3 \mathrm{H}), 3.87(\mathrm{~s}, 3 \mathrm{H}), 3.77(\mathrm{q}, J=7.1 \mathrm{~Hz}, 2 \mathrm{H}), 2.74(\mathrm{br}, 2 \mathrm{H}), 1.99-1.93(\mathrm{~m}$, 1H), $1.79-1.73(\mathrm{~m}, 1 \mathrm{H}), 1.35(\mathrm{~d}, J=7.1 \mathrm{~Hz}, 3 \mathrm{H}), 1.00(\mathrm{dd}, J=13.9,7.0 \mathrm{~Hz}, 2 \mathrm{H}), 0.85(\mathrm{t}, J=7.1 \mathrm{~Hz}, 3 \mathrm{H}), 0.69(\mathrm{t}, J=7.3$ $\mathrm{Hz}, 3 \mathrm{H}), 0.55(\mathrm{dt}, J=19.8,7.3 \mathrm{~Hz}, 2 \mathrm{H}) .{ }^{13} \mathrm{C} \mathrm{NMR}\left(126 \mathrm{MHz}, \mathrm{CDCl}_{3}\right) \delta 170.2,166.4,159.3,159.0,149.3,148.9,146.5$ $140.8,139.8,139.6,138.7,137.8,133.7,133.3,133.1,131.9_{1}, 131.9_{2}, 129.9,129.2,129.0,128.7,128.6,125.2,124.8$ $119.6,119.3,115.9,115.2,113.6,113.1,60.9,59.8,55.6,55.5,52.4,44.1,38.9,25.8,22.7,14.3,13.81,13.82 . M S$ (m/z, ESI): Calcd. for Chemical Formula: $\mathrm{C}_{44} \mathrm{H}_{43} \mathrm{CINaO}_{6}{ }^{+}[\mathrm{M}+\mathrm{Na}]^{+}:$725.2640, Found: 725.2638. HPLC analysis of the reaction product: Daicel Chiralpak IA, hexane/iso-propanol $=95: 5,1.0 \mathrm{~mL} / \mathrm{min}, \lambda=330 \mathrm{~nm}$, retention time: 13.94 $\min$ (minor) and $15.36 \mathrm{~min}$ (major).

\section{(R)-2-((2-(4-(tert-butyl)phenyl)-7-chloro-9-methyl-9H-fluoren-9-yl)oxy)acetic acid (49)}

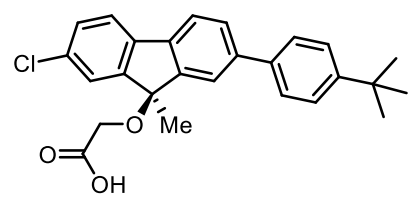

White solid, $74 \mathrm{mg}, 70 \%$ yield, $[\alpha]_{D^{20}}=-18.33\left(c 0.06 \mathrm{CHCl}_{3}\right)$ for $98.5: 1.5$ er. ${ }^{1} \mathrm{H}$ NMR $\left(400 \mathrm{MHz} \mathrm{CDCl}_{3}\right) \delta 7.71-7.63(\mathrm{~m}, 3 \mathrm{H}), 7.60-7.54(\mathrm{~m}, 3 \mathrm{H}), 7.51-7.47(\mathrm{~m}$, $3 \mathrm{H}), 7.39(\mathrm{dd}, J=8.1,2.0 \mathrm{~Hz}, 1 \mathrm{H}), 3.50(\mathrm{~s}, 2 \mathrm{H}), 1.82(\mathrm{~s}, 3 \mathrm{H}), 1.37(\mathrm{~s}, 9 \mathrm{H}) .{ }^{13} \mathrm{C}$ NMR $(126$

$\left.\mathrm{MHz}_{\mathrm{CDCl}}\right) \delta 170.6,151.1,147.1,145.4,141.9,138.4,137.8,137.7,134.2,130.2,128.9,126.9,126.1,124.4,122.4$ 121.5, 120.8, 85.5, 61.8, 31.5, 26.2. HRMS (m/z, ESI): Calcd. for Chemical Formula $\mathrm{C}_{26} \mathrm{H}_{24} \mathrm{ClO}_{3}{ }^{-}[\mathrm{M}-\mathrm{H}]^{-}: 419.1419$, Found: 419.1413. HPLC analysis of the reaction product: Daicel Chiralpak IA, hexane/iso-propanol $=97: 3,1.0 \mathrm{~mL} / \mathrm{min}, \lambda=$ $304 \mathrm{~nm}$, retention time: $15.88 \mathrm{~min}$ (major) and $13.73 \mathrm{~min}$ (minor). 
(R)-2-((9-butyl-2-(4-(tert-butyl)phenyl)-7-chloro-9H-fluoren-9-yl)oxy)acetic acid (50)

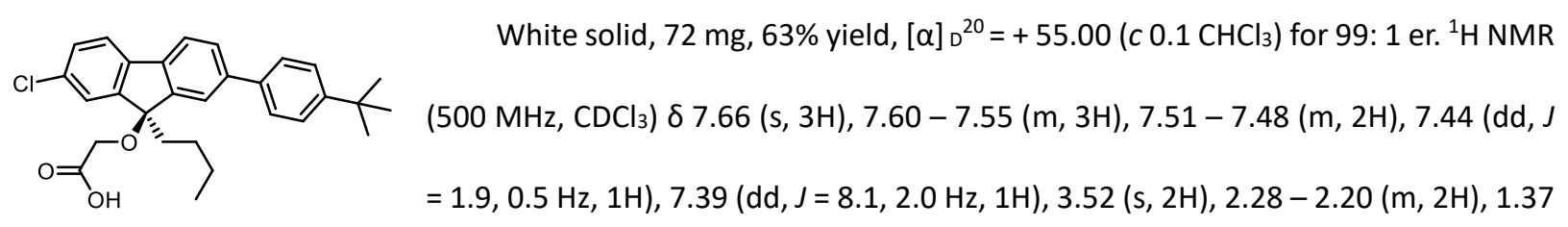

$(\mathrm{s}, 9 \mathrm{H}), 1.21-1.14(\mathrm{~m}, 2 \mathrm{H}), 0.89-0.84(\mathrm{~m}, 2 \mathrm{H}), 0.74(\mathrm{t}, J=7.4 \mathrm{~Hz}, 3 \mathrm{H}) .{ }^{13} \mathrm{C}$ NMR $\left(126 \mathrm{MHz}, \mathrm{CDCl}_{3}\right) \delta$ 171.8, 151.1, $146.2,144.5,141.6,139.2,138.7,137.7,134.1,130.1,128.7,126.9,126.1,124.5,122.6,121.3,120.7,88.8,61.6$, 39.2, 34.7, 31.5, 25.7, 22.9, 13.9. HRMS (m/z, ESI): Calcd. for Chemical Formula $\mathrm{C}_{29} \mathrm{H}_{30} \mathrm{ClO}_{3}{ }^{-}[\mathrm{M}-\mathrm{H}]:$ : 461.1889, Found: 461.1888. HPLC analysis of the reaction product: Daicel Chiralpak IA, hexane $/$ iso-propanol $=95: 5,1.0 \mathrm{~mL} / \mathrm{min}, \lambda=$ $304 \mathrm{~nm}$, retention time: $9.15 \mathrm{~min}$ (major) and $10.18 \mathrm{~min}$ (minor). 


\section{Control experiments}

1. The role of $\mathrm{K}^{+}$

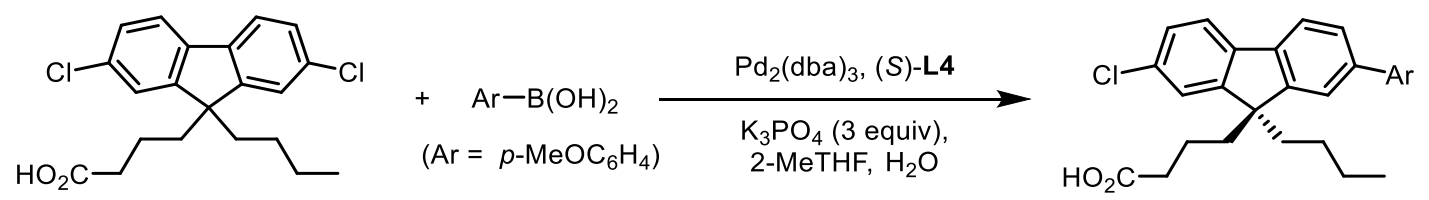

condition:

1. no additive $: 94 \%$ conv., $96: 4$ er

2. 1.0 equiv 18 -crown-6: $89 \%$ conv., $81: 19$ er

3. 3.0 equiv 18 -crown- $6: 88 \%$ conv., $78: 22$ er

4. 9.0 equiv 18 -crown-6: $84 \%$ conv., $61: 39$ er

Procedure: To the mixture of 4-(9-butyl-2,7-dichloro-9H-fluoren-9-yl)butanoic acid (0.1 mmol), $\mathrm{K}_{3} \mathrm{PO}_{4}$ (3.0 equiv) and 18-crown-6 were added $1.5 \mathrm{~mL}$ 2-MeTHF and the resulting reaction mixture was stirring for $20 \mathrm{~min}$, followed by addition of $p-\mathrm{MeOPhB}(\mathrm{OH})_{2}$ (1.2 equiv), (S)-L4 and $\mathrm{Pd}_{2} \mathrm{dba}_{3}$ complex solution (1.0 mol\%), and water following the general procedure. Conversion was determined by analysis of the ${ }^{1} \mathrm{H}$ NMR spectrum of crude reaction mixture.

2. The roles of the phosphonate group of ligand and the carboxylate group of the substrate.<smiles>CCCCC1(CCCC(=O)O)c2cc(Cl)ccc2-c2ccc(Cl)cc21</smiles><smiles>[R]OC(=O)CCCC1(CCCC)c2cc(Cl)ccc2-c2ccc(Cl)cc21</smiles>

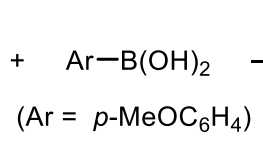$$
\text { (S }
$$

-L4 94\% conv., 96:4 er

(R)-L1 87\% conv., 56:44 er

(S)-L3: $90 \%$ conv., 83:17 er
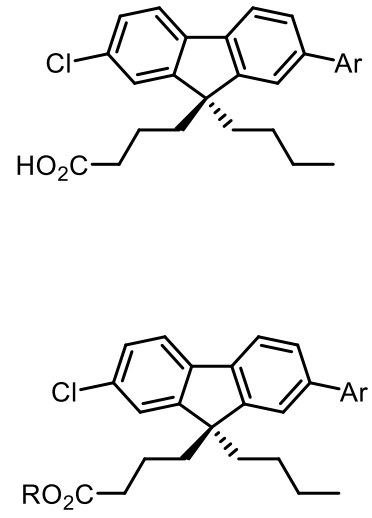

$\mathrm{R}=$

$\mathrm{H}: 67 \%$ yield, $96: 4 \mathrm{er}$

$\mathrm{K}$ : $61 \%$ yield, $96: 4 \mathrm{er}$

Et: $42 \%$ yield, $50: 50$ er 
Procedure: Potassium salt of 4-(9-butyl-2,7-dichloro-9H-fluoren-9-yl)butanoic acid was prepared by stirring of 4-(9-butyl-2,7-dichloro-9H-fluoren-9-yl)butanoic acid (151 mg, $0.4 \mathrm{mmol}$ ) and tBuOK (45.2 mg, $0.4 \mathrm{mmol}) \mathrm{in} 2.0 \mathrm{~mL}$ THF at $60^{\circ} \mathrm{C}$ for $1 \mathrm{~h}$, followed by removal of the solvent under reduced pressure to give the desired product as a white solid.

The reactions were carried out on $0.1 \mathrm{mmol}$ scale following the general procedure. Conversions were determined by analysis of ${ }^{1} \mathrm{H}$ NMR spectra of crude reaction mixtures. Yields were calculated based on the weights of products isolated using column chromatography.

3. Competition experiments
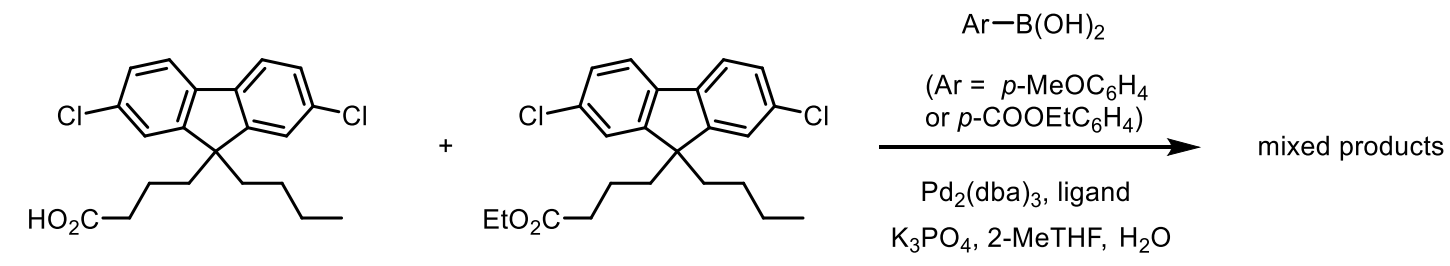

(ligand: RuPhos or (S)-L4)

Procedure: To a mixture of 4-(9-butyl-2,7-dichloro-9H-fluoren-9-yl)butanoic acid (37.3 mg, $0.1 \mathrm{mmol}$ ), ethyl 4-(9-butyl-2,7-dichloro-9H-fluoren-9-yl)butanoate $(40.5 \mathrm{mg}, 0.1 \mathrm{mmol})$, and $\mathrm{K}_{3} \mathrm{PO}_{4}(63.8 \mathrm{mg}, 0.3 \mathrm{mmol})$ were added $3 \mathrm{~mL}$ 2-MeTHF and $50 \mu \mathrm{L} \mathrm{H}$ O. The resulting reaction mixture was stirring for $20 \mathrm{~min}$, followed by addition of $p$ $\mathrm{MeOC}_{6} \mathrm{H}_{4} \mathrm{~B}(\mathrm{OH})_{2}(15.2 \mathrm{mg}, 0.1 \mathrm{mmol})$ or $p-\mathrm{COOEtC}_{6} \mathrm{H}_{4} \mathrm{~B}(\mathrm{OH})_{2}(19.4 \mathrm{mg}, 0.1 \mathrm{mmol})$, then $1.0 \mathrm{~mL}$ 2-MeTHF solution of (S)-L4 (1.3 mg, $0.022 \mathrm{mmol}$ ) or RuPhos (1.03 mg, $0.0022 \mathrm{mmol})$ and Pd $2 \mathrm{dba}_{3}(0.9 \mathrm{mg}, 0.001 \mathrm{mmol})$ complex was added. The resulting reaction mixture was stirred at $60^{\circ} \mathrm{C}$ for $20 \mathrm{~h}$. Conversion of each substrate was determined by analysis of the ${ }^{1} \mathrm{H}$ NMR spectra of the resulting mixtures. 
Characterization data of the coupling product of ethyl ester $\mathbf{5 3}$ under the optimized reaction conditions.

Ethyl (R)-4-(9-butyl-7-chloro-9-(4-ethoxy-4-oxobutyl)-9H-fluoren-2-yl)benzoate (coupling product of 53)

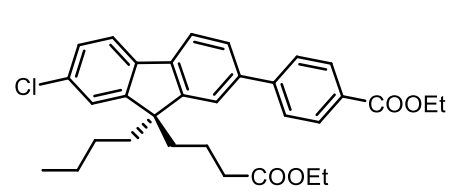

White solid, $21.7 \mathrm{mg}, 42 \%$ yield. ${ }^{1} \mathrm{H}$ NMR $\left(500 \mathrm{MHz}, \mathrm{CDCl}_{3}\right) \delta 8.18-8.13(\mathrm{~m}, 2 \mathrm{H})$, $7.77-7.73(\mathrm{~m}, 3 \mathrm{H}), 7.65(\mathrm{ddd}, J=14.5,7.5,1.7 \mathrm{~Hz}, 2 \mathrm{H}), 7.59(\mathrm{~d}, J=1.2 \mathrm{~Hz}, 1 \mathrm{H}), 7.36$ $(\mathrm{dd}, J=7.1,1.8 \mathrm{~Hz}, 2 \mathrm{H}), 4.44(\mathrm{q}, J=7.1 \mathrm{~Hz}, 2 \mathrm{H}), 4.04(\mathrm{q}, J=7.1,2 \mathrm{H}), 2.13-1.96(\mathrm{~m}$,

$6 \mathrm{H}), 1.45(\mathrm{t}, J=7.1 \mathrm{~Hz}, 3 \mathrm{H}), 1.18(\mathrm{t}, J=7.1 \mathrm{~Hz}, 3 \mathrm{H}), 1.14-1.12(\mathrm{~m}, 2 \mathrm{H}), 1.05-0.98(\mathrm{~m}, 2 \mathrm{H}), 0.71(\mathrm{t}, J=7.4 \mathrm{~Hz}, 3 \mathrm{H}), 0.69$

- 0.62 (m, 2H). ${ }^{13} \mathrm{C}$ NMR $\left(126 \mathrm{MHz}, \mathrm{CDCl}_{3}\right) \delta 173.2,166.6,152.3,150.7,145.7,140.2,139.3,139.1,133.3,130.1$

$129.2,127.5,127.1,126.6,123.3,121.6,121.0,120.3,61.0,60.2,55.3,40.2,39.5,34.3,25.8,22.9,19.4,14.4,14.2$

13.8. MS (m/z, ESI): Calcd. for Chemical Formula: Chemical Formula: $\mathrm{C}_{32} \mathrm{H}_{35} \mathrm{ClO}_{4}{ }^{+}[\mathrm{M}+\mathrm{H}]^{+}: 519.2302$, Found: 519.2304 .

HPLC analysis of the product: Daicel Chiralpak IA, hexane/iso-propanol $=99: 1,1.0 \mathrm{~mL} / \mathrm{min}, \lambda=330 \mathrm{~nm}$, retention time: $7.16 \mathrm{~min}$ and $7.54 \mathrm{~min} .50: 50$ er. 


\section{Synthetic applications}

\section{(S)-4-(9-butyl-2-(4-(ethoxycarbonyl)phenyl)-7-(4,4,5,5-tetramethyl-1,3,2-dioxaborolan-2-yl)-9H-fluoren-9-}

\section{yl)butanoic acid (54)}

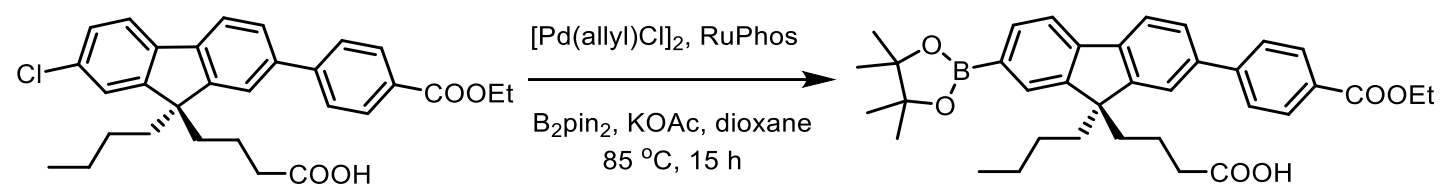

Under $\mathrm{N}_{2}$ atmosphere, to a mixture of $[\mathrm{Pd}(\mathrm{allyl}) \mathrm{Cl}]_{2}(3.6 \mathrm{mg}, 0.01 \mathrm{mmol})$ and RuPhos $(10.3 \mathrm{mg}, 0.022 \mathrm{mmol})$ was added $0.5 \mathrm{~mL}$ dioxane and the resulting solution was stirred for $20 \mathrm{~min}$ before use. To a mixture of $(R)-4$-(9-butyl-2chloro-7-(4-(ethoxycarbonyl)phenyl)-9H-fluoren-9-yl)butanoic acid 3 (49.1 mg, $0.1 \mathrm{mmol}), B_{2}$ pinz $(38.0 \mathrm{mg}, 0.15$ $\mathrm{mmol}$ ) and $\mathrm{KOAC}(19.6 \mathrm{mg}, 0.2 \mathrm{mmol})$ was added $1.0 \mathrm{~mL}$ dioxane and followed with $0.1 \mathrm{~mL}$ metal-ligand complex solution. ${ }^{[13]}$ The resulting reaction mixture was stirred at $85{ }^{\circ} \mathrm{C}$ for $15 \mathrm{~h}$. After the reaction was completed, the reaction was quenched with water, neutralized to $\mathrm{pH}$ 3-5 with $1 \mathrm{M} \mathrm{HCl}$ and extracted with ethyl acetate $(3 \mathrm{~mL} \mathrm{X} \mathrm{3)}$ for three times. The combined organic phases were washed with brine, dried over $\mathrm{Na}_{2} \mathrm{SO}_{4}$ and concentrated in vacuo. The crude materials were purified by flash column chromatography with hexane and ethyl acetate (6:1-1:1) to give the desired products as a white solid $39.0 \mathrm{mg}$ in $67 \%$ yield. $[\alpha]_{\mathrm{D}}{ }^{20}=+9.50\left(c 0.020 \mathrm{CHCl}_{3}\right)$ for $97: 3$ er. ${ }^{1} \mathrm{H}$ NMR $(500$ $\left.\mathrm{MHz}, \mathrm{CDCl}_{3}\right) \delta 8.14(\mathrm{~d}, J=8.4 \mathrm{~Hz}, 2 \mathrm{H}), 7.85(\mathrm{dd}, J=7.5,0.8 \mathrm{~Hz}, 1 \mathrm{H}), 7.81(\mathrm{~d}, J=7.9 \mathrm{~Hz}, 1 \mathrm{H}), 7.75(\mathrm{dd}, J=16.9,8.6 \mathrm{~Hz}$, 4H), $7.63(\mathrm{dd}, J=7.9,1.6 \mathrm{~Hz}, 1 \mathrm{H}), 7.59(\mathrm{~d}, J=1.2 \mathrm{~Hz}, 1 \mathrm{H}), 4.43(\mathrm{q}, J=7.1 \mathrm{~Hz}, 2 \mathrm{H}), 2.12(\mathrm{dt}, J=20.0,7.3 \mathrm{~Hz}, 4 \mathrm{H}), 2.07$ $-2.03(\mathrm{~m}, 2 \mathrm{H}), 1.44(\mathrm{t}, J=7.1 \mathrm{~Hz}, 3 \mathrm{H}), 1.41(\mathrm{~s}, 12 \mathrm{H}), 1.09(\mathrm{dd}, J=14.1,7.1 \mathrm{~Hz}, 2 \mathrm{H}), 0.98(\mathrm{dd}, J=13.1,7.2 \mathrm{~Hz}, 2 \mathrm{H}), 0.70$ - $0.59(\mathrm{~m}, 5 \mathrm{H}) .{ }^{13} \mathrm{C}$ NMR $\left(126 \mathrm{MHz}, \mathrm{CDCl}_{3}\right) \delta 177.4,166.6,151.4,149.5,145.9,143.5,141.1,139.5,134.1,130.1$, $129.1,128.8,127.1,126.5,121.7,120.7,119.4,83.8,61.0,55.0,40.2,39.4,33.7,25.8,24.96,24.9,23.0,19.2,14.4$, 13.8. HRMS (m/z, ESI): Calcd. for Chemical Formula: $\mathrm{C}_{36} \mathrm{H}_{44} \mathrm{BO}_{6}{ }^{+}[\mathrm{M}+\mathrm{H}]^{+}$: 583.3014 , Found: 583.2954. HPLC analysis of the reaction product: Daicel Chiralpak IH, hexane/iso-propanol $=95: 5,1.0 \mathrm{~mL} / \mathrm{min}, \lambda=338 \mathrm{~nm}$, retention time: $26.09 \mathrm{~min}$ (major) and $40.06 \mathrm{~min}$ (minor). 
(R)-4-(9-butyl-2-(4-(diphenylamino)phenyl)-7-(4-(ethoxycarbonyl)phenyl)-9H-fluoren-9-yl)butanoic acid (55)

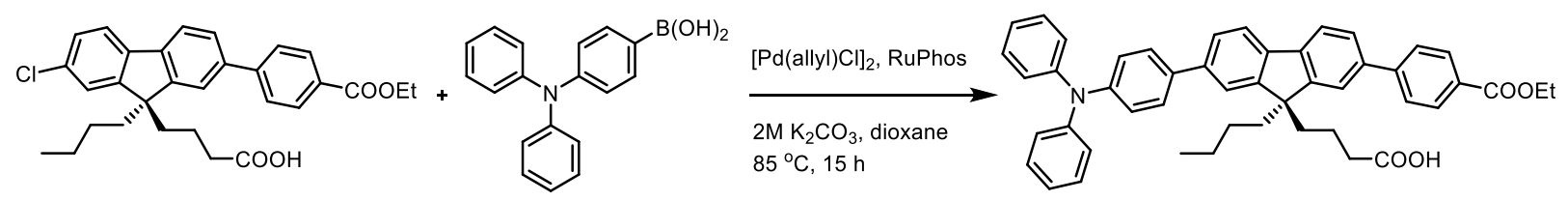

Under $\mathrm{N}_{2}$ atmosphere, to a mixture of $[\mathrm{Pd}(\mathrm{allyl}) \mathrm{Cl}]_{2}(3.6 \mathrm{mg}, 0.01 \mathrm{mmol})$ and RuPhos $(10.3 \mathrm{mg}, 0.022 \mathrm{mmol})$ was added $0.5 \mathrm{~mL}$ dioxane and the resulting solution was stirred for $20 \mathrm{~min}$ before use. To a mixture of (R)-4-(9-butyl-2chloro-7-(4-(ethoxycarbonyl)phenyl)-9H-fluoren-9-yl)butanoic acid $\quad 3 \quad(49.1 \quad \mathrm{mg}, \quad 0.1 \quad \mathrm{mmol})$, and (4(diphenylamino)phenyl)boronic acid $(31.8 \mathrm{mg}, 0.11 \mathrm{mg}$ ) was added $1.0 \mathrm{~mL}$ dioxane and followed with $0.1 \mathrm{~mL}$ metalligand complex solution, then $0.15 \mathrm{~mL} 2 \mathrm{M} \mathrm{K}_{2} \mathrm{CO}_{3}(41.4 \mathrm{mg}, 0.3 \mathrm{mmol})$ was added. The resulting reaction mixture was stirred at $85^{\circ} \mathrm{C}$ for $15 \mathrm{~h}$. After the reaction was completed, the reaction was quenched with water, neutralized to $\mathrm{pH}$ 3-5 with $1 \mathrm{M} \mathrm{HCl}$ and extracted with ethyl acetate $(3 \mathrm{~mL} \times 3)$ for three times. The combined organic phases were washed with brine, dried over $\mathrm{Na}_{2} \mathrm{SO}_{4}$ and concentrated in vacuo. The crude materials were purified by flash column chromatography with hexane and ethyl acetate (6:1-1:1) to give the desired products $50.2 \mathrm{mg}$ in $72 \%$ yield as a white solid, $[\alpha]_{D}{ }^{20}=+27.00\left(c 0.020 \mathrm{CHCl}_{3}\right)$ for $97: 3$ er. ${ }^{1} \mathrm{H} \mathrm{NMR}\left(500 \mathrm{MHz}, \mathrm{CDCl}_{3}\right) \delta 8.19-8.11(\mathrm{~m}, 2 \mathrm{H}), 7.79(\mathrm{dd}, J=7.7,6.6$ $\mathrm{Hz}, 2 \mathrm{H}), 7.76-7.71(\mathrm{~m}, 2 \mathrm{H}), 7.63(\mathrm{dd}, J=7.9,1.6 \mathrm{~Hz}, 1 \mathrm{H}), 7.58(\mathrm{ddd}, J=6.6,5.9,4.3 \mathrm{~Hz}, 5 \mathrm{H}), 7.32-7.28(\mathrm{~m}, 4 \mathrm{H}), 7.23$ $-7.12(\mathrm{~m}, 6 \mathrm{H}), 7.09-7.02(\mathrm{~m}, 2 \mathrm{H}), 4.43(\mathrm{q}, J=7.1 \mathrm{~Hz}, 2 \mathrm{H}), 2.17-2.09(\mathrm{~m}, 4 \mathrm{H}), 2.08-2.03(\mathrm{~m}, 2 \mathrm{H}), 1.44(\mathrm{t}, J=7.1$ $\mathrm{Hz}, 3 \mathrm{H}), 1.11(\mathrm{dd}, J=14.8,7.4 \mathrm{~Hz}, 2 \mathrm{H}), 1.08-1.02(\mathrm{~m}, 2 \mathrm{H}), 0.75-0.66(\mathrm{~m}, 5 \mathrm{H}) .{ }^{13} \mathrm{C} \mathrm{NMR}\left(126 \mathrm{MHz}, \mathrm{CDCl}_{3}\right) \delta 166.6$, 151.0, 151.0, 147.7, 147.2, 145.9, 141.0, 140.0, 139.3, 138.9, 135.4, 130.1, 129.3, 129.0, 127.8, 127.0, 126.5, 125.9, $124.4,124.0,123.0,121.6,121.0,120.3,120.2,61.0,55.0,40.4,39.5,33.7,25.9,23.0,19.3,14.4,13.8 . \mathrm{HRMS}(\mathrm{m} / \mathrm{z}$, ESI): Calcd. for Chemical Formula: $\mathrm{C}_{48} \mathrm{H}_{46} \mathrm{NO}_{4}{ }^{+}[\mathrm{M}+\mathrm{H}]^{+}:$700.3785, Found: 700.3738. HPLC analysis of the reaction product: Daicel Chiralpak IH, hexane/iso-propanol $=94: 6,1.0 \mathrm{~mL} / \mathrm{min}, \lambda=350 \mathrm{~nm}$, retention time:7.66 min (major) and $32.51 \mathrm{~min}$ (minor). 
(R)-4-(9-butyl-2-(4-(ethoxycarbonyl)phenyl)-7-(methyl(phenyl)amino)-9H-fluoren-9-yl)butanoic acid (56)
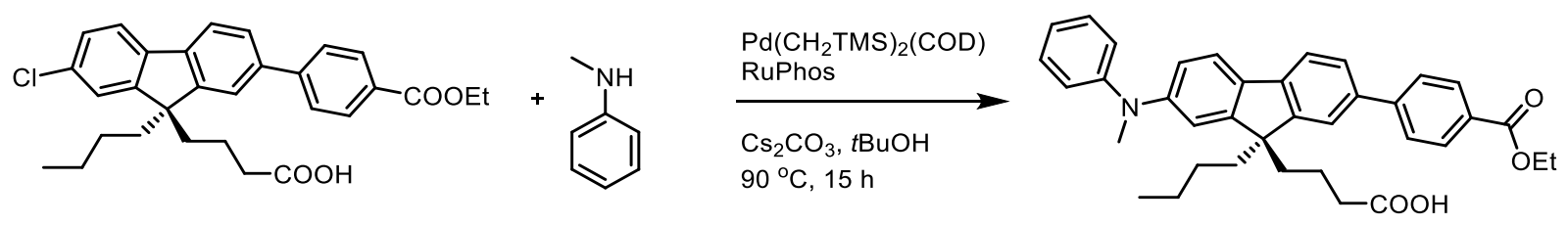

Under $\mathrm{N}_{2}$ atmosphere, to a mixture of $\mathrm{Pd}\left(\mathrm{CH}_{2} \mathrm{TMS}\right)_{2}(\mathrm{COD})(9.5 \mathrm{mg}, 0.025 \mathrm{mmol})$ and RuPhos $(16.5 \mathrm{mg}, 0.035$ mmol) was added $0.5 \mathrm{~mL}$ dioxane and the resulting solution was stirred for $20 \mathrm{~min}$ before use. Then $0.1 \mathrm{~mL} \mathrm{metal}$ ligand complex solution was added to a $1.0 \mathrm{~mL} t \mathrm{BuOH}$ solution of (R)-4-(9-butyl-2-chloro-7-(4(ethoxycarbonyl)phenyl)-9H-fluoren-9-yl)butanoic acid 3 (49.1 mg, $0.1 \mathrm{mmol})$, methylaniline $(12.9 \mathrm{mg}, 0.12 \mathrm{mmol})$ and $\mathrm{Cs}_{2} \mathrm{CO}_{3}(48.9 \mathrm{mg}, 0.15 \mathrm{mmol}) .{ }^{[14]}$ Then the resulting reaction mixture was stirred at $90{ }^{\circ} \mathrm{C}$ for $15 \mathrm{~h}$. The reaction was quenched with water, neutralized to $\mathrm{pH} 1 \sim 2$ with $1 \mathrm{M} \mathrm{HCl}$ and extracted with ethyl acetate $(3 \mathrm{~mL} X 3)$ for three times. The combined organic phases were washed with brine, dried over $\mathrm{Na}_{2} \mathrm{SO}_{4}$ and concentrated in vacuo. Then the crude product was purified by flash column chromatography with hexane and ethyl acetate (6:1-1:1) to give the desired products $44.1 \mathrm{mg}$ in $79 \%$ yield as a light-yellow solid, $[\alpha]_{\mathrm{D}}{ }^{20}=+4.00\left(c 0.030 \mathrm{CHCl}_{3}\right)$ for $97: 3$ er. ${ }^{1} \mathrm{H}$ NMR $(500$ $\left.\mathrm{MHz}, \mathrm{CDCl}_{3}\right) \delta 8.14-8.13(\mathrm{~m}, 2 \mathrm{H}), 7.72(\mathrm{dt}, J=16.8,5.9 \mathrm{~Hz}, 3 \mathrm{H}), 7.65-7.58(\mathrm{~m}, 2 \mathrm{H}), 7.55(\mathrm{~s}, 1 \mathrm{H}), 7.34-7.28(\mathrm{~m}, 2 \mathrm{H})$, $7.16-6.86(\mathrm{~m}, 5 \mathrm{H}), 4.43(\mathrm{q}, J=7.1 \mathrm{~Hz}, 2 \mathrm{H}), 3.41(\mathrm{~s}, 3 \mathrm{H}), 2.13-1.94(\mathrm{~m}, 6 \mathrm{H}), 1.44(\mathrm{t}, J=7.1 \mathrm{~Hz}, 3 \mathrm{H}), 1.11-1.06(\mathrm{~m}$, 4H), $0.74-0.67$ (m, 5H). $\left.{ }^{13} \mathrm{C} \mathrm{NMR} \mathrm{(126} \mathrm{MHz,} \mathrm{CDCl}_{3}\right) \delta 178.1,166.7,151.7,150.5,150.2,149.2,148.8,146.0,141.4$, $137.8,134.1,130.1,129.2,128.8,127.5,127.2,127.0,126.9,126.5,122.9,121.5,121.4,121.2,120.7,120.3,120.1$, 119.7, 119.4, 115.3, 60.9, 54.9, 40.6, 40.2, 39.5, 33.9, 25.9, 23.0, 19.3, 19.2, 14.4, 13.8. MS (m/z, ESI): Calcd. for Chemical Formula: $\mathrm{C}_{37} \mathrm{H}_{38} \mathrm{NO}_{4}{ }^{+}[\mathrm{M}+\mathrm{H}]^{+}: 560.2806$, Found: 560.2803. HPLC analysis of the reaction product: Daicel Chiralpak $\mathrm{IH}$, hexane/iso-propanol $=95: 5,1.0 \mathrm{~mL} / \mathrm{min}, \lambda=346 \mathrm{~nm}$, retention time:8.42 $\mathrm{min}$ (major) and $11.72 \mathrm{~min}$ (minor). 
to 60$)$

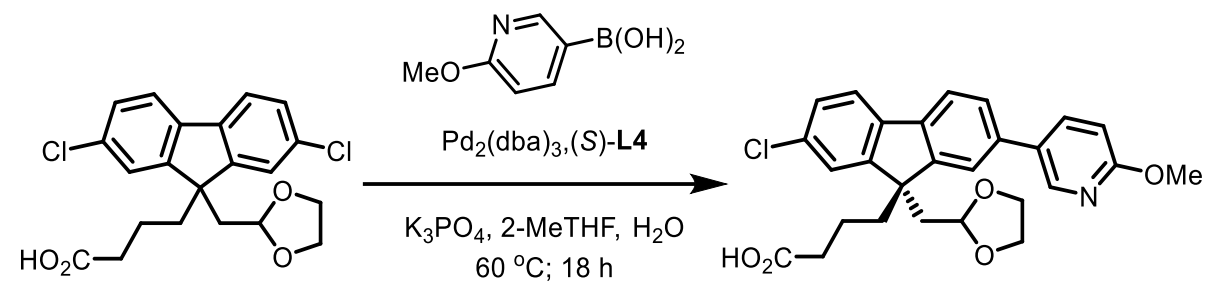

Under $\mathrm{N}_{2}$ atmosphere, to a mixture of $\mathrm{Pd}_{2} \mathrm{dba}_{3}(4.6 \mathrm{mg}, 0.005 \mathrm{mmol})$ and $(S)-\mathrm{L} 4(5.9 \mathrm{mg}, 0.01 \mathrm{mmol})$ was added $1 \mathrm{~mL} 2$-MeTHF and the resulting solution was stirred for $20 \mathrm{~min}$ before use. Then the metal-ligand complex solution was added to a $9 \mathrm{~mL}$ 2-MeTHF solution of 2-Methoxy-5-pyridineboronic acid (92 mg, $0.6 \mathrm{mmol})$, 4-(9-((1,3dioxolan-2-yl)methyl)-2,7-dichloro-9H-fluoren-9-yl)butanoic acid (203 mg, $0.5 \mathrm{mmol}$ ) and $\mathrm{K}_{3} \mathrm{PO}_{4}(1.06 \mathrm{~g}, 5 \mathrm{mmol})$, followed by $0.82 \mathrm{~mL} \mathrm{H} 2 \mathrm{O}$. Then the resulting reaction mixture was stirred at $60^{\circ} \mathrm{C}$ for $18 \mathrm{~h}$. The reaction was quenched with water, neutralized to $\mathrm{pH} \mathrm{3-5} \mathrm{with} 1 \mathrm{M} \mathrm{HCl}$ and extracted with ethyl acetate $(30 \mathrm{~mL} \times 3)$ for three times. The combined organic phases were washed with brine, dried over $\mathrm{Na}_{2} \mathrm{SO}_{4}$ and concentrated in vacuo. The crude materials were purified by flash column chromatography with hexane and acetone(20:1-3:1) to give the desired products 136 mg in $57 \%$ yield as a white solid. ${ }^{1} \mathrm{H} \mathrm{NMR}\left(400 \mathrm{MHz}, \mathrm{CDCl}_{3}\right) \delta 8.5-8.4(\mathrm{~m}, 1 \mathrm{H}), 7.9(\mathrm{dd}, J=8.6,2.6 \mathrm{~Hz}, 1 \mathrm{H}), 7.7(\mathrm{dd}, J$ $=7.8,0.7 \mathrm{~Hz}, 1 \mathrm{H}), 7.6(\mathrm{~d}, J=8.1 \mathrm{~Hz}, 1 \mathrm{H}), 7.5-7.5(\mathrm{~m}, 2 \mathrm{H}), 7.4(\mathrm{~d}, J=1.9 \mathrm{~Hz}, 1 \mathrm{H}), 7.3(\mathrm{dd}, J=8.1,1.9 \mathrm{~Hz}, 1 \mathrm{H}), 6.8(\mathrm{dd}$, $J=8.7,0.7 \mathrm{~Hz}, 1 \mathrm{H}), 4.3(\mathrm{t}, \mathrm{J}=4.7 \mathrm{~Hz}, 1 \mathrm{H}), 4.0(\mathrm{~s}, 3 \mathrm{H}), 3.8-3.7(\mathrm{~m}, 2 \mathrm{H}), 3.6-3.5(\mathrm{~m}, 2 \mathrm{H}), 2.4-2.3(\mathrm{~m}, 2 \mathrm{H}), 2.2-2.0$ (m, 4H), $1.0-0.9(\mathrm{~m}, 2 \mathrm{H}) .{ }^{13} \mathrm{C} \mathrm{NMR}\left(101 \mathrm{MHz}, \mathrm{CDCl}_{3}\right) \delta 177.7,163.5,151.2,149.8,144.8,139.2,138.9,138.1,137.0$ 133.2, 130.6, 127.9, 126.3, 124.1, 122.1, 121.1, 120.5, 111.0, 102.2, 64.6, 64.6, 54.1, 52.7, 43.8, 39.4, 33.7, 18.9. $[\alpha]_{\mathrm{D}^{20}}=-8.45\left(\right.$ ( $\left.1.0 \mathrm{CHCl}_{3}\right)$ for 95:5 er. HRMS (m/z, ESI): Calcd. for Chemical Formula: $\mathrm{C}_{27} \mathrm{H}_{25} \mathrm{ClNO}_{5}{ }^{-}[\mathrm{M}-\mathrm{H}]^{-}: 478.1427$, Found: 478.1425. HPLC analysis of the reaction product: Daicel Chiralpak IA, hexane/iso-propanol = 80: 20, 1.0 $\mathrm{mL} / \mathrm{min}, \lambda=322 \mathrm{~nm}$, retention time: $7.10 \mathrm{~min}$ (major) and $5.88 \mathrm{~min}$ (minor). 
(R)-4-(9-((1,3-dioxolan-2-yl)methyl)-2-(3,6-dihydro-2H-pyran-4-yl)-7-(6-methoxypyridin-3-yl)-9H-fluoren-9-

yl)butanoic acid (60)

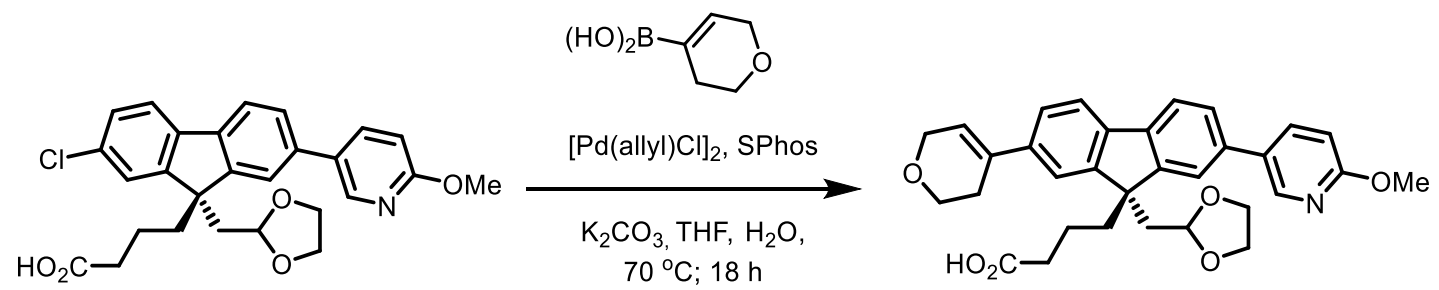

Under $\mathrm{N}_{2}$ atmosphere, to a mixture of $\left[\mathrm{Pd}(\text { allyl)Cl}]_{2}(4.8 \mathrm{mg}, 0.013 \mathrm{mmol})\right.$ and SPhos $(10.6 \mathrm{mg}, 0.026 \mathrm{mmol})$

was added $1 \mathrm{~mL}$ THF and the resulting solution was stirred for $20 \mathrm{~min}$ before use. Then the metal-ligand complex solution was added to a $4 \mathrm{~mL}$ THF solution of 3,6-Dihydro-2H-pyran-4-boronic acid pinacol ester (109 mg, $0.52 \mathrm{mmol}$ ), (S)-4-(9-((1,3-dioxolan-2-yl)methyl)-2-chloro-7-(6-methoxypyridin-3-yl)-9H-fluoren-9-yl)butanoic acid (126 mg, 0.26 $\mathrm{mmol})$ and $\mathrm{K}_{2} \mathrm{CO}_{3}(179 \mathrm{Mg}, 1.3 \mathrm{mmol})$, followed by $0.5 \mathrm{~mL} \mathrm{H}{ }_{2} \mathrm{O}$. Then the resulting reaction mixture was stirred at 70 ${ }^{\circ} \mathrm{C}$ for $18 \mathrm{~h}$. The reaction was quenched with water, neutralized to $\mathrm{pH}$ 3-5 with $1 \mathrm{M} \mathrm{HCl}$ and extracted with ethyl acetate $(30 \mathrm{~mL} \times 3)$ for three times. The combined organic phases were washed with brine, dried over $\mathrm{Na}_{2} \mathrm{SO}_{4}$ and concentrated in vacuo. The crude materials were purified by flash column chromatography with hexane and acetone(20:1-1:1) to give the desired products $125 \mathrm{mg}$ in $91 \%$ yield as a white solid. ${ }^{1} \mathrm{H} N M R\left(400 \mathrm{MHz}, \mathrm{CDCl}_{3}\right) \delta 8.45$ (dd, $J=2.6,0.8 \mathrm{~Hz}, 1 \mathrm{H}), 7.87(\mathrm{dd}, J=8.6,2.5 \mathrm{~Hz}, 1 \mathrm{H}), 7.73(\mathrm{~d}, J=8.0 \mathrm{~Hz}, 1 \mathrm{H}), 7.67(\mathrm{~d}, J=7.9 \mathrm{~Hz}, 1 \mathrm{H}), 7.54-7.47(\mathrm{~m}$, $2 \mathrm{H}), 7.45-7.38(\mathrm{~m}, 2 \mathrm{H}), 6.84(\mathrm{dd}, J=8.6,0.7 \mathrm{~Hz}, 1 \mathrm{H}), 6.18-6.20(\mathrm{~m}, 1 \mathrm{H}), 4.39-4.33(\mathrm{~m}, 3 \mathrm{H}), 3.99(\mathrm{~s}, 3 \mathrm{H}), 3.96(\mathrm{t}, J$ $=5.4 \mathrm{~Hz}, 2 \mathrm{H}), 3.77-3.70(\mathrm{~m}, 2 \mathrm{H}), 3.58-3.51(\mathrm{~m}, 2 \mathrm{H}), 2.57-2.60(\mathrm{~m}, 2 \mathrm{H}), 2.35(\mathrm{~d}, J=4.8 \mathrm{~Hz}, 2 \mathrm{H}), 2.06-2.14(\mathrm{~m}$, 4H), $0.92-0.98(\mathrm{~m}, 2 \mathrm{H}) .{ }^{13} \mathrm{C}$ NMR $\left(101 \mathrm{MHz}, \mathrm{CDCl}_{3}\right) \delta 177.8,163.4,150.2,149.6,144.8,139.9,139.6,139.6,138.1$, $136.5,134.4,130.8,126.2,124.2,122.5,122.1,120.4,120.0,119.8,110.9,102.4,66.0,64.6,64.5,54.0,52.5,44.0$, 39.4, 33.8, 27.5, 19.0. $[\alpha]_{D}^{20}=-1.69\left(c 0.13 \mathrm{CHCl}_{3}\right)$ for 94.5:5.5 er. HRMS (m/z, ESI): Calcd. for Chemical Formula: $\mathrm{C}_{32} \mathrm{H}_{32} \mathrm{NO}_{6}{ }^{-}[\mathrm{M}-\mathrm{H}]^{-}: 526.2235$, Found: 526.2235. HPLC analysis of the reaction product: Daicel Chiralpak IA, hexane/isopropanol $=80: 20,1.0 \mathrm{~mL} / \mathrm{min}, \lambda=325 \mathrm{~nm}$, retention time: $10.96 \mathrm{~min}$ (major) and $8.12 \mathrm{~min}$ (minor). 

(precursor to ent-60)

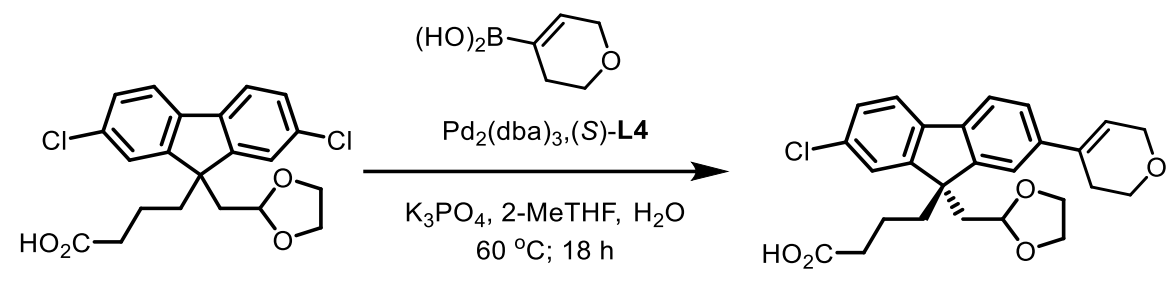

Under $\mathrm{N}_{2}$ atmosphere, to a mixture of $\mathrm{Pd}_{2} \mathrm{dba}_{3}(3.76 \mathrm{mg}, 0.00411 \mathrm{mmol})$ and $(\mathrm{S})-\mathrm{L} 4(4.84 \mathrm{mg}, 0.00822 \mathrm{mmol})$ was added $1 \mathrm{~mL} 2$-MeTHF and the resulting solution was stirred for $20 \mathrm{~min}$ before use. Then the metal-ligand complex solution was added to a $7.2 \mathrm{~mL}$ 2-MeTHF solution of 3,6-Dihydro-2H-pyran-4-boronic acid pinacol ester (102 mg, $0.493 \mathrm{mmol}), 4-(9-((1,3-d i o x o l a n-2-y \mathrm{l})$ methyl)-2,7-dichloro-9H-fluoren-9-yl)butanoic acid (167 mg, $0.411 \mathrm{mmol}$ ) and $\mathrm{K}_{3} \mathrm{PO}_{4}(871 \mathrm{mg}, 4.11 \mathrm{mmol})$, followed by $0.67 \mathrm{~mL} \mathrm{H} \mathrm{H}_{2} \mathrm{O}$. Then the resulting reaction mixture was stirred at $60{ }^{\circ} \mathrm{C}$ for 18 h. The reaction was quenched with water, neutralized to $\mathrm{pH} \mathrm{3-5} \mathrm{with} 1 \mathrm{M} \mathrm{HCl}$ and extracted with ethyl acetate (15 $\mathrm{mL} X 3$ ) for three times. The combined organic phases were washed with brine, dried over $\mathrm{Na}_{2} \mathrm{SO}_{4}$ and concentrated in vacuo. The crude materials were purified by flash column chromatography with hexane and acetone (20:1-3:1) to give the desired products $98 \mathrm{mg}$ in $53 \%$ yield as a white solid. ${ }^{1} \mathrm{H} \mathrm{NMR}\left(500 \mathrm{MHz}, \mathrm{CDCl}_{3}\right) \delta 7.63-7.57(\mathrm{~m}, 2 \mathrm{H}), 7.40$ $-7.35(\mathrm{~m}, 3 \mathrm{H}), 7.31(\mathrm{dd}, \mathrm{J}=8.1,1.9 \mathrm{~Hz}, 1 \mathrm{H}), 6.17-6.19(\mathrm{~m}, 1 \mathrm{H}), 4.29-4.35(\mathrm{~m}, 2 \mathrm{H}), 4.29(\mathrm{t}, \mathrm{J}=4.7 \mathrm{~Hz}, 1 \mathrm{H}), 3.95(\mathrm{t}, \mathrm{J}=$ $5.5 \mathrm{~Hz}, 2 \mathrm{H}), 3.73-3.75(\mathrm{~m}, 2 \mathrm{H}), 3.52-3.55(\mathrm{~m}, 2 \mathrm{H}), 2.55-2.59(\mathrm{~m}, 2 \mathrm{H}), 2.31(\mathrm{t}, \mathrm{J}=4.8 \mathrm{~Hz}, 2 \mathrm{H}), 2.06-2.10(\mathrm{~m}, 4 \mathrm{H}), 0.91$ $-0.85(\mathrm{~m}, 2 \mathrm{H}) .{ }^{13} \mathrm{C}$ NMR $\left(126 \mathrm{MHz}, \mathrm{CDCl}_{3}\right) \delta 177.7,151.2,149.1,139.8,139.12,139.05,134.3,132.9,127.8,124.3$, $124.1,122.7,120.9,119.9,119.8,102.2,68.1,66.0,64.6,64.5,52.6,43.8,39.4,33.6,27.5,18.8 .[\alpha]_{D}{ }^{20}=-10.9(c 1.0$ $\mathrm{CHCl}_{3}$ ) for $95: 5$ er. HRMS (m/z, ESI): Calcd. for Chemical Formula: $\mathrm{C}_{26} \mathrm{H}_{26} \mathrm{ClO}_{5}[\mathrm{M}-\mathrm{H}]=$ : 454.1474, Found: 453.1478. HPLC analysis of the reaction product: Daicel Chiralpak IA, hexane/iso-propanol $=95: 5,1.0 \mathrm{~mL} / \mathrm{min}, \lambda=318 \mathrm{~nm}$, retention time: $20.44 \mathrm{~min}$ (major) and $24.34 \mathrm{~min}$ (minor). 
(S)-4-(9-((1,3-dioxolan-2-yl)methyl)-2-(3,6-dihydro-2H-pyran-4-yl)-7-(6-methoxypyridin-3-yl)-9H-fluoren-9-

yl)butanoic acid (ent-60)
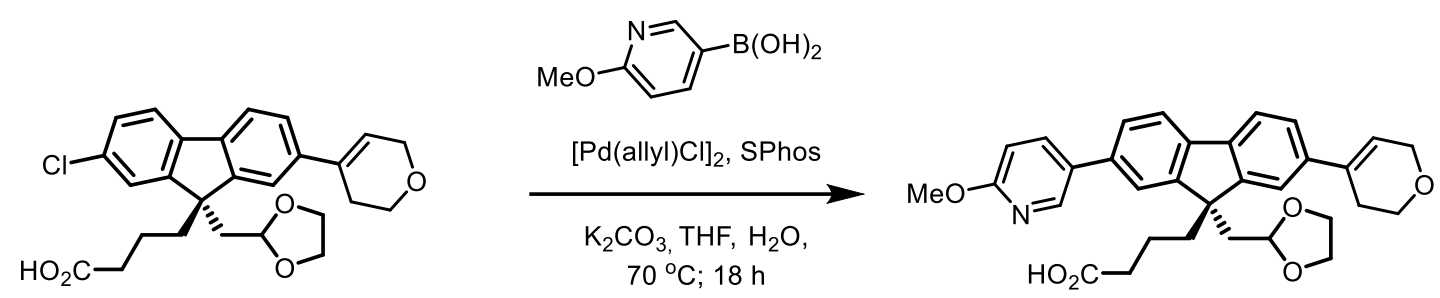

Under $\mathrm{N}_{2}$ atmosphere, to a mixture of $[\mathrm{Pd}(\text { allyl }) \mathrm{Cl}]_{2}(3.2 \mathrm{mg}, 0.0088 \mathrm{mmol})$ and SPhos $(7.2 \mathrm{mg}, 0.0176 \mathrm{mmol})$

was added $0.5 \mathrm{~mL}$ THF and the resulting solution was stirred for $20 \mathrm{~min}$ before use. Then the metal-ligand complex solution was added to a $2.5 \mathrm{~mL}$ THF solution of 2-Methoxy-5-pyridineboronic acid (54 mg, $0.352 \mathrm{mmol}),(S)-4-(9-$ ((1,3-dioxolan-2-yl)methyl)-2-chloro-7-(3,6-dihydro-2H-pyran-4-yl)-9H-fluoren-9-yl)butanoic acid (80 mg, 0.176 $\mathrm{mmol})$ and $\mathrm{K}_{2} \mathrm{CO}_{3}(121 \mathrm{mg}, 0.88 \mathrm{mmol})$, followed by $0.4 \mathrm{~mL} \mathrm{H} \mathrm{H}_{2} \mathrm{O}$. Then the resulting reaction mixture was stirred at $70^{\circ} \mathrm{C}$ for $18 \mathrm{~h}$. The reaction was quenched with water, neutralized to $\mathrm{pH}$ 3-5 with $1 \mathrm{M} \mathrm{HCl}$ and extracted with ethyl acetate $(30 \mathrm{~mL} \times 3)$ for three times. The combined organic phases were washed with brine, dried over $\mathrm{Na}_{2} \mathrm{SO}_{4}$ and concentrated in vacuo. The crude materials were purified by flash column chromatography with hexane and acetone (20:1-1:1) to give the desired products $80 \mathrm{mg}$ in $86 \%$ yield as a white solid. $[\alpha]_{\mathrm{D}}^{20}=+2.4\left(\right.$ c $\left.1.0 \mathrm{CHCl}_{3}\right)$ for $95: 5$ er. HRMS (m/z, ESI): Calcd. for Chemical Formula: $\mathrm{C}_{32} \mathrm{H}_{32} \mathrm{NO}_{6}{ }^{-}[\mathrm{M}-\mathrm{H}]: 526.2235$, Found: 526.2235 . HPLC analysis of the reaction product: Daicel Chiralpak IA, hexane/iso-propanol $=80: 20,1.0 \mathrm{~mL} / \mathrm{min}, \lambda=324 \mathrm{~nm}$, retention time: 8.44 $\min$ (major) and $11.55 \mathrm{~min}$ (minor). 
Methyl (R)-3-amino-2'-(3,6-dihydro-2H-pyran-4-yl)-7'-(6-methoxypyridin-3-yl)spiro[cyclohexane-1,9'-fluoren]-3ene-4-carboxylate (61)
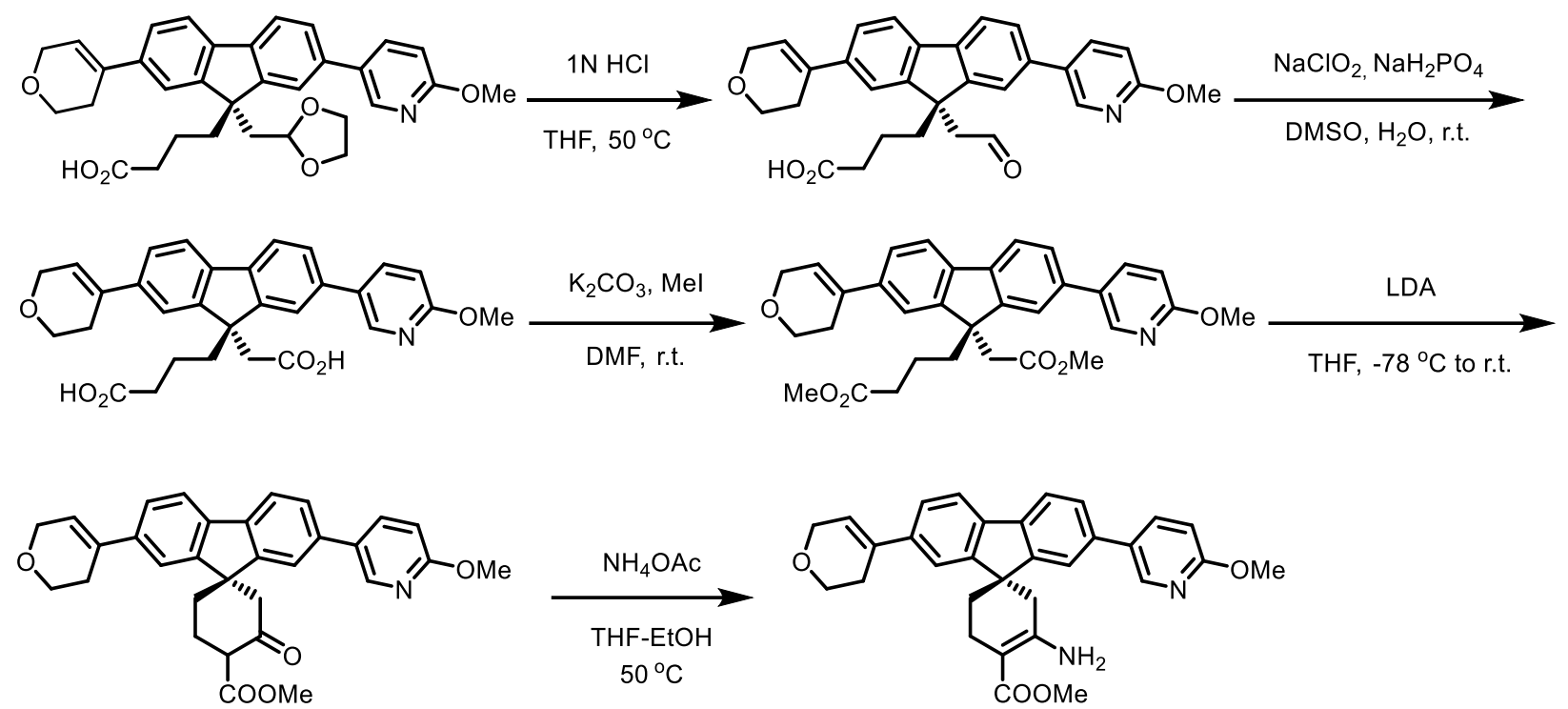

(R)-4-(9-((1,3-dioxolan-2-yl)methyl)-2-(3,6-dihydro-2H-pyran-4-yl)-7-(6-methoxypyridin-3-yl)-9H-fluoren-9-

yl)butanoic acid (120 mg, $0.23 \mathrm{mmol}$ ) was dissolved in $1.0 \mathrm{~mL} \mathrm{THF}$ and $1 \mathrm{~mL} 1 \mathrm{M} \mathrm{HCl}$ was added. The reaction mixtures were stirred at $50{ }^{\circ} \mathrm{C}$ overnight and solvents were removed under reduced pressure. Water was added and the reaction solution was extracted with ethyl acetate $(10 \mathrm{~mL} X 3)$ three times. The combined organic phases were washed with brine, dried over $\mathrm{Na}_{2} \mathrm{SO}_{4}$, and concentrated in vacuo. The crude product was directly subjected to the next step without further purification.

Under $\mathrm{N}_{2}$ atmosphere, the above mixture and $\mathrm{NaH}_{2} \mathrm{PO}_{4}(115 \mathrm{mg}, 0.96 \mathrm{mmol})$ were dissolved in DMSO (2 mL) and $\mathrm{H}_{2} \mathrm{O}(0.7 \mathrm{~mL}) . \mathrm{NaClO}_{2}(32.4 \mathrm{mg}, 0.36 \mathrm{mmol})$ in $\mathrm{H}_{2} \mathrm{O}(0.7 \mathrm{~mL})$ was added dropwise into the above solution at room temperature ${ }^{[15]}$. After addition was completed, the resulting reaction mixture was stirred at room temperature overnight. The reaction was quenched with $1 \mathrm{M} \mathrm{HCl}$ solution to make $\mathrm{pH}=2$, and extracted with ethyl acetate three times. The combined organic phases were washed with brine, dried over $\mathrm{Na}_{2} \mathrm{SO}_{4}$, and concentrated in vacuo. The crude mixture was directly subjected to the next step without further purification.

Under $\mathrm{N}_{2}$ atmosphere, the above mixture and $\mathrm{K}_{2} \mathrm{CO}_{3}(138 \mathrm{mg}, 1 \mathrm{mmol})$ were dissolved in DMF ( $\left.3 \mathrm{~mL}\right)$. Mel ( $86 \mathrm{mg}, 0.60 \mathrm{mmol}$ ) was added dropwise into the above solution at room temperature. After addition was completed, 
the resulting reaction mixture was stirred at room temperature for $2 \mathrm{~h}$. The reaction was quenched with saturated $\mathrm{NH}_{4} \mathrm{Cl}$ solution and extracted with ethyl acetate three times. The combined organic phases were washed with brine, dried over $\mathrm{Na}_{2} \mathrm{SO}_{4}$, and concentrated in vacuo. The crude mixture was purified by column chromatography on silica gel (hexane: acetone $=20: 1$ ) to afford the product (40 mg, 33\% yield over three steps) as a white solid.

Under $\quad \mathrm{N}_{2}$ atmosphere, methyl (R)-4-(2-(3,6-dihydro-2H-pyran-4-yl)-9-(2-methoxy-2-oxoethyl)-7-(6methoxypyridin-3-yl)-9H-fluoren-9-yl)butanoate (40 mg, $0.076 \mathrm{mmol}$ ) was dissolved in THF (1 mL). LDA (2M, $76 \mu \mathrm{L}$, $0.152 \mathrm{mmol}$ ) was added dropwise into the above solution at $-78^{\circ} \mathrm{C}$. After addition was completed, the resulting reaction mixture was allowed to warm to room temperature and stirred for $3 h^{[16]}$. The reaction was quenched with saturated $\mathrm{NH}_{4} \mathrm{Cl}$ solution and extracted with ethyl acetate three times. The combined organic phases were washed with brine, dried over $\mathrm{Na}_{2} \mathrm{SO}_{4}$, and concentrated in vacuo. The crude mixture was directly subjected to the next step without further purification.

The above mixture and $\mathrm{NH}_{4} \mathrm{OAc}(23 \mathrm{mg}, 0.31 \mathrm{mmol})$ were dissolved in THF $(0.5 \mathrm{~mL})$ and EtOH $(1 \mathrm{~mL})$. The reaction mixture was stirred at $50{ }^{\circ} \mathrm{C}$ overnight ${ }^{[17]}$. The reaction was quenched with $\mathrm{H}_{2} \mathrm{O}$ and extracted with ethyl acetate three times. The combined organic phases were washed with brine, dried over $\mathrm{Na}_{2} \mathrm{SO}_{4}$, and concentrated in vacuo. The crude mixture was purified by column chromatography on silica gel (hexane: ethyl acetate $=10: 1-5: 1$ ) to afford the target compound (14 mg, 37\% yield over two steps) as a white solid. ${ }^{1} \mathrm{H} \mathrm{NMR}(400 \mathrm{MHz}, \mathrm{CDCl} 3) \delta 8.41(\mathrm{~d}$, $J=2.5 \mathrm{~Hz}, 1 \mathrm{H}), 7.83-7.76(\mathrm{~m}, 2 \mathrm{H}), 7.72(\mathrm{~d}, \mathrm{~J}=7.9 \mathrm{~Hz}, 1 \mathrm{H}), 7.56-7.51(\mathrm{~m}, 2 \mathrm{H}), 7.47-7.40(\mathrm{~m}, 2 \mathrm{H}), 6.84(\mathrm{~d}, \mathrm{~J}=8.6$ $\mathrm{Hz}, 1 \mathrm{H}), 6.14-6.16(\mathrm{~m}, 1 \mathrm{H}), 4.34-4.38(\mathrm{~m} 2 \mathrm{H}), 4.01(\mathrm{~d}, \mathrm{~J}=1.1 \mathrm{~Hz}, 3 \mathrm{H}), 3.96(\mathrm{t}, \mathrm{J}=5.4 \mathrm{~Hz}, 2 \mathrm{H}), 3.79(\mathrm{~s}, 3 \mathrm{H}), 2.66-$ $2.71(\mathrm{~m}, 2 \mathrm{H}), 2.63-2.52(\mathrm{~m}, 4 \mathrm{H}), 1.92-1.82(\mathrm{~m}, 2 \mathrm{H}) .{ }^{13} \mathrm{C} \mathrm{NMR}\left(101 \mathrm{MHz}, \mathrm{CDCl}_{3}\right) \delta 170.5,155.7,152.1,151.5,140.1$, $138.8,138.4,137.9,134.4,130.5,126.5,124.5,122.8,122.2,120.6,120.1,120.0,111.1,91.5,66.1,64.6,53.9,51.0$, 48.8, 39.5, 32.9, 27.6, 22.3. $[\alpha]_{\mathrm{D}}{ }^{20}=+8.3\left(\right.$ c $\left.1.0 \mathrm{CHCl}_{3}\right)$ for 94.5:5.5 er. HRMS (m/z, ESI): Calcd. for Chemical Formula: $\mathrm{C}_{31} \mathrm{H}_{31} \mathrm{~N}_{2} \mathrm{O}_{4}{ }^{+}[\mathrm{M}+\mathrm{H}]^{+}:$495.2278, Found: 495.2276. HPLC analysis of the reaction product: Daicel Chiralpak IA, hexane/iso-propanol = 90: 10, $1.0 \mathrm{~mL} / \mathrm{min}, \lambda=324 \mathrm{~nm}$, retention time: $50.46 \mathrm{~min}$ (major) and $66.92 \mathrm{~min}$ (minor). 

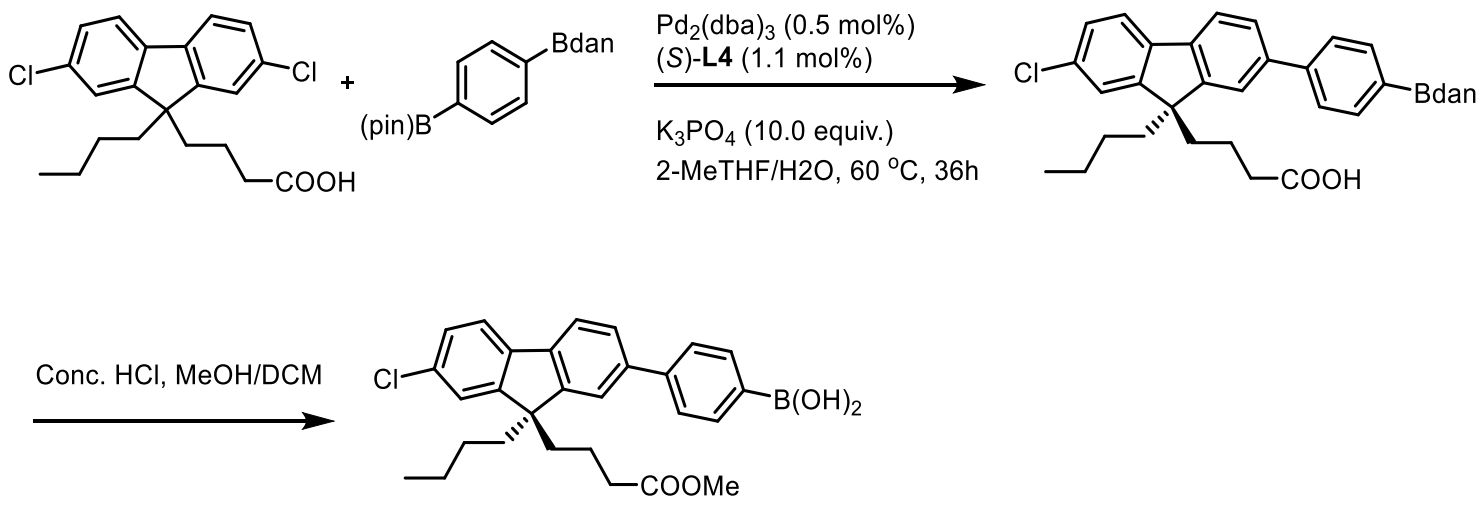

\section{(R)-4-(2-(4-(1H-naphtho[1,8-de][1,3,2]diazaborinin-2(3H)-yl)phenyl)-9-butyl-7-chloro-9H-fluoren-9-yl)butanoic} $\operatorname{acid}(62)$

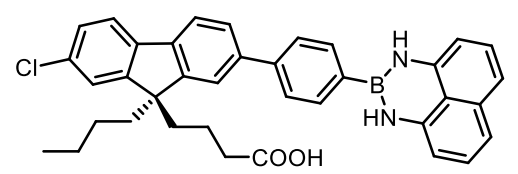

Under $\mathrm{N}_{2}$ atmosphere, to a mixture of $\mathrm{Pd}_{2} \mathrm{dba}_{3}(4.6 \mathrm{mg}, 0.005 \mathrm{mmol})$ and

(S)-L4 (6.4 mg, $0.011 \mathrm{mmol})$ was added $1.0 \mathrm{~mL}$ 2-MeTHF and the resulting solution was stirred for $20 \mathrm{~min}$ before use. Then metal-ligand complex solution was added to a $20 \mathrm{~mL}$ 2-MeTHF solution of 4-(9-butyl-2,7-dichloro-9H-fluoren-9-yl)butanoic acid 51 (377.6 mmol, $1.0 \quad \mathrm{mmol}), \quad$ 2-(4-(4,4,5,5-tetramethyl-1,3,2-dioxaborolan-2-yl)phenyl)-2,3-dihydro-1H-naphtho[1,8de][1,3,2]diazaborinine $(0.407 \mathrm{~g}, 1.1 \mathrm{mmol})$ and $\mathrm{K}_{3} \mathrm{PO}_{4}(2.12 \mathrm{~g}, 10.0 \mathrm{mmol})$, followed by $1.5 \mathrm{~mL} \mathrm{H}_{2} \mathrm{O}$. Then the resulting reaction mixture was stirred at $60^{\circ} \mathrm{C}$ for $36 \mathrm{~h}$. The reaction was quenched with water, neutralized to $\mathrm{pH}$ 35 with $1 \mathrm{M} \mathrm{HCl}$ and extracted with ethyl acetate for three times. The combined organic phases were washed with brine, dried over $\mathrm{Na}_{2} \mathrm{SO}_{4}$ and concentrated in vacuo. The crude materials were purified by flash column chromatography with hexane and ethyl acetate (6:1-1:1) to give the desired products $0.36 \mathrm{~g}$ in $61 \%$ yield as a brown solid. ${ }^{1} \mathrm{H}$ NMR $\left(500 \mathrm{MHz}, \mathrm{CDCl}_{3}\right) \delta 7.79-7.69(\mathrm{~m}, 5 \mathrm{H}), 7.66-7.60(\mathrm{~m}, 2 \mathrm{H}), 7.58-7.57(\mathrm{~m}, 1 \mathrm{H}), 7.36-7.32(\mathrm{~m}, 2 \mathrm{H})$, $7.19(\mathrm{t}, J=7.8 \mathrm{~Hz}, 2 \mathrm{H}), 7.11(\mathrm{~d}, J=8.2 \mathrm{~Hz}, 2 \mathrm{H}), 6.46(\mathrm{~d}, J=7.2 \mathrm{~Hz}, 2 \mathrm{H}), 6.08(\mathrm{~s}, 2 \mathrm{H}), 2.16-1.98(\mathrm{~m}, 6 \mathrm{H}), 1.12(\mathrm{dt}, J=$ 14.9, 7.4 Hz, 2H), $1.03(\mathrm{dt}, J=10.8,5.1 \mathrm{~Hz}, 2 \mathrm{H}), 0.73-0.64(\mathrm{~m}, 5 \mathrm{H}) .{ }^{13} \mathrm{C} \mathrm{NMR}\left(126 \mathrm{MHz}, \mathrm{CDCl}_{3}\right) \delta 178.5,152.1,150.5$, 143.1, 141.1, 140.1, 139.7, 139.3, 136.4, 133.1, 132.0, 127.7, 127.5, 127.0, 126.5, 123.3, 121.5, 121.0, 120.3, 117.9, 106.1, 55.2, 40.2, 39.4, 33.8, 25.8, 22.9, 19.2, 13.8. HRMS (m/z, ESI): Calcd. for Chemical Formula: $\mathrm{C}_{37} \mathrm{H}_{35} \mathrm{ClBN}_{2} \mathrm{O}_{2}{ }^{+}$ $[\mathrm{M}+\mathrm{H}]^{+}: 585.2475$, Found: 585.2486 . 


\section{(R)-(4-(9-butyl-7-chloro-9-(4-methoxy-4-oxobutyl)-9H-fluoren-2-yl)phenyl)boronic acid (63)}

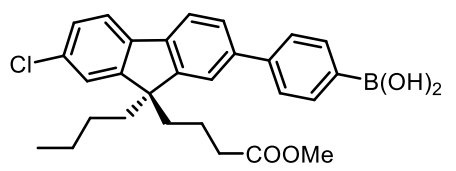

(R)-4-(2-(4-(1H-naphtho[1,8-de][1,3,2]diazaborinin-2(3H)-yl)phenyl)-9-butyl-7chloro-9H-fluoren-9-yl)butanoic acid (30 mg) was dissolved in $0.75 \mathrm{~mL} \mathrm{DCM}$ and $0.75 \mathrm{~mL} \mathrm{MeOH}$, then $20 \mu \mathrm{L}$ conc. $\mathrm{HCl}$ was added. The reaction mixture was stirred at room temperature for $15 \mathrm{~h}^{[18]}$. Then the reaction was quenched by water and extracted with $\mathrm{DCM}(4 \mathrm{~mL} \times 3)$ for three times. The combined organic phases were washed with brine, dried over $\mathrm{Na}_{2} \mathrm{SO}_{4}$ and concentrated in vacuo. The crude materials were purified by flash column chromatography to give the desired product as a white solid 16.3 $\mathrm{mg}$ in $67 \%$ yield. $[\alpha]_{D^{20}}=+3.20\left(c 0.080 \mathrm{CHCl}_{3}\right)$ for $97: 3$ er. ${ }^{1} \mathrm{H}$ NMR (500 MHz, Acetone) $\delta 8.01(\mathrm{~d}, J=8.2 \mathrm{~Hz}, 2 \mathrm{H}), 7.92$ (d, $J=7.9 \mathrm{~Hz}, 1 \mathrm{H}), 7.87(\mathrm{~d}, J=8.1 \mathrm{~Hz}, 1 \mathrm{H}), 7.84(\mathrm{~d}, J=1.3 \mathrm{~Hz}, 1 \mathrm{H}), 7.77(\mathrm{~d}, J=8.2 \mathrm{~Hz}, 2 \mathrm{H}), 7.74(\mathrm{dd}, J=7.9,1.7 \mathrm{~Hz}, 1 \mathrm{H})$, $7.55(\mathrm{~d}, J=1.9 \mathrm{~Hz}, 1 \mathrm{H}), 7.42(\mathrm{dd}, J=8.1,1.9 \mathrm{~Hz}, 1 \mathrm{H}), 3.50(\mathrm{~s}, 3 \mathrm{H}), 2.28-2.09(\mathrm{~m}, 6 \mathrm{H}), 1.12(\mathrm{dd}, J=14.8,7.4 \mathrm{~Hz}, 2 \mathrm{H})$, $0.99-0.92(\mathrm{~m}, 2 \mathrm{H}), 0.70-0.61$ (m, 5H). ${ }^{13} \mathrm{C}$ NMR (126 MHz, Acetone) $\delta 172.8,152.7,150.8,142.8,140.5,139.8$, $139.5,134.8,127.3,126.3,126.1,123.3,121.5,121.2,120.4,50.5,39.8,38.9,33.4,25.9,22.7,19.5,13.2$. HRMS (m/z, ESI): Calcd. for Chemical Formula: $\mathrm{C}_{28} \mathrm{H}_{31} \mathrm{BClO}_{4}{ }^{+}[\mathrm{M}+\mathrm{H}]^{+}:$477.1998, Found: 477.2008. HPLC analysis of the reaction product: Daicel Chiralpak IA, hexane/iso-propanol $=97: 3,1.0 \mathrm{~mL} / \mathrm{min}, \lambda=333 \mathrm{~nm}$, retention time: 14.24 min (major) and $14.94 \mathrm{~min}$ (minor). 


\section{4,4'-((9R,9'R)-1,4-phenylenebis(9-butyl-7-chloro-9H-fluorene-2,9-diyl))dibutyric acid (64)}

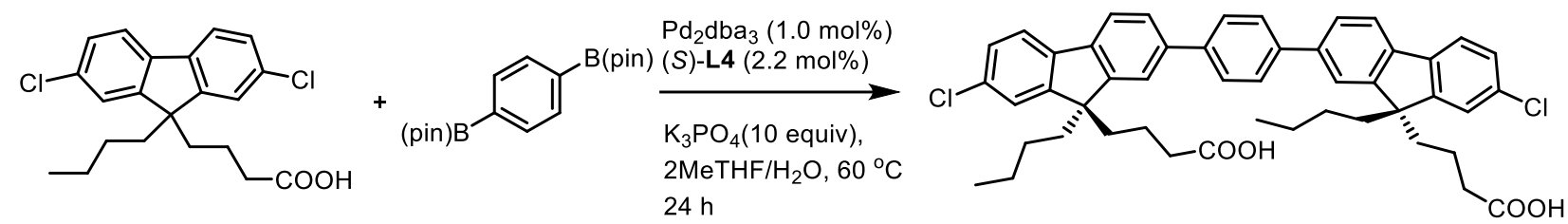

Under $\mathrm{N}_{2}$ atmosphere, to a mixture of $\mathrm{Pd}_{2} \mathrm{dba}_{3}(3.0 \mathrm{mg}, 0.0033 \mathrm{mmol})$ and $(\mathrm{S})-\mathrm{L} 4(4.0 \mathrm{mg}, 0.0066 \mathrm{mmol})$ was added $1.0 \mathrm{~mL}$ 2-MeTHF and the resulting solution was stirred for $20 \mathrm{~min}$ before use. Then the metal-ligand complex solution was added to a $19.0 \mathrm{~mL}$ 2-MeTHF solution of 4-(9-butyl-2,7-dichloro-9H-fluoren-9-yl)butanoic acid 51 (377.3

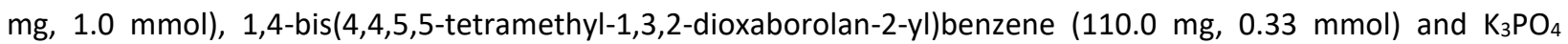
(2.123 g, $10.0 \mathrm{mmol})$, followed by $1.65 \mathrm{~mL} \mathrm{H} 2 \mathrm{O}$. Then the resulting reaction mixture was stirred at $60^{\circ} \mathrm{C}$ for $36 \mathrm{~h}$. The reaction was quenched with water, neutralized to $\mathrm{pH}$ 3-5 with $1 \mathrm{M} \mathrm{HCl}$ and extracted with ethyl acetate (3 $\mathrm{mL} X 3$ ) for three times. The combined organic phases were washed with brine, dried over $\mathrm{Na}_{2} \mathrm{SO}_{4}$ and concentrated in vacuo. Then the crude product was dissolved in $1.0 \mathrm{~mL}$ EtOH again and $10 \mu \mathrm{L}$ conc. $\mathrm{H}_{2} \mathrm{SO}_{4}$ was added and the resulting reaction mixture was heated at $70^{\circ} \mathrm{C}$ for $12 \mathrm{~h}$. The reaction was quenched with water and extracted with ethyl acetate $(2 \mathrm{~mL} \times 3)$ for three times. The combined organic phases were washed with brine, dried over $\mathrm{Na}_{2} \mathrm{SO}_{4}$ and concentrated in vacuo. The crude materials were purified by flash column chromatography with hexane and ethyl acetate $(6: 1-1: 1)$ to give the desired products $112.1 \mathrm{mg}$ in $42 \%$ yield as a white solid, $[\alpha]_{\mathrm{D}}^{20}=-76.00\left(c 0.010 \mathrm{CHCl}_{3}\right)$, 99:1 er. Diethyl ester product: ${ }^{1} \mathrm{H}$ NMR $\left(500 \mathrm{MHz}, \mathrm{CDCl}_{3}\right) \delta 7.79(\mathrm{~s}, 4 \mathrm{H}), 7.77(\mathrm{~d}, J=7.9 \mathrm{~Hz}, 2 \mathrm{H}), 7.70-7.65(\mathrm{~m}, 4 \mathrm{H})$, $7.61(\mathrm{~d}, J=1.2 \mathrm{~Hz}, 2 \mathrm{H}), 7.39-7.32(\mathrm{~m}, 4 \mathrm{H}), 4.09-4.02(\mathrm{~m}, 4 \mathrm{H}), 2.14-1.99(\mathrm{~m}, 12 \mathrm{H}), 1.20(\mathrm{t}, J=7.1 \mathrm{~Hz}, 6 \mathrm{H}), 1.14-$ $1.12(\mathrm{~m}, 4 \mathrm{H}), 1.09-1.01(\mathrm{~m}, 4 \mathrm{H}), 0.74-0.67(\mathrm{~m}, 10 \mathrm{H}) .{ }^{13} \mathrm{C} \mathrm{NMR}\left(126 \mathrm{MHz}, \mathrm{CDCl}_{3}\right) \delta 173.3,152.3,150.6,140.3,140.0$ $139.5,139.4,133.0,127.6,127.5,126.3,123.3,121.4,120.9,120.2,60.2,55.2,40.2,39.6,34.4,25.9,23.0,19.5,14.2$, 13.8. MS (m/z, ESI): Calcd. for Chemical Formula: $\mathrm{C}_{52} \mathrm{H}_{56} \mathrm{Cl}_{2} \mathrm{O}_{4}^{-}[\mathrm{M}-\mathrm{H}]^{-}: 837.3448$, Found: 837.3440. HPLC analysis of the dicarboxylic acid, prepared through hydrolysis of the above diethyl ester product under aqueous basic conditions ( $\mathrm{NaOH}$ in water-THF): Daicel Chiralpak IH, hexane/iso-propanol =98: $2,1.0 \mathrm{~mL} / \mathrm{min}, \lambda=329 \mathrm{~nm}$, retention time: $35.09 \mathrm{~min}$ (major) and $48.22 \mathrm{~min}$ (minor). 
(meso)-4,4'-((9R,9'S)-1,4-phenylenebis(9-butyl-7-chloro-9H-fluorene-2,9-diyl))dibutyric acid (meso-64)
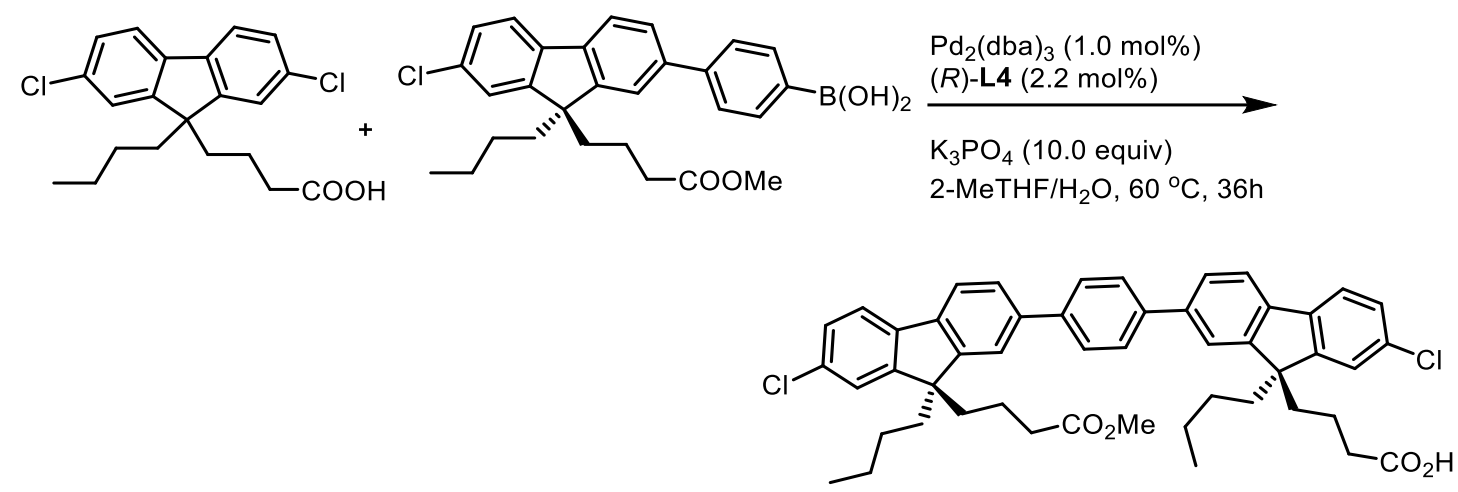

Under $\mathrm{N}_{2}$ atmosphere, to a mixture of $\mathrm{Pd}_{2} \mathrm{dba}_{3}(0.3 \mathrm{mg}, 0.00033 \mathrm{mmol})$ and $(R)-\mathrm{L} 4(0.43 \mathrm{mg}, 0.0073 \mathrm{mmol})$ was added $0.3 \mathrm{~mL}$ 2-MeTHF and the resulting solution was stirred for $20 \mathrm{~min}$ before use. Then the metal-ligand complex solution was added to a $0.3 \mathrm{~mL}$ 2-MeTHF solution of (R)-(4-(9-butyl-7-chloro-9-(4-methoxy-4-oxobutyl)-9H-fluoren2-yl)phenyl)boronic acid (13.7 mg, $0.033 \mathrm{mmol}$ ), 4-(9-butyl-2,7-dichloro-9H-fluoren-9-yl)butanoic acid (37.7 mg, 0.1 $\mathrm{mmol})$ and $\mathrm{K}_{3} \mathrm{PO}_{4}(77.3 \mathrm{mg}, 3.3 \mathrm{mmol})$, followed by $0.055 \mathrm{~mL} \mathrm{H} \mathrm{H}_{2} \mathrm{O}$. Then the resulting reaction mixture was stirred at $60{ }^{\circ} \mathrm{C}$ for $36 \mathrm{~h}$. The reaction was quenched with water, neutralized to $\mathrm{pH}$ 3-5 with $1 \mathrm{M} \mathrm{HCl}$ and extracted with ethyl acetate $(3 \mathrm{~mL} \times 3)$ for three times. The combined organic phases were washed with brine, dried over $\mathrm{Na}_{2} \mathrm{SO}_{4}$ and concentrated in vacuo. The crude materials were purified by flash column chromatography with hexane and ethyl acetate (6:1-1:1) to give the desired products $26.2 \mathrm{mg}$ in $34 \%$ yield as a white solid. Mono methyl ester product: ${ }^{1} \mathrm{H}$ $\operatorname{NMR}\left(500 \mathrm{MHz}, \mathrm{CDCl}_{3}\right) \delta 7.80-7.74(\mathrm{~m}, 6 \mathrm{H}), 7.66(\mathrm{dd}, J=8.5,4.6 \mathrm{~Hz}, 4 \mathrm{H}), 7.61(\mathrm{~s}, 2 \mathrm{H}), 7.36(\mathrm{dq}, J=4.1,2.0 \mathrm{~Hz}, 4 \mathrm{H})$, $3.59(\mathrm{~s}, 3 \mathrm{H}), 2.15-2.00(\mathrm{~m}, 12 \mathrm{H}), 1.15-1.10(\mathrm{~m}, 4 \mathrm{H}), 1.07-1.02(\mathrm{~m}, 4 \mathrm{H}), 0.74-0.66(\mathrm{~m}, 10 \mathrm{H}) .{ }^{13} \mathrm{C} \mathrm{NMR}(126 \mathrm{MHz}$, $\mathrm{CDCl}_{3}$ ) $\delta 177.4,173.7,152.2,152.1,150.6,150.5,140.3,140.0,139.4,139.3,133.1,127.6,127.5,126.3,126.3,123.3$ $121.4,120.9,120.2,55.2,51.4,40.2,39.6,39.4,34.1,33.7,31.9,29.4,25.9,23.0,19.5,19.2,13.8 . \mathrm{MS}$ (m/z, ESI): Calcd. for Chemical Formula: $\mathrm{C}_{49} \mathrm{H}_{50} \mathrm{Cl}_{2} \mathrm{O}_{4} \mathrm{Na}^{+}[\mathrm{M}+\mathrm{Na}]^{+}: 795.2978$, Found: 795.2974. HPLC analysis of the mesodicarboxylic acid, prepared through hydrolysis of the above methyl ester product under aqueous basic conditions ( $\mathrm{NaOH}$ in water-THF): Daicel Chiralpak IH, hexane/iso-propanol =98: 2,1.0 mL/min, $\lambda=329 \mathrm{~nm}$, retention time: $32.9 \min$. 
(R)-4-(9-butyl-2-chloro-7-(4-cyanophenyl)-9H-fluoren-9-yl)butanoic acid (precursor to 65)
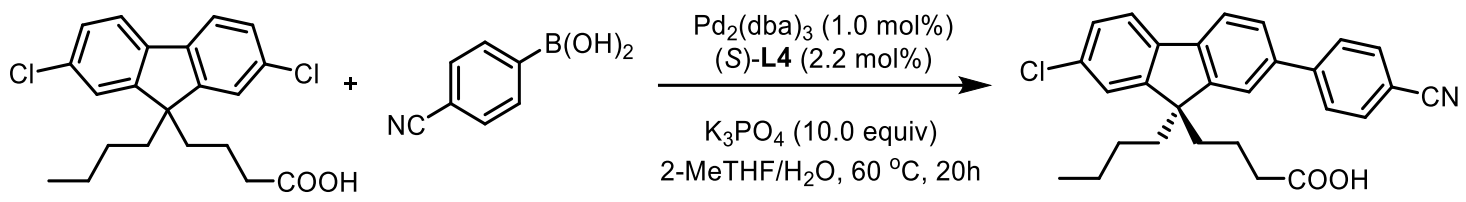

Under $\mathrm{N}_{2}$ atmosphere, to a mixture of $\mathrm{Pd}_{2} \mathrm{dba}_{3}(2.3 \mathrm{mg}, 0.0025 \mathrm{mmol})$ and $(S)-\mathrm{L} 4(3.2 \mathrm{mg}, 0.0055 \mathrm{mmol})$ was added $1.0 \mathrm{~mL}$ 2-MeTHF and the resulting solution was stirred for $20 \mathrm{~min}$ before use. Then metal-ligand complex solution was added to a $3 \mathrm{~mL}$ 2-MeTHF solution of 4-(9-butyl-2,7-dichloro-9H-fluoren-9-yl)butanoic acid 51 (188.8 mmol, $0.5 \mathrm{mmol}$ ), (4-cyanophenyl)boronic acid ( $162 \mathrm{mg}, 1.1 \mathrm{mmol}$ ) and $\mathrm{K}_{3} \mathrm{PO}_{4}(1.01 \mathrm{~g}, 10.0 \mathrm{mmol}$ ), followed by 0.4 $\mathrm{mL} \mathrm{H} \mathrm{H}_{2} \mathrm{O}$. Then the resulting reaction mixture was stirred at $60^{\circ} \mathrm{C}$ for $20 \mathrm{~h}$. The reaction was quenched with water, neutralized to $\mathrm{pH} \mathrm{3-5} \mathrm{with} 1 \mathrm{M} \mathrm{HCl}$ and extracted with ethyl acetate for three times. The combined organic phases were washed with brine, dried over $\mathrm{Na}_{2} \mathrm{SO}_{4}$ and concentrated in vacuo. The crude materials were purified by flash column chromatography with hexane and ethyl acetate (6:1-1:1) to give the desired products $0.132 \mathrm{~g}$ in $60 \%$ yield as a white solid. $[\alpha]_{D}{ }^{20}=+3.60\left(c 1.0 \mathrm{CHCl}_{3}\right)$ for 95.5:4.5 er. ${ }^{1} \mathrm{H} \mathrm{NMR}\left(400 \mathrm{MHz}, \mathrm{CDCl}_{3}\right) \delta 7.77-7.69(\mathrm{~m}, 5 \mathrm{H}), 7.67-7.63$ (m, 1H), $7.57(\mathrm{dd}, \mathrm{J}=7.9,1.7 \mathrm{~Hz}, 1 \mathrm{H}), 7.53-7.49(\mathrm{~m}, 1 \mathrm{H}), 7.34(\mathrm{~d}, \mathrm{~J}=7.5 \mathrm{~Hz}, 2 \mathrm{H}), 2.15-2.02(\mathrm{~m}, 4 \mathrm{H}), 1.96-2.01(\mathrm{~m}$, 2H), $1.12-1.04(\mathrm{~m}, 2 \mathrm{H}), 0.94-0.98(\mathrm{~m}, 2 \mathrm{H}), 0.68(\mathrm{t}, \mathrm{J}=7.3 \mathrm{~Hz}, 3 \mathrm{H}), 0.61(\mathrm{t}, \mathrm{J}=7.3 \mathrm{~Hz}, 2 \mathrm{H}) .{ }^{13} \mathrm{C} \mathrm{NMR}\left(101 \mathrm{MHz}, \mathrm{CDCl}_{3}\right)$ $\delta 178.2,152.1,150.7,145.8,140.7,138.9,138.4,133.6,132.6,127.8,127.7,126.7,123.3,121.6,121.2,120.5,119.0$ 110.8, 55.3, 40.2, 39.3, 33.6, 25.8, 22.9, 19.0, 13.8. HRMS (m/z, ESI): Calcd. for Chemical Formula: $\mathrm{C}_{28} \mathrm{H}_{25} \mathrm{CINO}_{2}{ }^{-}$[M$\mathrm{H}]=$ : 442.1579, Found: 442.1572. HPLC analysis of the reaction product: Daicel Chiralpak IA, hexane/iso-propanol = 97: $3,1.0 \mathrm{~mL} / \mathrm{min}, \lambda=322 \mathrm{~nm}$, retention time: $13.85 \mathrm{~min}$ (major) and $15.68 \mathrm{~min}$ (minor).

(R)-4-(9-butyl-7-chloro-9-(4-hydroxybutyl)-9H-fluoren-2-yl)benzonitrile (precursor to 65)

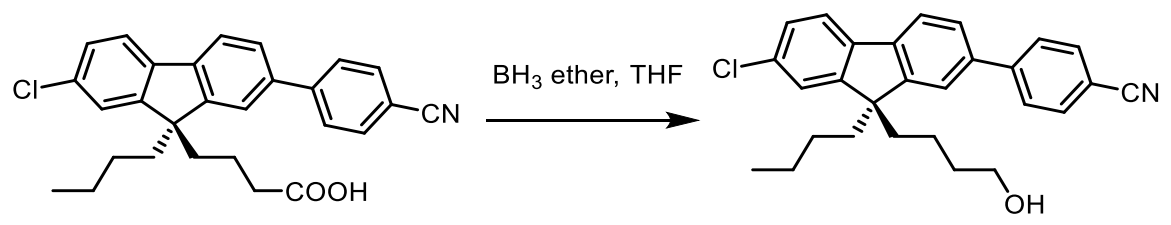

Under $\mathrm{N}_{2}$ atmosphere, to a solution of (R)-4-(9-butyl-7-chloro-9-(4-hydroxybutyl)-9H-fluoren-2yl)benzonitrile (60 mg, $15 \mathrm{mmol}$ ) in anhydrous $1.0 \mathrm{~mL}$ THF was added $\mathrm{BH}_{3}$-ether solution $(67 \mu \mathrm{L}, 0.134 \mathrm{mmol}, 2 \mathrm{~mol} / \mathrm{L})$ dropwise at $-78^{\circ} \mathrm{C}$. After addition was completed, the reaction mixture was stirred at this temperature for $20 \mathrm{~min}$. 
Then the reaction was transferred to $-15^{\circ} \mathrm{C}$ and stirred for $12 \mathrm{~h}^{[19]}$. The reaction was quenched with water and extracted with ethyl acetate for three times. The combined organic phases were washed with brine, dried over $\mathrm{Na}_{2} \mathrm{SO}_{4}$ and concentrated in vacuo. The crude materials were purified by flash column chromatography with hexane and ethyl acetate (6:1-1:1) to give the desired products $42 \mathrm{mg}$ in $73 \%$ yield as a white solid. ${ }^{1} \mathrm{H} \mathrm{NMR}(400 \mathrm{MHz}, \mathrm{CDCl} 3)$ $\delta 7.77-7.72(\mathrm{~m}, 5 \mathrm{H}), 7.66-7.63(\mathrm{~m}, 1 \mathrm{H}), 7.58(\mathrm{dd}, \mathrm{J}=7.9,1.7 \mathrm{~Hz}, 1 \mathrm{H}), 7.52(\mathrm{dd}, \mathrm{J}=1.7,0.6 \mathrm{~Hz}, 1 \mathrm{H}), 7.36-7.32(\mathrm{~m}$, $2 \mathrm{H}), 3.45-3.39(\mathrm{~m}, 2 \mathrm{H}), 2.09-1.97(\mathrm{~m}, 4 \mathrm{H}), 1.38-1.34(\mathrm{~m}, 2 \mathrm{H}), 1.10(q, \mathrm{~J}=7.4 \mathrm{~Hz}, 2 \mathrm{H}), 0.76-0.66(\mathrm{~m}, 5 \mathrm{H}), 0.65-$ 0.57 (m, 2H). ${ }^{13} \mathrm{C}$ NMR $\left(126 \mathrm{MHz}, \mathrm{CDCl}_{3}\right) \delta 152.8,151.4,146.0,141.0,139.1,138.5,133.8,132.8,128.0,127.7,126.7$, $123.5,121.6,121.3,120.6,119.2,111.0,62.8,55.7,40.4,40.3,33.2,26.1,23.2,20.4,14.0$.

\section{(R)-4-(9-butyl-7-chloro-9-(4-(2-(2-methoxyethoxy)ethoxy)butyl)-9H-fluoren-2-yl)benzonitrile (65)}

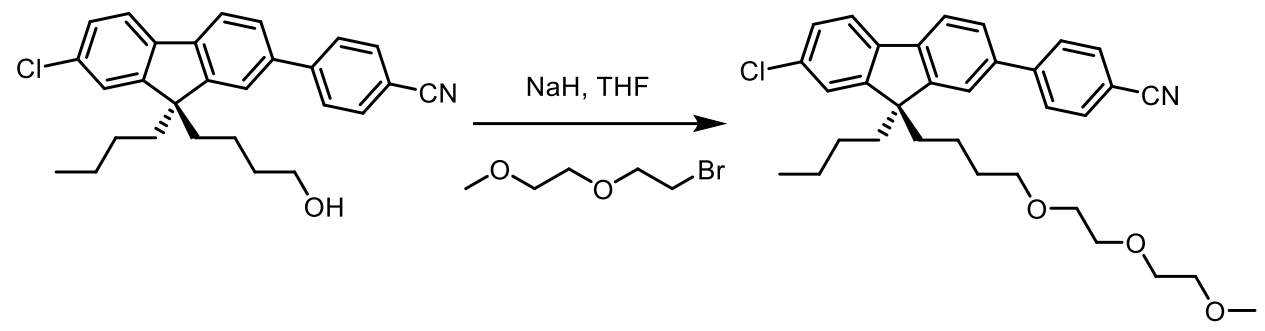

Under $\mathrm{N}_{2}$ atmosphere, to a solution of (R)-4-(9-butyl-7-chloro-9-(4-(2-(2-methoxyethoxy)ethoxy)butyl)-9Hfluoren-2-yl)benzonitrile (15 mg, $0.035 \mathrm{mmol}$ ) in anhydrous $1.0 \mathrm{~mL}$ THF was added $\mathrm{NaH}\left(2 \mathrm{mg}, 0.052 \mathrm{mmol}\right.$ ) at $0{ }^{\circ} \mathrm{C}$. After addition was completed, the reaction mixture was stirred at room temperature for 1 hour. Then 1-bromo-2-(2methoxyethoxy)ethane $(9.6 \mathrm{mg}, 0.052 \mathrm{mmol})$ was added dropwise at room temperature and the reaction mixture was heated to $45^{\circ} \mathrm{C}$ and stirred for $24 \mathrm{~h}$. The reaction was quenched with water and extracted with ethyl acetate for three times. The combined organic phases were washed with brine, dried over $\mathrm{Na}_{2} \mathrm{SO}_{4}$ and concentrated in vacuo. The crude materials were purified by flash column chromatography with hexane and ethyl acetate (6:1-1:1) to give the desired products $10 \mathrm{mg}$ in $54 \%$ yield as a white solid. $[\alpha]_{\mathrm{D}}{ }^{20}=+32\left(c 0.6 \mathrm{CHCl}_{3}\right)$ for $95: 5 \mathrm{er} .{ }^{1} \mathrm{H} \mathrm{NMR}(400 \mathrm{MHz}$, $\left.\mathrm{CDCl}_{3}\right) \delta 7.77-7.72(\mathrm{~m}, 5 \mathrm{H}), 7.64(\mathrm{~d}, \mathrm{~J}=8.7 \mathrm{~Hz}, 1 \mathrm{H}), 7.58(\mathrm{dd}, \mathrm{J}=7.9,1.7 \mathrm{~Hz}, 1 \mathrm{H}), 7.52(\mathrm{dd}, \mathrm{J}=1.7,0.7 \mathrm{~Hz}, 1 \mathrm{H}), 7.35$ $-7.31(\mathrm{~m}, 2 \mathrm{H}), 3.56-3.50(\mathrm{~m}, 4 \mathrm{H}), 3.50-3.46(\mathrm{~m}, 2 \mathrm{H}), 3.45-3.42(\mathrm{~m}, 2 \mathrm{H}), 3.34(\mathrm{~s}, 3 \mathrm{H}), 3.22(\mathrm{t}, \mathrm{J}=6.7 \mathrm{~Hz}, 2 \mathrm{H}), 1.97$ - $2.04(\mathrm{~m}, 4 \mathrm{H}), 1.37(\mathrm{dt}, \mathrm{J}=15.2,6.9 \mathrm{~Hz}, 2 \mathrm{H}), 1.09(\mathrm{q}, \mathrm{J}=7.4 \mathrm{~Hz}, 2 \mathrm{H}), 0.68(\mathrm{t}, \mathrm{J}=7.3 \mathrm{~Hz}, 5 \mathrm{H}), 0.61(\mathrm{t}, \mathrm{J}=7.4 \mathrm{~Hz}, 2 \mathrm{H}) .{ }^{13} \mathrm{C}$ NMR $\left(126 \mathrm{MHz}, \mathrm{CDCl}_{3}\right) \delta 152.9,151.5,146.1,141.0,139.1,138.5,133.7,132.8,128.0,127.6,126.7,123.6,121.7$ 
121.3, 120.6, 119.2, 111.0, 72.1, 71.2, 70.7, 70.7, 70.2, 59.2, 55.7, 40.4, 40.3, 30.0, 26.1, 23.2, 20.7, 14.0. HRMS (m/z, ESI): Calcd. for Chemical Formula: $\mathrm{C}_{33} \mathrm{H}_{39} \mathrm{ClNO}_{3}{ }^{+}[\mathrm{M}+\mathrm{H}]^{+}: 536.2613$, Found: 523.2611 . HPLC analysis of the reaction product: Daicel Chiralpak IA, hexane/iso-propanol = 99: 1, $0.6 \mathrm{~mL} / \mathrm{min}, \lambda=322 \mathrm{~nm}$, retention time: 35.54 min (major) and $33.84 \mathrm{~min}$ (minor). 


\section{References}

[1] Park, Y.; Jeon, I.; Shin, S.; Min, J.; Lee, P. H. J. Org. Chem. 2013, 78, 10209.

[2] Fleckenstein, C. A.; Plenio, H. Chem. Eur. J. 2007, 13, 2701.

[3] Chen, J.-J.; Onogi, S.; Hsieh, Y.-C.; Hsiao, C.-C.; Higashibayashi, S.; Sakurai, H.; Wu, Y.-T. Adv. Synth. Catal. 2012, 354, 1551.

[4] Voortman, T. P.; Chiechi, R. C. ACS Appl. Mater. Interfaces 2015, 7, 28006.

[5] Gaylord, B. S.; Bartholomew, G. P.; Baldocchi, R. A.; Hong, J. W.; Huisman, W. H.; Liang, Y.; Nguyen, T.; Tran, L. P.; Wheeler, J. M.; Plamer, A. C. V.; Uckert, F. P., WO 2011091086 A1, 28 July 2011.

[6] Bihovsky, R.; Levinson, B. L.; Loewi, R. C.; Erhardt, P. W.; Polokoff, M. A. J. Med. Chem. 1995, $38,2119$.

[7] Sivets, G. G. Carbohydrate Research 2020, 488, 107901.

[8] a) Ostermann, N.; Ruedisser, S.; Ehrhardt, C.; Breitenstein, W.; Marzinzik, A.; Jacoby, E.; Vangrevelinghe, E.; Ottl, J.; Klumpp, M.; Hartwieg, J. C.; Cumin, F.; Hassiepen, U.; Trappe, J.; Sedrani, R.; Geisse, S.; Gerhartz, B.; Richert, P.; Francotte, E.; Wagner, T.; Kromer, M.; Kosaka, T.; Webb, R. L.; Rigel, D. F.; Maibaum, J.; Baeschlin, D. K. J. Med. Chem. 2013, 56, 2196; b) Cochran, B. M. Synlett. 2016, 27, 245.

[9] Jepsen, T. H.; Larsen, M.; Jørgensen, M.; Solanko, K. A.; Bond, A. D.; Kadziola, A.; Nielsen, M. B. Eur. J. Org. Chem. 2011, 53.

[10] Yang, F.; Zhou, B. H.; Chen, P.; Zou, D.; Luo, Q. N.; Ren, W. Z.; Li, L. L.; Fan, L. M.; Li, J. Molecules, $2018,23,1922$.

[11] Gormisky, P. E.; White, M. C. J. Am. Chem. Soc. 2011, 133, 12584.

[12] Trujillo, John I.; Gopalan, Aravamudan S. Tetrahedron Lett. 1993, 34, 7355.

[13] Ishiyama, T.; Ishida, K.; Miyaura, N. Tetrahedron 2001, 57, 9813.

[14] Maiti, D.; Fors, B. P.; Henderson, J. L.; Nakamura, Y.; Buchwald, S. L. Chem. Sci. 2011, 2, 57.

[15] Dalcanale, E.; Montanari, F. J. Org. Chem. 1986, 51, 567.

[16] Schepens, W.; Haver, D. V.; Vandewalle, M.; Bouillon, R.; Verstuyf, A.; De Clercq, P. J. Org. Lett. $2006,8,4247$.

[17] Litvic, M.; Filipan, M.; Pogorelic, I.; Cepanec, I. Green Chem. 2005, 7, 771.

[18] Noguchi, H.; Shioda, T.; Chou, C.-M.; Suginome, M. Org. Lett. 2008, 10, 377.

[19] Yoon, N. M.; Pak, C. S.; Brown, H. C.; Krishnamurthy, S.; Stocky, T. P. J. Org. Chem 1973, 38, 2786. 


\section{NMR spectra}

${ }^{1} \mathrm{H}$ NMR $\left(500 \mathrm{MHz}, \mathrm{CDCl}_{3}\right)$

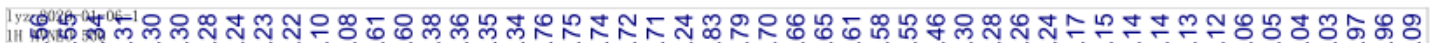

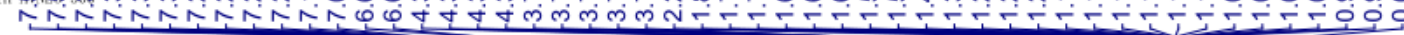

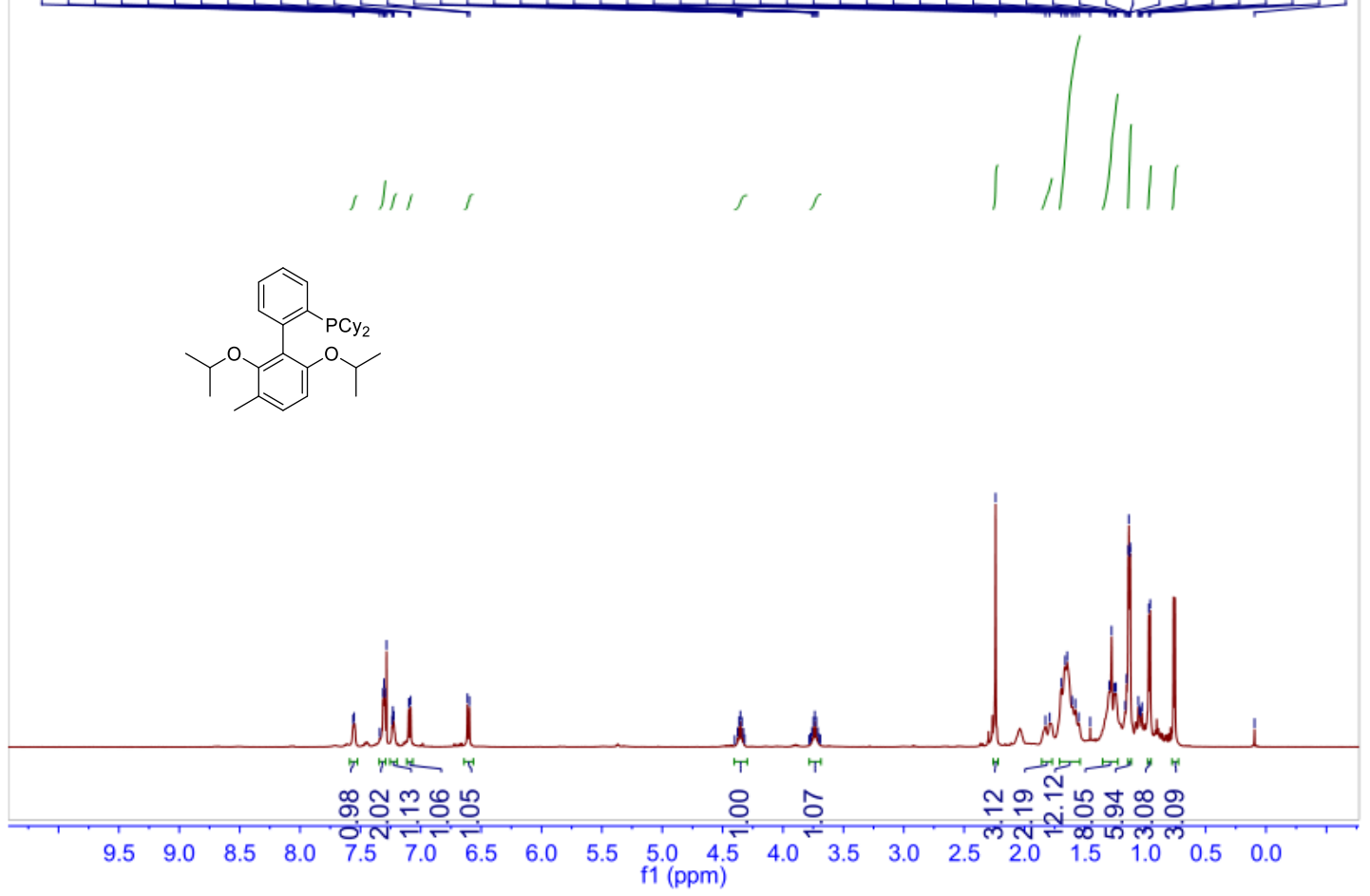

${ }^{13} \mathrm{C}$ NMR (126 MHz, $\mathrm{CDCl}_{3}$ )

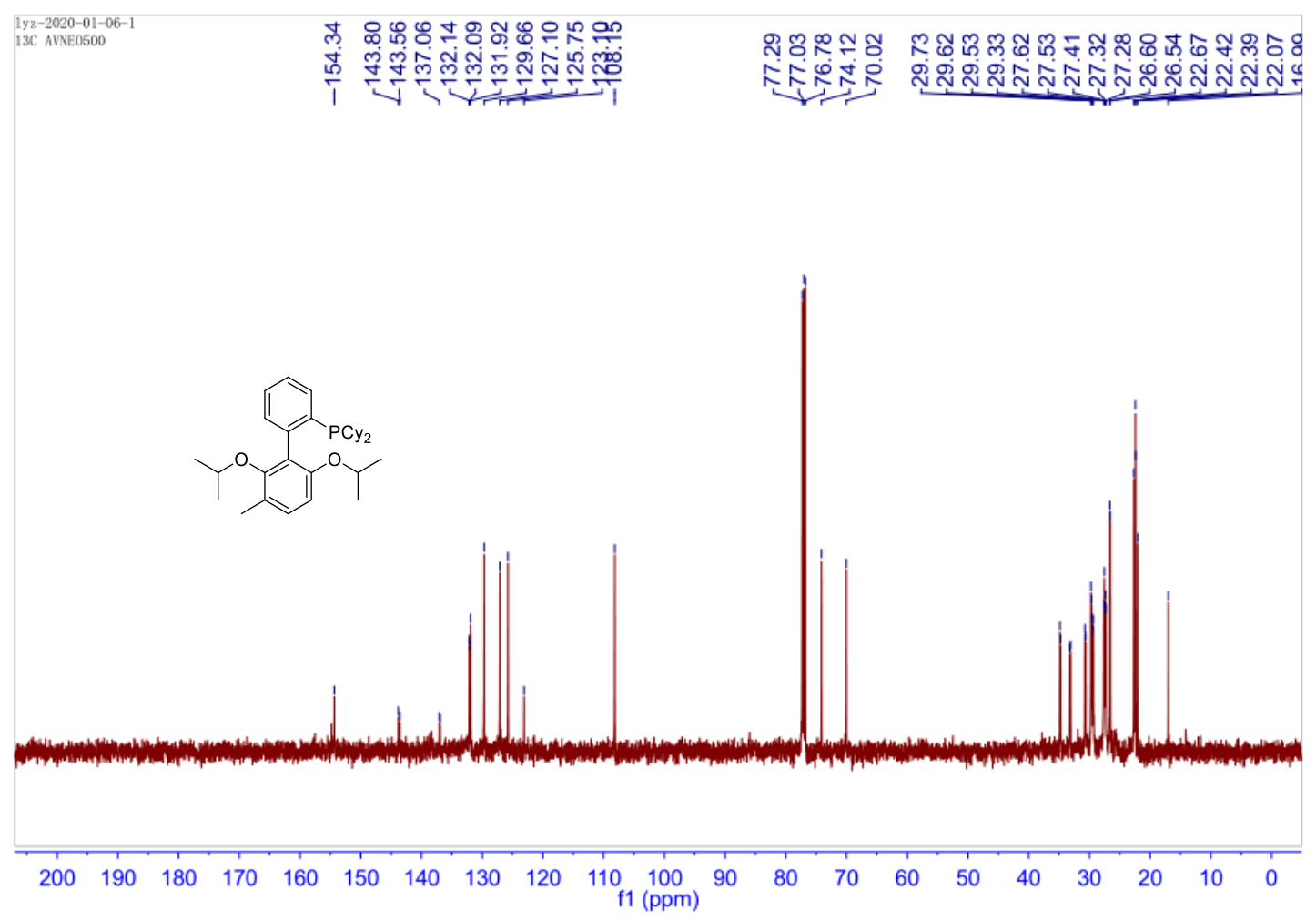




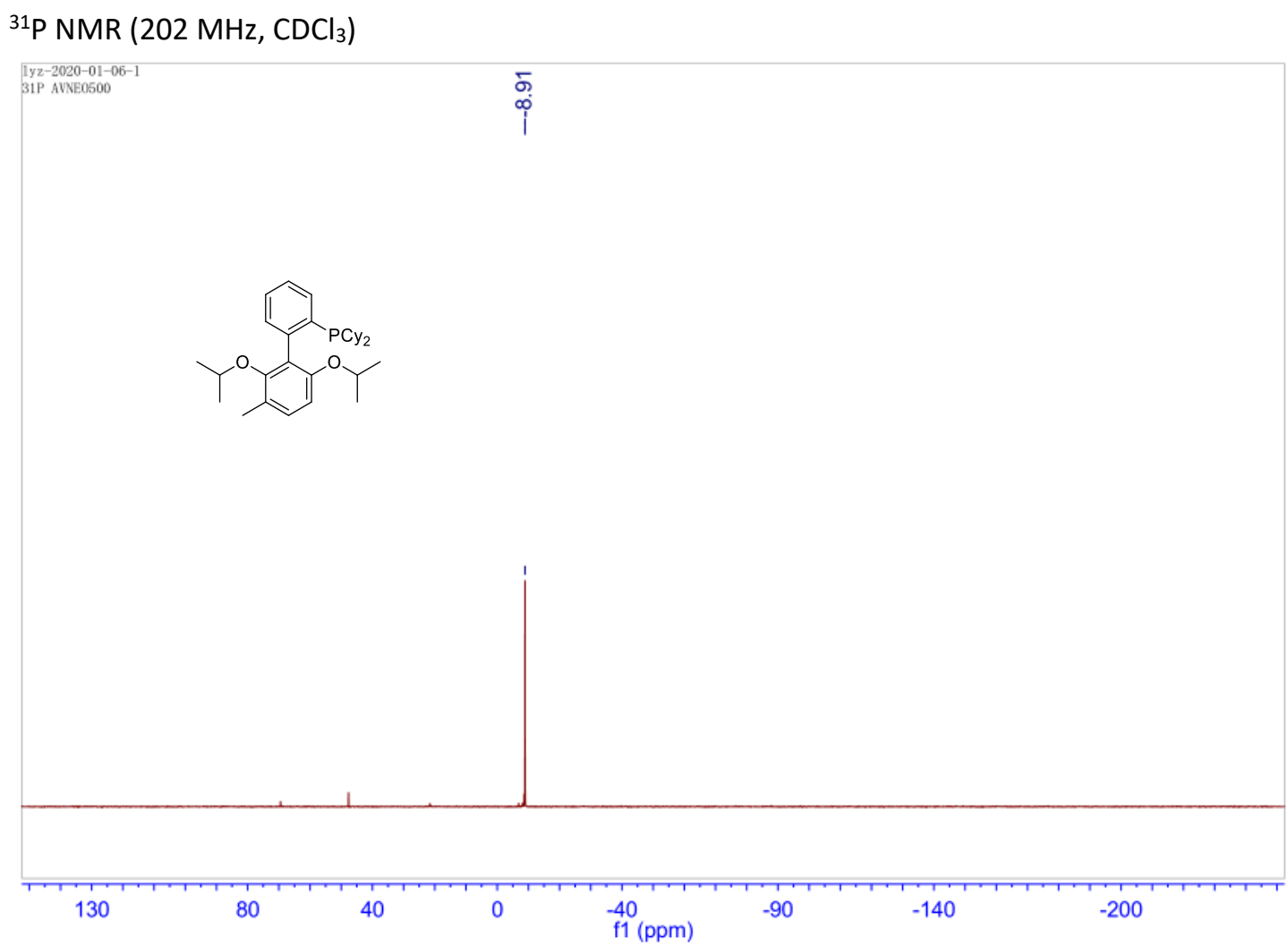


${ }^{1} \mathrm{H}$ NMR (500 MHz, $\left.\mathrm{CDCl}_{3}\right)$

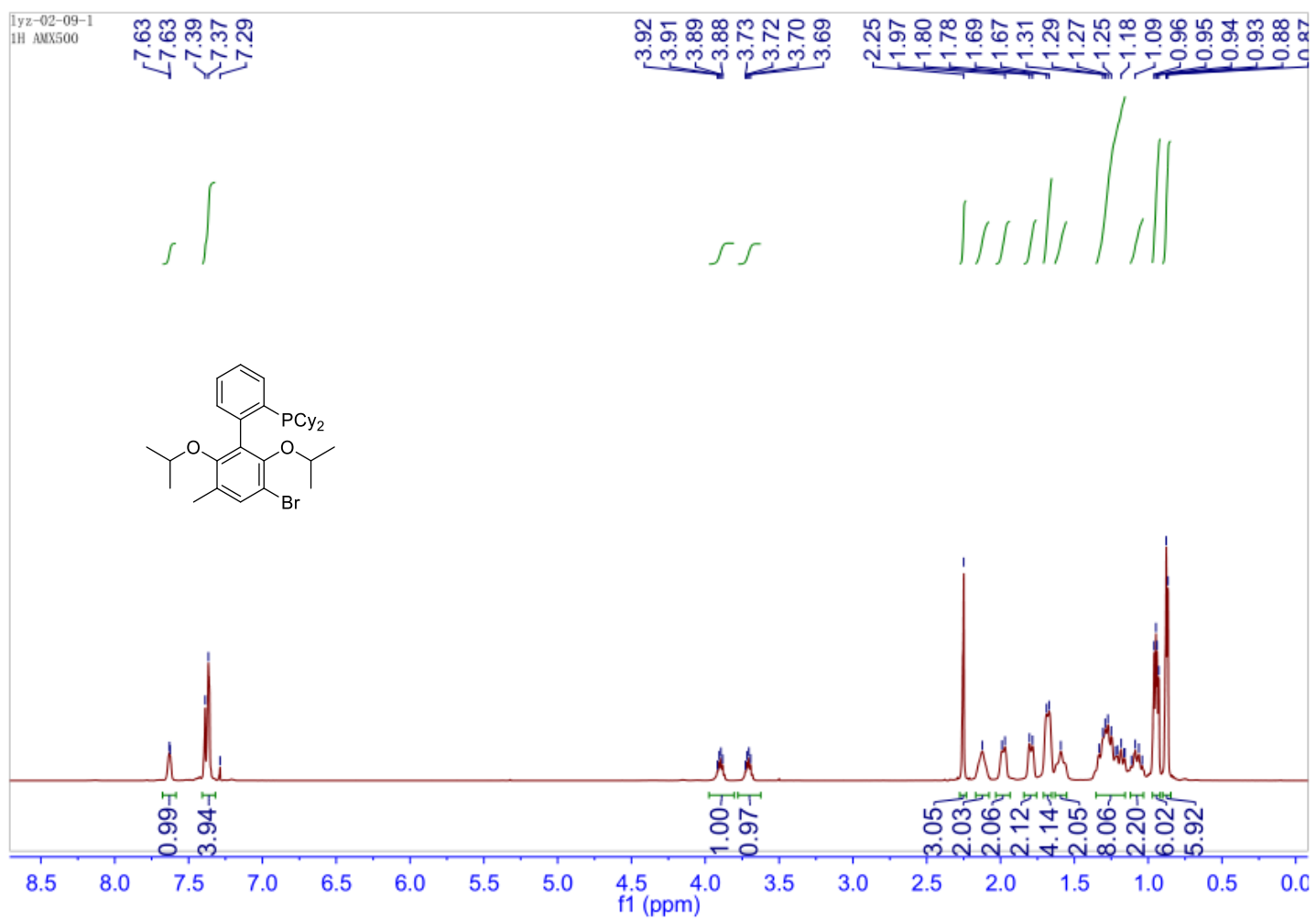

${ }^{13} \mathrm{C} \mathrm{NMR}\left(126 \mathrm{MHz}, \mathrm{CDCl}_{3}\right)$

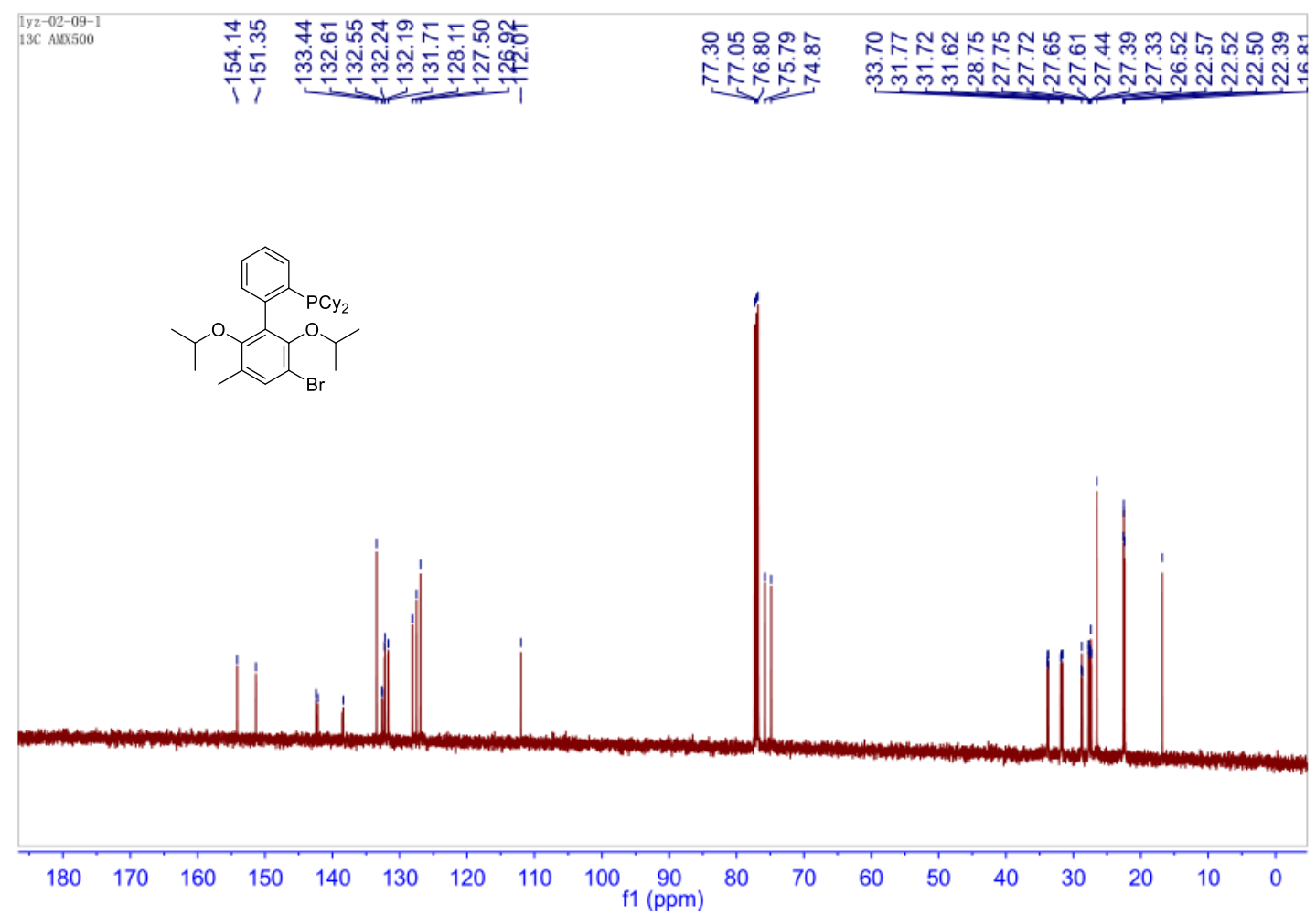




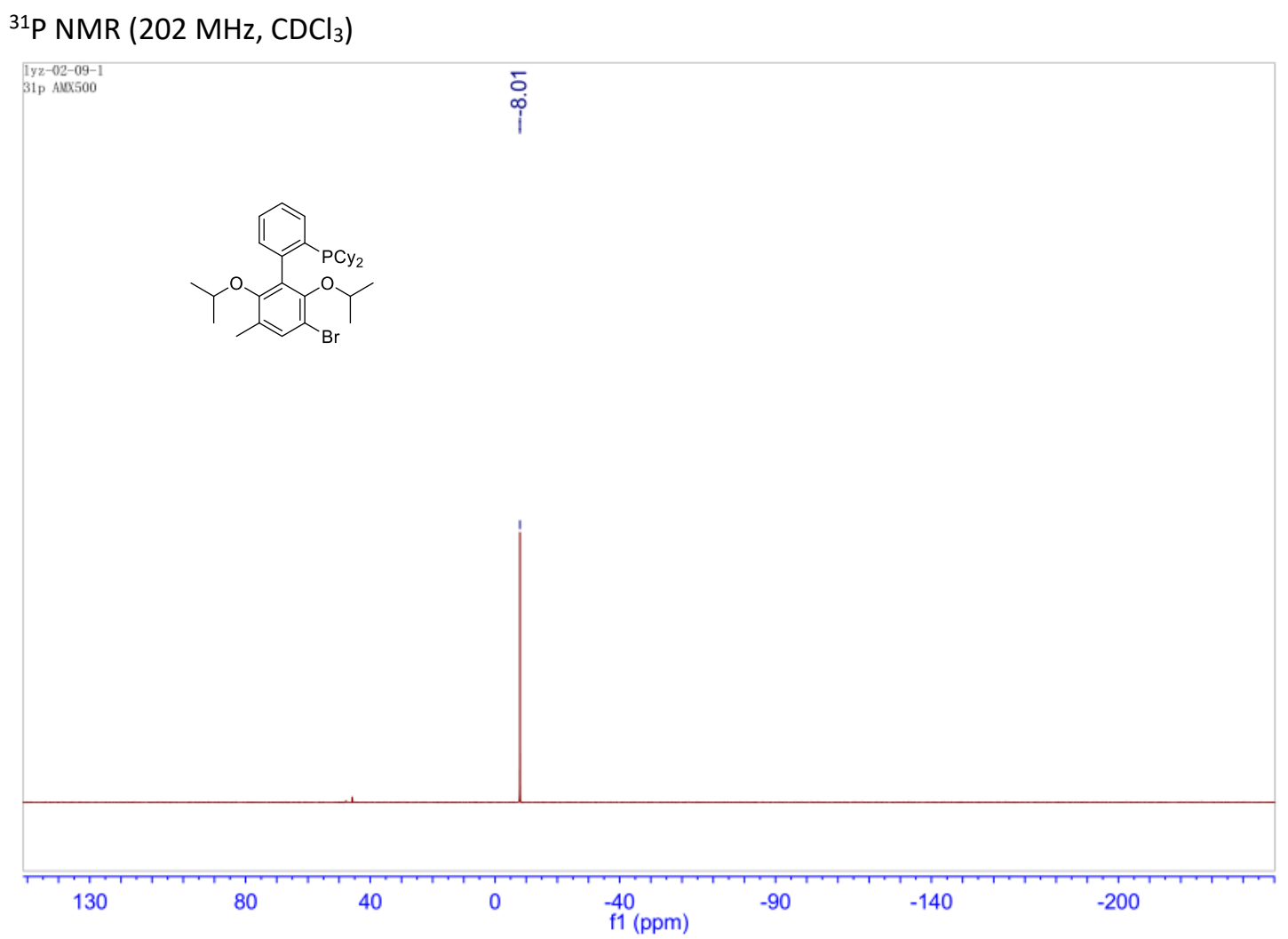


$\left(R, S_{S}\right)$-L2: ${ }^{1} \mathrm{H}$ NMR $\left(500 \mathrm{MHz}, \mathrm{CDCl}_{3}\right)$

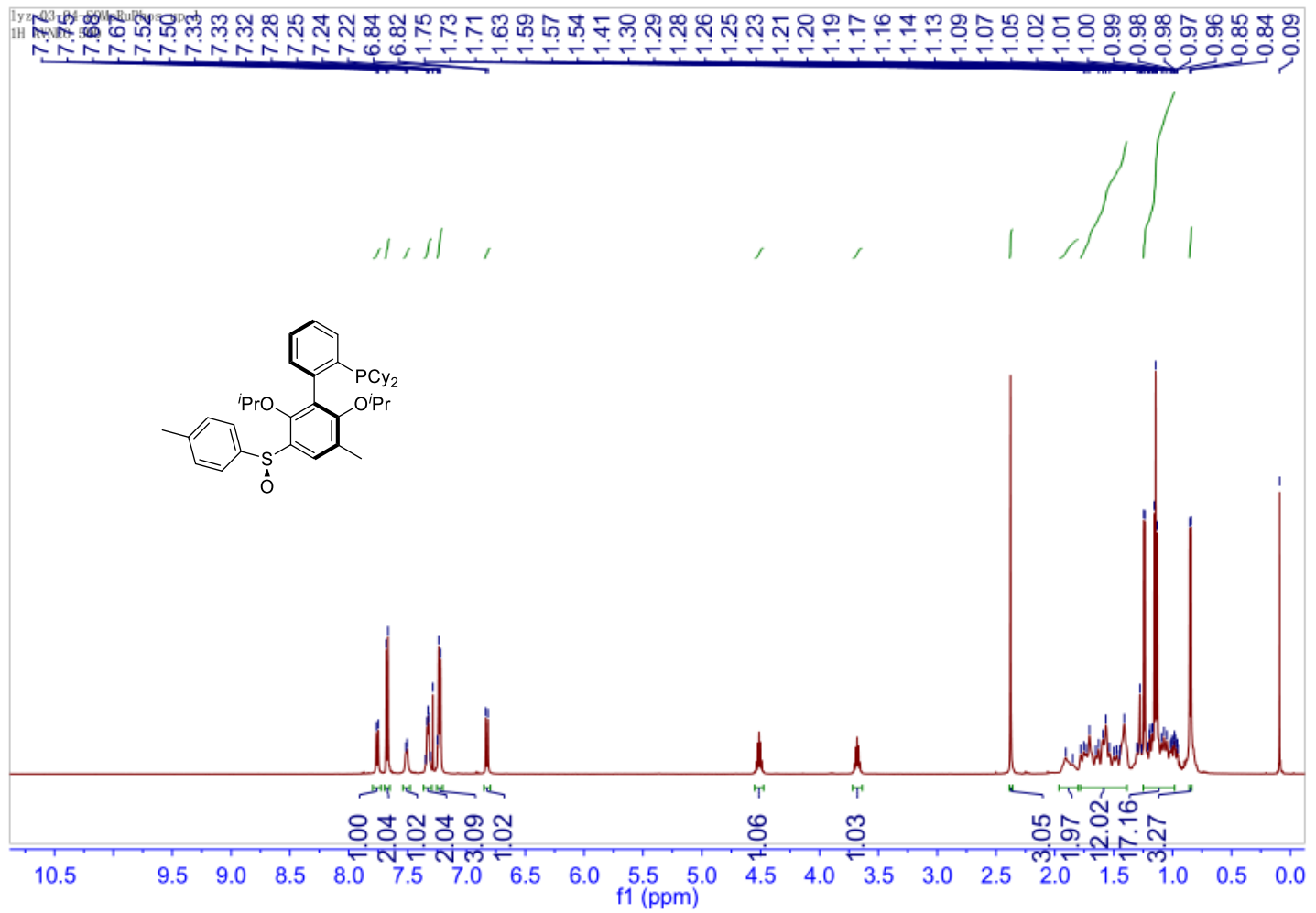

${ }^{13} \mathrm{C}$ NMR (126 MHz, $\mathrm{CDCl}_{3}$ )

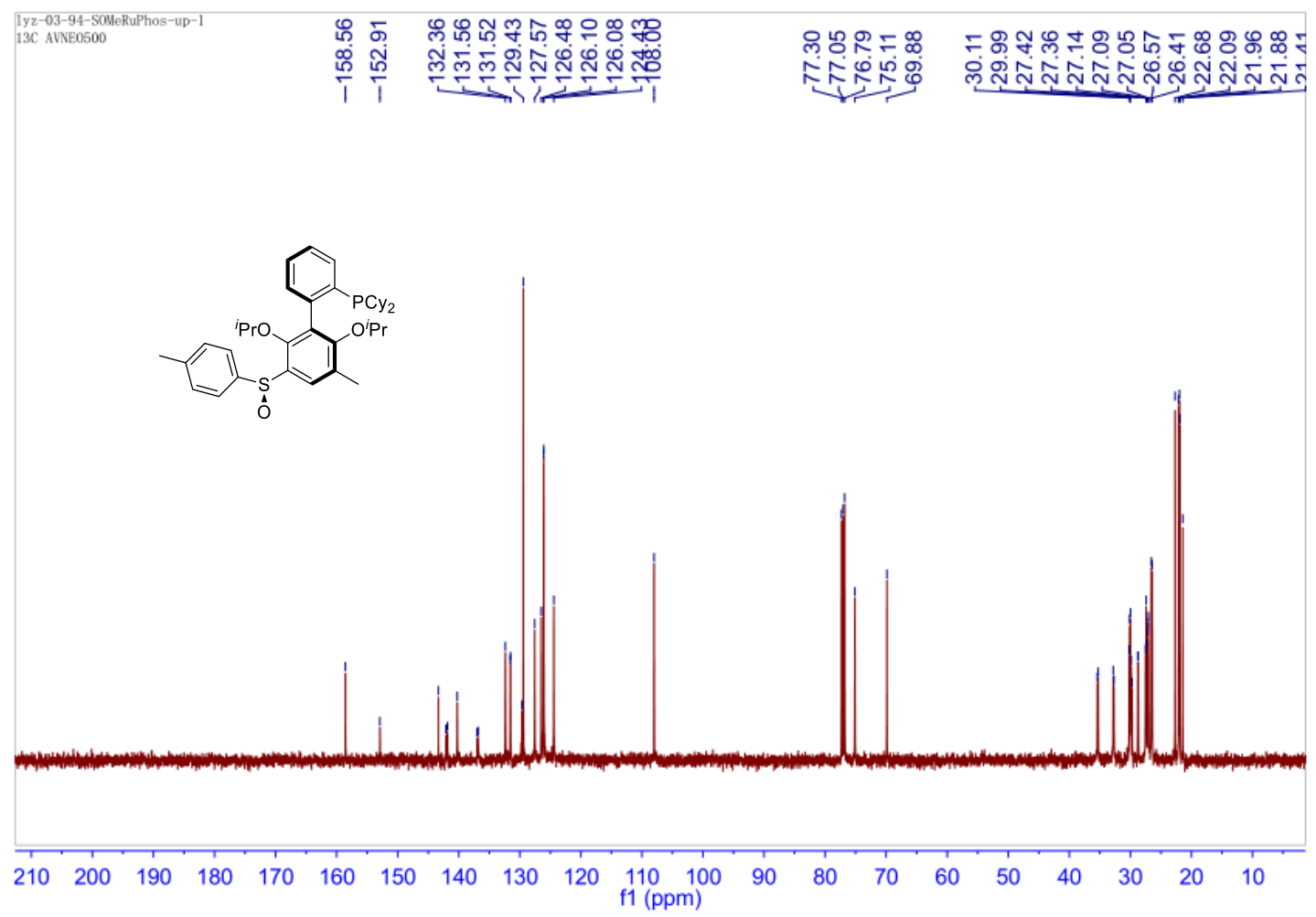


${ }^{31} \mathrm{P}$ NMR (202 MHz, $\left.\mathrm{CDCl}_{3}\right)$

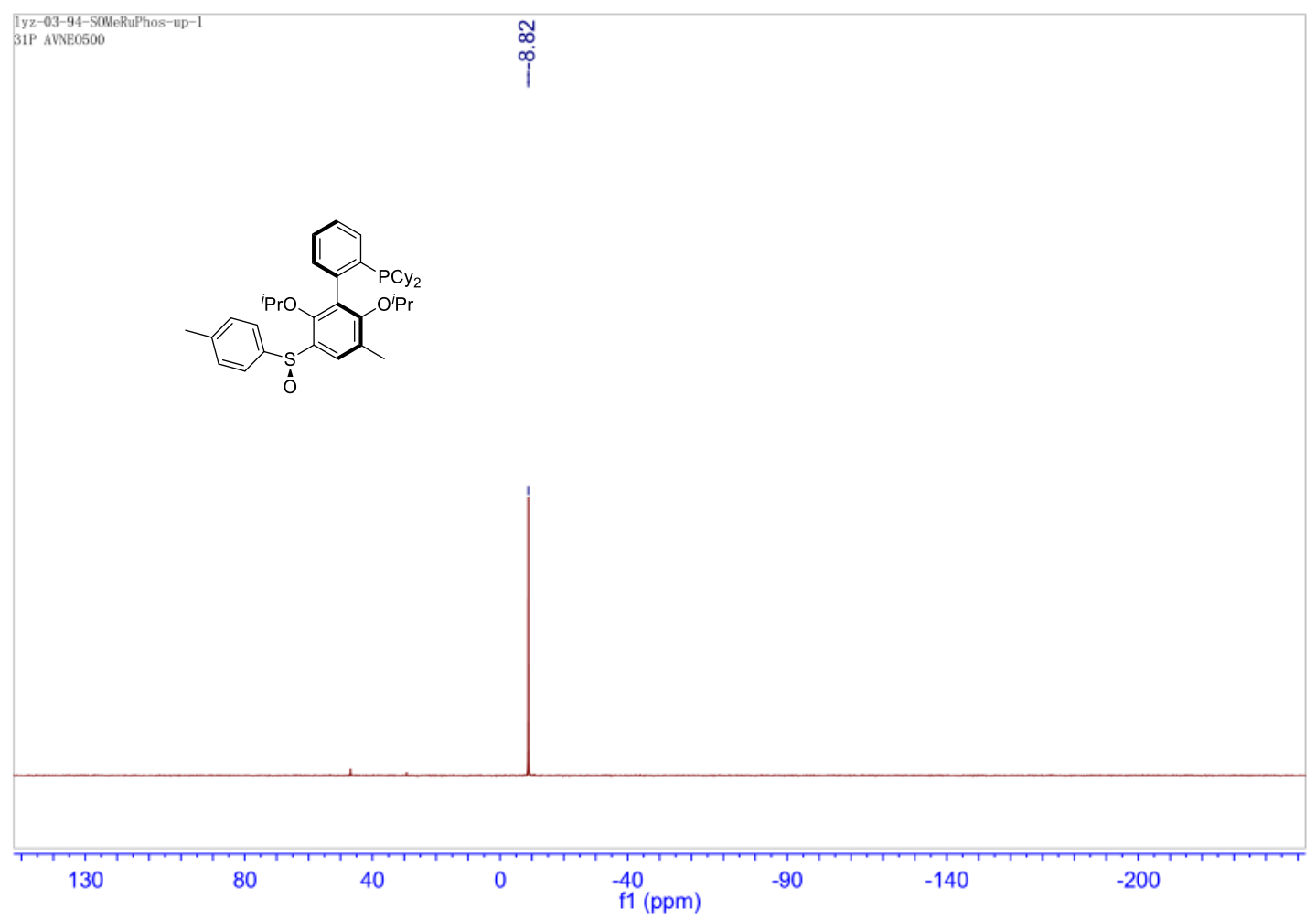


$\left(S, S_{S}\right)$-L2: ${ }^{1} \mathrm{H}$ NMR $\left(500 \mathrm{MHz}, \mathrm{CDCl}_{3}\right)$

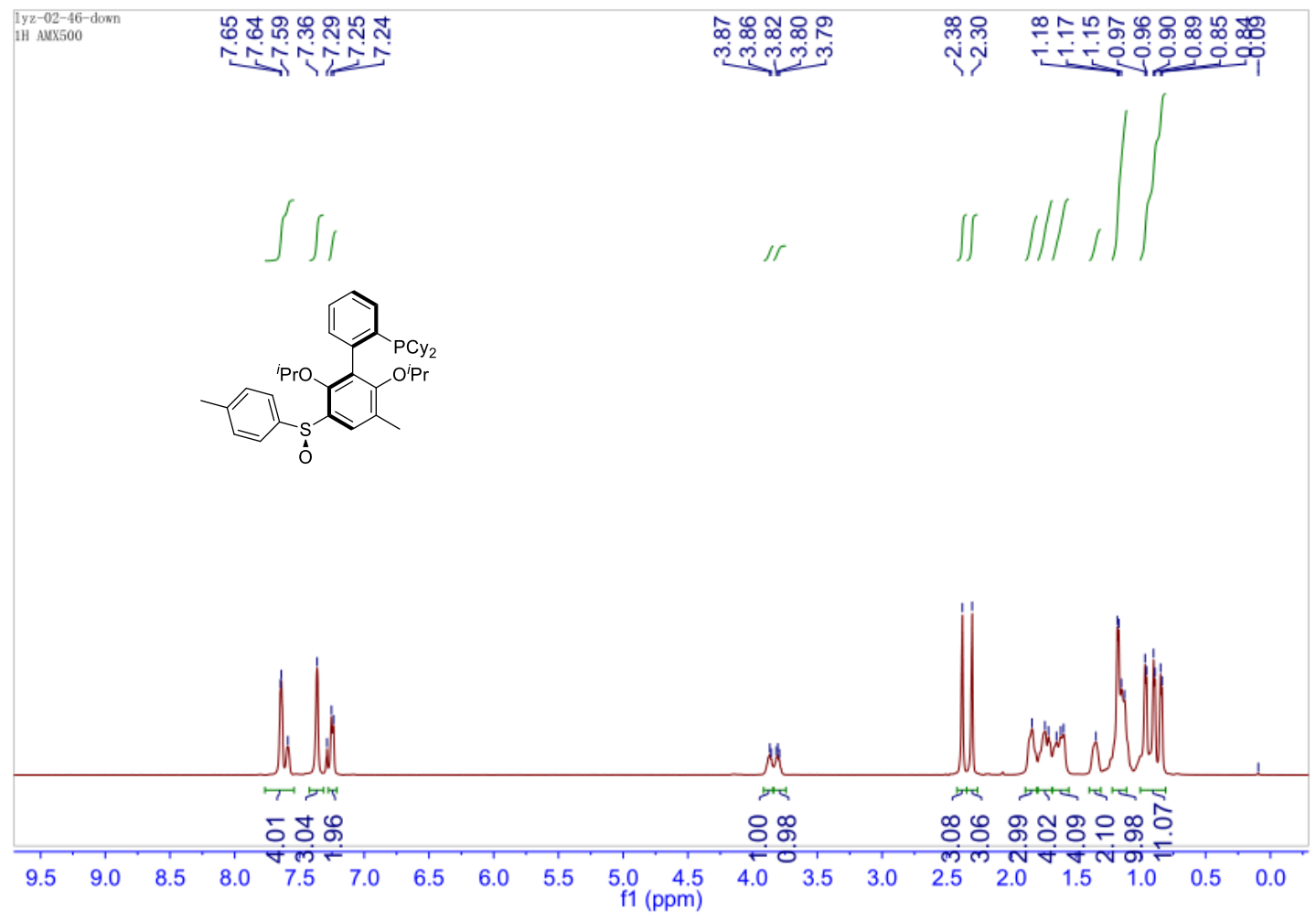

${ }^{13} \mathrm{C}$ NMR $\left(126 \mathrm{MHz}, \mathrm{CDCl}_{3}\right)$

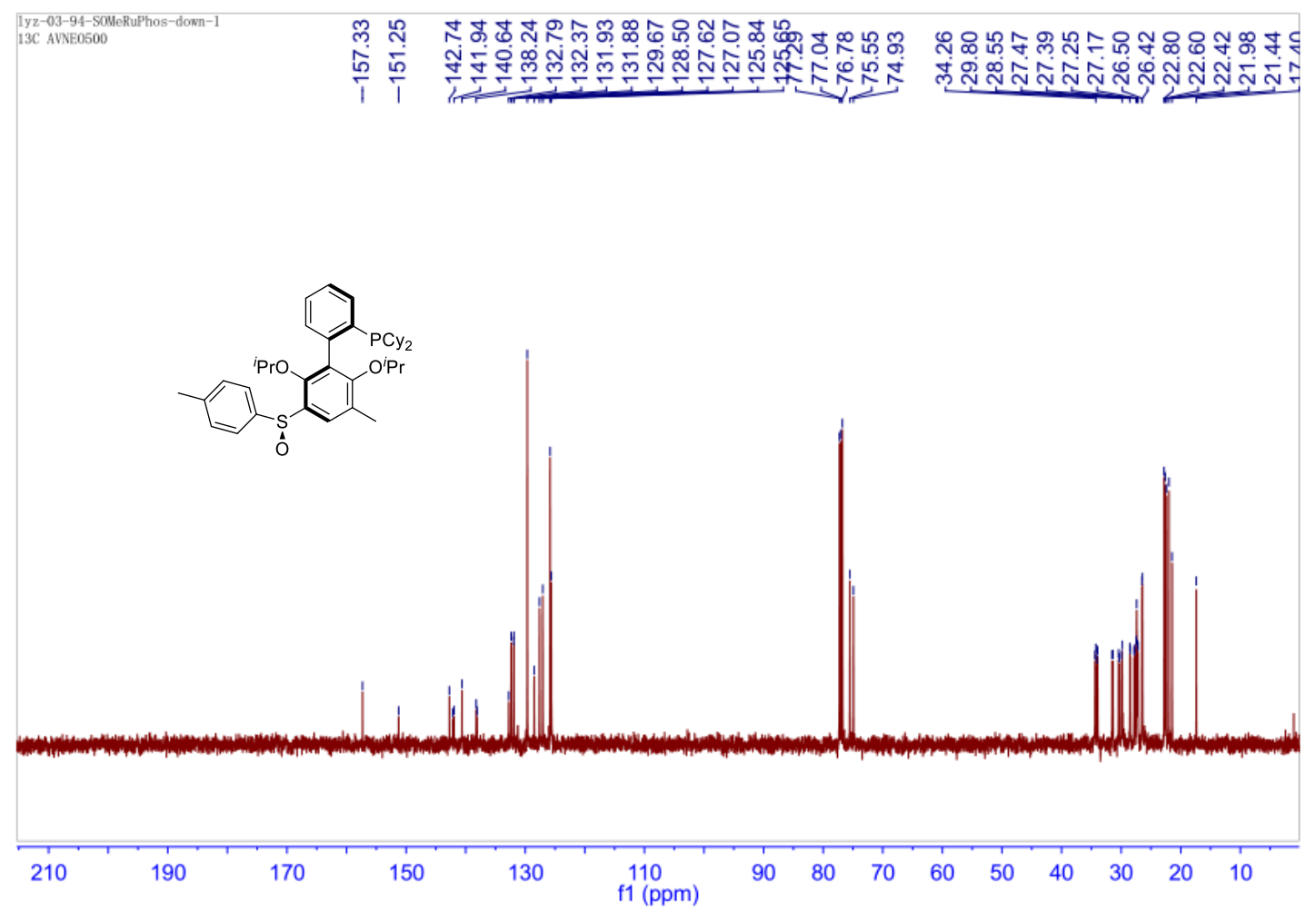




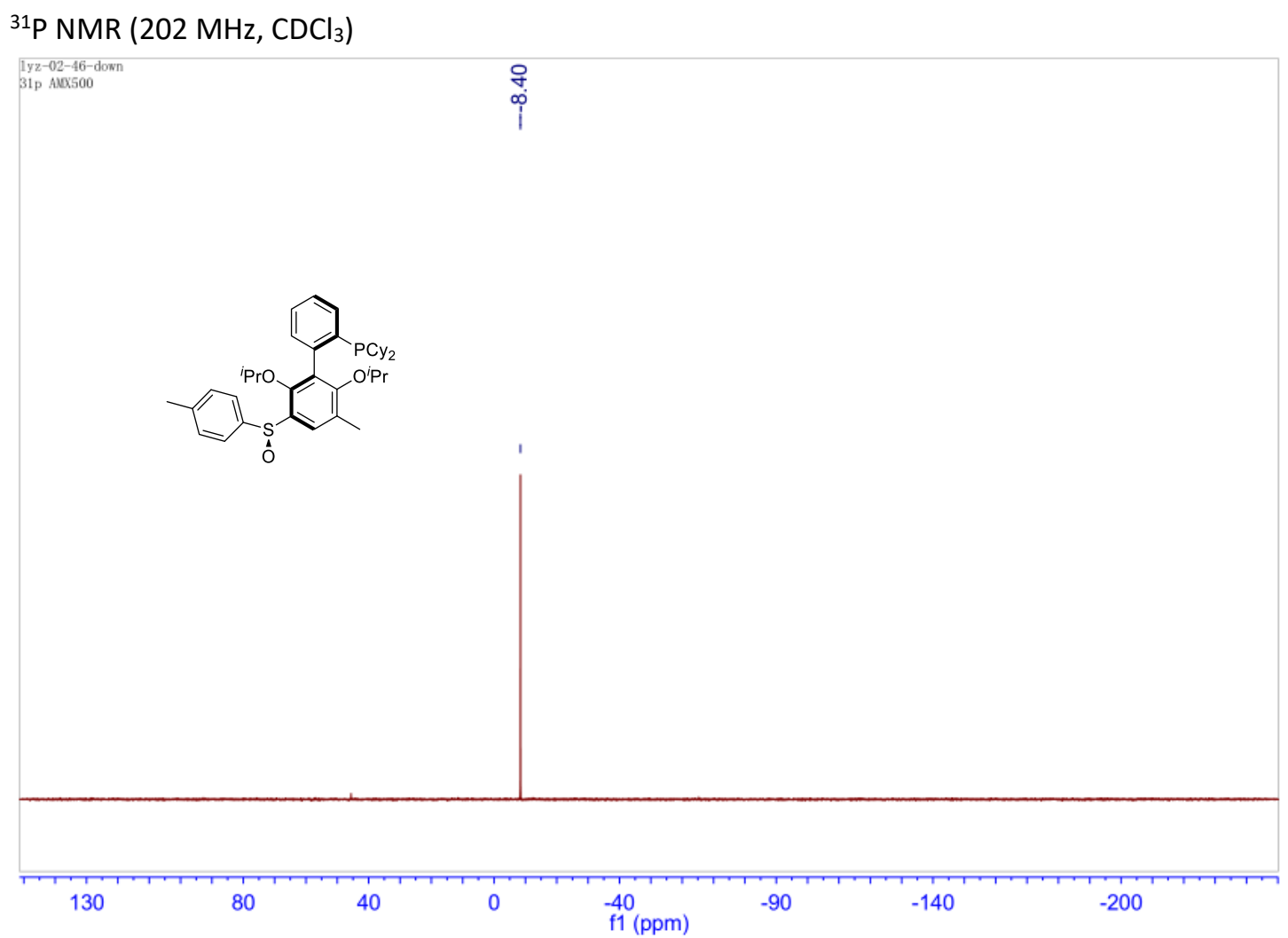


${ }^{1} \mathrm{H}$ NMR $\left(500 \mathrm{MHz} \mathrm{CDCl}_{3}\right)$

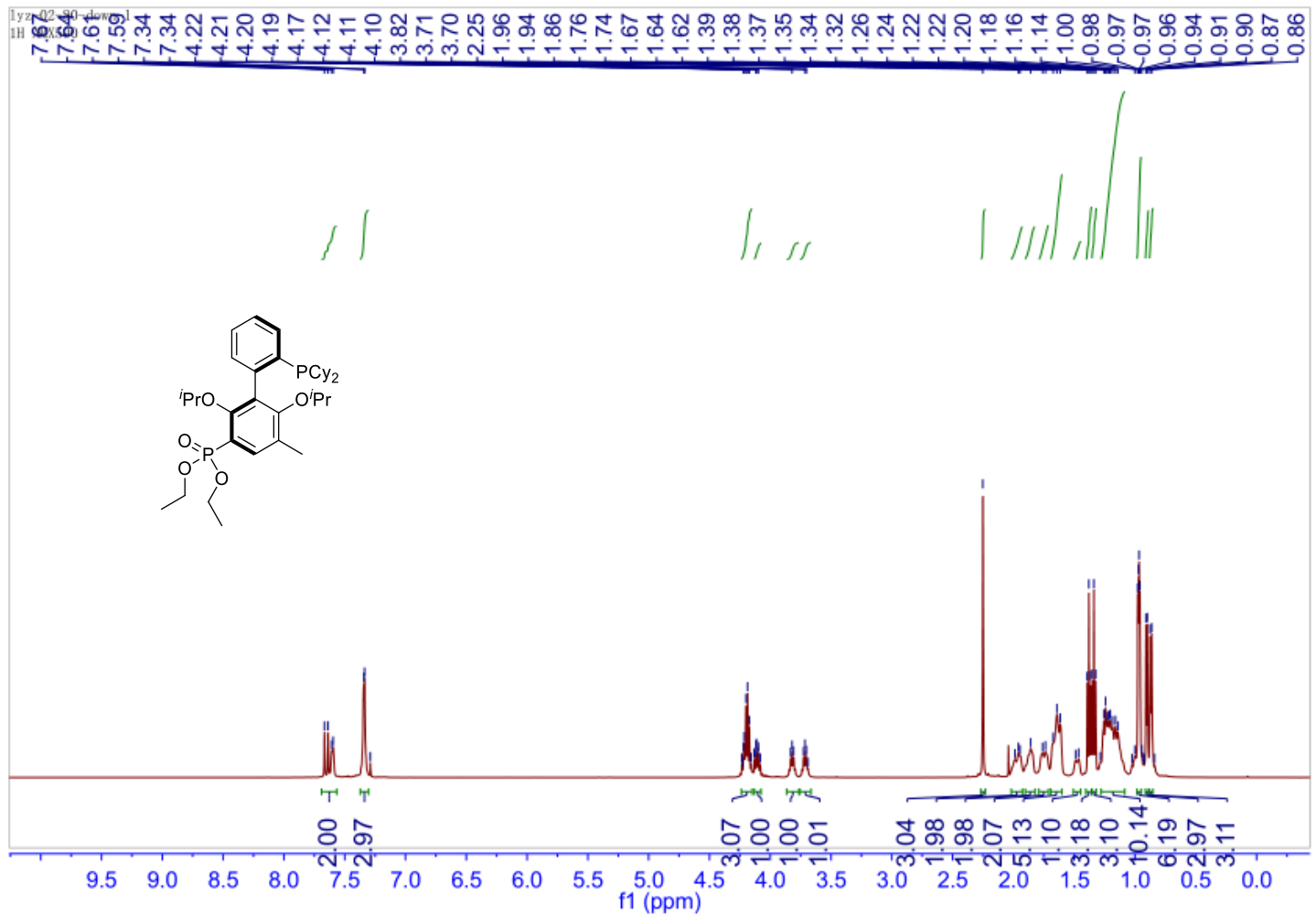

${ }^{13} \mathrm{C}$ NMR $\left(126 \mathrm{MHz}, \mathrm{CDCl}_{3}\right)$

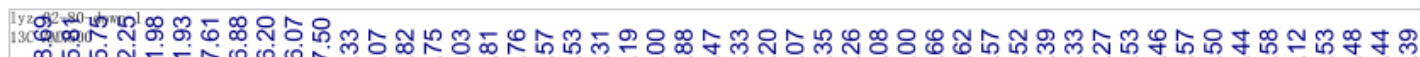

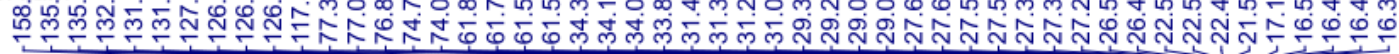

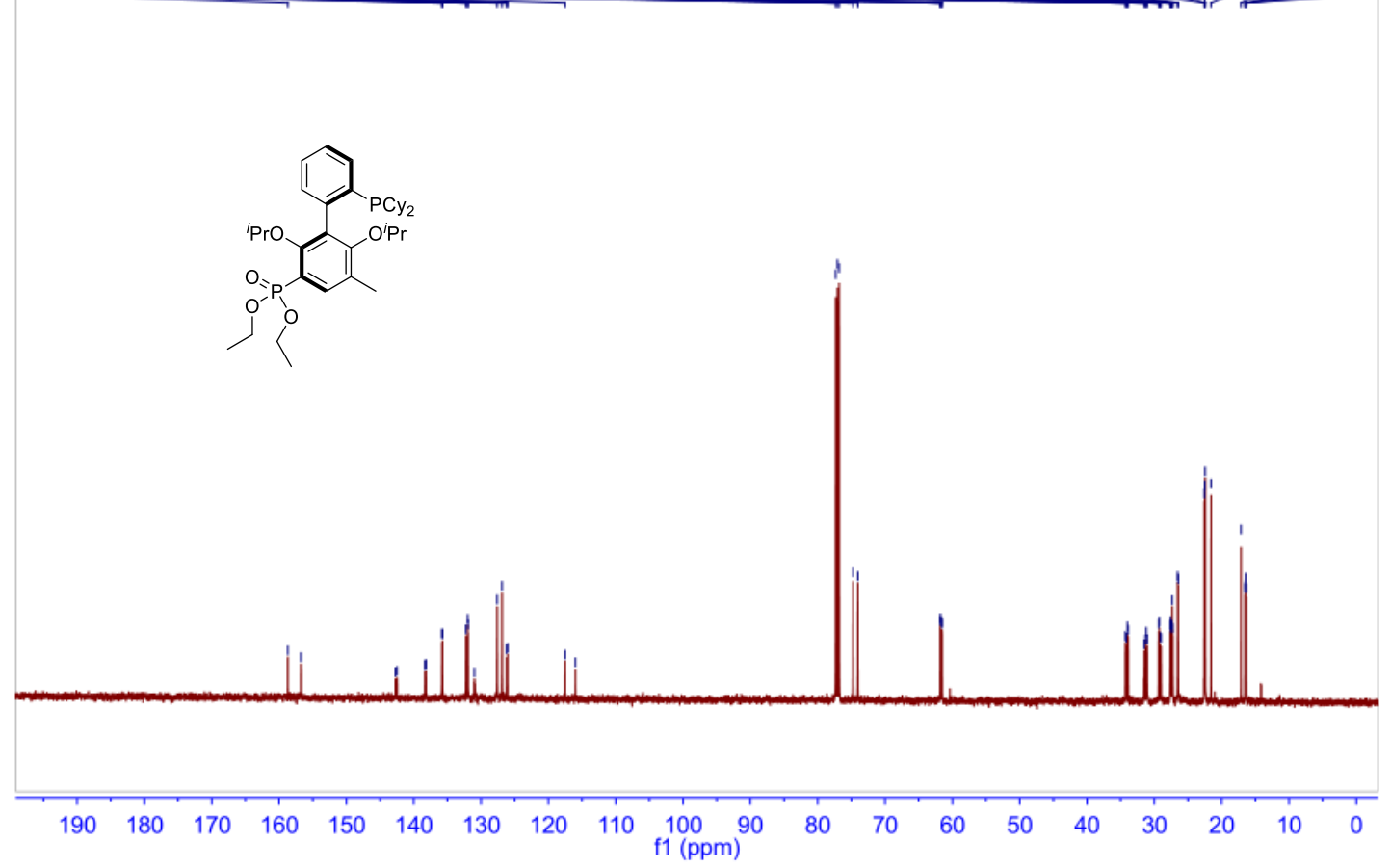




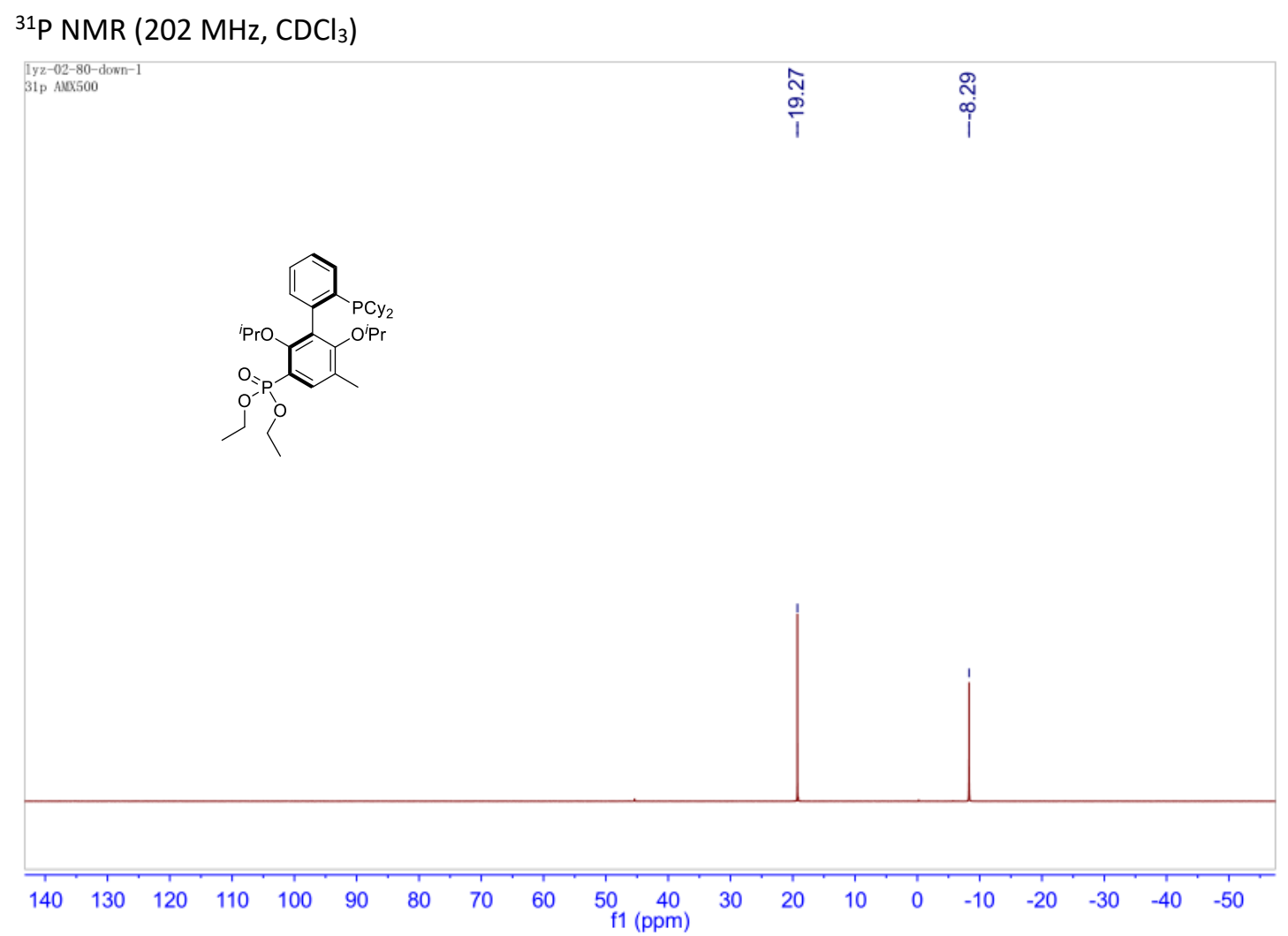


${ }^{1} \mathrm{H}$ NMR $\left(500 \mathrm{MHz}, \mathrm{CDCl}_{3}\right)$ of $(S)-\mathrm{L} 4$

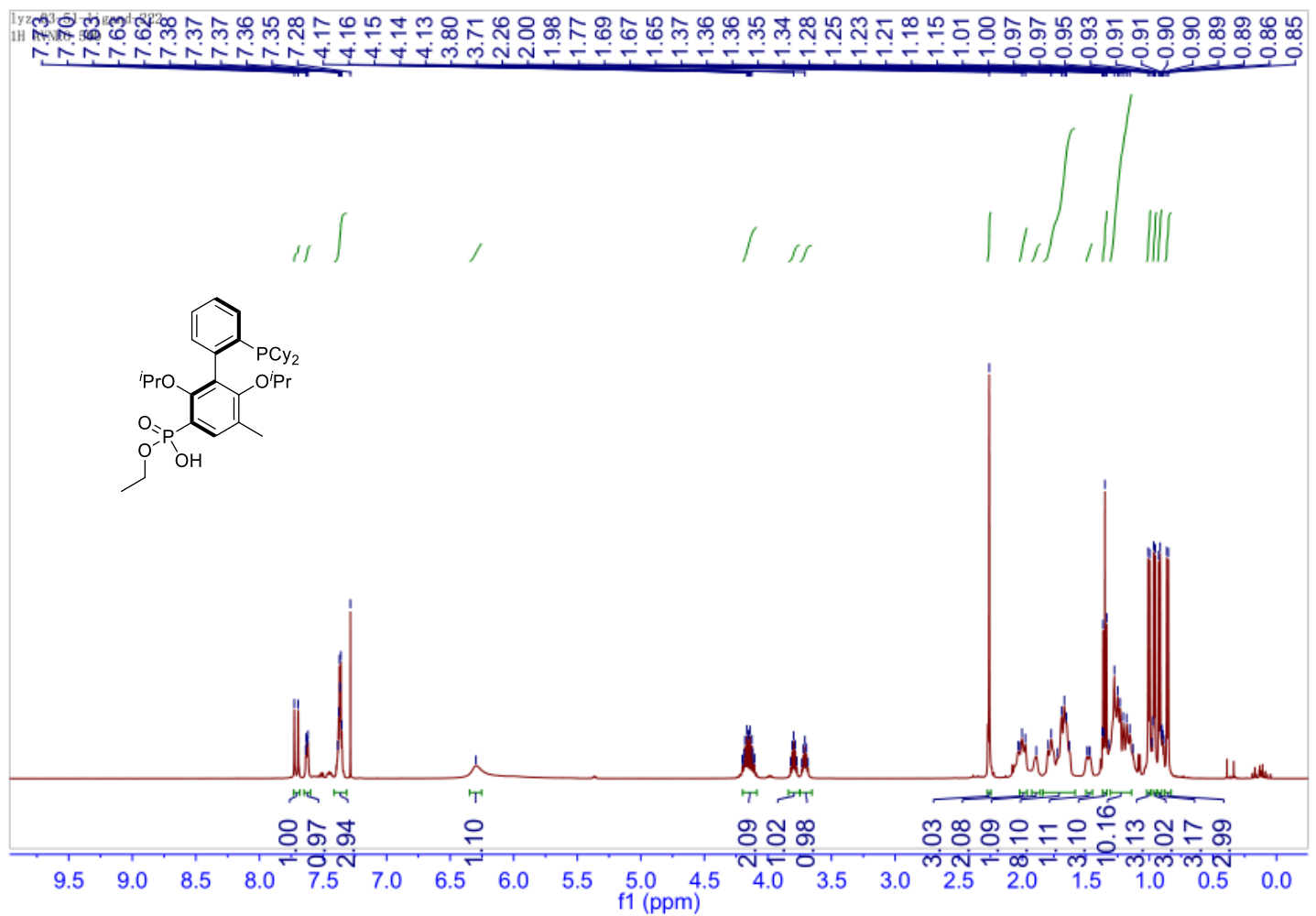

${ }^{13} \mathrm{C}$ NMR (126 MHz, $\mathrm{CDCl}_{3}$ ) of (S)-L4

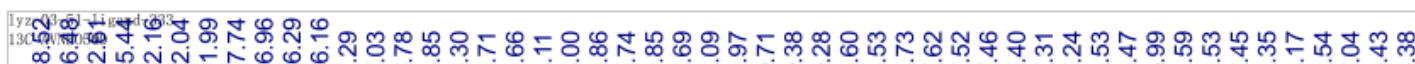

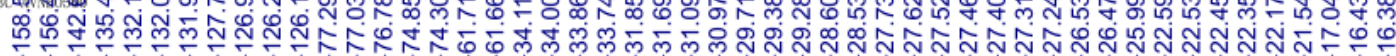<smiles>CCOc1c(C)cc(P(=O)(O)OCC)c(OC#N)c1-c1ccccc1O</smiles>

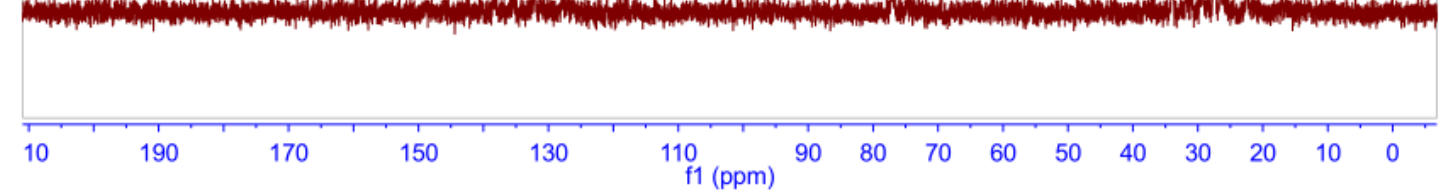




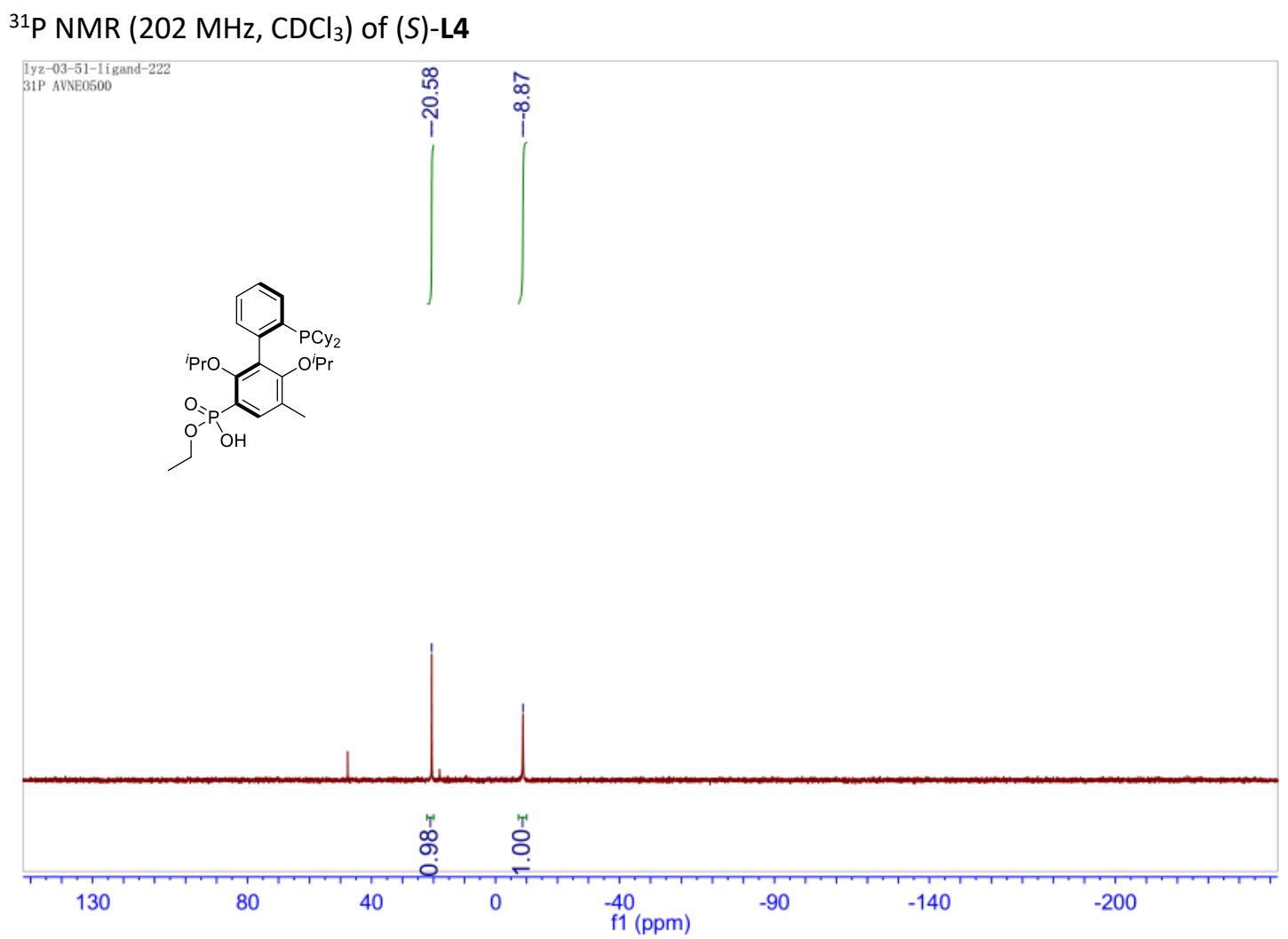


${ }^{1} \mathrm{H}$ NMR $\left(500 \mathrm{MHz}, \mathrm{CDCl}_{3}\right)$

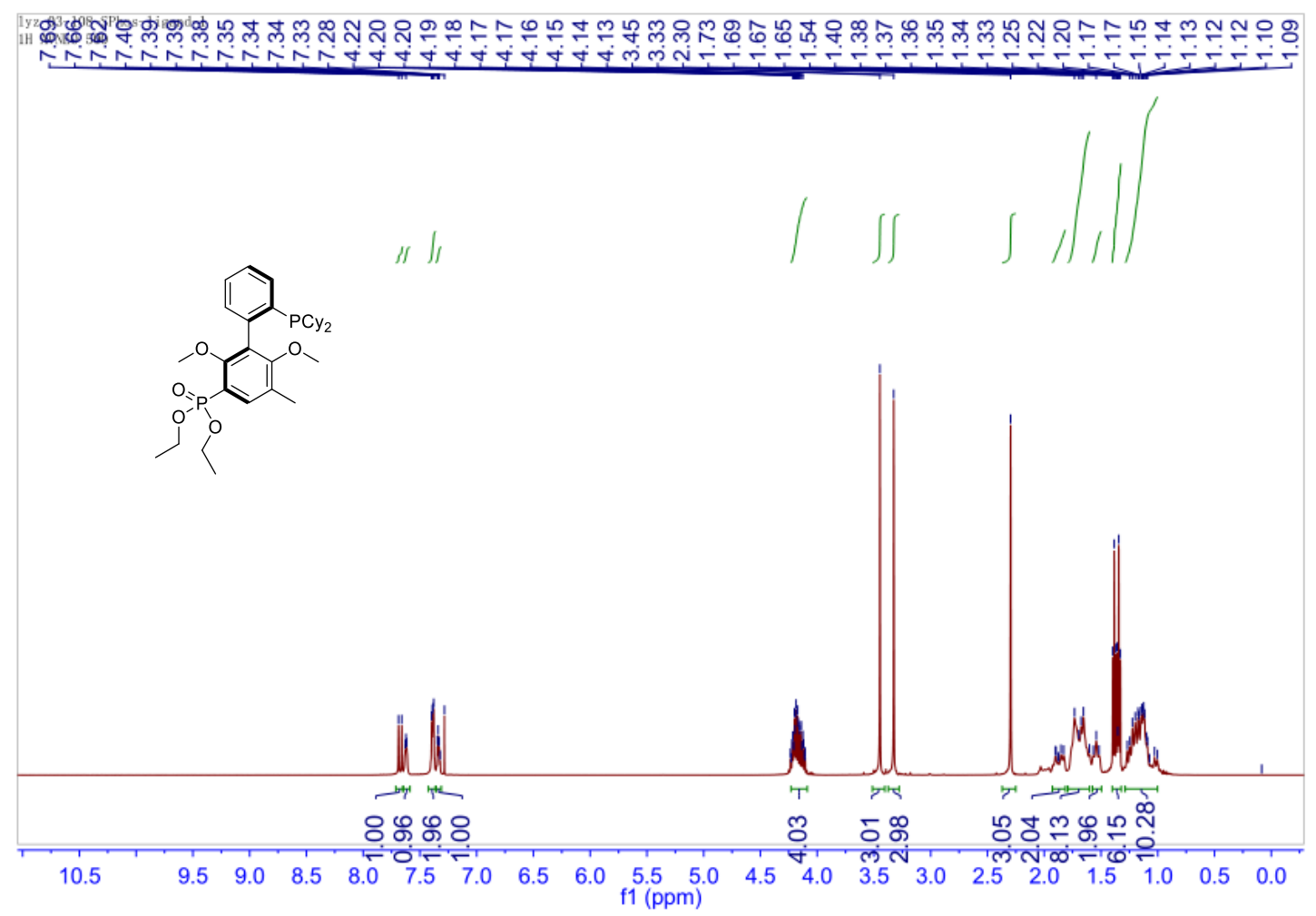

${ }^{13} \mathrm{C}$ NMR (126 MHz, $\left.\mathrm{CDCl}_{3}\right)$

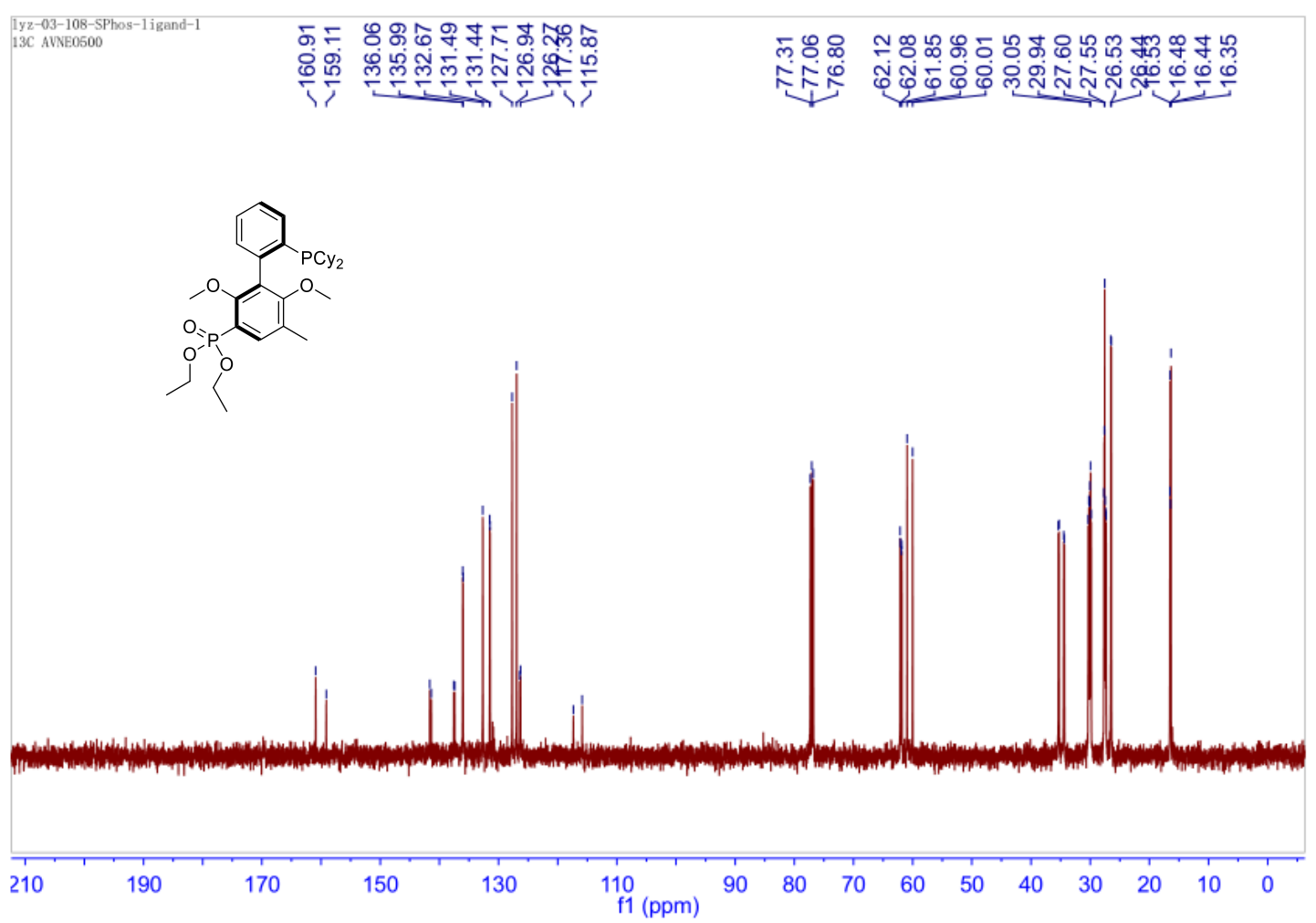




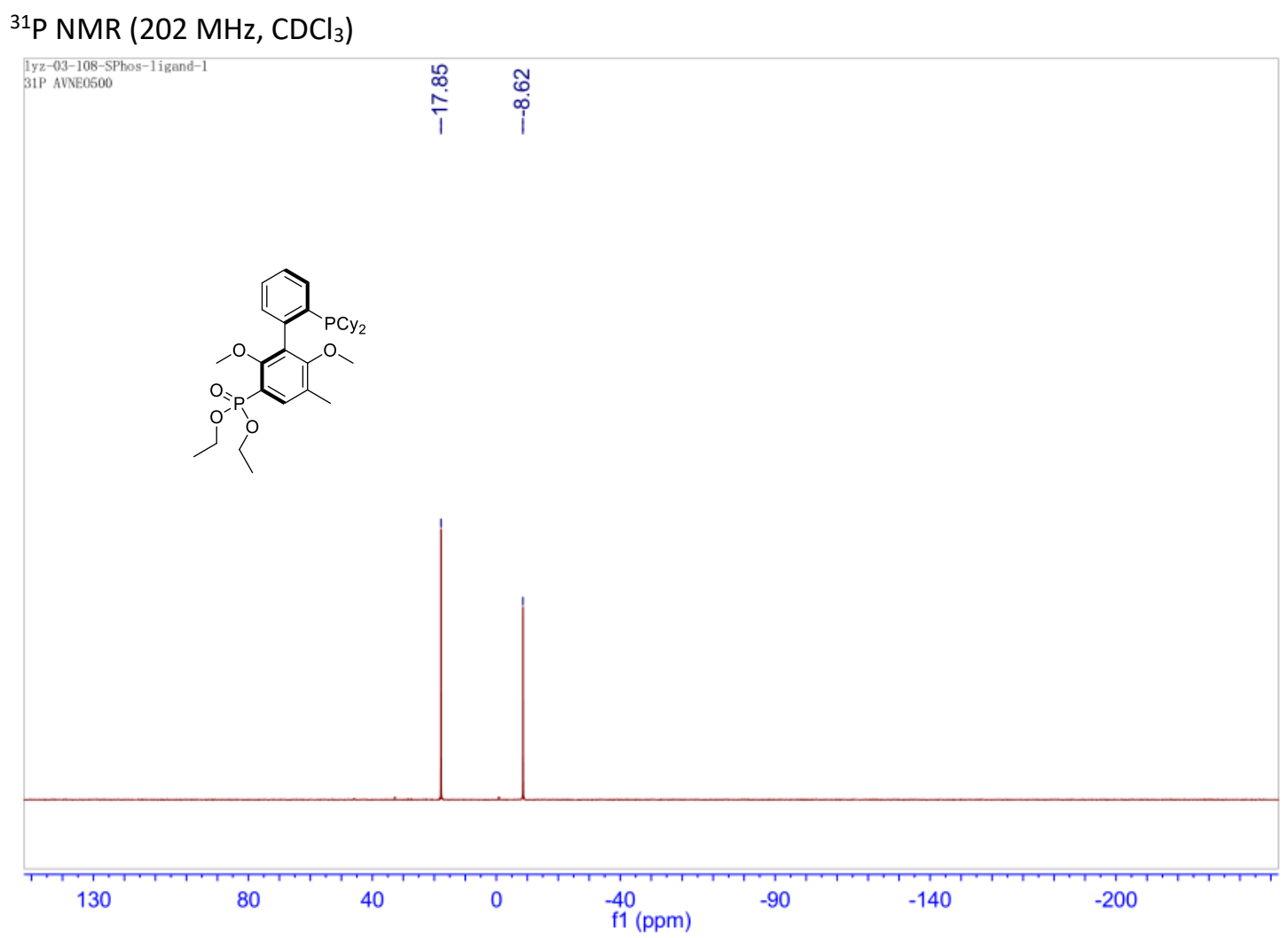


${ }^{1} \mathrm{H}$ NMR (500 MHz, CDCl 3 ) of (S)-L5

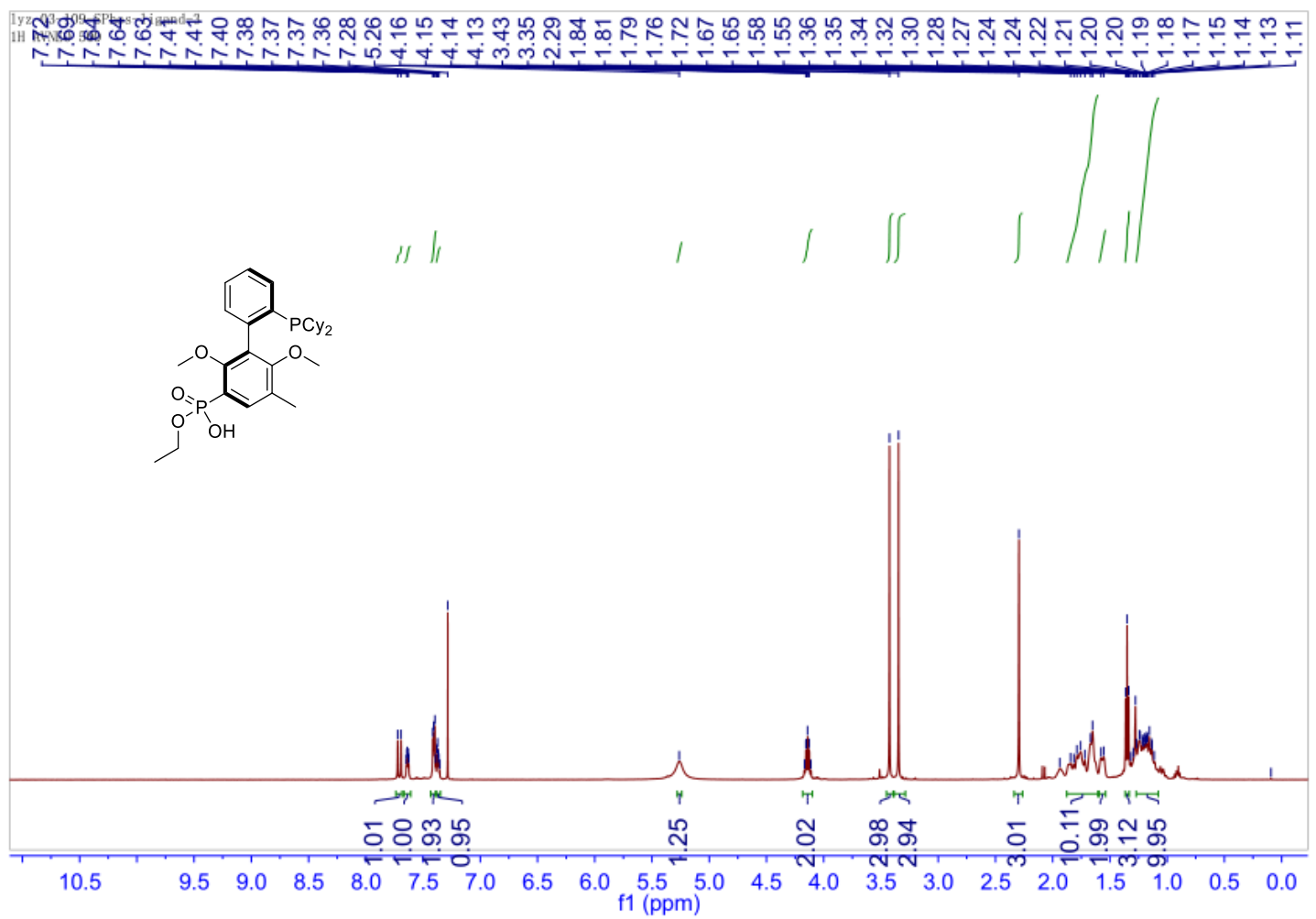

${ }^{13} \mathrm{C}$ NMR (126 MHz, $\mathrm{CDCl}_{3}$ ) of (S)- $\mathbf{L} \mathbf{5}$

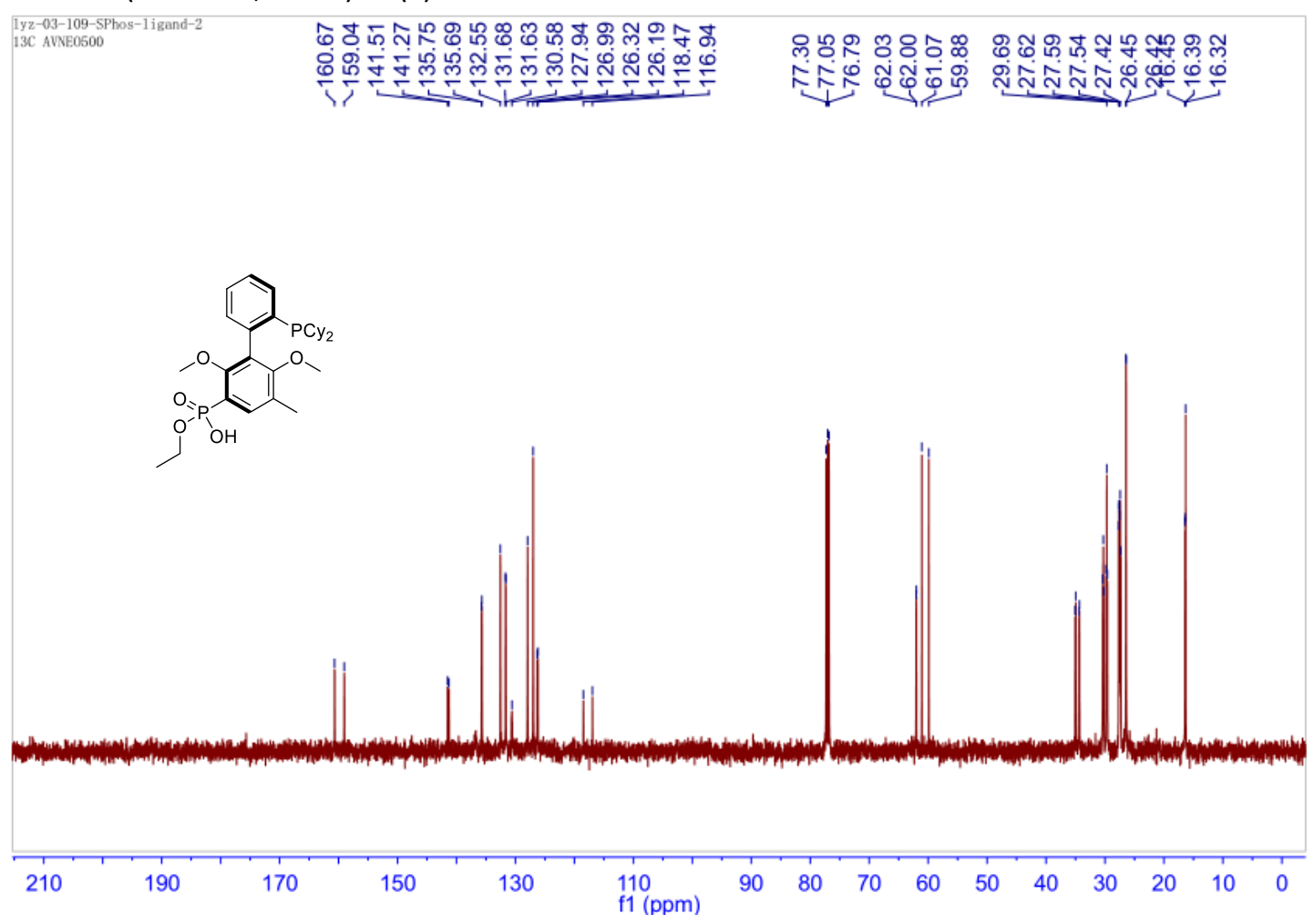




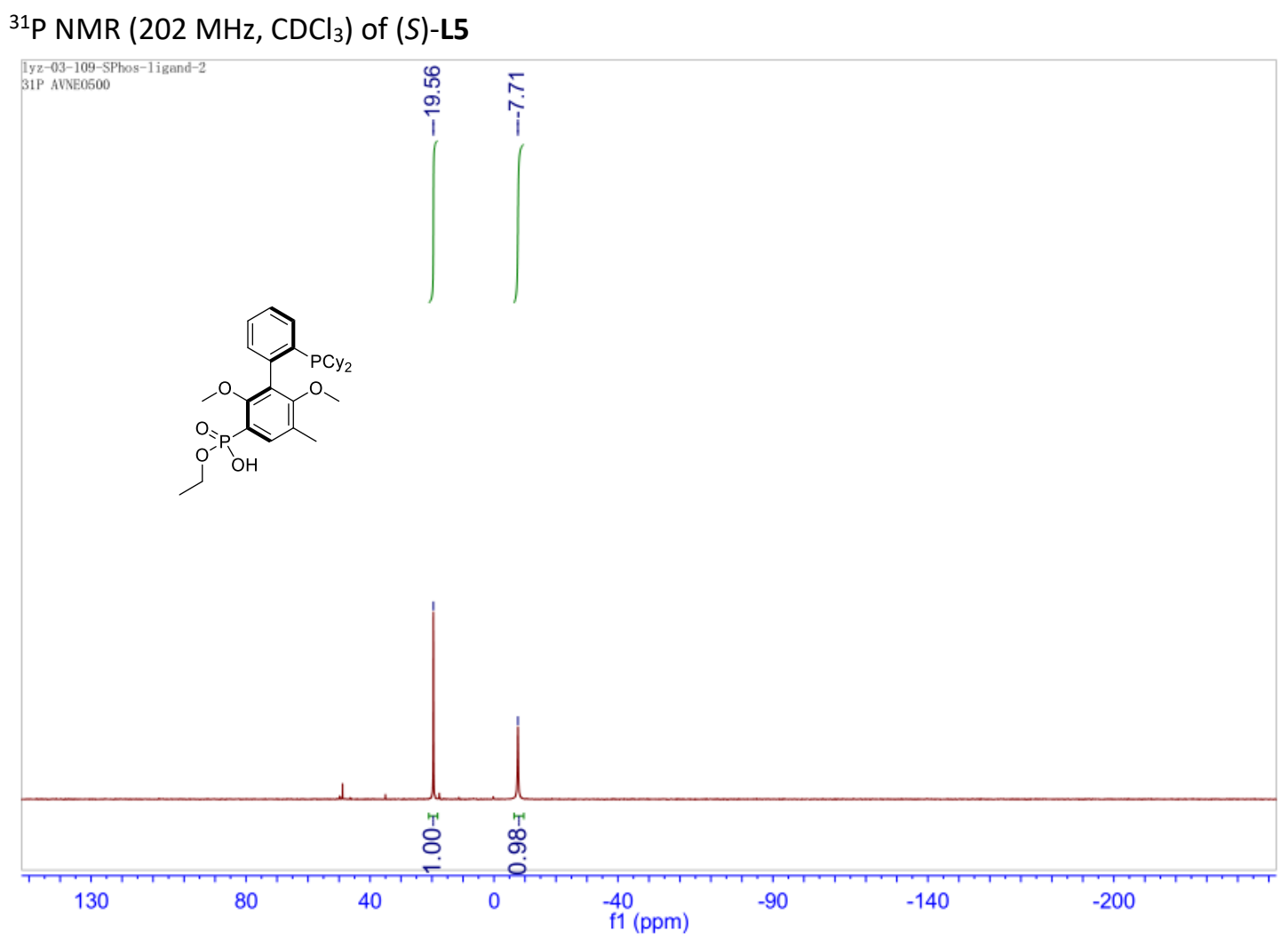


${ }^{1} \mathrm{H}$ NMR (500 MHz, $\mathrm{CDCl}_{3}$ )

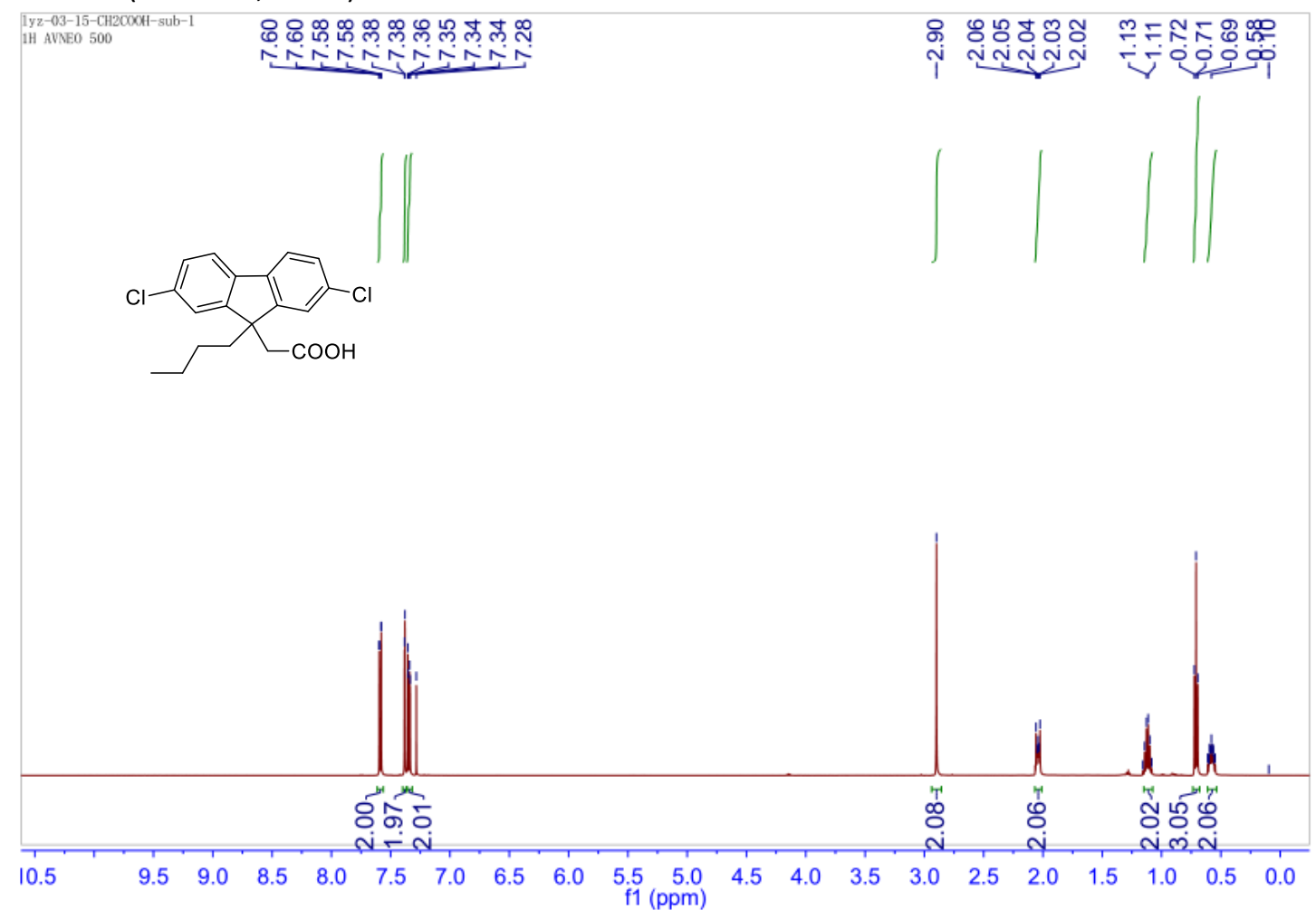

\section{${ }^{13} \mathrm{C}$ NMR $\left(126 \mathrm{MHz}, \mathrm{CDCl}_{3}\right)$}

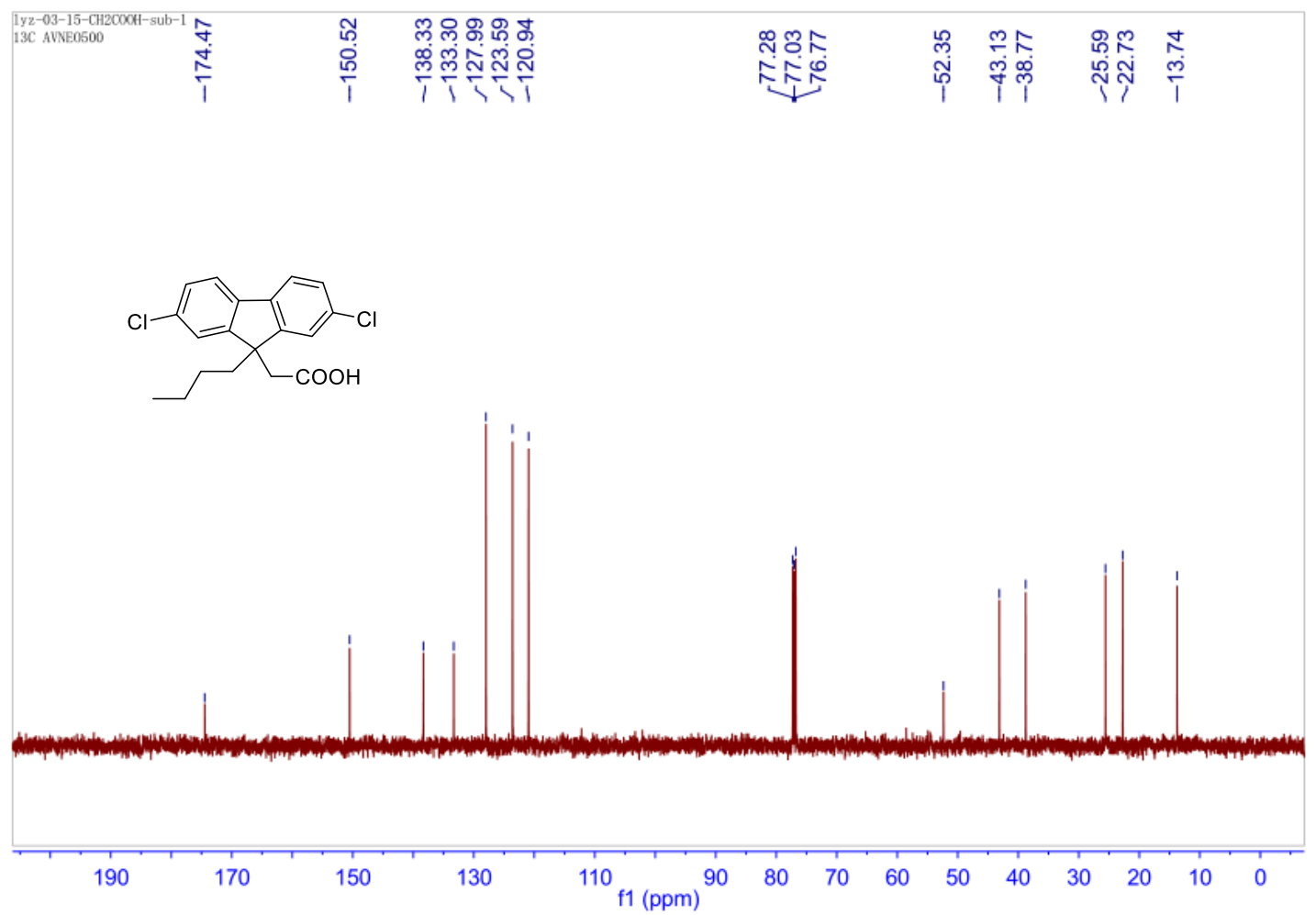


${ }^{1} \mathrm{H}$ NMR (300 MHz, $\mathrm{CDCl}_{3}$ )

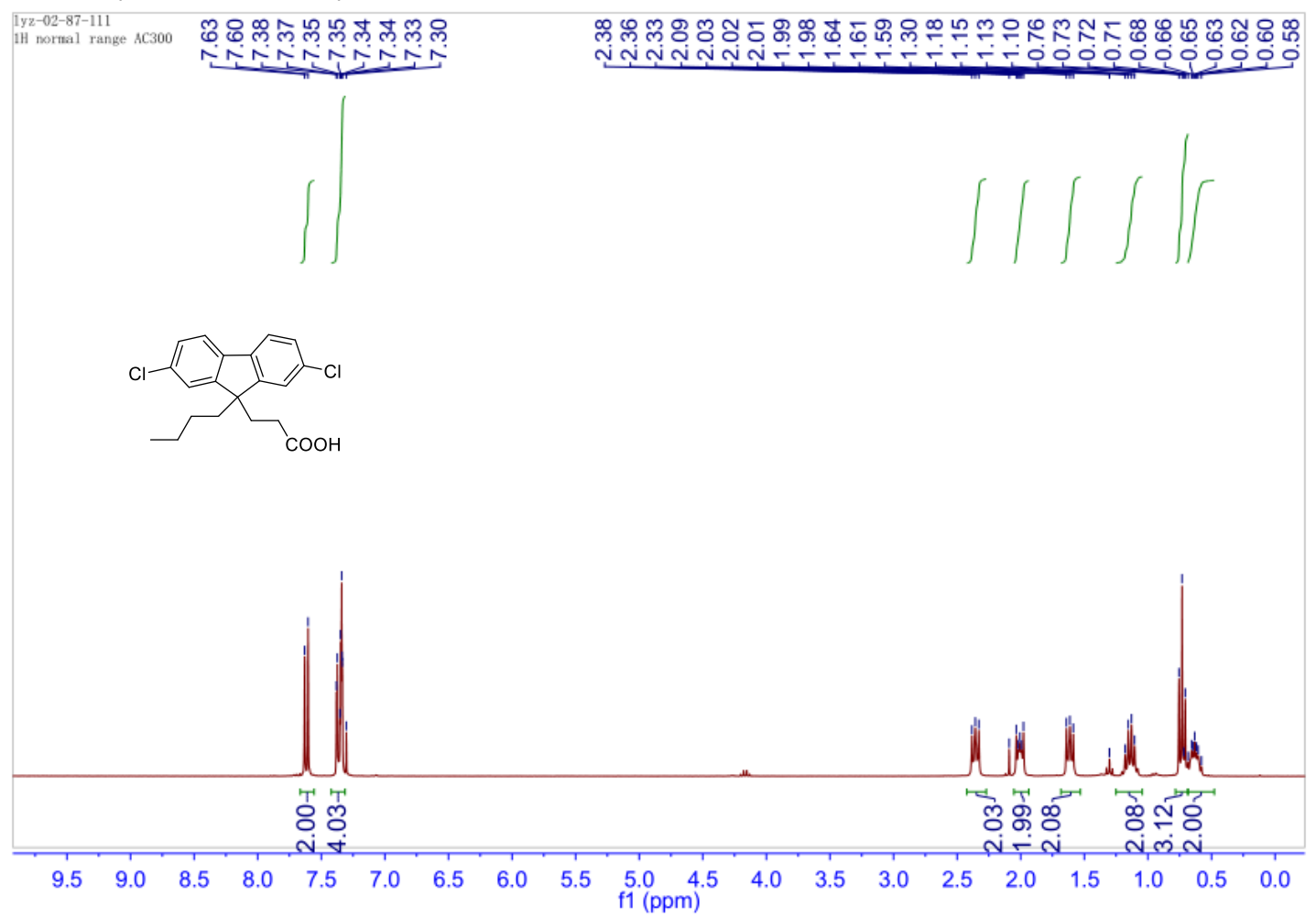

${ }^{13} \mathrm{C}$ NMR $\left(75 \mathrm{MHz}, \mathrm{CDCl}_{3}\right)$

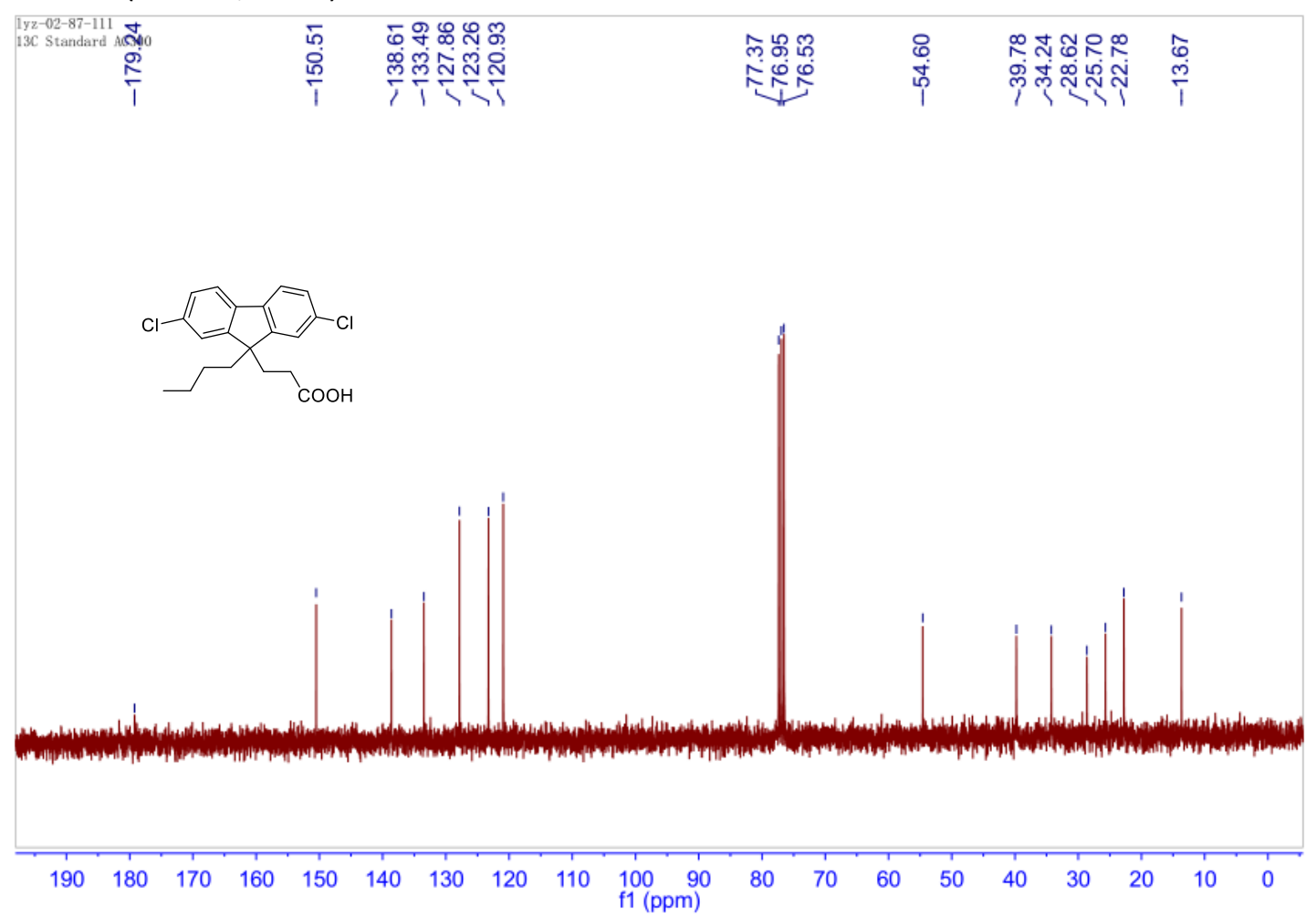


${ }^{1} \mathrm{H}$ NMR (500 MHz, $\mathrm{CDCl}_{3}$ )-51

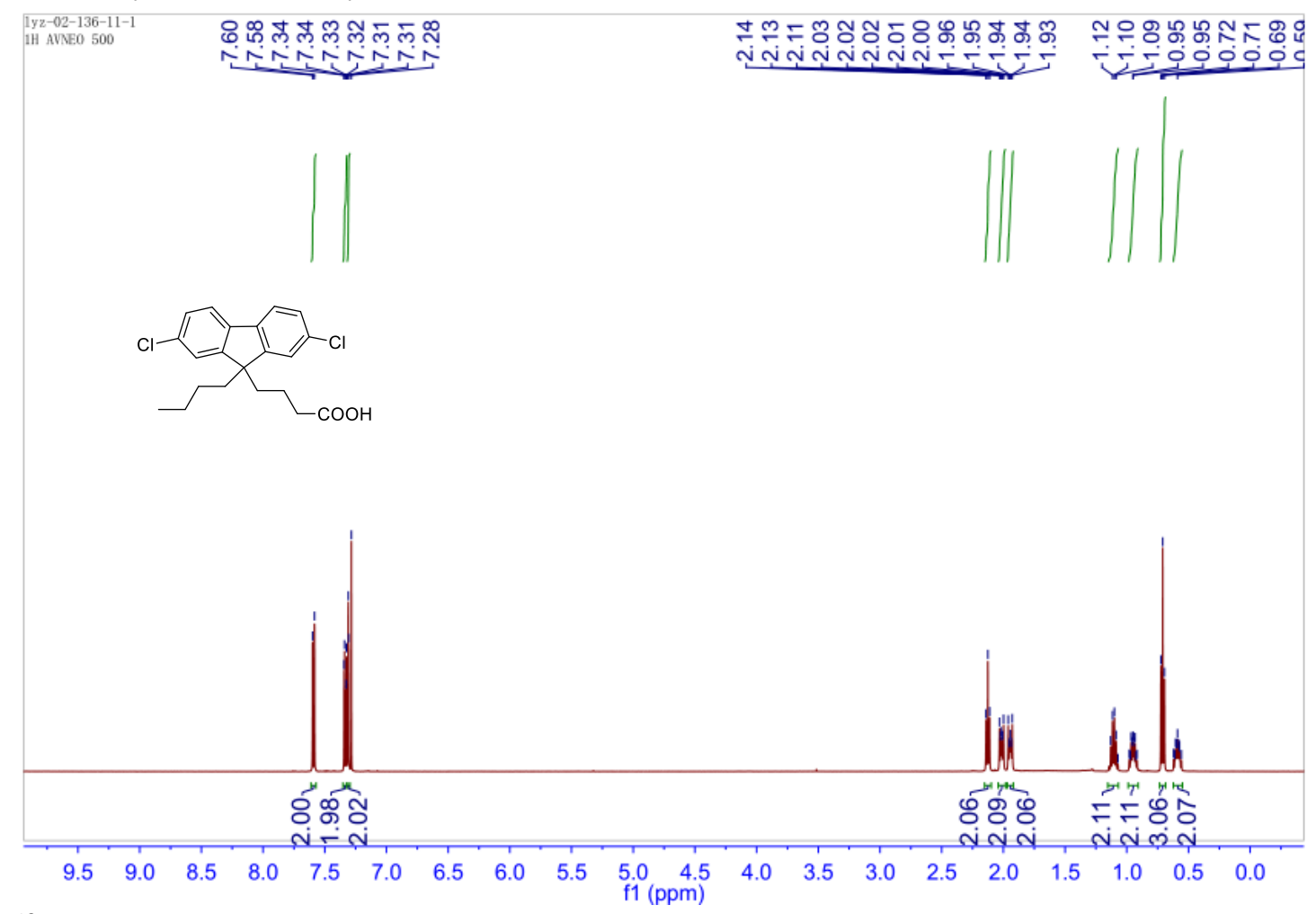

${ }^{13} \mathrm{C}$ NMR (126 MHz, $\left.\mathrm{CDCl}_{3}\right)-51$

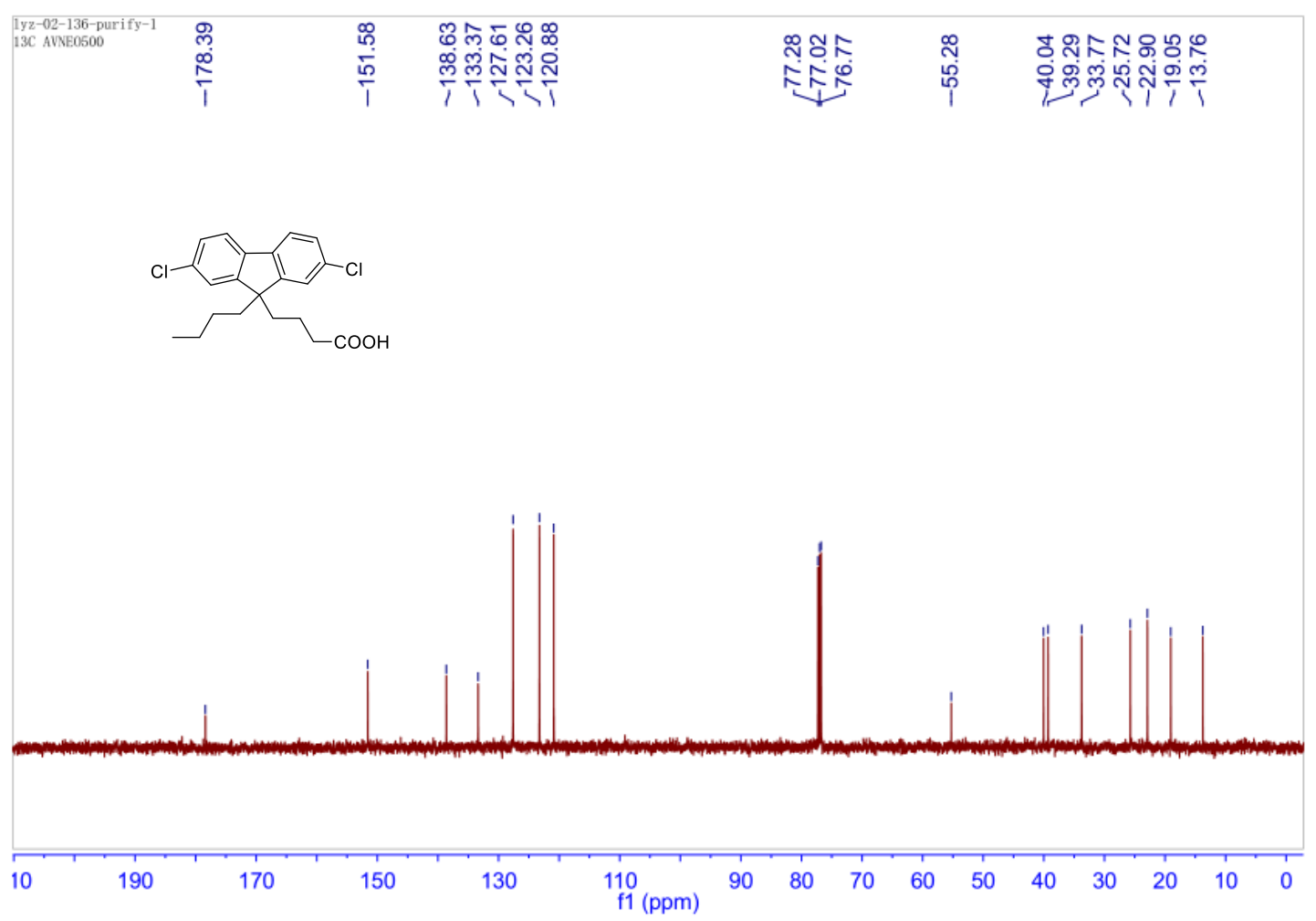


${ }^{1} \mathrm{H}$ NMR $\left(500 \mathrm{MHz}, \mathrm{CDCl}_{3}\right)-53$

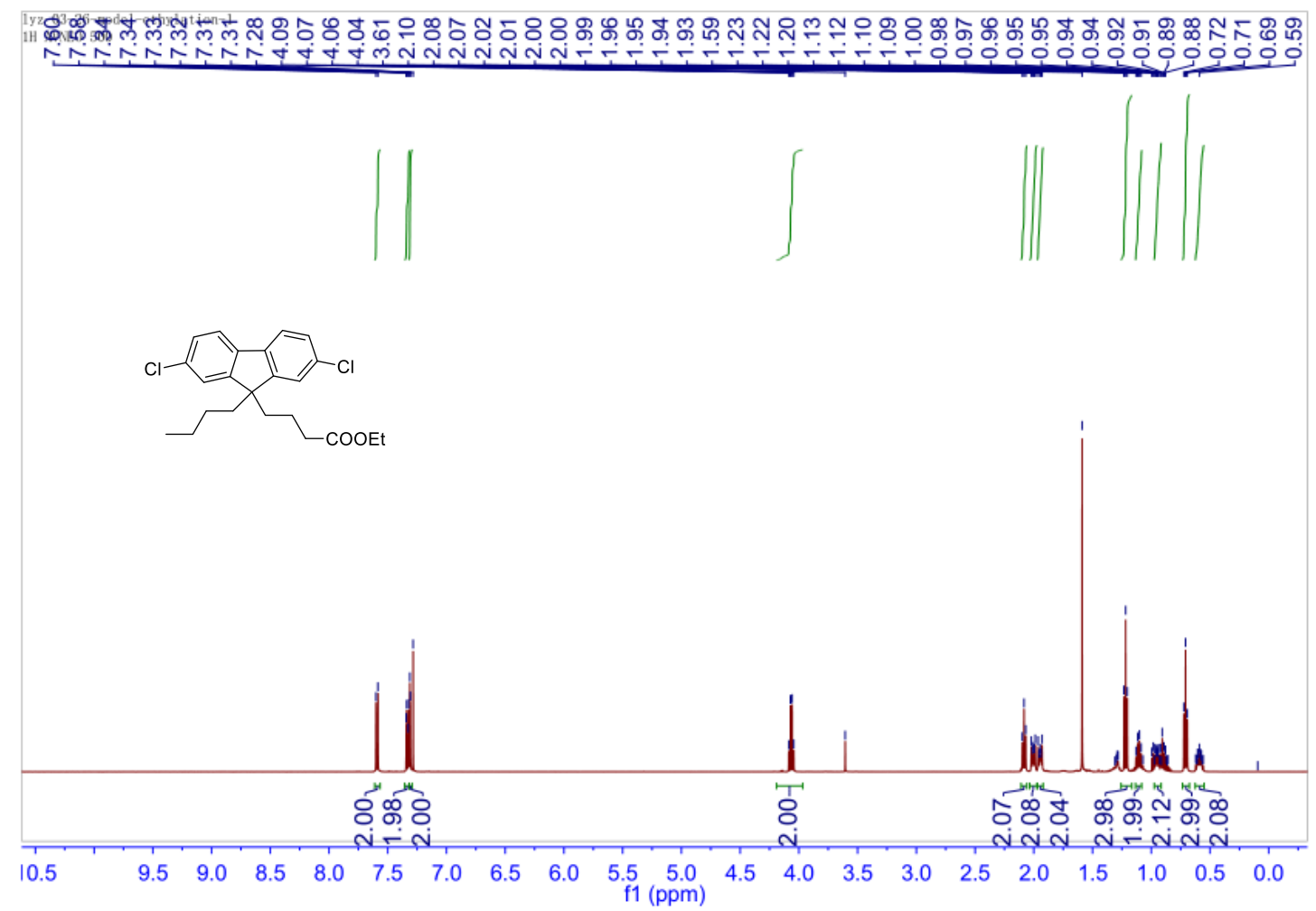

${ }^{13} \mathrm{C}$ NMR (126 MHz, $\left.\mathrm{CDCl}_{3}\right)-53$

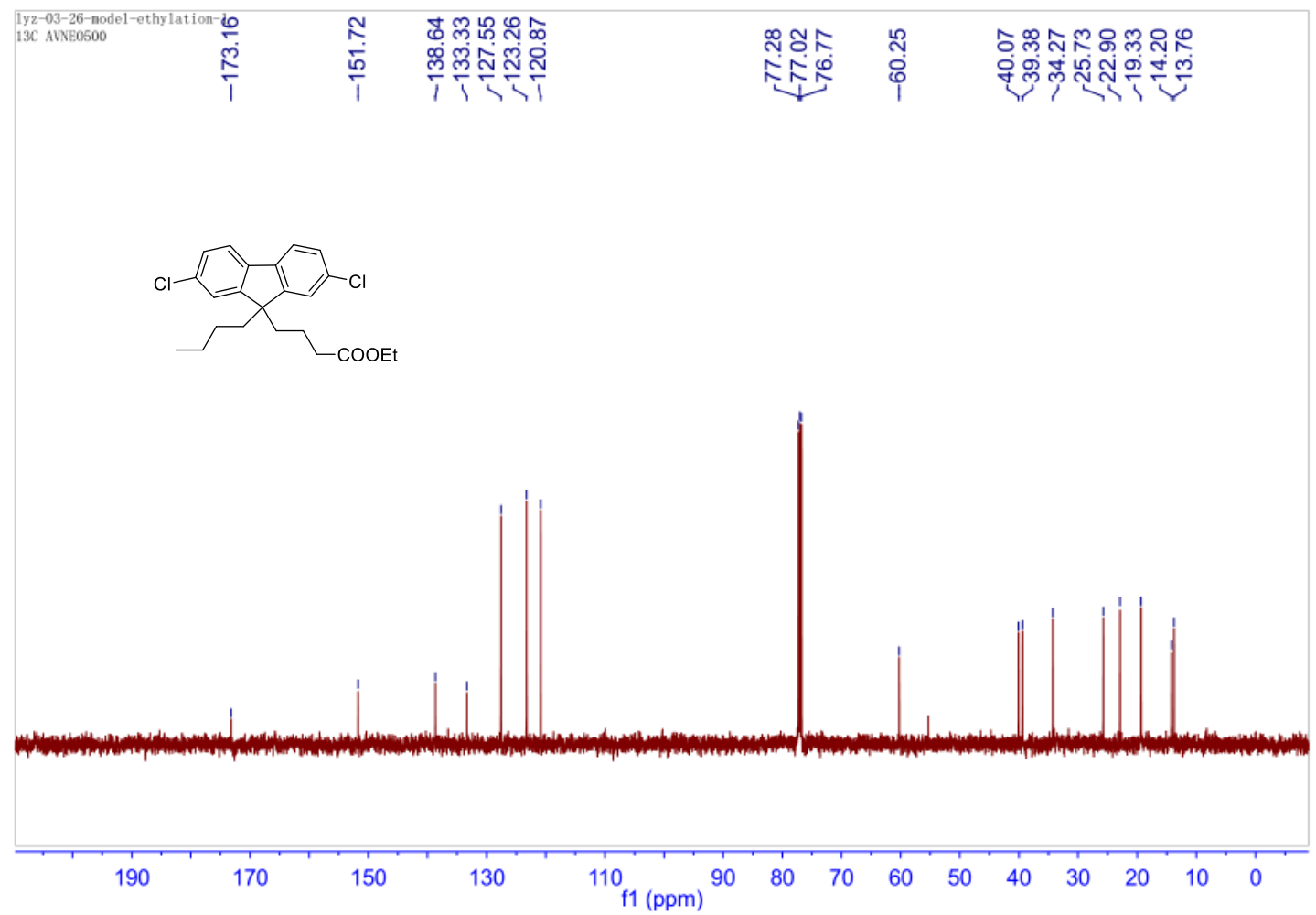


${ }^{1} \mathrm{H}$ NMR (500 MHz, $\mathrm{CDCl}_{3}$ )

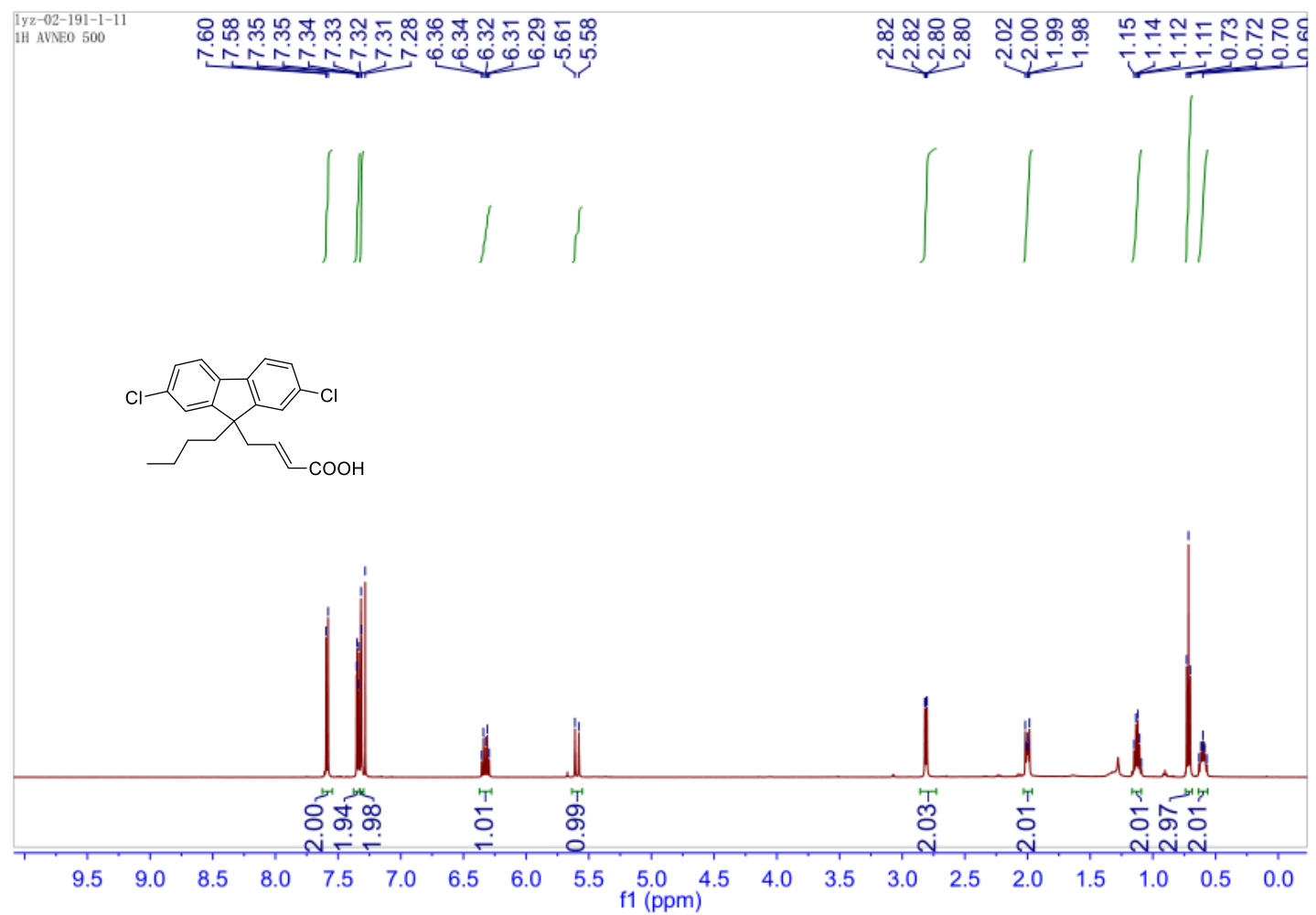

${ }^{13} \mathrm{C}$ NMR (126 MHz, $\left.\mathrm{CDCl}_{3}\right)$

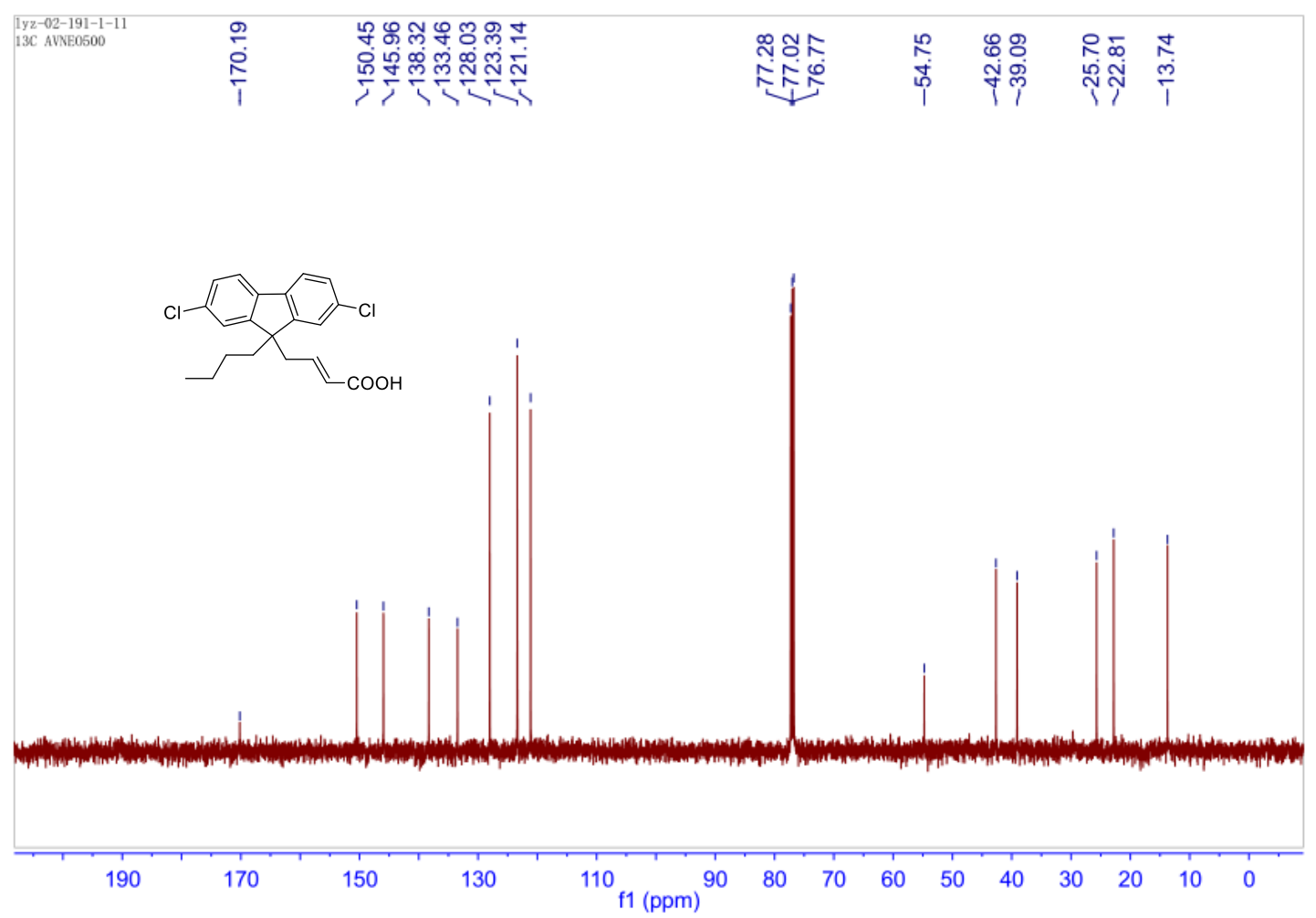


${ }^{1} \mathrm{H}$ NMR (500 MHz, $\left.\mathrm{CDCl}_{3}\right)$

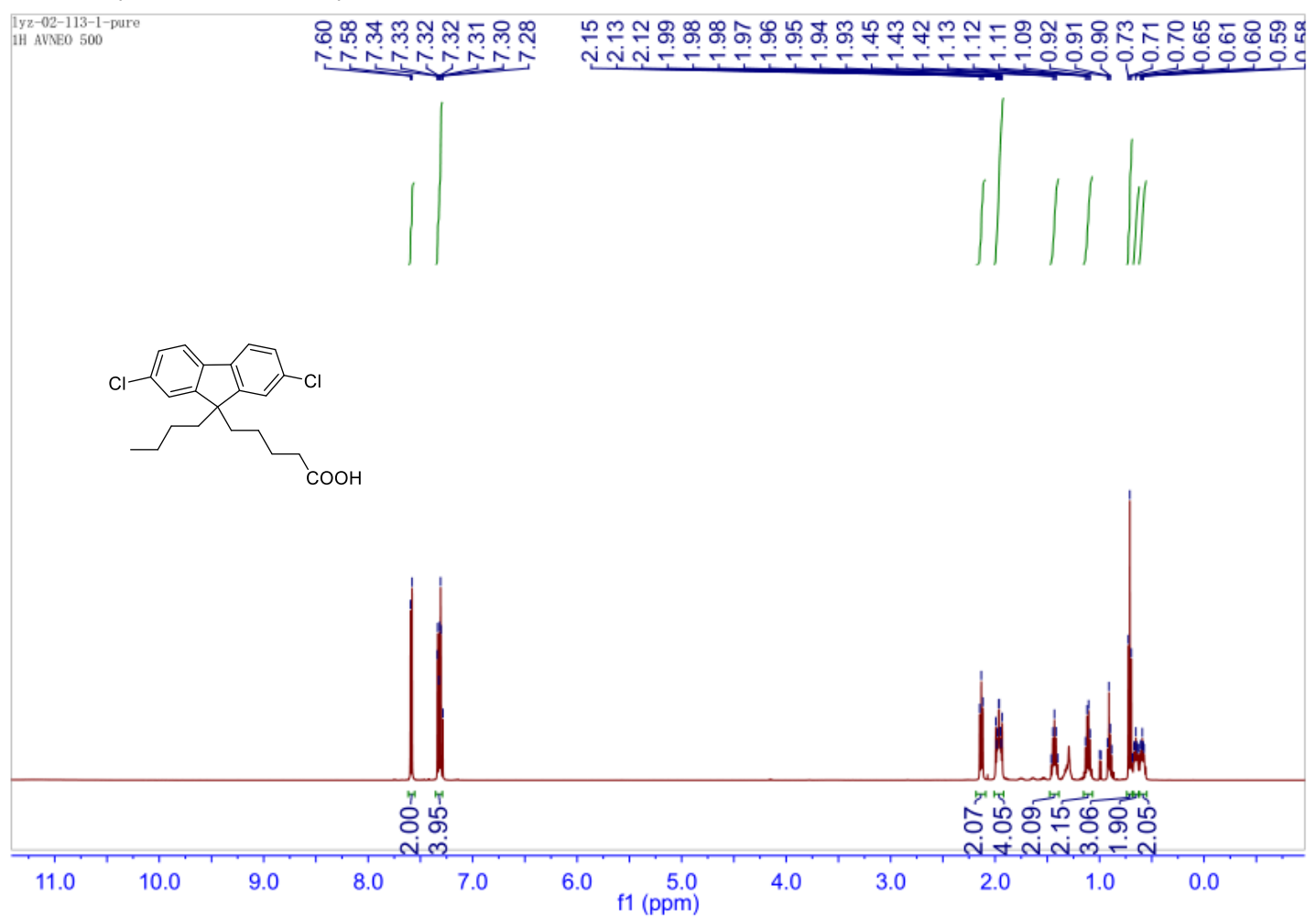

${ }^{13} \mathrm{C}$ NMR (126 MHz, $\mathrm{CDCl}_{3}$ )

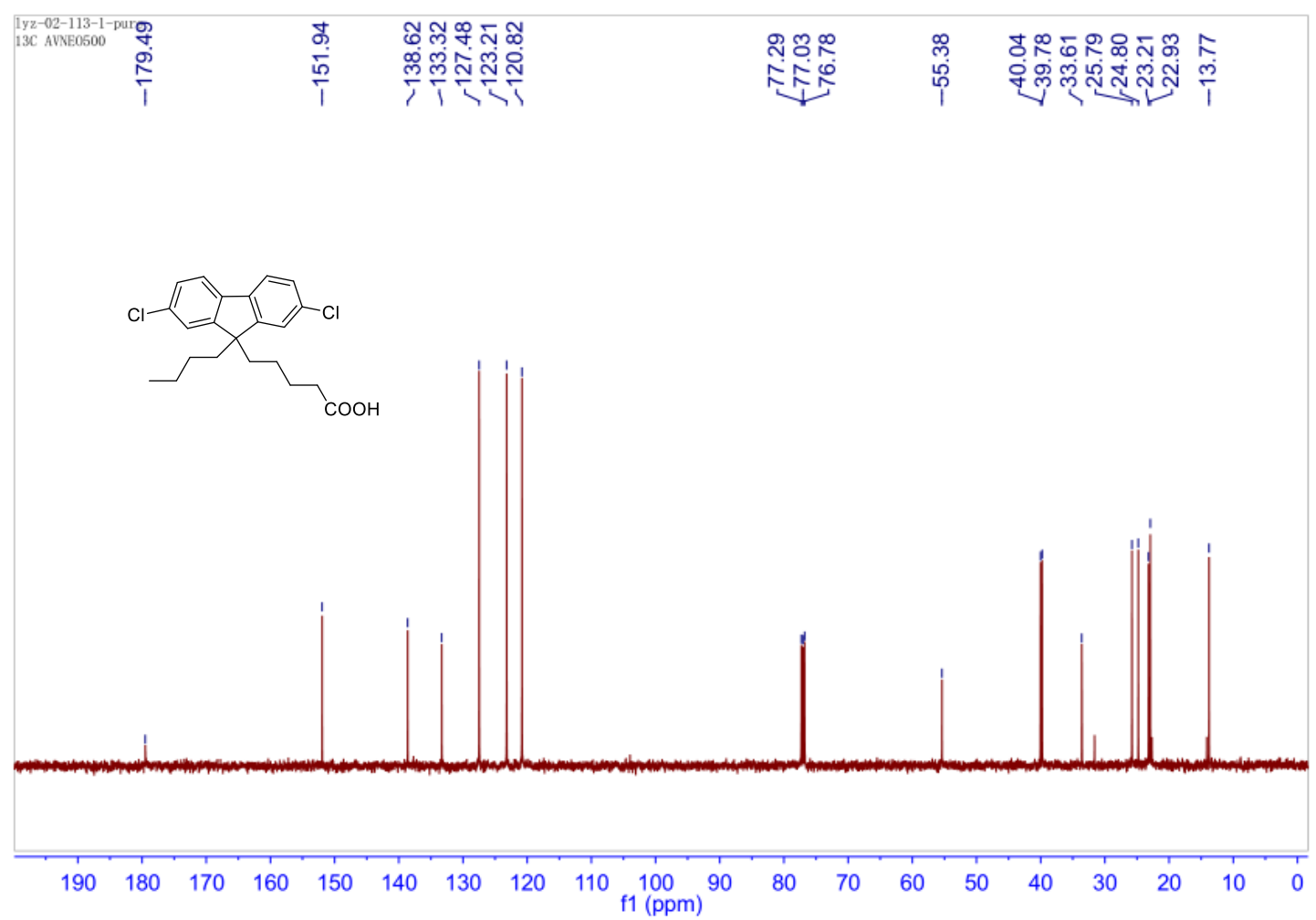


${ }^{1} \mathrm{H}$ NMR $\left(300 \mathrm{MHz}, \mathrm{CDCl}_{3}\right)$

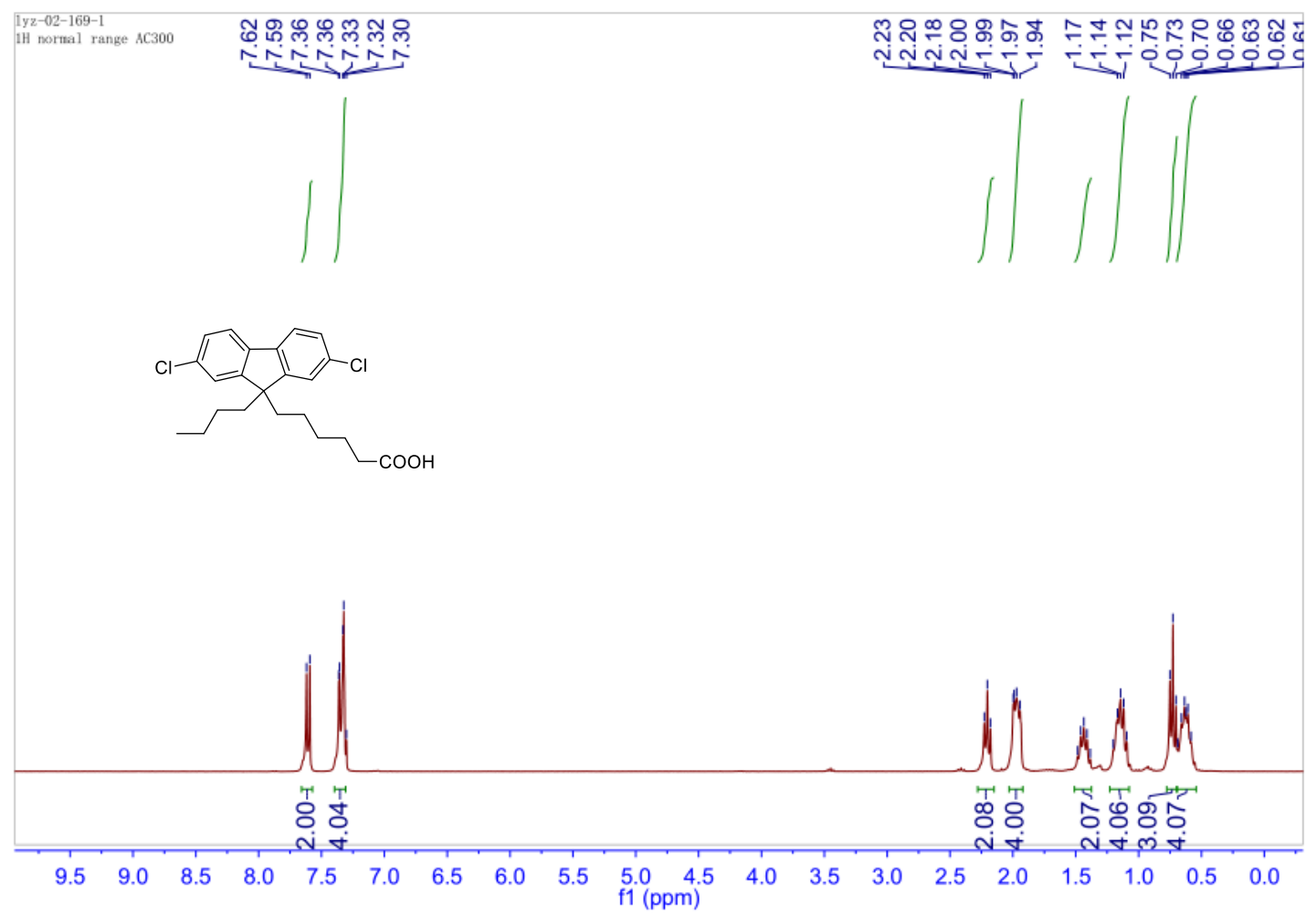

${ }^{13} \mathrm{C}$ NMR $\left(75 \mathrm{MHz}, \mathrm{CDCl}_{3}\right)$

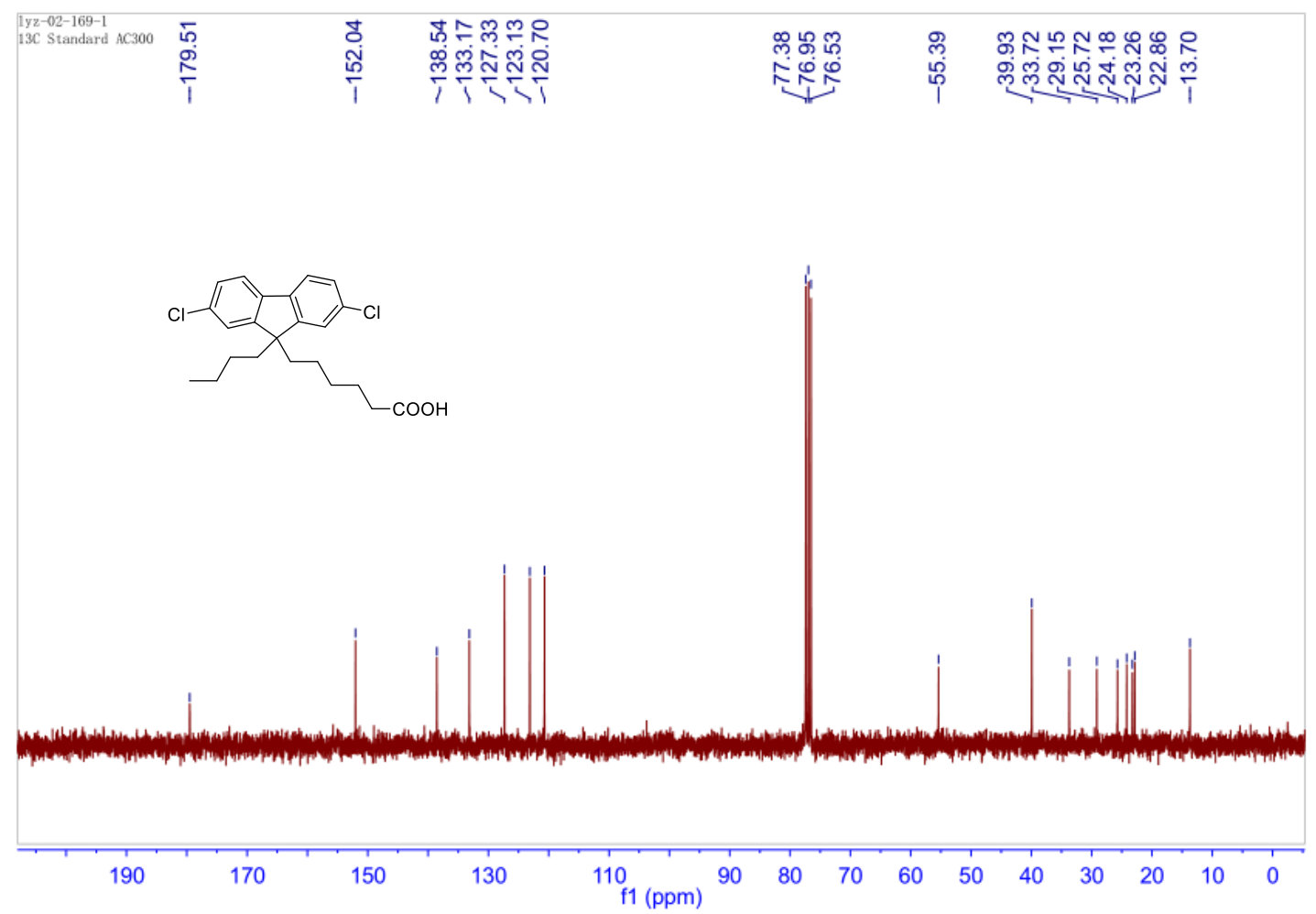


${ }^{1} \mathrm{H}$ NMR (500 MHz, $\mathrm{CDCl}_{3}$ )

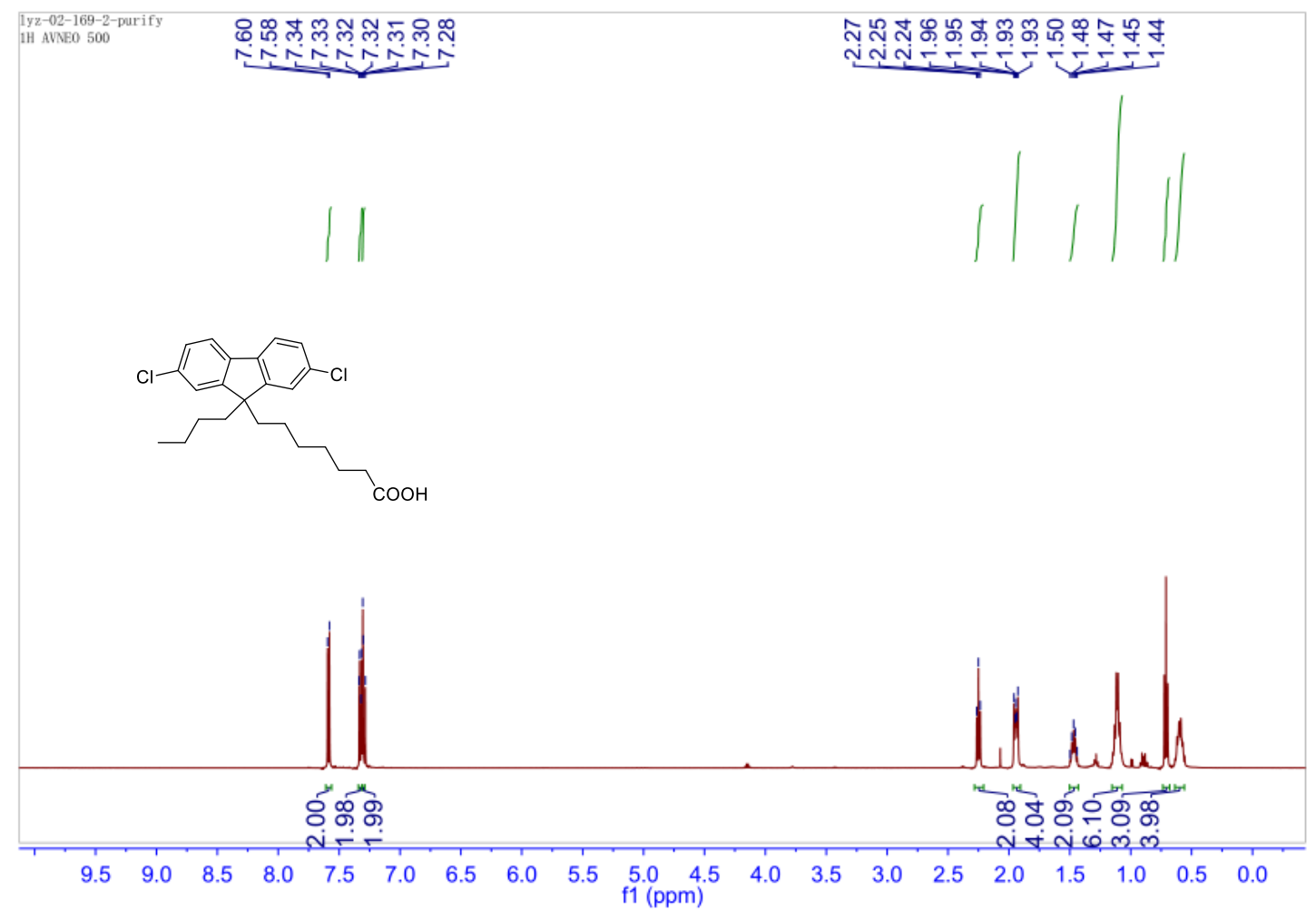

${ }^{13} \mathrm{C}$ NMR (126 MHz, $\left.\mathrm{CDCl}_{3}\right)$

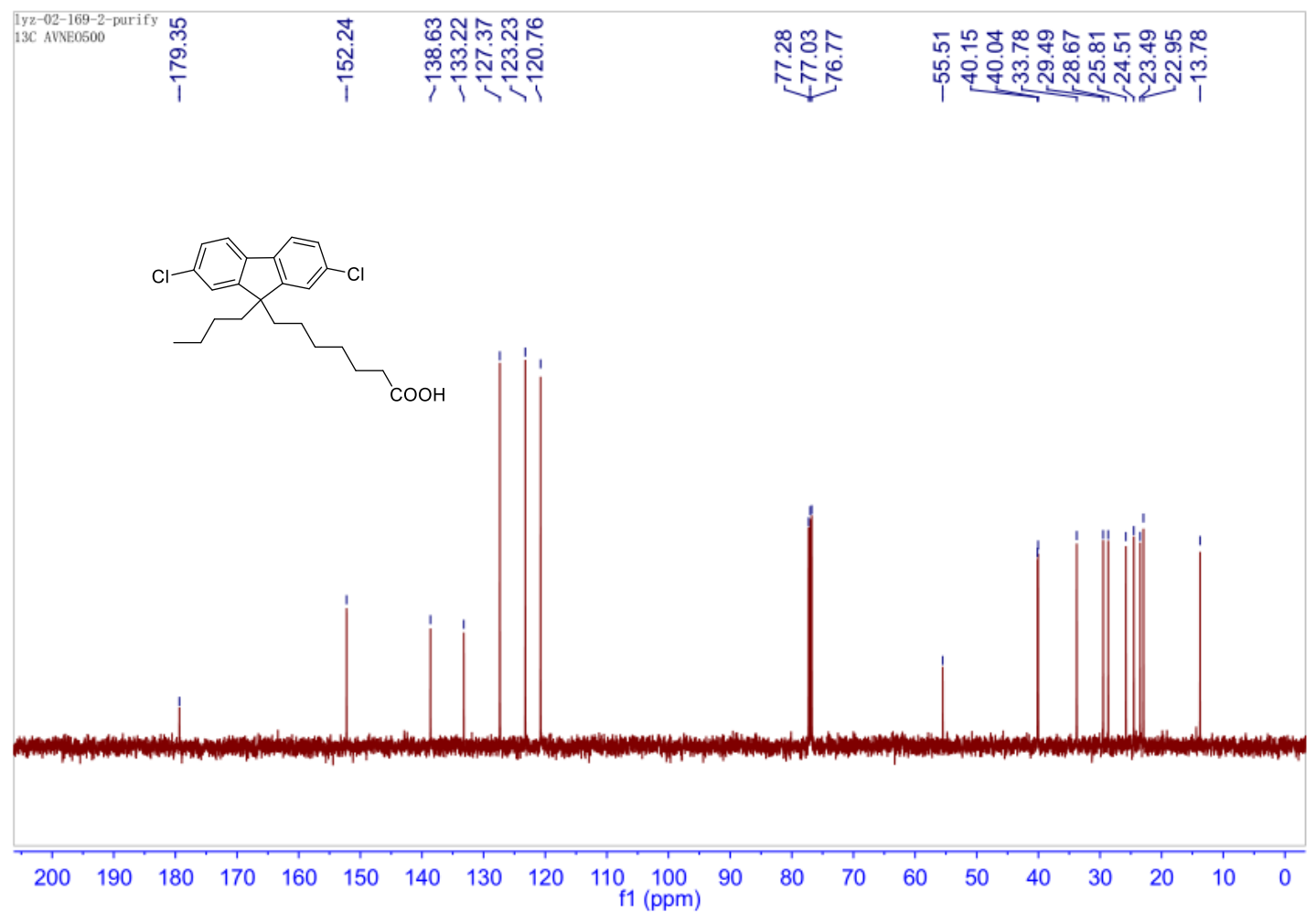


${ }^{1} \mathrm{H}$ NMR $\left(500 \mathrm{MHz}, \mathrm{CDCl}_{3}\right)$

1yz-03-43-c7000H-sub-2

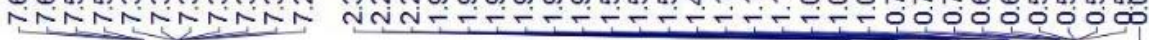
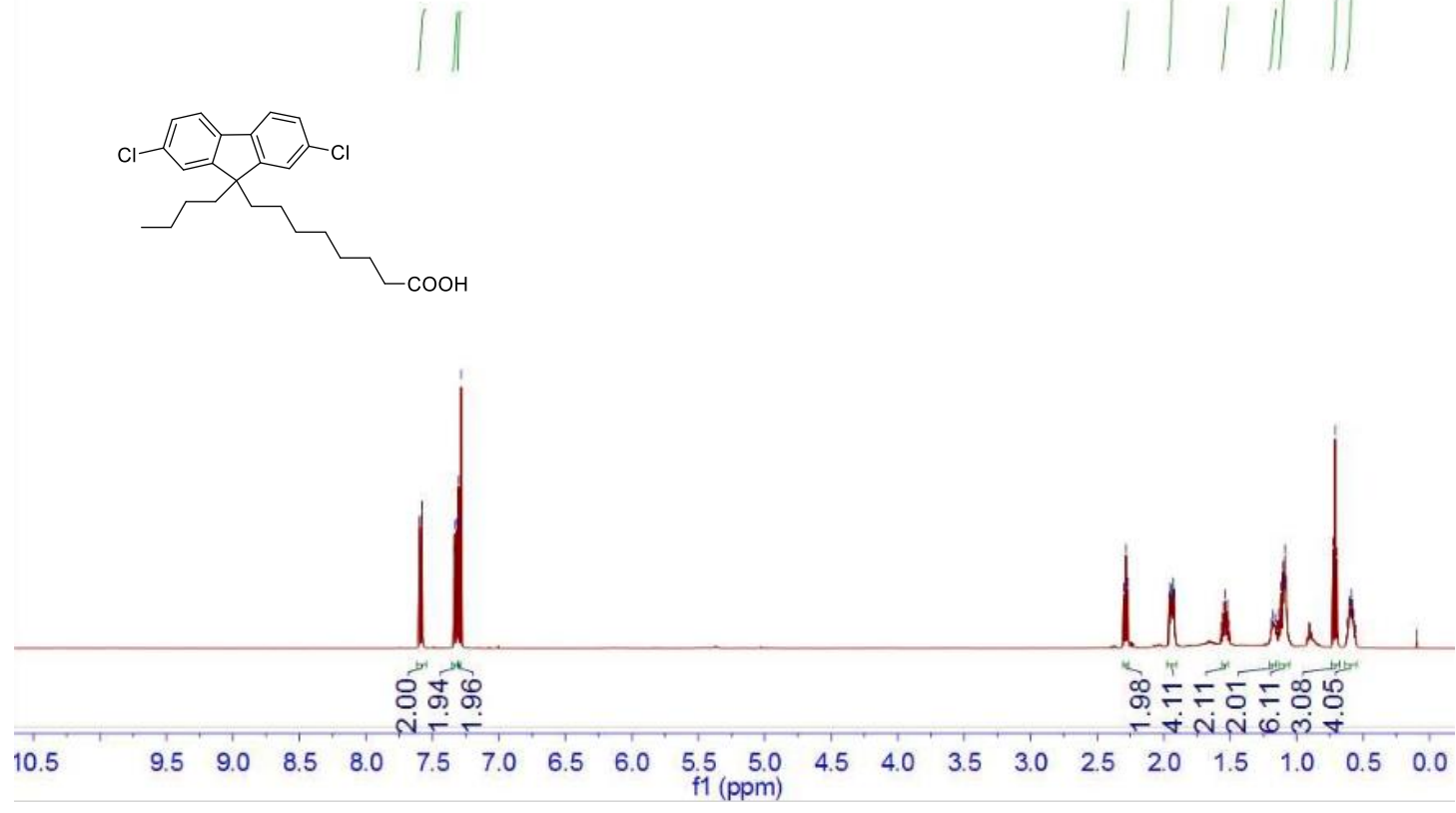

${ }^{13} \mathrm{C} \mathrm{NMR}\left(126 \mathrm{MHz}, \mathrm{CDCl}_{3}\right)$

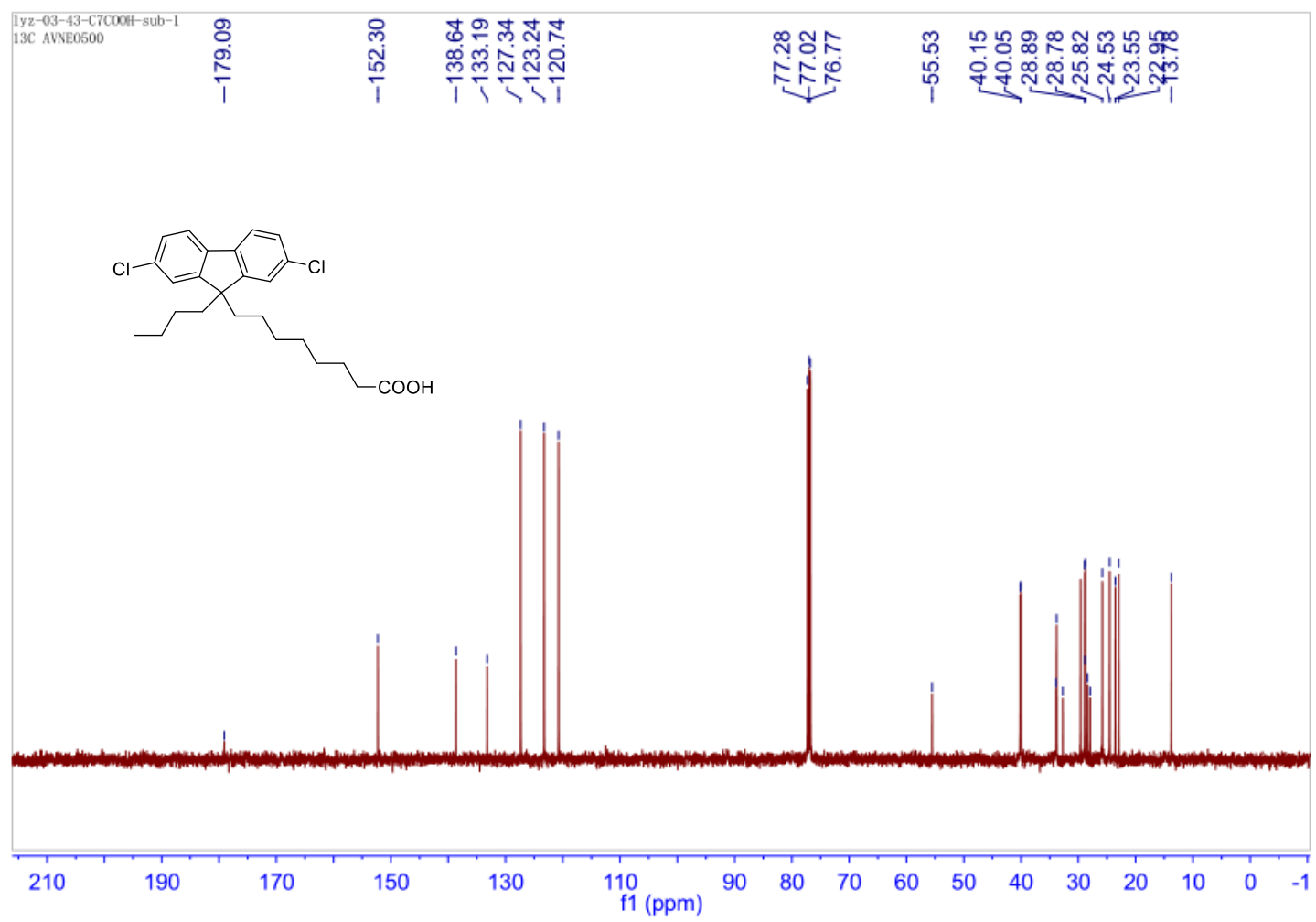


${ }^{1} \mathrm{H}$ NMR (500 MHz, $\mathrm{CDCl}_{3}$ )

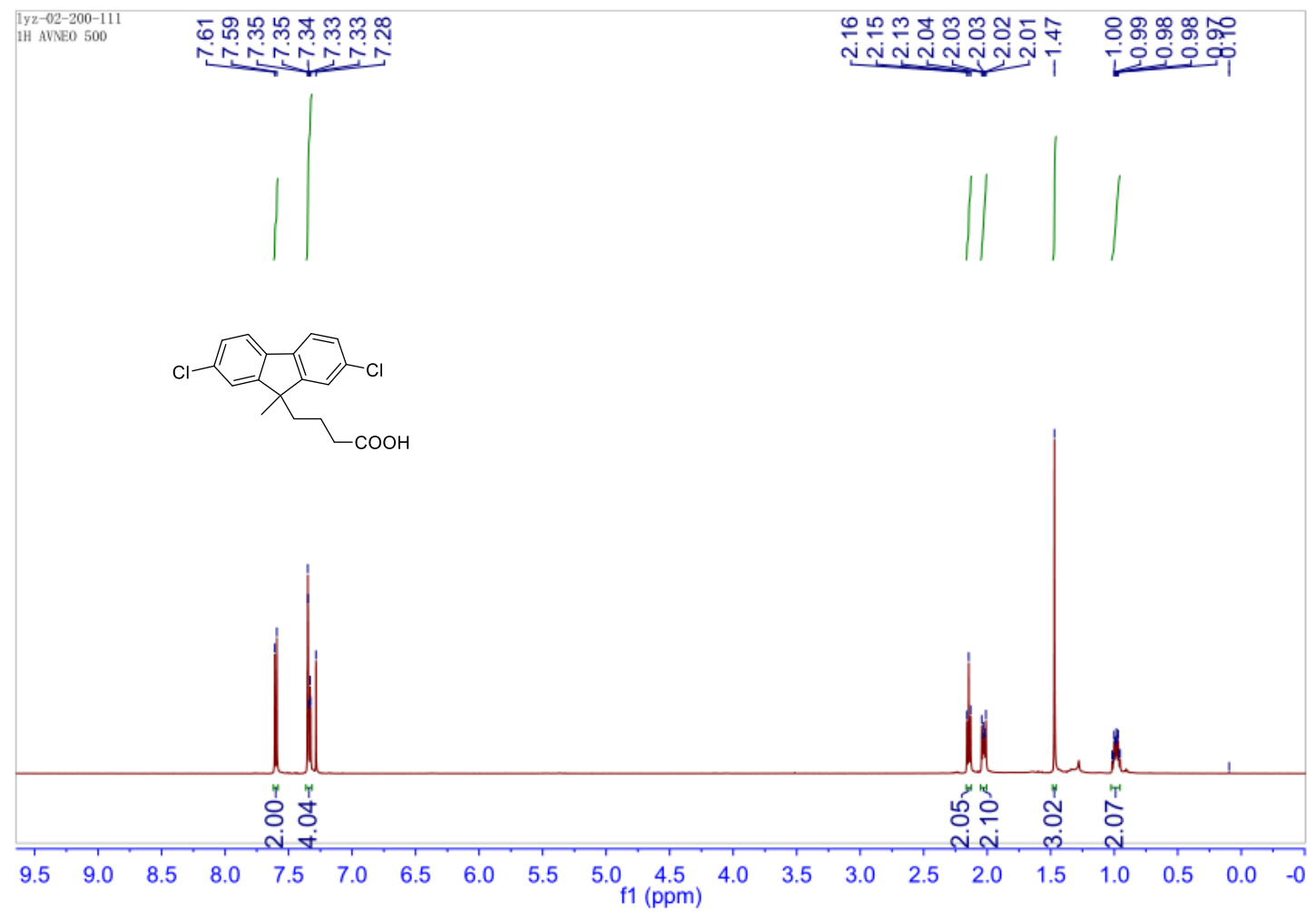

${ }^{13} \mathrm{C} \mathrm{NMR}\left(126 \mathrm{MHz}, \mathrm{CDCl}_{3}\right)$

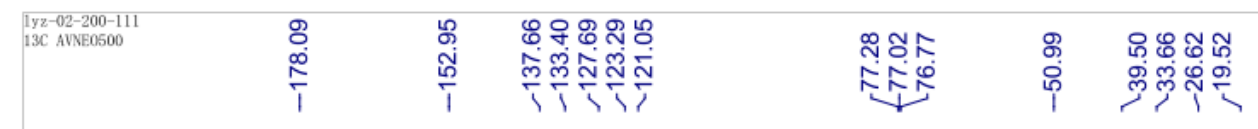
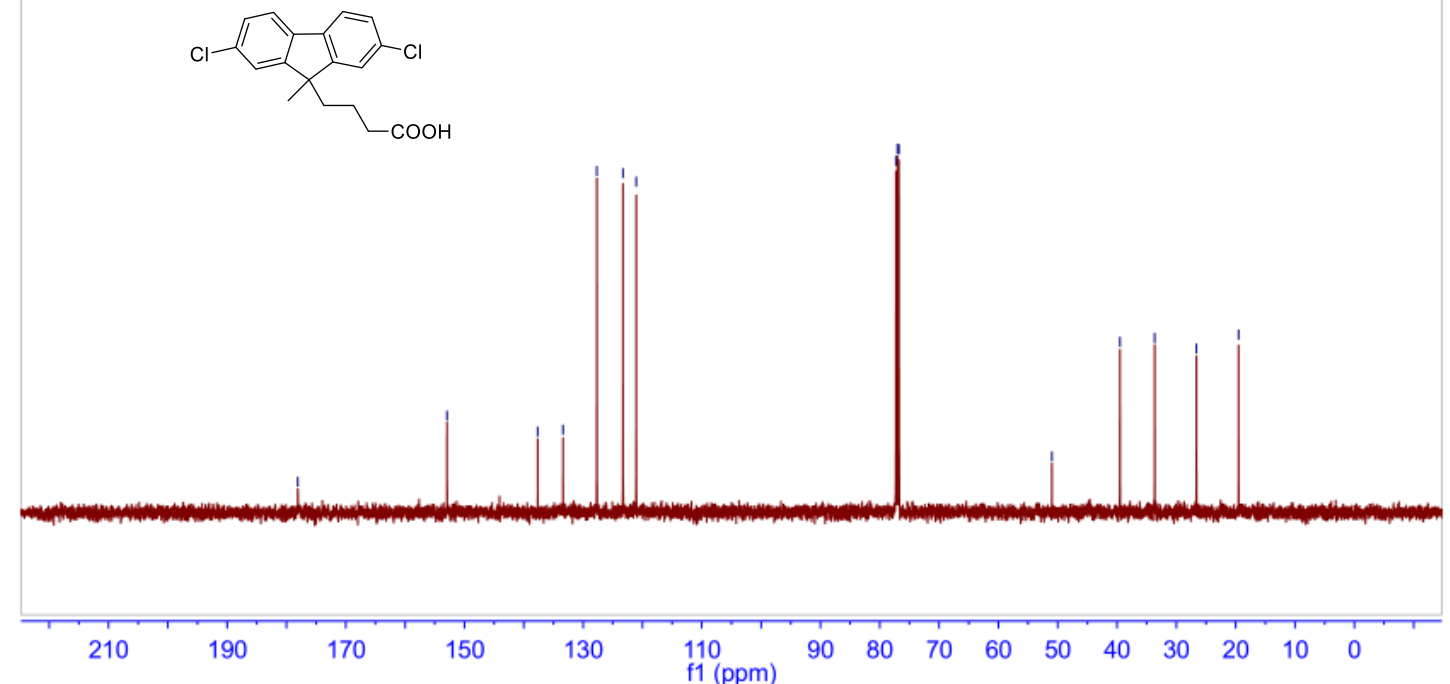
${ }^{1} \mathrm{H}$ NMR $\left(500 \mathrm{MHz}, \mathrm{CDCl}_{3}\right)$

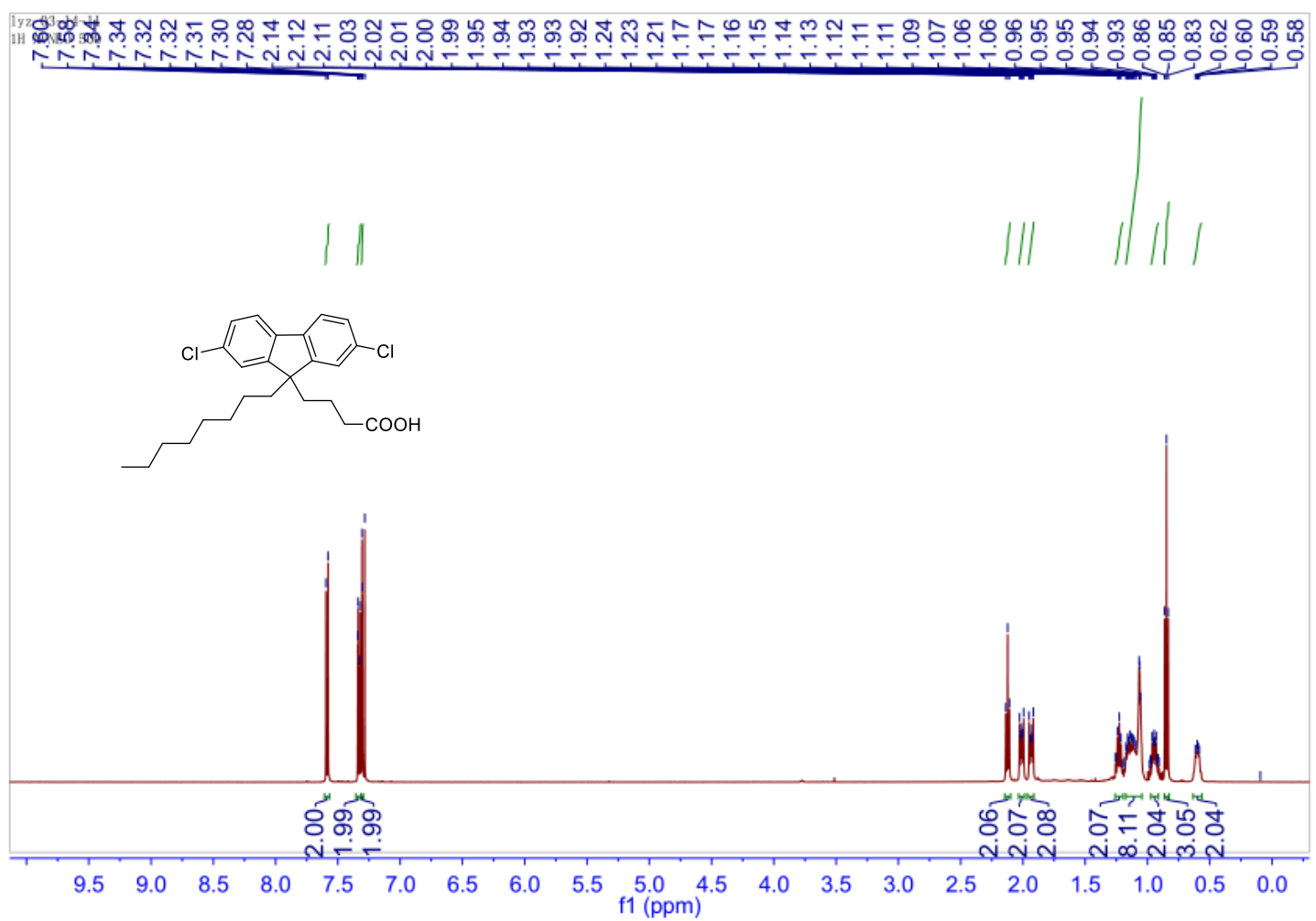

${ }^{13} \mathrm{C}$ NMR (126 MHz, $\mathrm{CDCl}_{3}$ )

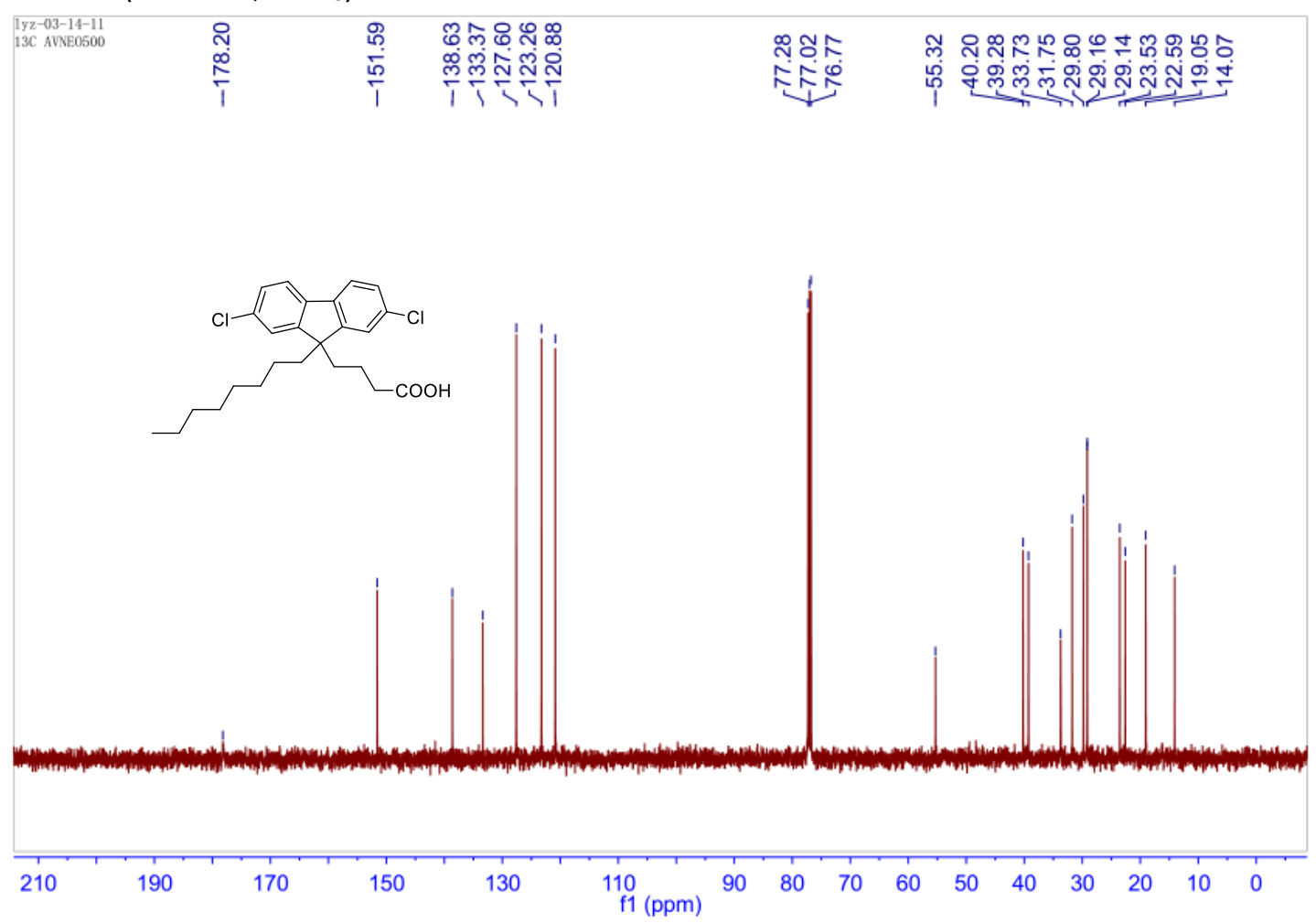


${ }^{1} \mathrm{H}$ NMR (500 MHz, CDCl 3 )

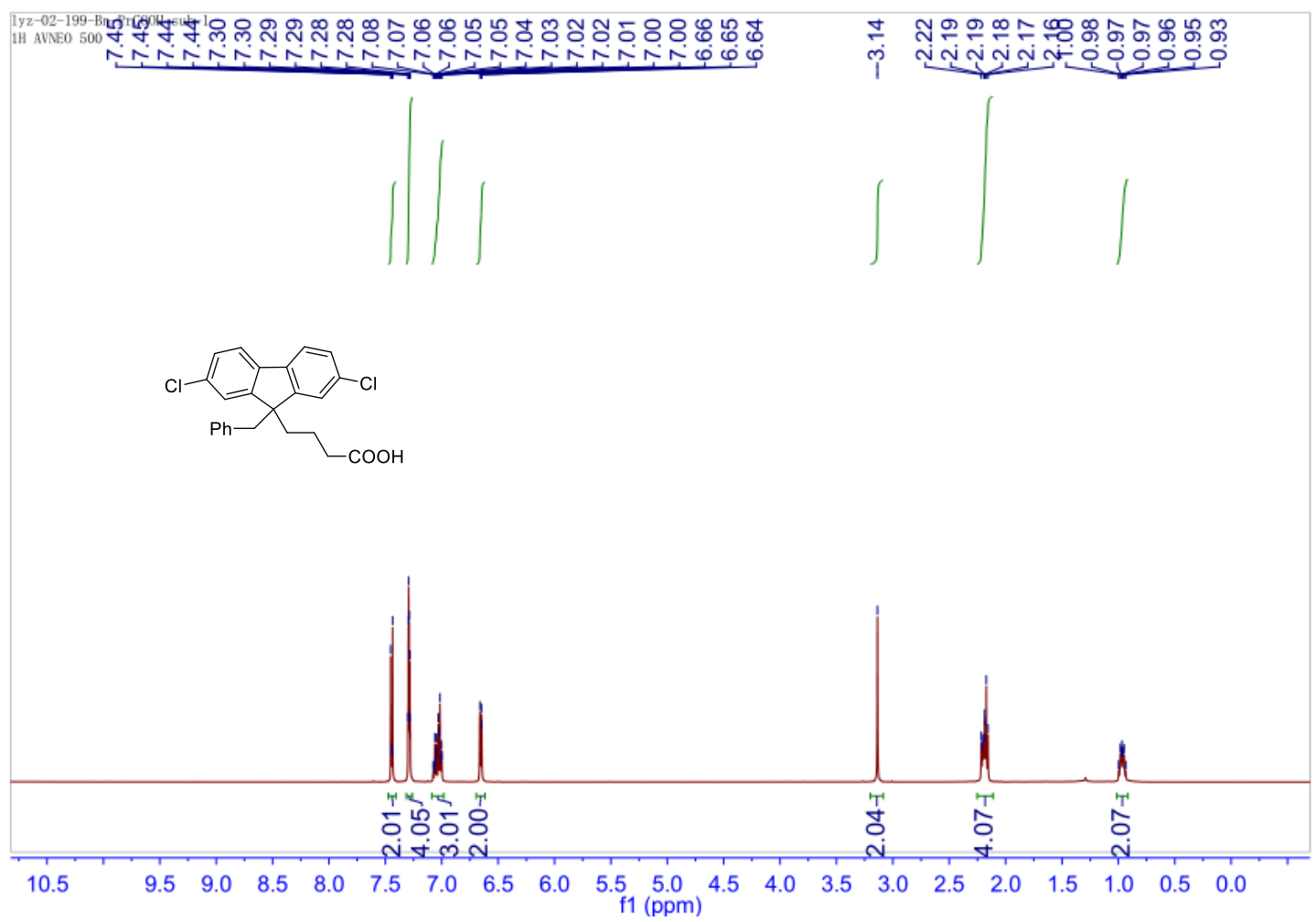

${ }^{13} \mathrm{C}$ NMR (126 MHz, $\left.\mathrm{CDCl}_{3}\right)$

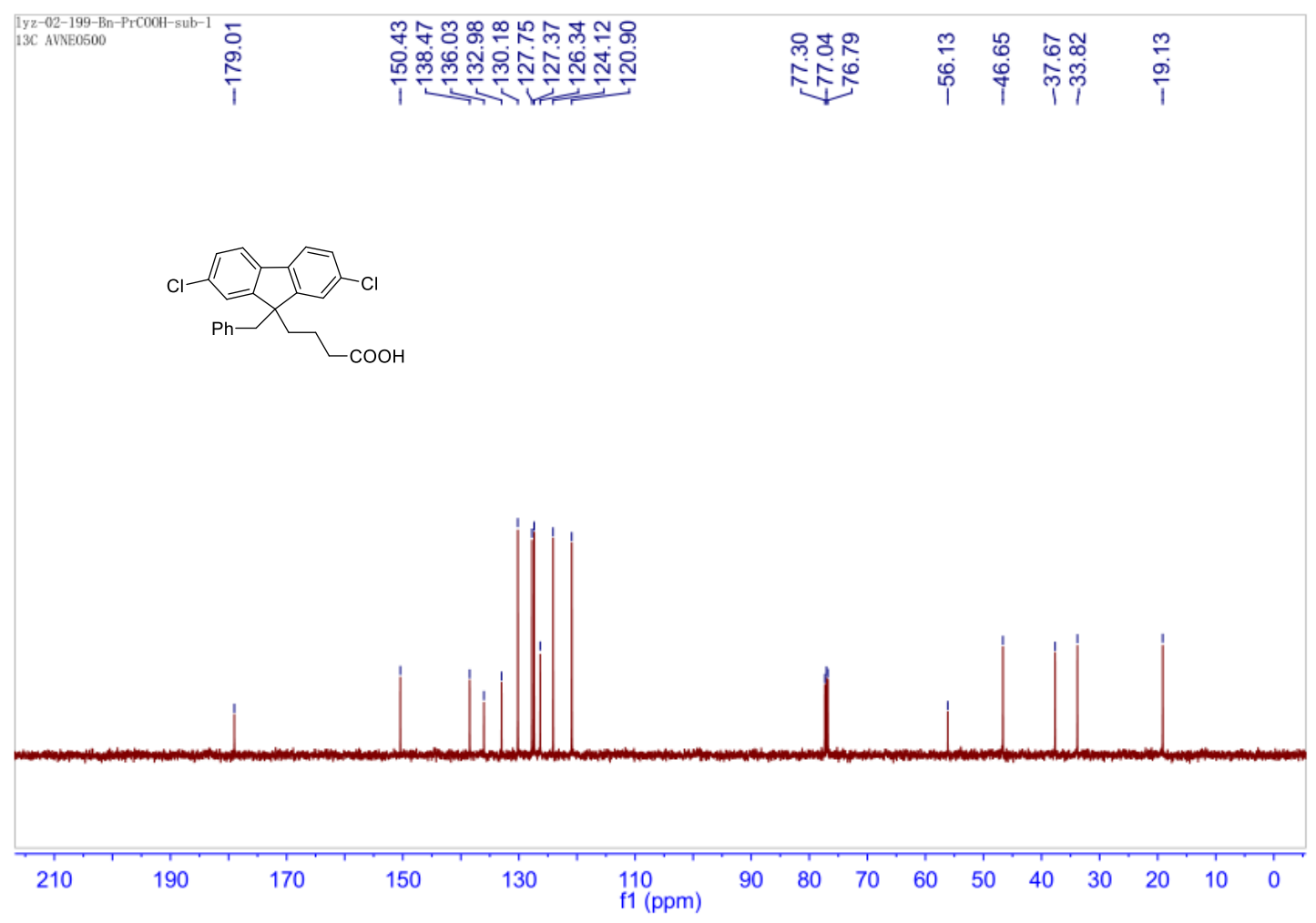


${ }^{1} \mathrm{H}$ NMR (500 MHz, $\mathrm{CDCl}_{3}$ )

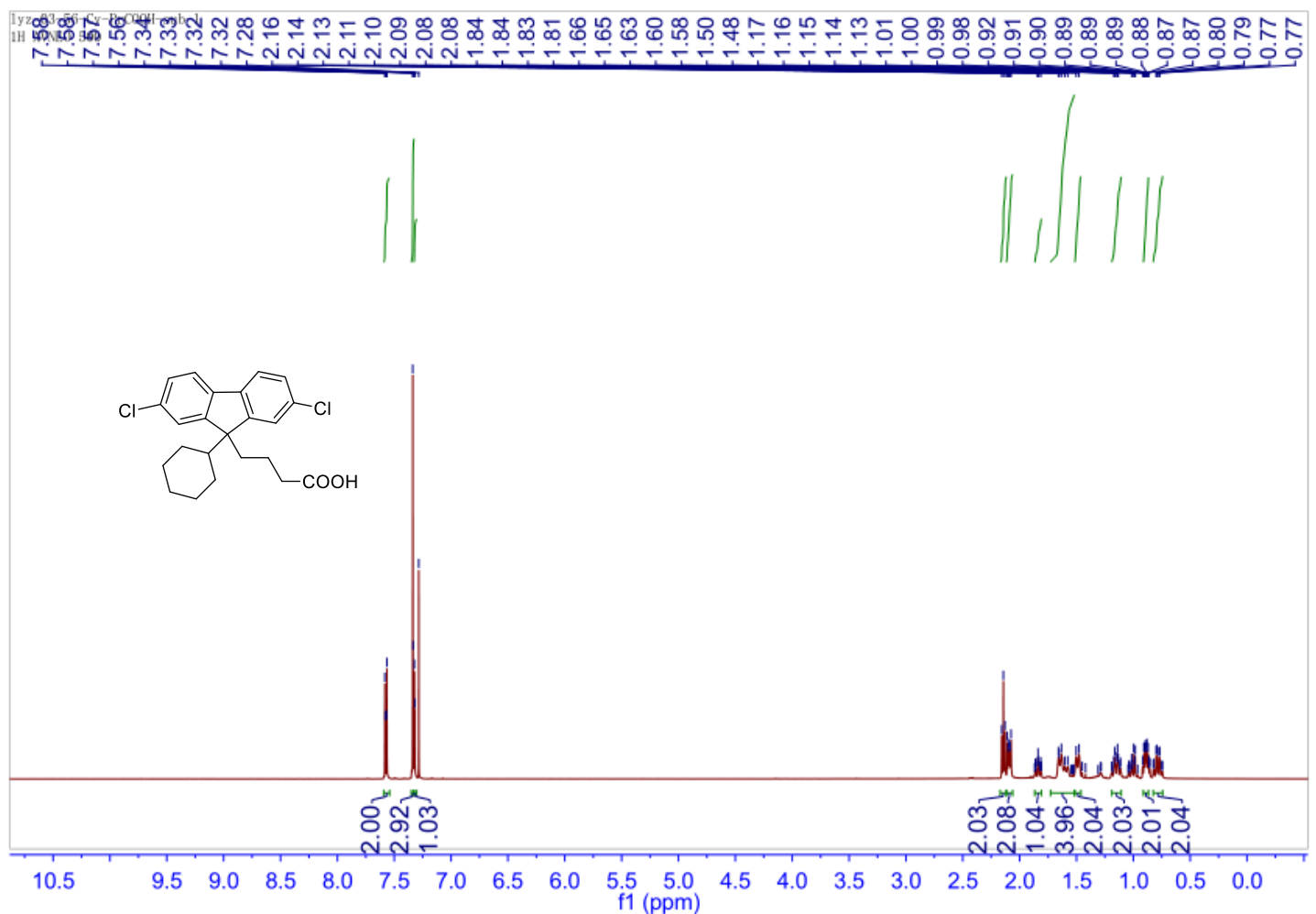

${ }^{13} \mathrm{C}$ NMR (126 MHz, $\left.\mathrm{CDCl}_{3}\right)$

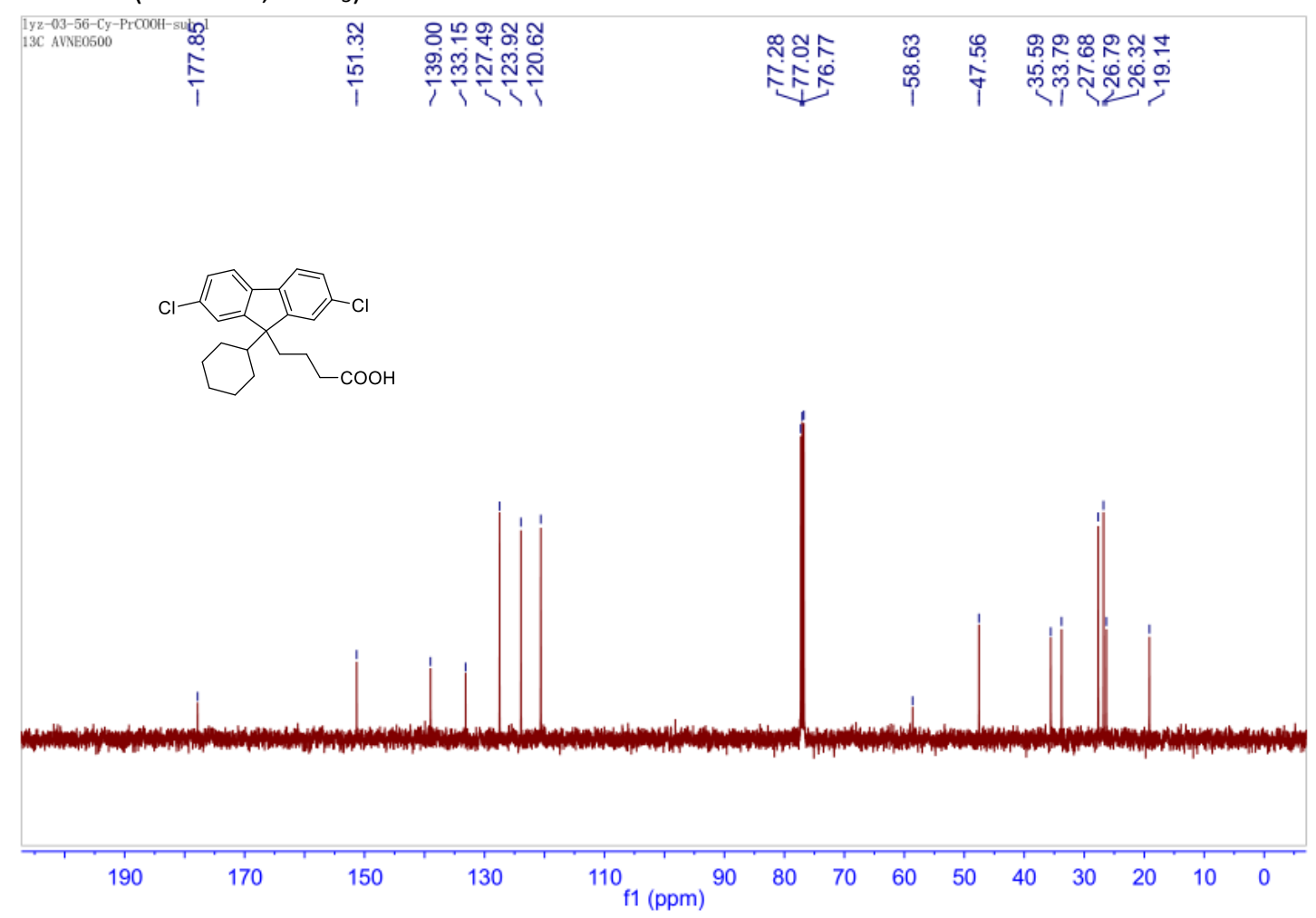




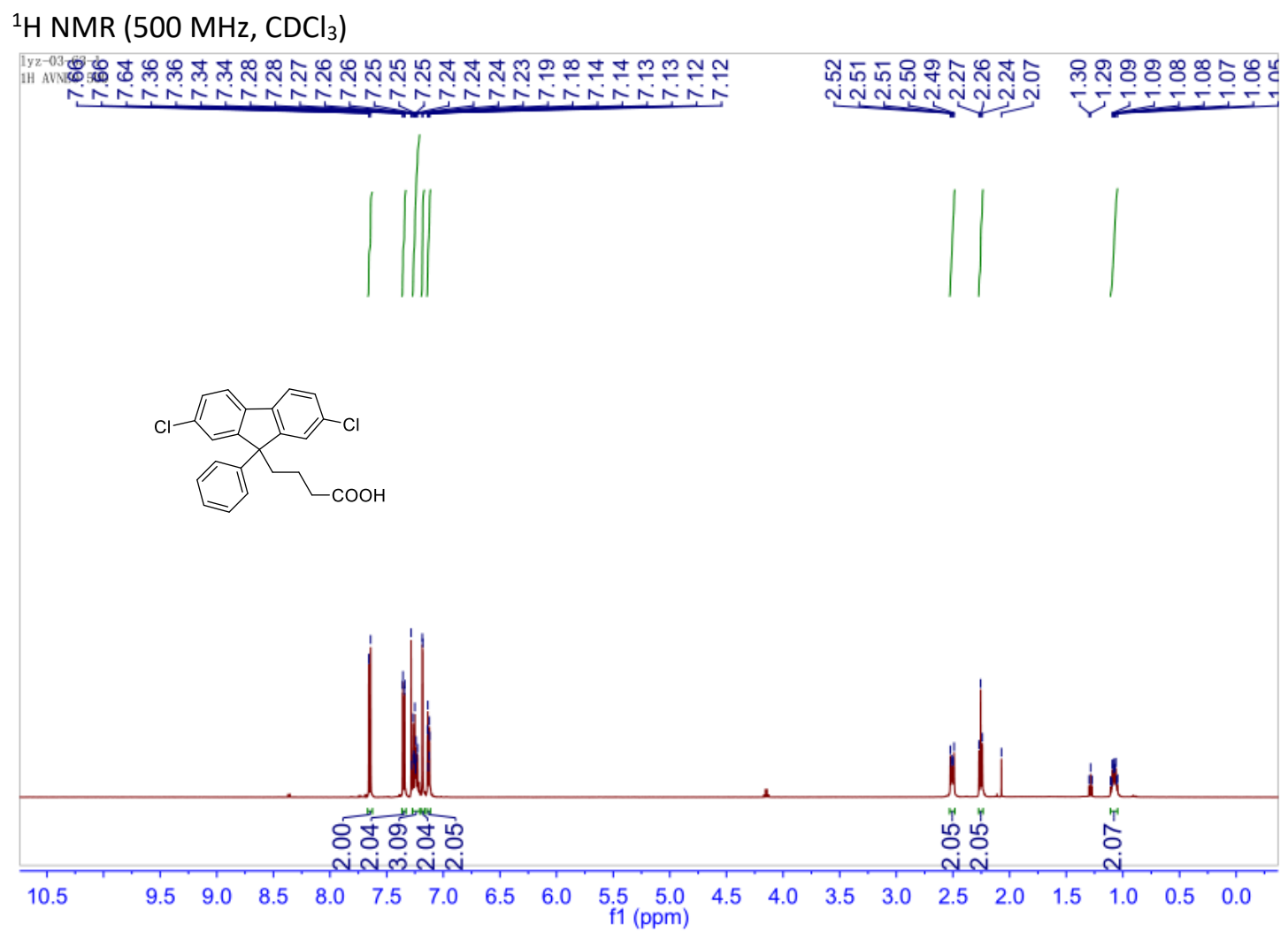

${ }^{13} \mathrm{C}$ NMR (126 MHz, $\left.\mathrm{CDCl}_{3}\right)$

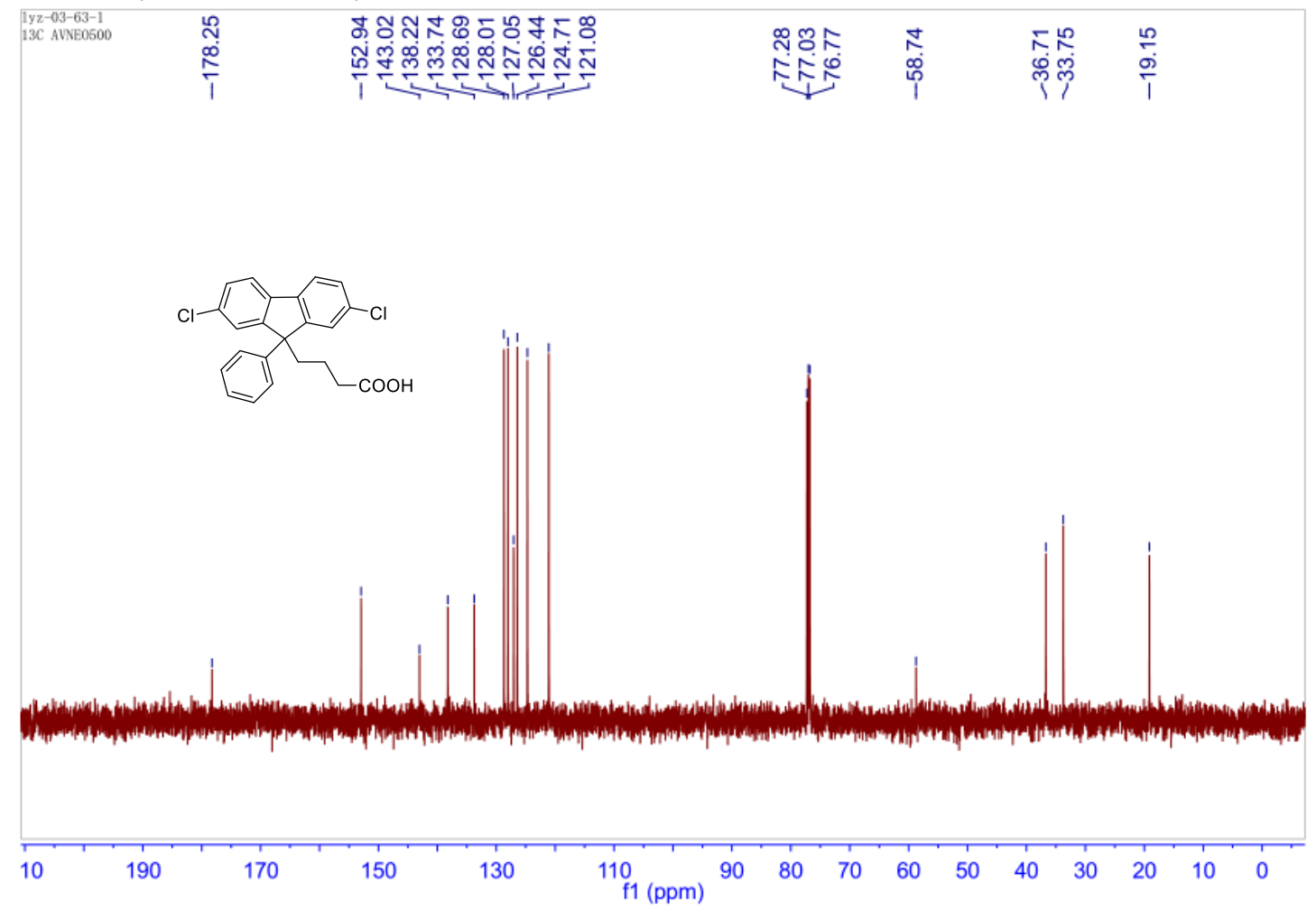


${ }^{1} \mathrm{H}$ NMR $\left(500 \mathrm{MHz}, \mathrm{CDCl}_{3}\right)$

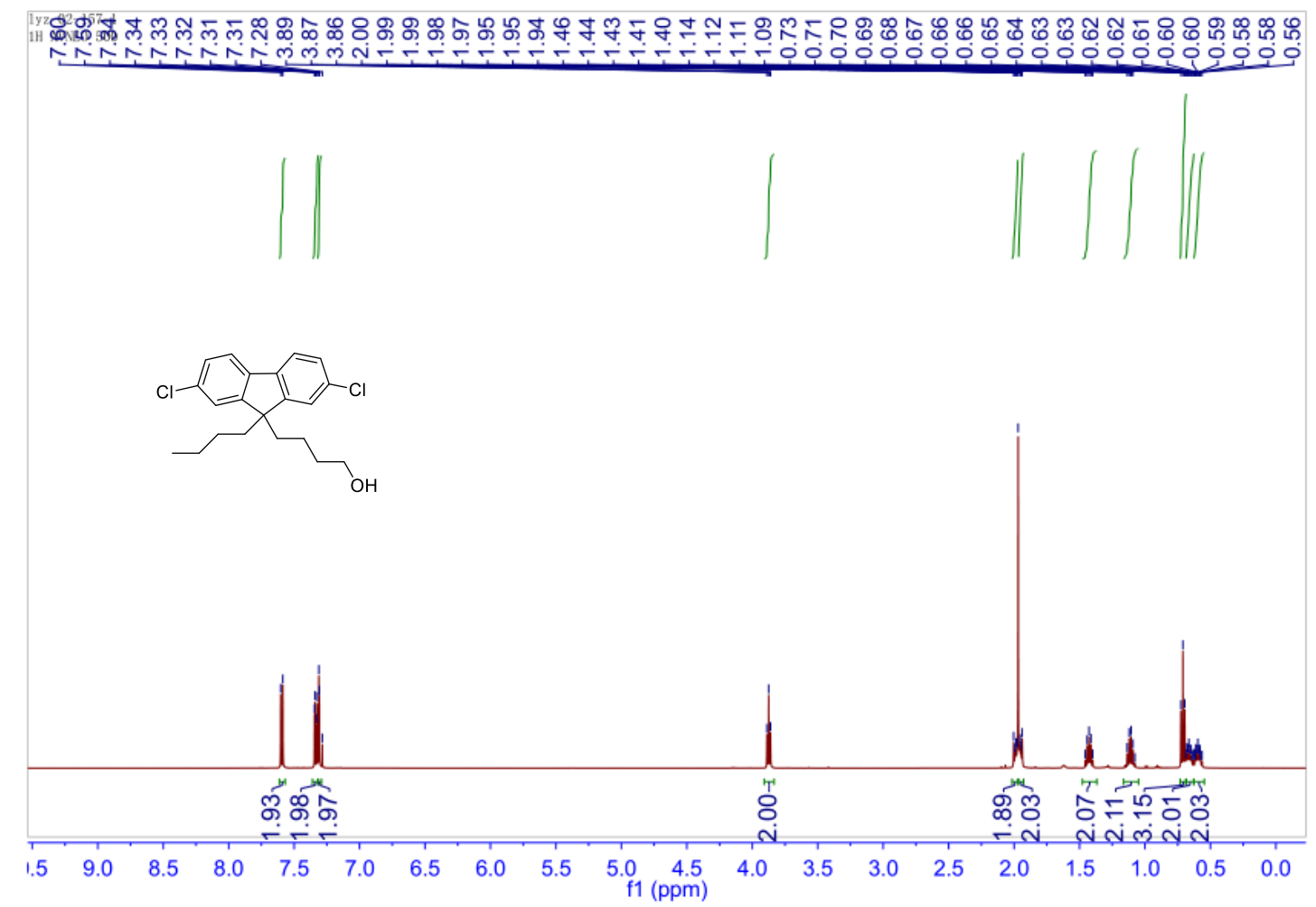

${ }^{13} \mathrm{C}$ NMR (126 MHz, $\left.\mathrm{CDCl}_{3}\right)$

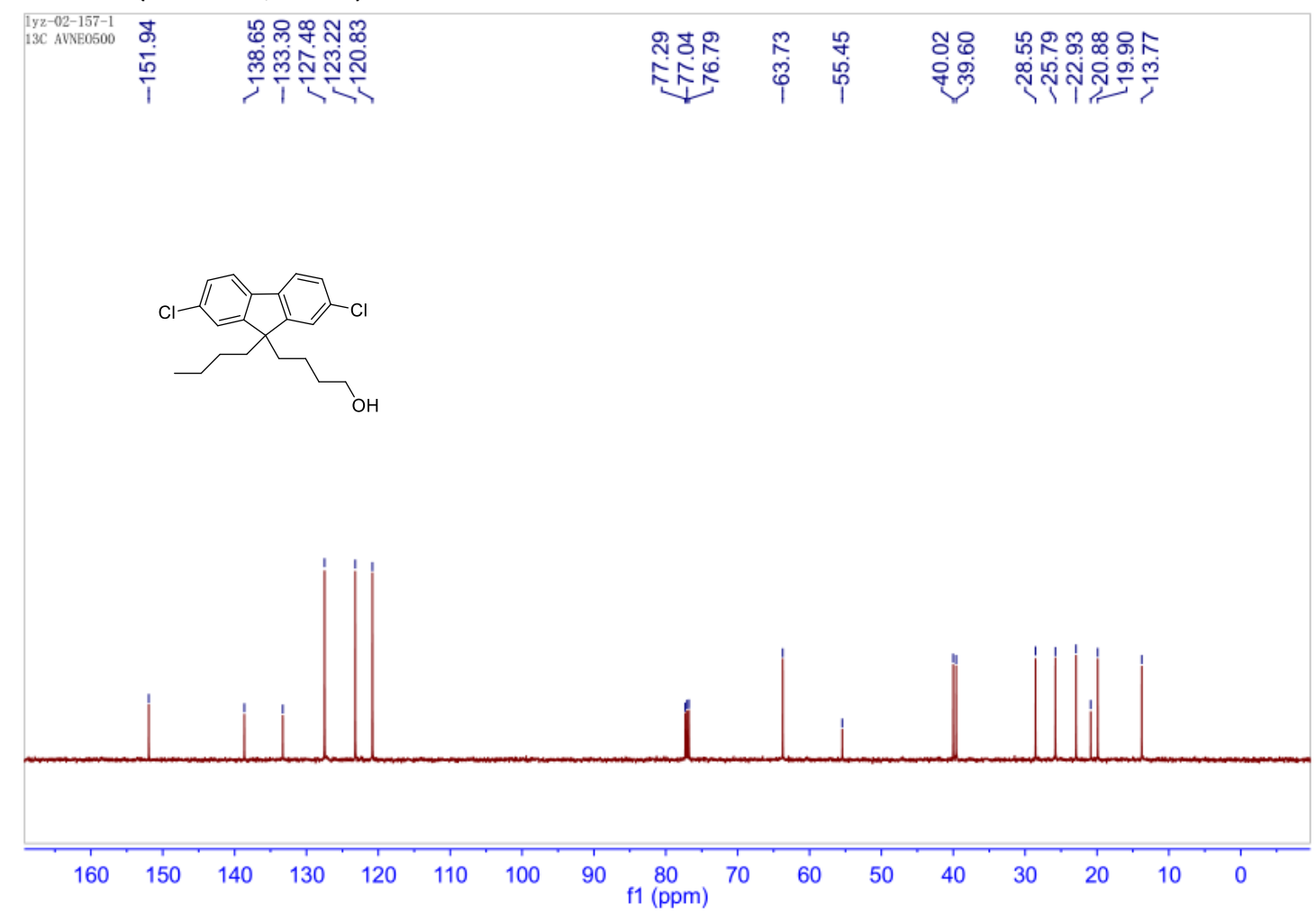




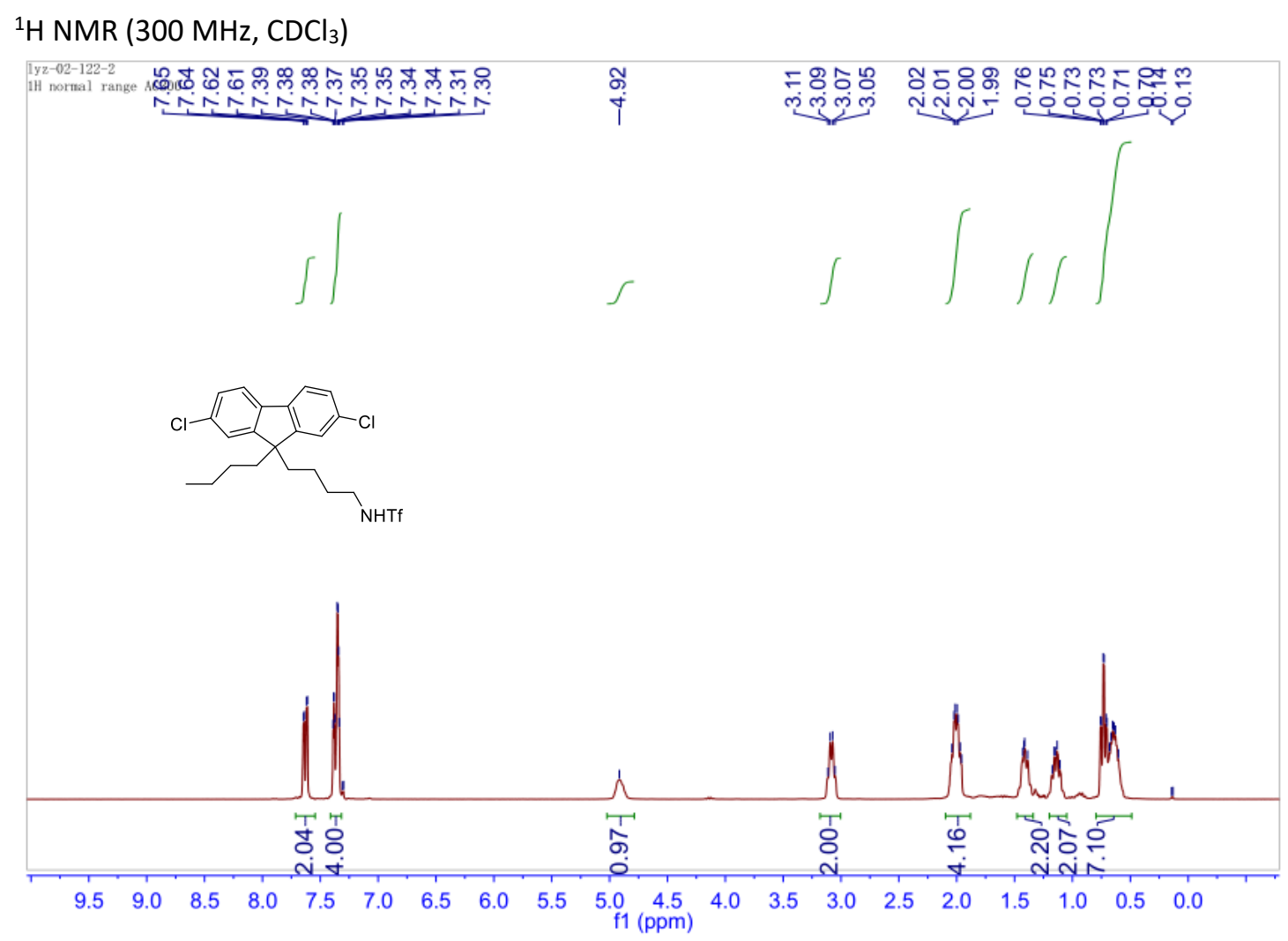

${ }^{13} \mathrm{C} \mathrm{NMR}\left(75 \mathrm{MHz}, \mathrm{CDCl}_{3}\right)$

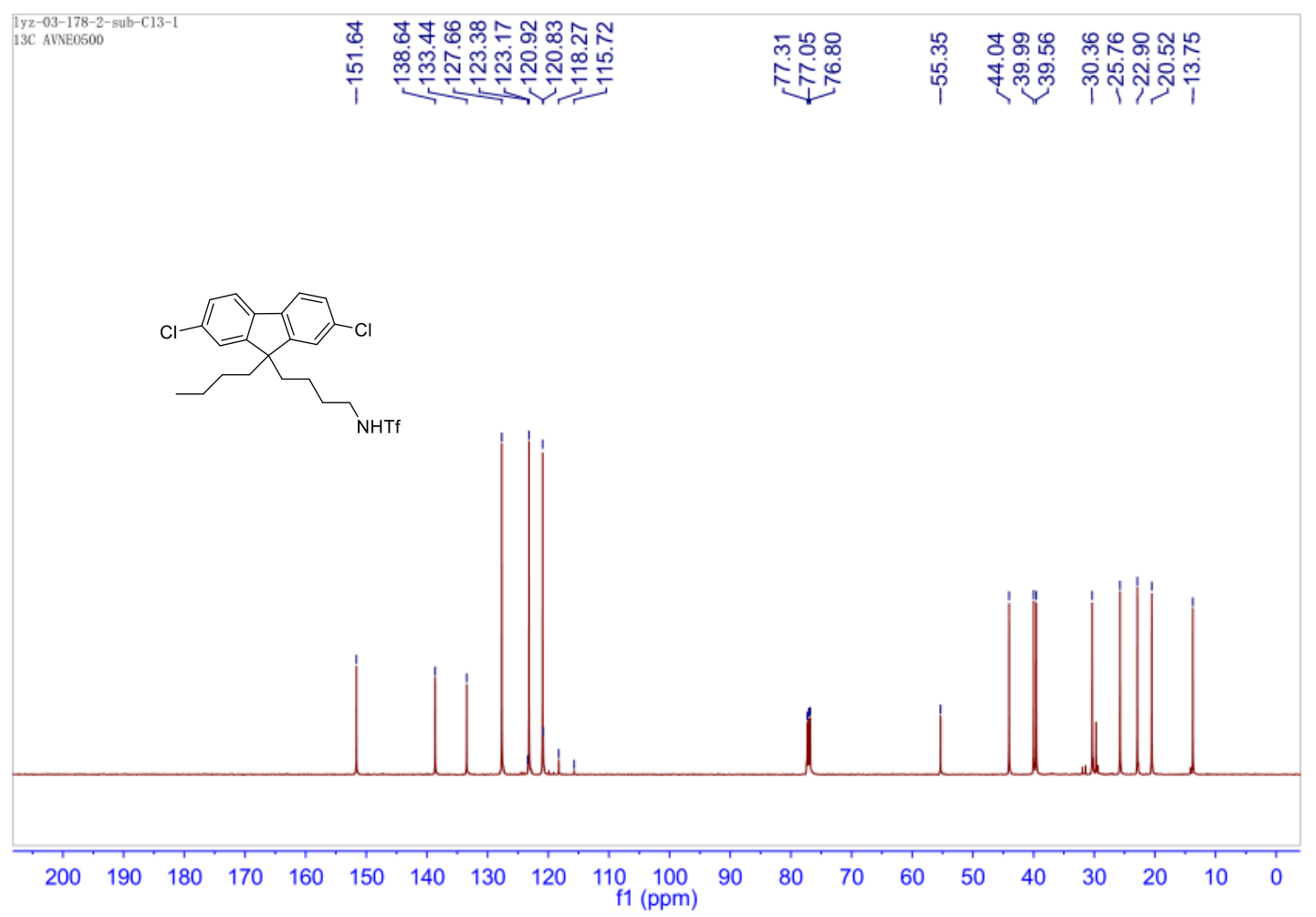


${ }^{19} \mathrm{~F}$ NMR (471 MHz, $\left.\mathrm{CDCl}_{3}\right)$

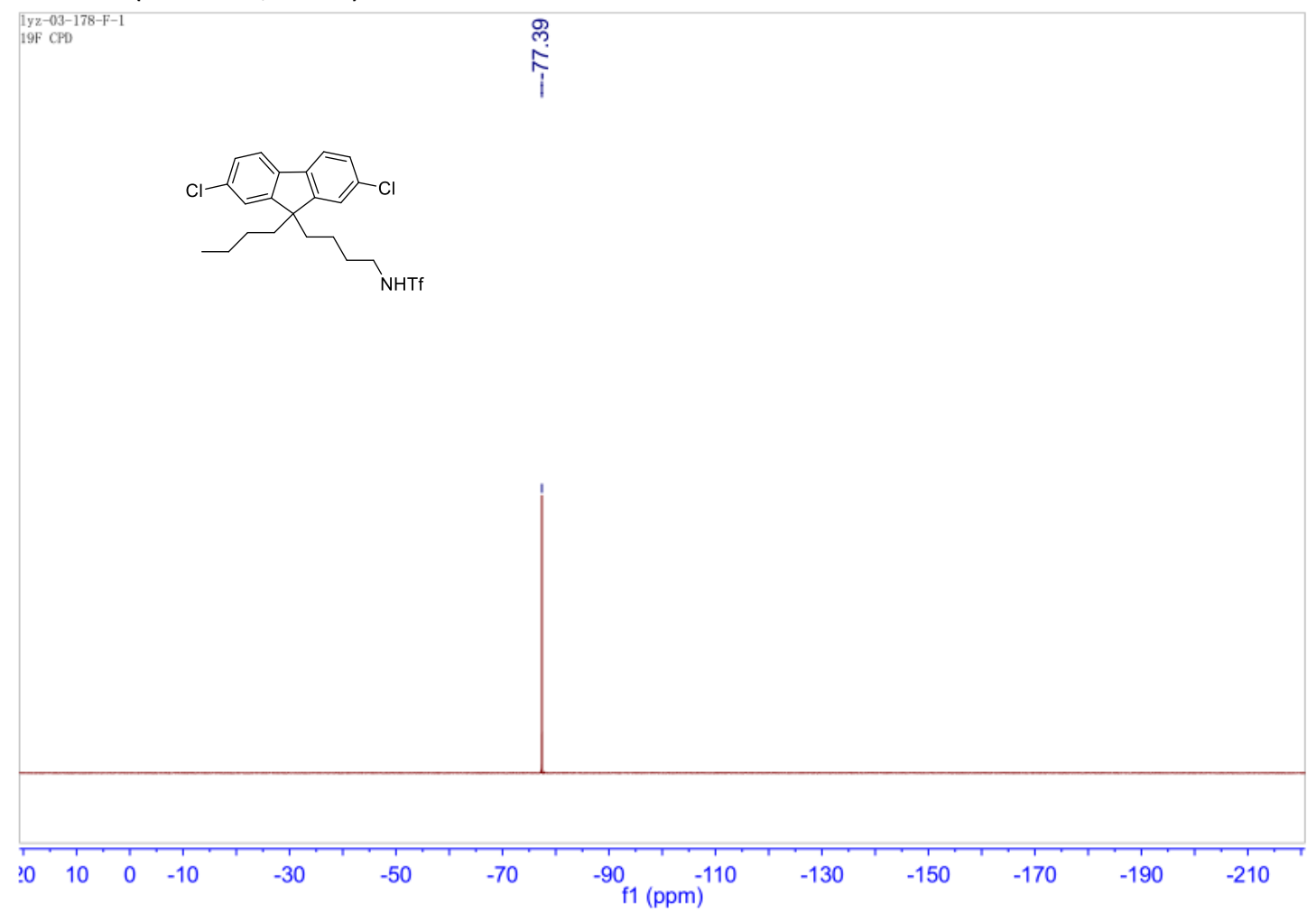


${ }^{1} \mathrm{H}$ NMR $\left(500 \mathrm{MHz}, \mathrm{CDCl}_{3}\right.$ )

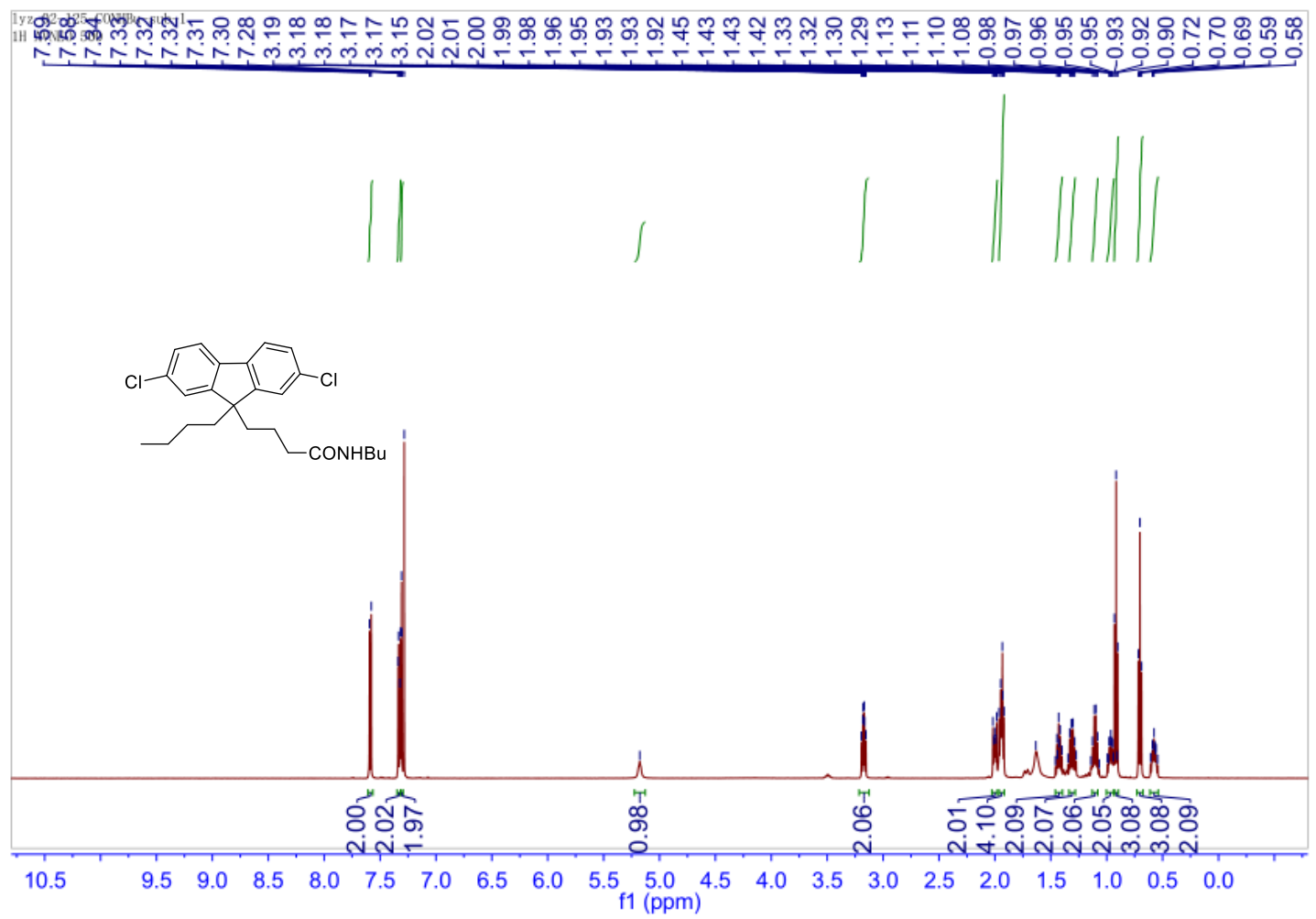

${ }^{13} \mathrm{C}$ NMR (126 MHz, $\mathrm{CDCl}_{3}$ )

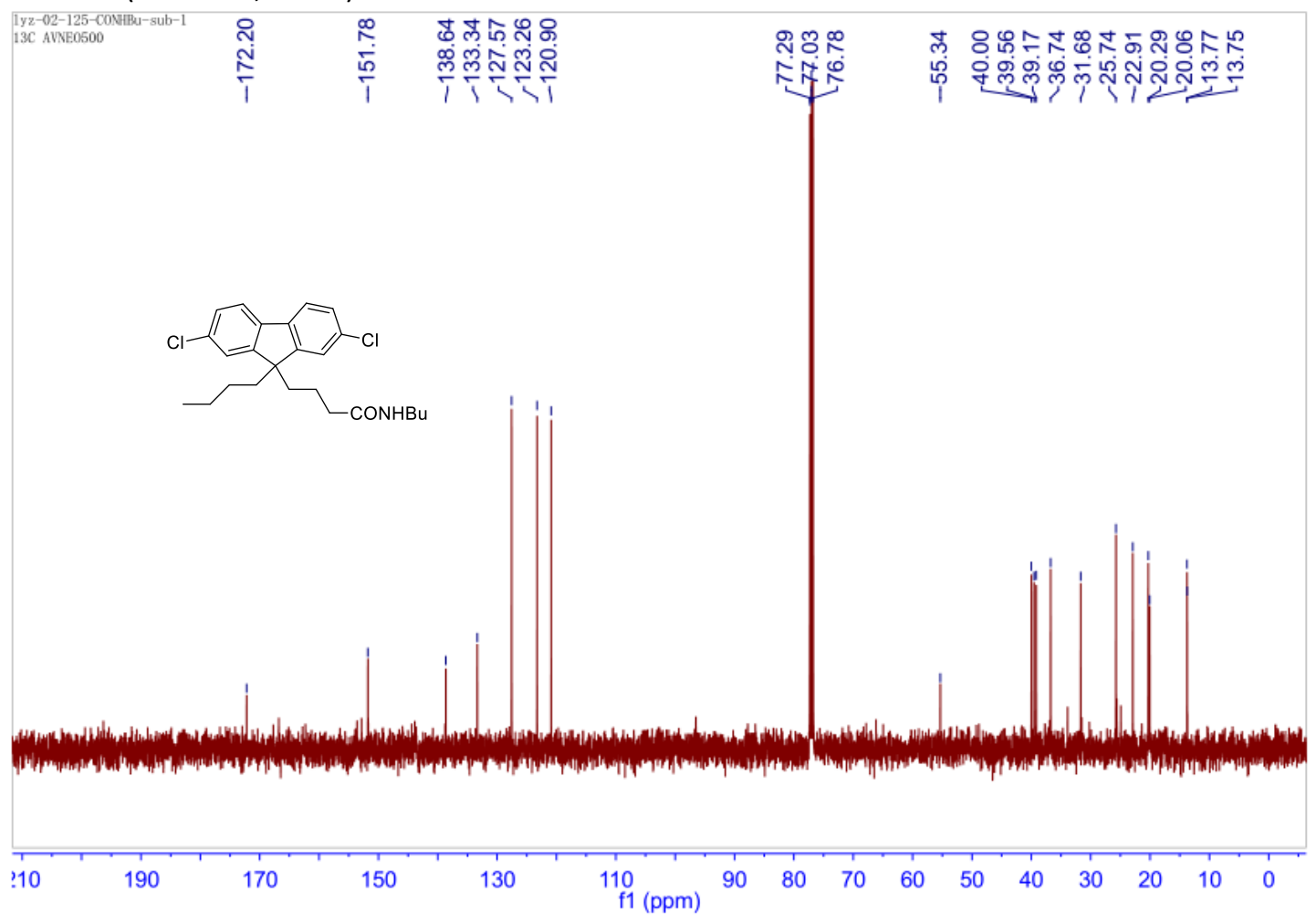




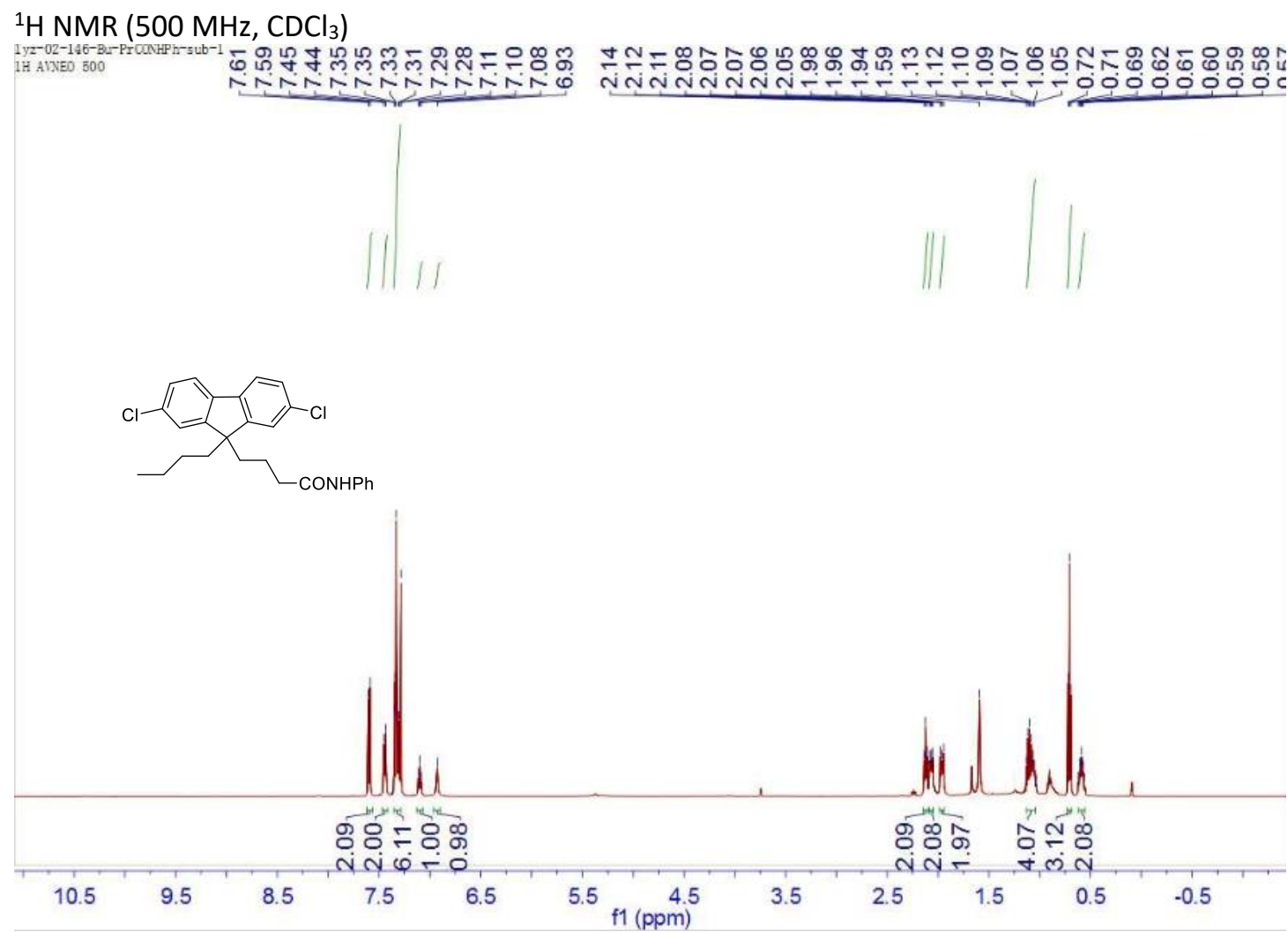

${ }^{13} \mathrm{C} \mathrm{NMR}\left(126 \mathrm{MHz}, \mathrm{CDCl}_{3}\right)$

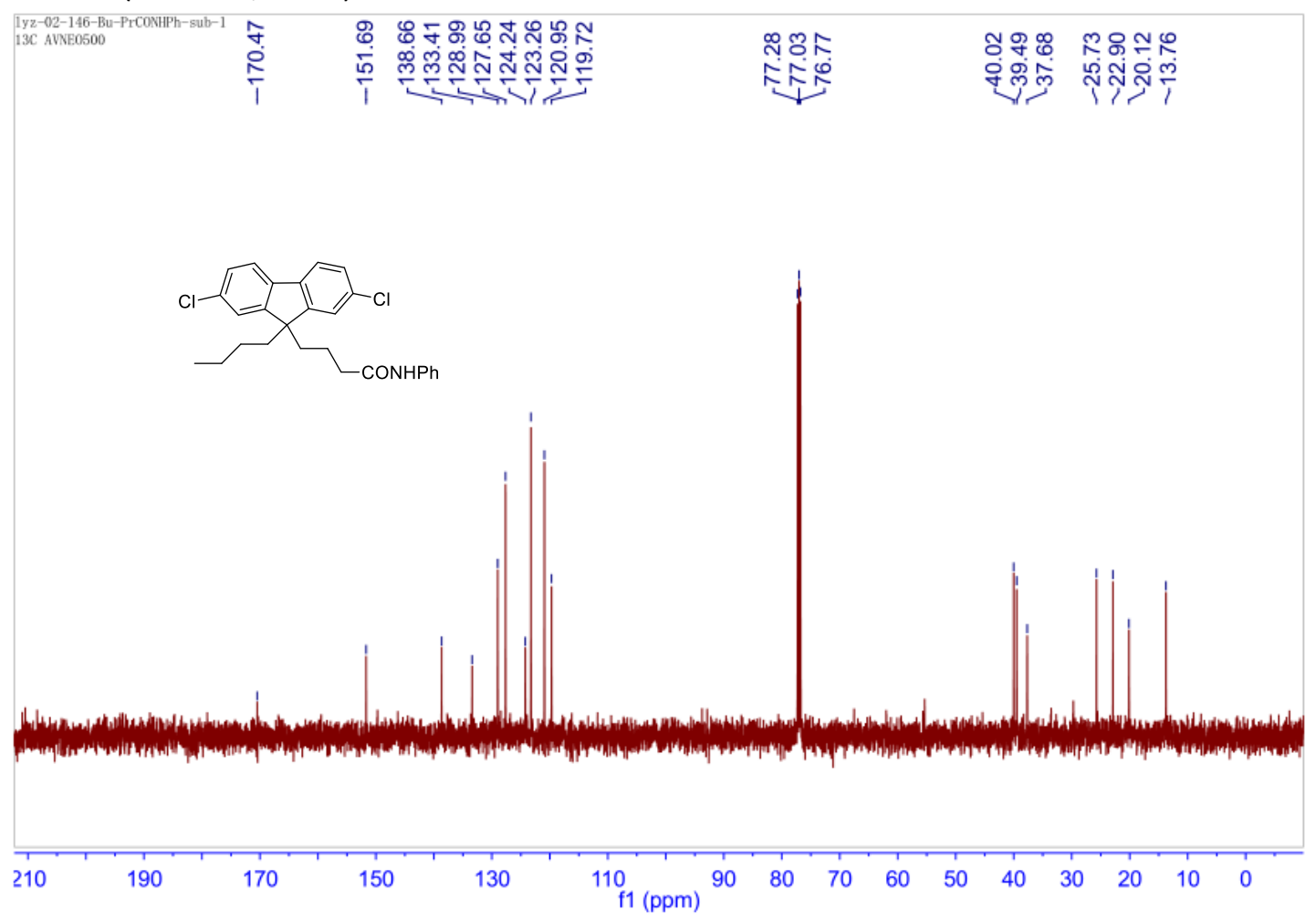


${ }^{1} \mathrm{H}$ NMR (500 MHz, $\mathrm{CDCl}_{3}$ )

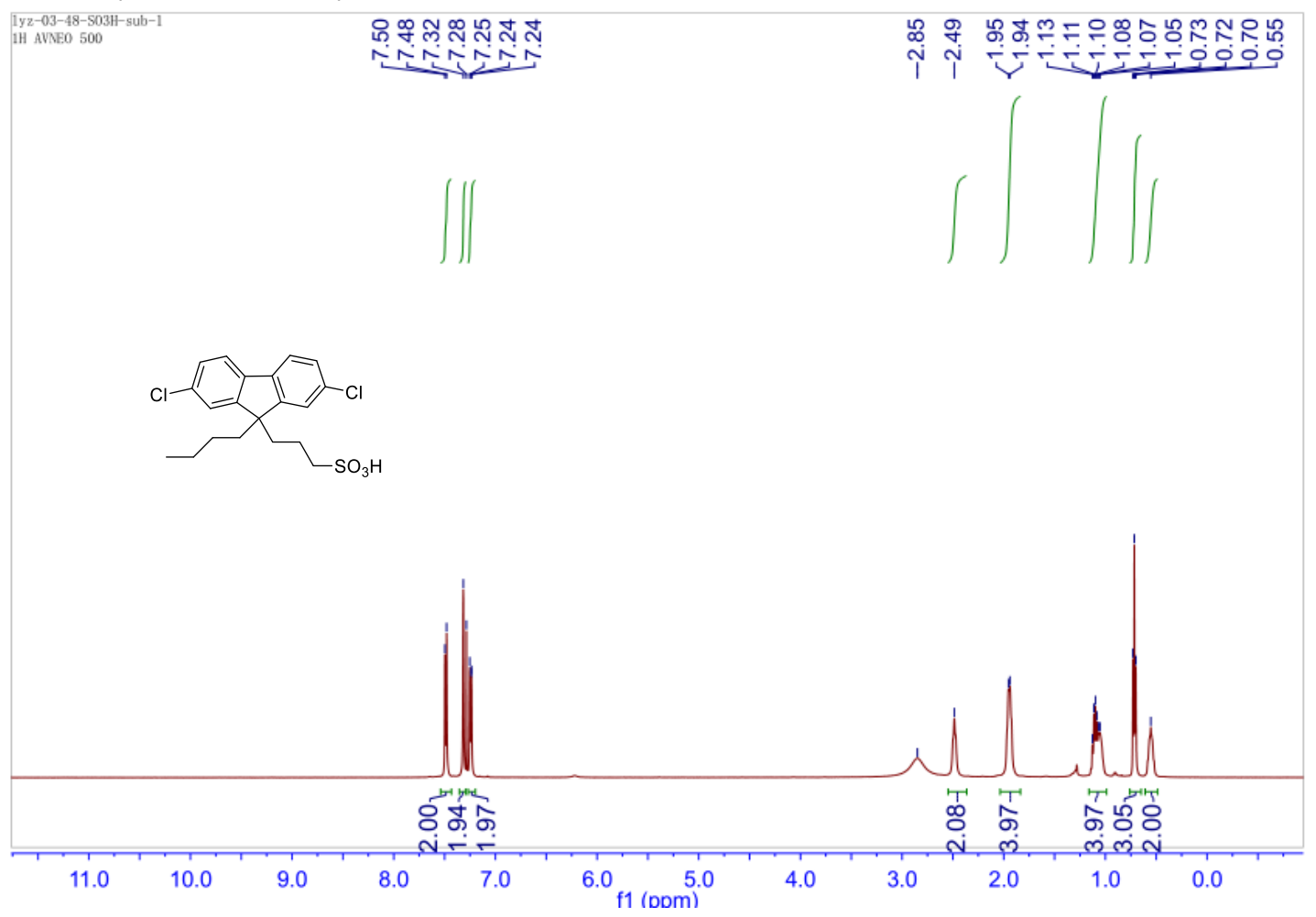

${ }^{13} \mathrm{C}$ NMR $\left(126 \mathrm{MHz}, \mathrm{CDCl}_{3}\right)$

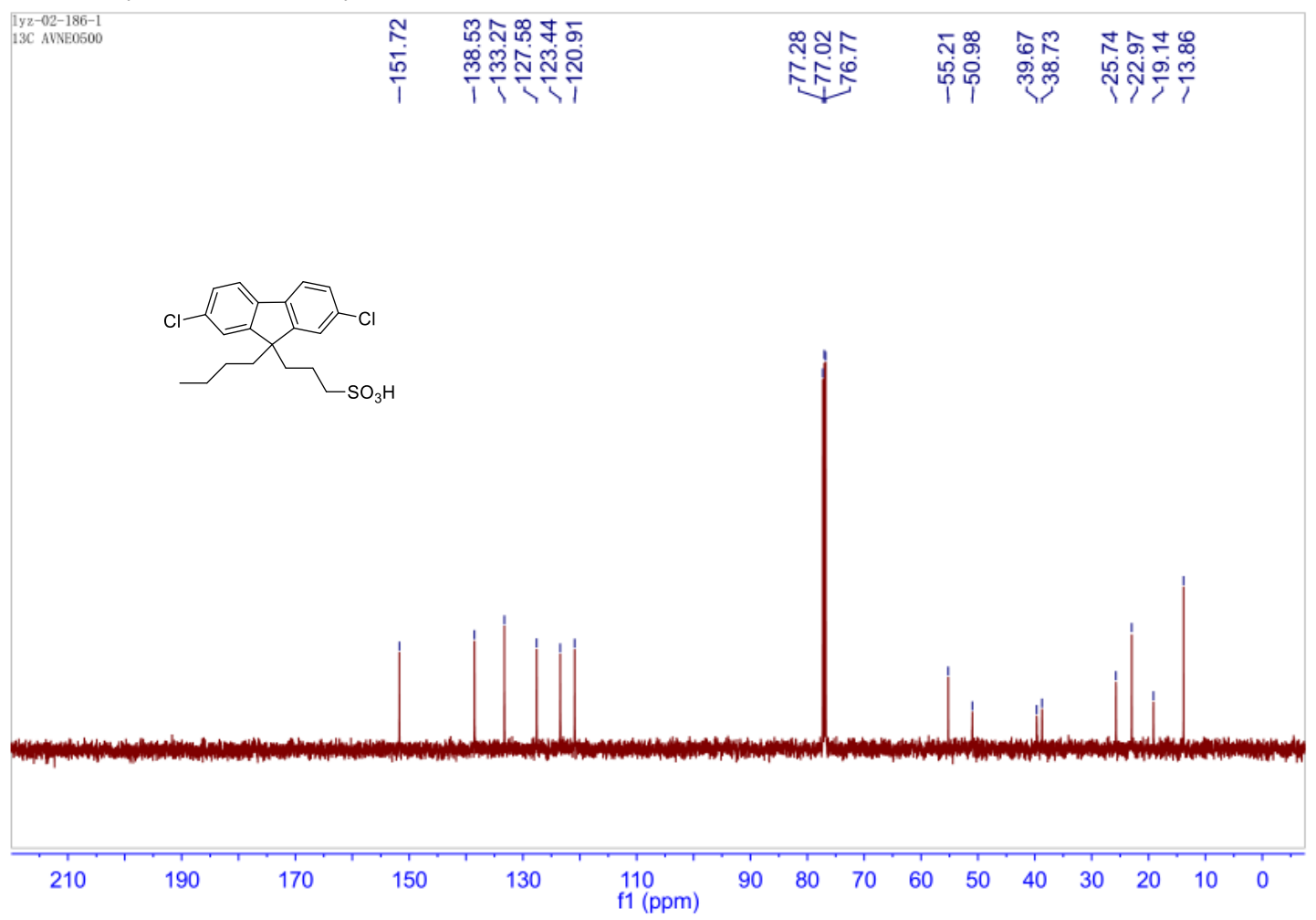


${ }^{1} \mathrm{H}$ NMR (500 MHz, $\mathrm{CDCl}_{3}$ )

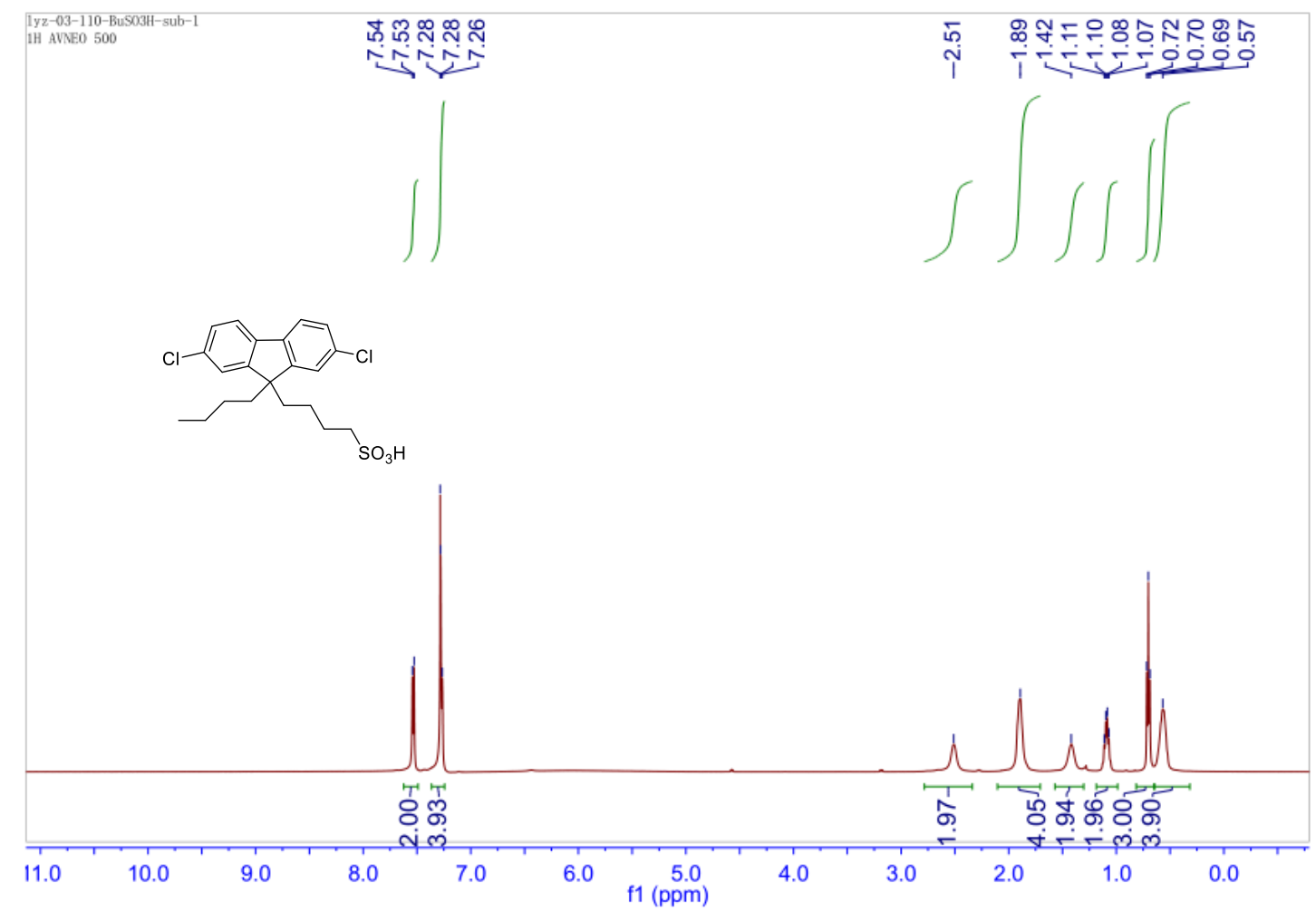

${ }^{13} \mathrm{C}$ NMR $\left(126 \mathrm{MHz}, \mathrm{CDCl}_{3}\right)$

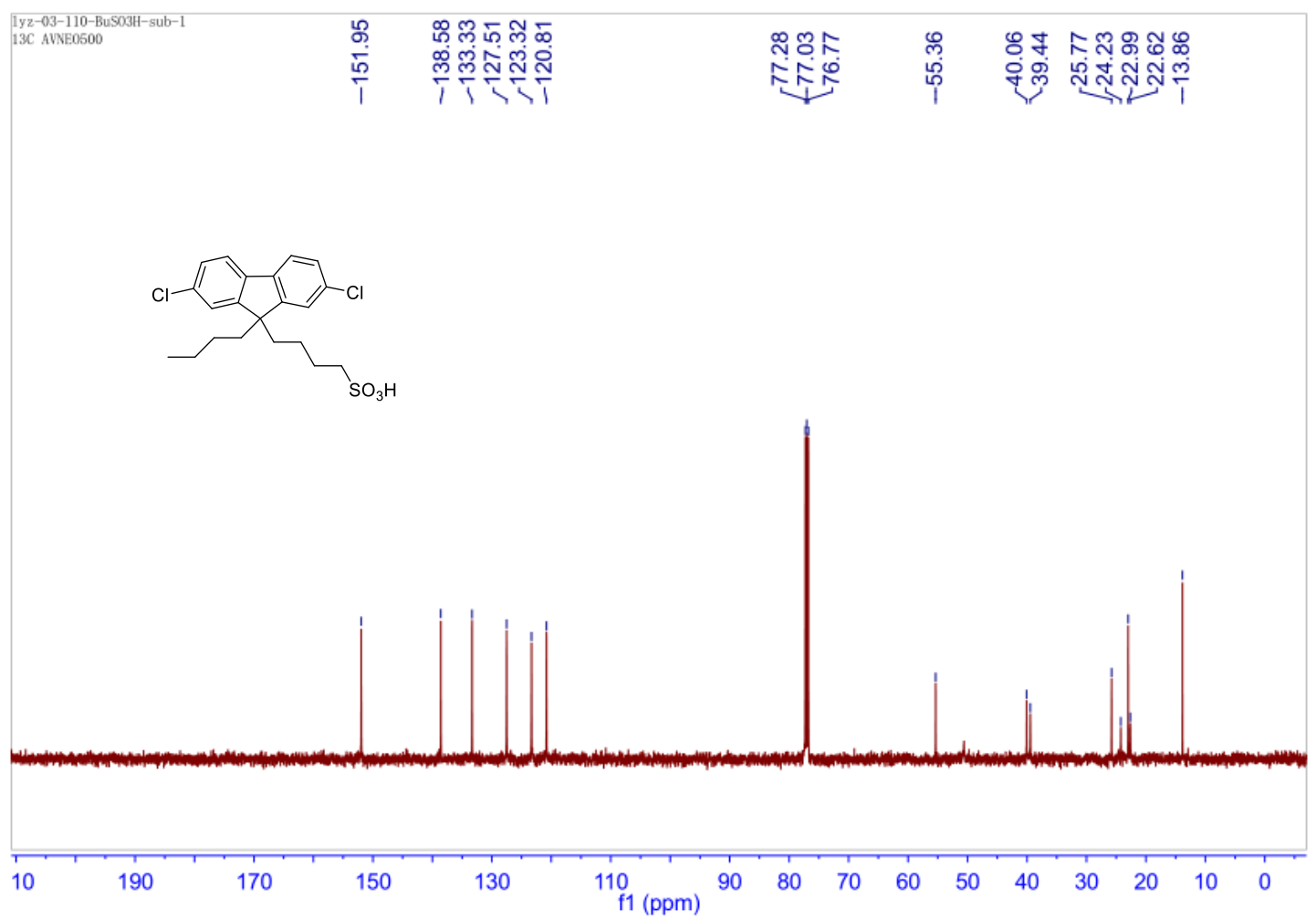


${ }^{1} \mathrm{H}$ NMR $\left(500 \mathrm{MHz}, \mathrm{CDCl}_{3}\right)$

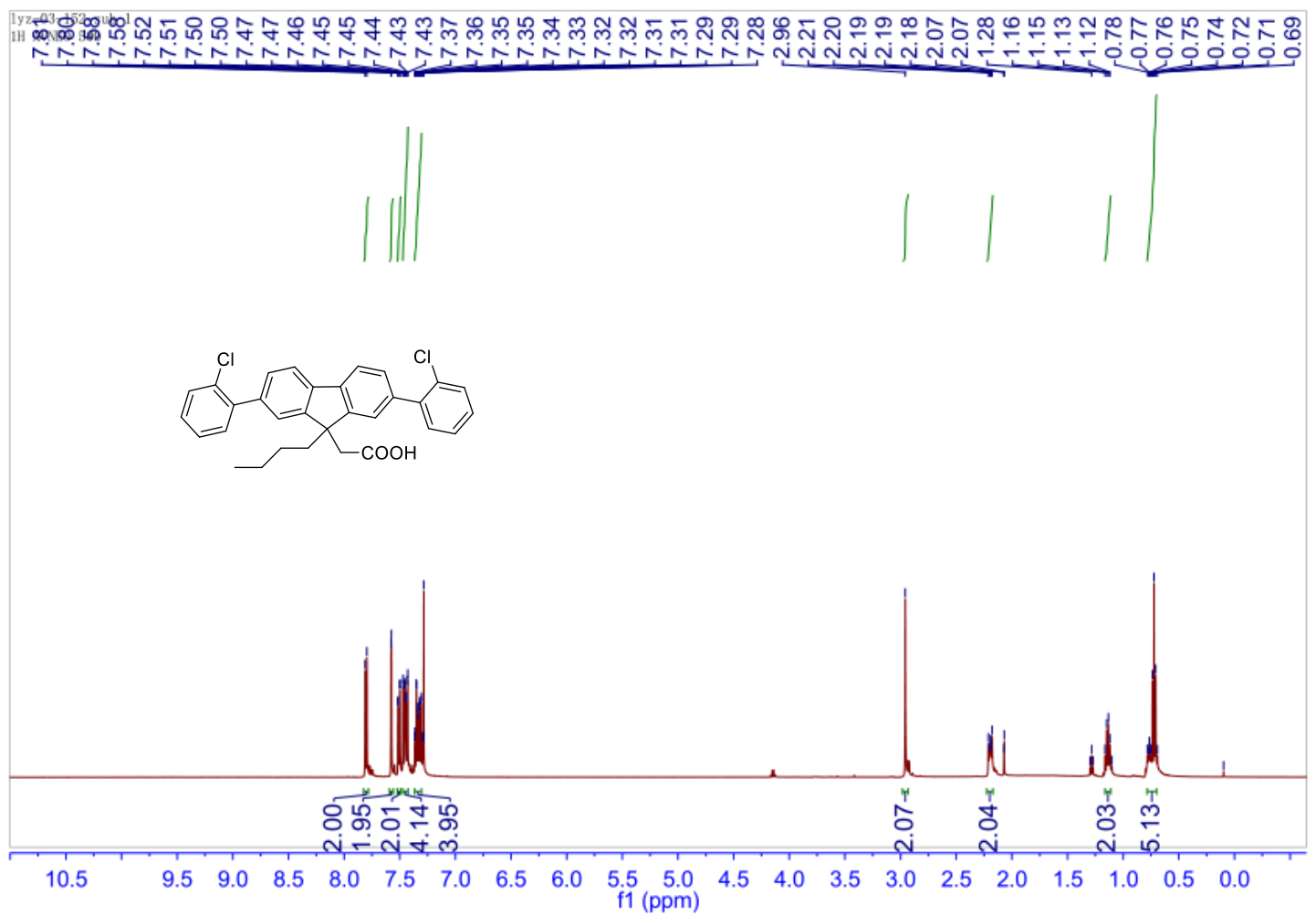

${ }^{13} \mathrm{C}$ NMR (126 MHz, $\left.\mathrm{CDCl}_{3}\right)$

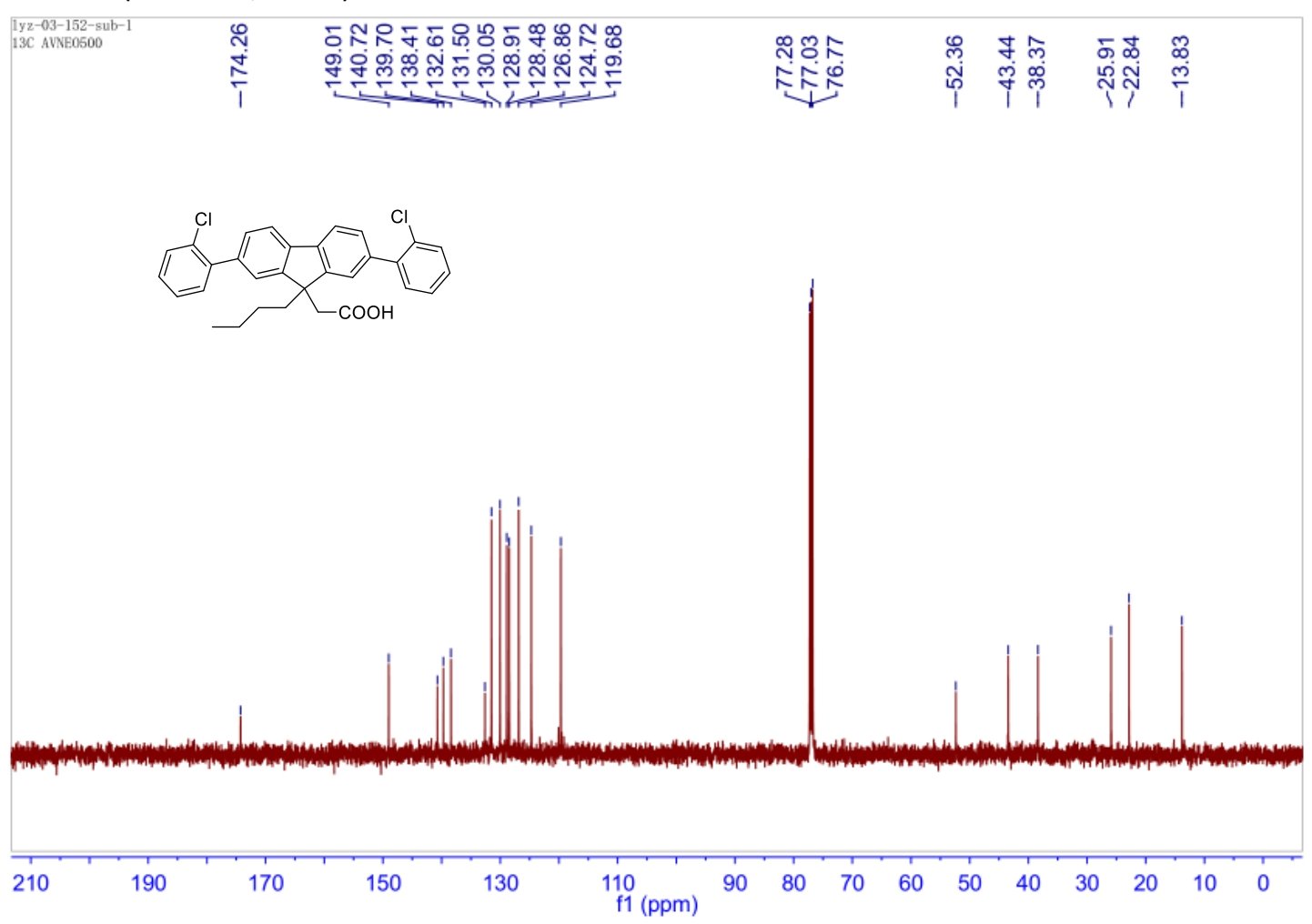


${ }^{1} \mathrm{H}$ NMR $\left(500 \mathrm{MHz}, \mathrm{CDCl}_{3}\right)$

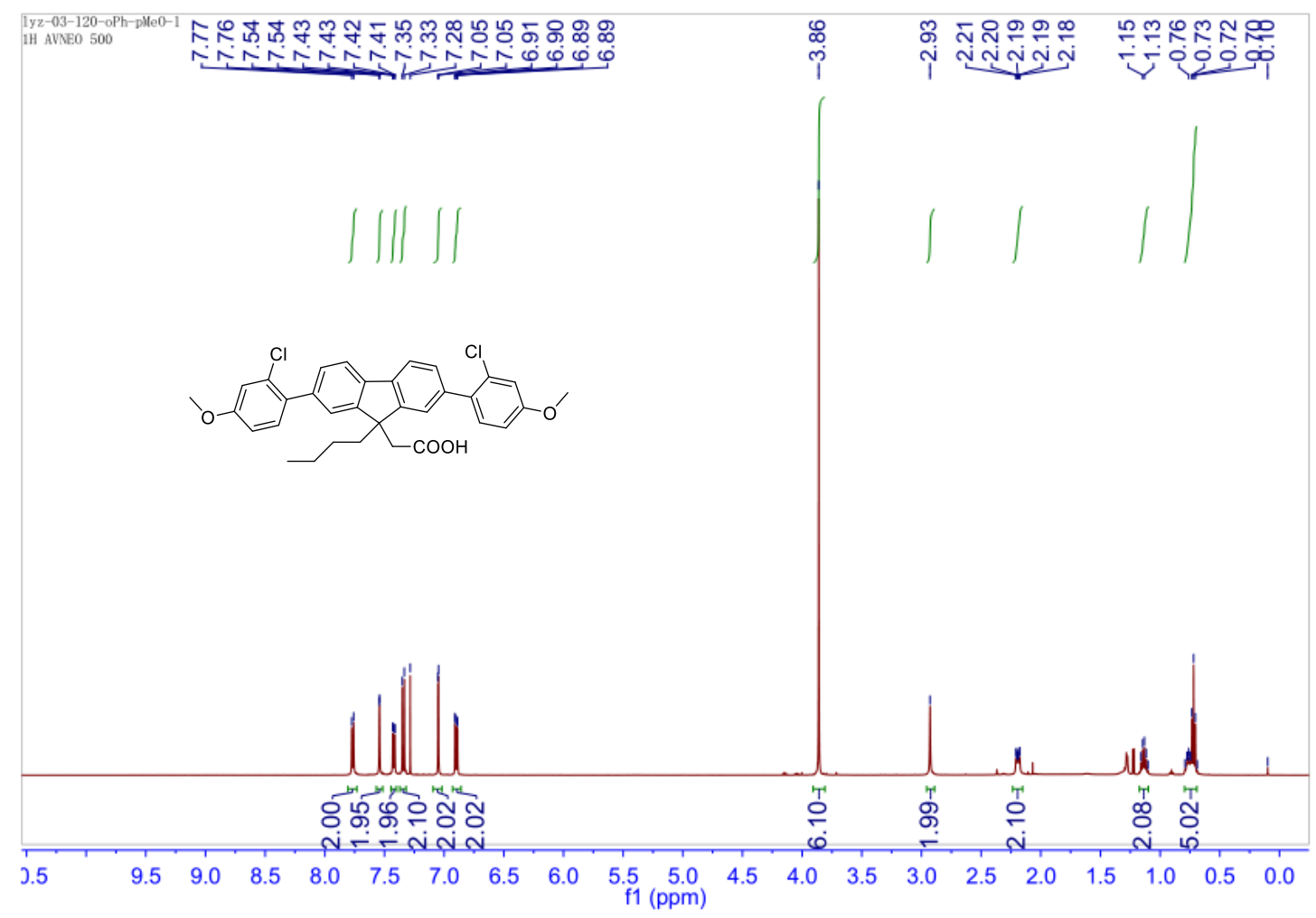

${ }^{13} \mathrm{C}$ NMR (126 MHz, $\mathrm{CDCl}_{3}$ )

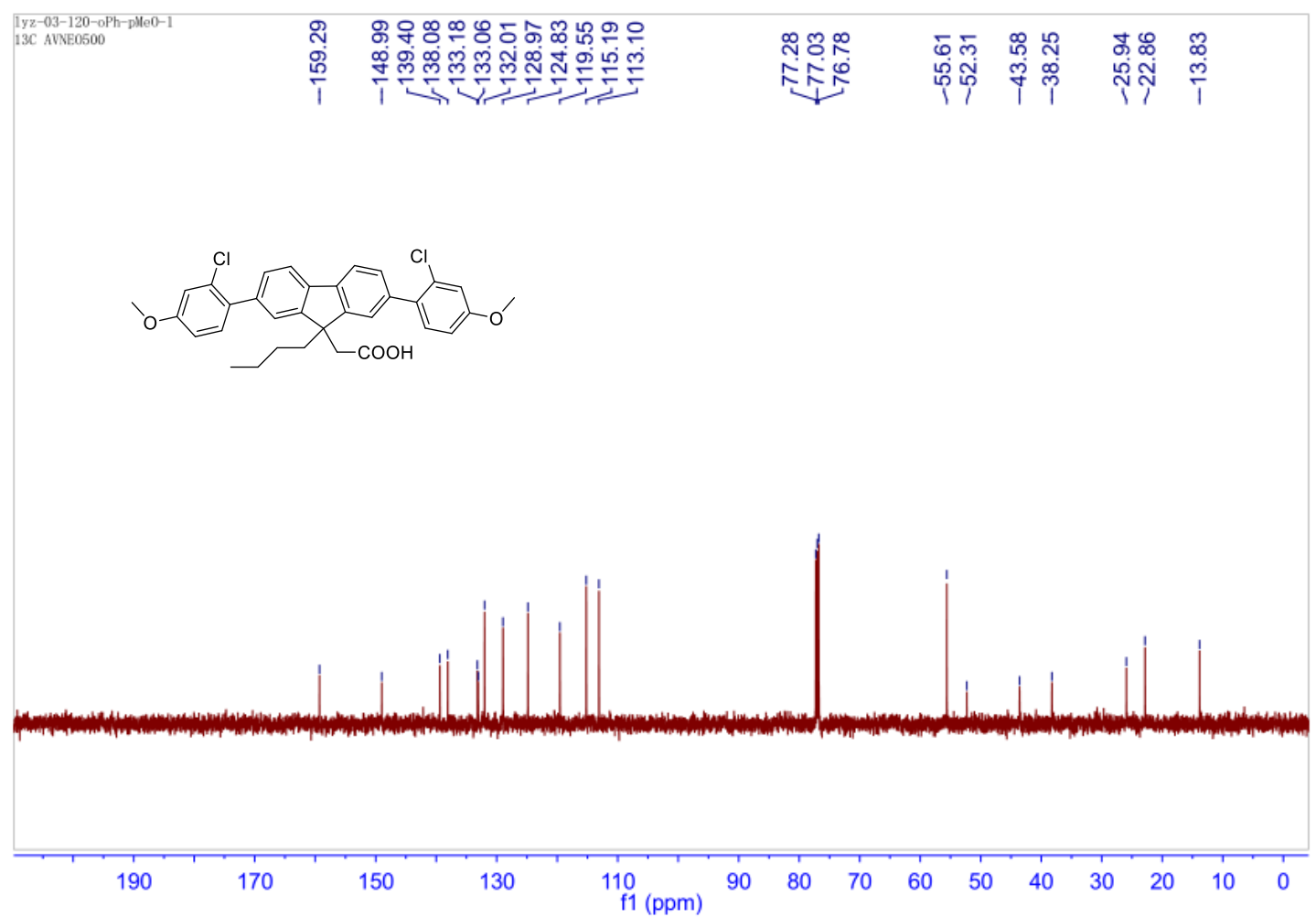


${ }^{1} \mathrm{H}$ NMR (500 MHz, $\left.\mathrm{CDCl}_{3}\right)$

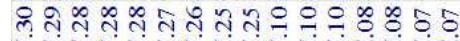

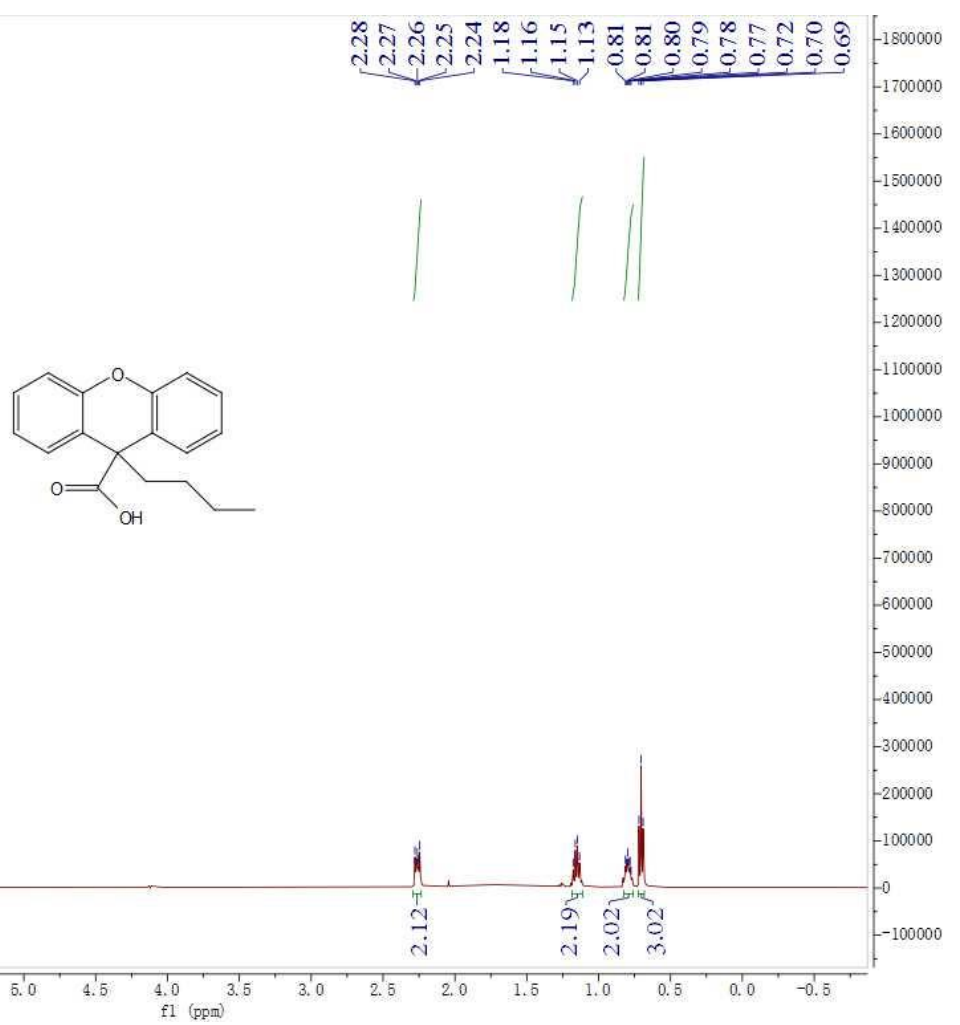

${ }^{13} \mathrm{C}$ NMR (126 MHz, $\left.\mathrm{CDCl}_{3}\right)$

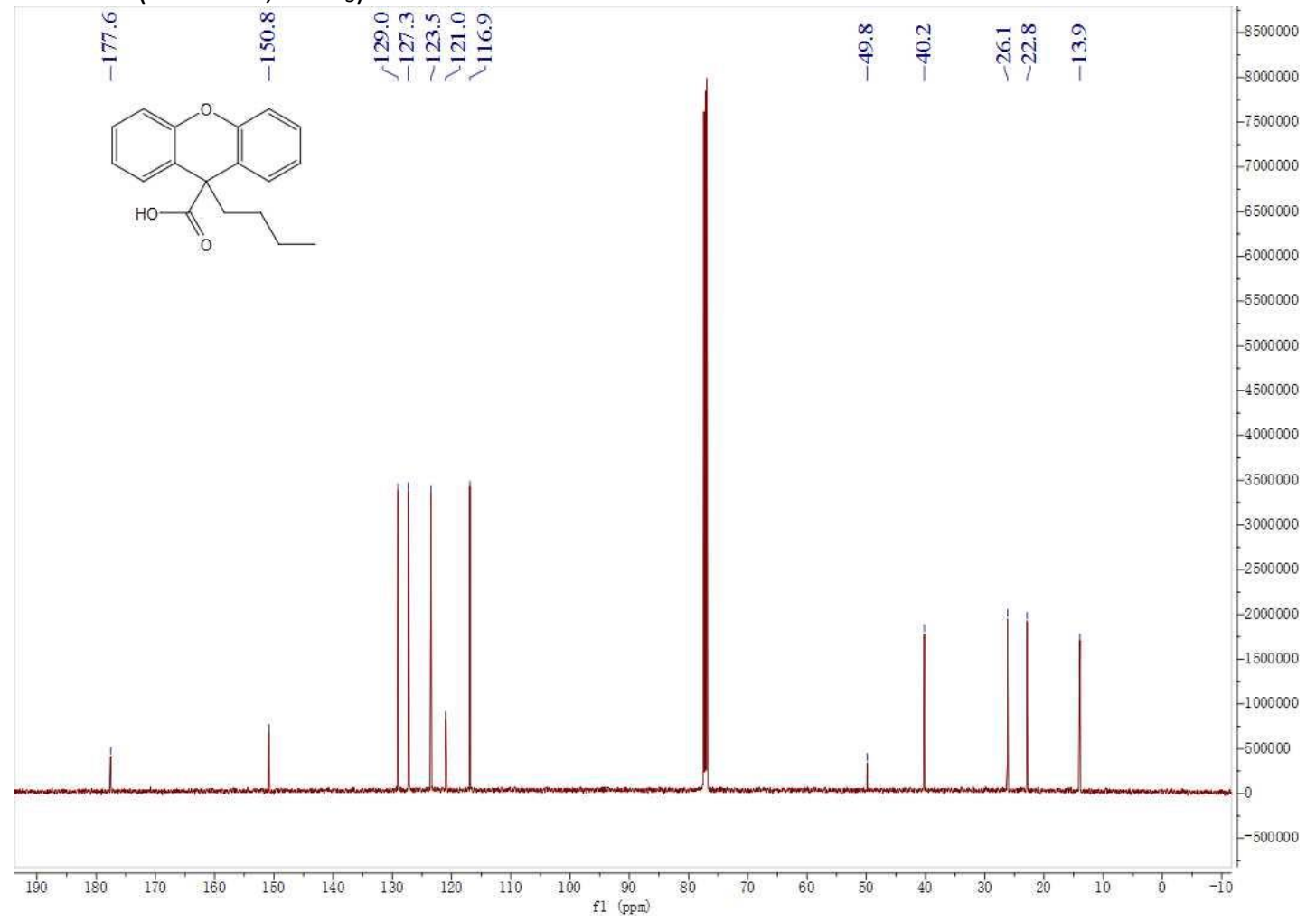




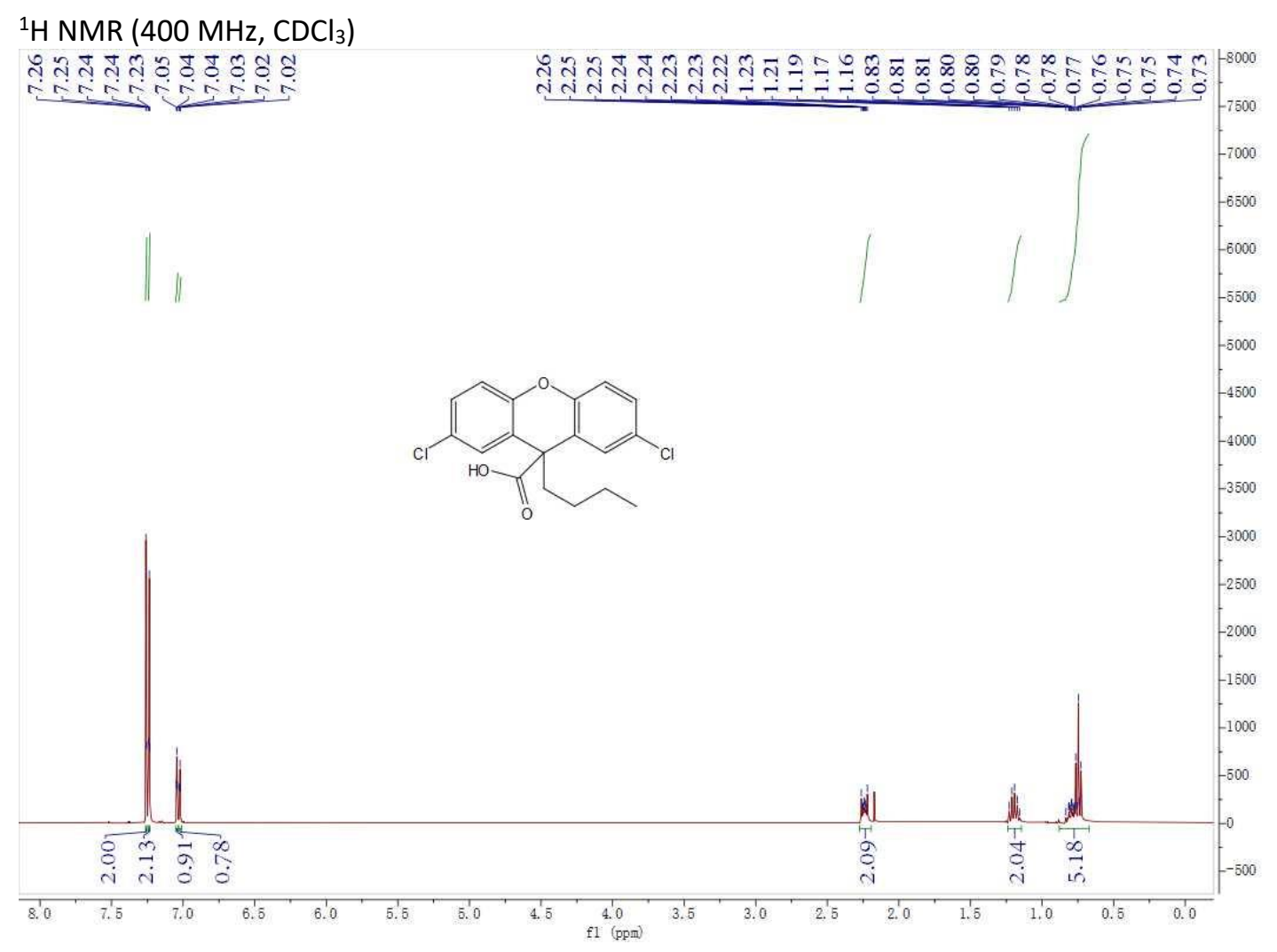

${ }^{13} \mathrm{C}$ NMR (126 MHz, $\left.\mathrm{CDCl}_{3}\right)$
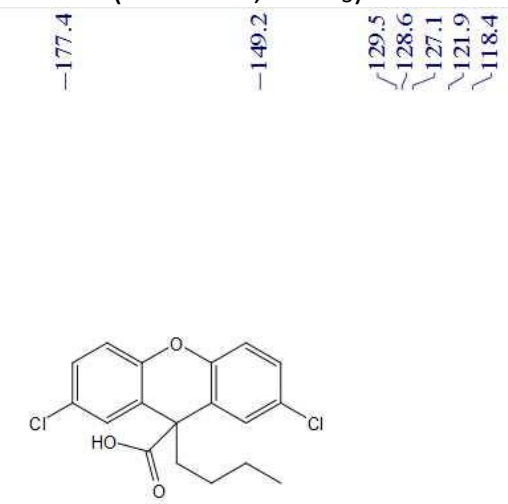

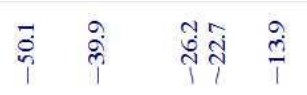

-1500000
-1400000
-1300000

100000

$-1000000$

$-900000$

$-800000$

$-700000$

600000

$-500000$

$-400000$
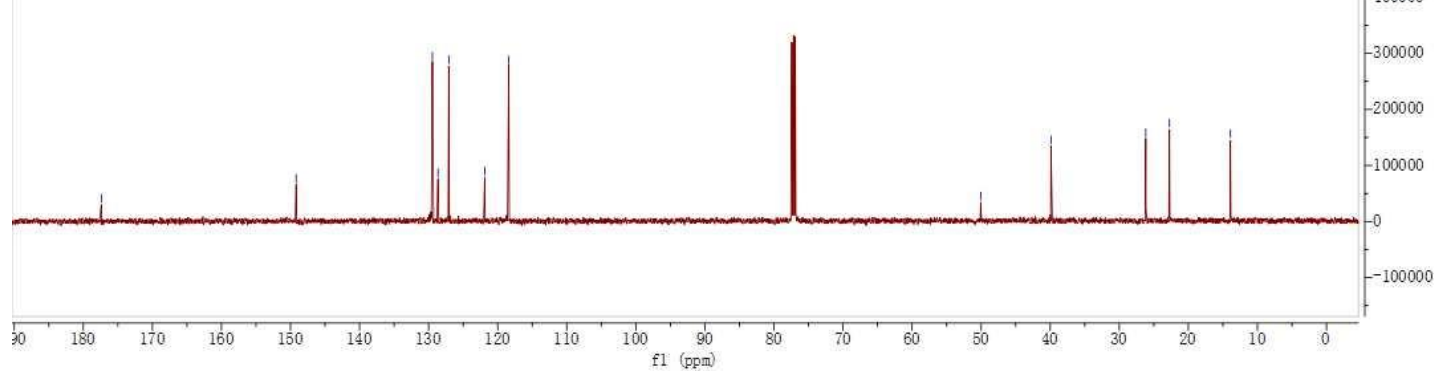


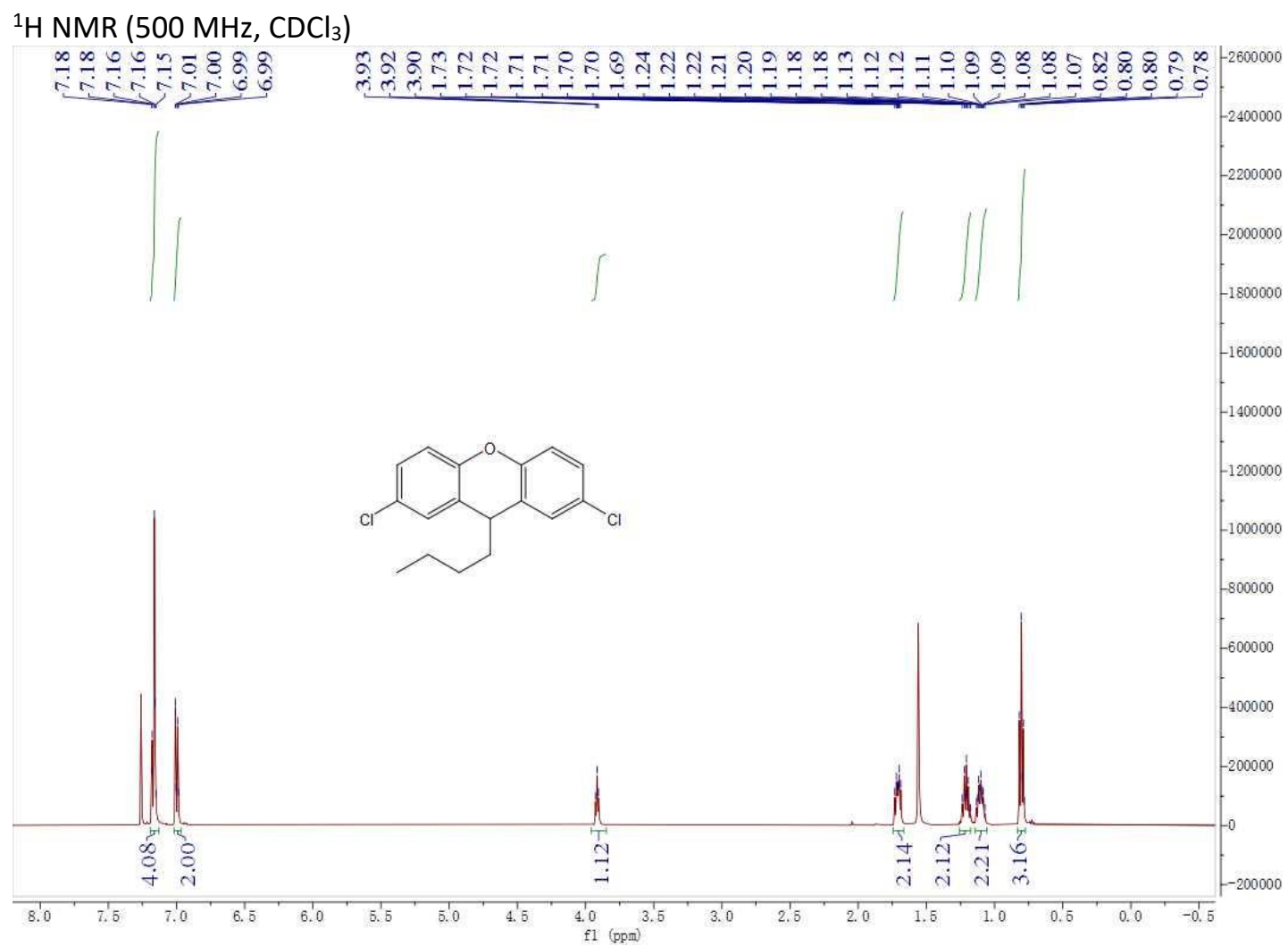

${ }^{13} \mathrm{C}$ NMR (126 MHz, $\left.\mathrm{CDCl}_{3}\right)$

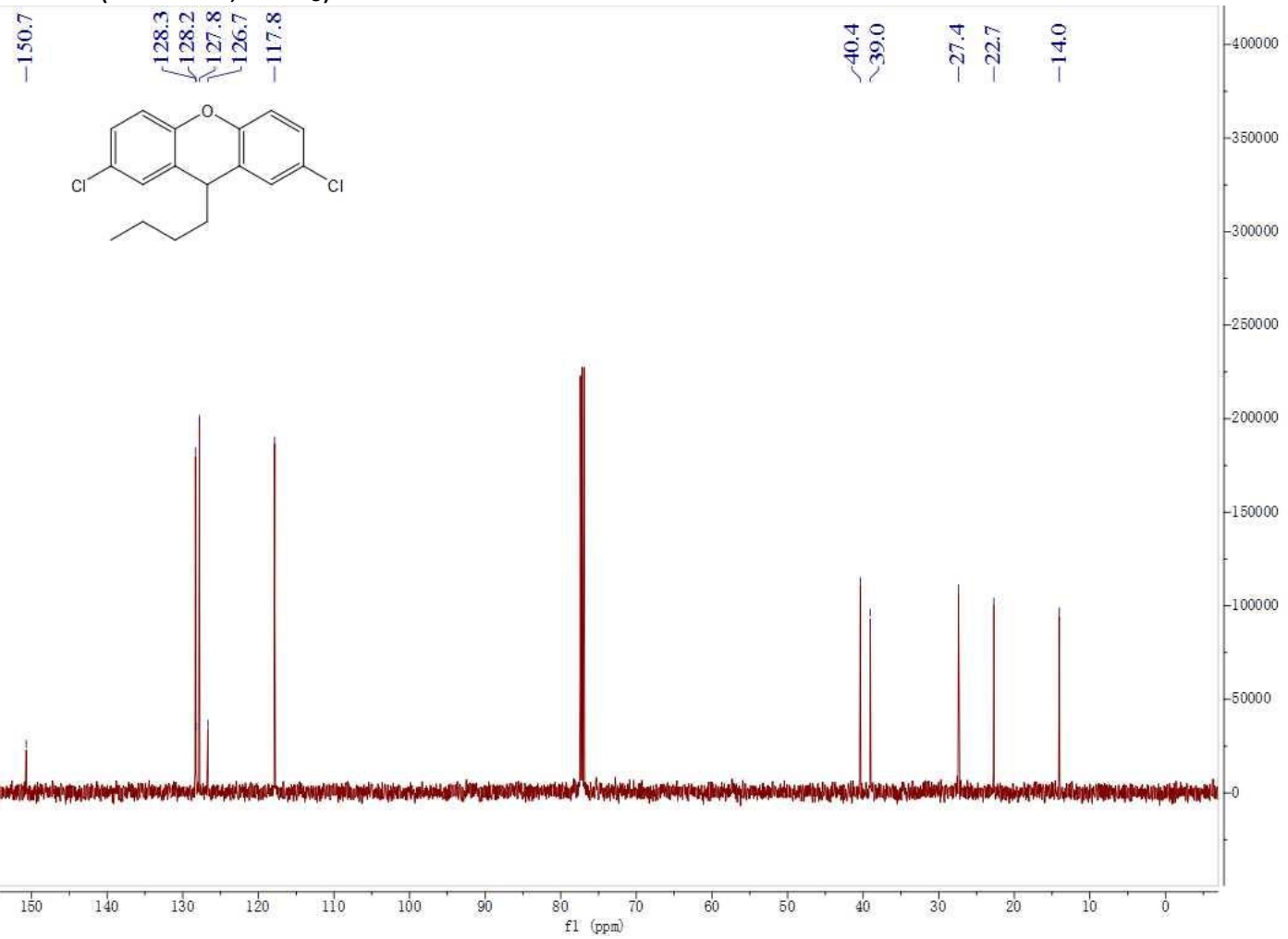


${ }^{1} \mathrm{H} \mathrm{NMR}\left(400 \mathrm{MHz}, \mathrm{CDCl}_{3}\right)$

สุ

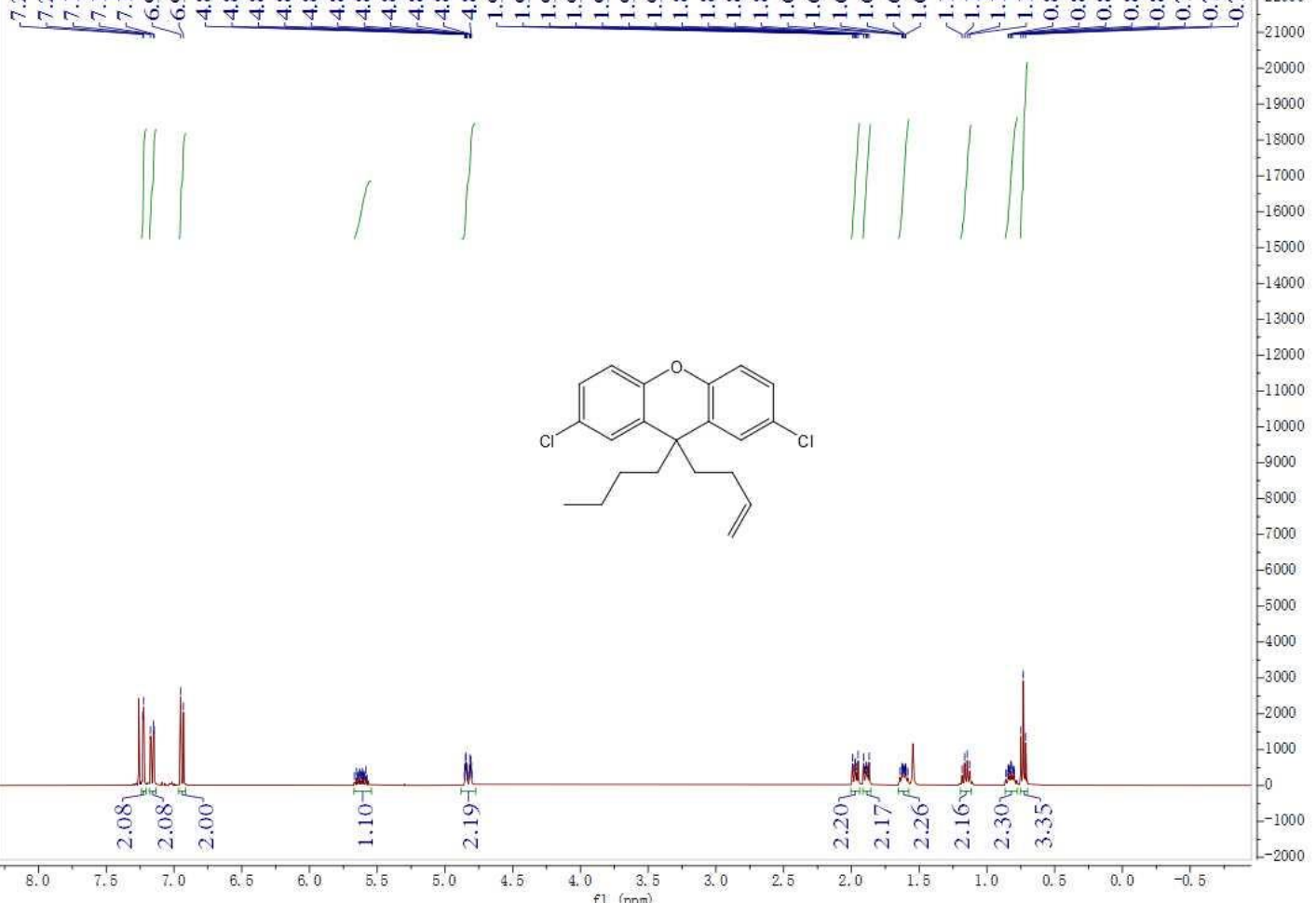

${ }^{13} \mathrm{C} \mathrm{NMR}\left(126 \mathrm{MHz}, \mathrm{CDCl}_{3}\right)$

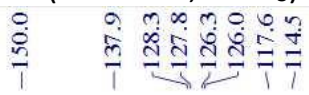

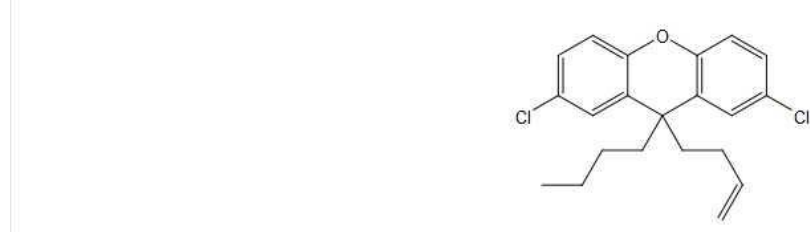

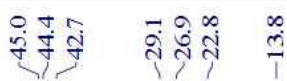

700000
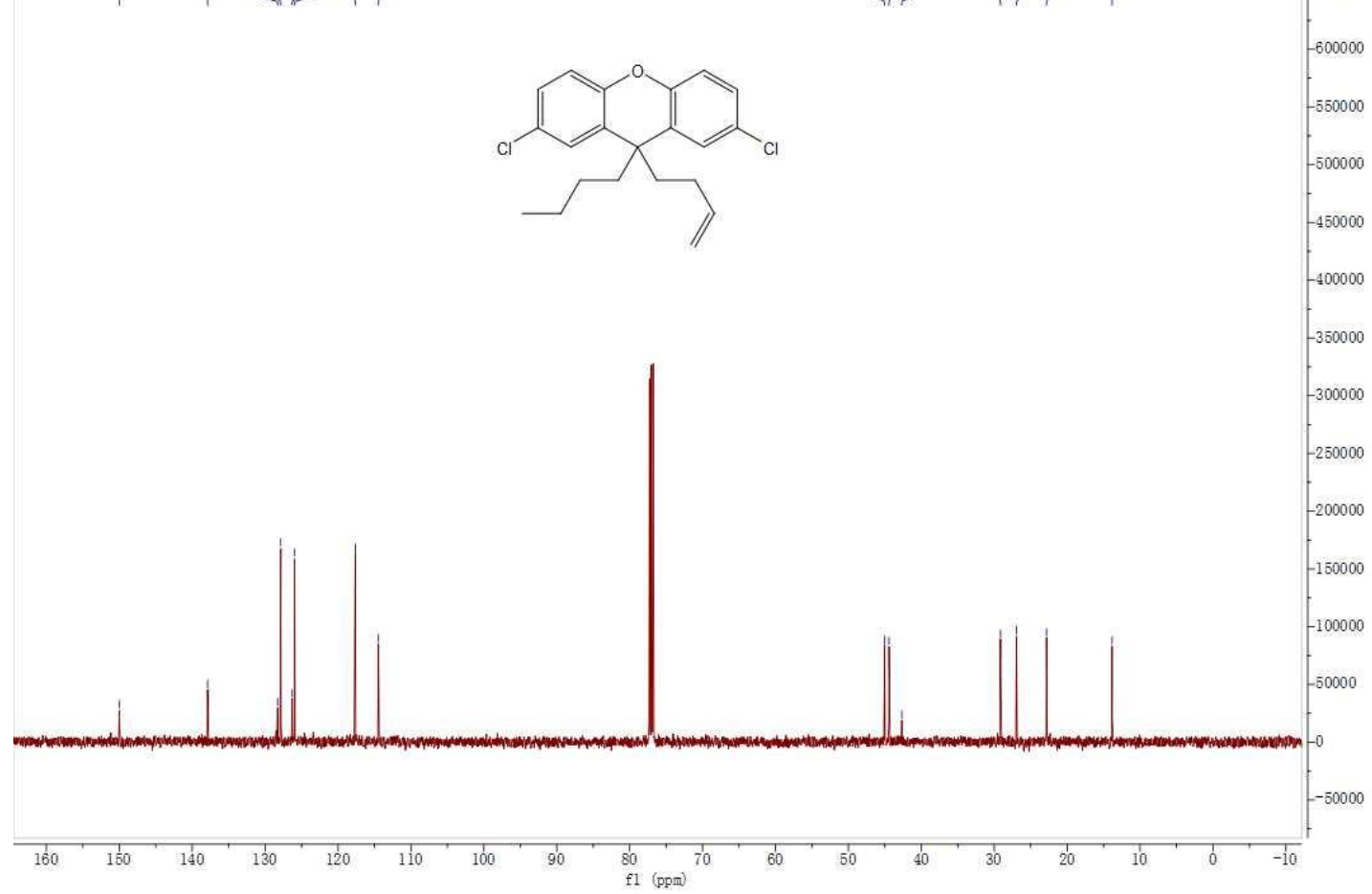
${ }^{1} \mathrm{H}$ NMR (500 MHz, $\mathrm{CDCl}_{3}$ )

तุ

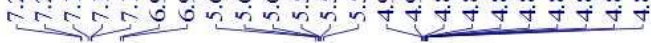

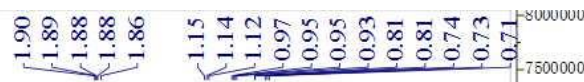

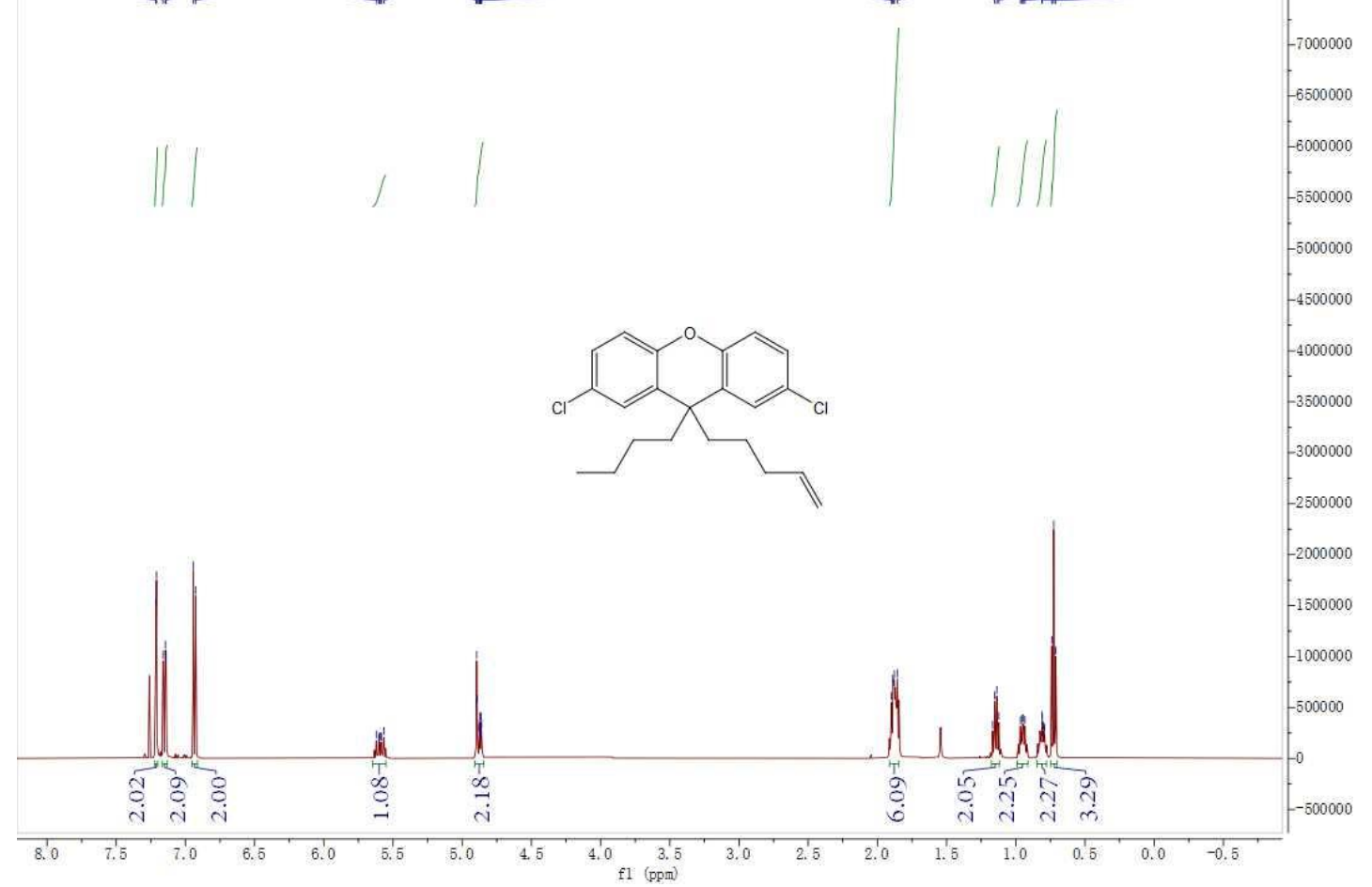

${ }^{13} \mathrm{C}$ NMR $\left(126 \mathrm{MHz}, \mathrm{CDCl}_{3}\right)$

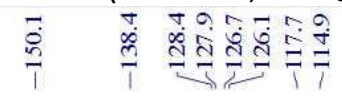

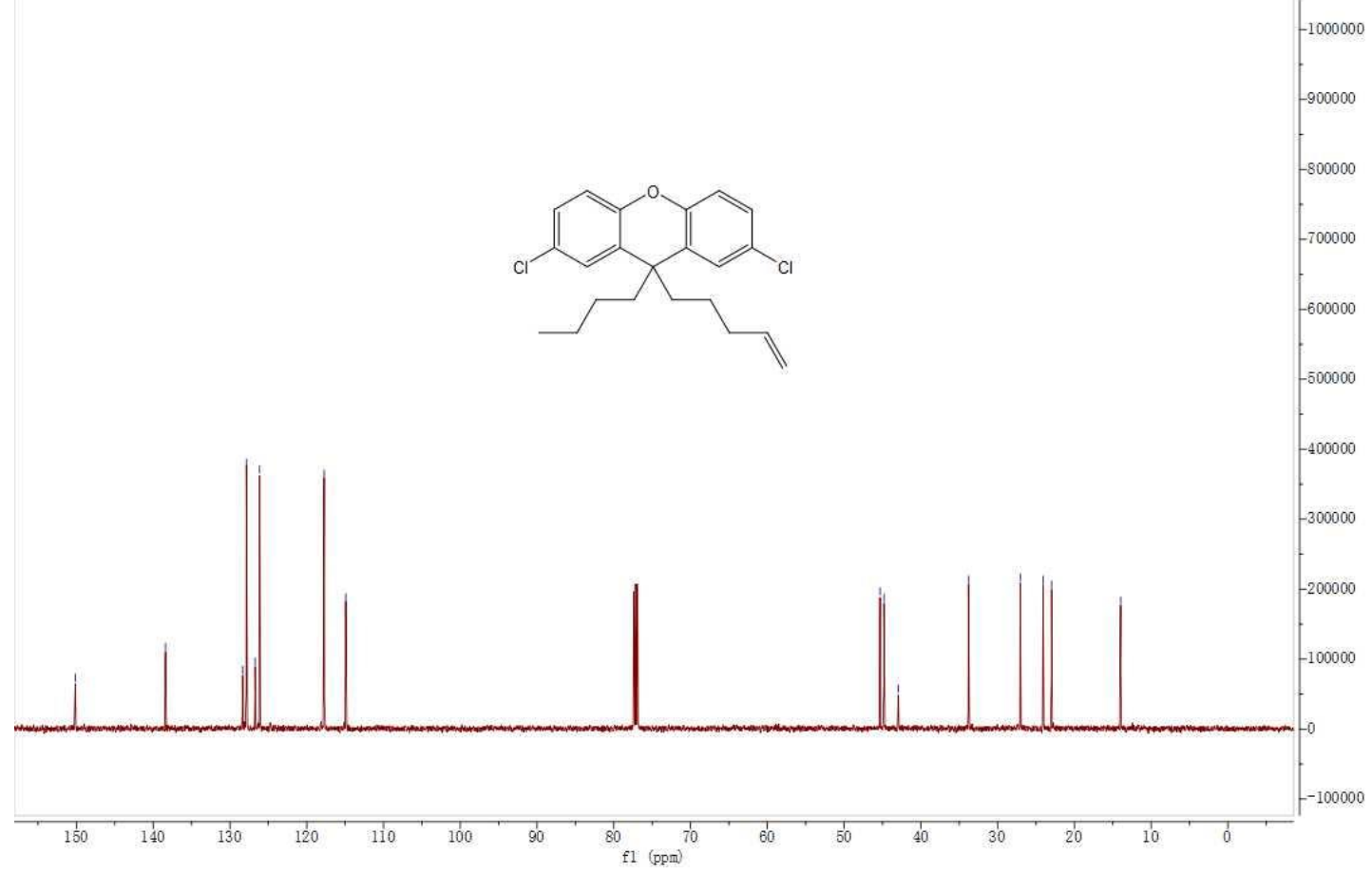




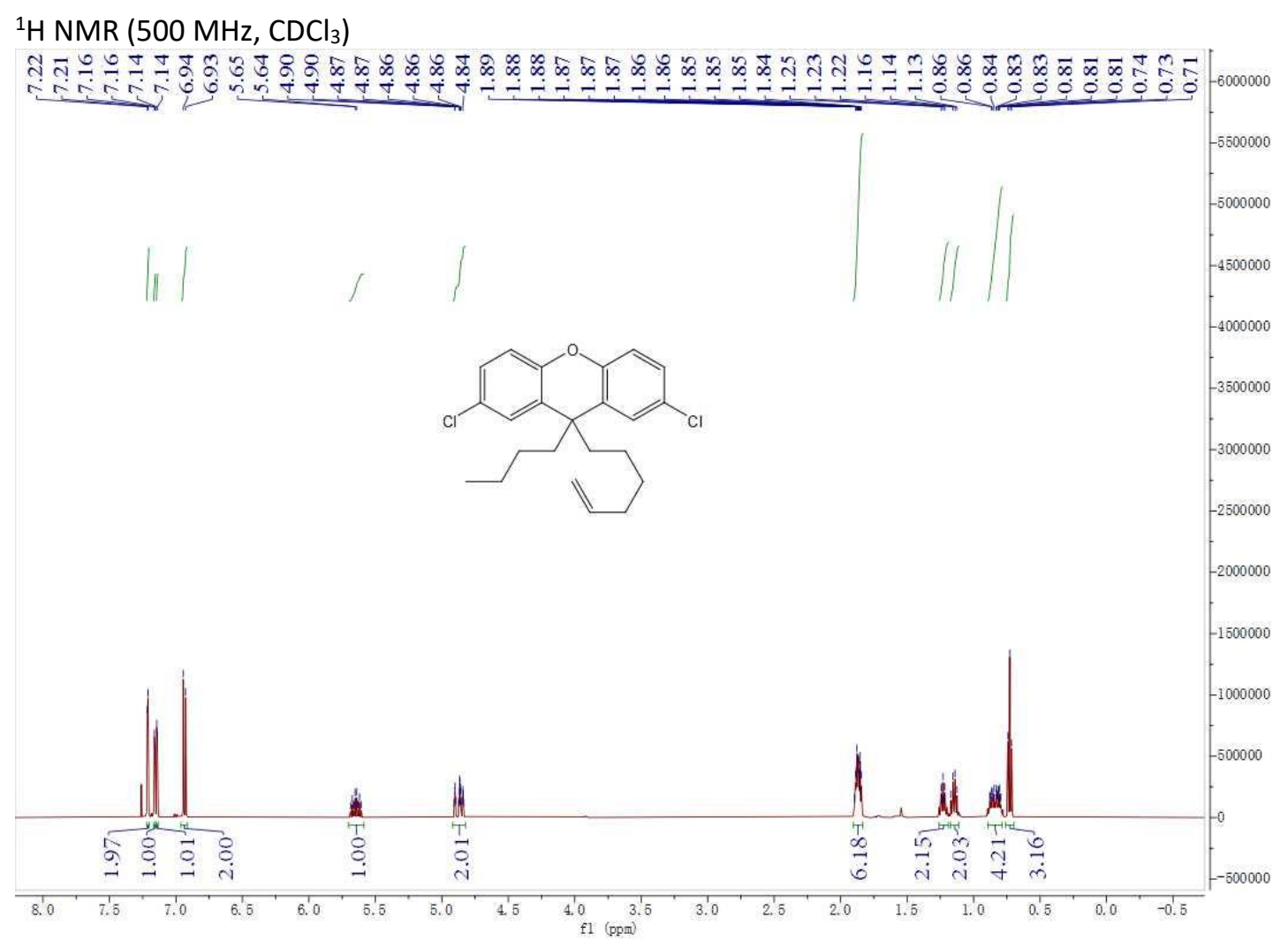

${ }^{13} \mathrm{C}$ NMR (126 MHz, $\mathrm{CDCl}_{3}$ )

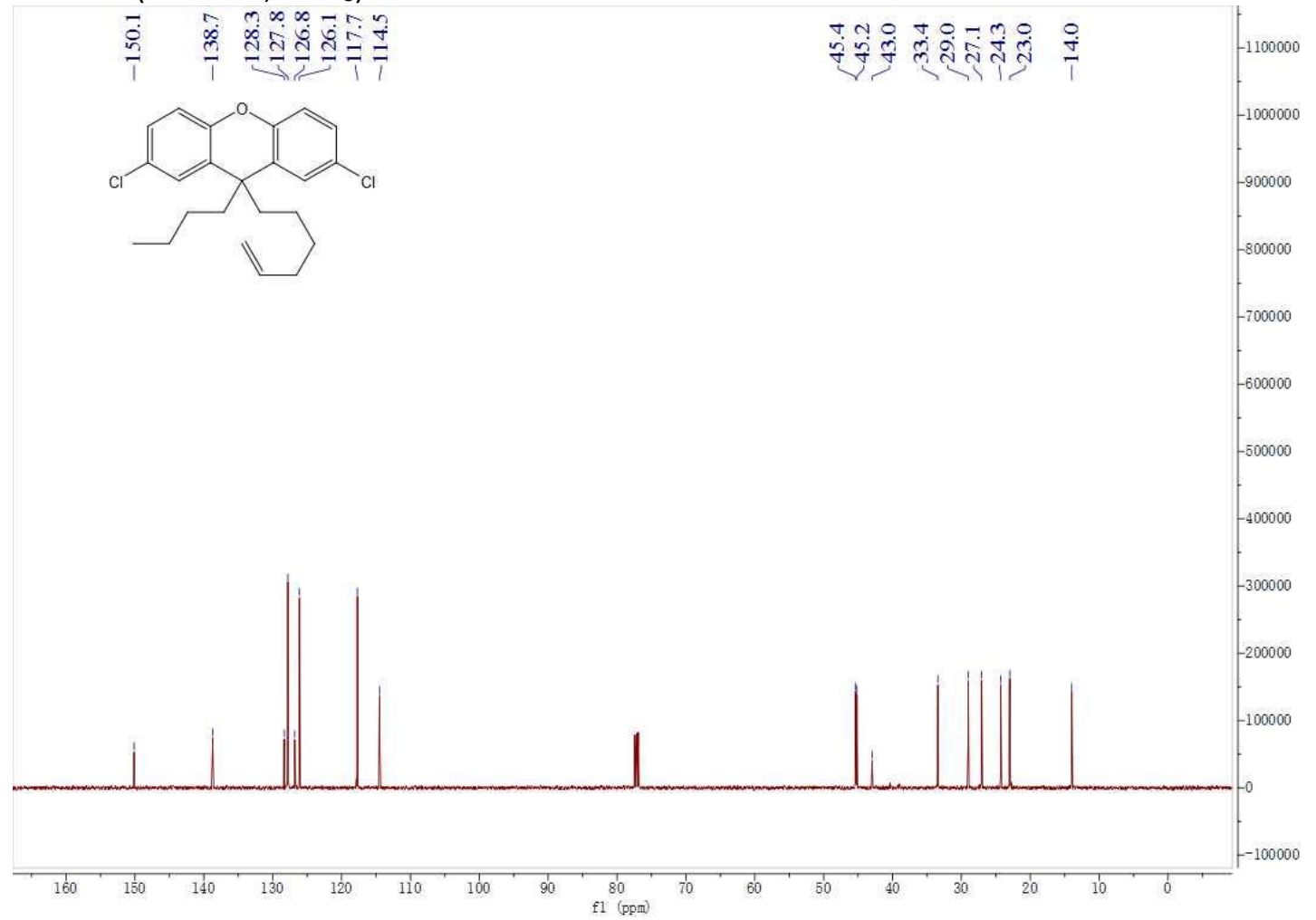


${ }^{1} \mathrm{H}$ NMR $\left(500 \mathrm{MHz}, \mathrm{CDCl}_{3}\right)$

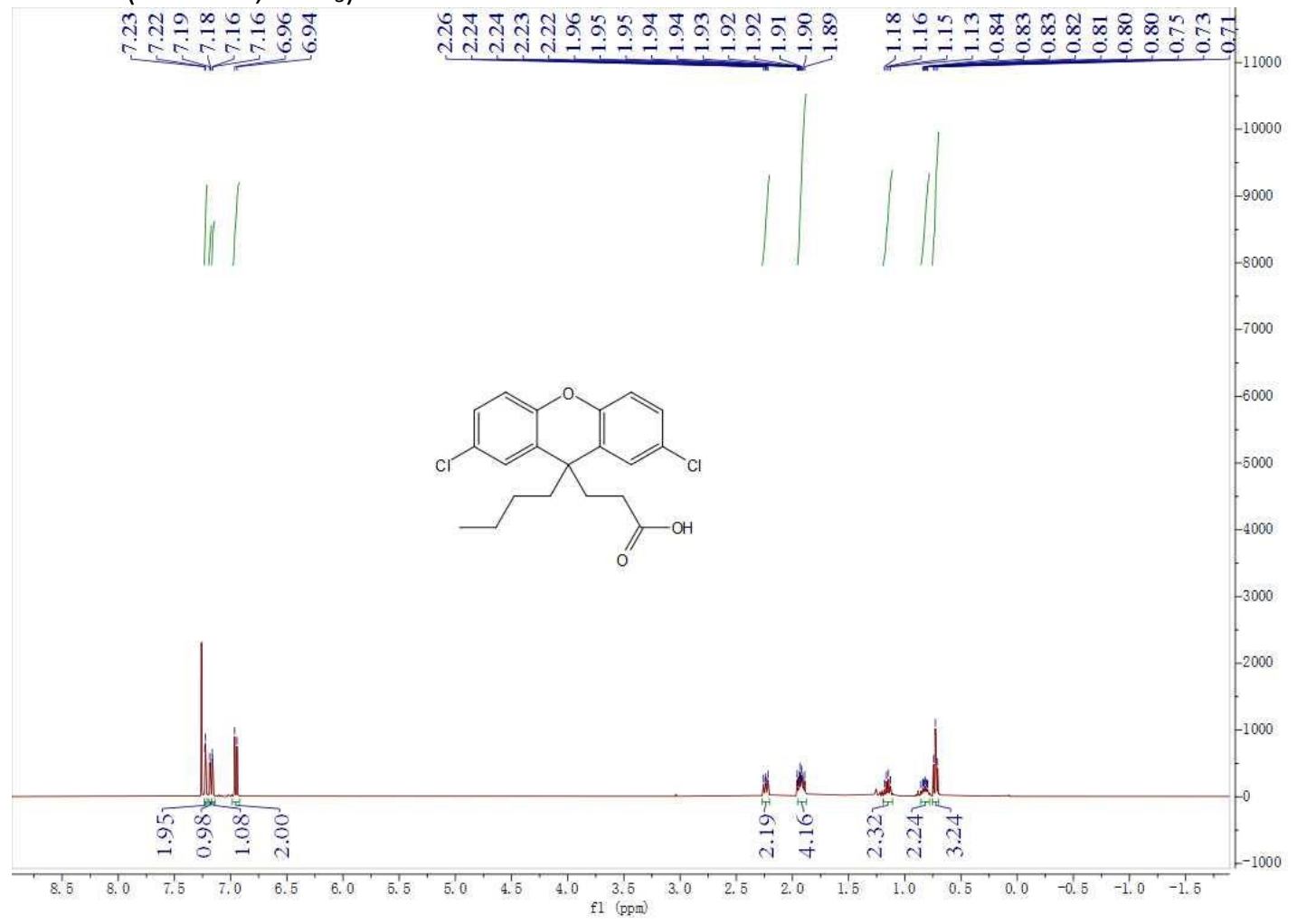

${ }^{13} \mathrm{C}$ NMR (126 MHz, $\left.\mathrm{CDCl}_{3}\right)$

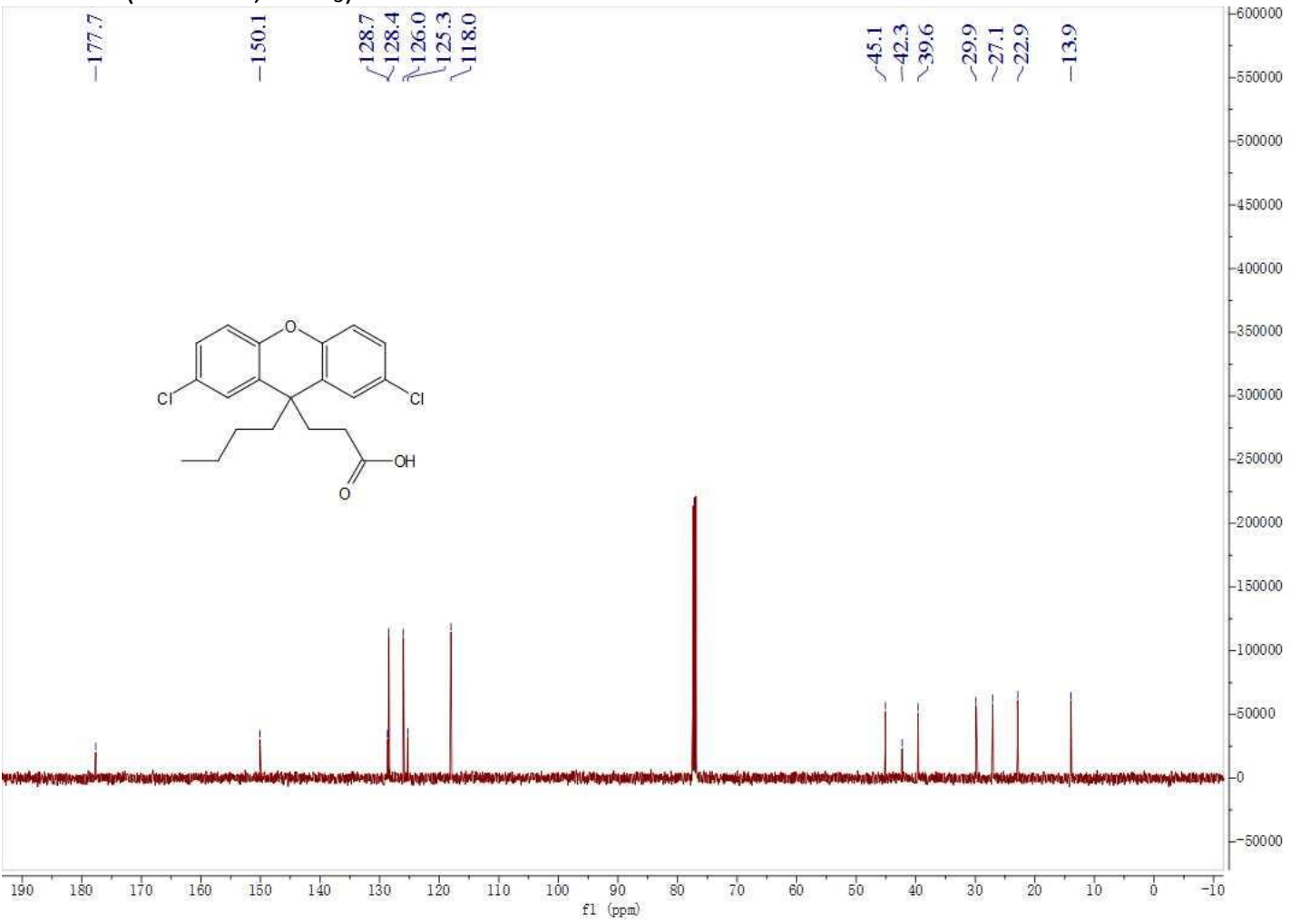




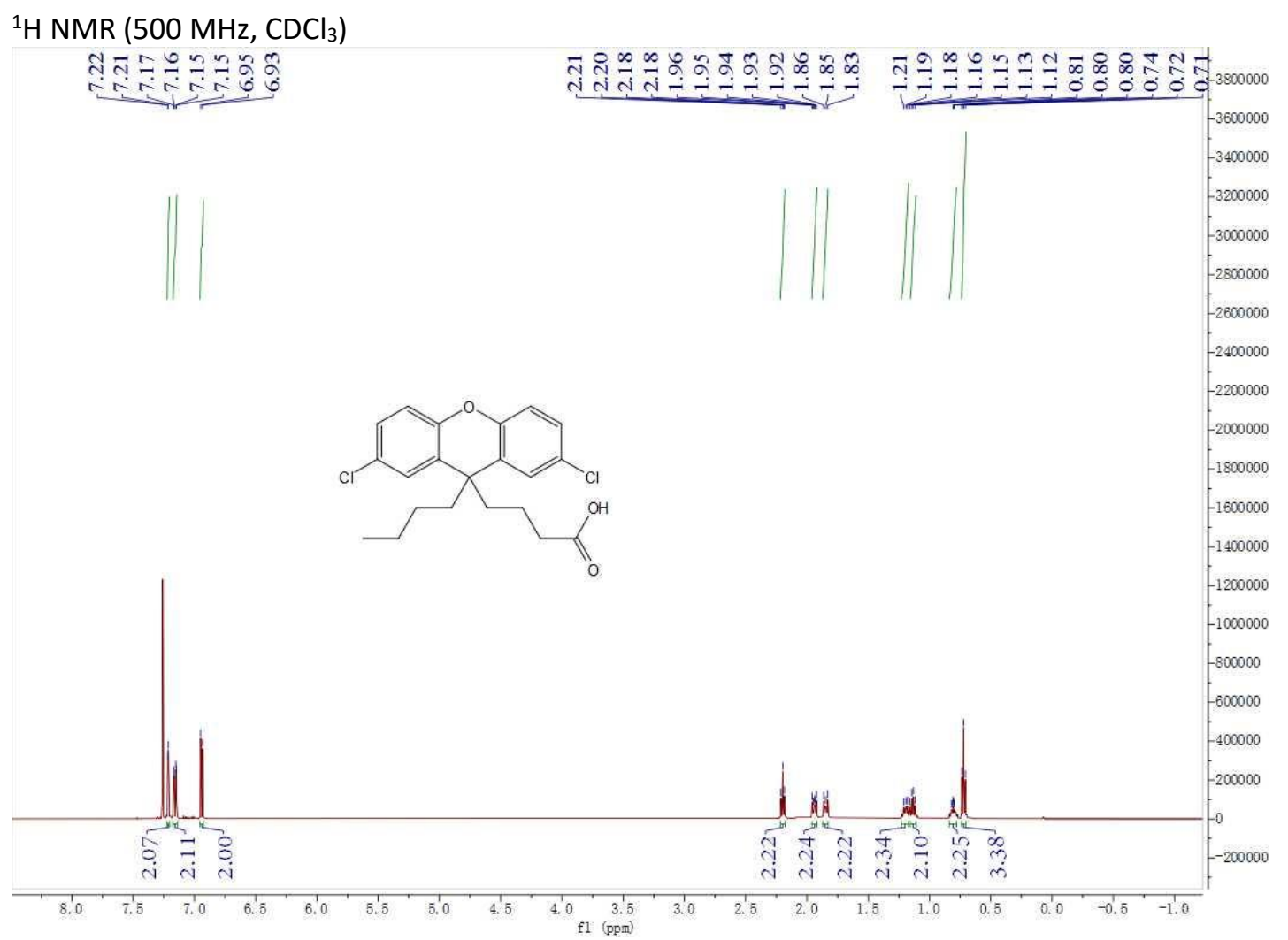

${ }^{13} \mathrm{C}$ NMR (126 MHz, $\left.\mathrm{CDCl}_{3}\right)$

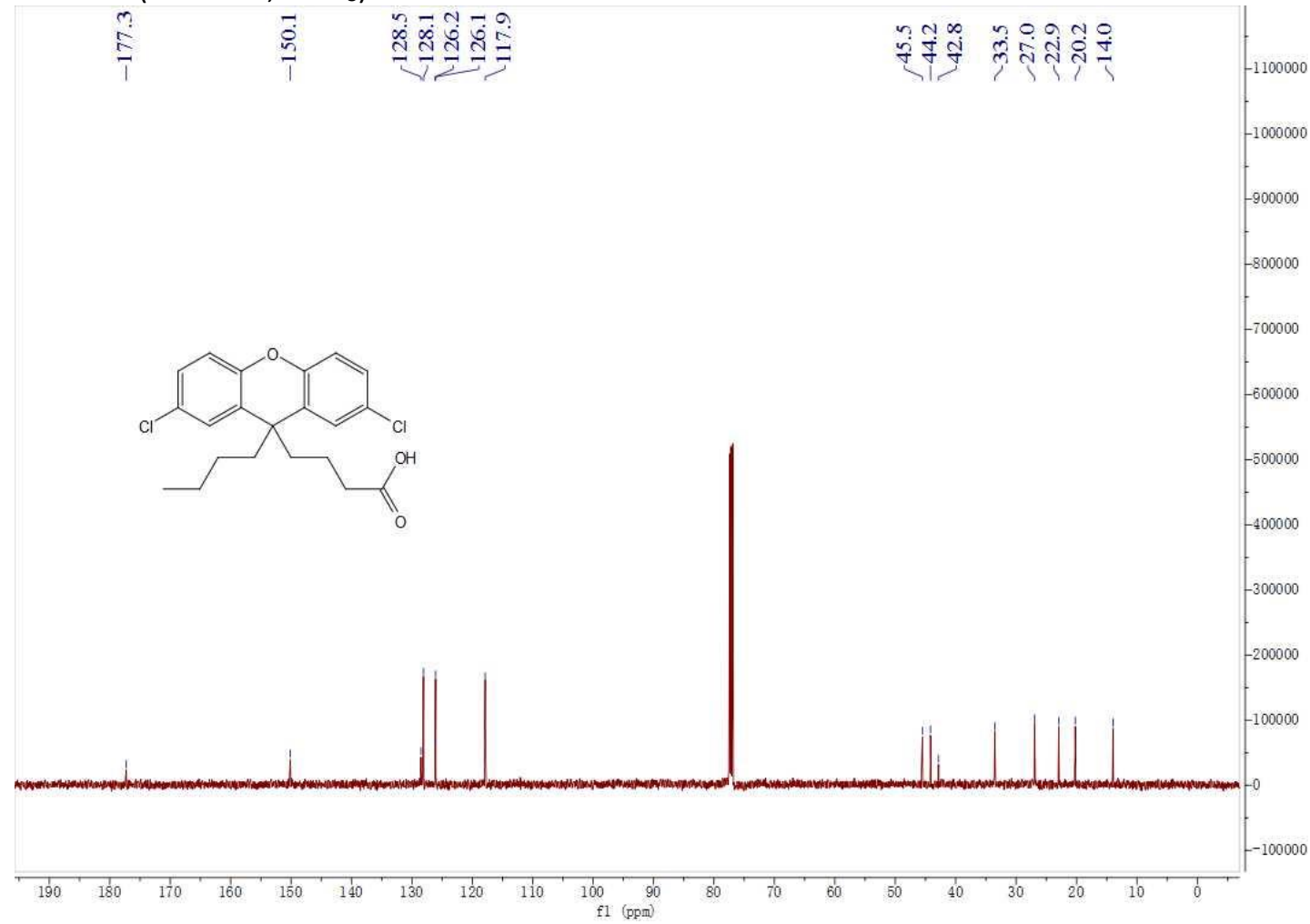


${ }^{1} \mathrm{H}$ NMR $\left(500 \mathrm{MHz}, \mathrm{CDCl}_{3}\right)$

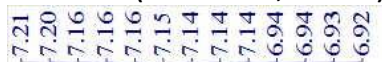

車

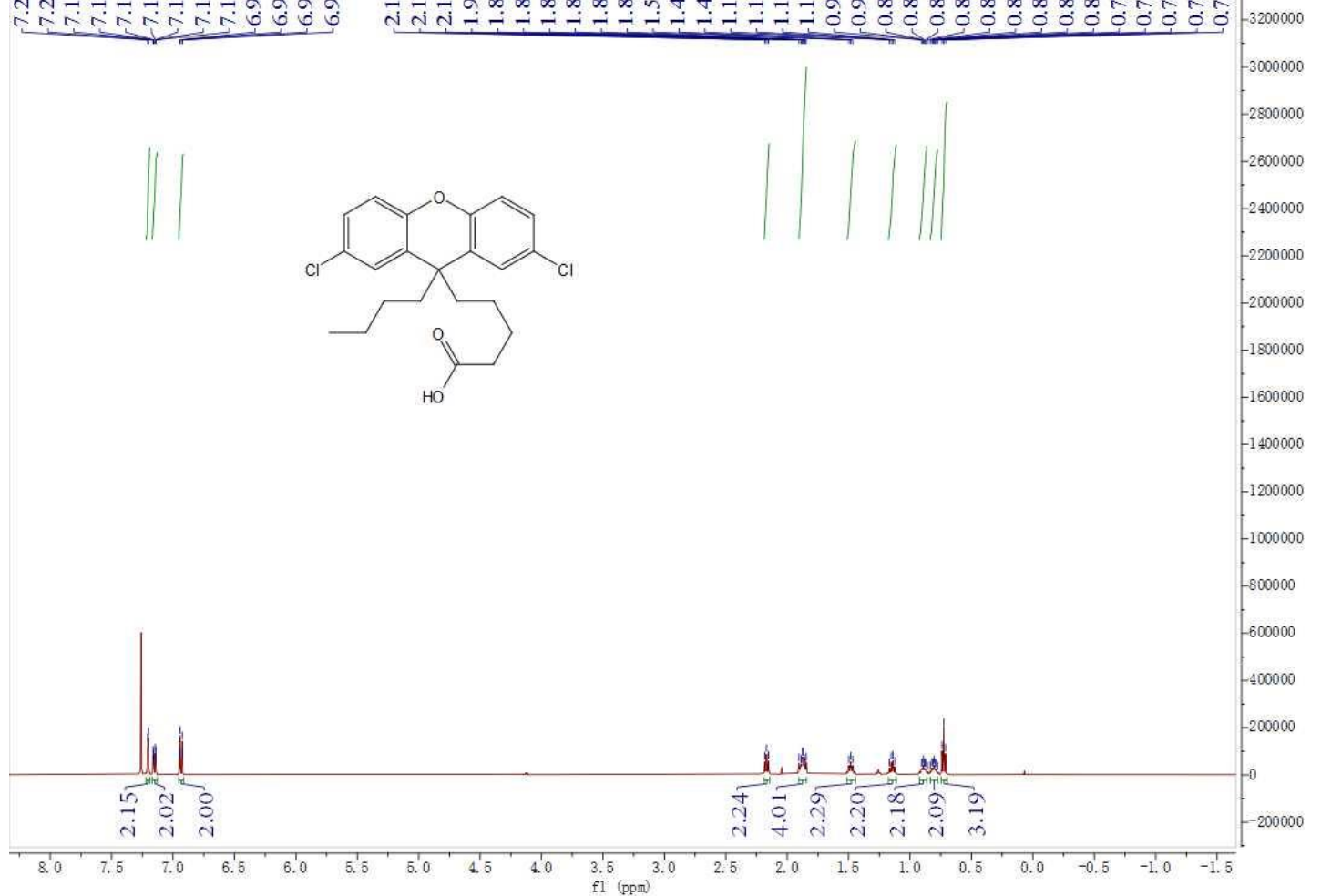

$\left.{ }^{13} \mathrm{C} \mathrm{NMR} \mathrm{(126} \mathrm{MHz,} \mathrm{CDCl}_{3}\right)$

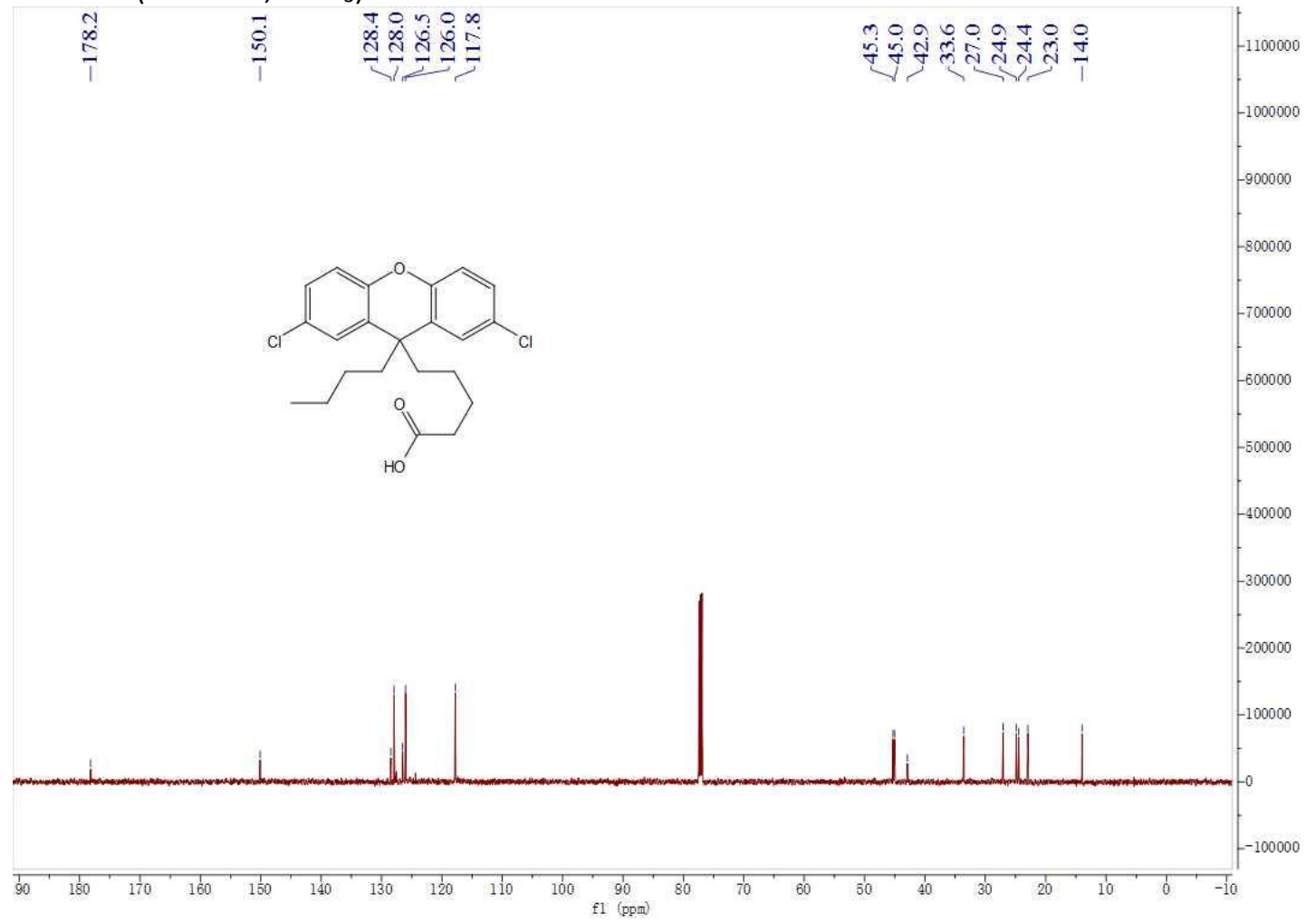


${ }^{1} \mathrm{H}$ NMR $\left(500 \mathrm{MHz}, \mathrm{CDCl}_{3}\right)$

चै चा ने शे ले ले ले के

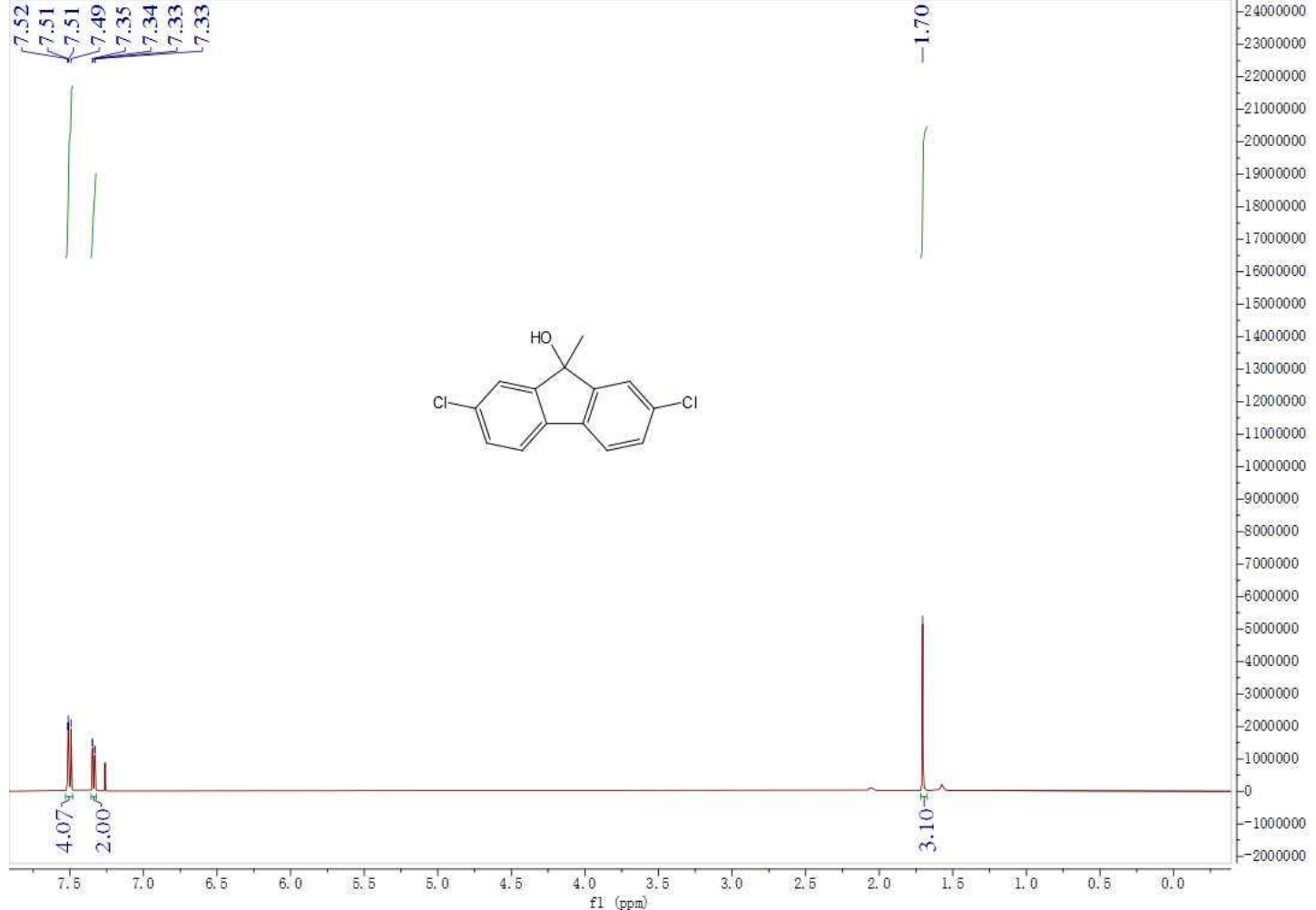

${ }^{13} \mathrm{C}$ NMR (126 MHz, $\left.\mathrm{CDCl}_{3}\right)$

ज)

$\stackrel{2}{1}$

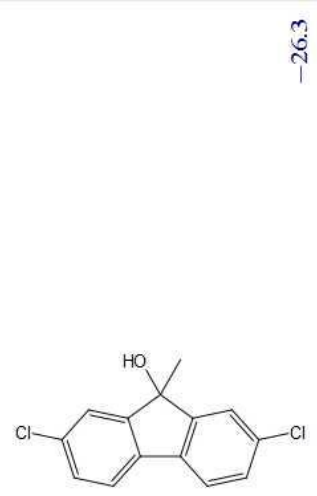

ใูำ

1600000
-1500000

$-1400000$

$-1400000$

$-1300000$

$-1200000$

$-1100000$

$-1000000$

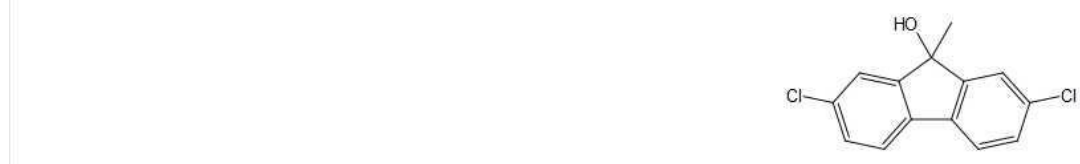

$-900000$

$-800000$

$-700000$

$-600000$

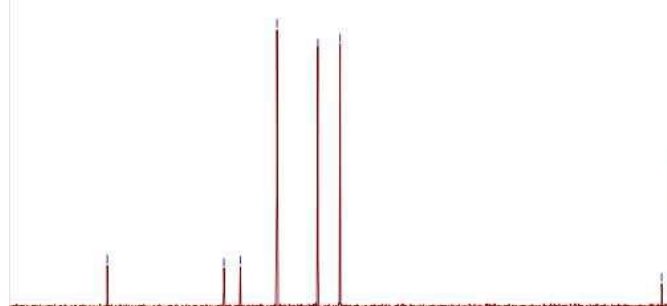

500000

$-400000$

$-300000$

$-300000$

$-200000$

$-100000$

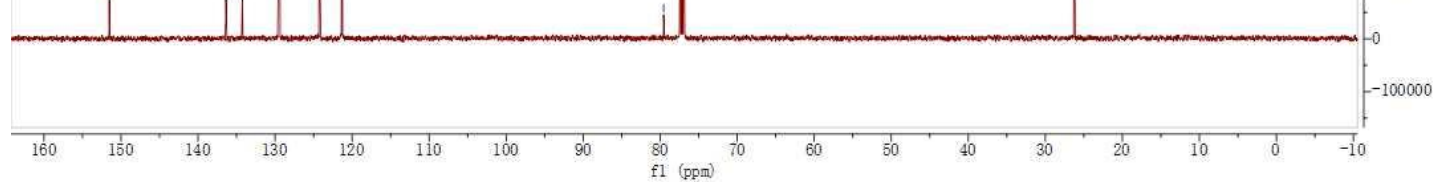




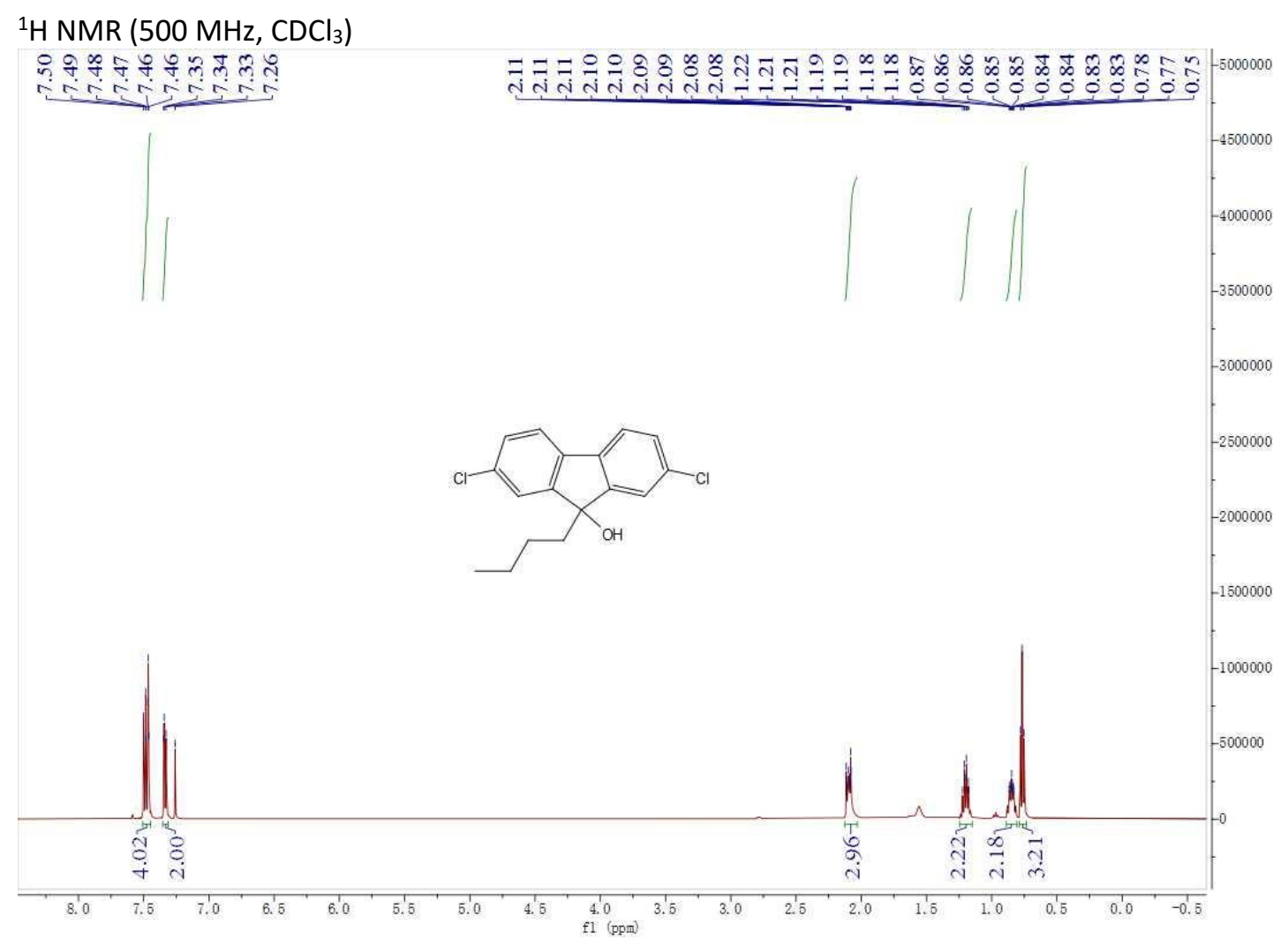

${ }^{13} \mathrm{C}$ NMR (126 MHz, $\left.\mathrm{CDCl}_{3}\right)$

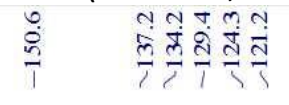
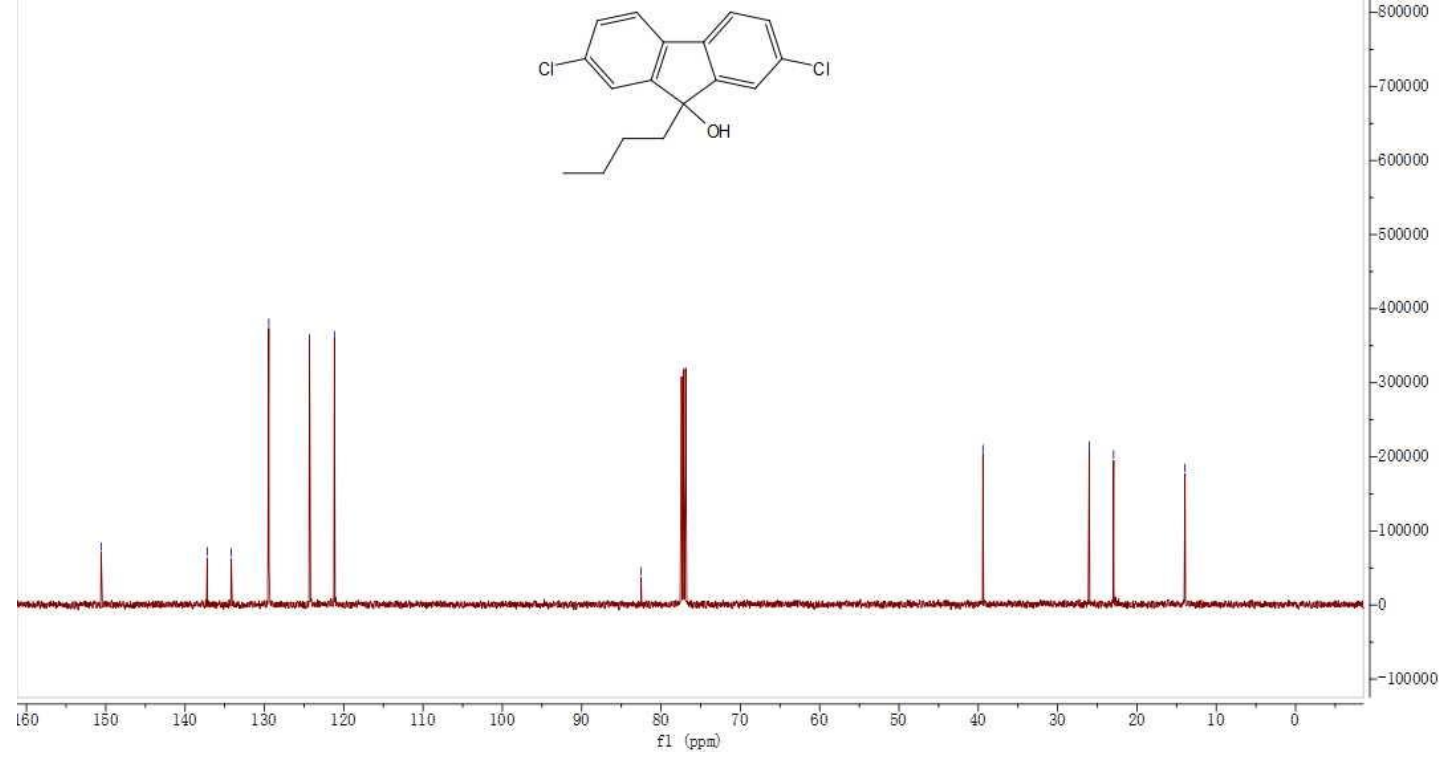
${ }^{1} \mathrm{H}$ NMR $\left(500 \mathrm{MHz}, \mathrm{CDCl}_{3}\right)$

क

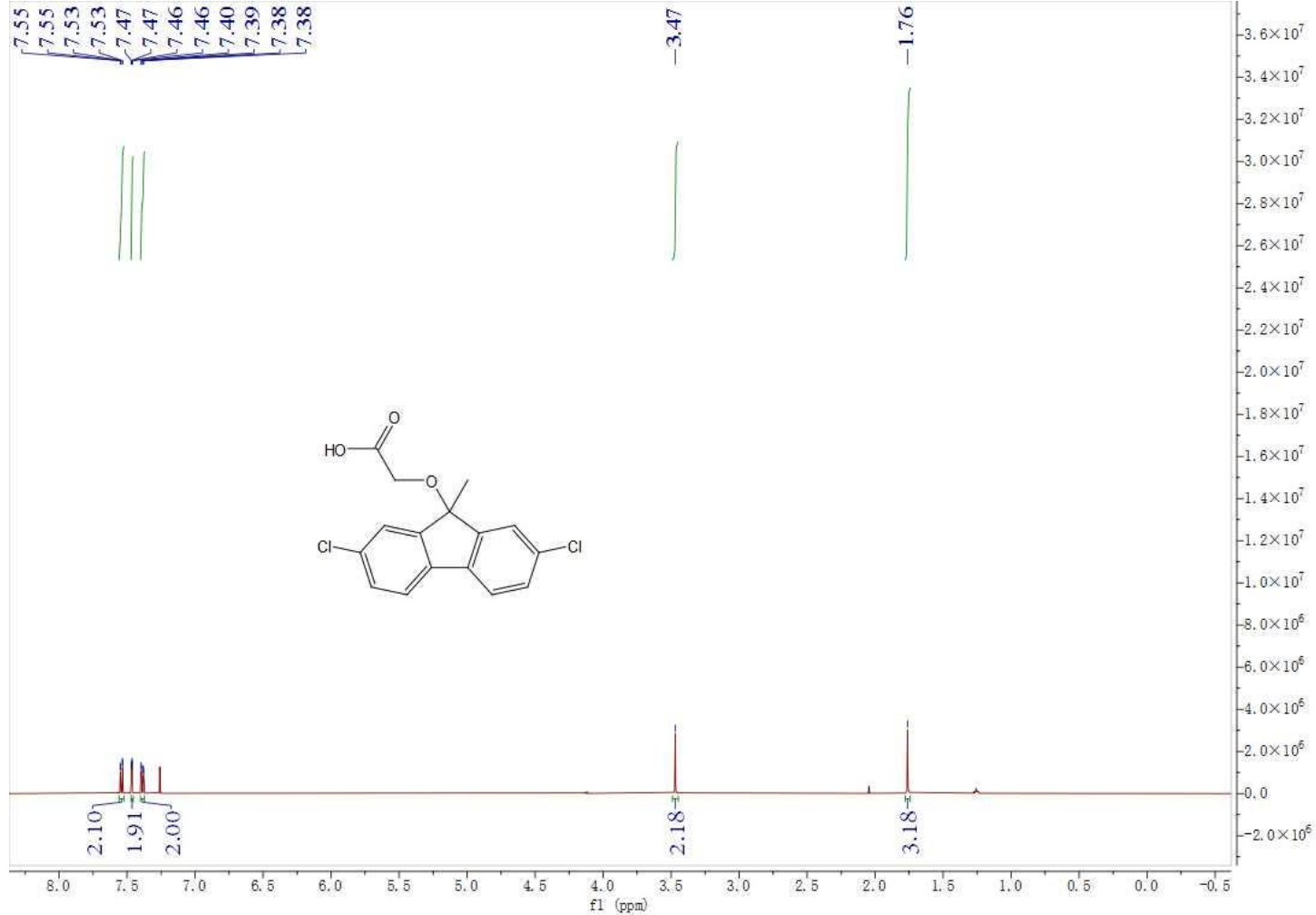

${ }^{13} \mathrm{C}$ NMR (126 MHz, $\left.\mathrm{CDCl}_{3}\right)$

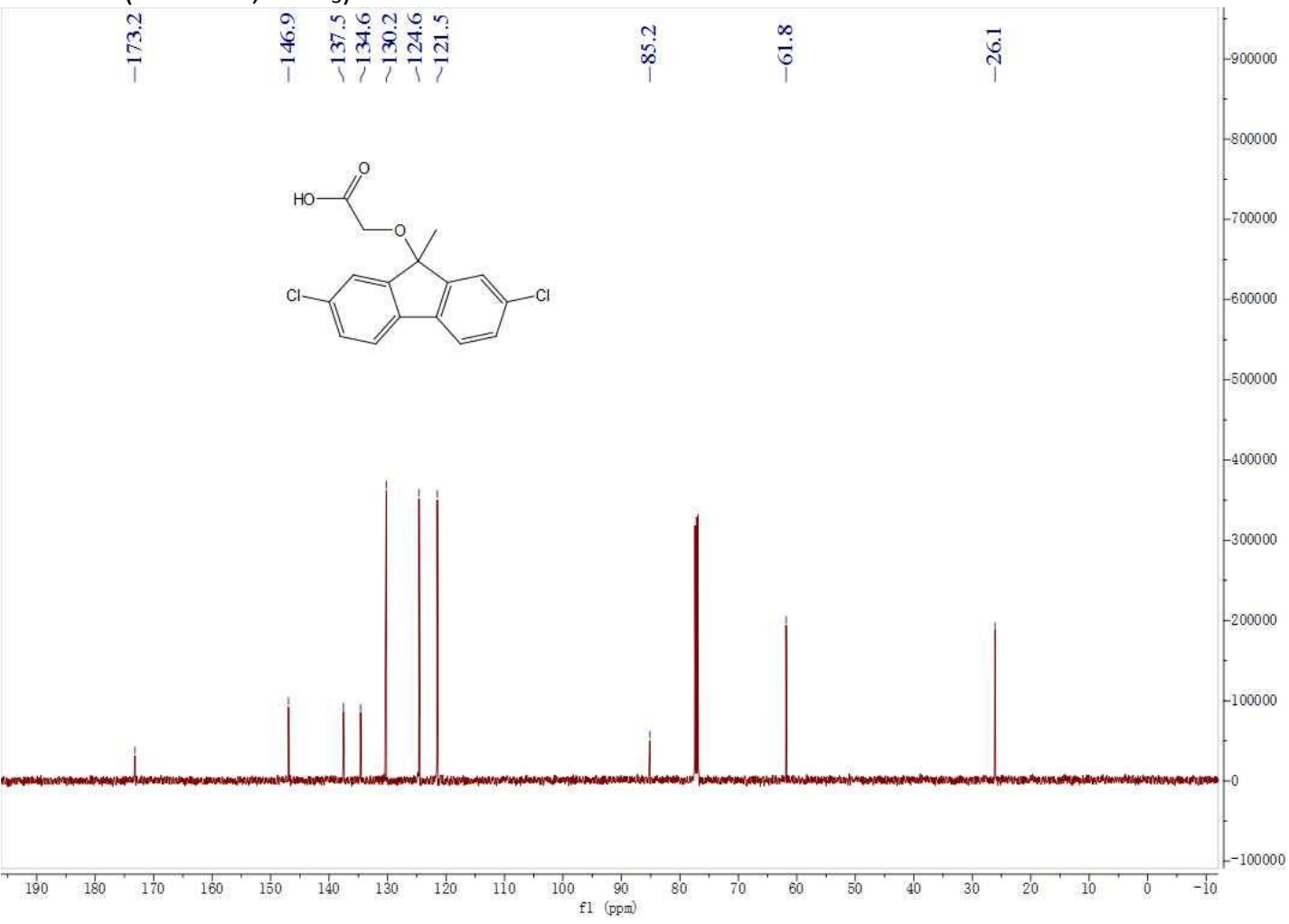


${ }^{1} \mathrm{H}$ NMR $\left(500 \mathrm{MHz}, \mathrm{CDCl}_{3}\right)$

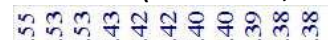

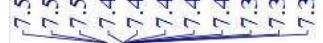

मำ ํำ

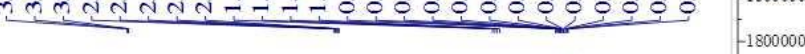
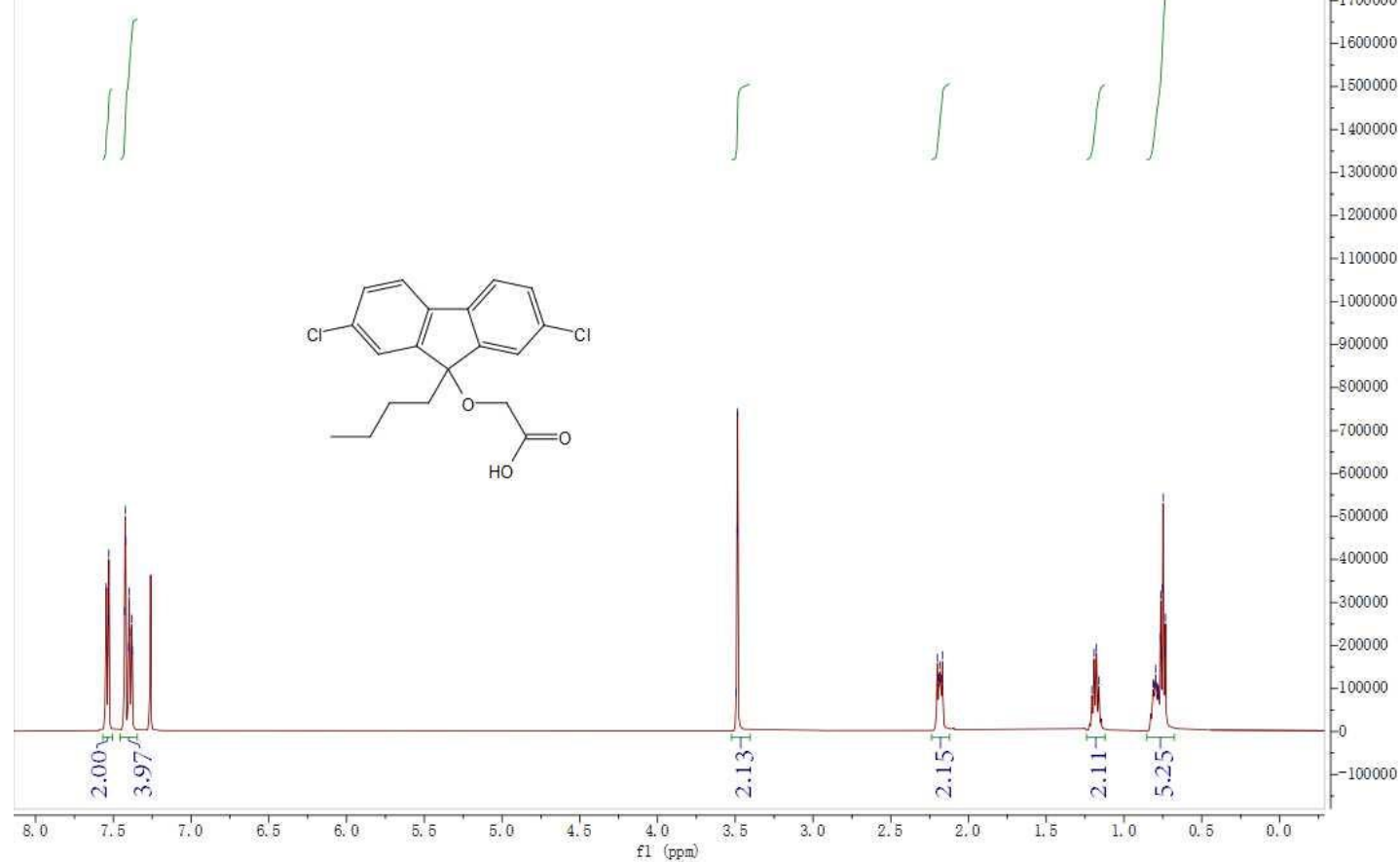

${ }^{13} \mathrm{C}$ NMR (126 MHz, $\mathrm{CDCl}_{3}$ )

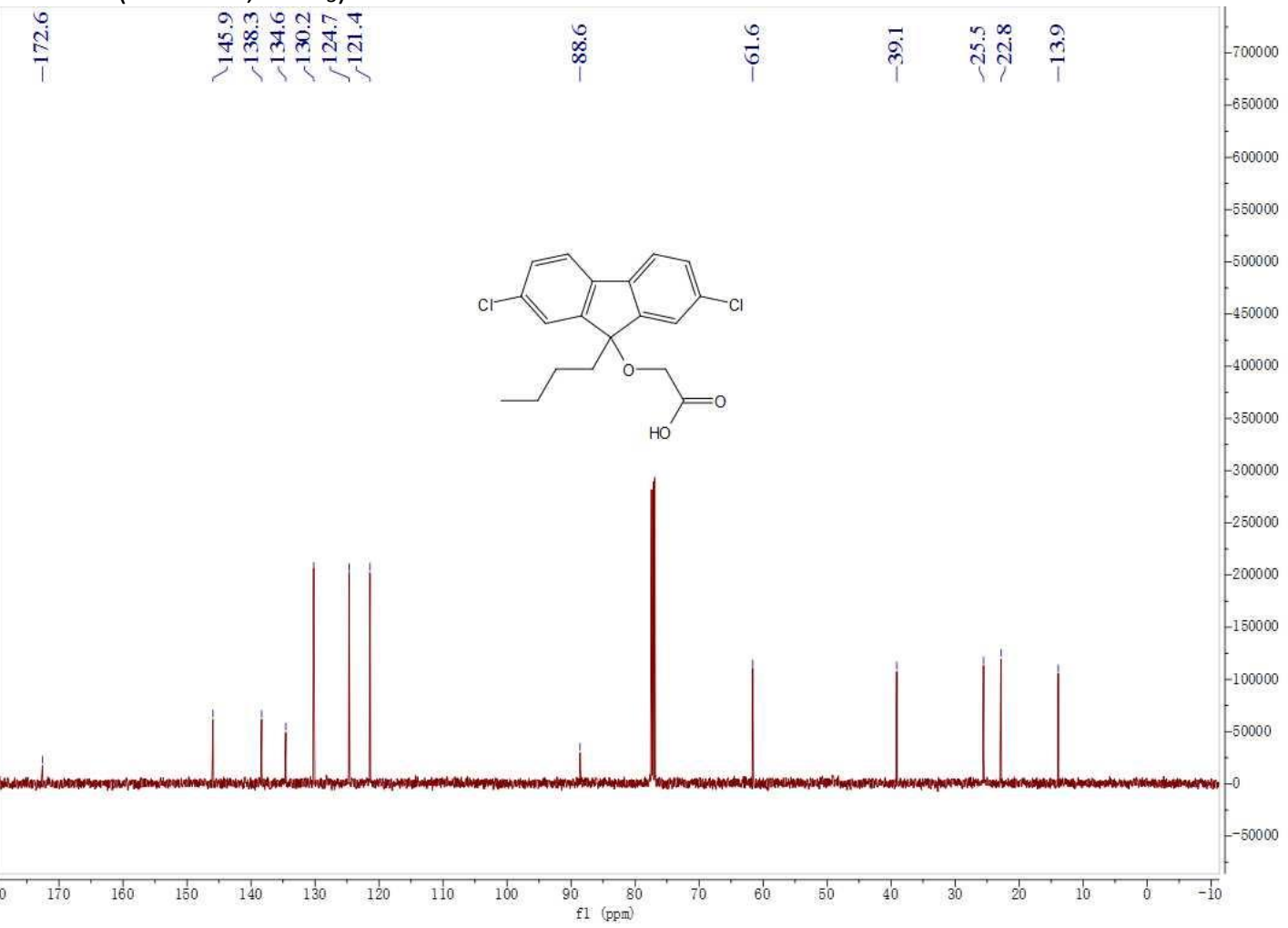


${ }^{1} \mathrm{H}$ NMR $\left(500 \mathrm{MHz}, \mathrm{CDCl}_{3}\right)-57$

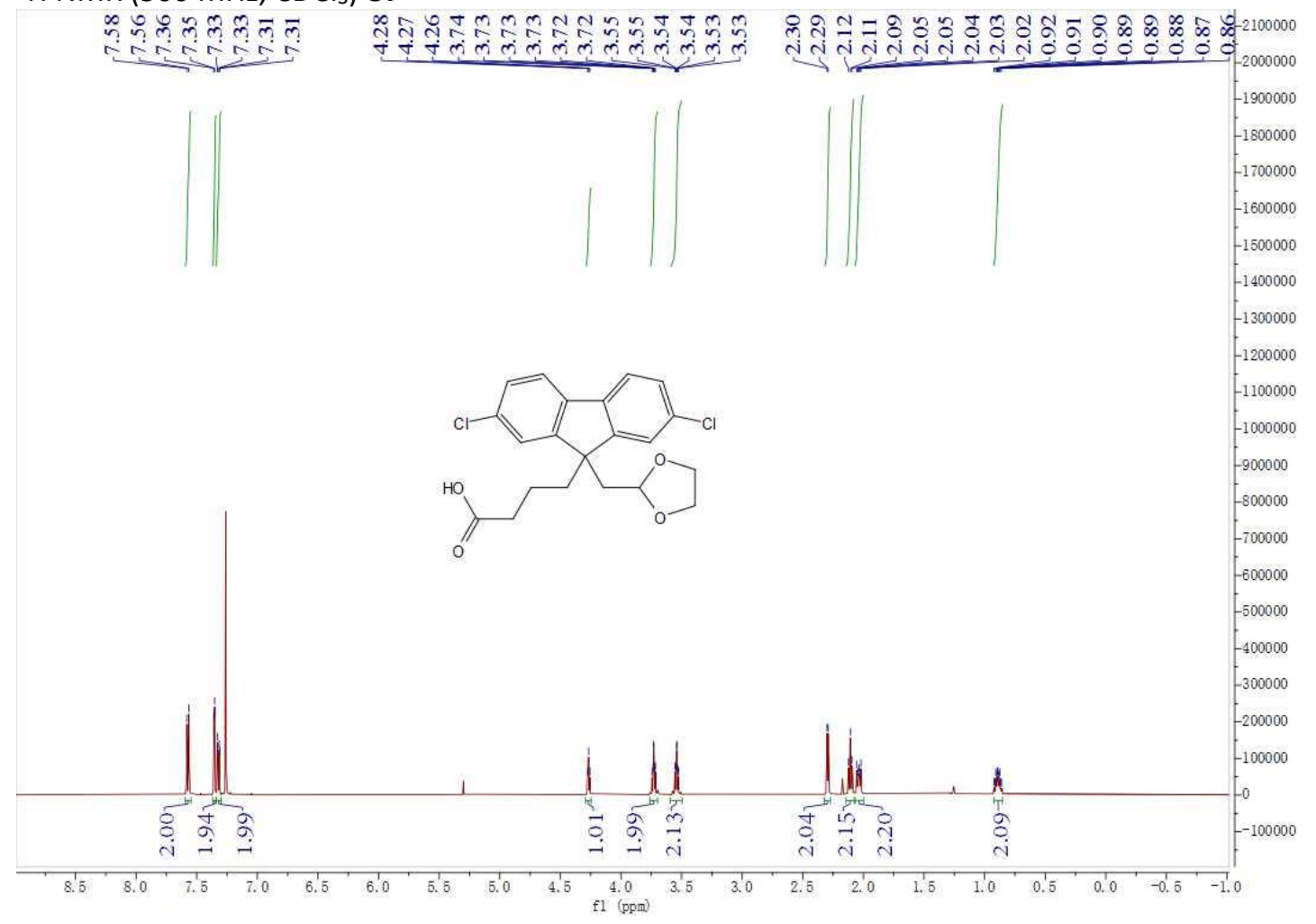

${ }^{13} \mathrm{C}$ NMR (101 MHz, $\left.\mathrm{CDCl}_{3}\right)-57$

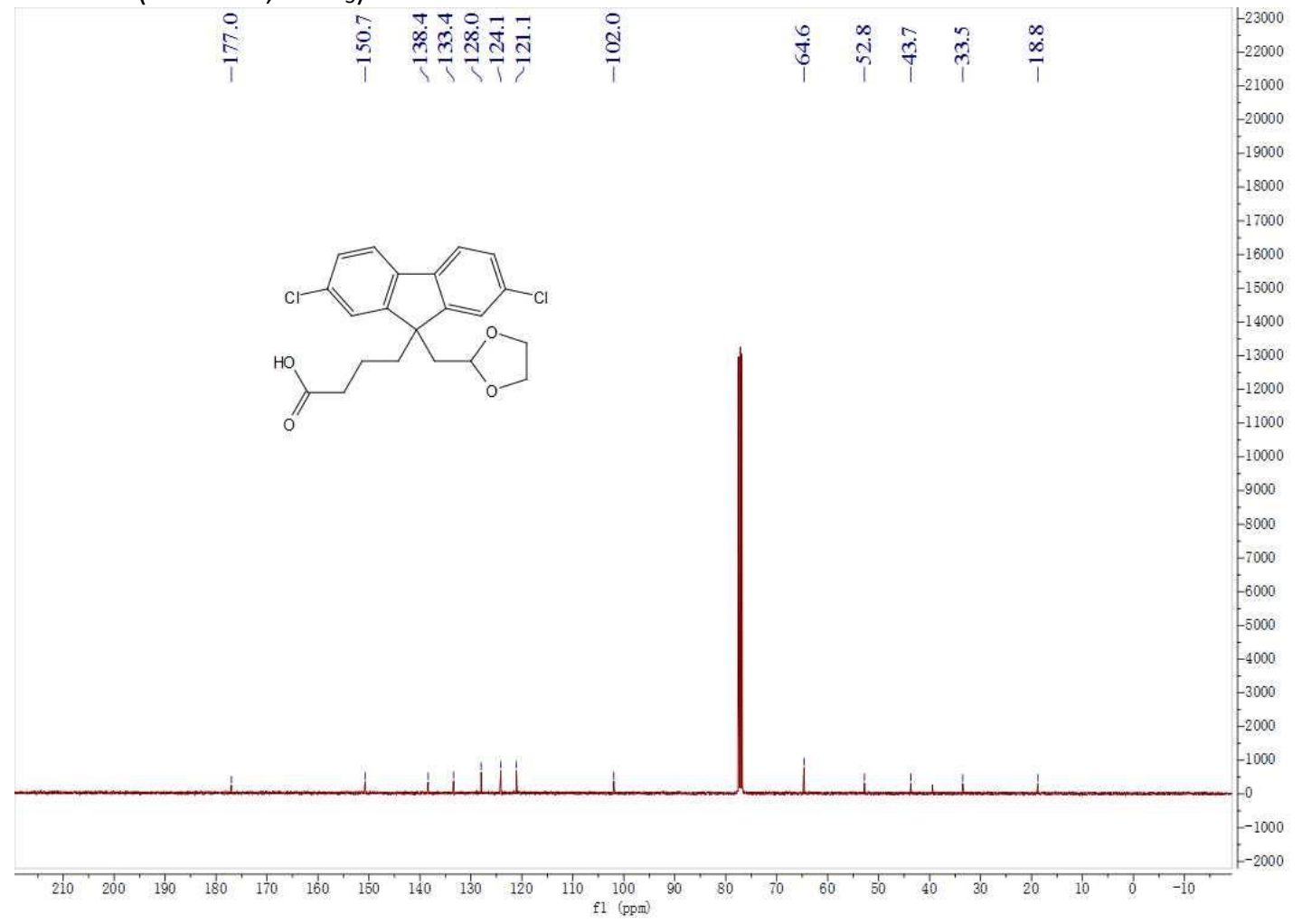


${ }^{1} \mathrm{H}$ NMR $\left(500 \mathrm{MHz}, \mathrm{CDCl}_{3}\right)-1$

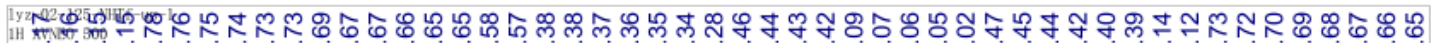
om

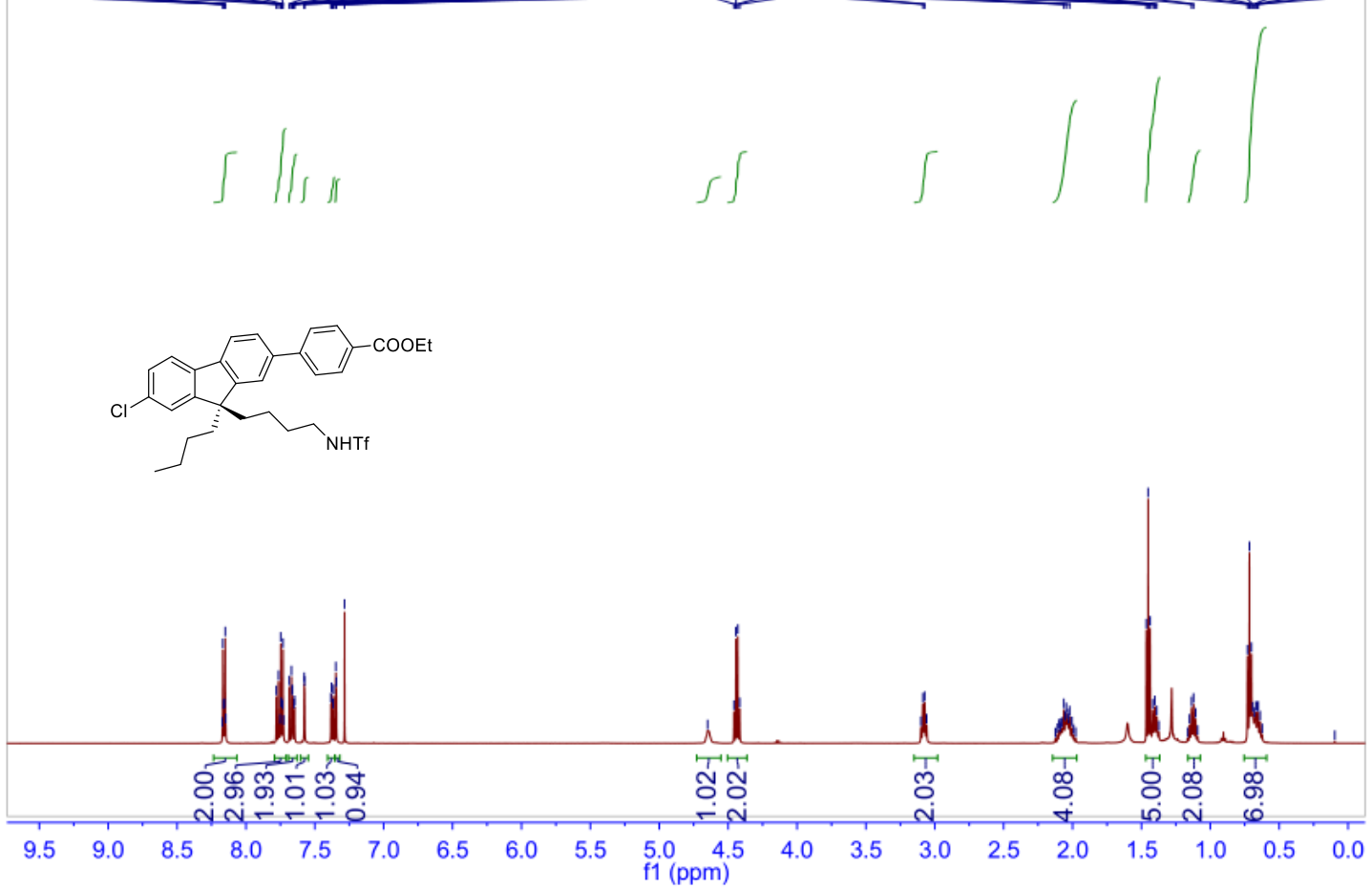

${ }^{13} \mathrm{C}$ NMR $\left(126 \mathrm{MHz}, \mathrm{CDCl}_{3}\right)-1$

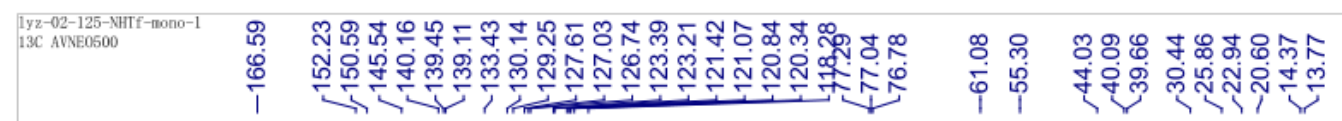

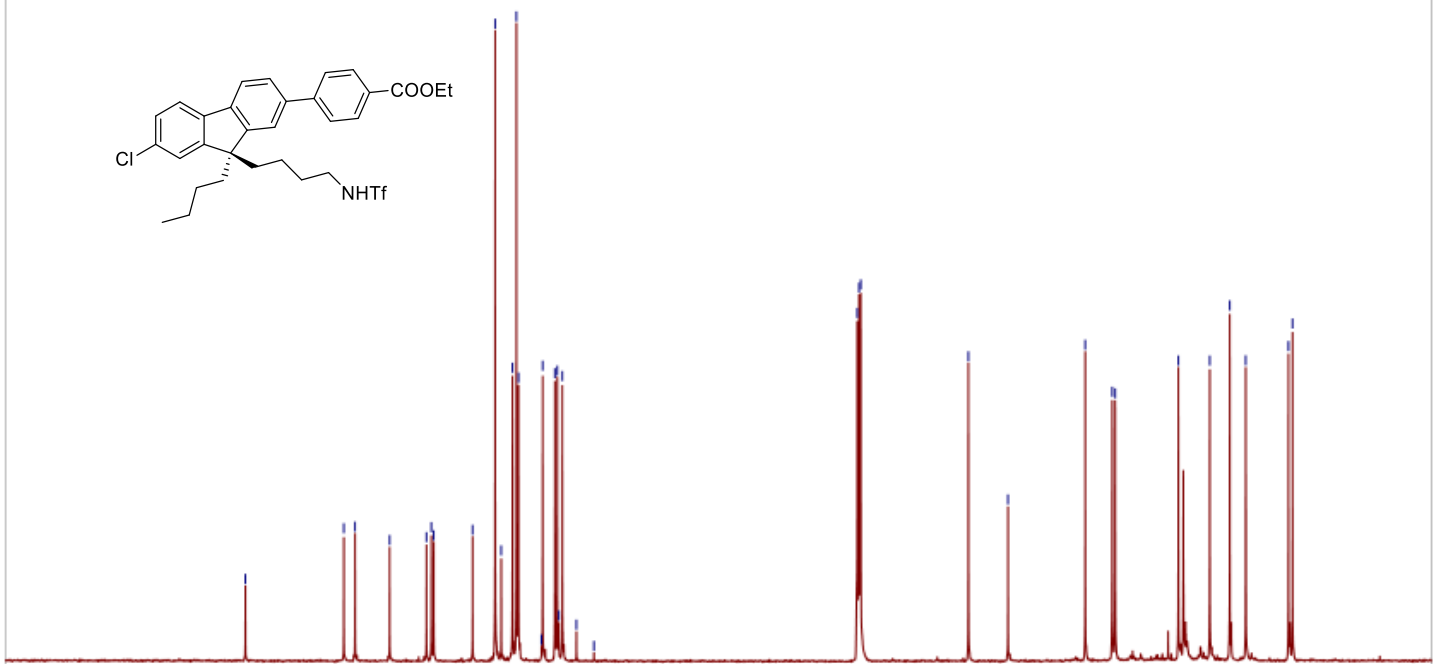

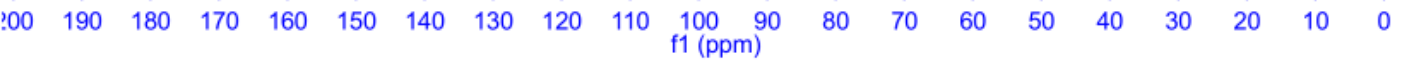


$\left.{ }^{19} \mathrm{~F} \mathrm{NMR} \mathrm{(471} \mathrm{MHz,} \mathrm{CDCl}_{3}\right)-1$

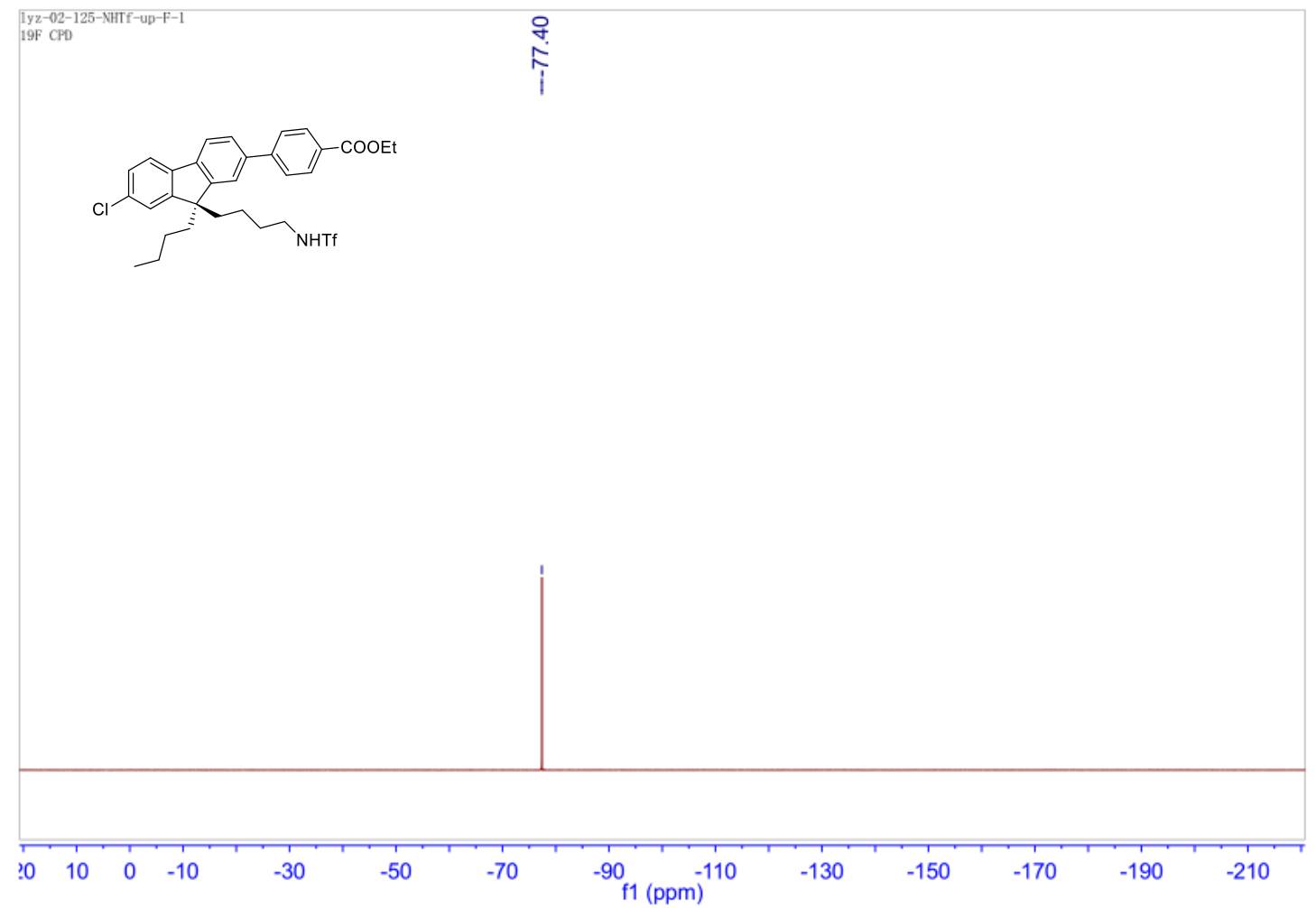


${ }^{1} \mathrm{H}$ NMR $\left(500 \mathrm{MHz}, \mathrm{CDCl}_{3}\right)-2$

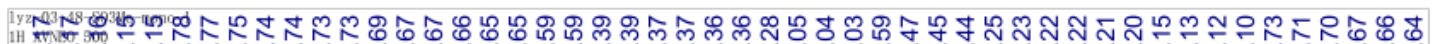

o

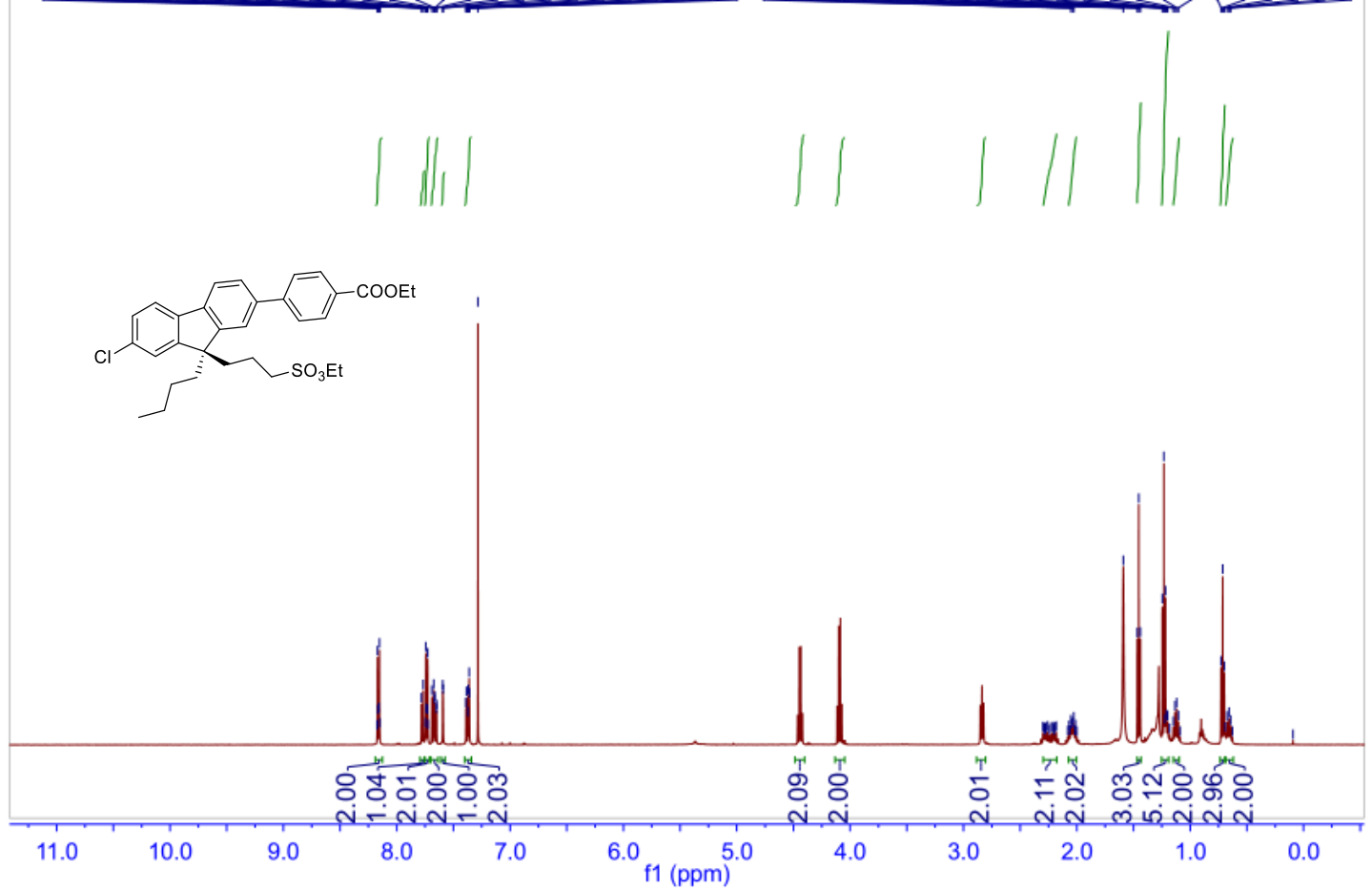

${ }^{13} \mathrm{C} \mathrm{NMR}\left(126 \mathrm{MHz}, \mathrm{CDCl}_{3}\right)-2$

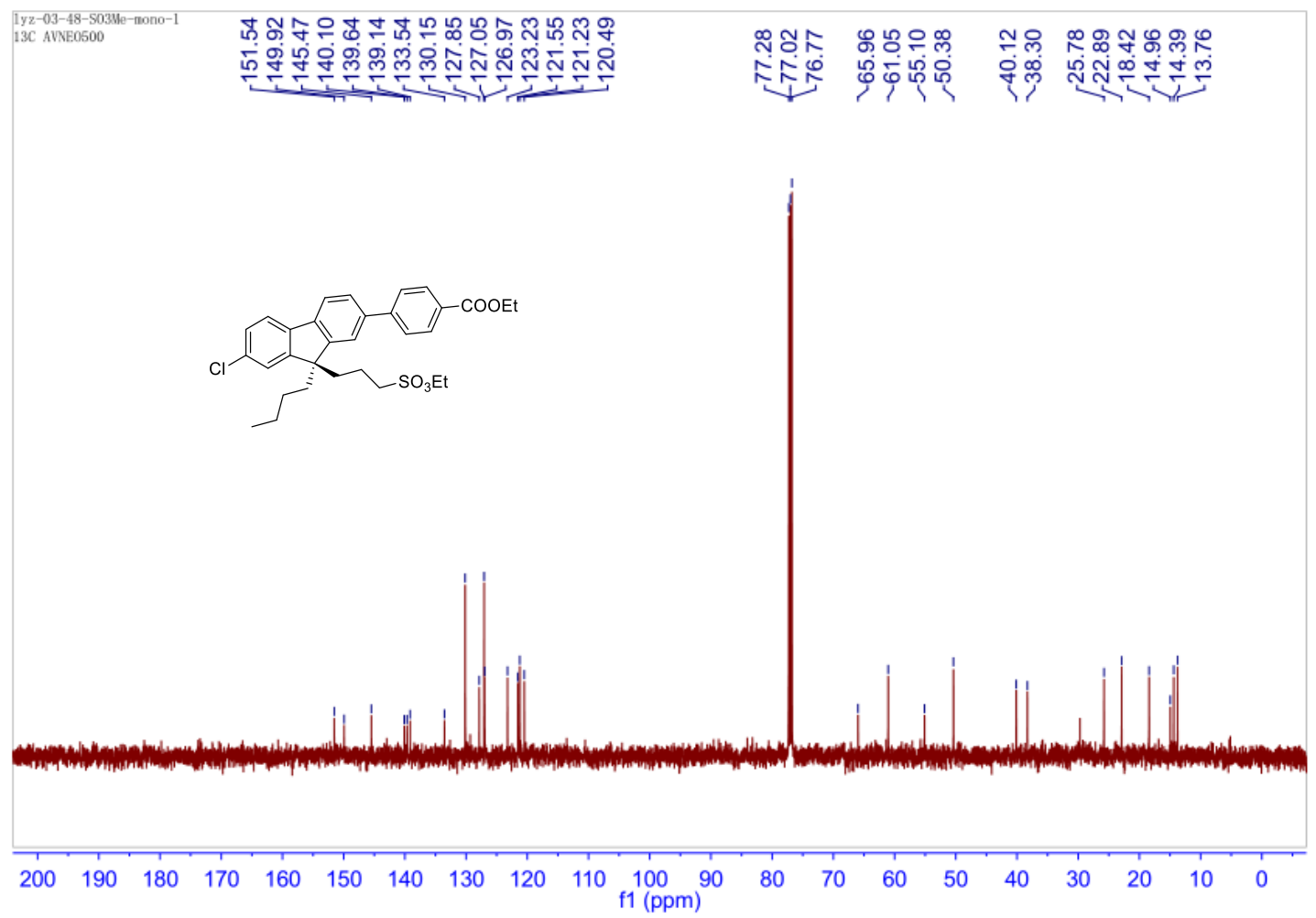


${ }^{1} \mathrm{H}$ NMR $\left(500 \mathrm{MHz}, \mathrm{CDCl}_{3}\right)-3$

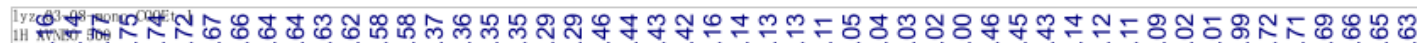

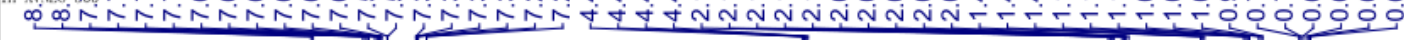

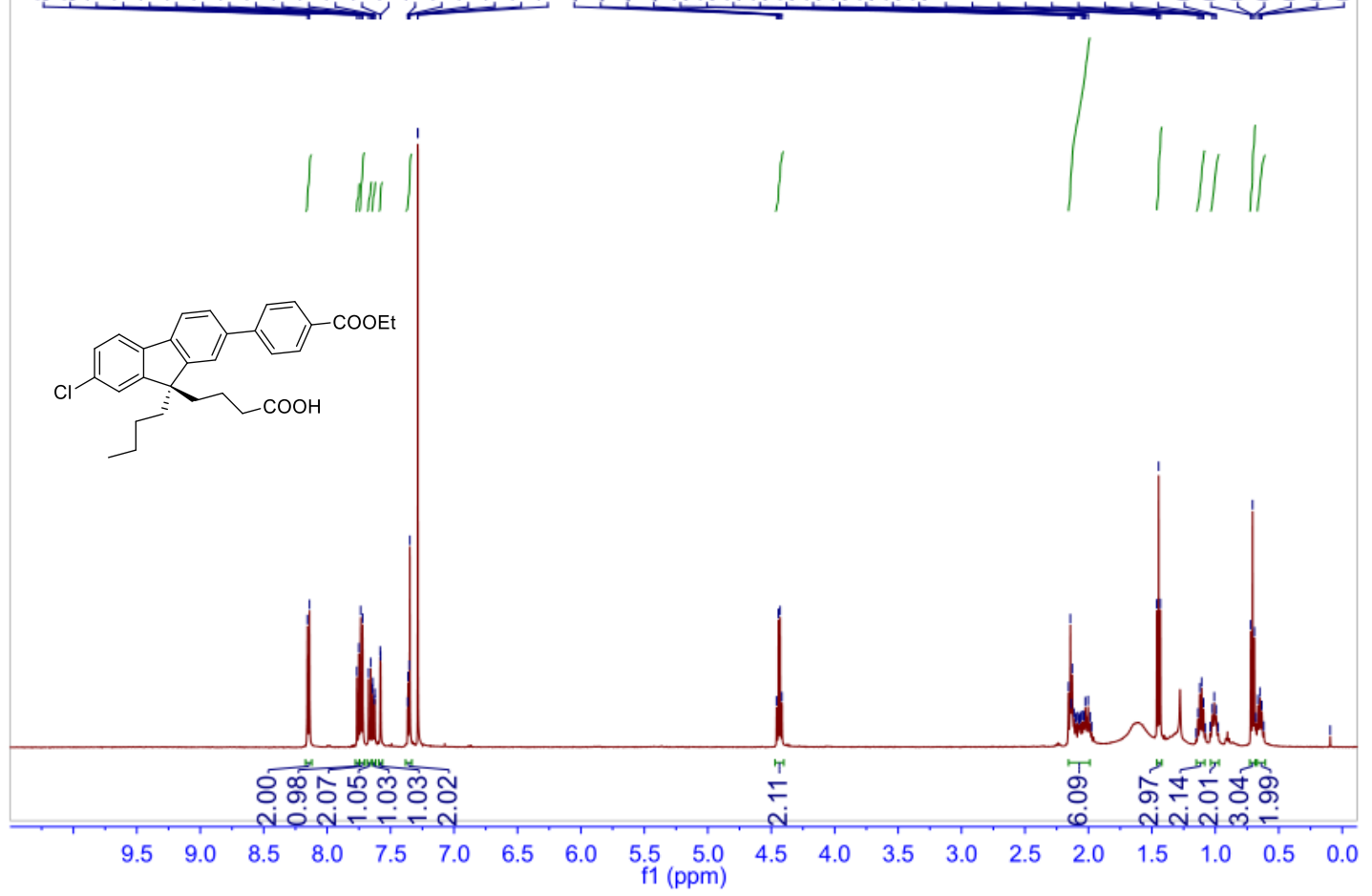

${ }^{13} \mathrm{C} \mathrm{NMR}\left(126 \mathrm{MHz}, \mathrm{CDCl}_{3}\right)-3$

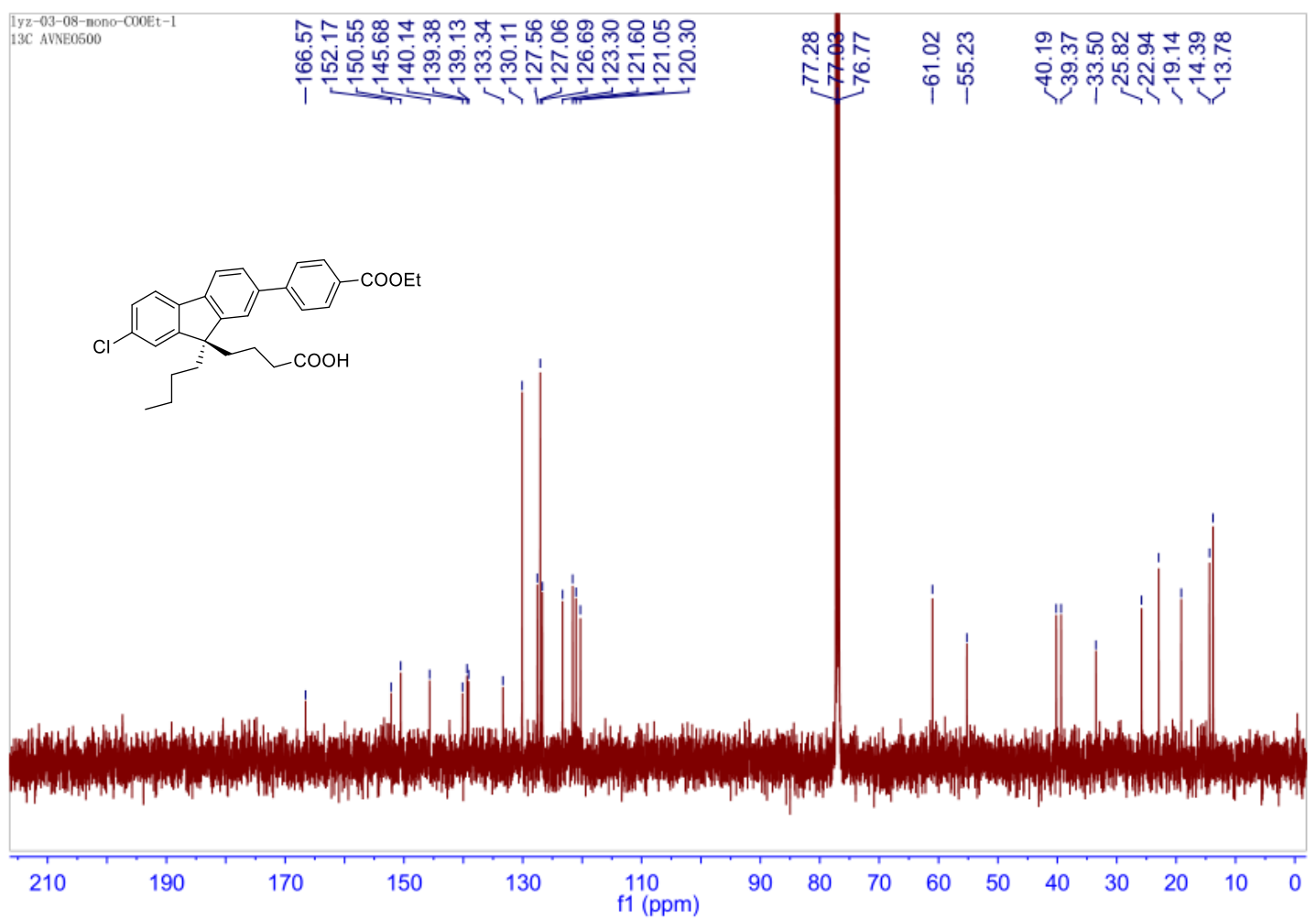


${ }^{1} \mathrm{H}$ NMR (500 MHz, $\mathrm{CDCl}_{3}$ ) -4

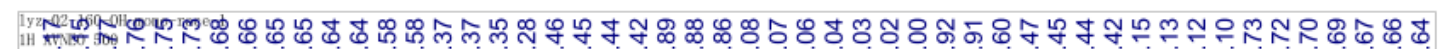

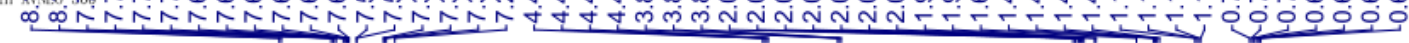

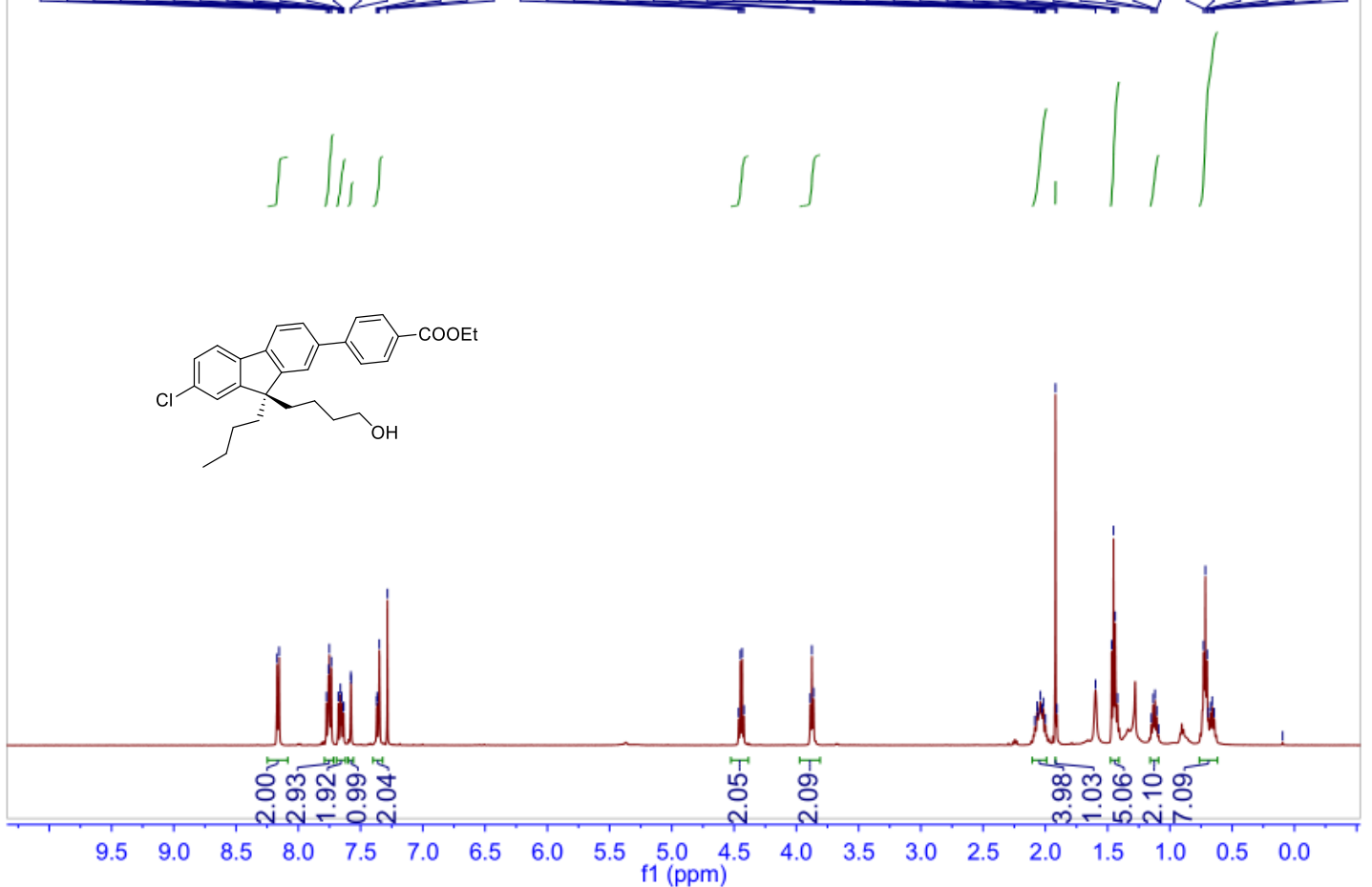

${ }^{13} \mathrm{C} \mathrm{NMR}\left(126 \mathrm{MHz}, \mathrm{CDCl}_{3}\right)-4$

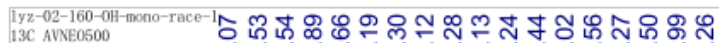

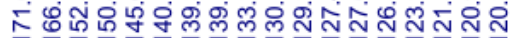
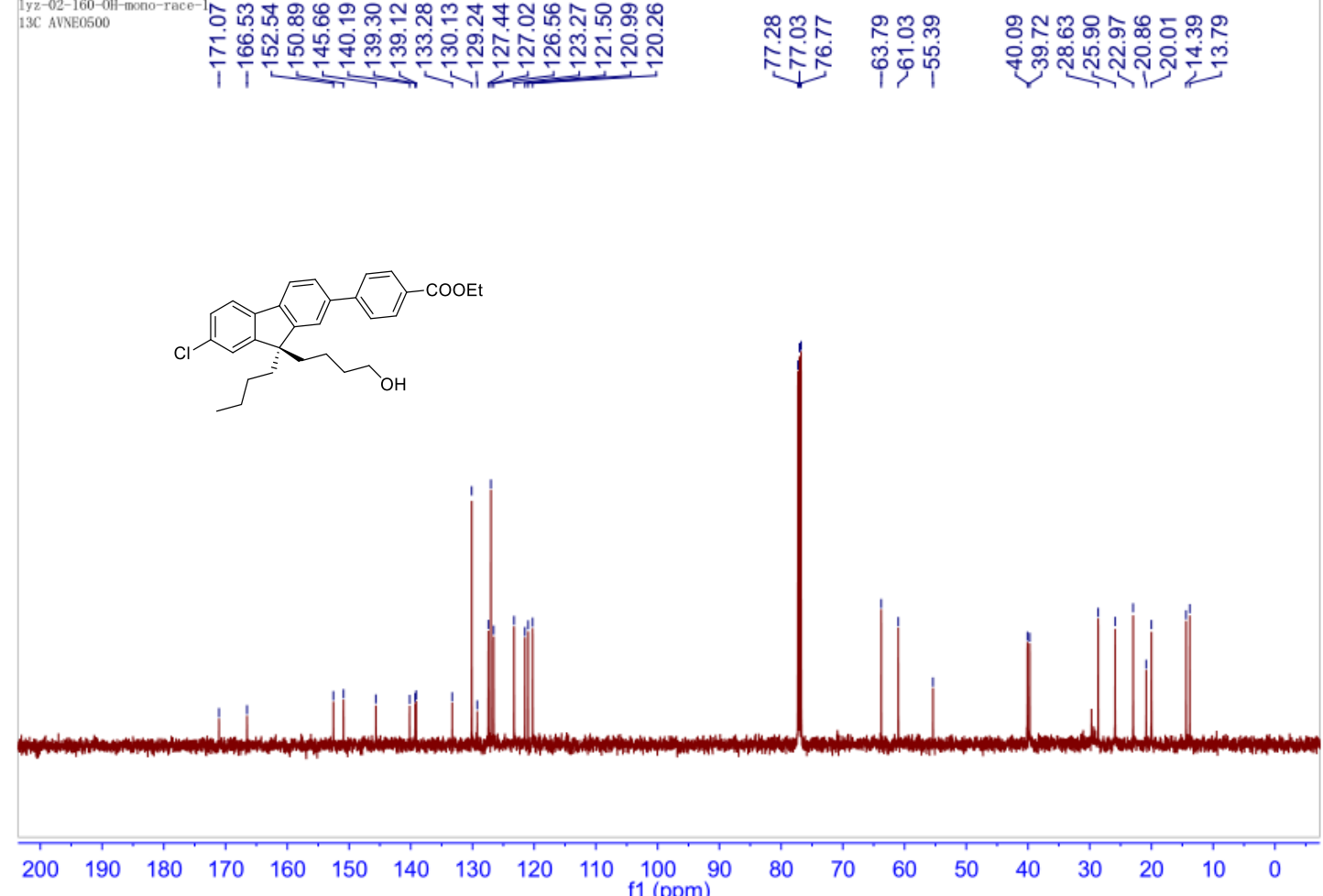
${ }^{1} \mathrm{H}$ NMR (500 MHz, CDCl 3 ) -5

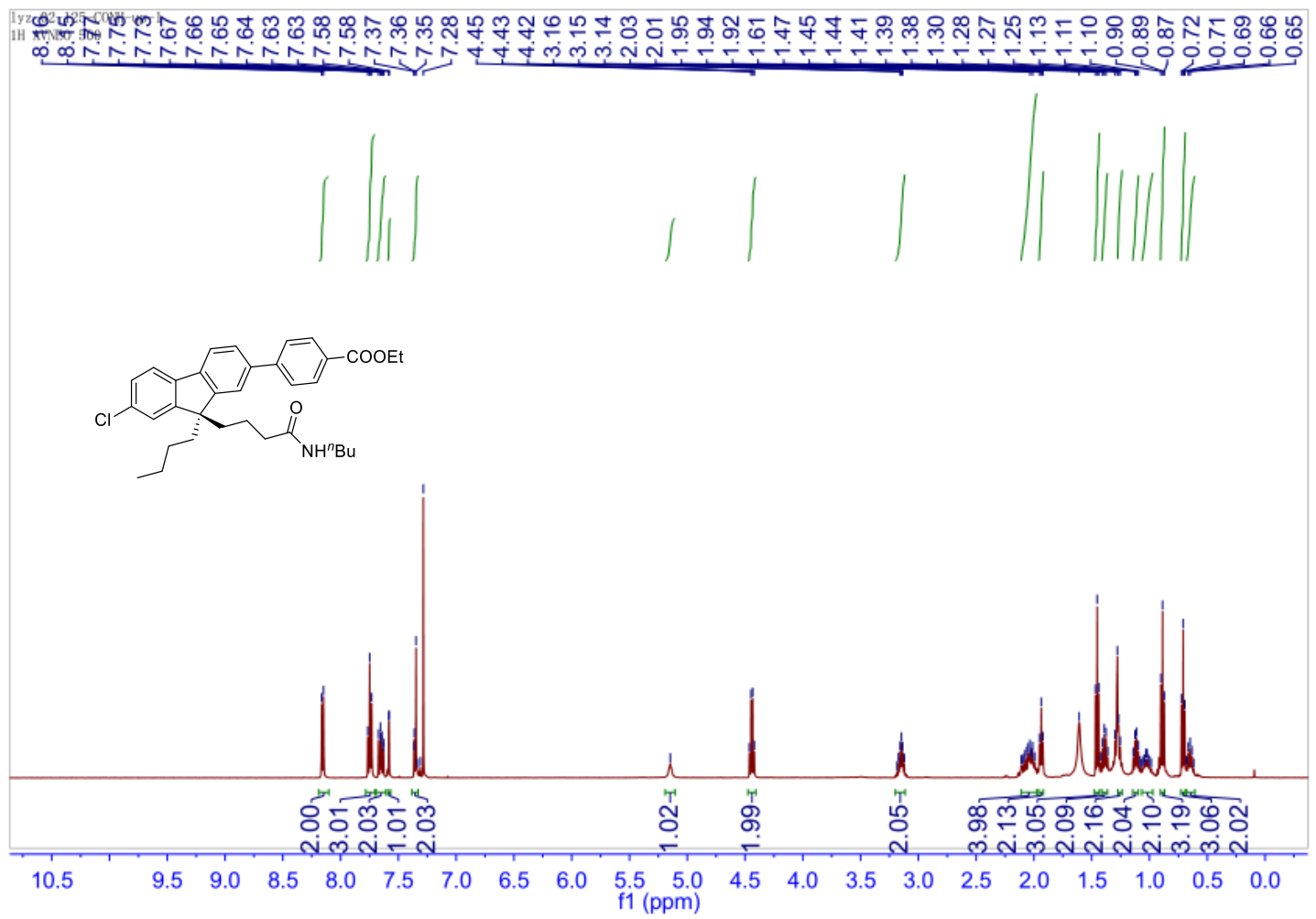

${ }^{13} \mathrm{C}$ NMR $\left(126 \mathrm{MHz}, \mathrm{CDCl}_{3}\right)-5$

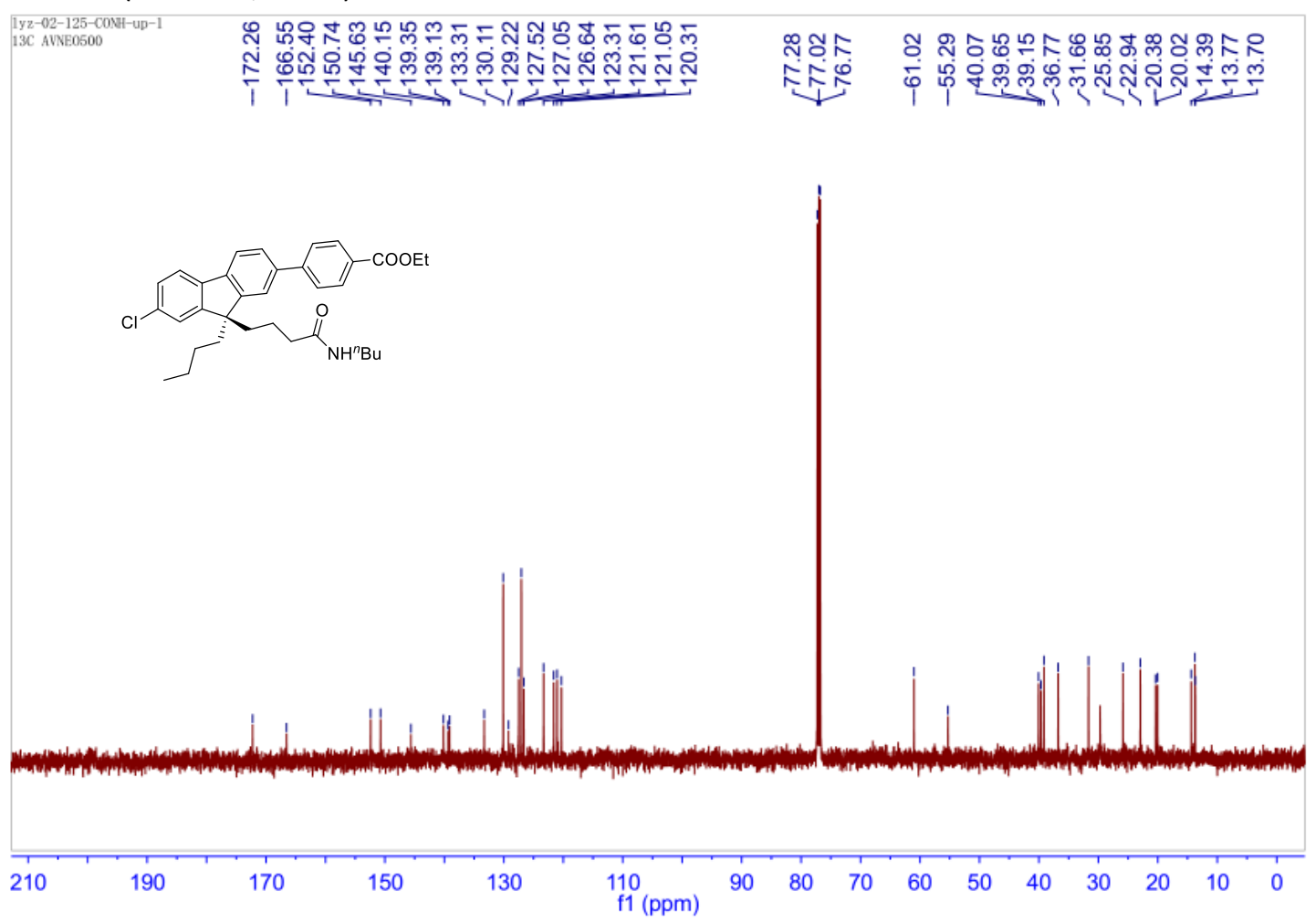


${ }^{1} \mathrm{H}$ NMR $\left(500 \mathrm{MHz}, \mathrm{CDCl}_{3}\right)-6$

rage zm

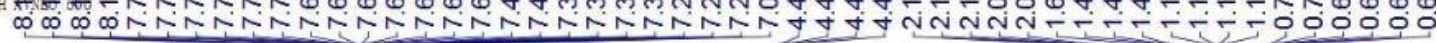
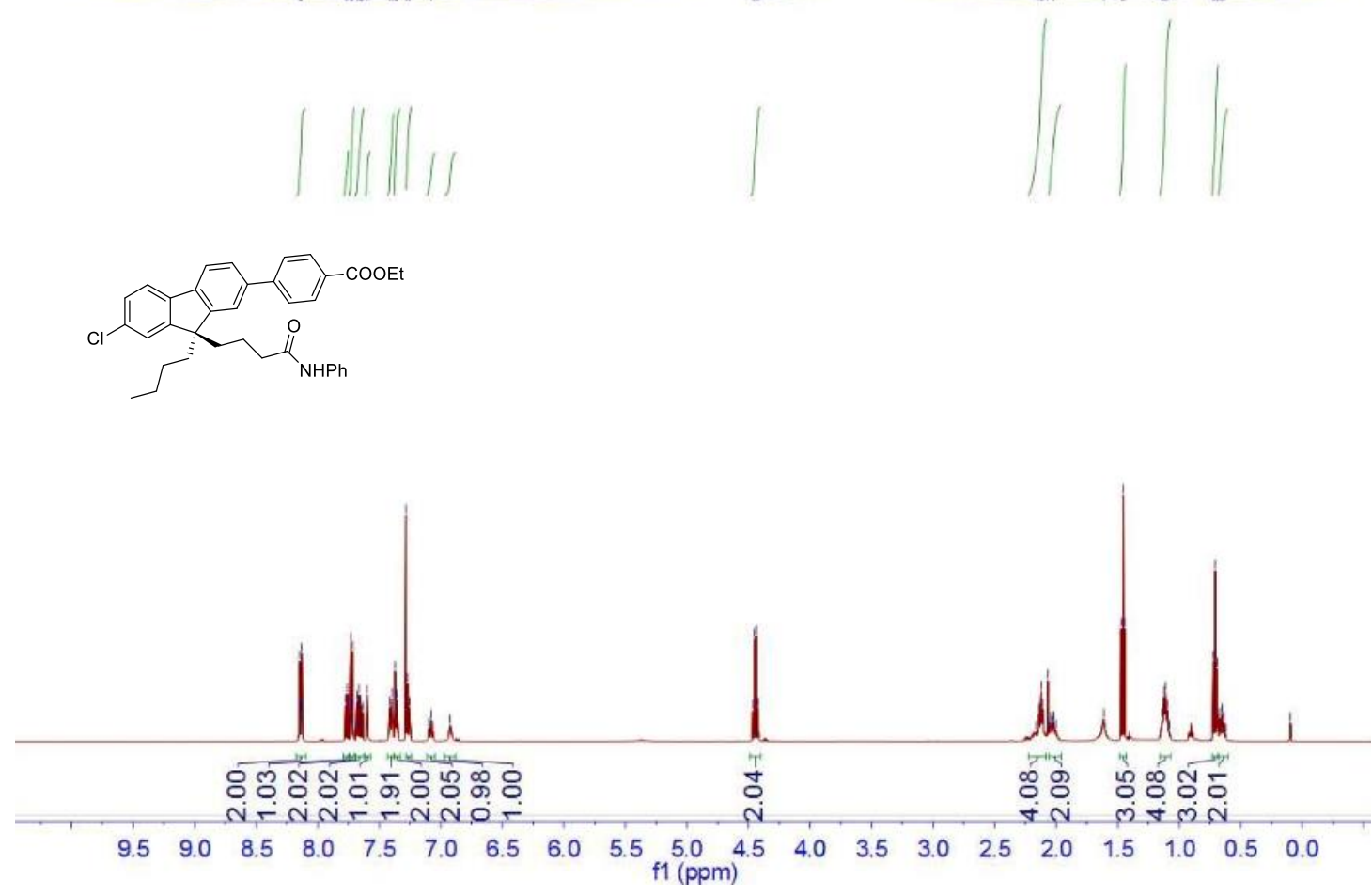

${ }^{13} \mathrm{C} \mathrm{NMR}\left(126 \mathrm{MHz} \mathrm{CDCl}_{3}\right)-6$

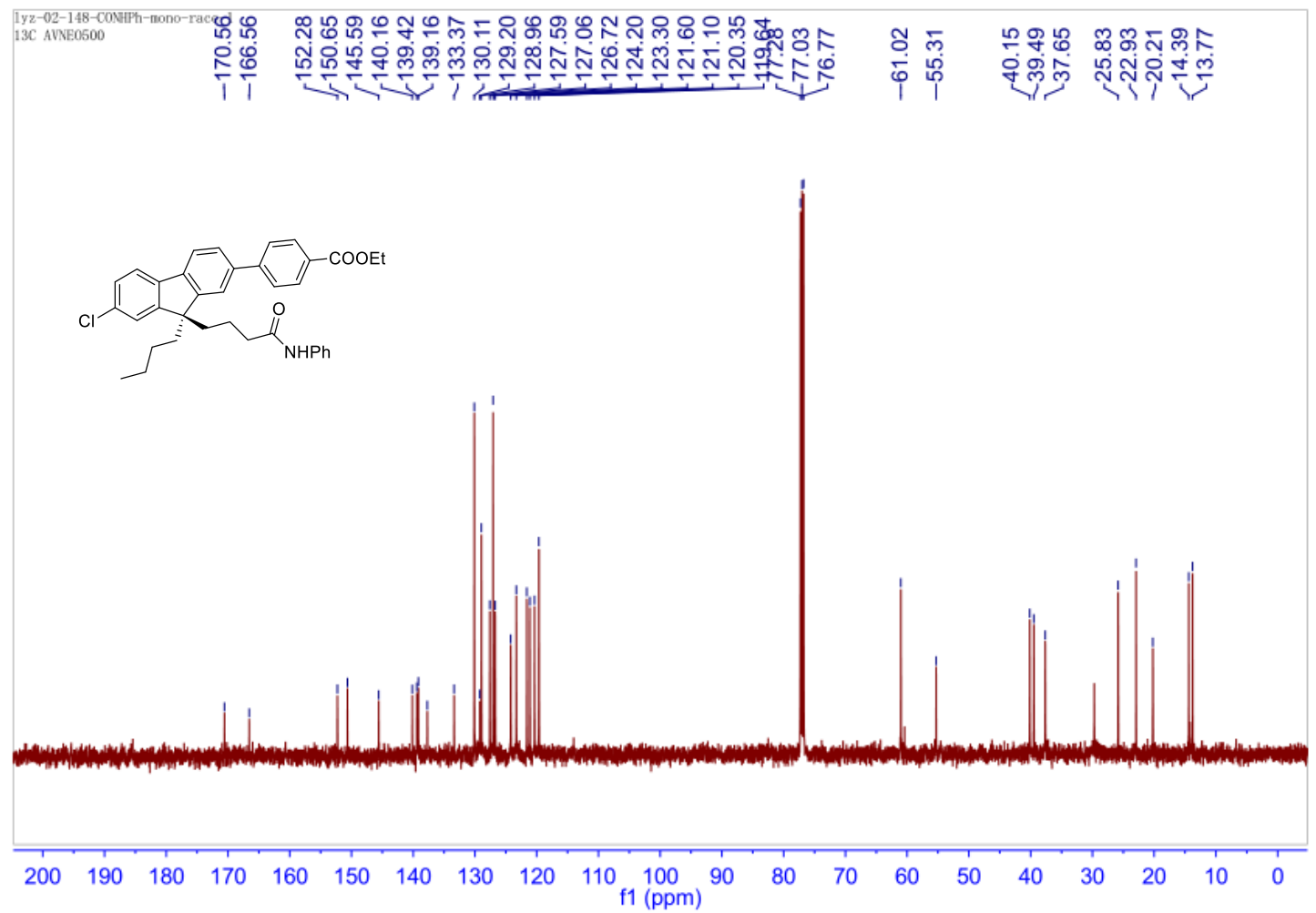


${ }^{1} \mathrm{H}$ NMR (500 MHz, $\mathrm{CDCl}_{3}$ ) -7

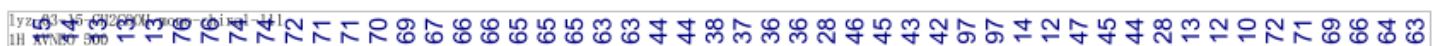

o
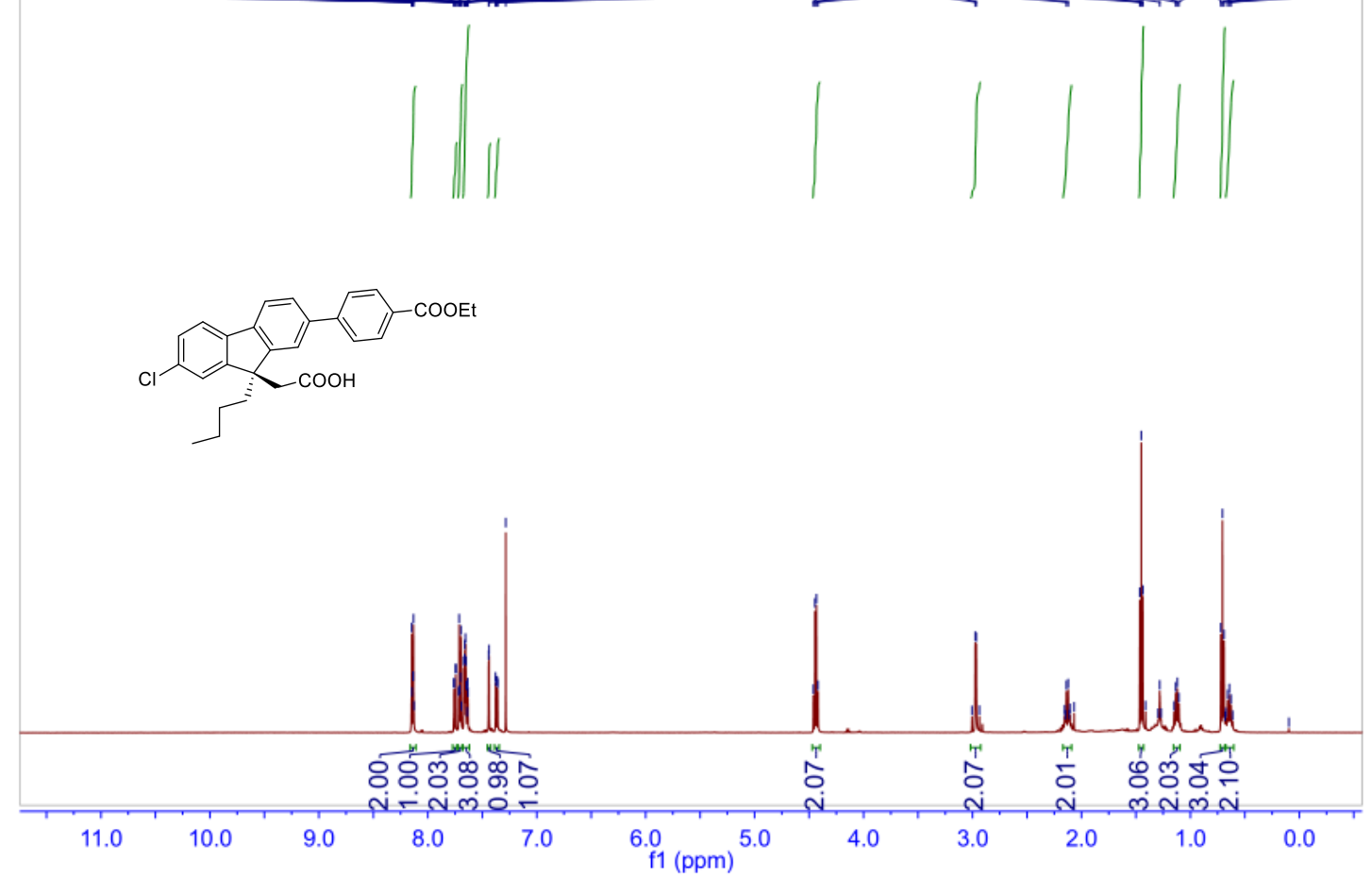

$\left.{ }^{13} \mathrm{C} \mathrm{NMR} \mathrm{(126} \mathrm{MHz,} \mathrm{CDCl}_{3}\right)-7$

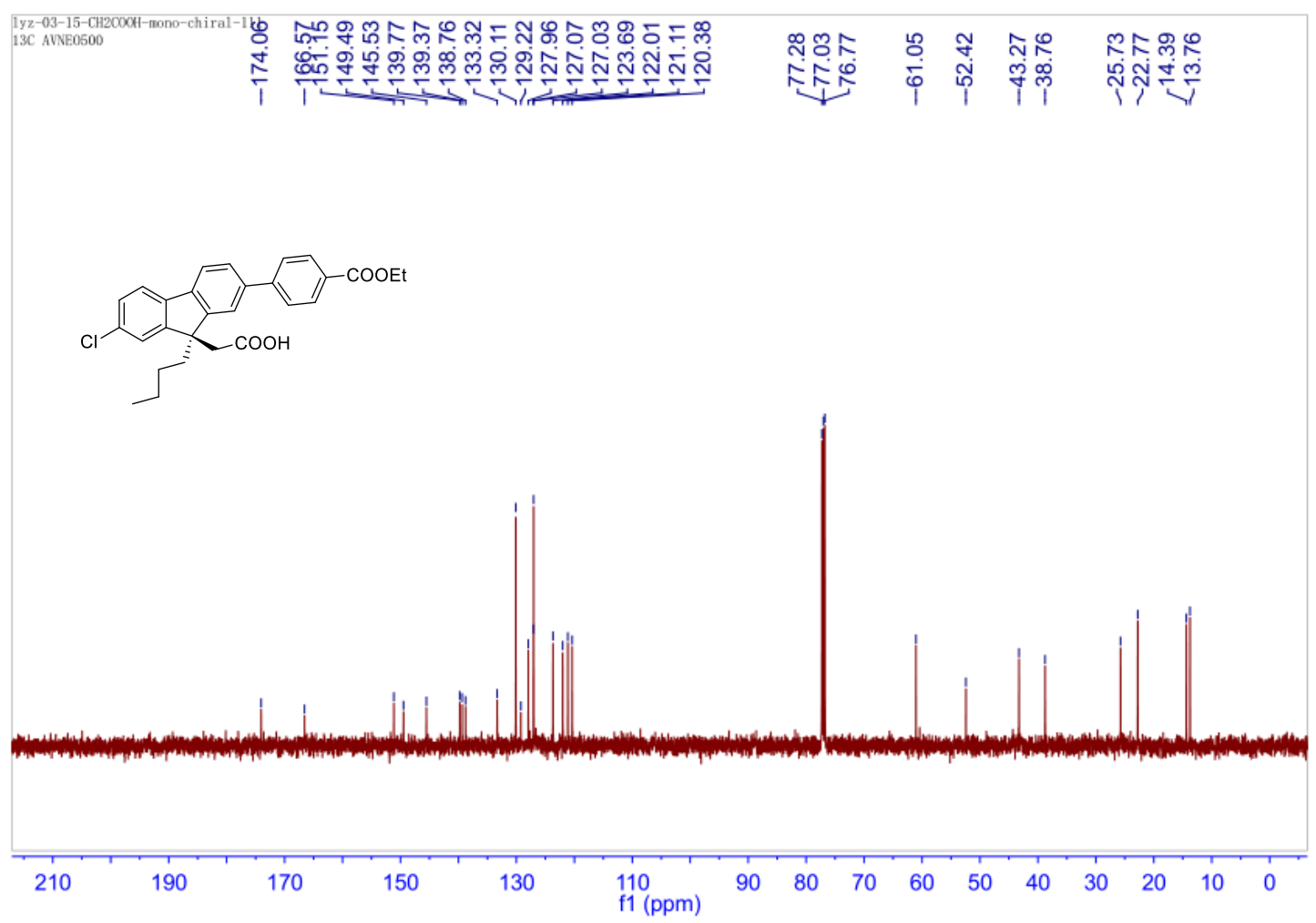


${ }^{1} \mathrm{H}$ NMR $\left(500 \mathrm{MHz}, \mathrm{CDCl}_{3}\right)-8$

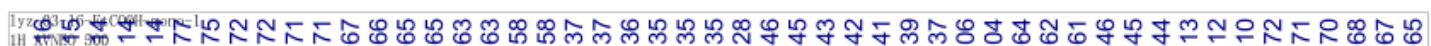
o

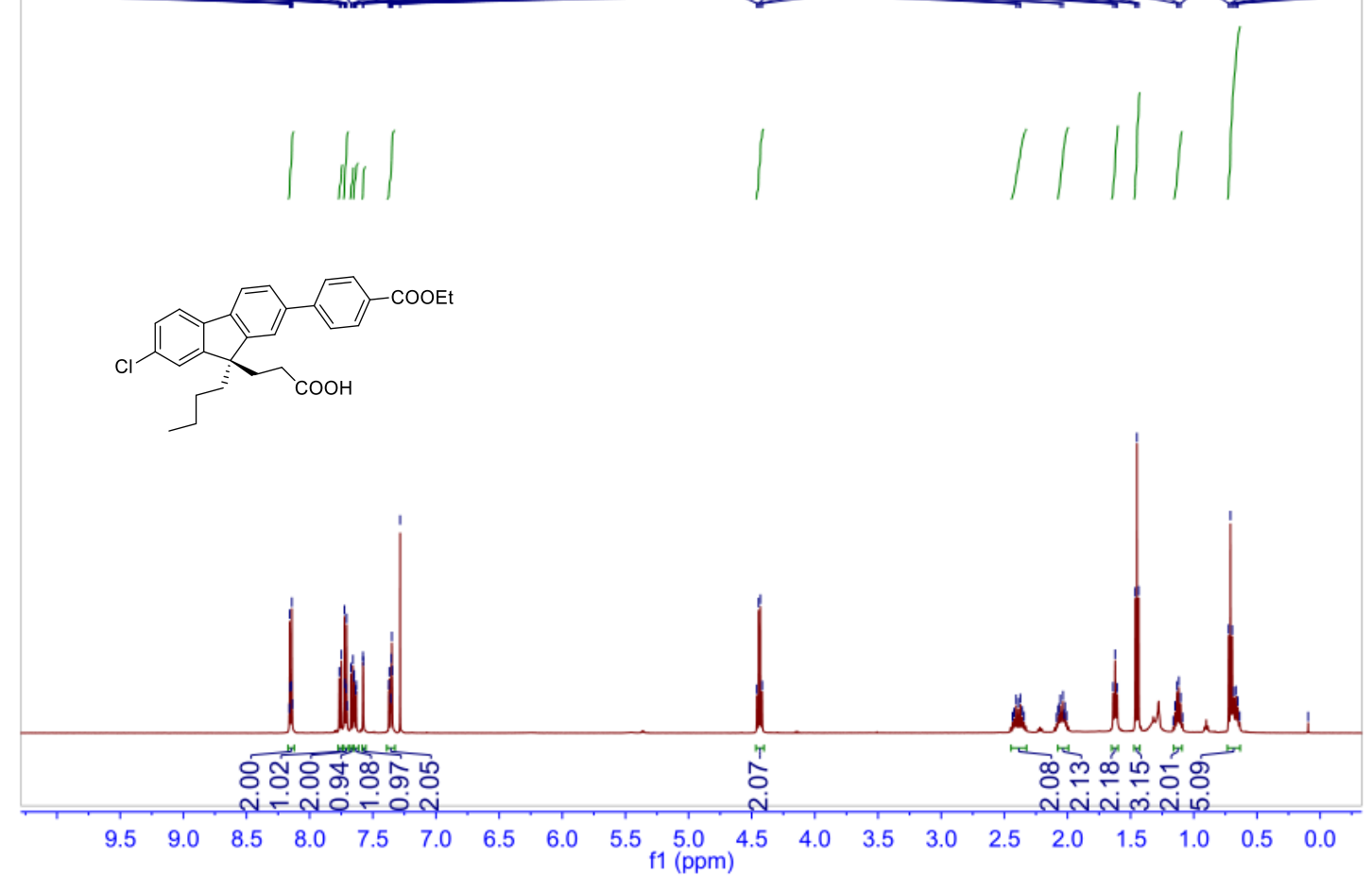

${ }^{13} \mathrm{C} \mathrm{NMR}\left(126 \mathrm{MHz}, \mathrm{CDCl}_{3}\right)-8$

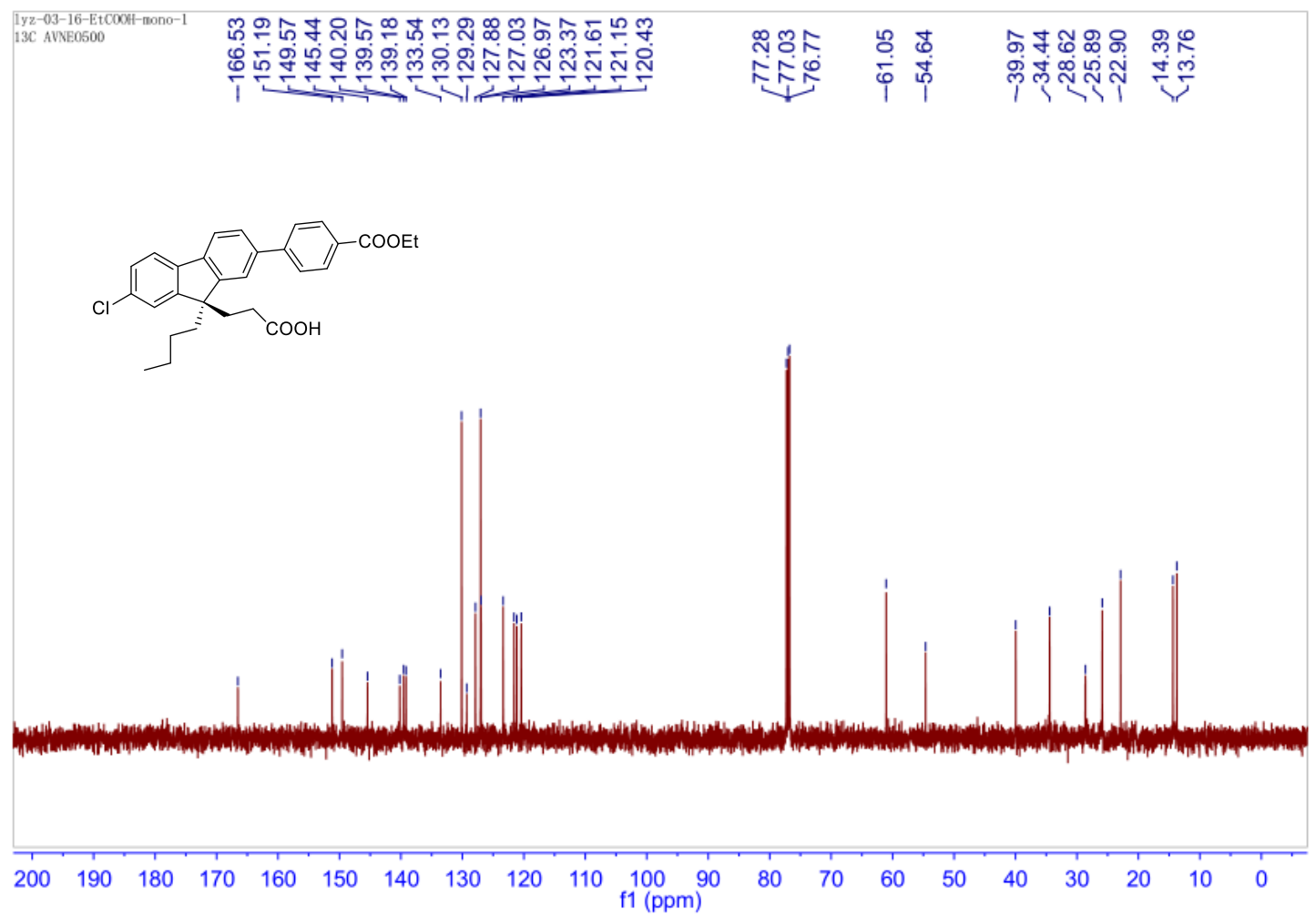


${ }^{1} \mathrm{H}$ NMR (300 MHz, $\mathrm{CDCl}_{3}$ ) -9

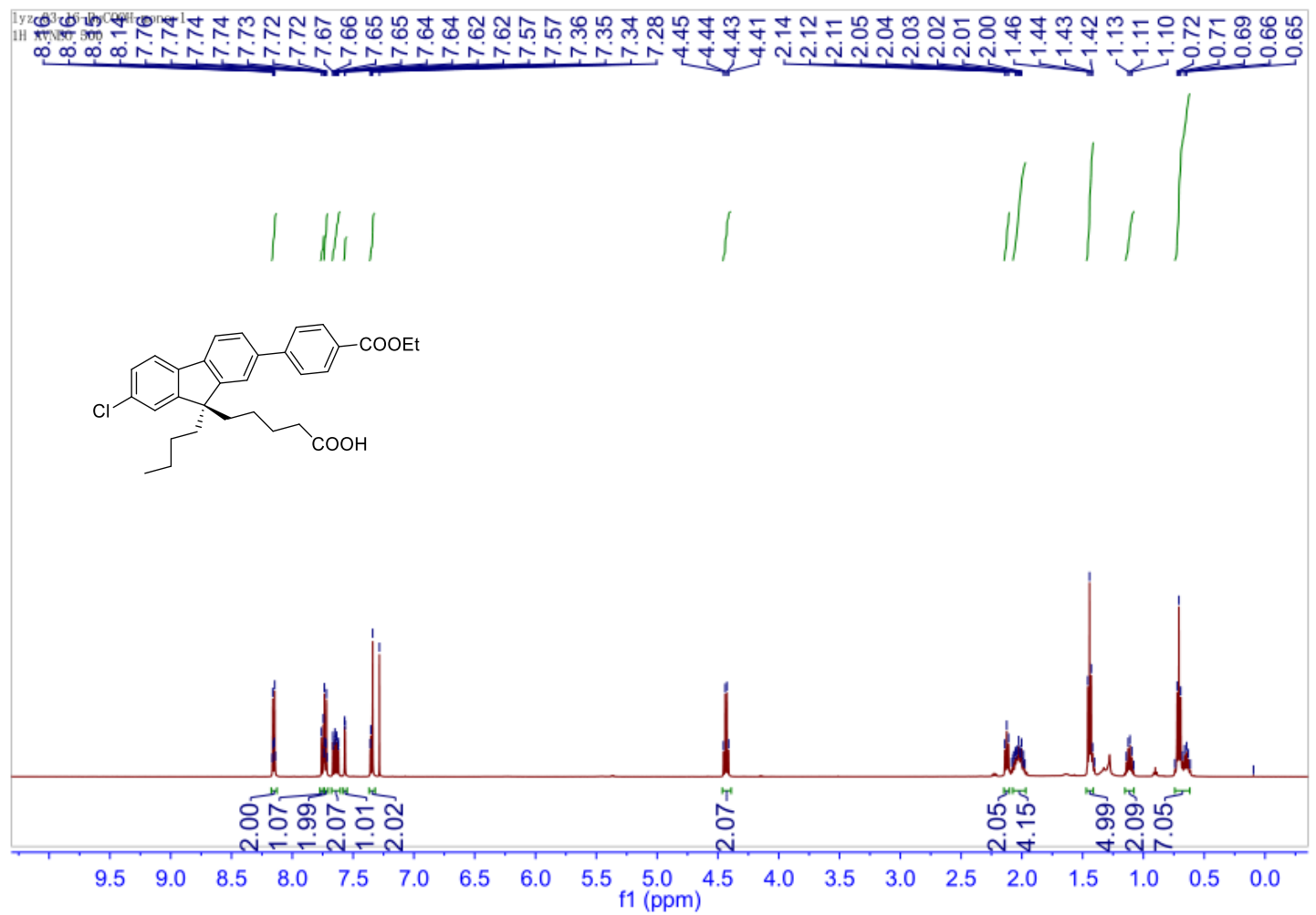

${ }^{13} \mathrm{C}$ NMR $\left(75 \mathrm{MHz}, \mathrm{CDCl}_{3}\right.$ ) -9

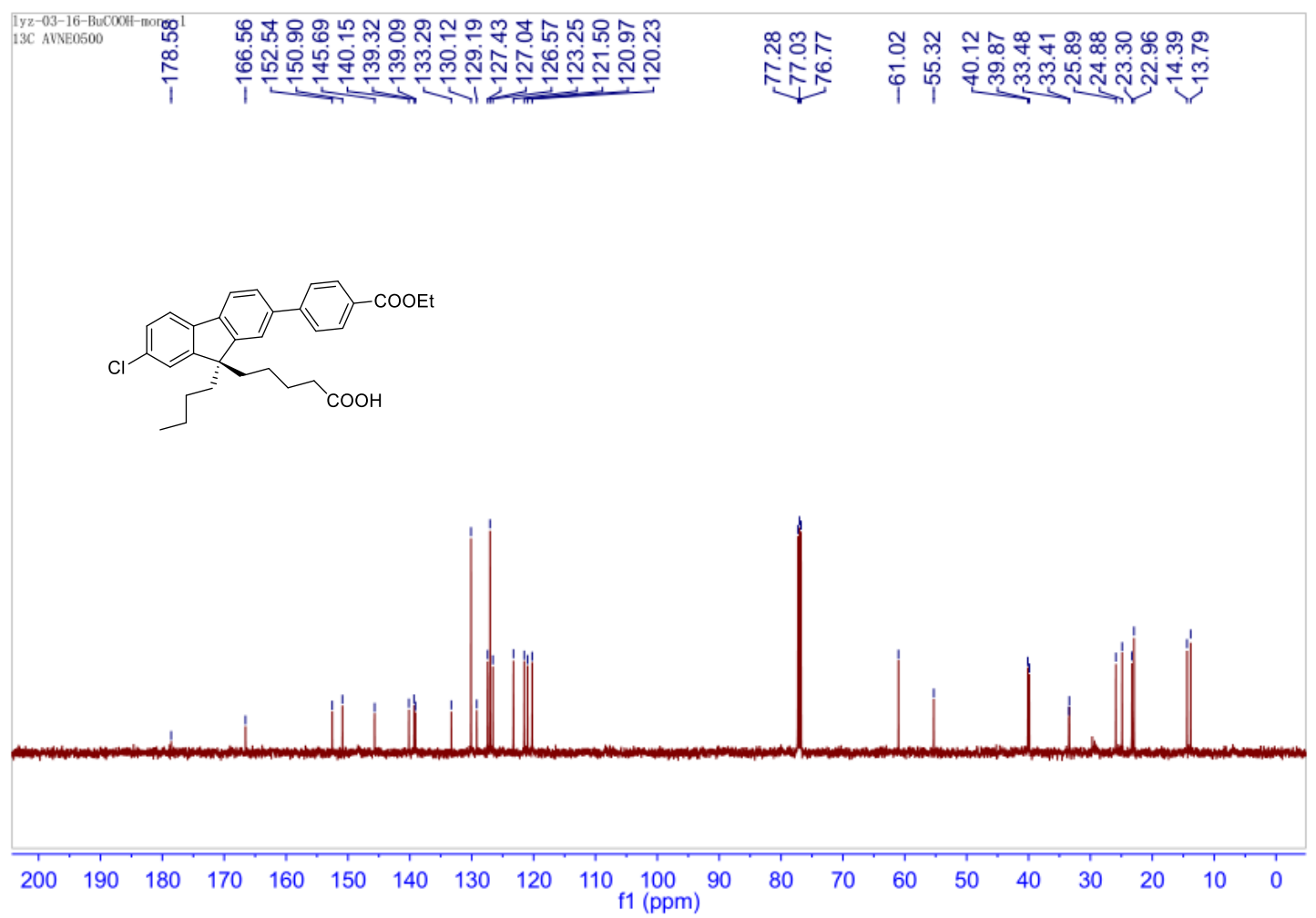


${ }^{1} \mathrm{H}$ NMR (500 MHz, $\left.\mathrm{CDCl}_{3}\right)-10$

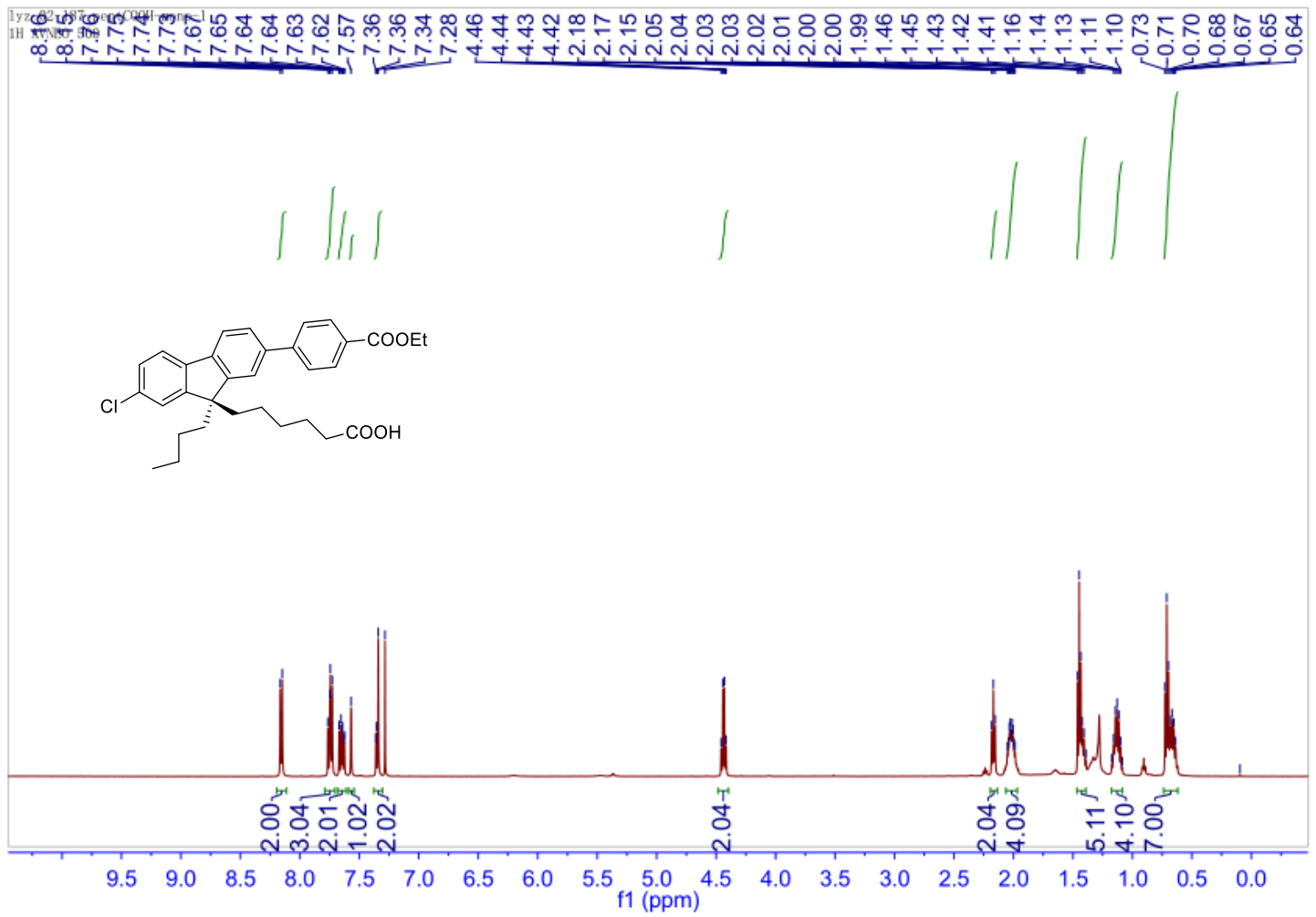

${ }^{13} \mathrm{C}$ NMR (126 MHz, $\left.\mathrm{CDCl}_{3}\right)-\mathbf{- 1 0}$

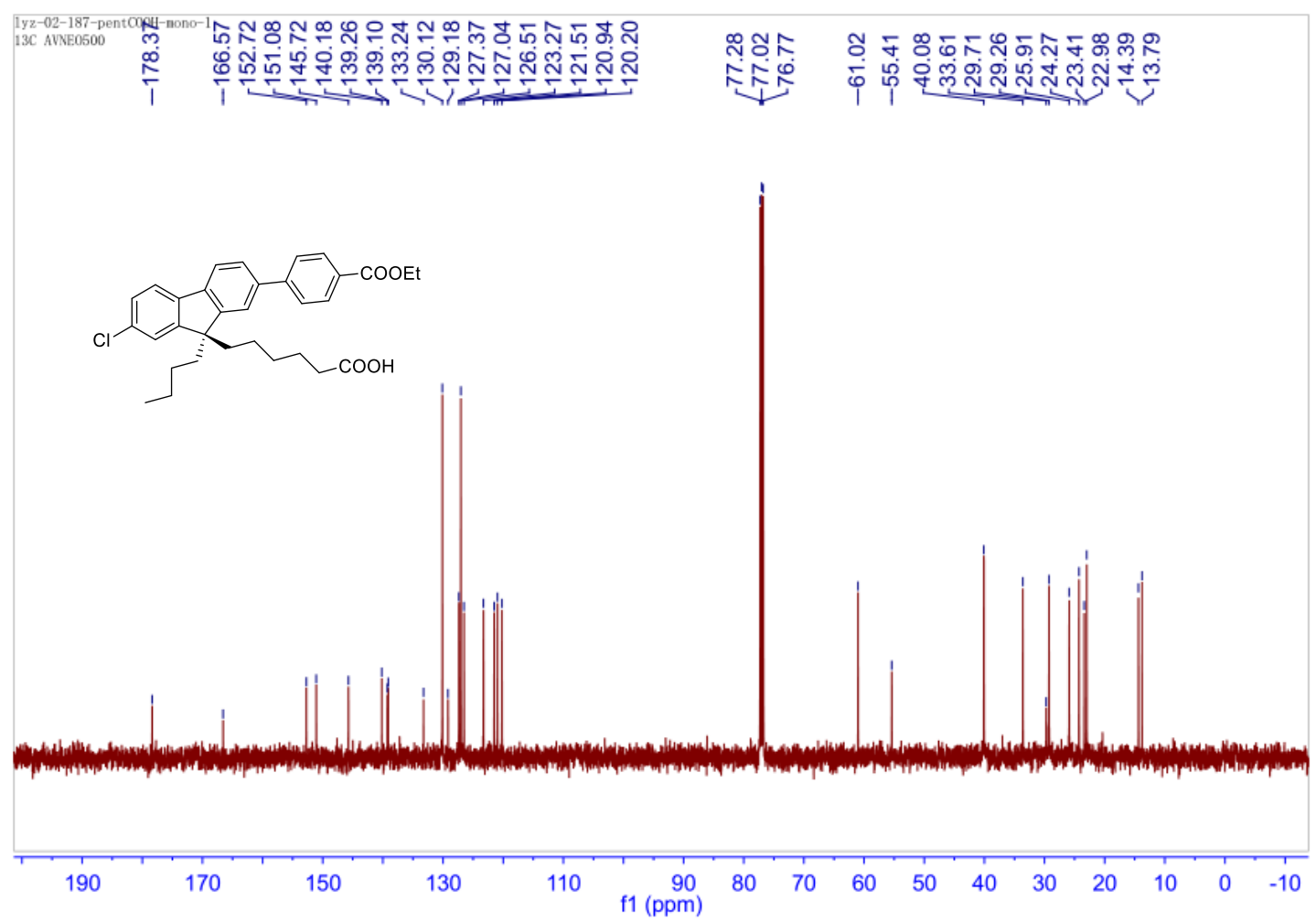


${ }^{1} \mathrm{H}$ NMR $\left(500 \mathrm{MHz}, \mathrm{CDCl}_{3}\right)-\mathbf{1 1}$

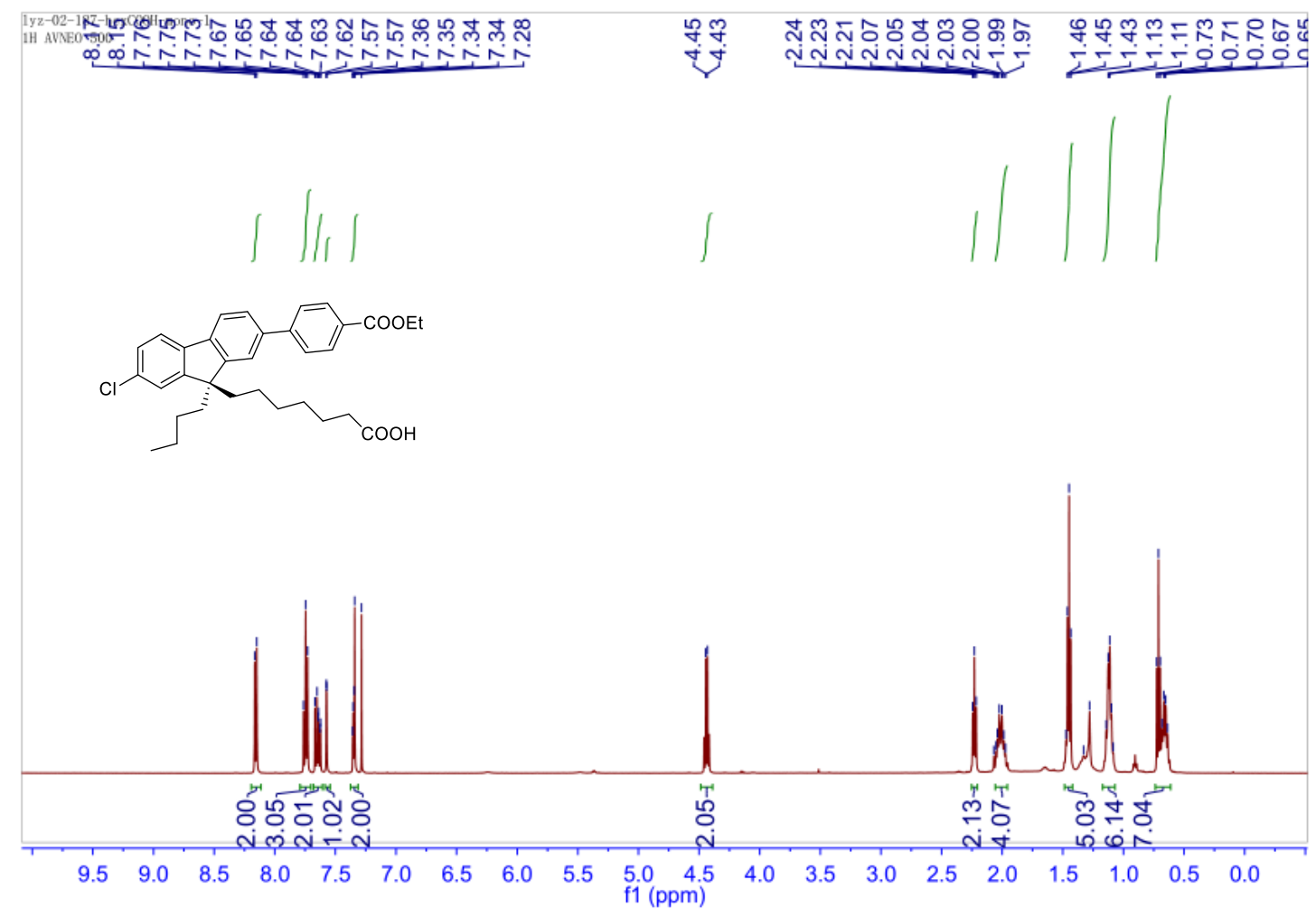

${ }^{13} \mathrm{C} \mathrm{NMR}\left(126 \mathrm{MHz}, \mathrm{CDCl}_{3}\right)-\mathbf{1 1}$

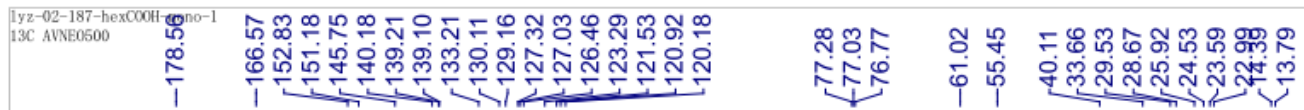
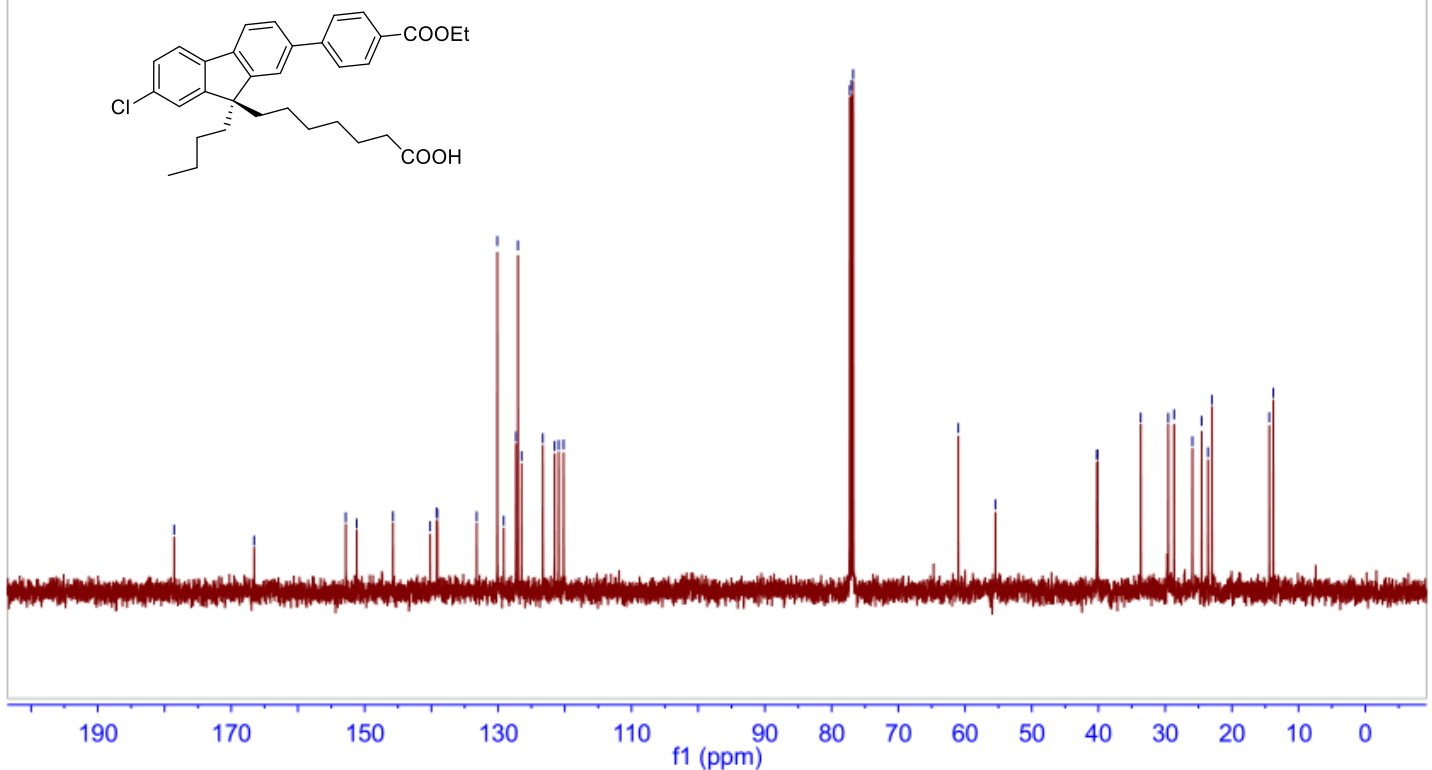
${ }^{1} \mathrm{H}$ NMR (500 MHz, $\mathrm{CDCl}_{3}$ ) -12

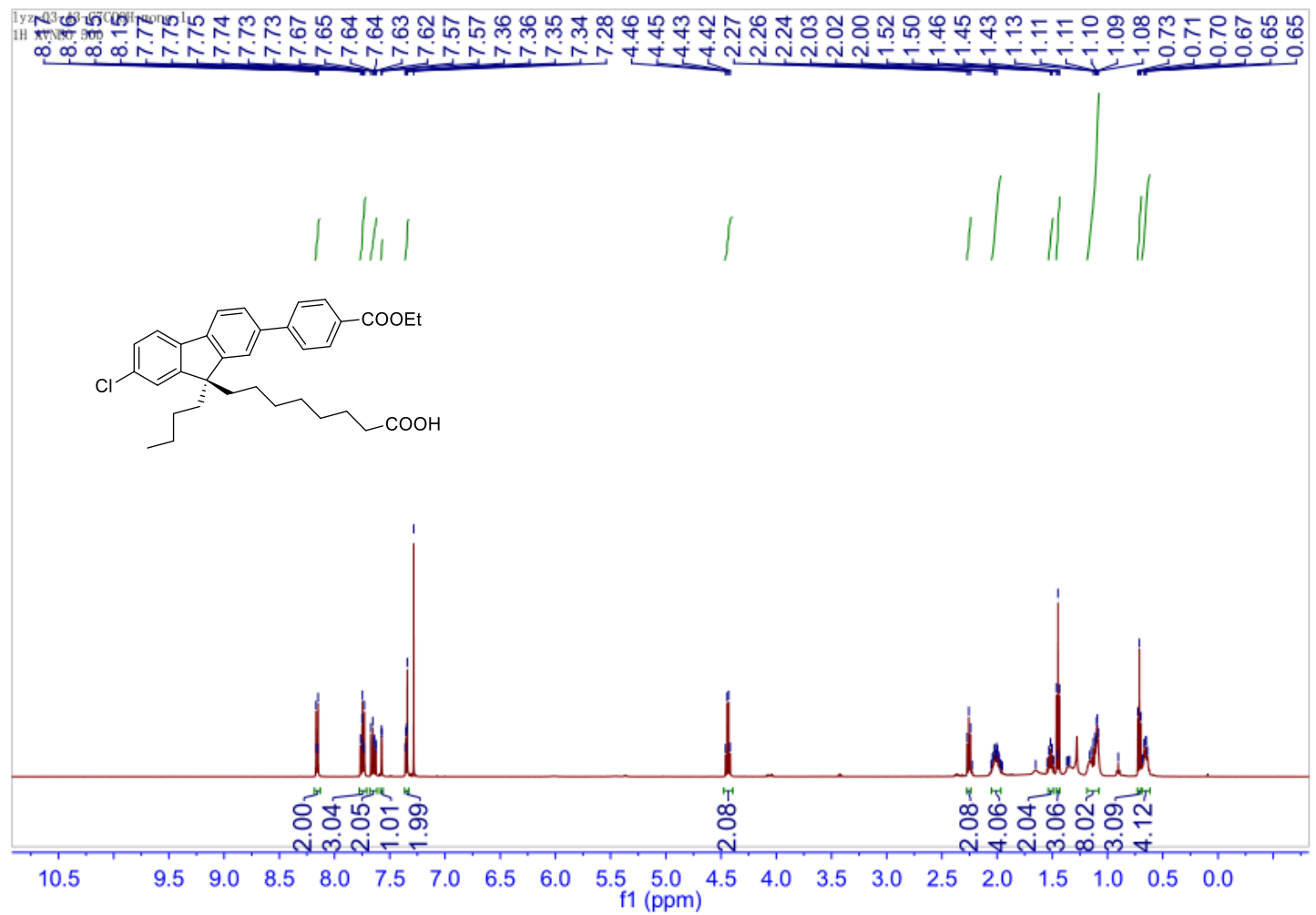

${ }^{13} \mathrm{C}$ NMR $\left(126 \mathrm{MHz}, \mathrm{CDCl}_{3}\right)-12$

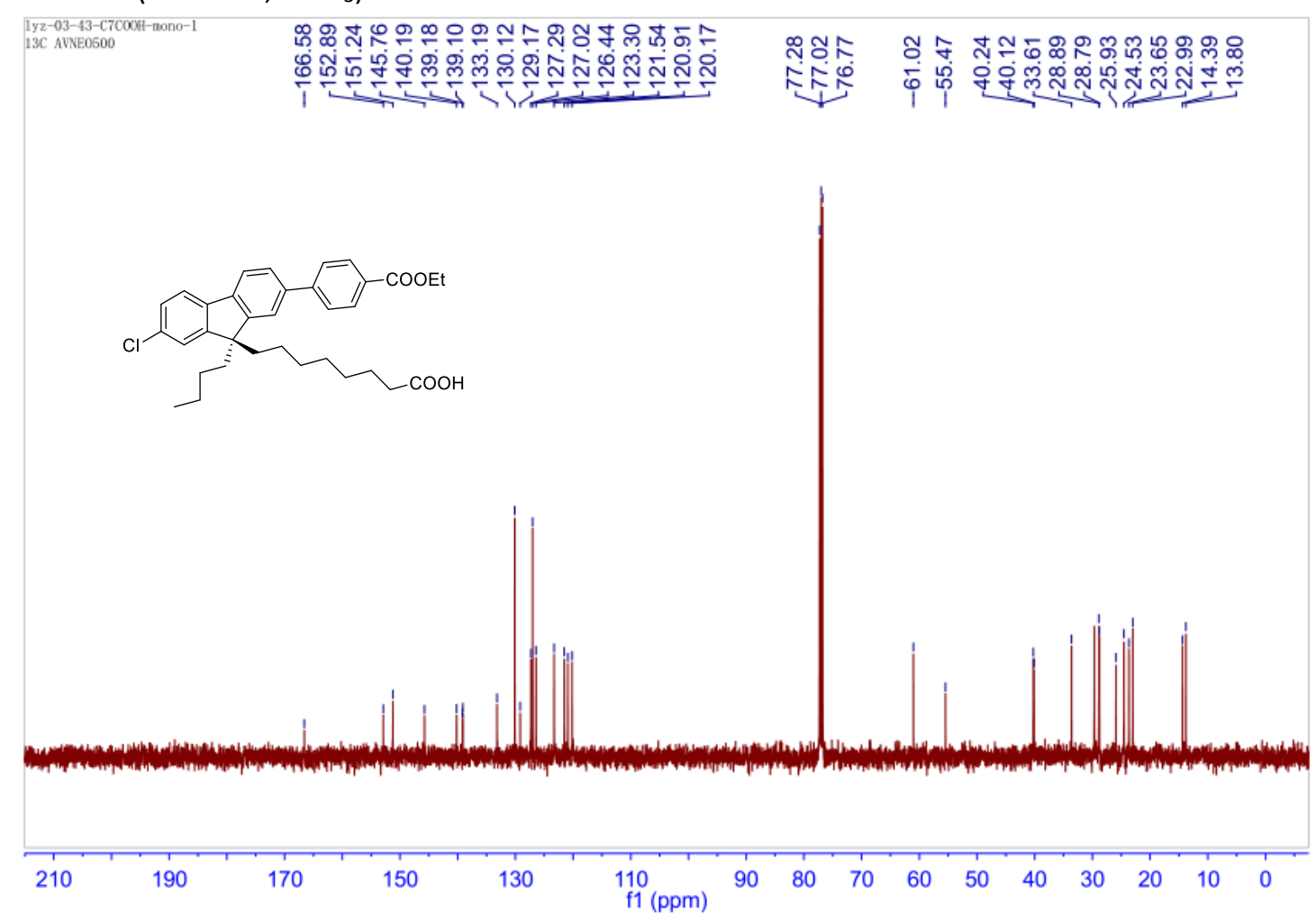


${ }^{1} \mathrm{H}$ NMR (500 MHz, $\mathrm{CDCl}_{3}$ ) -13

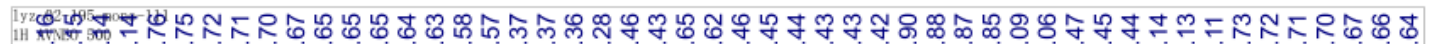
on

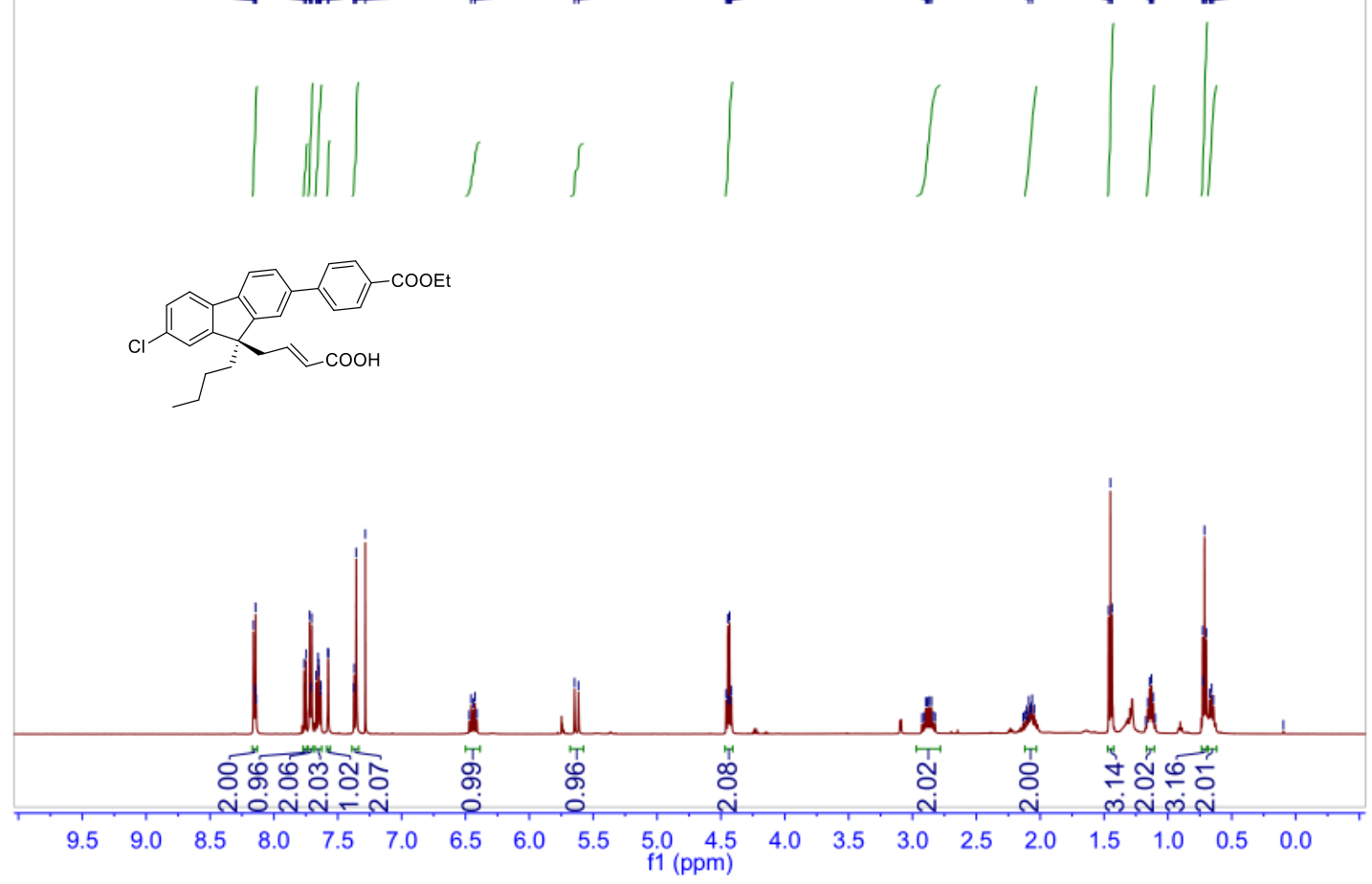

${ }^{13} \mathrm{C}$ NMR (126 MHz, CDCl $)$ - 13

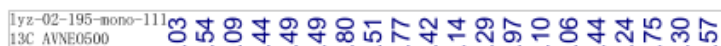

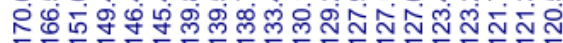

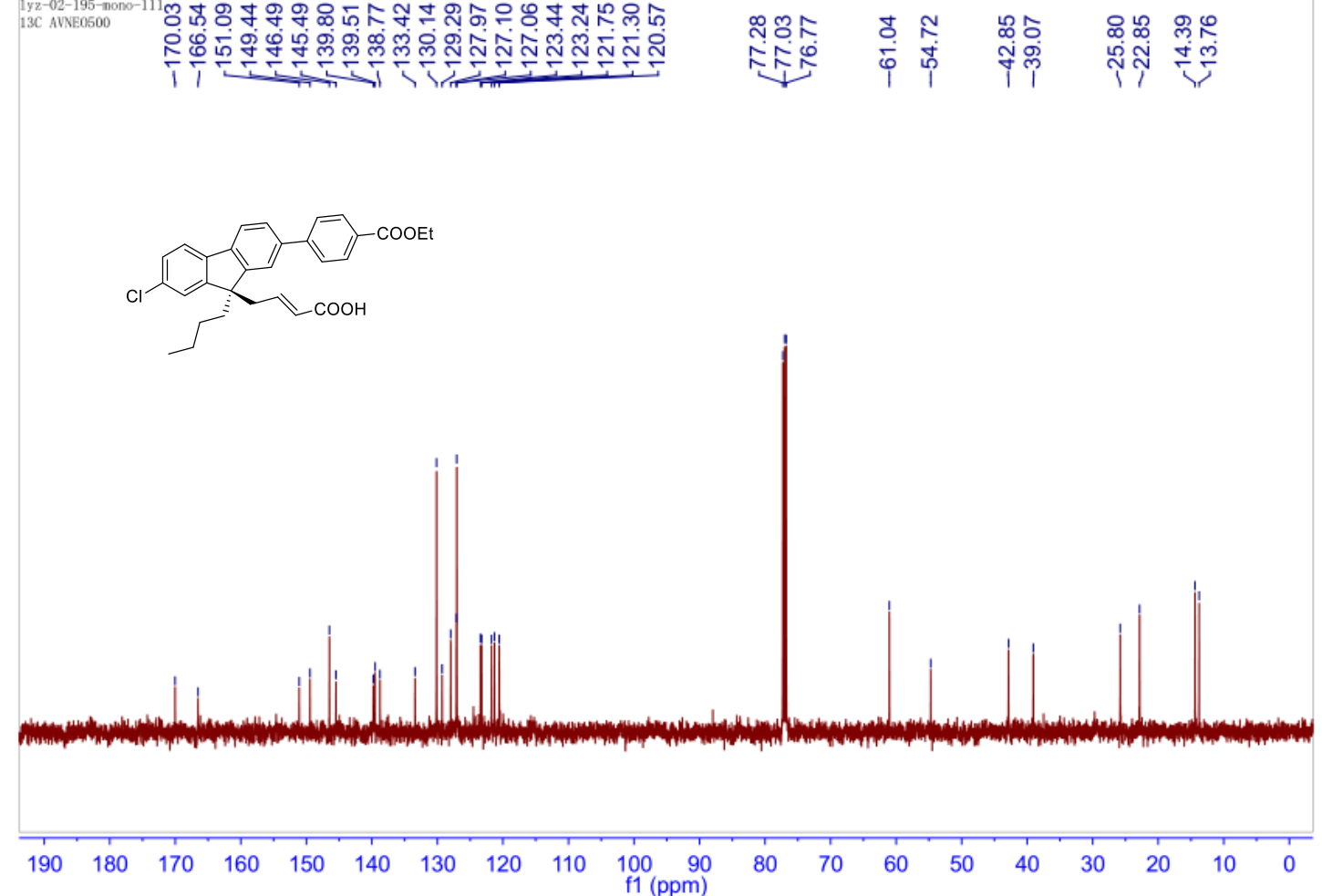


${ }^{1} \mathrm{H}$ NMR (500 MHz, $\mathrm{CDCl}_{3}$ ) -14

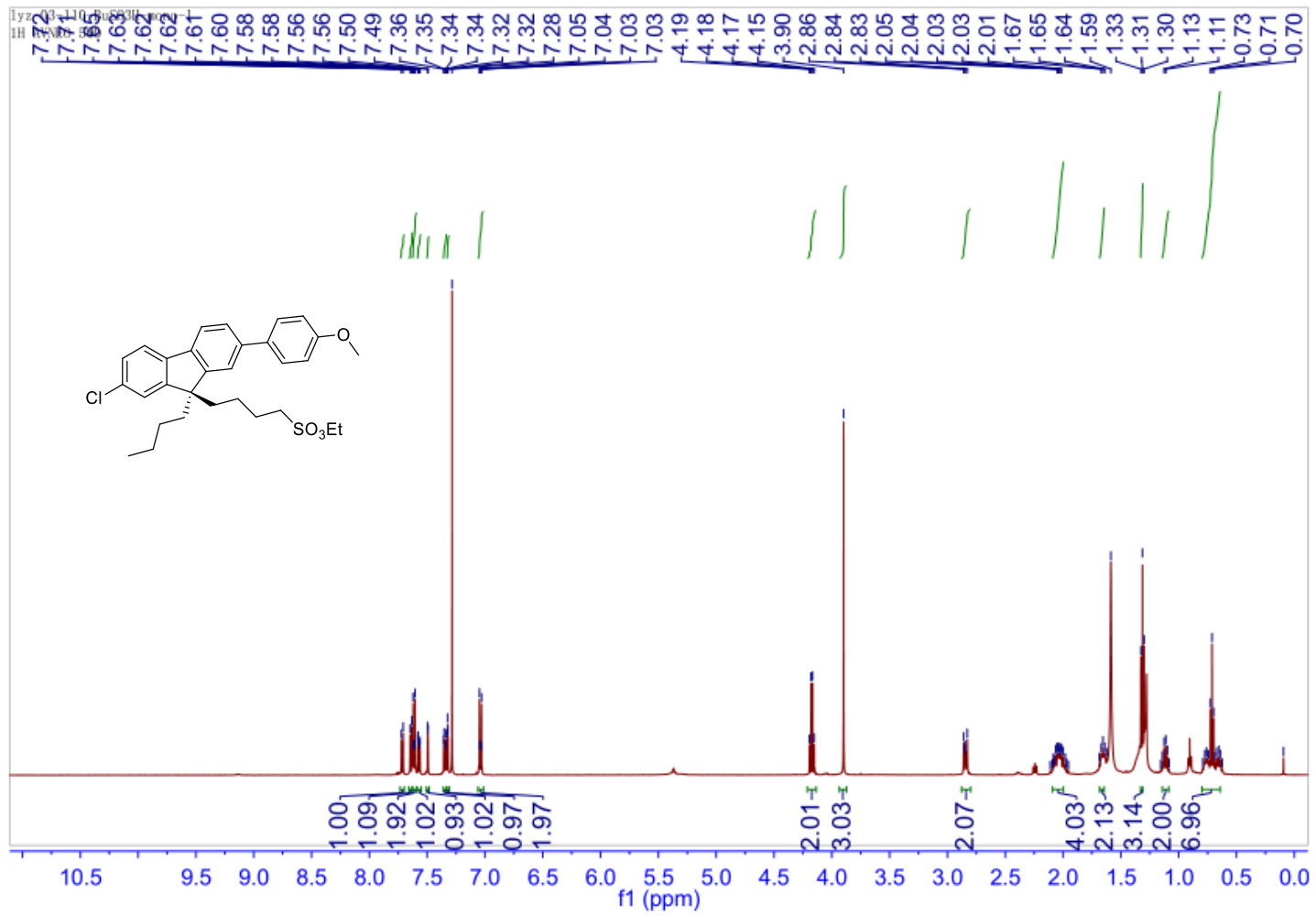

${ }^{13} \mathrm{C}$ NMR (126 MHz, $\mathrm{CDCl}_{3}$ ) -14

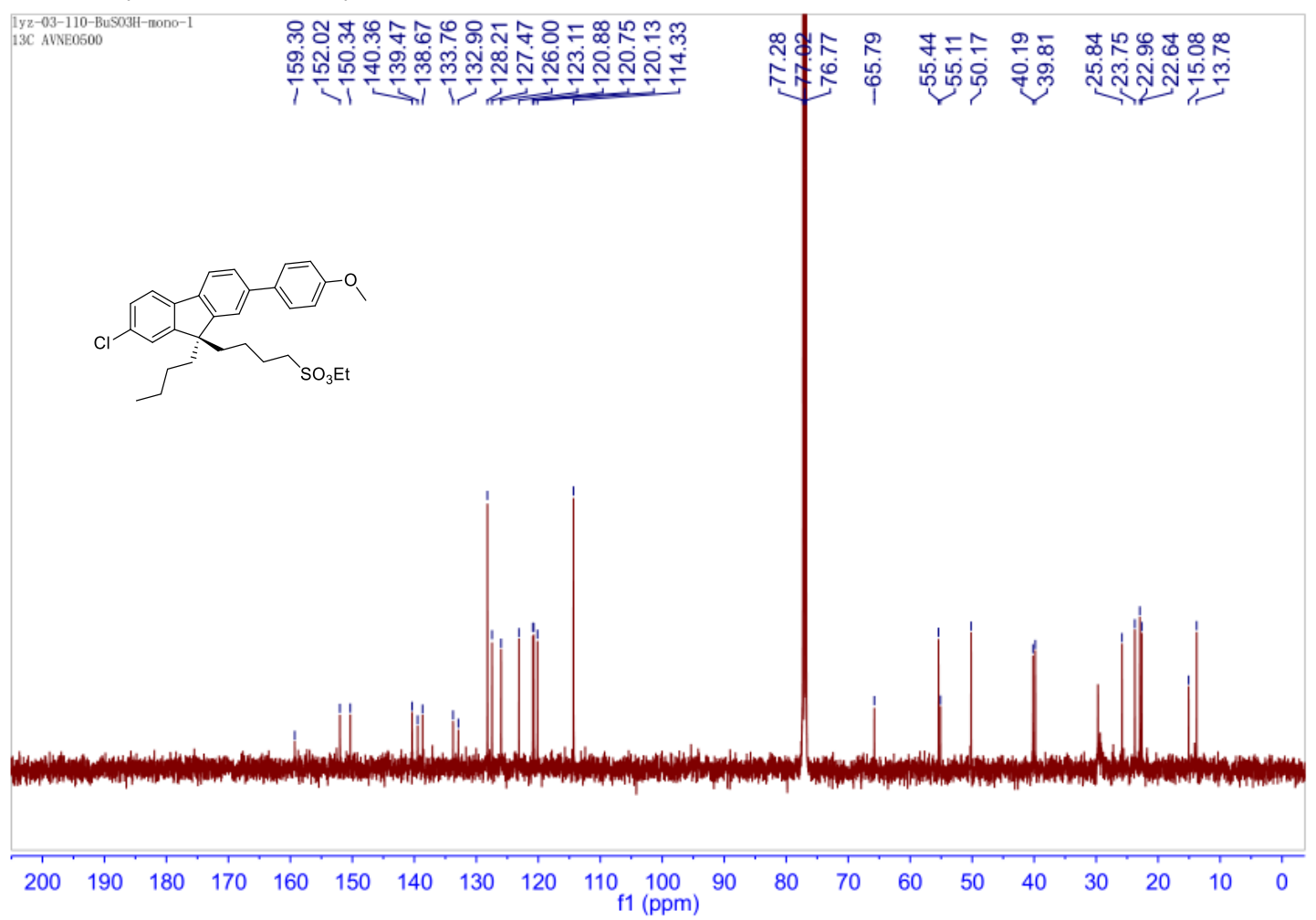


${ }^{1} \mathrm{H}$ NMR $\left(500 \mathrm{MHz}, \mathrm{CDCl}_{3}\right)-15$

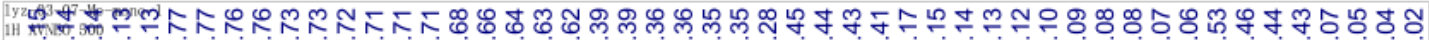

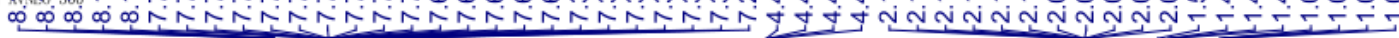

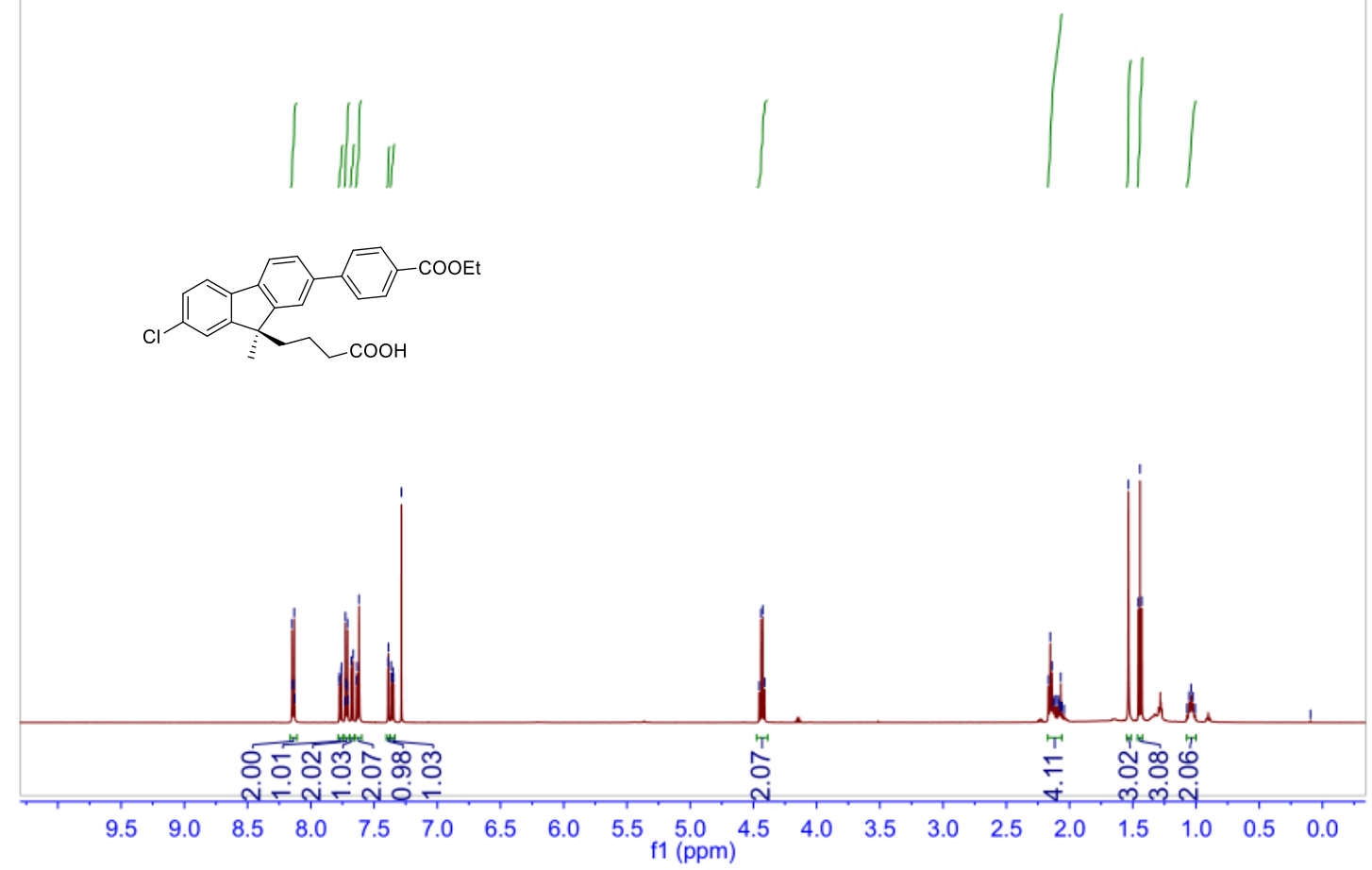

${ }^{13} \mathrm{C} \mathrm{NMR}\left(126 \mathrm{MHz}, \mathrm{CDCl}_{3}\right)-15$

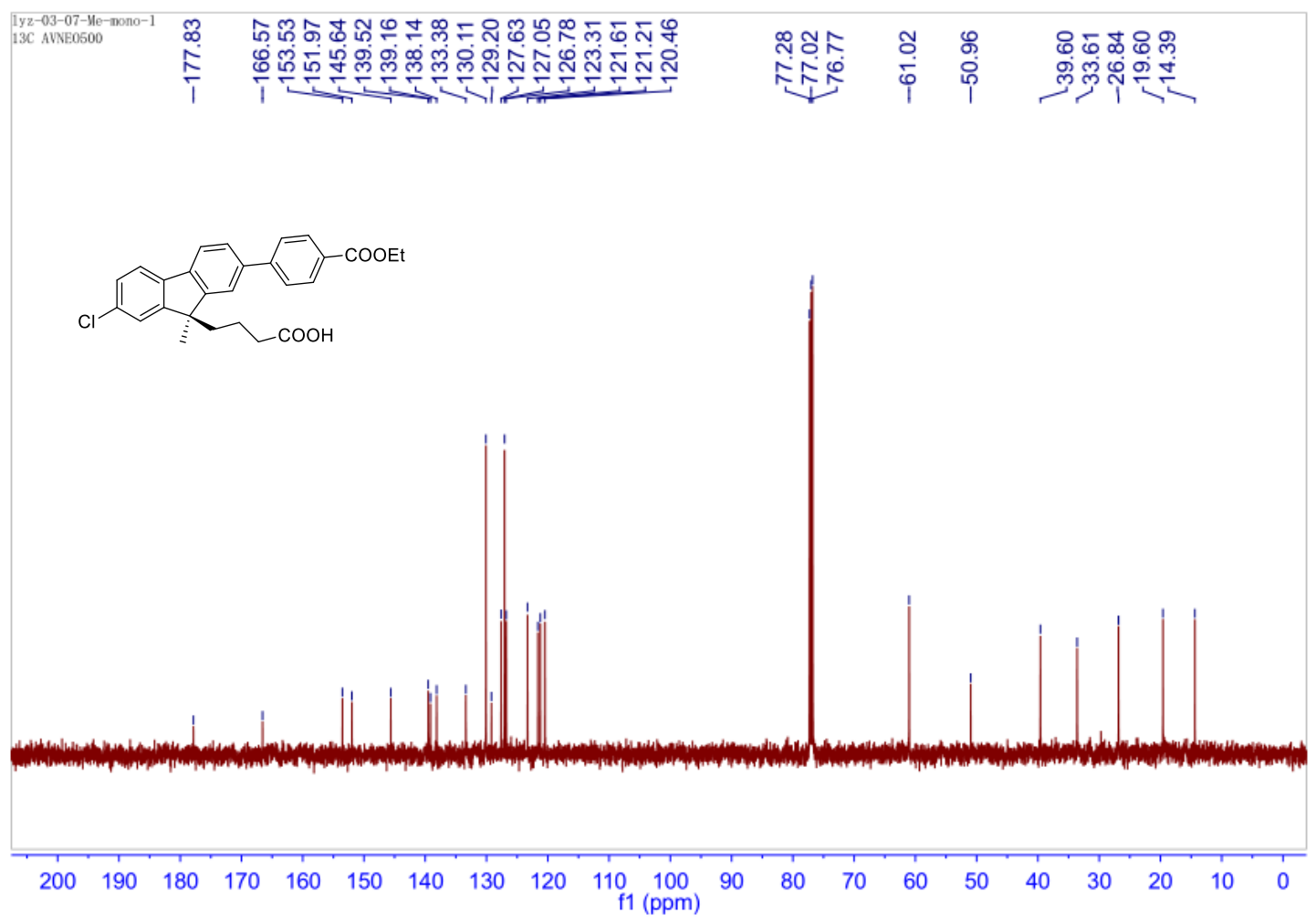


${ }^{1} \mathrm{H}$ NMR (500 MHz, $\mathrm{CDCl}_{3}$ ) -16

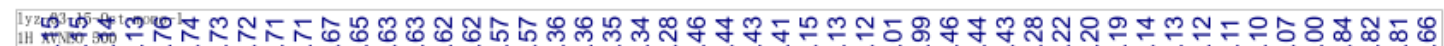

o

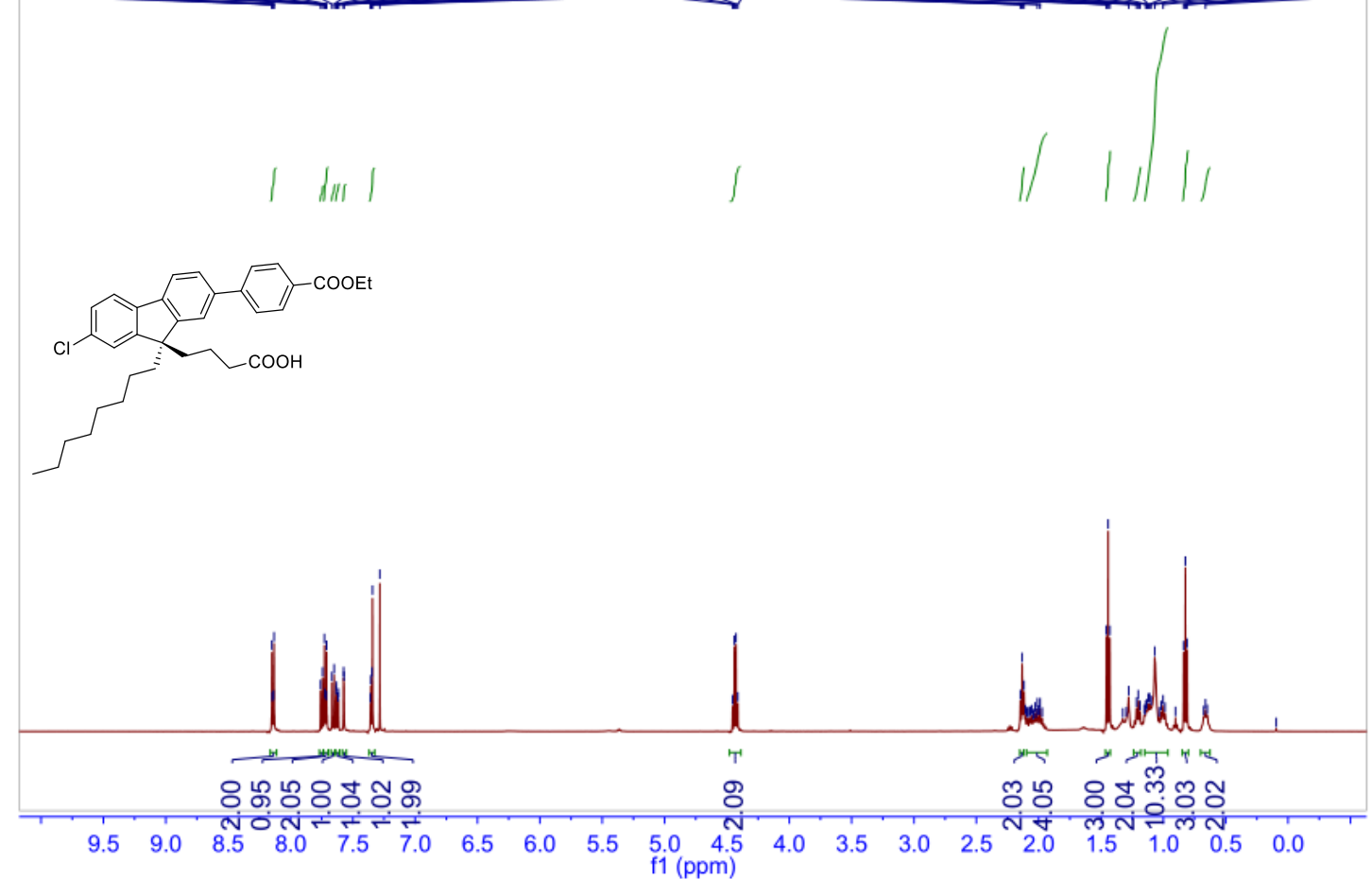

$\left.{ }^{13} \mathrm{C} \mathrm{NMR} \mathrm{(126} \mathrm{MHz,} \mathrm{CDCl}_{3}\right)-16$

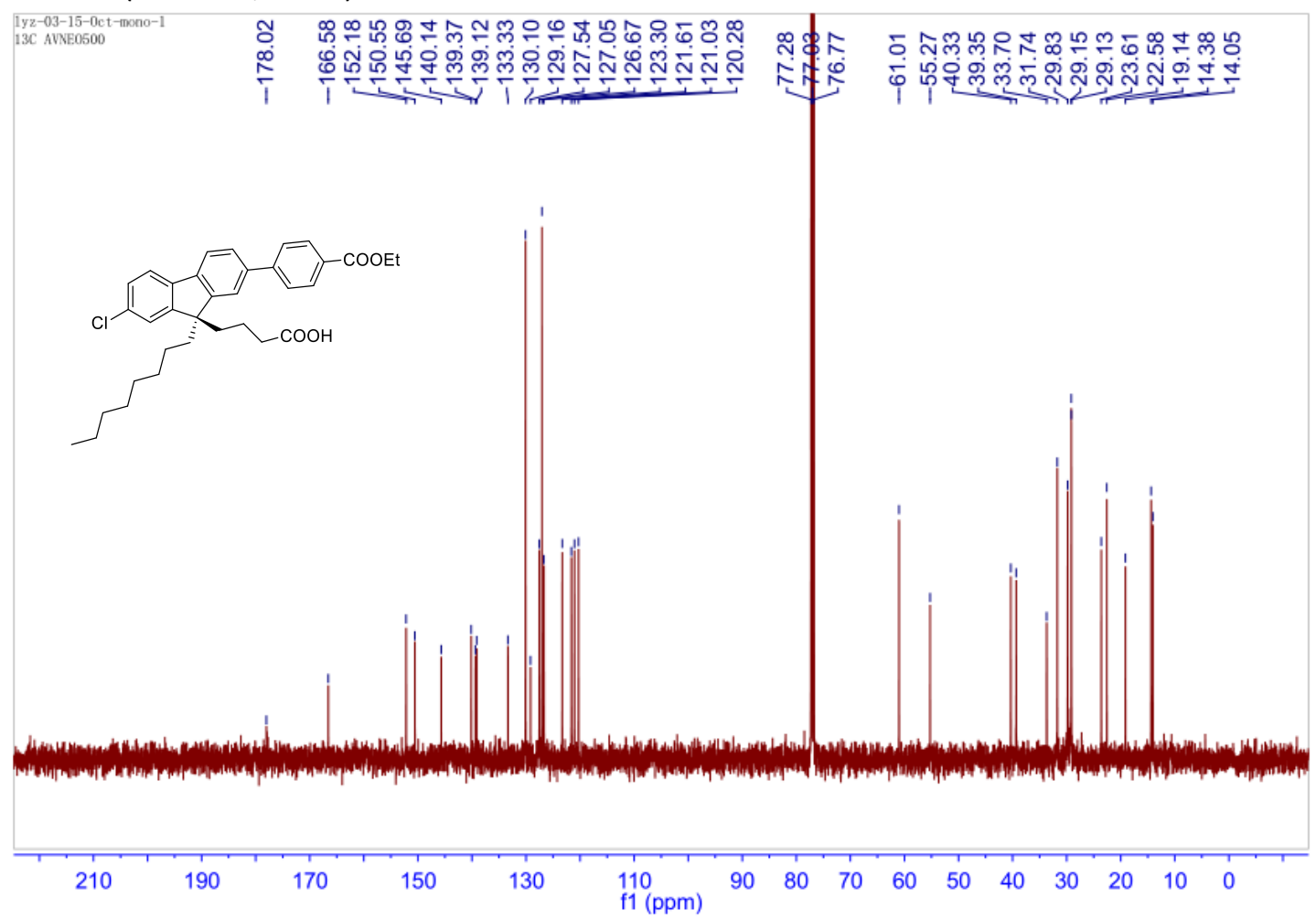


${ }^{1} \mathrm{H}$ NMR (500 MHz, $\mathrm{CDCl}_{3}$ ) -17

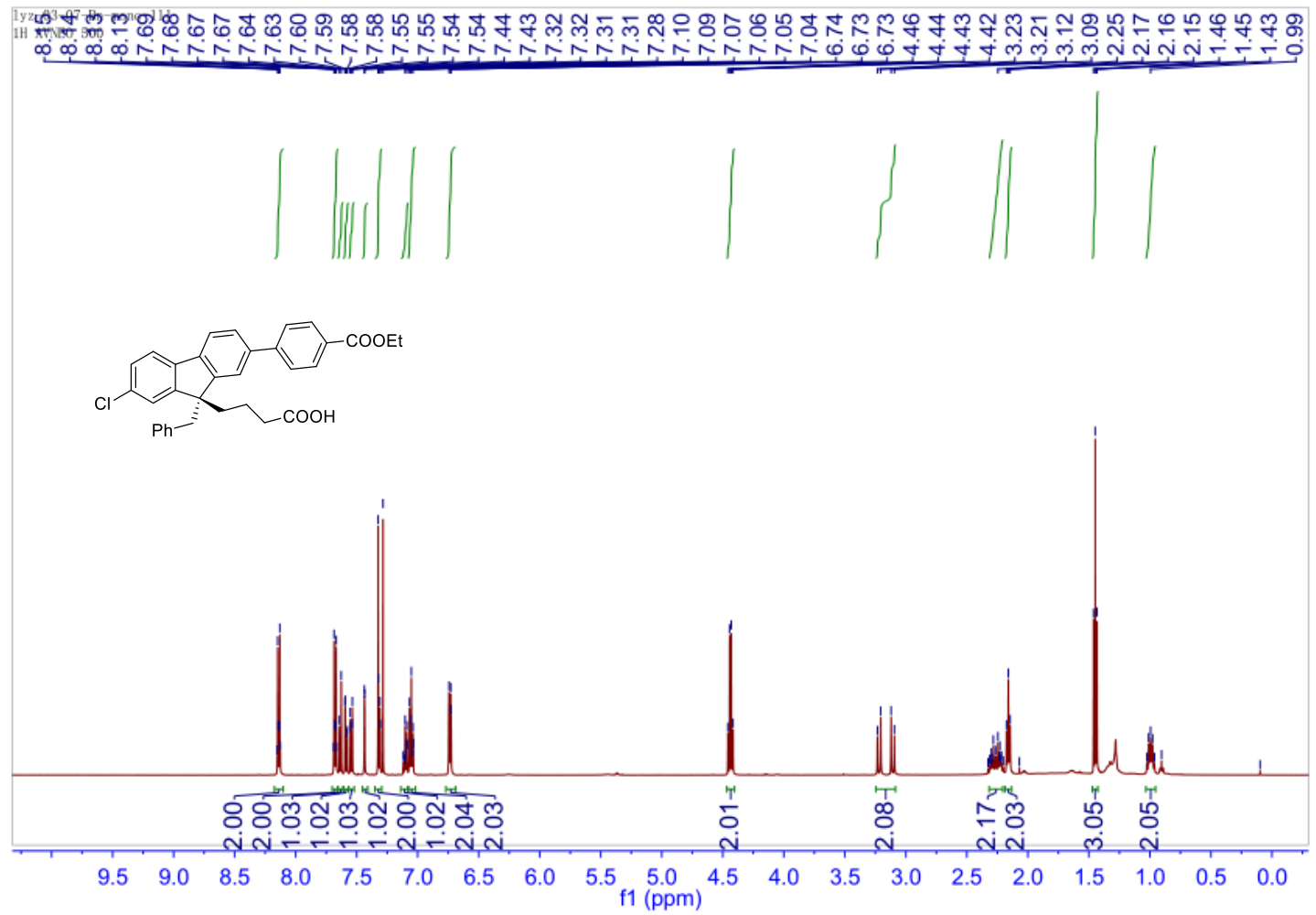

${ }^{13} \mathrm{C}$ NMR (126 MHz, CDCl $)$-17

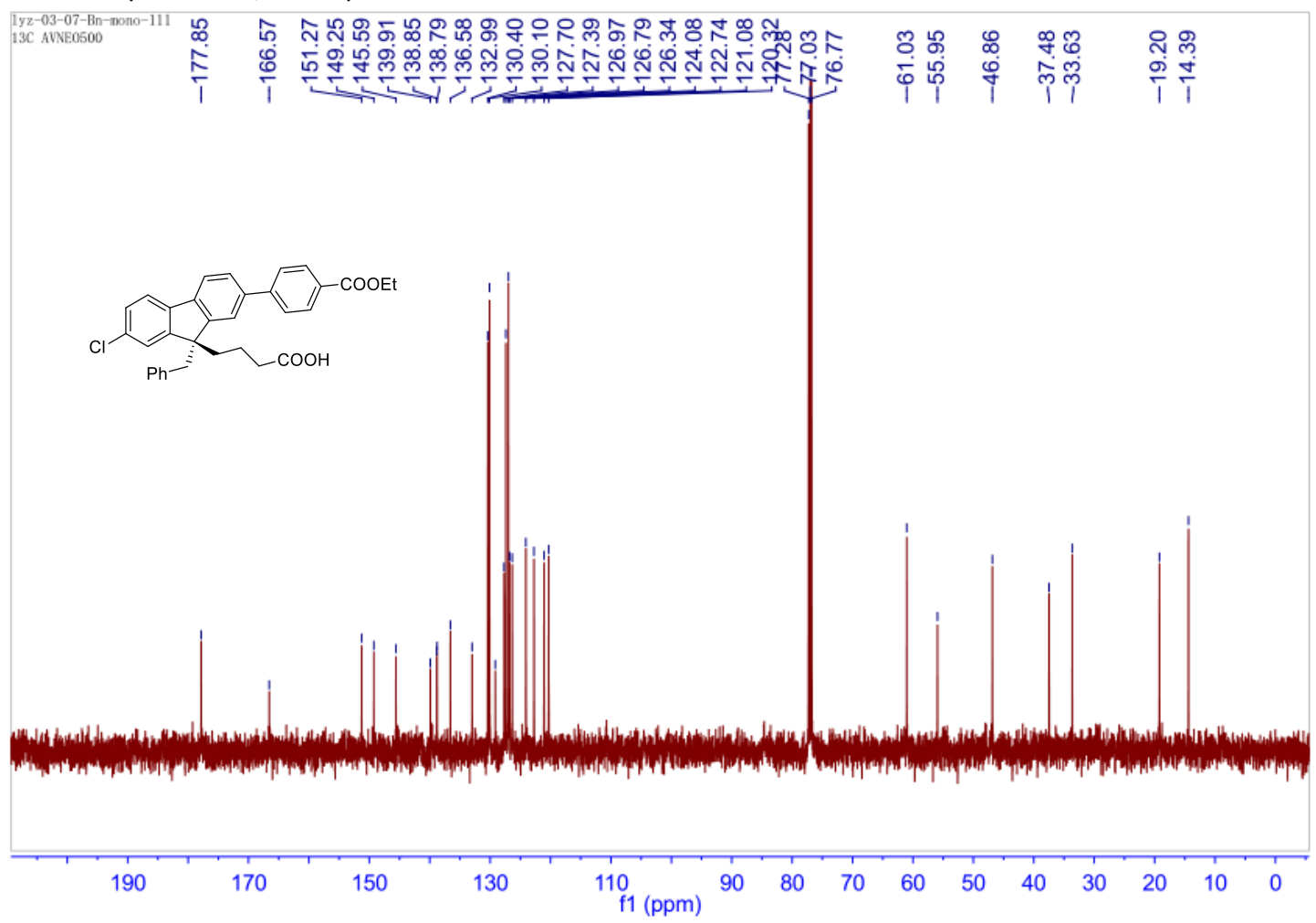


${ }^{1} \mathrm{H}$ NMR $\left(500 \mathrm{MHz}, \mathrm{CDCl}_{3}\right) \mathbf{- 1 8}$

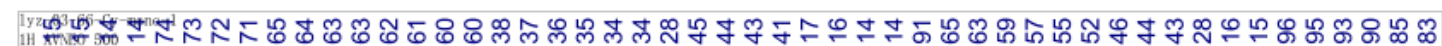

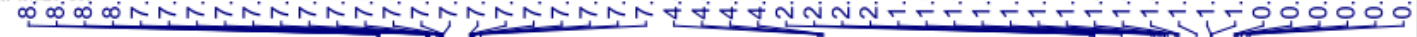

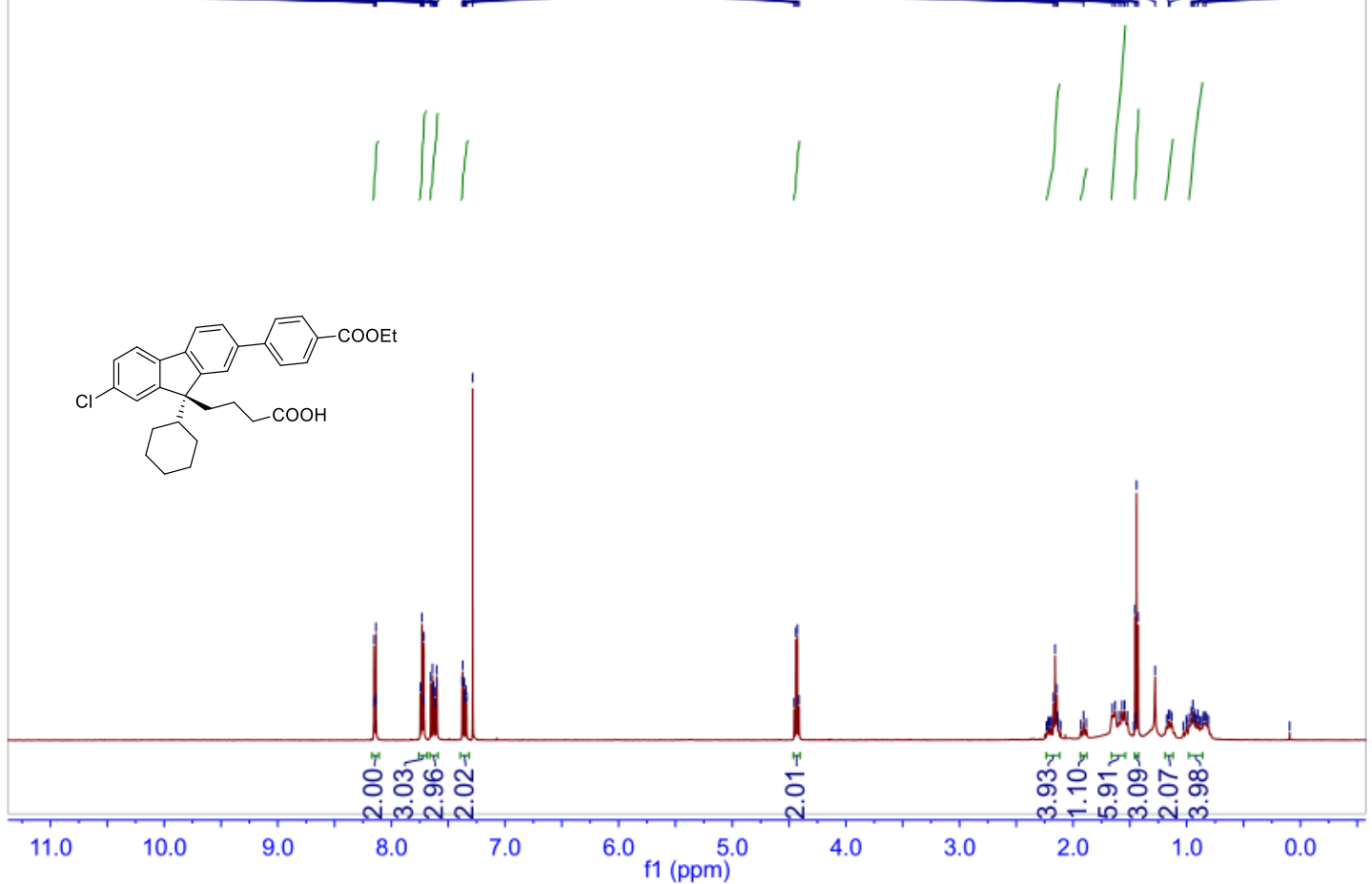

${ }^{13} \mathrm{C}$ NMR $\left(126 \mathrm{MHz}, \mathrm{CDCl}_{3}\right)-\mathbf{1 8}$

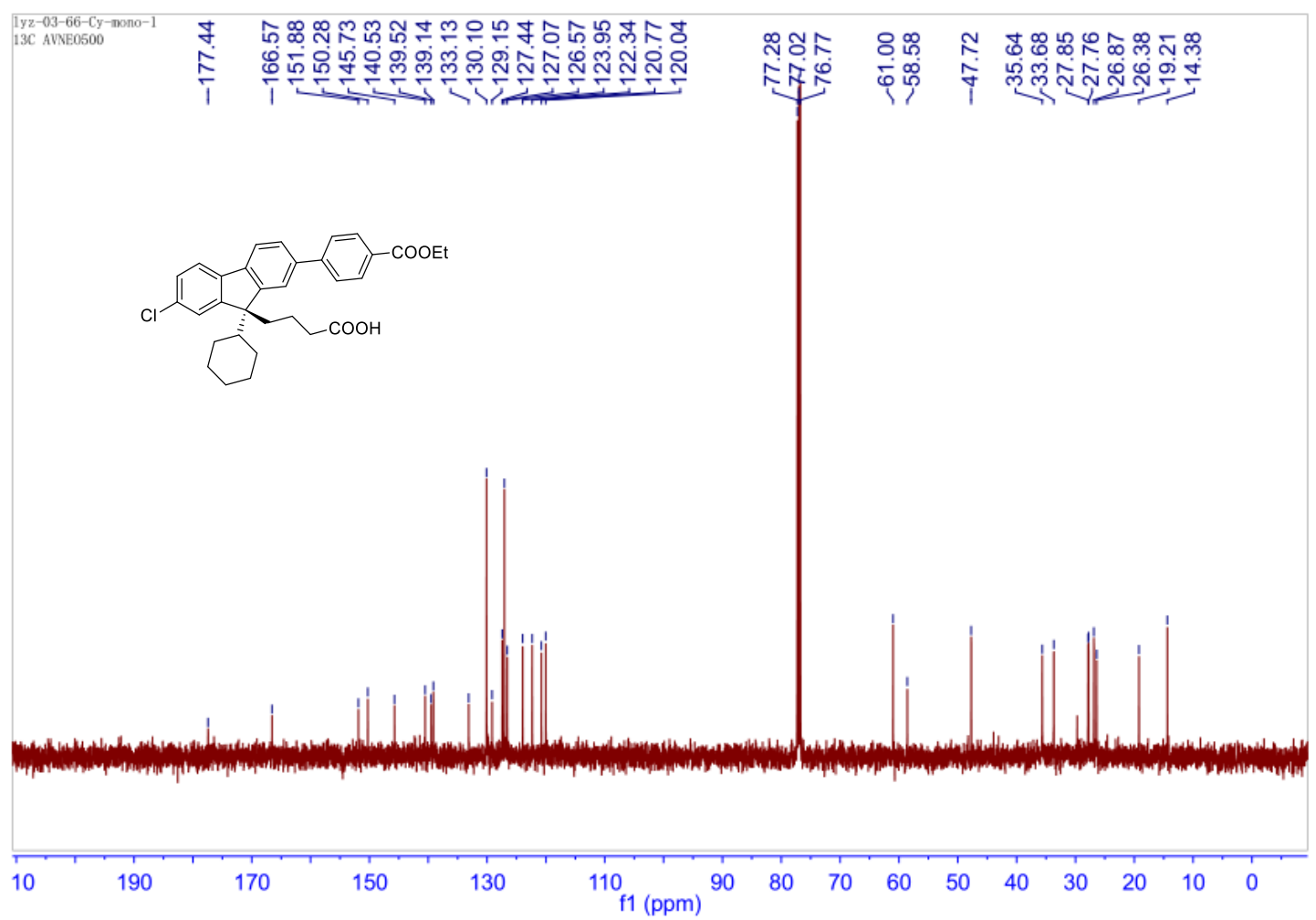


${ }^{1} \mathrm{H}$ NMR $\left(500 \mathrm{MHz}, \mathrm{CDCl}_{3}\right)-19$

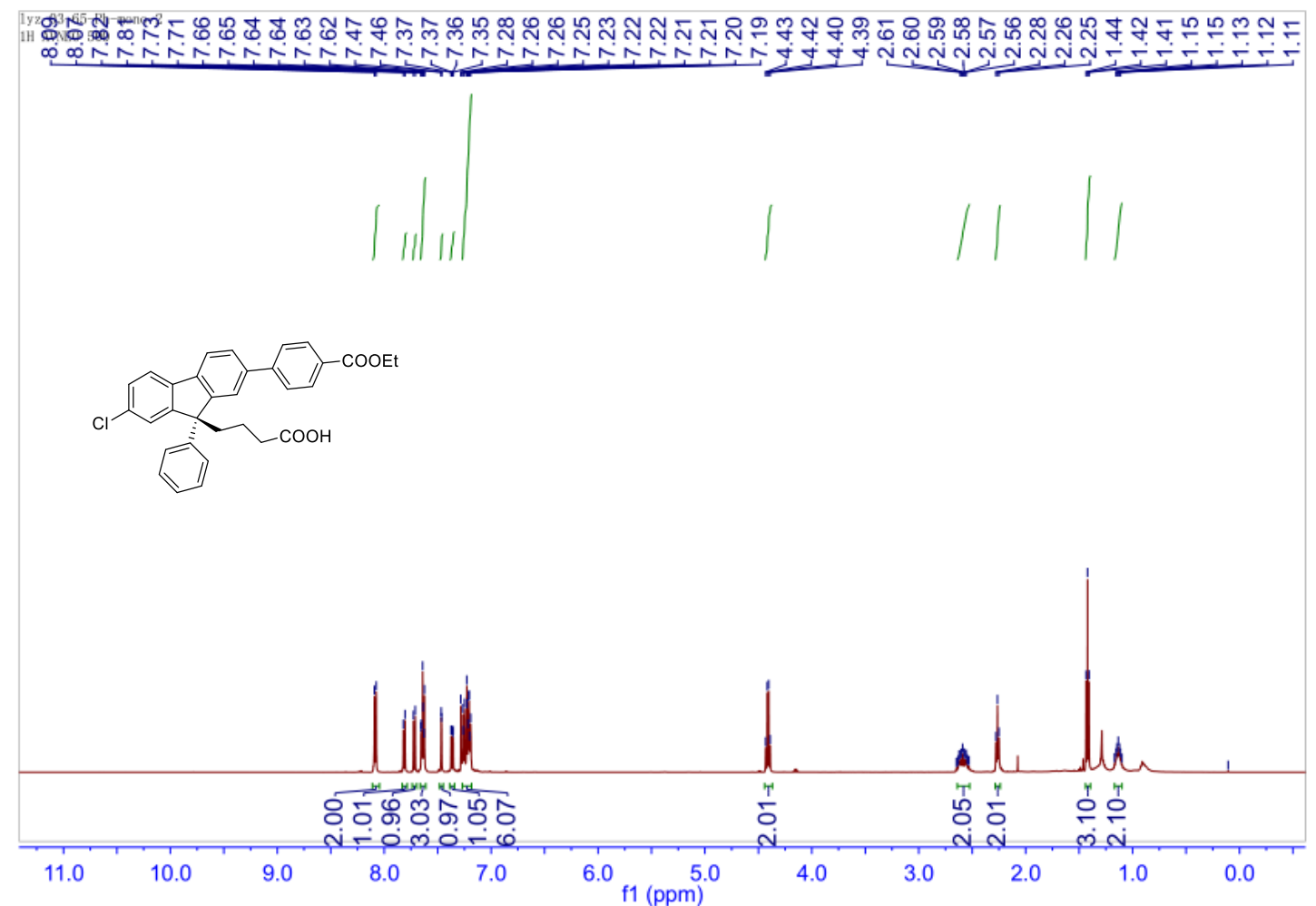

${ }^{13} \mathrm{C}$ NMR $\left(126 \mathrm{MHz}, \mathrm{CDCl}_{3}\right)-19$

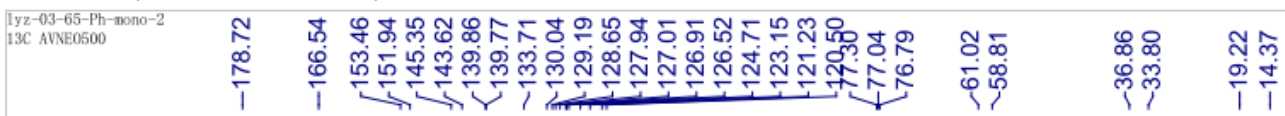

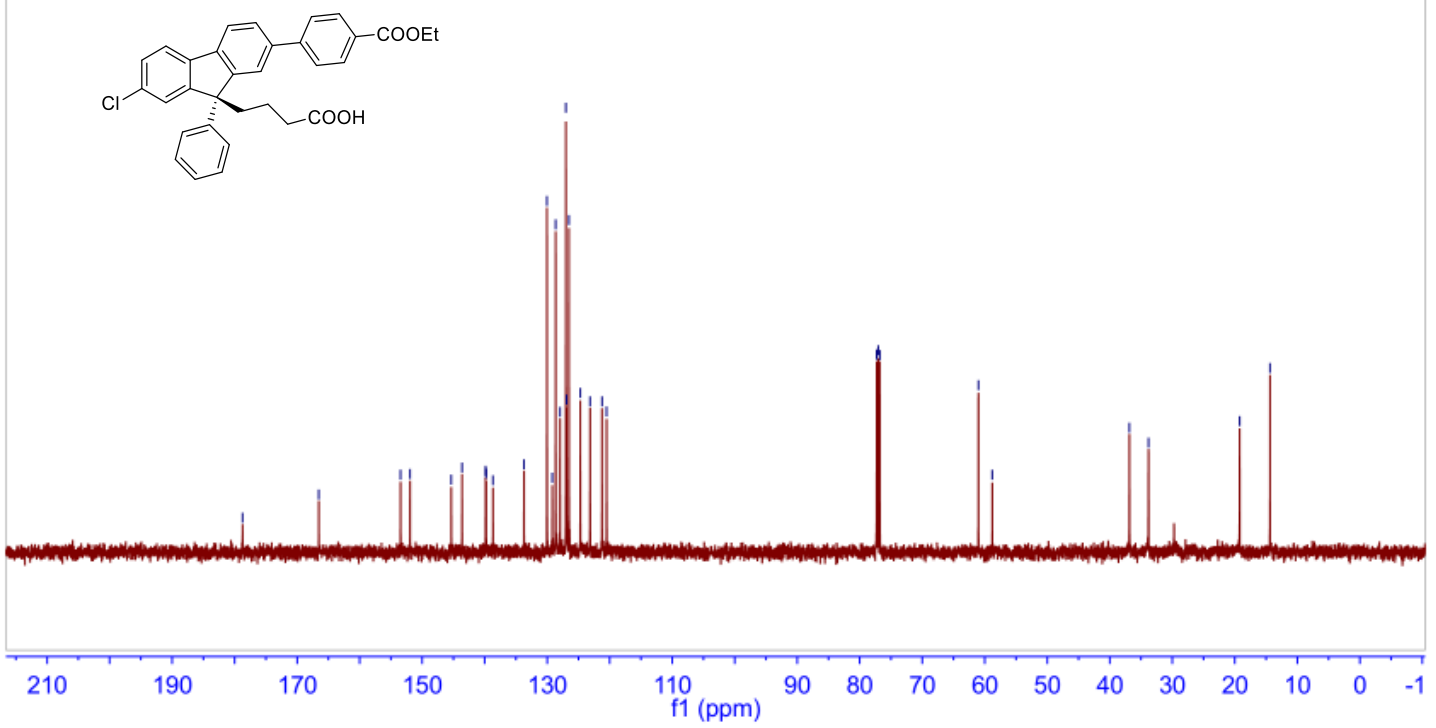


${ }^{1} \mathrm{H}$ NMR (500 MHz, $\left.\mathrm{CDCl}_{3}\right)-20$

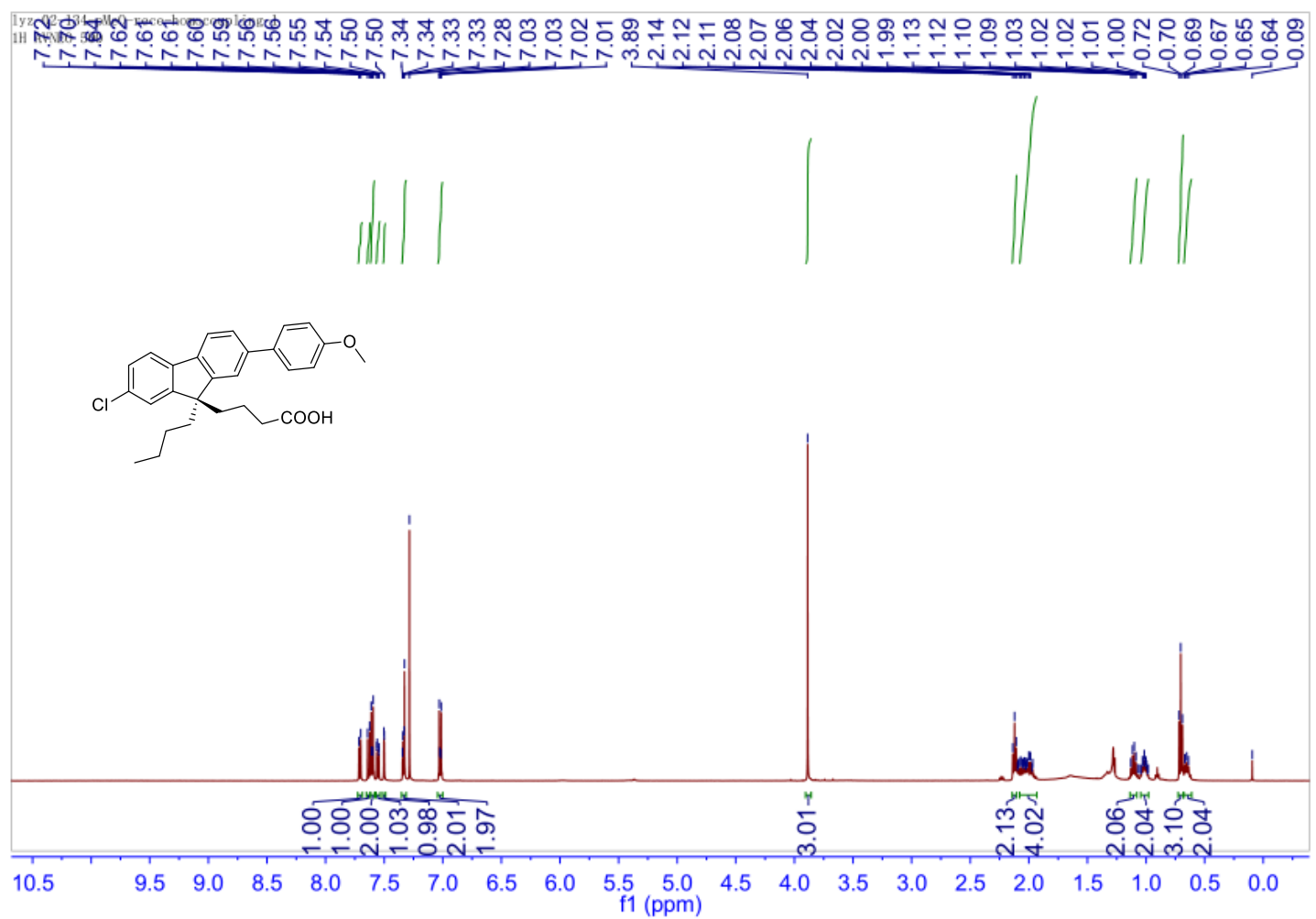

${ }^{13} \mathrm{C} \mathrm{NMR}\left(126 \mathrm{MHz}, \mathrm{CDCl}_{3}\right)-20$

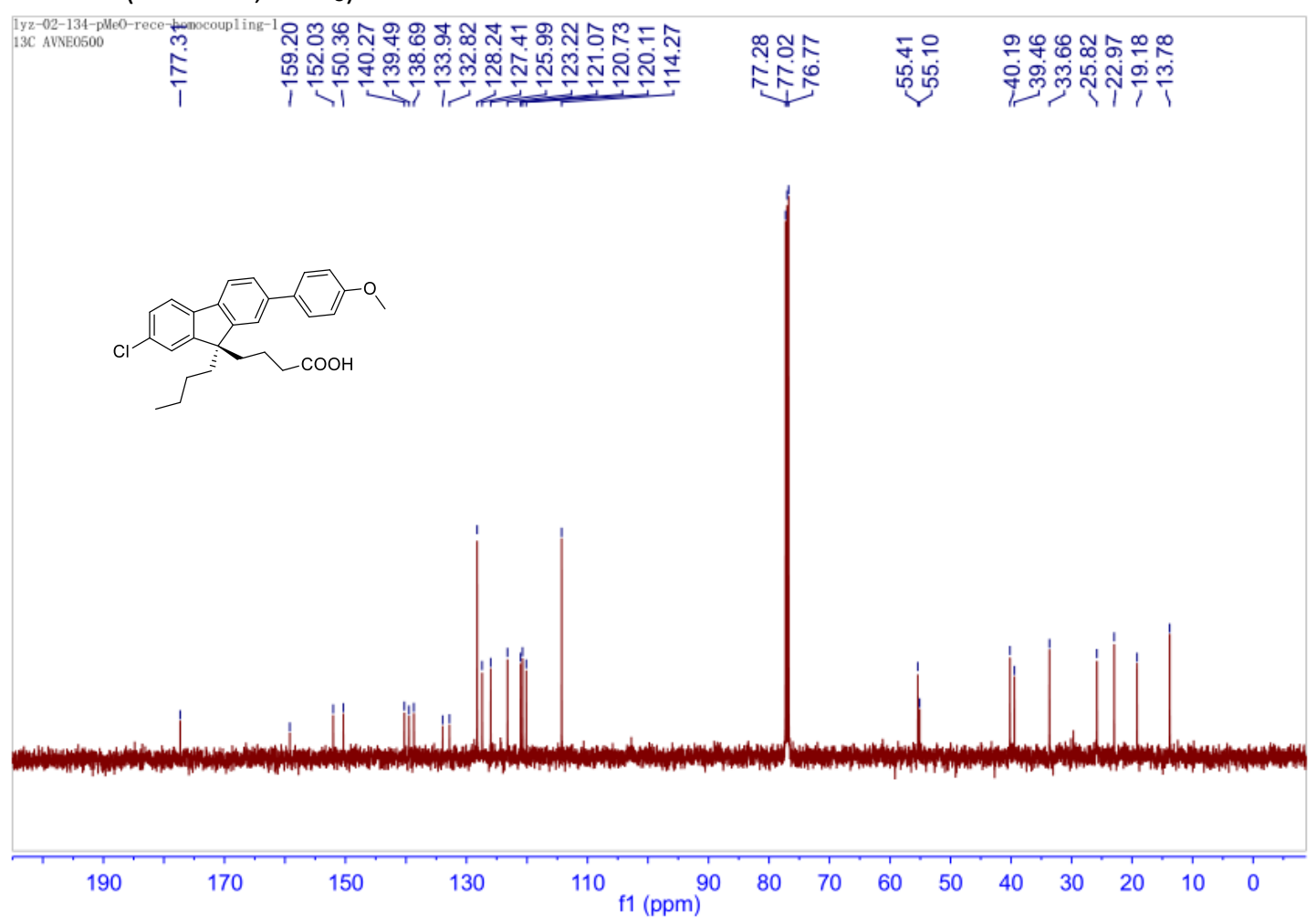


${ }^{1} \mathrm{H}$ NMR $\left(500 \mathrm{MHz}, \mathrm{CDCl}_{3}\right)-21$

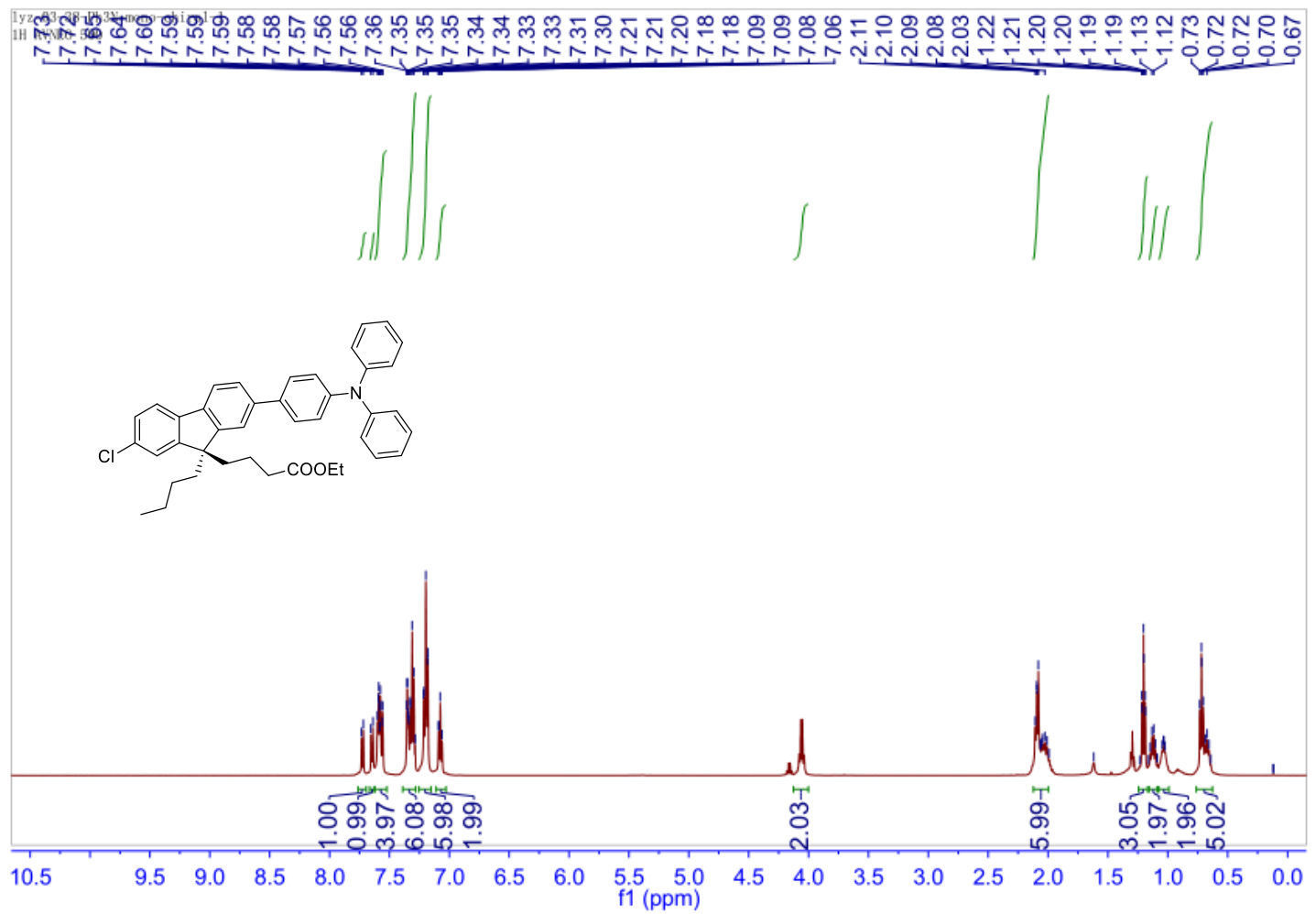

${ }^{13} \mathrm{C} \mathrm{NMR}\left(126 \mathrm{MHz}, \mathrm{CDCl}_{3}\right)-21$

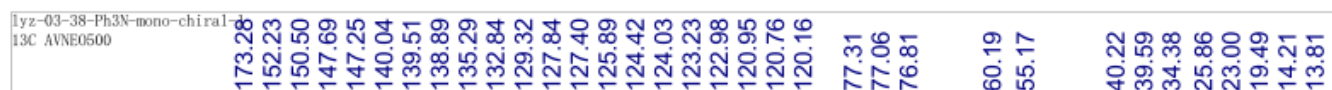

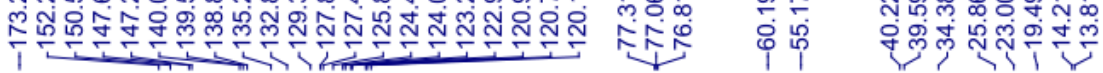

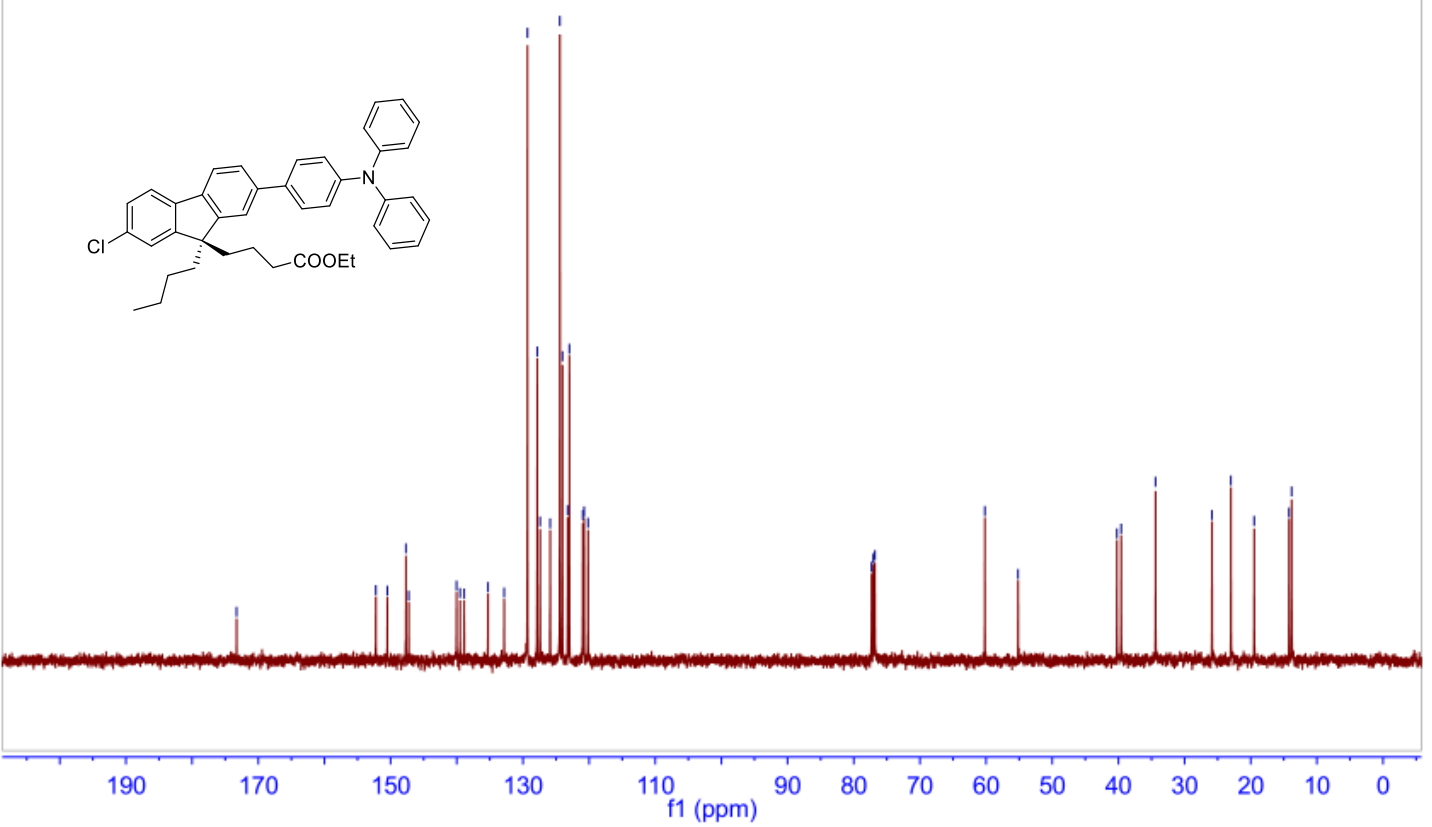


${ }^{1} \mathrm{H}$ NMR $\left(500 \mathrm{MHz}, \mathrm{CDCl}_{3}\right)-22$

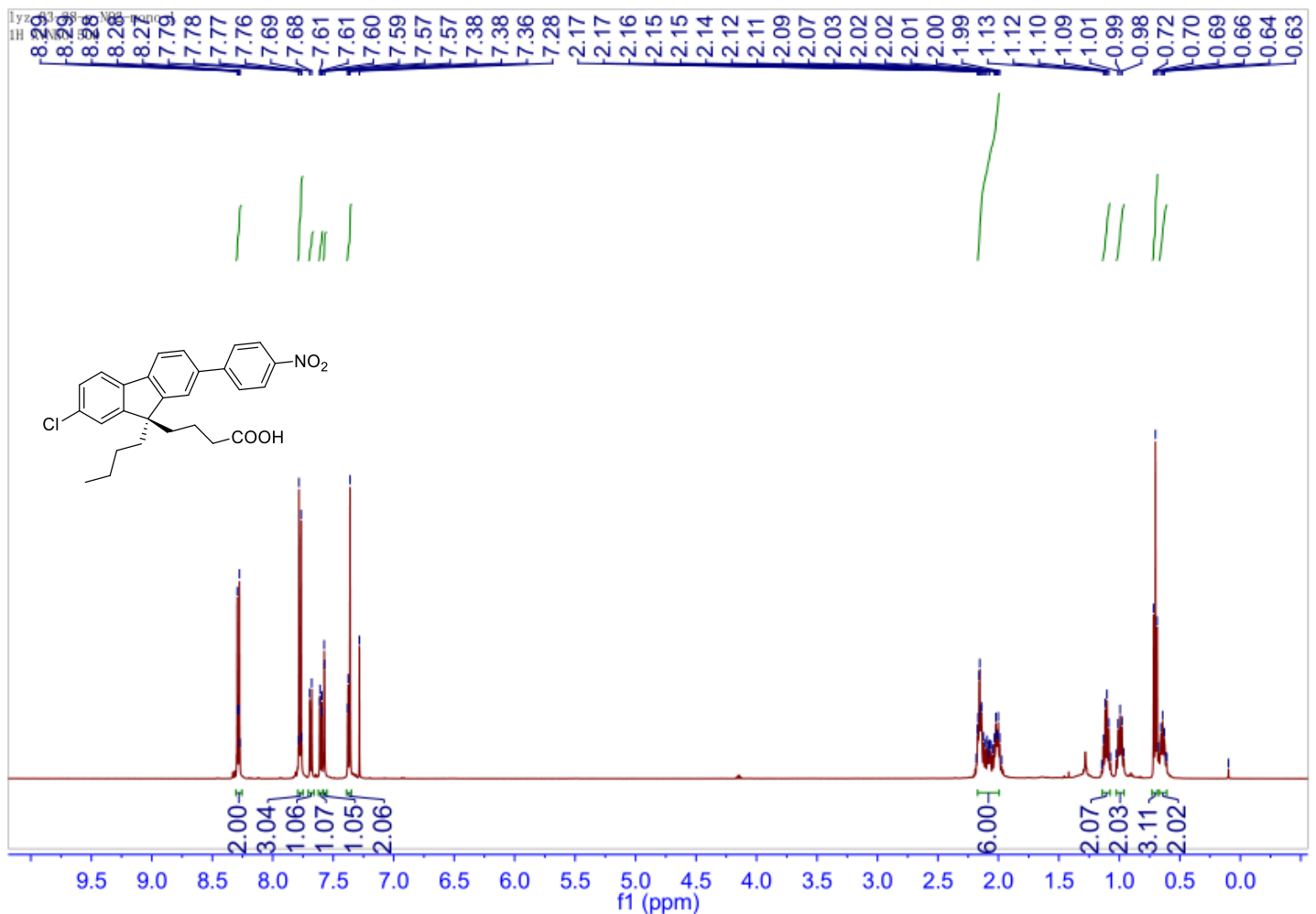

$\left.{ }^{13} \mathrm{C} \mathrm{NMR} \mathrm{(126} \mathrm{MHz,} \mathrm{CDCl}_{3}\right)-22$

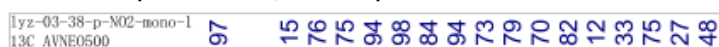
13C ANEO500

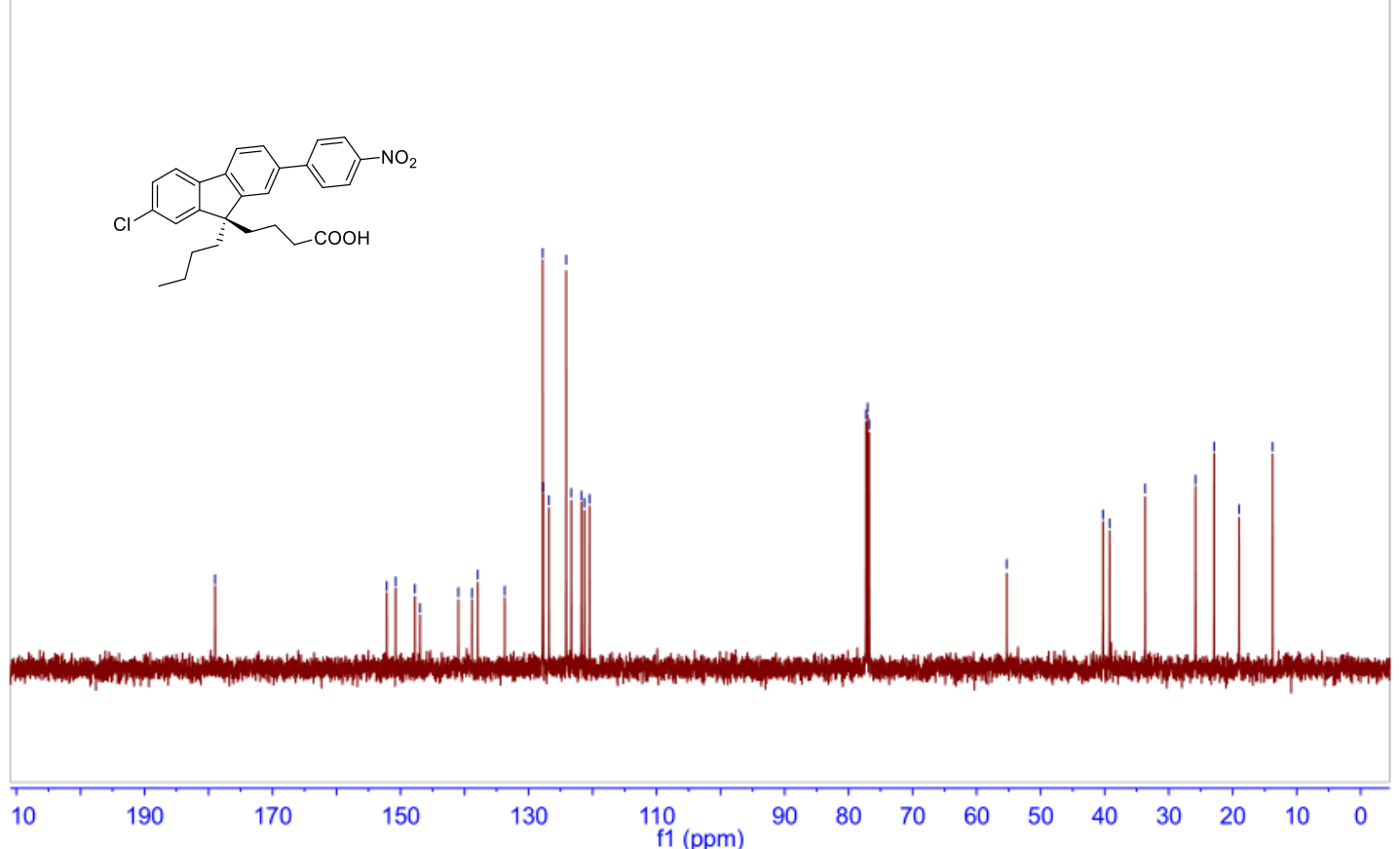


${ }^{1} \mathrm{H}$ NMR $\left(500 \mathrm{MHz}, \mathrm{CDCl}_{3}\right)-23$

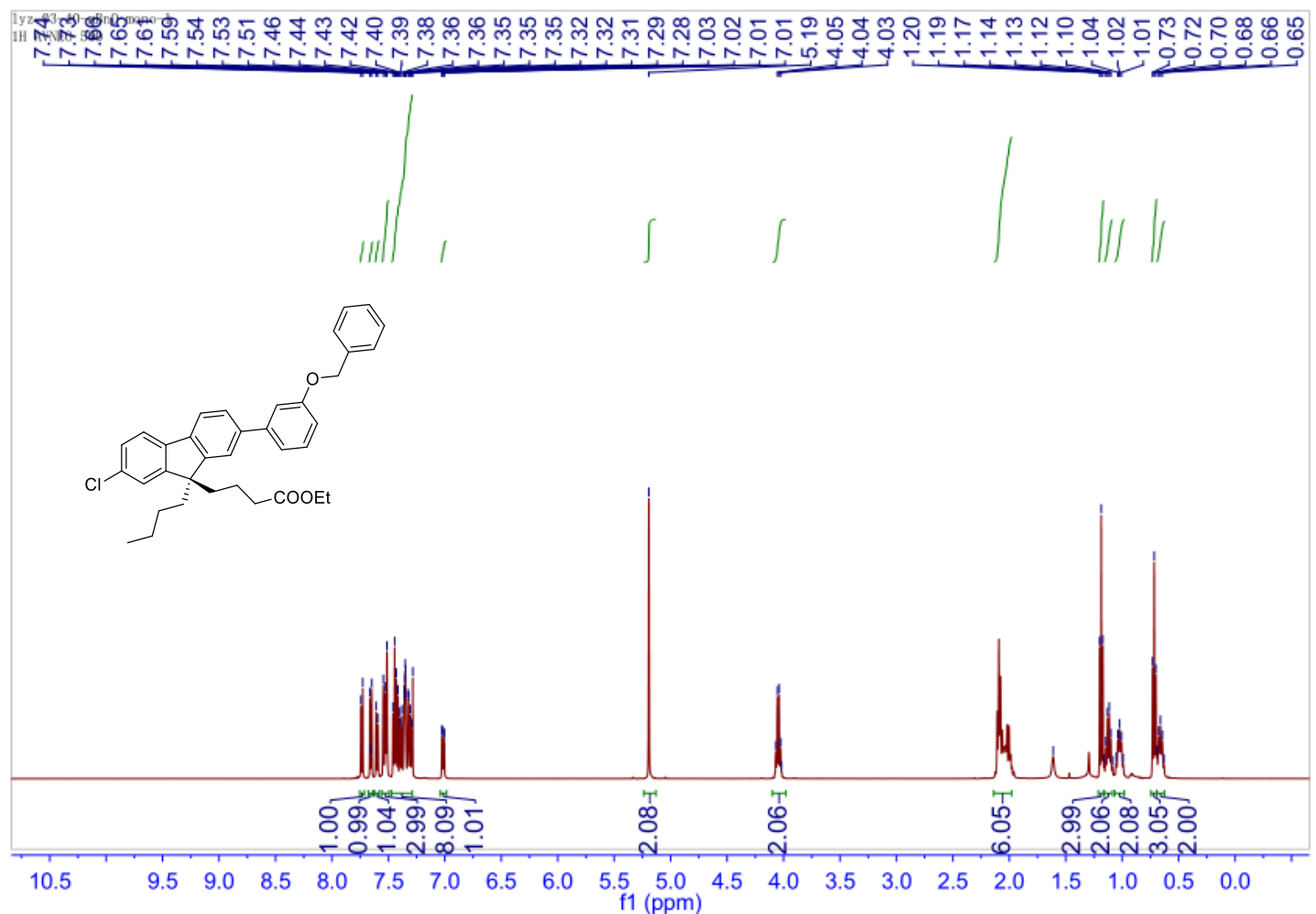

${ }^{13} \mathrm{C} \mathrm{NMR} \mathrm{(126} \mathrm{MHz,} \mathrm{CDCl}_{3}$ )-23

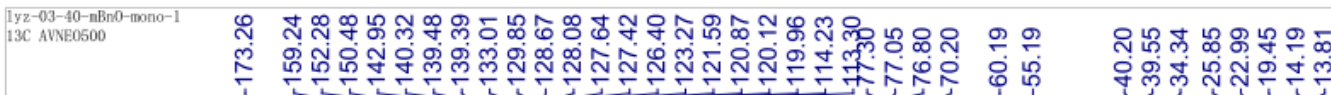

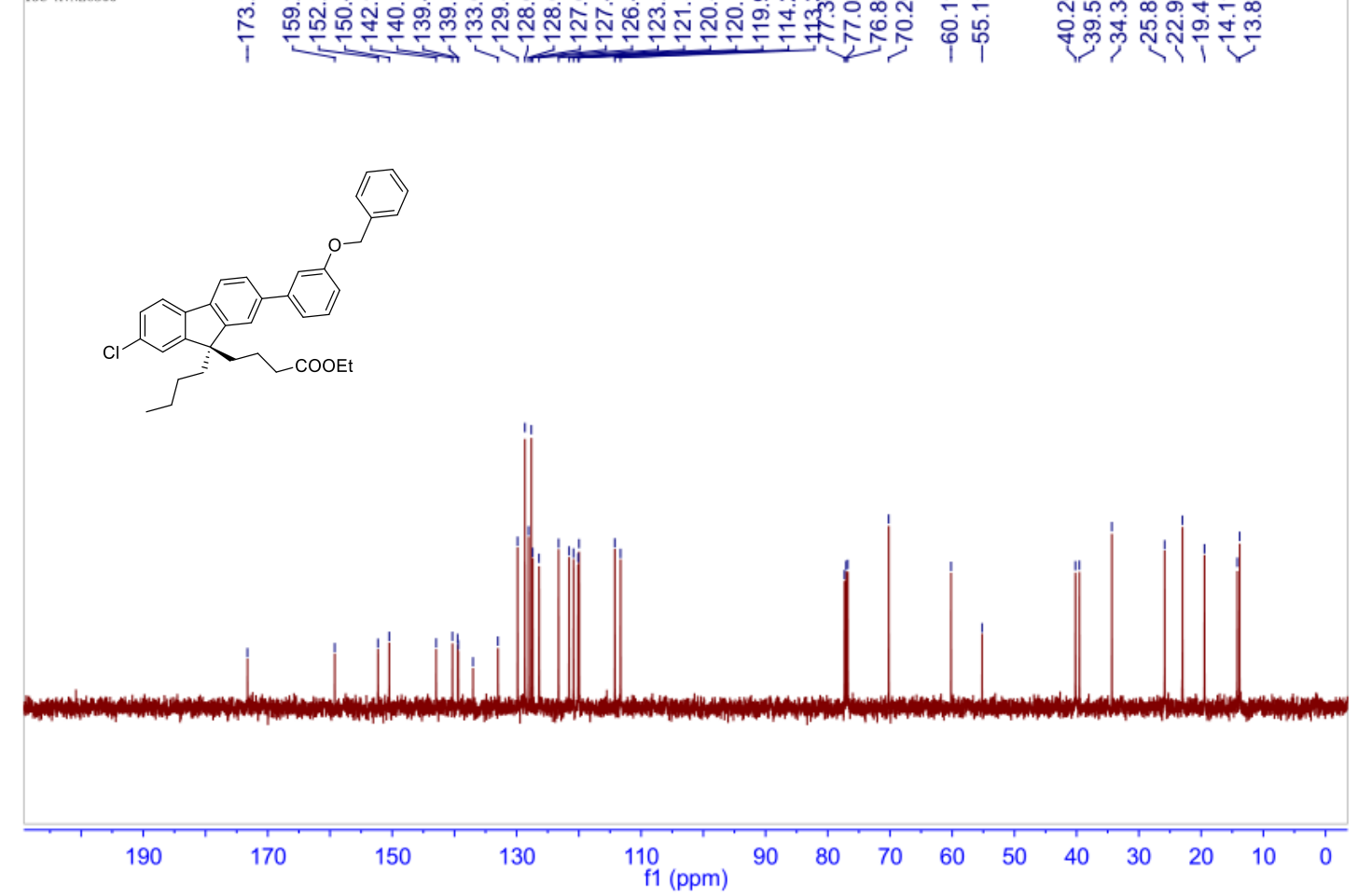




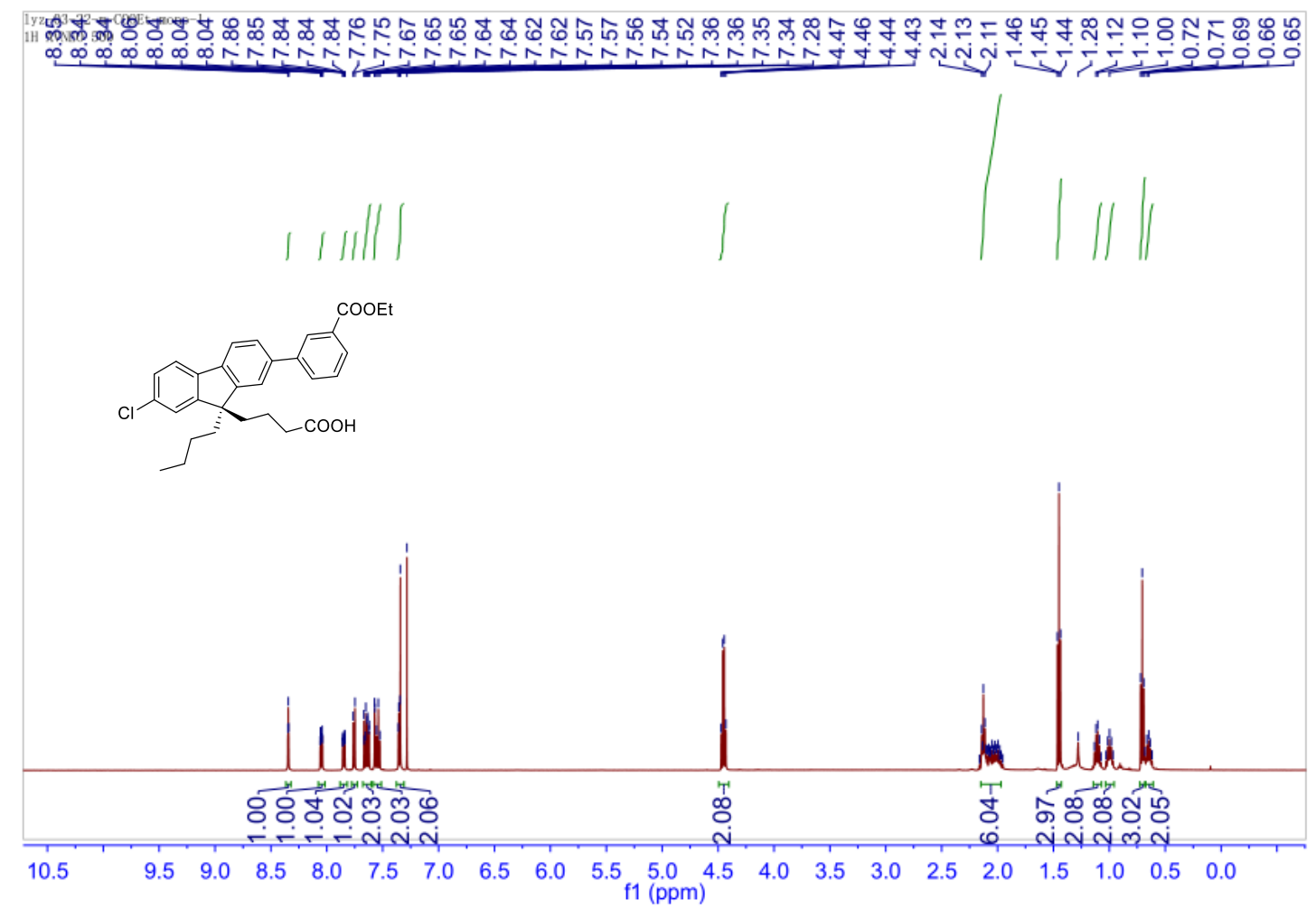

${ }^{13} \mathrm{C} \mathrm{NMR} \mathrm{(126} \mathrm{MHz,} \mathrm{CDCl}_{3}$ )-24

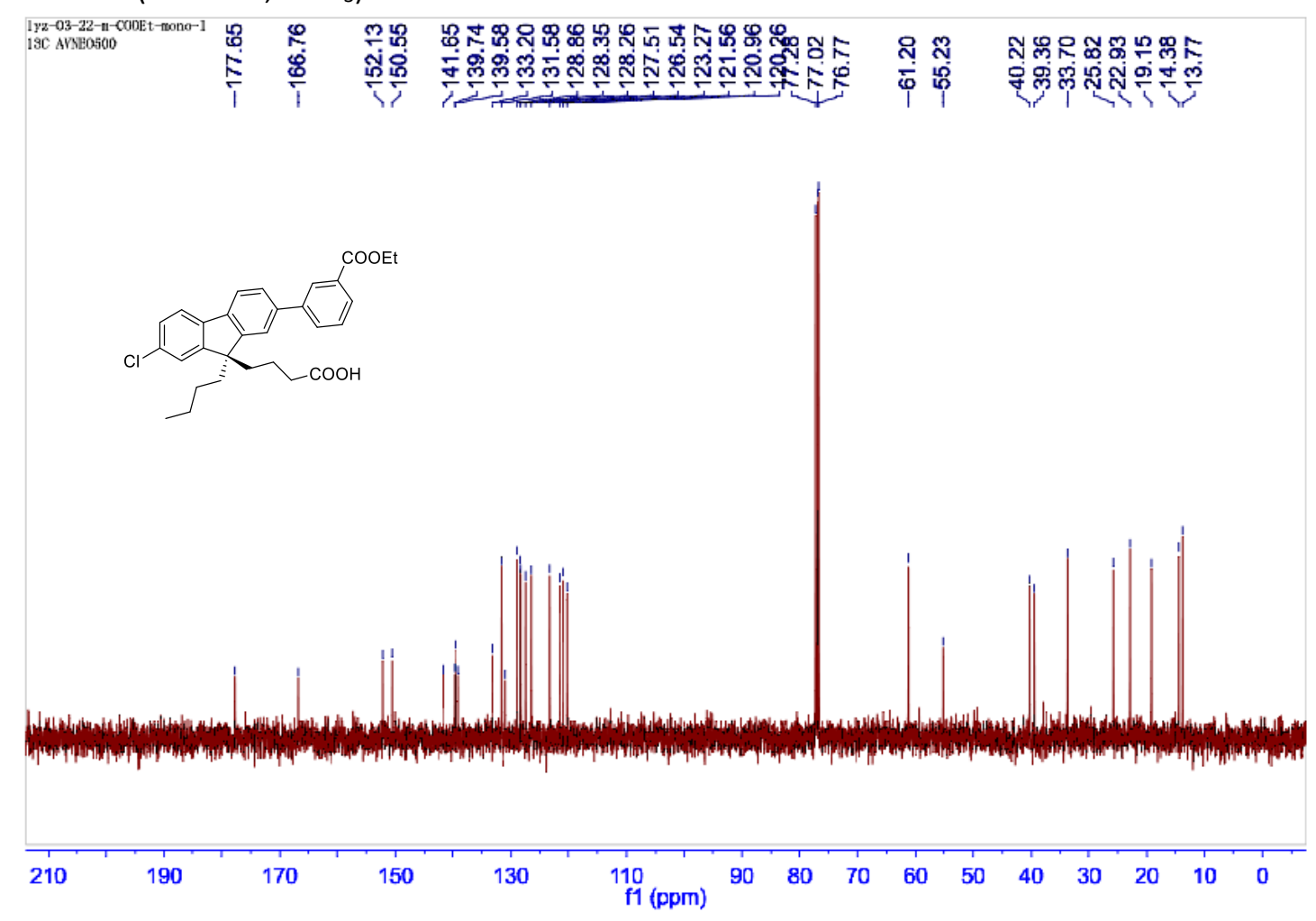


${ }^{1} \mathrm{H}$ NMR (500 MHz, $\mathrm{CDCl}_{3}$ )-25

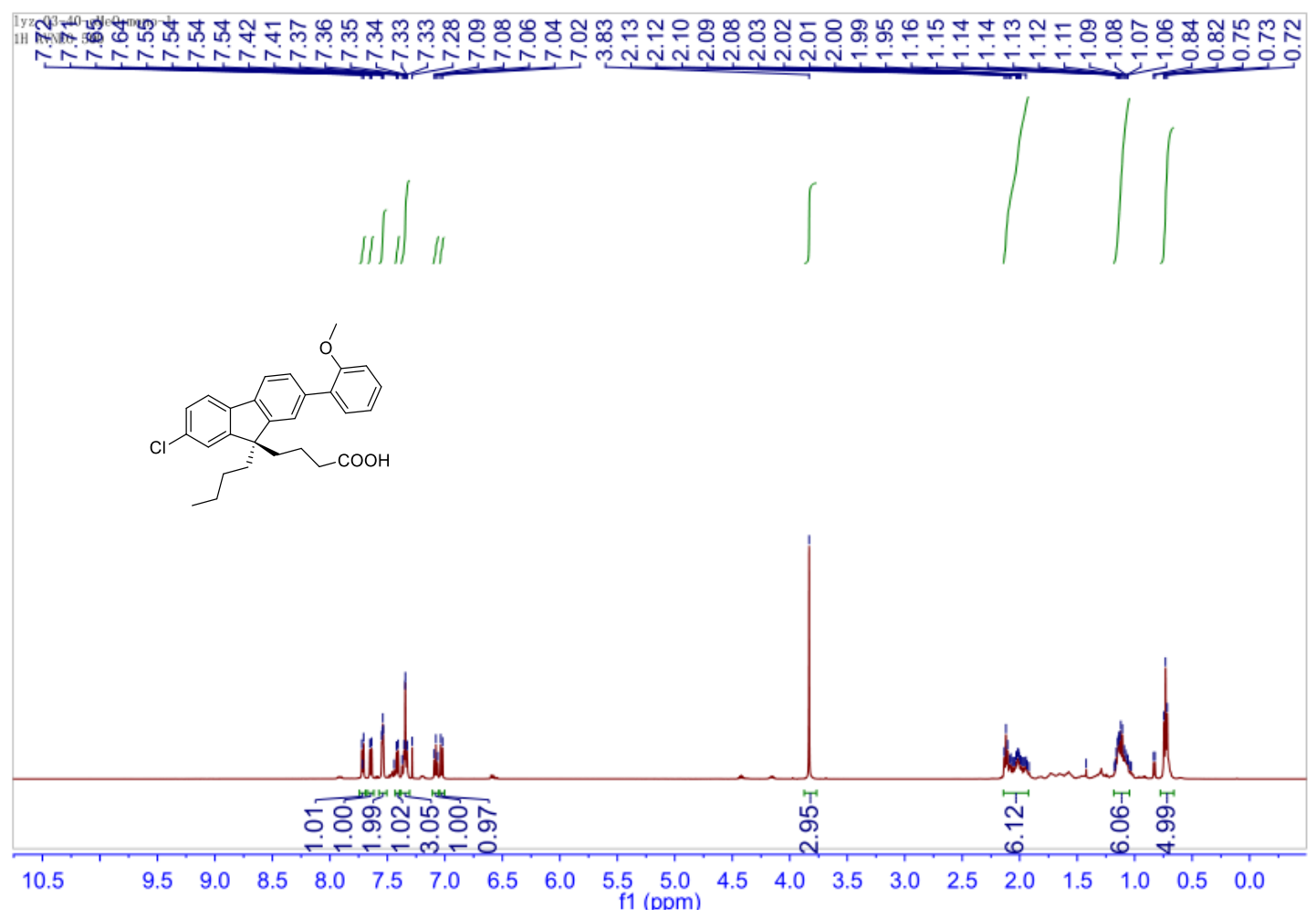

${ }^{13} \mathrm{C} \mathrm{NMR}\left(126 \mathrm{MHz}, \mathrm{CDCl}_{3}\right)-25$

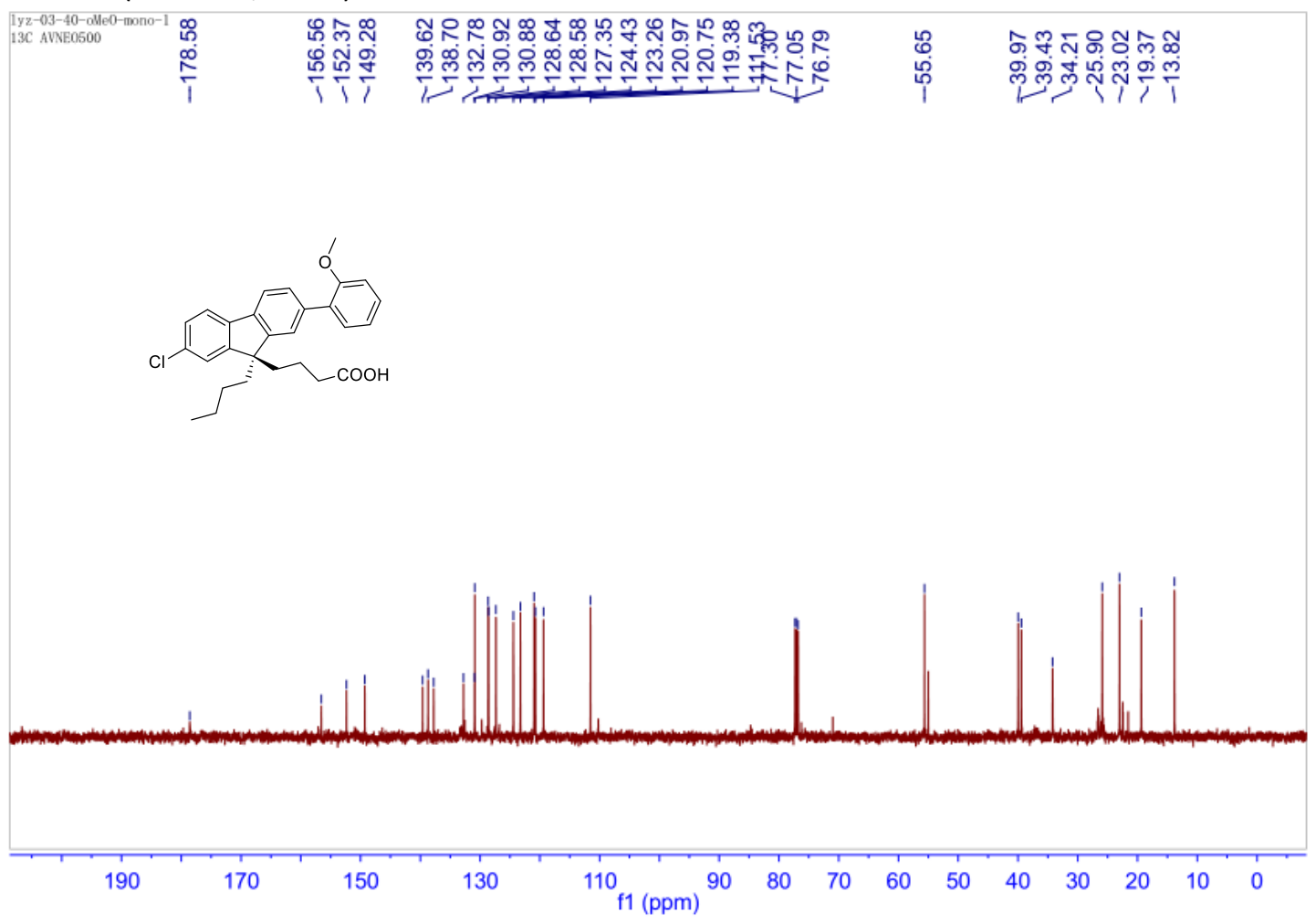


${ }^{1} \mathrm{H}$ NMR (500 MHz, $\left.\mathrm{CDCl}_{3}\right)-26$

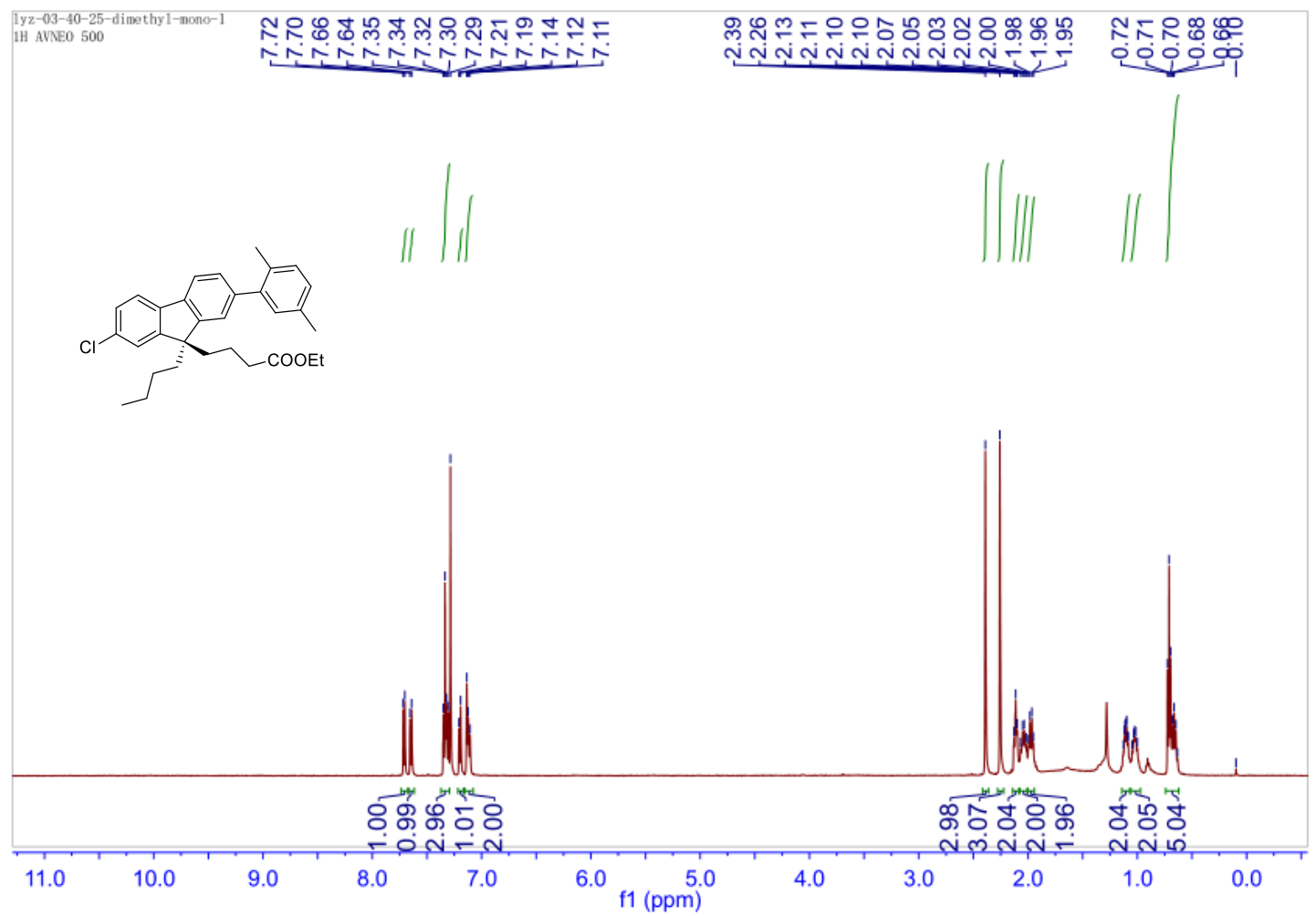

${ }^{13} \mathrm{C} \mathrm{NMR}\left(126 \mathrm{MHz}, \mathrm{CDCl}_{3}\right)-26$

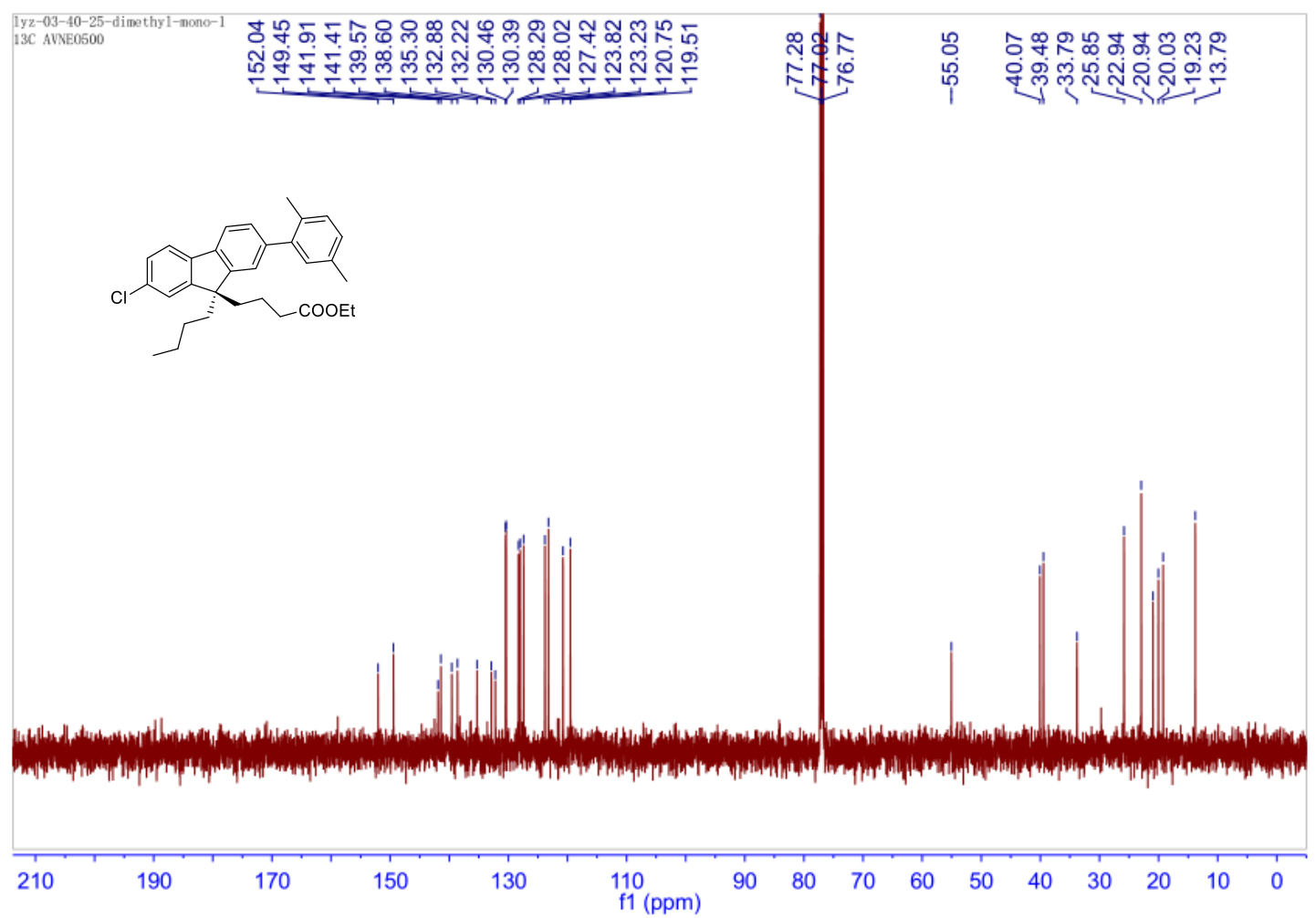


${ }^{1} \mathrm{H}$ NMR $\left(500 \mathrm{MHz}, \mathrm{CDCl}_{3}\right)-27$

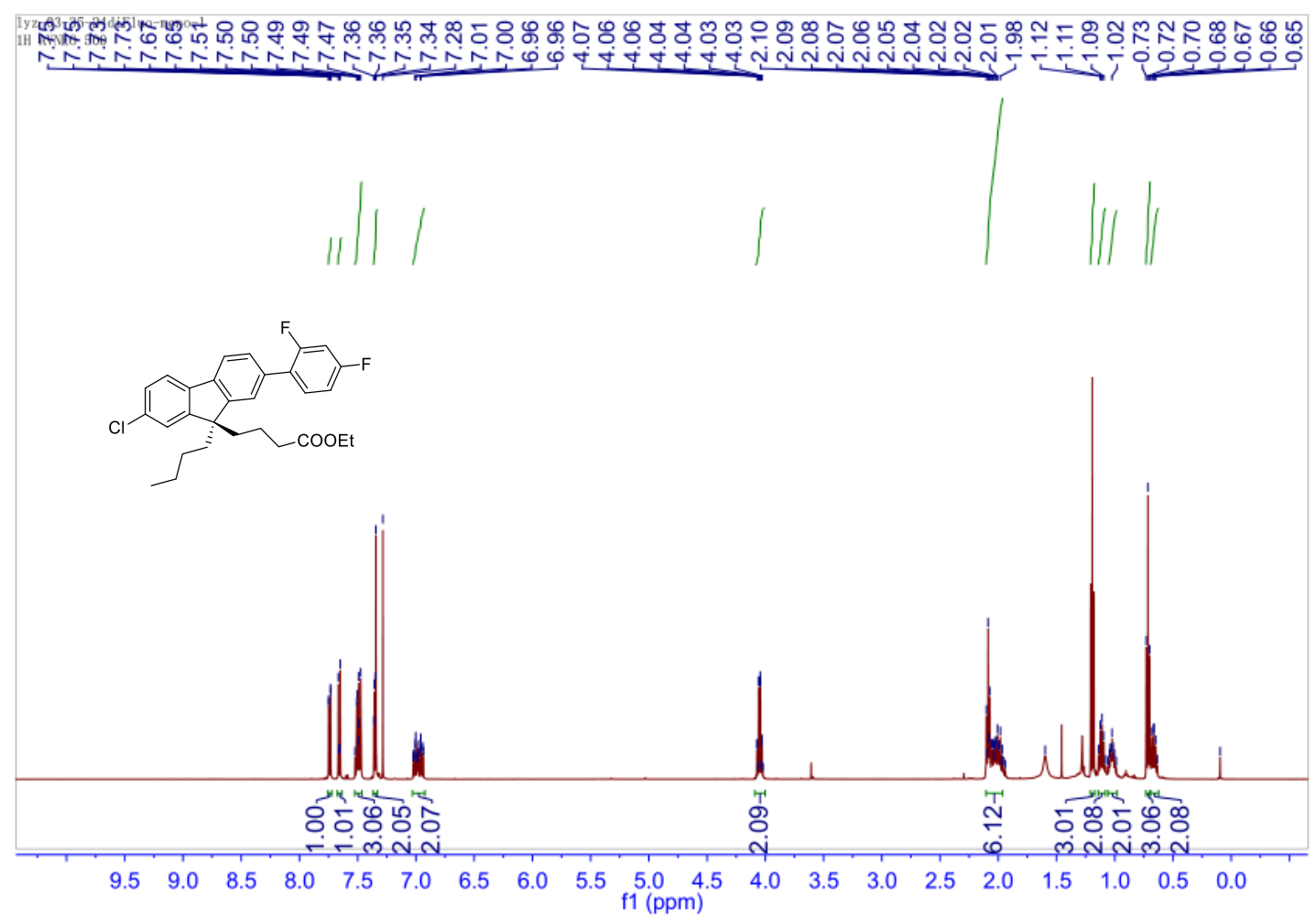

${ }^{13} \mathrm{C} \mathrm{NMR}\left(126 \mathrm{MHz}, \mathrm{CDCl}_{3}\right)-27$

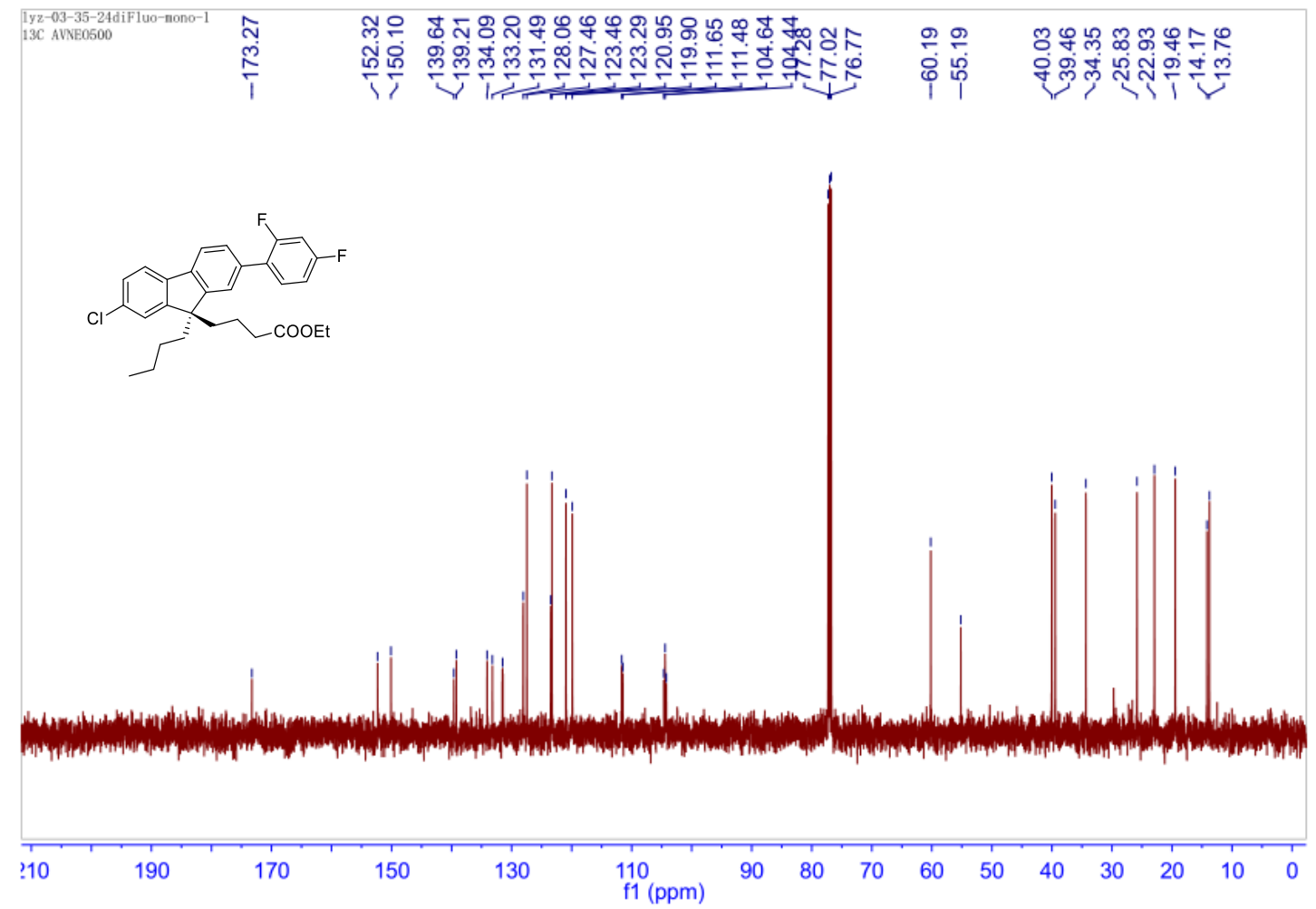




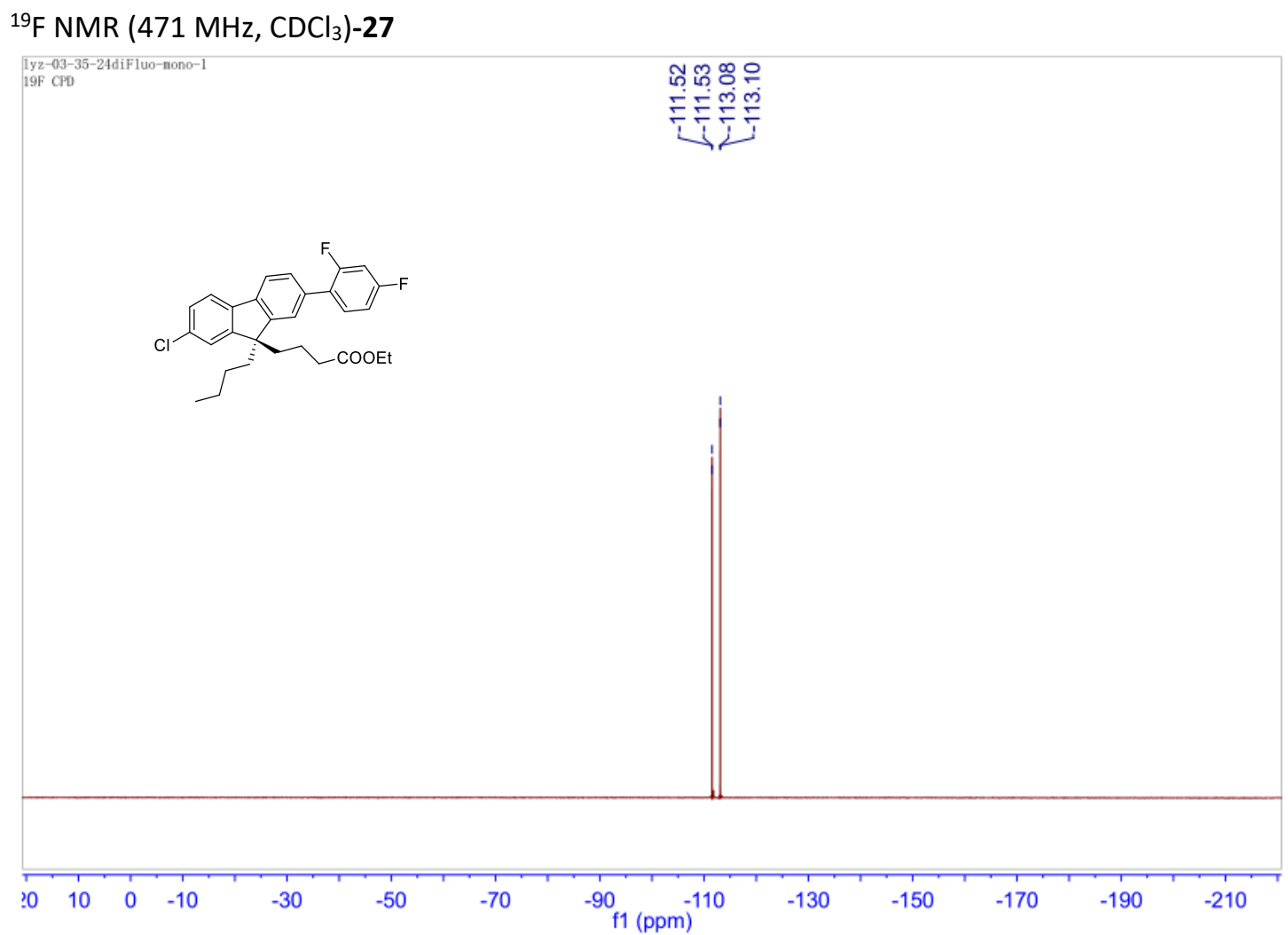


${ }^{1} \mathrm{H}$ NMR (500 MHz, $\mathrm{CDCl}_{3}$ )-28

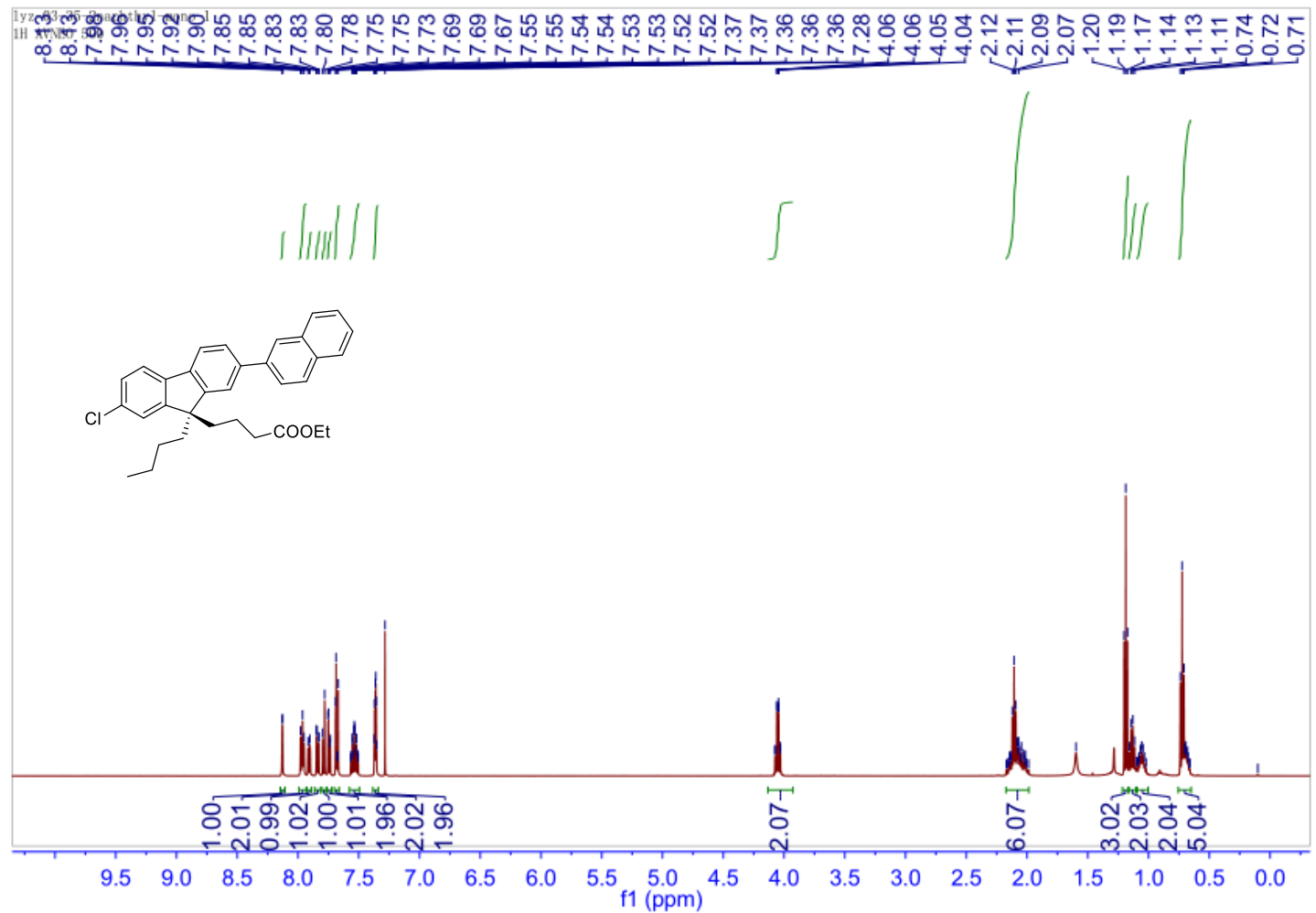

${ }^{13} \mathrm{C} \mathrm{NMR}\left(126 \mathrm{MHz}, \mathrm{CDCl}_{3}\right)-28$

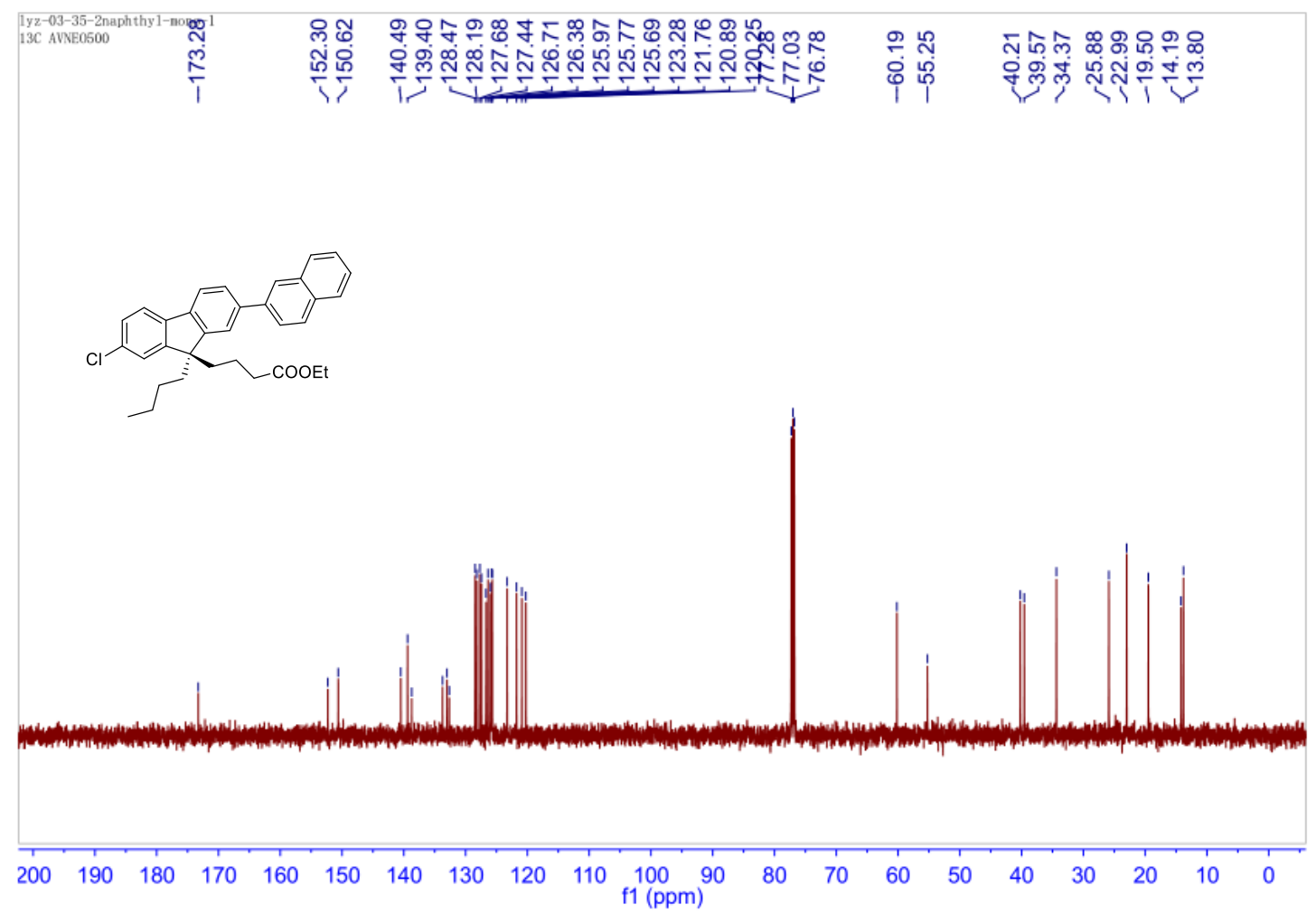


${ }^{1} \mathrm{H}$ NMR (500 MHz, $\mathrm{CDCl}_{3}$ )-29

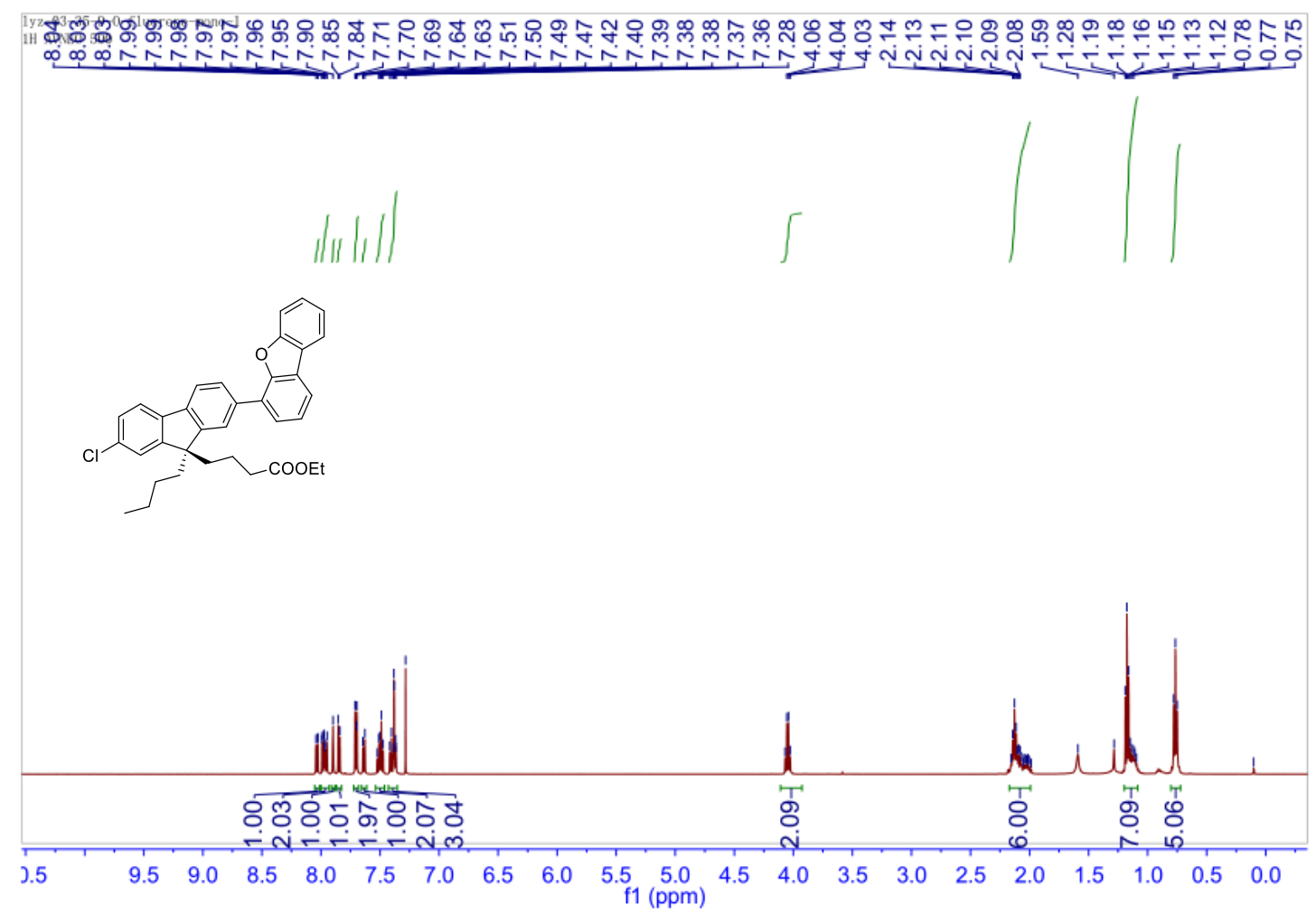

${ }^{13} \mathrm{C}$ NMR (126 MHz, $\left.\mathrm{CDCl}_{3}\right)-29$

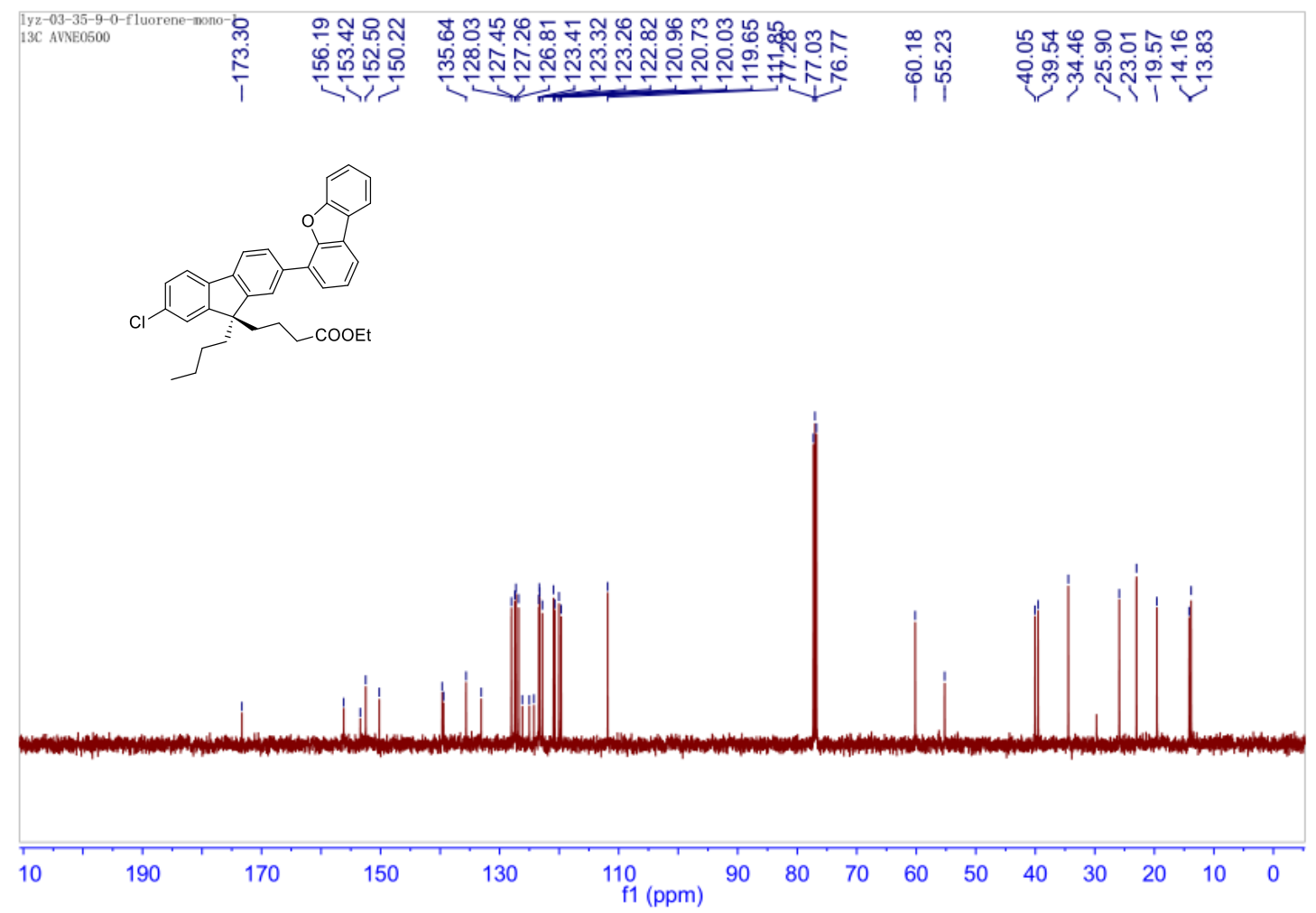


${ }^{1} \mathrm{H}$ NMR $\left(500 \mathrm{MHz}, \mathrm{CDCl}_{3}\right)-30$

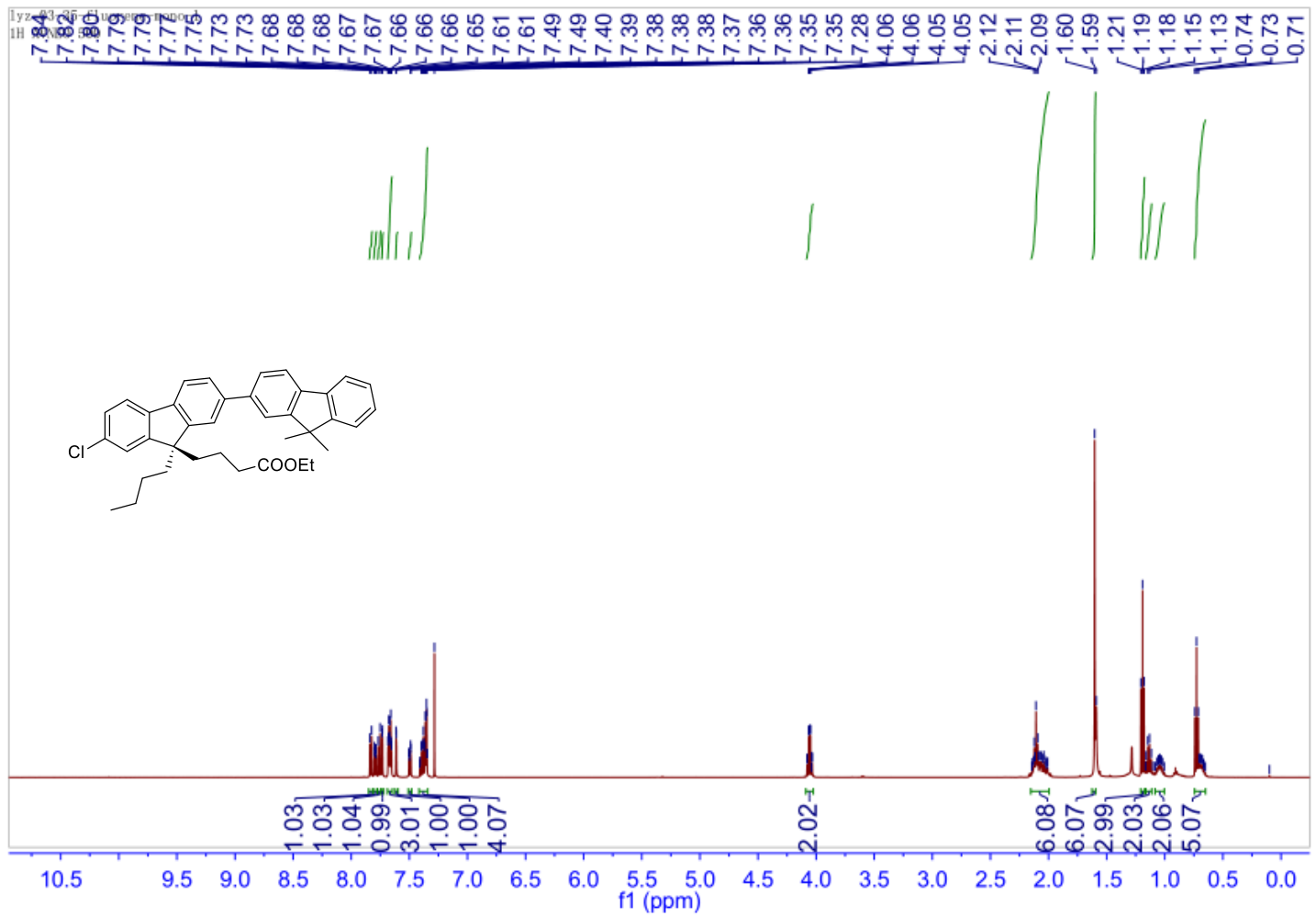

${ }^{13} \mathrm{C} \mathrm{NMR}\left(126 \mathrm{MHz}, \mathrm{CDCl}_{3}\right)-30$

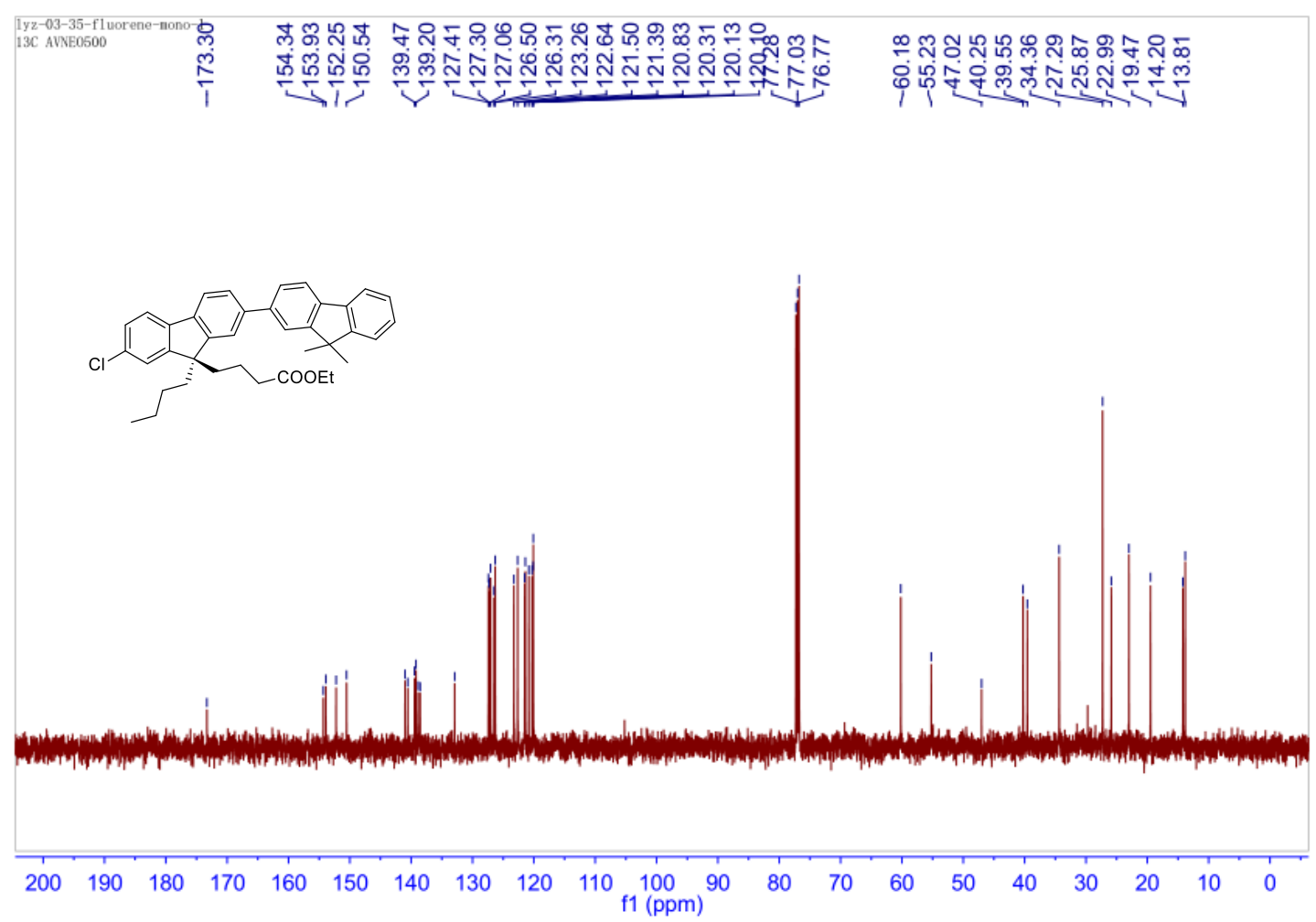


${ }^{1} \mathrm{H}$ NMR (500 MHz, $\mathrm{CDCl}_{3}$ )-31

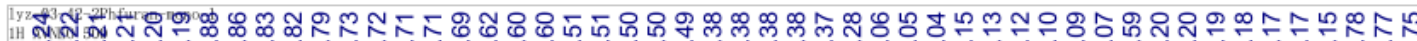
品

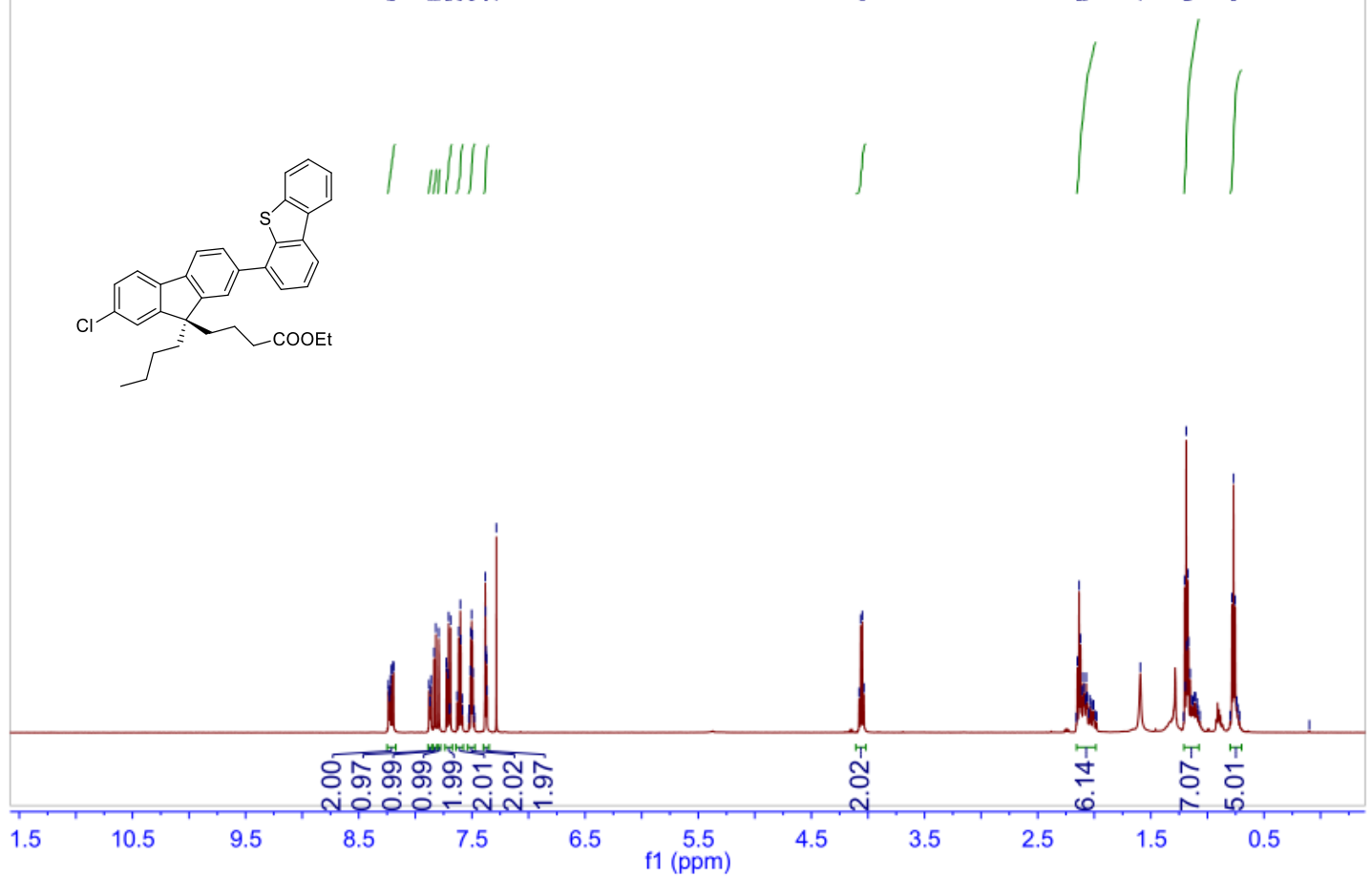

${ }^{13} \mathrm{C} \mathrm{NMR}\left(126 \mathrm{MHz}, \mathrm{CDCl}_{3}\right)-31$

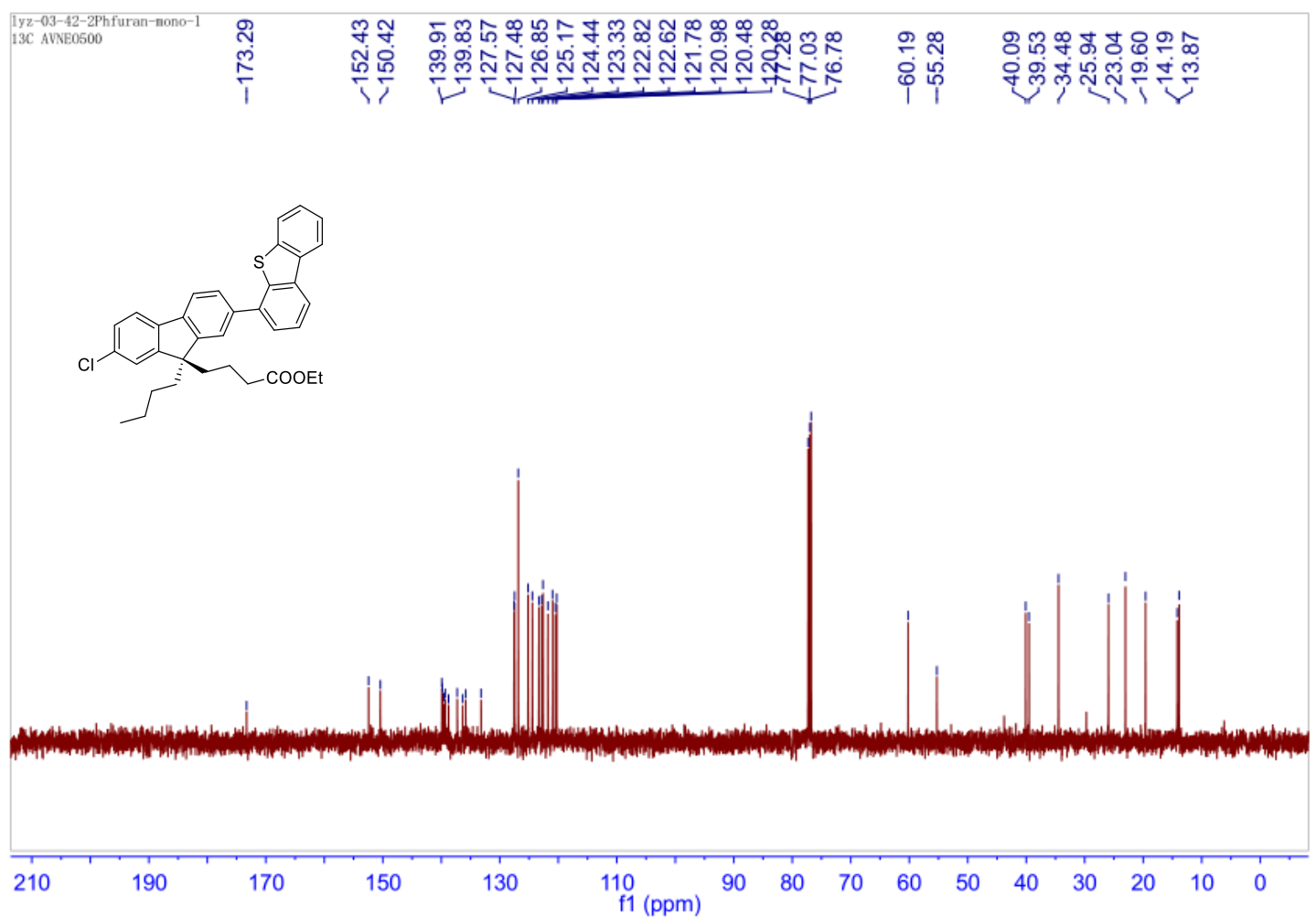


${ }^{1} \mathrm{H}$ NMR (500 MHz, $\mathrm{CDCl}_{3}$ )-32

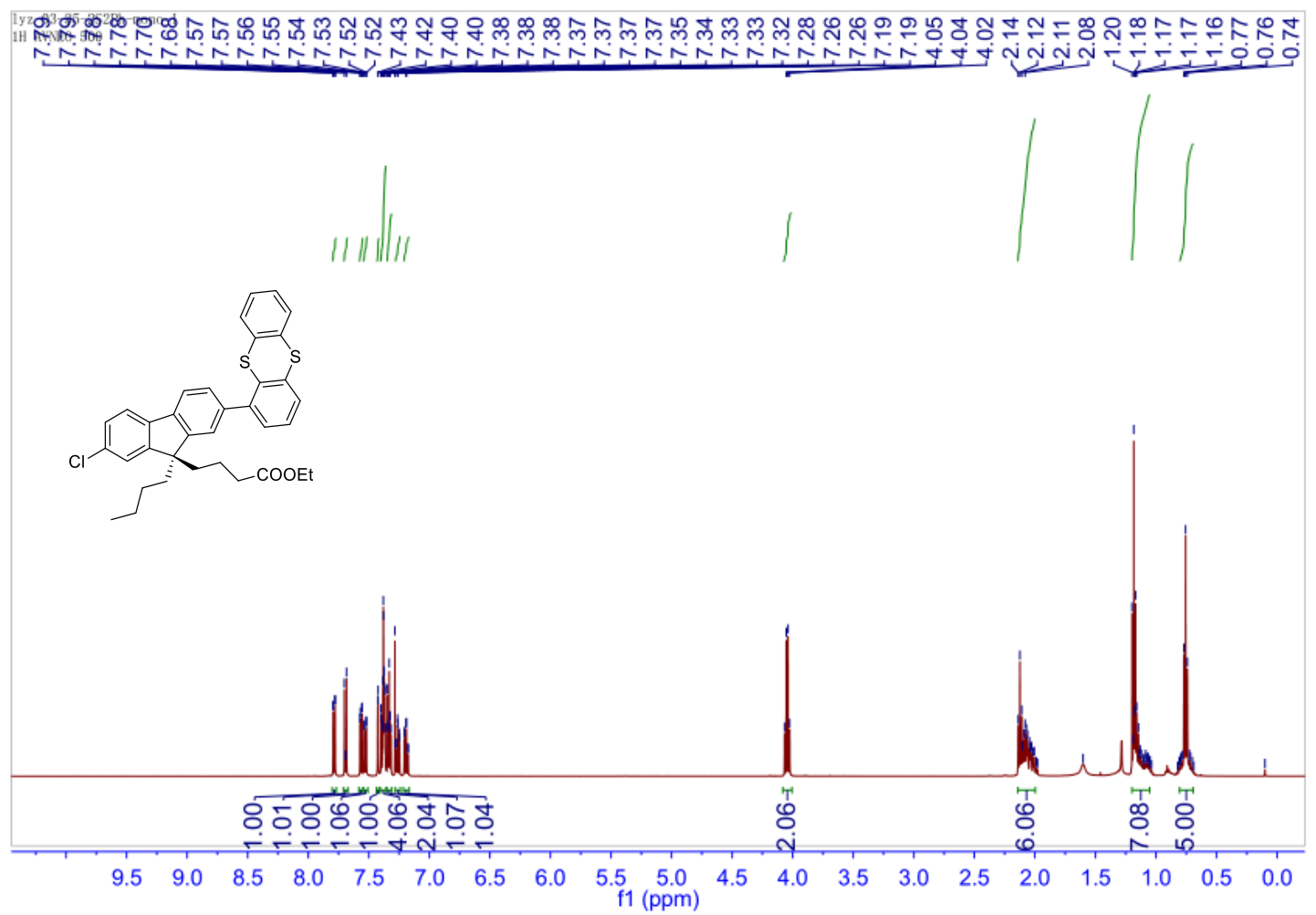

$\left.{ }^{13} \mathrm{C} \mathrm{NMR} \mathrm{(126} \mathrm{MHz,} \mathrm{CDCl}_{3}\right)-32$

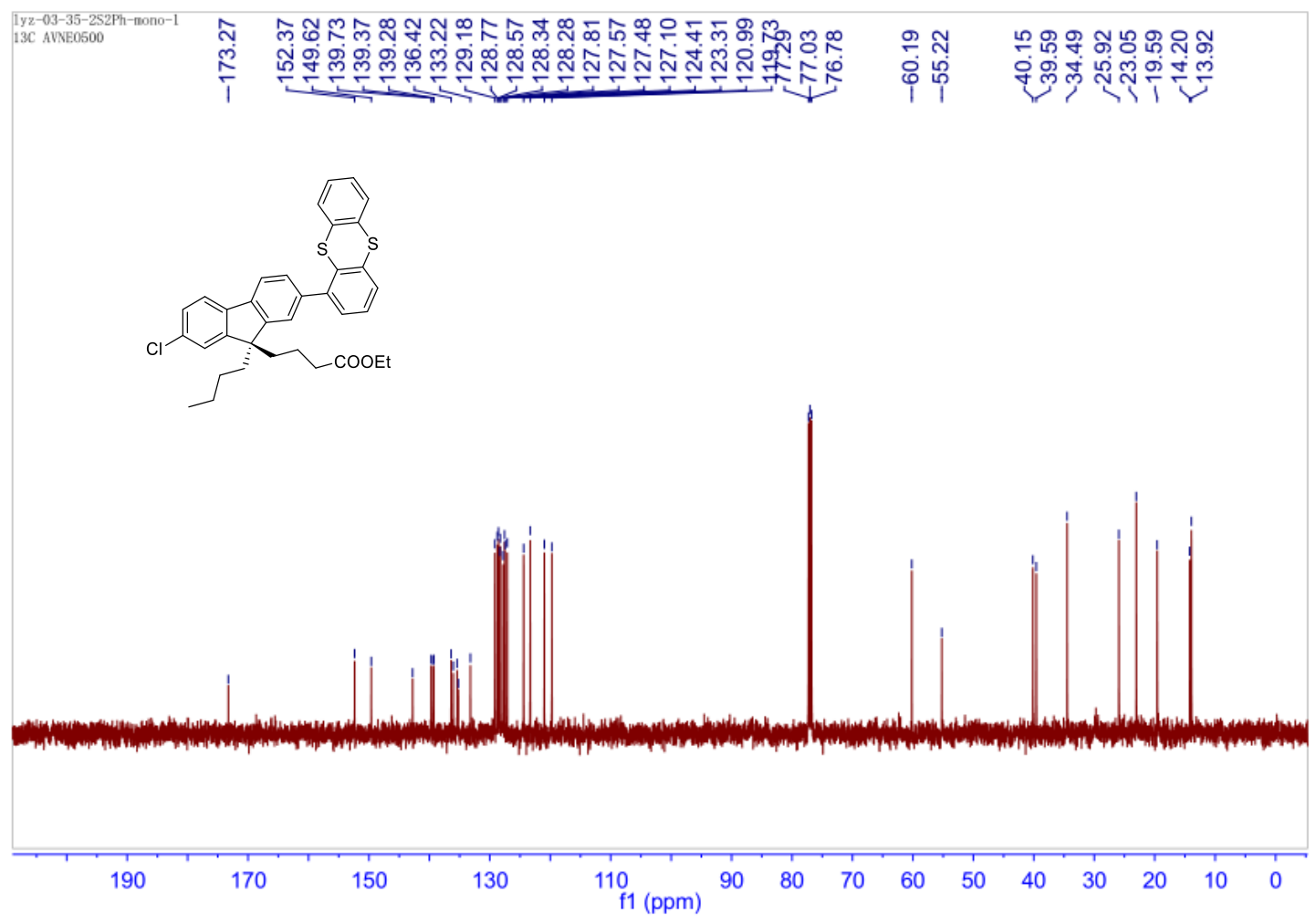


${ }^{1} \mathrm{H}$ NMR (500 MHz, $\mathrm{CDCl}_{3}$ )-33

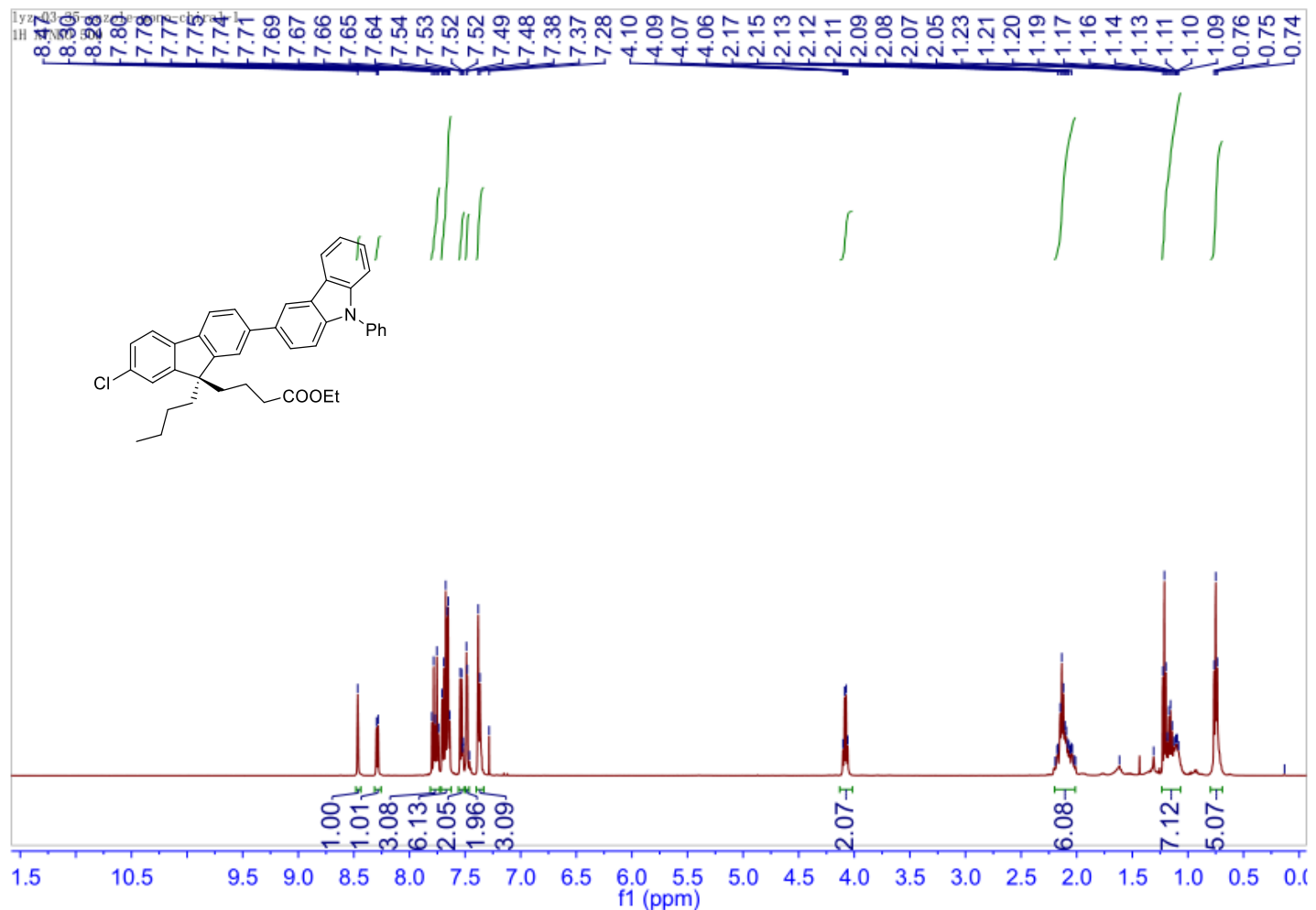

${ }^{13} \mathrm{C}$ NMR (126 MHz, $\left.\mathrm{CDCl}_{3}\right)-33$

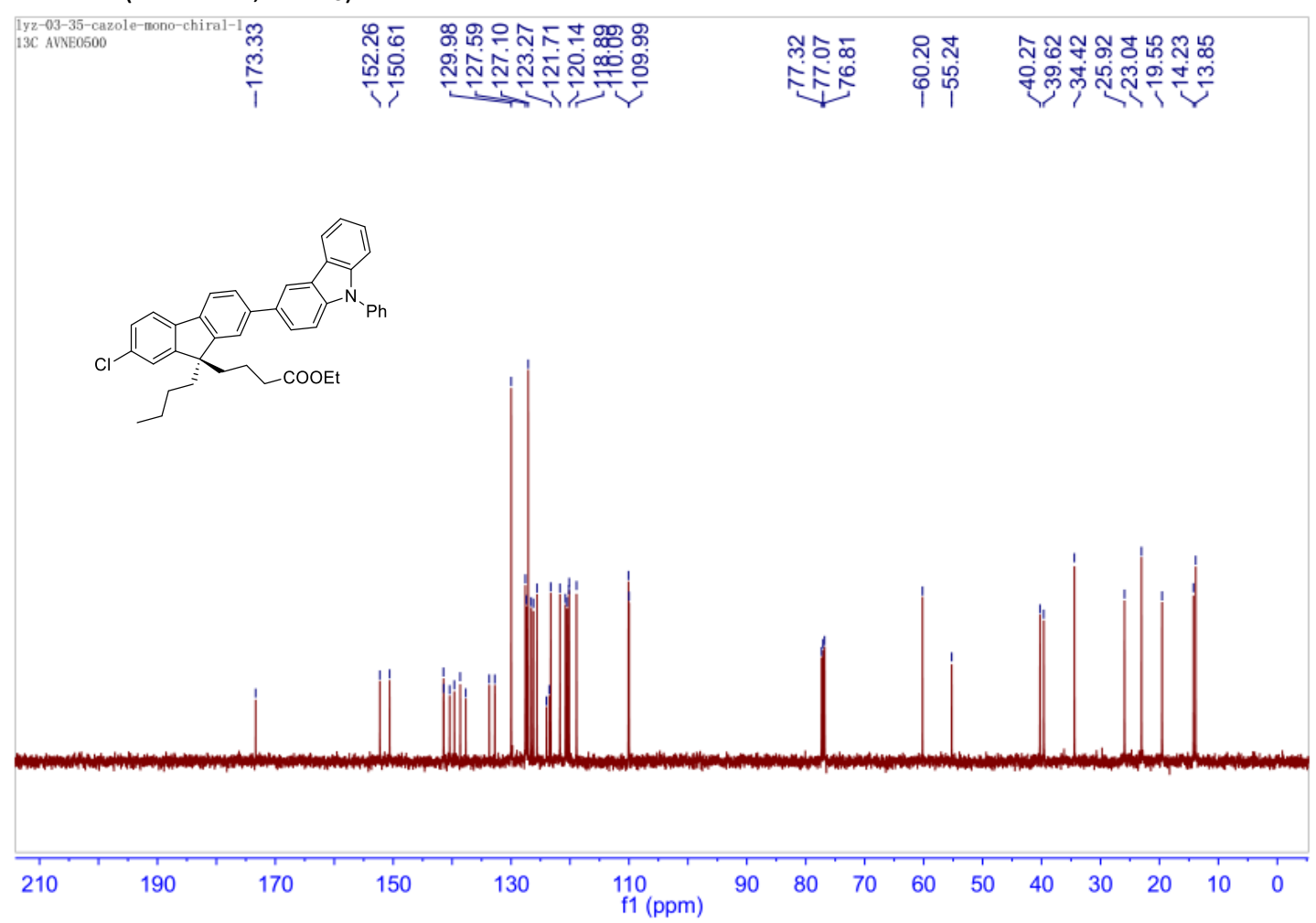


${ }^{1} \mathrm{H}$ NMR $\left(500 \mathrm{MHz}, \mathrm{CDCl}_{3}\right)-34$

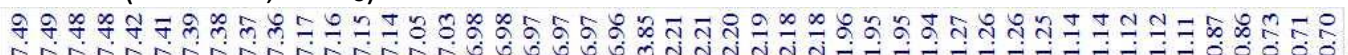

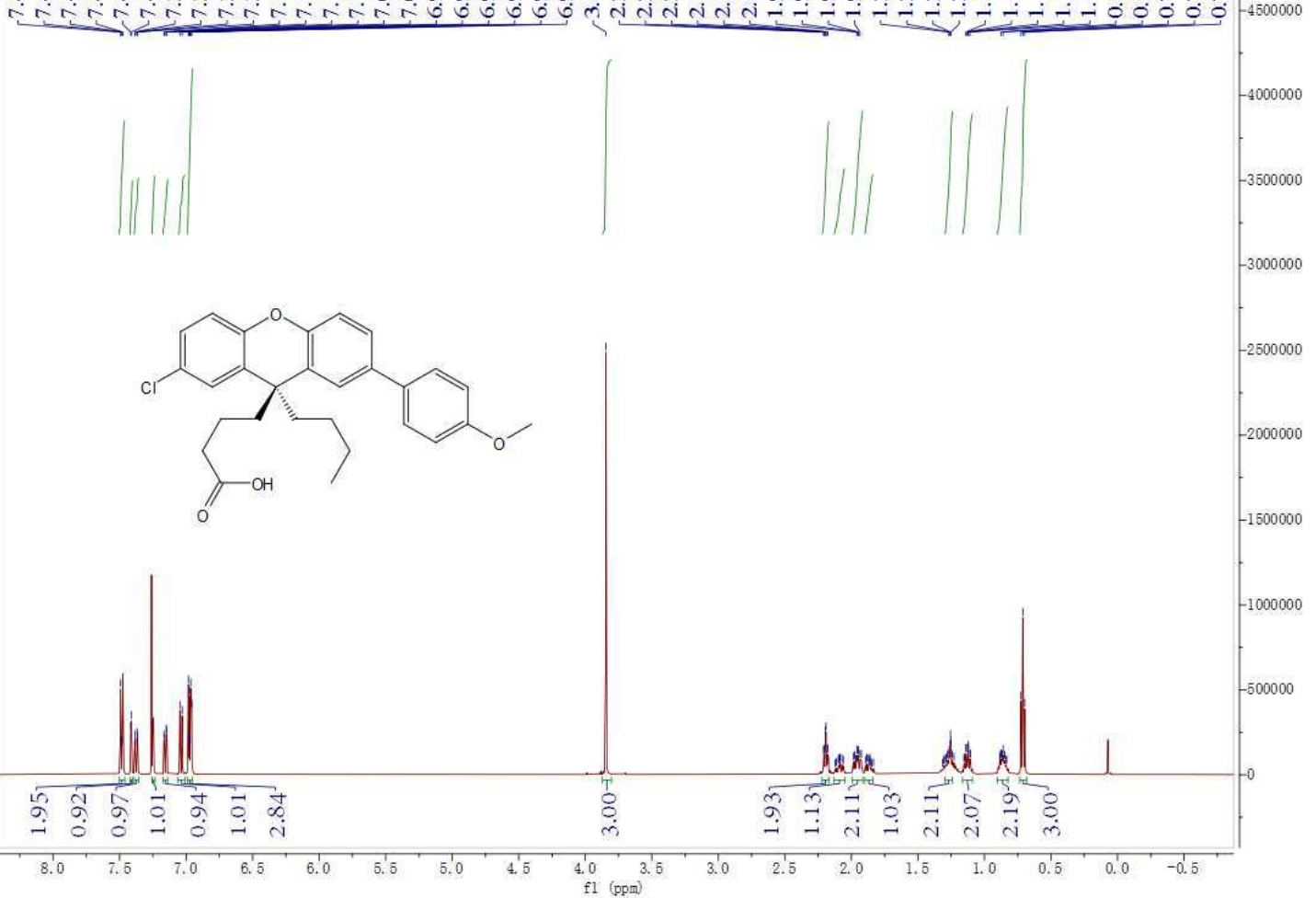

${ }^{13} \mathrm{C}$ NMR (126 MHz, $\left.\mathrm{CDCl}_{3}\right)-34$

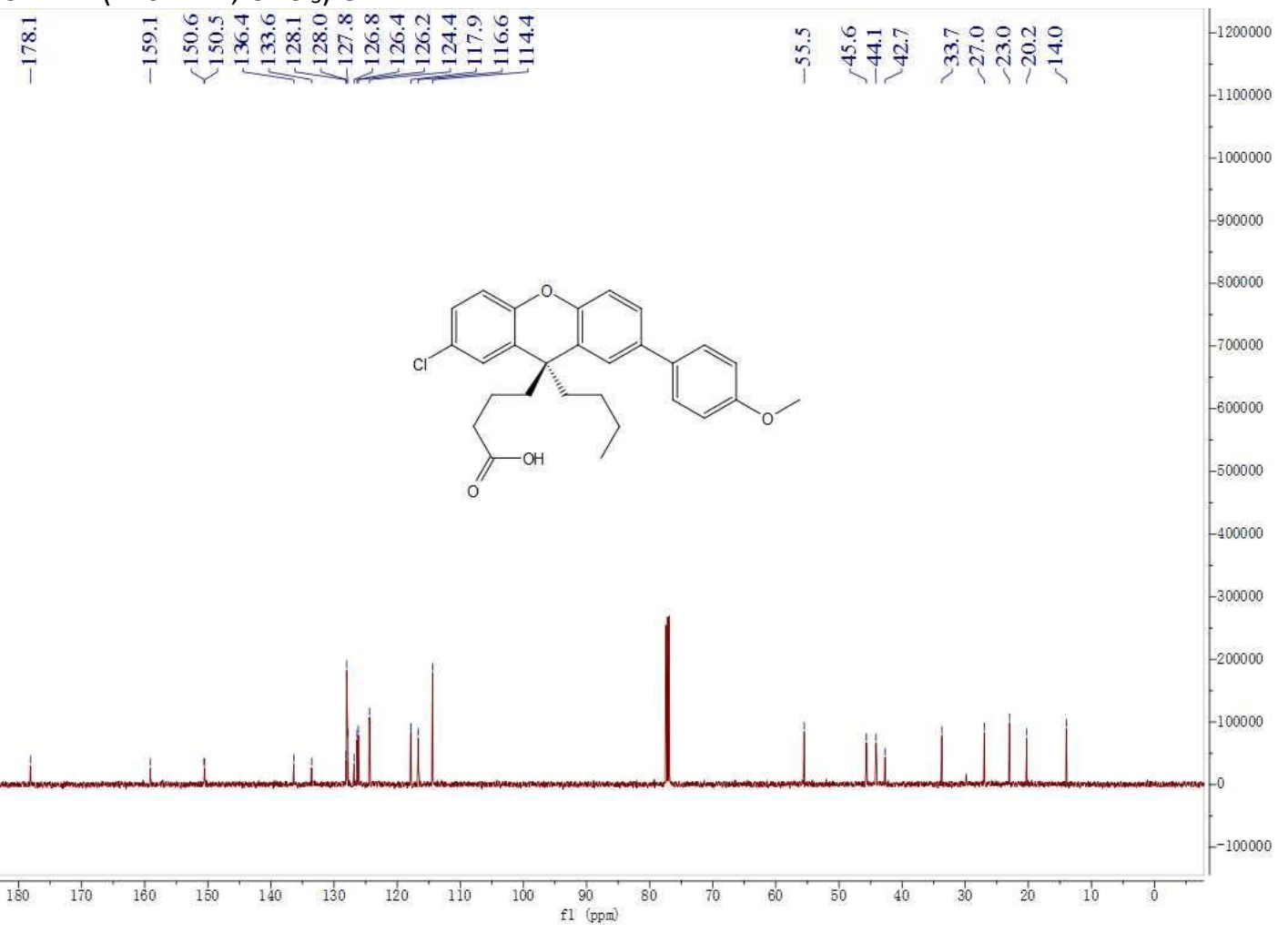


${ }^{1} \mathrm{H} \mathrm{NMR}\left(500 \mathrm{MHz}, \mathrm{CDCl}_{3}\right)-35$

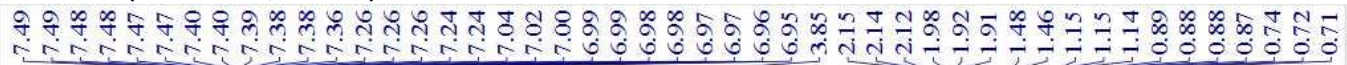

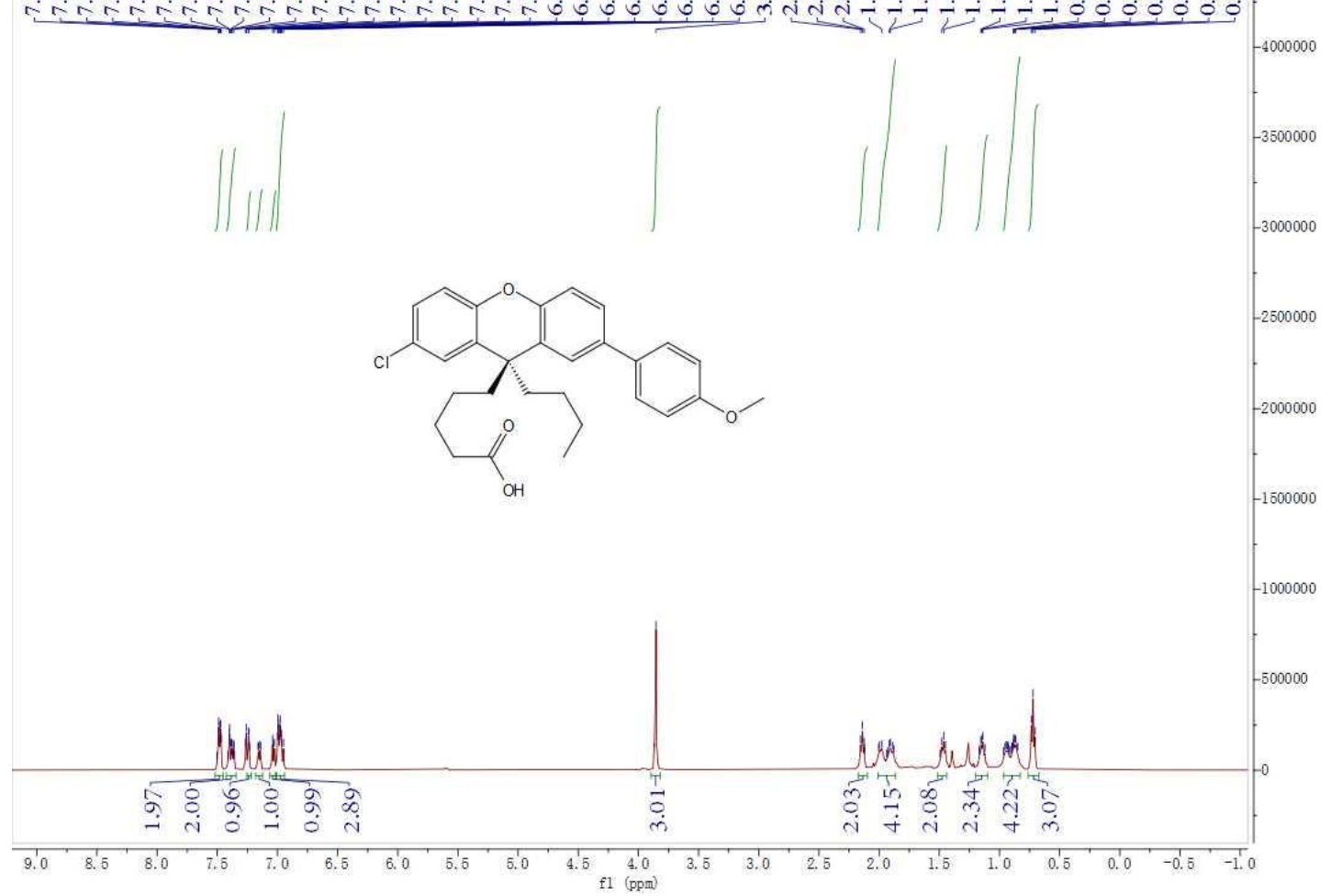

${ }^{13} \mathrm{C} \mathrm{NMR}\left(126 \mathrm{MHz}, \mathrm{CDCl}_{3}\right)-35$

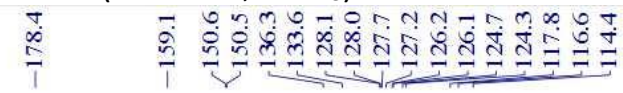

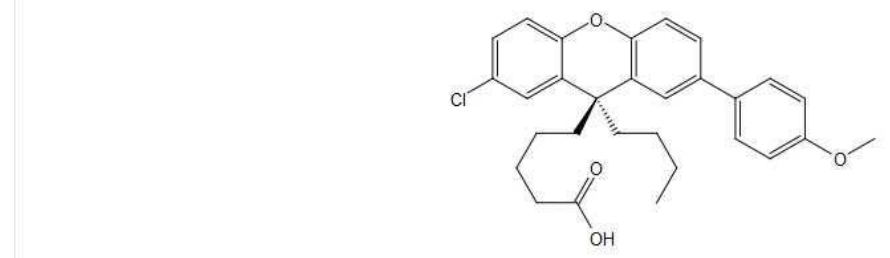

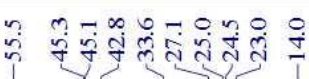

$-1300000$

1200000

1100000

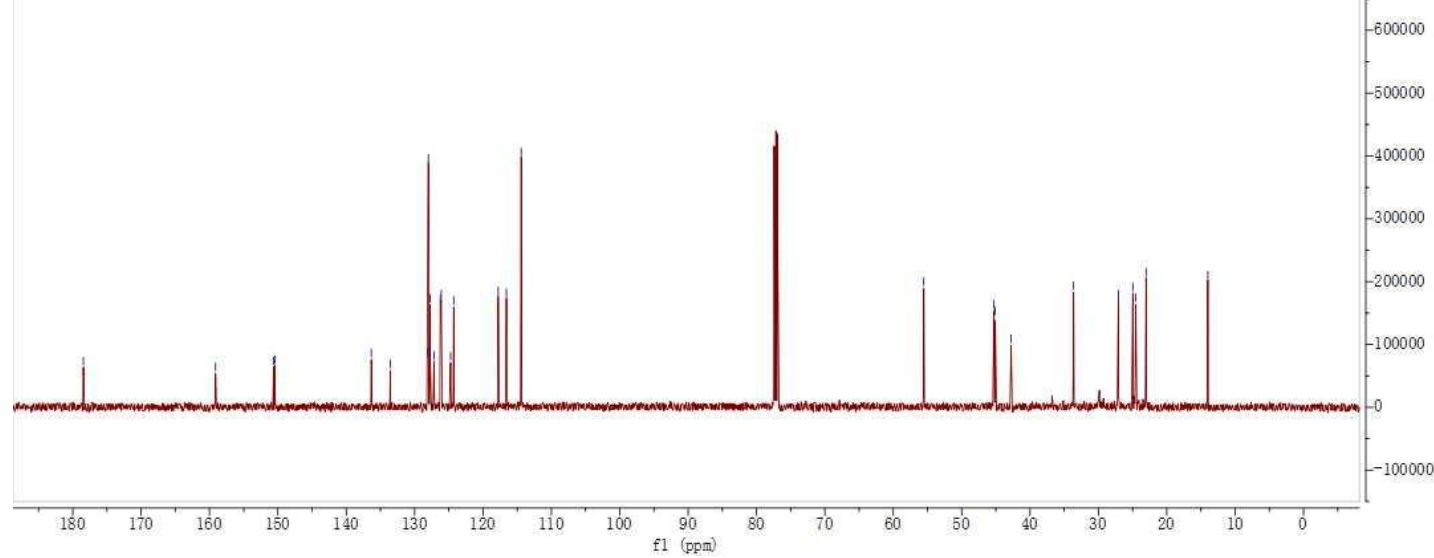




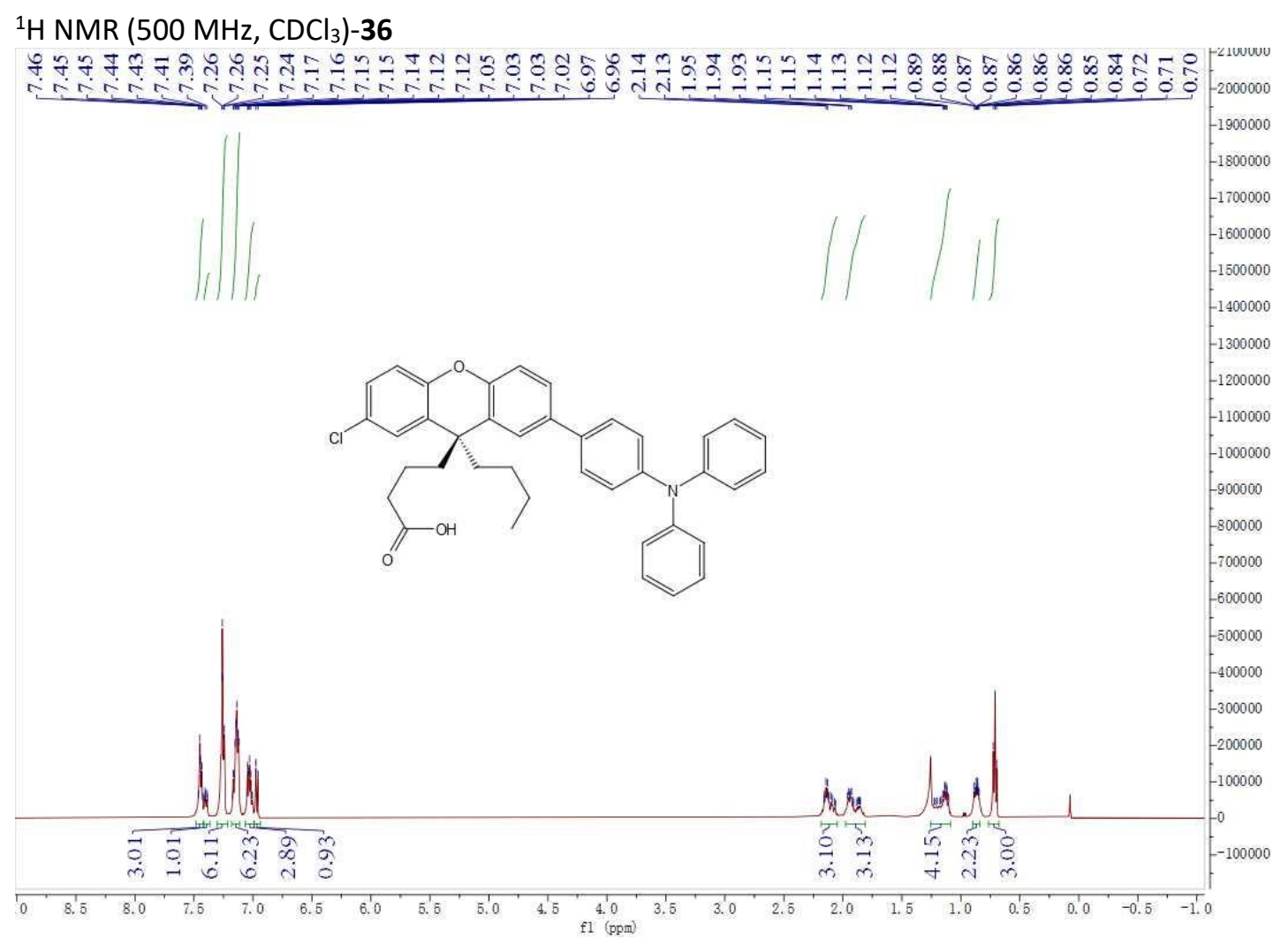

${ }^{13} \mathrm{C}$ NMR (126 MHz, $\left.\mathrm{CDCl}_{3}\right)-36$

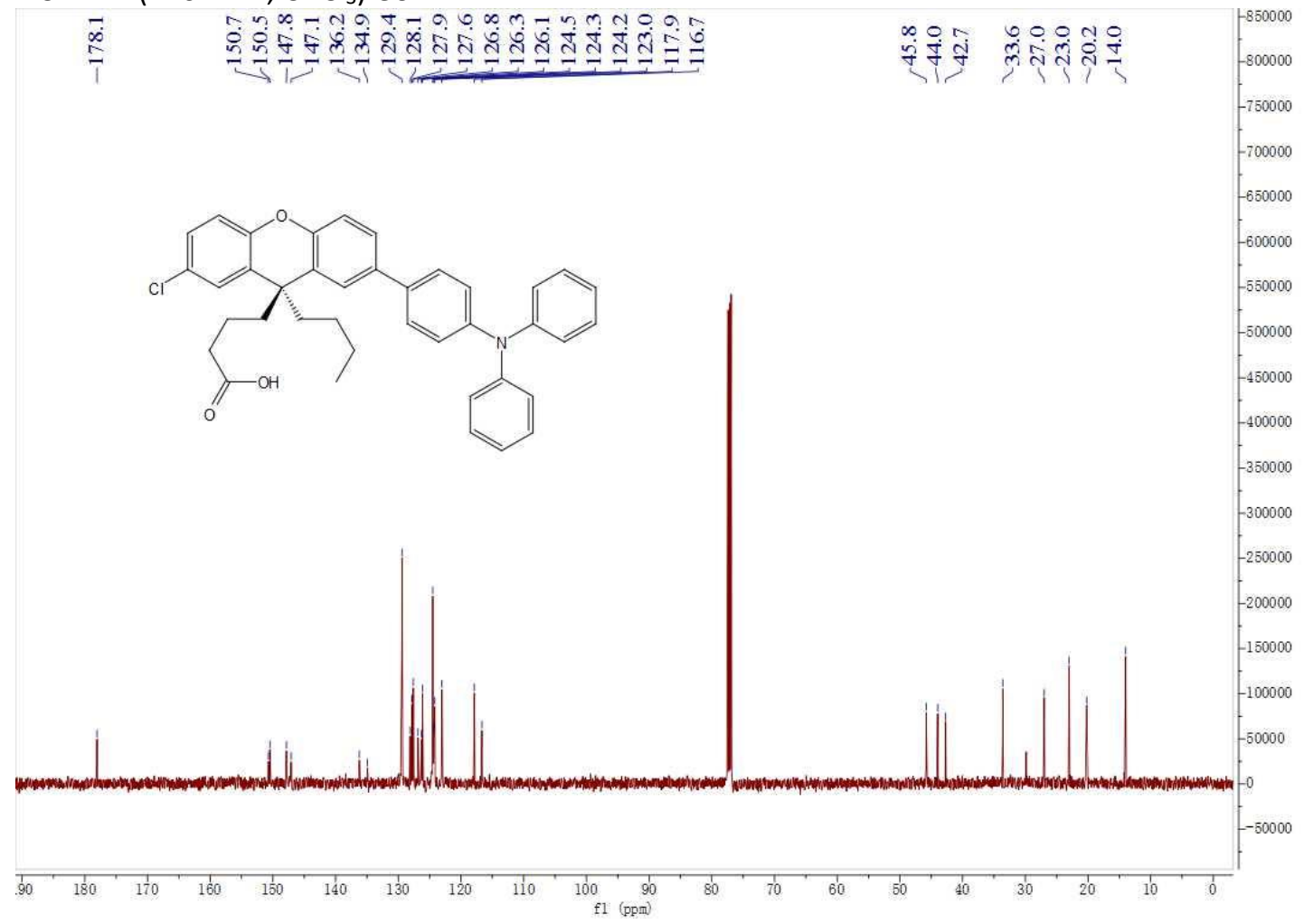


${ }^{1} \mathrm{H}$ NMR (500 MHz, $\left.\mathrm{CDCl}_{3}\right)-37$

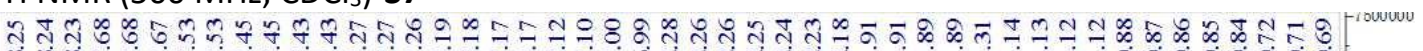

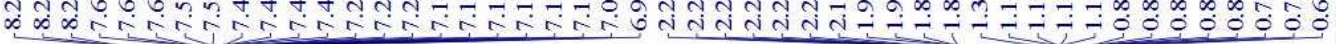

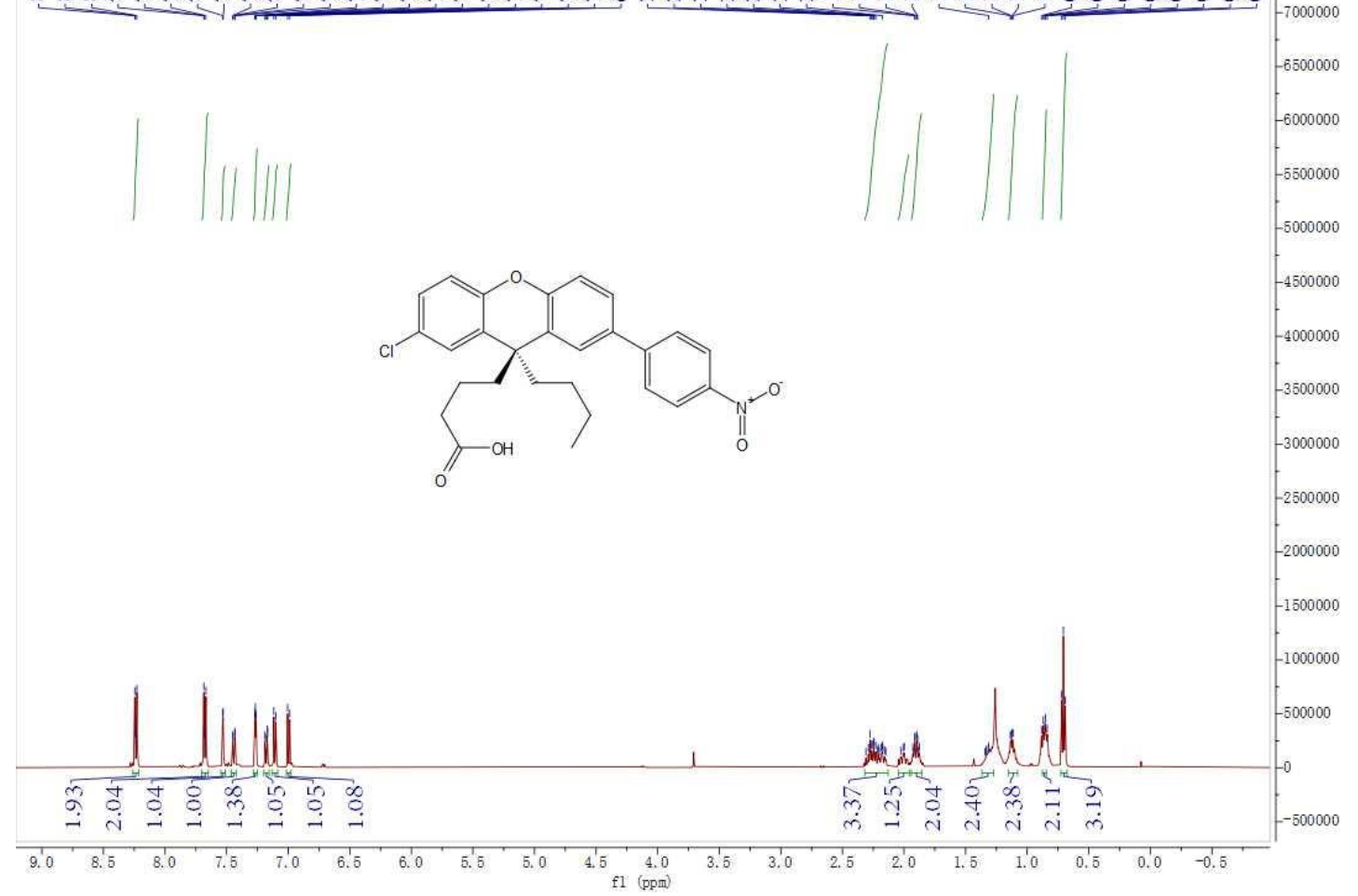

${ }^{13} \mathrm{C} \mathrm{NMR}\left(126 \mathrm{MHz}, \mathrm{CDCl}_{3}\right)-37$

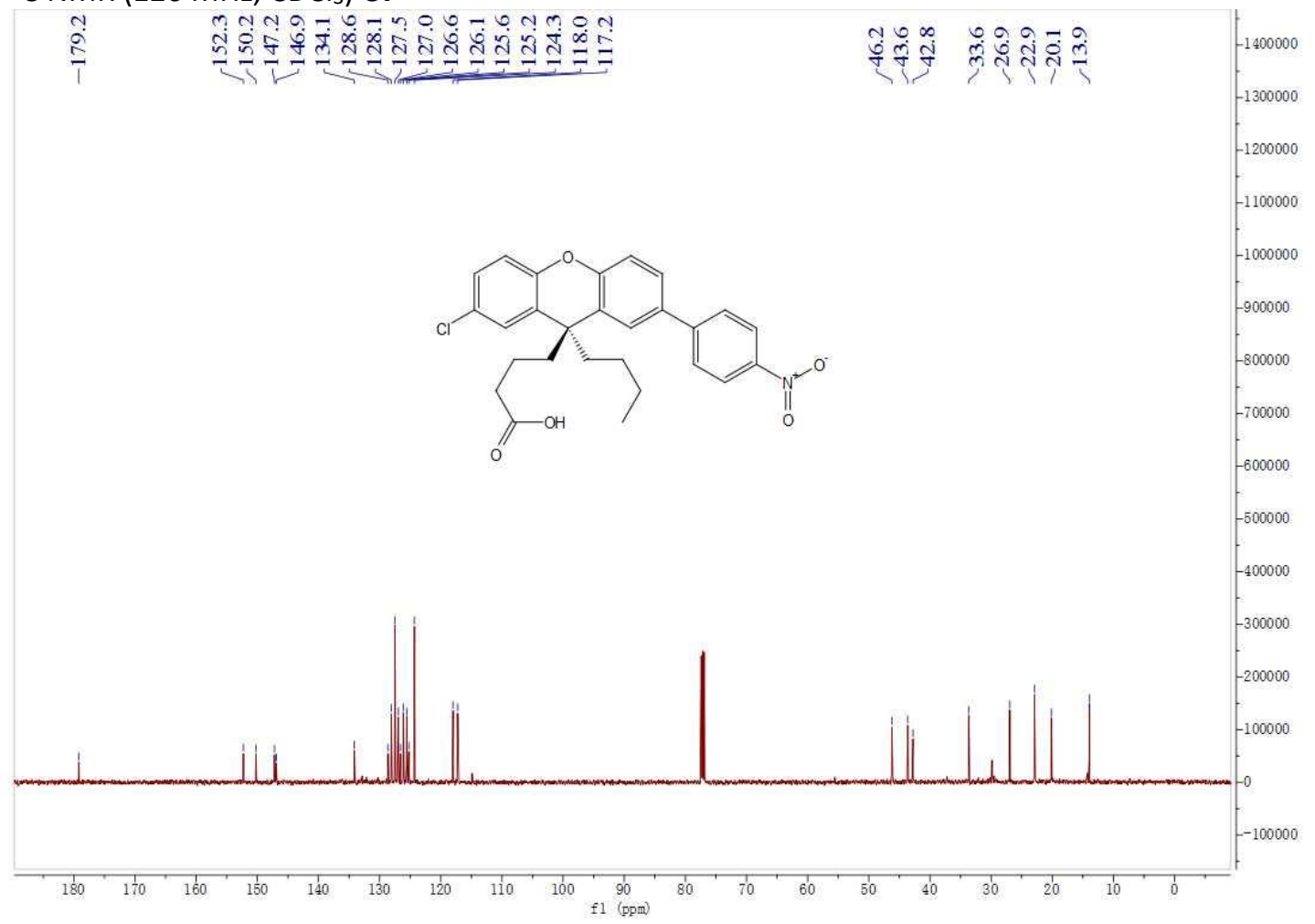


${ }^{1} \mathrm{H}$ NMR (500 MHz, $\mathrm{CDCl}_{3}$ )-38

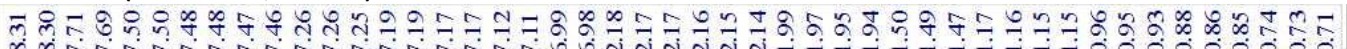

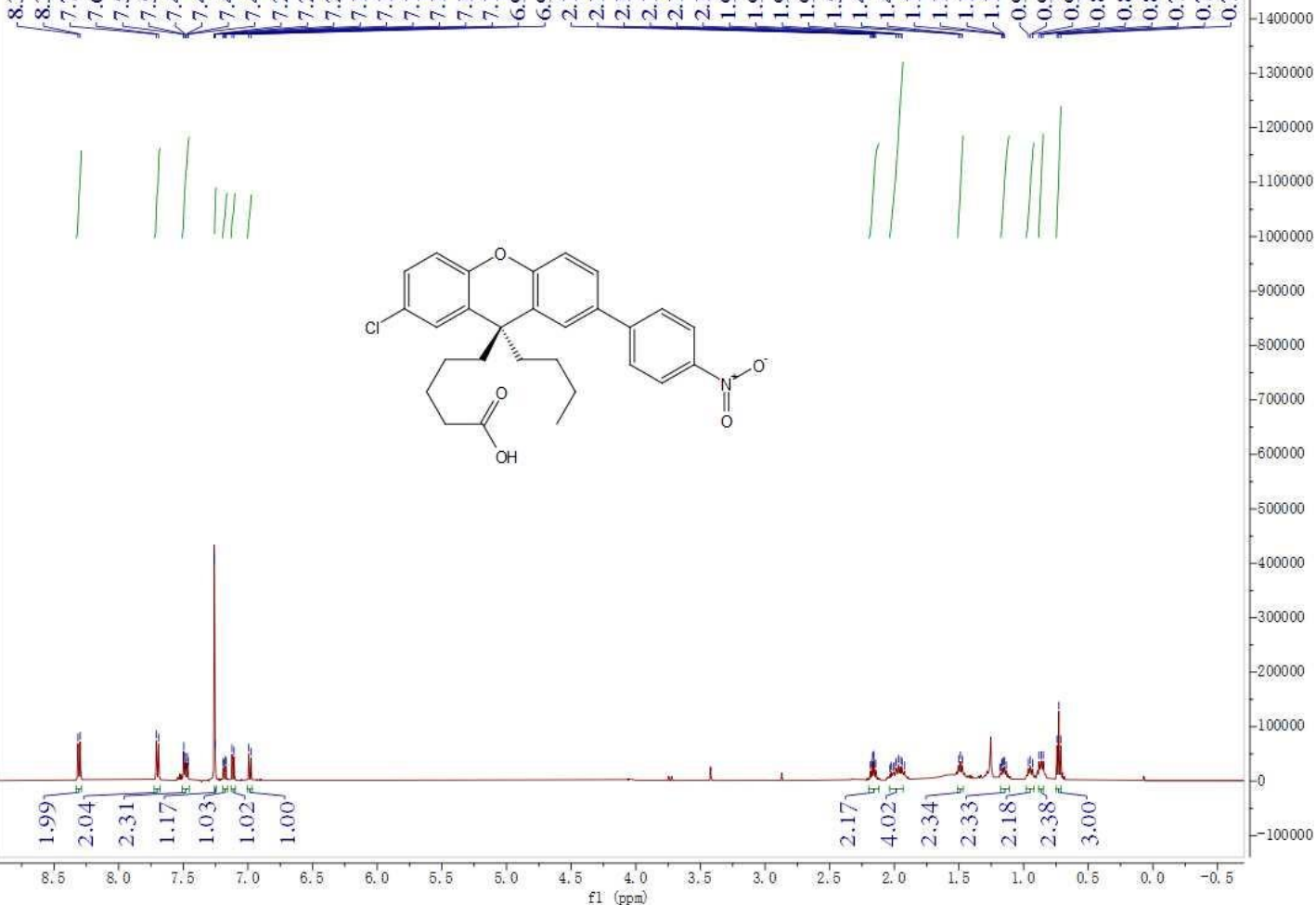

${ }^{13} \mathrm{C}$ NMR $\left(126 \mathrm{MHz}, \mathrm{CDCl}_{3}\right)-38$

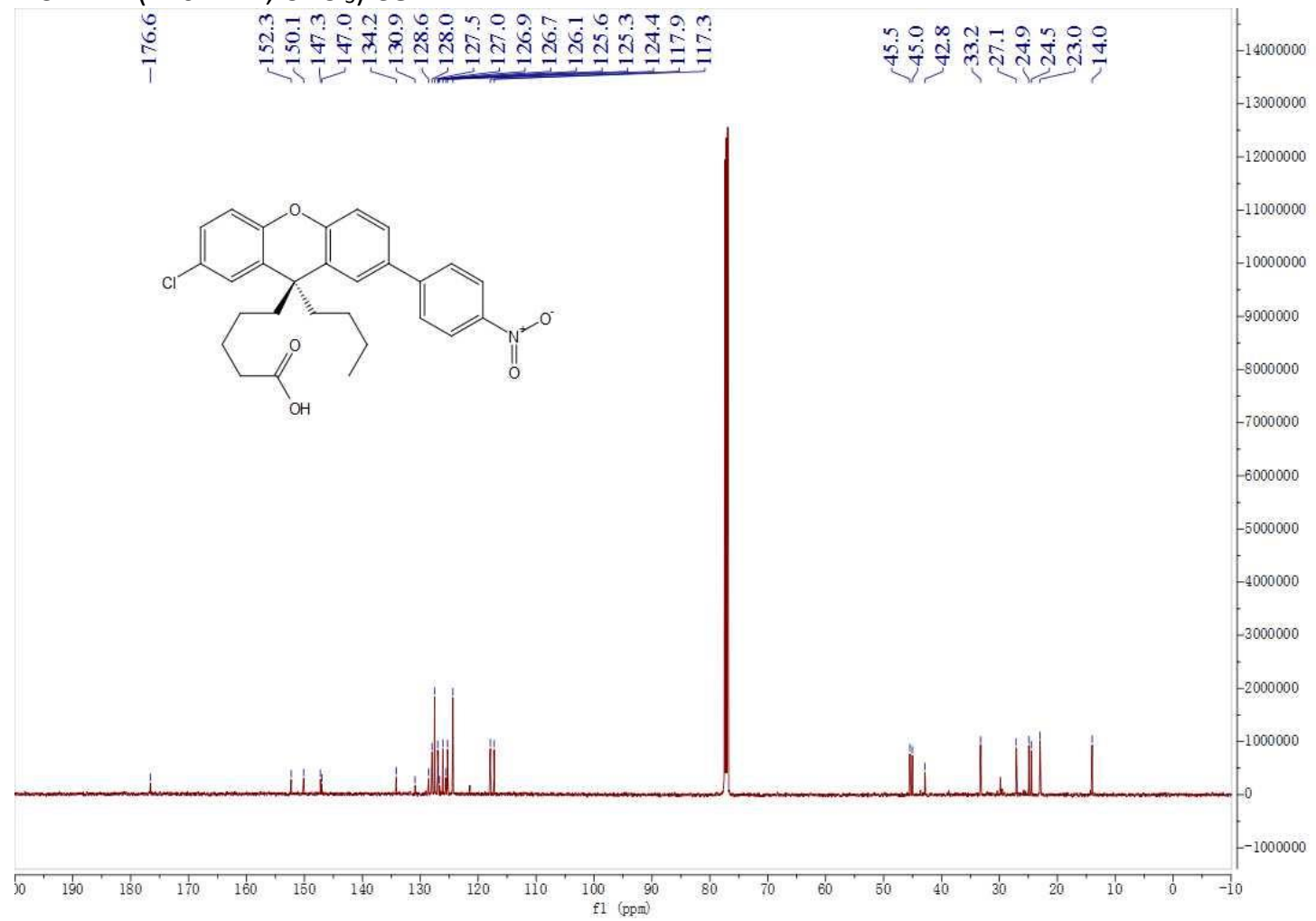


${ }^{1} \mathrm{H}$ NMR (500 MHz, $\left.\mathrm{CDCl}_{3}\right)$-39

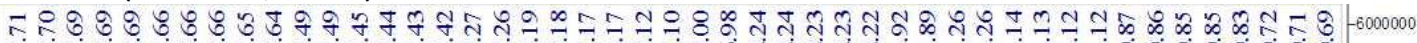

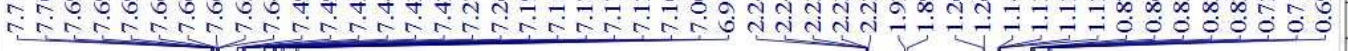

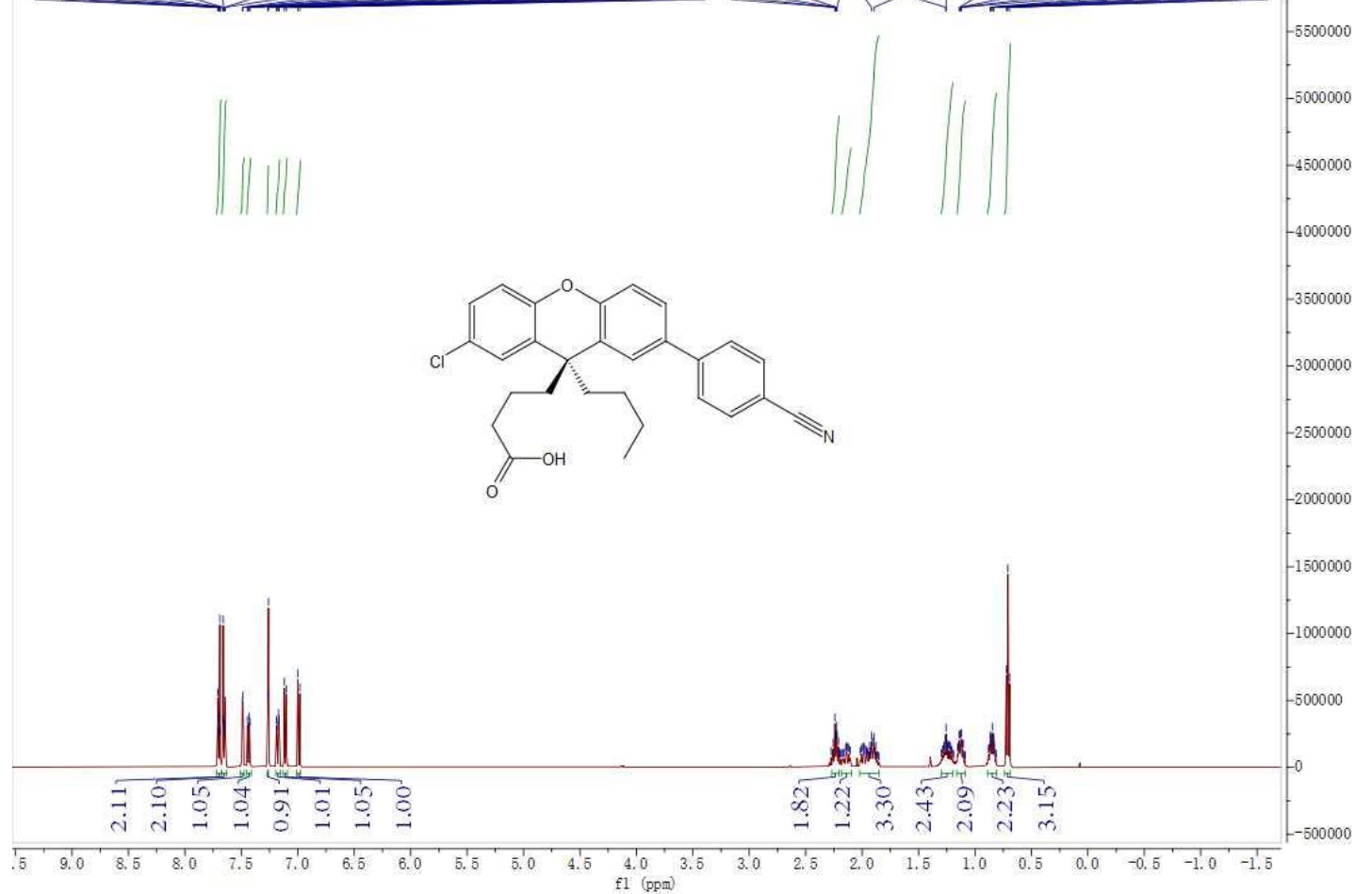

$\left.{ }^{13} \mathrm{C} \mathrm{NMR} \mathrm{(126} \mathrm{MHz,} \mathrm{CDCl}_{3}\right)-39$

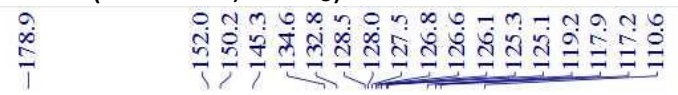

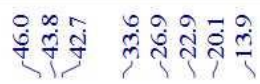

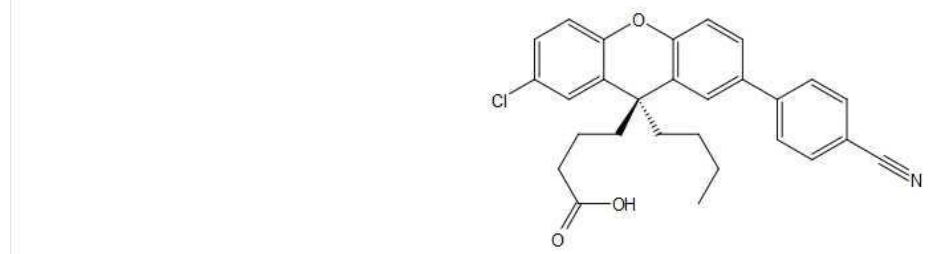

$-1100000$

$-1000000$

900000

00000

00000

600000

$-500000$

400000

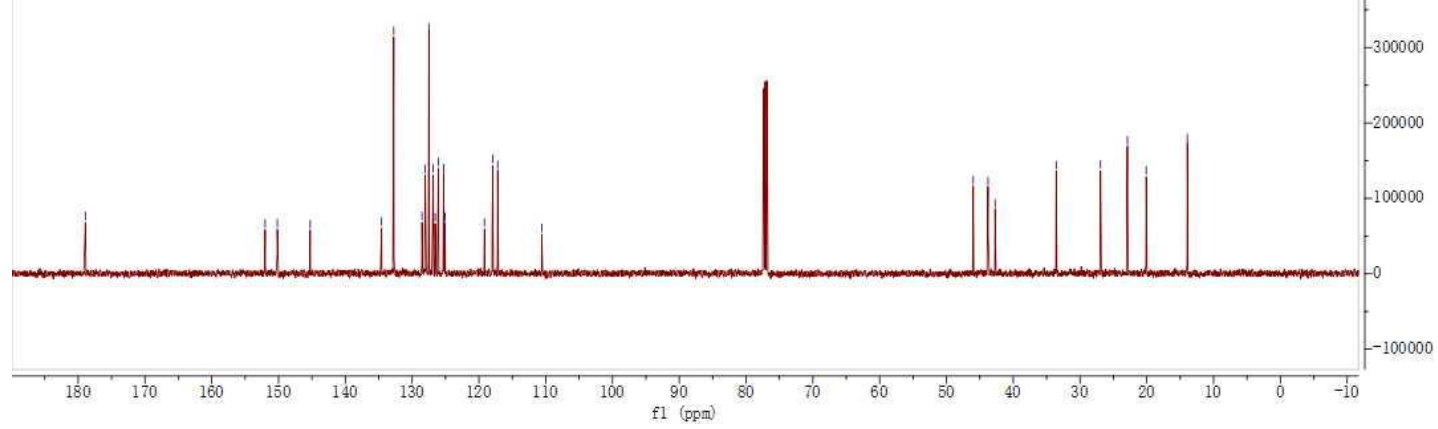


${ }^{1} \mathrm{H}$ NMR $\left(500 \mathrm{MHz}, \mathrm{CDCl}_{3}\right)-40$

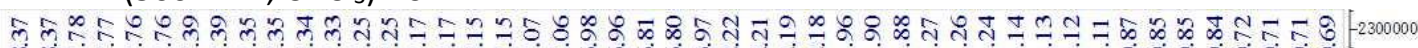
on

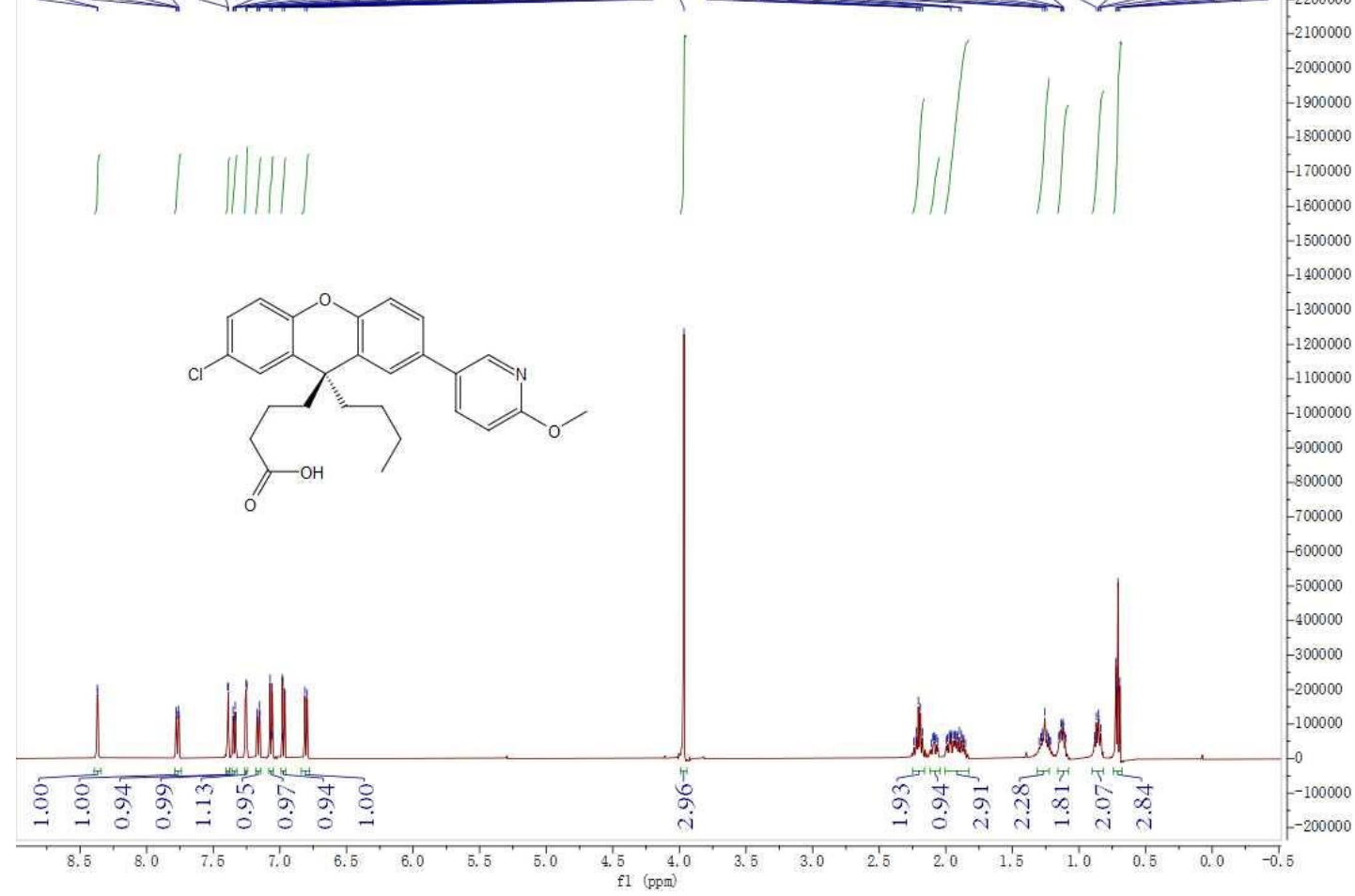

${ }^{13} \mathrm{C} \mathrm{NMR}\left(101 \mathrm{MHz}, \mathrm{CDCl}_{3}\right)-40$

落
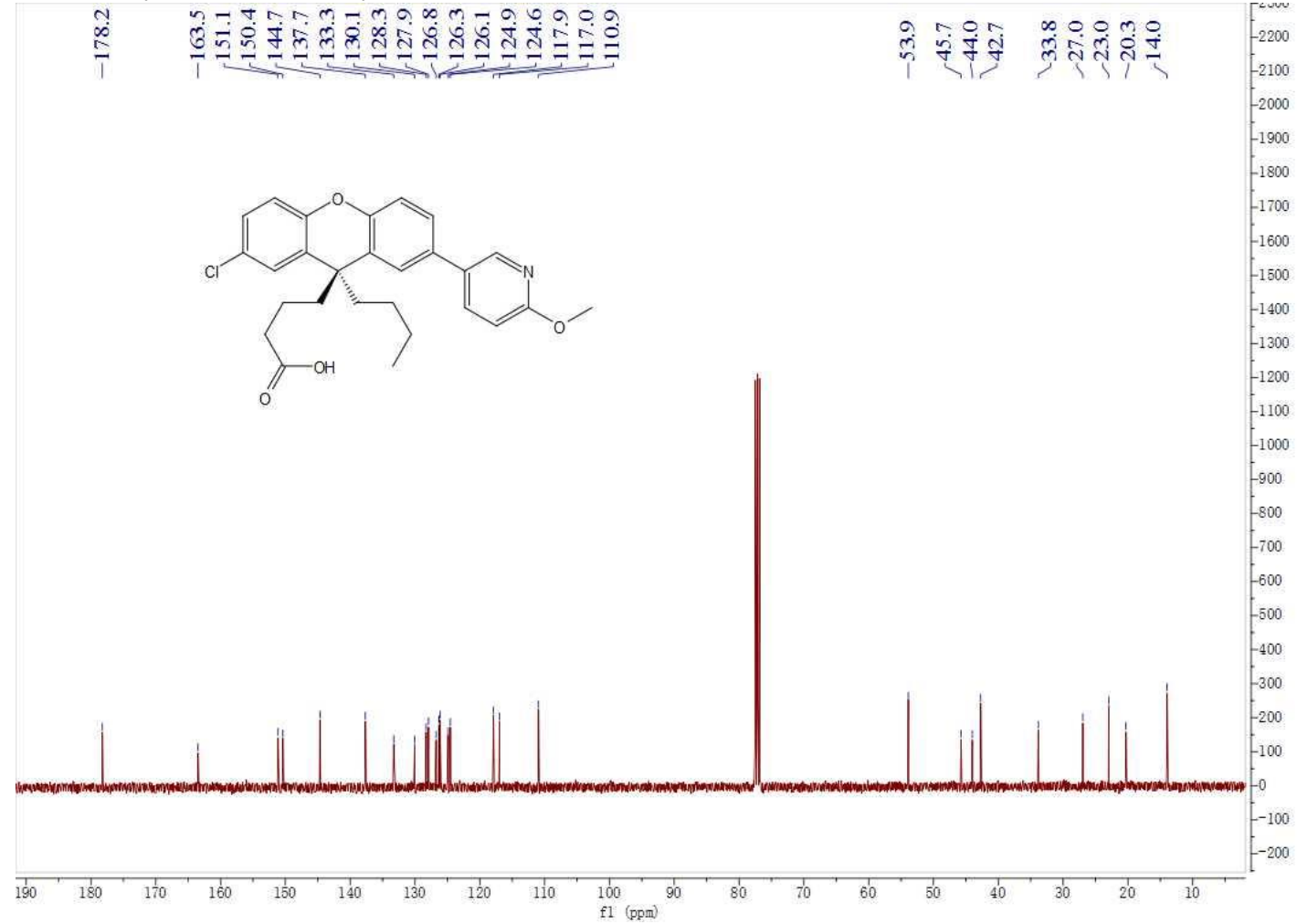
$\left.{ }^{1} \mathrm{H} \mathrm{NMR} \mathrm{(400} \mathrm{MHz,} \mathrm{CDCl}_{3}\right)-41$

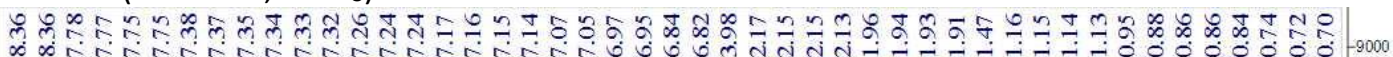

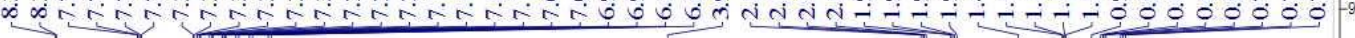

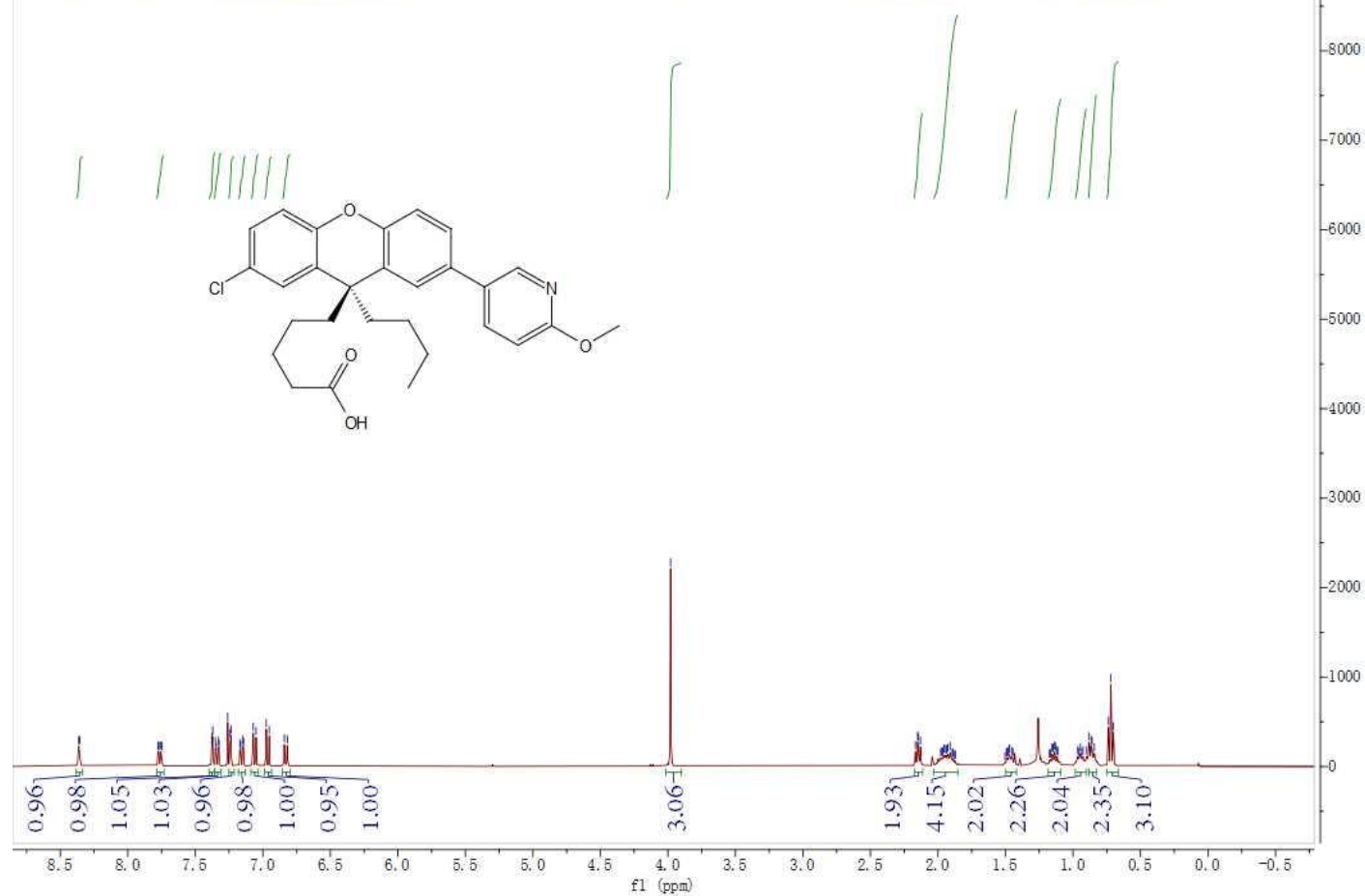

${ }^{13} \mathrm{C} \mathrm{NMR}\left(126 \mathrm{MHz}, \mathrm{CDCl}_{3}\right)-41$

$\begin{array}{lll}1 & 1 \\ 0 & 8 & 0 \\ 0\end{array}$
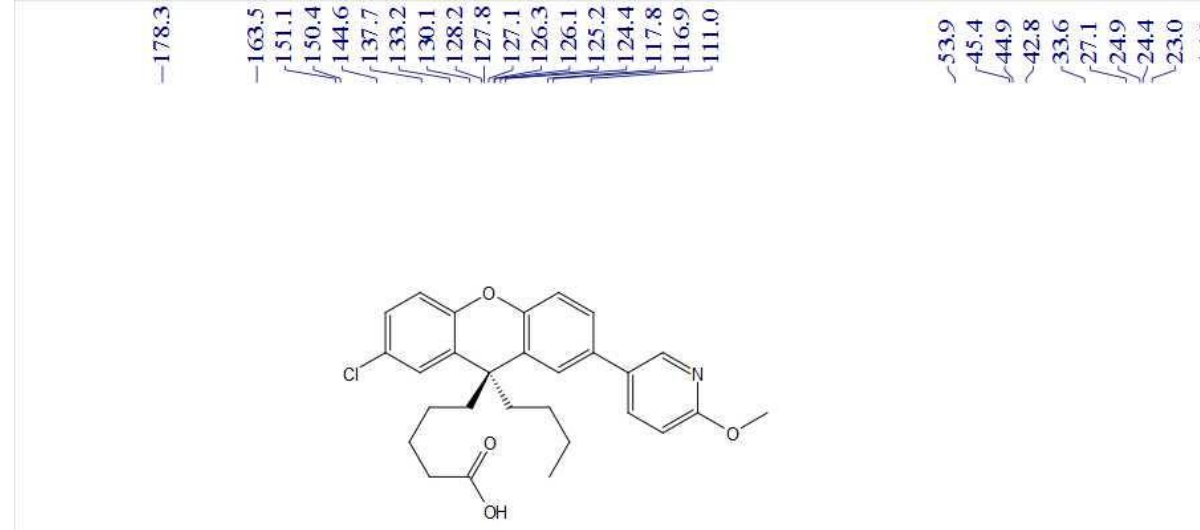

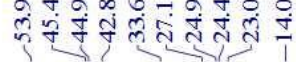

450000

0000

300000

$-250000$

200000

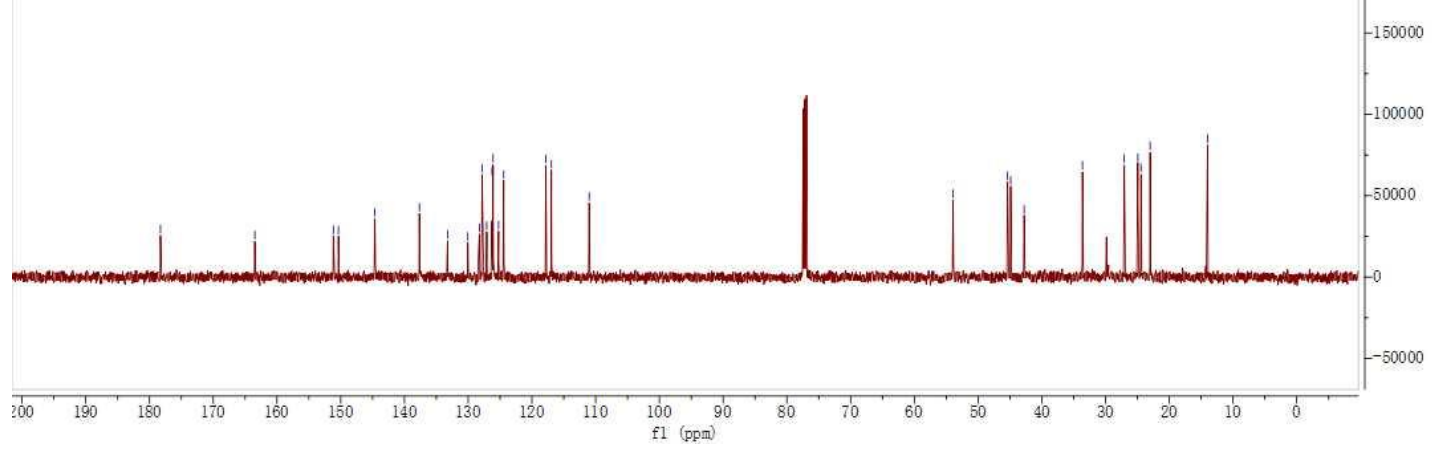




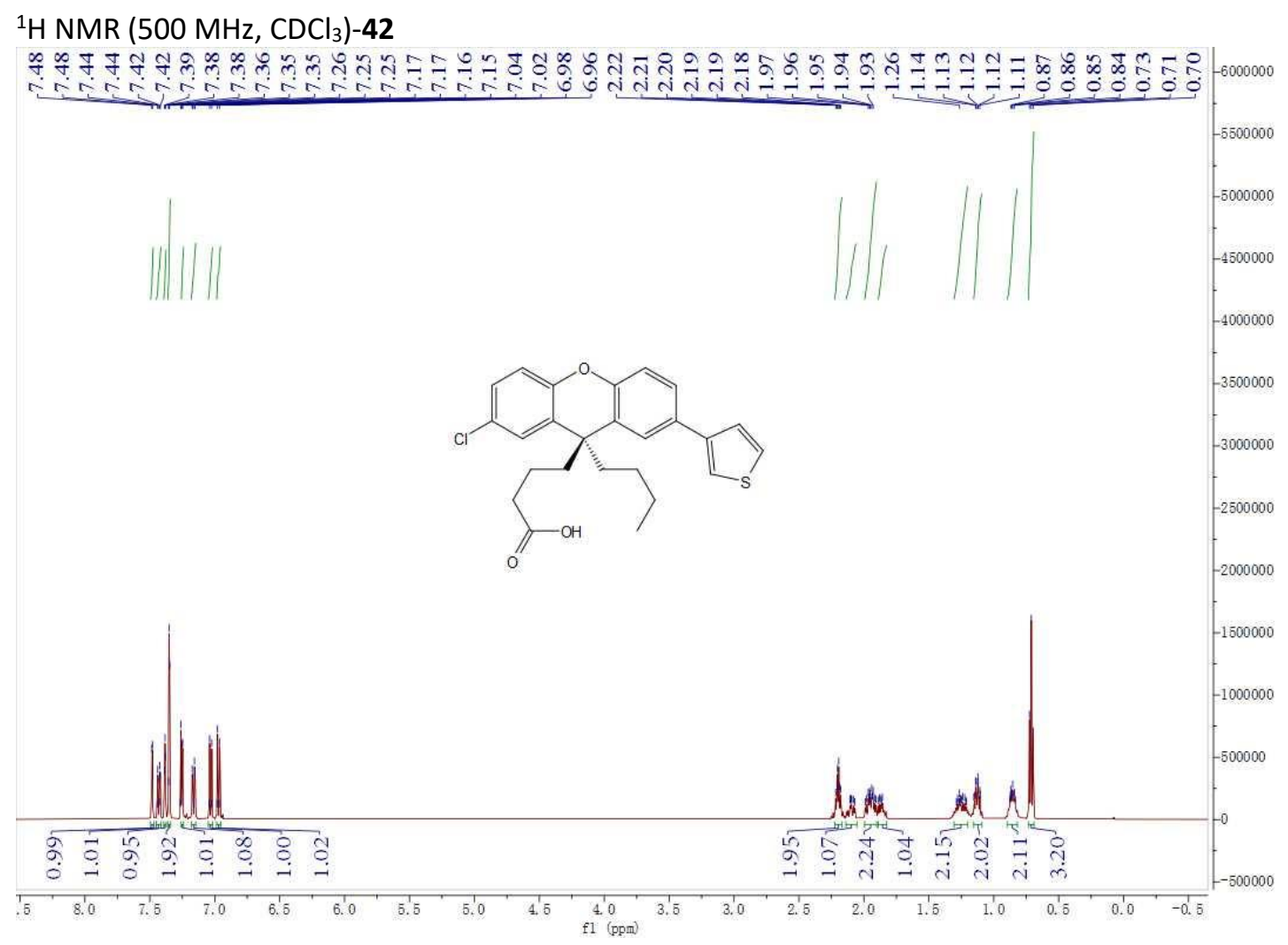

${ }^{13} \mathrm{C} \mathrm{NMR}\left(126 \mathrm{MHz}, \mathrm{CDCl}_{3}\right)-42$

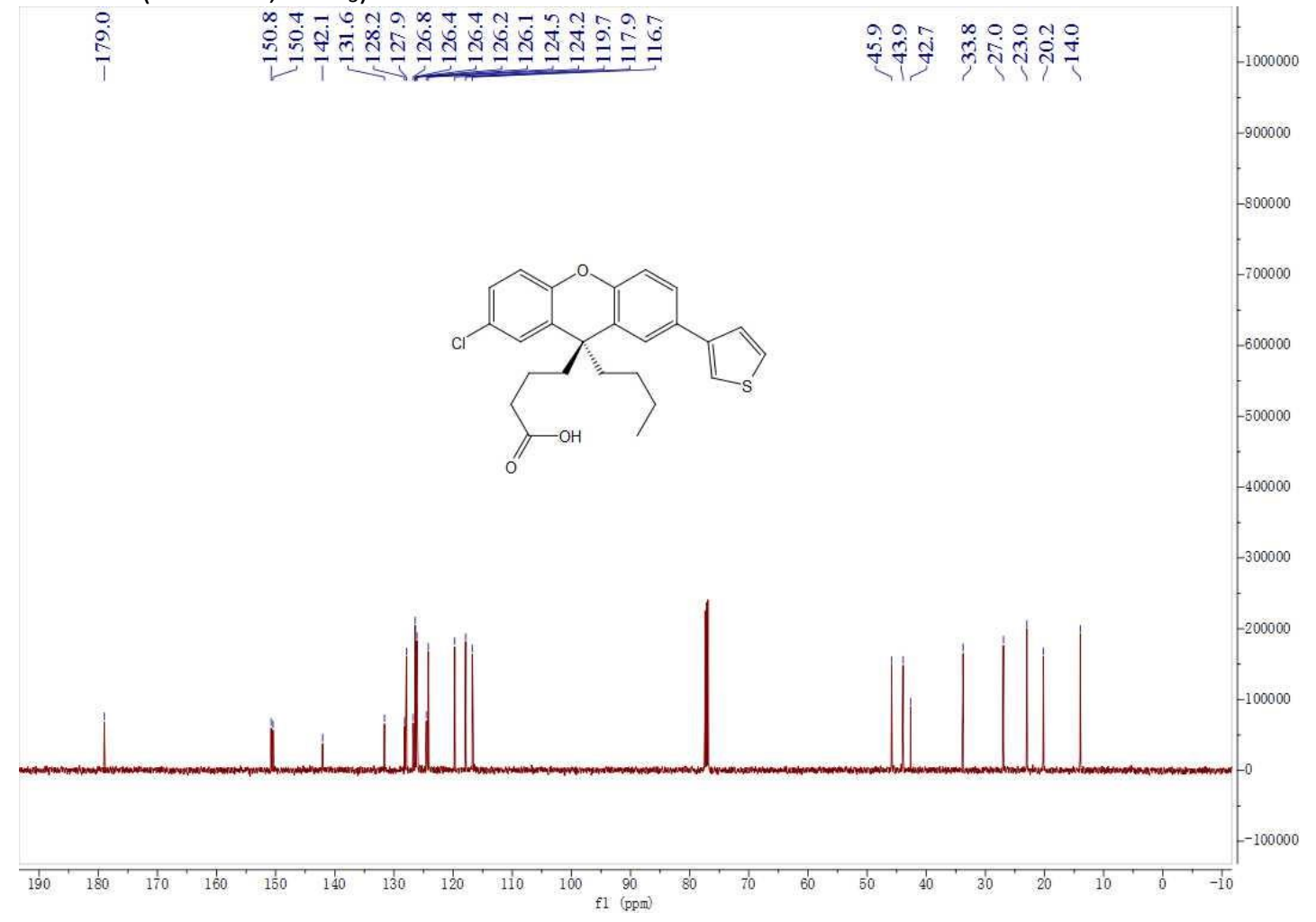


$\left.{ }^{1} \mathrm{H} \mathrm{NMR} \mathrm{(500} \mathrm{MHz,} \mathrm{CDCl}_{3}\right)-43$

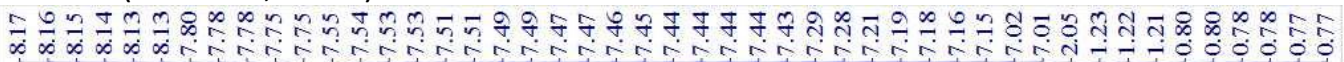

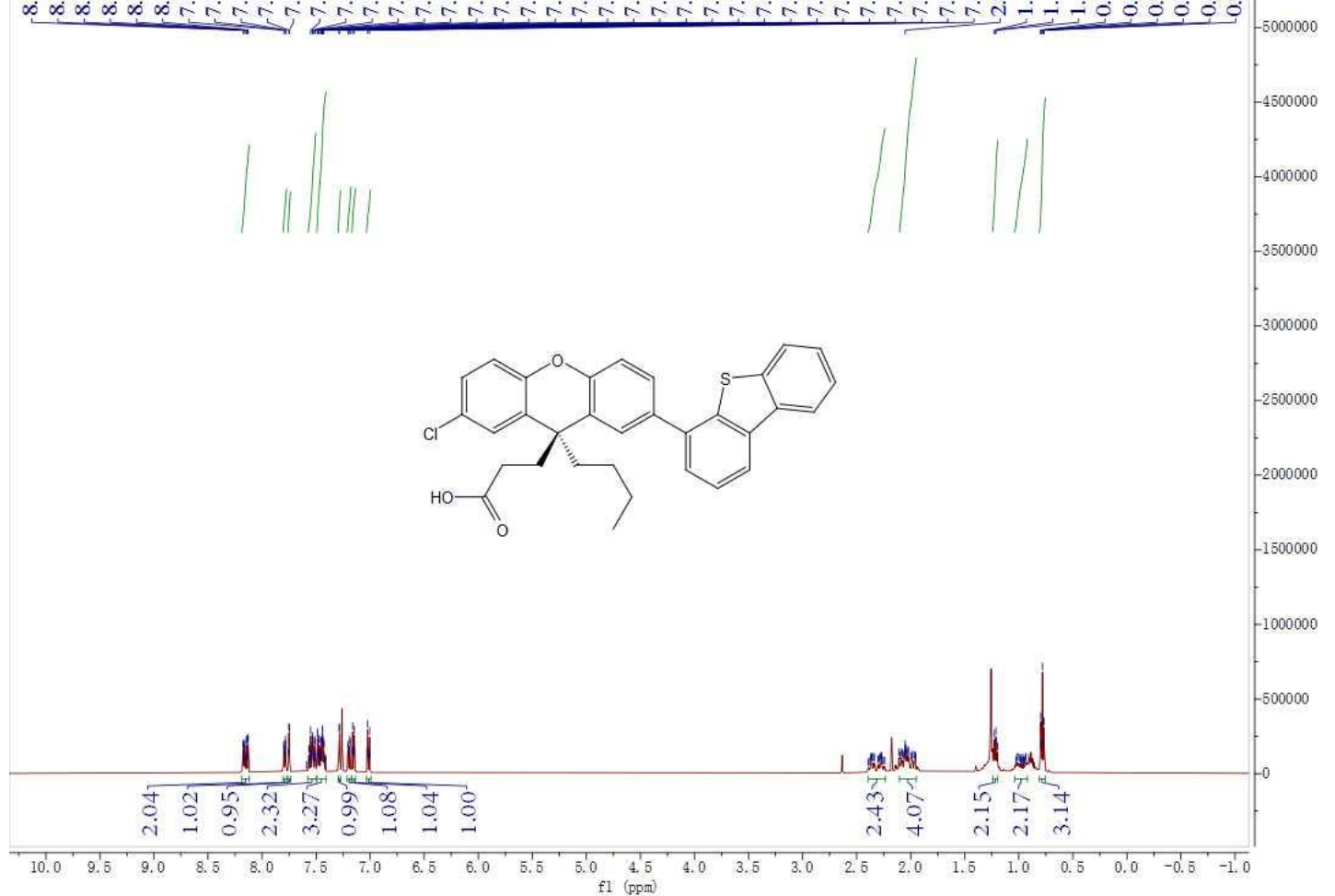

${ }^{13} \mathrm{C}$ NMR (126 MHz, $\left.\mathrm{CDCl}_{3}\right)-43$

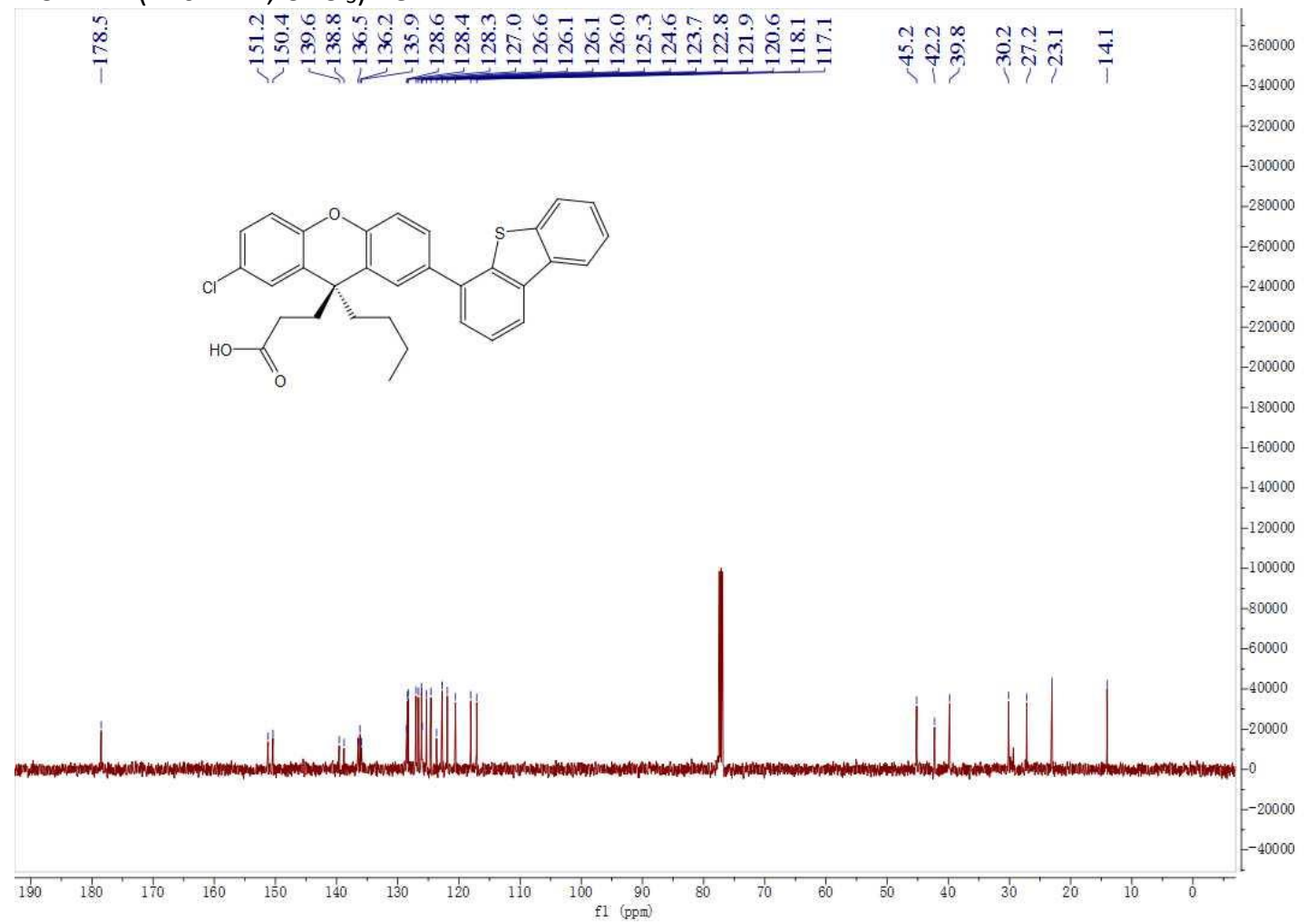


${ }^{1} \mathrm{H} \mathrm{NMR}\left(500 \mathrm{MHz}, \mathrm{CDCl}_{3}\right)-44$

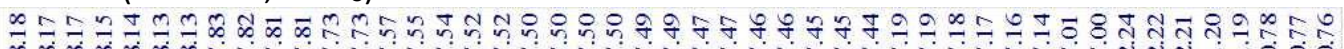

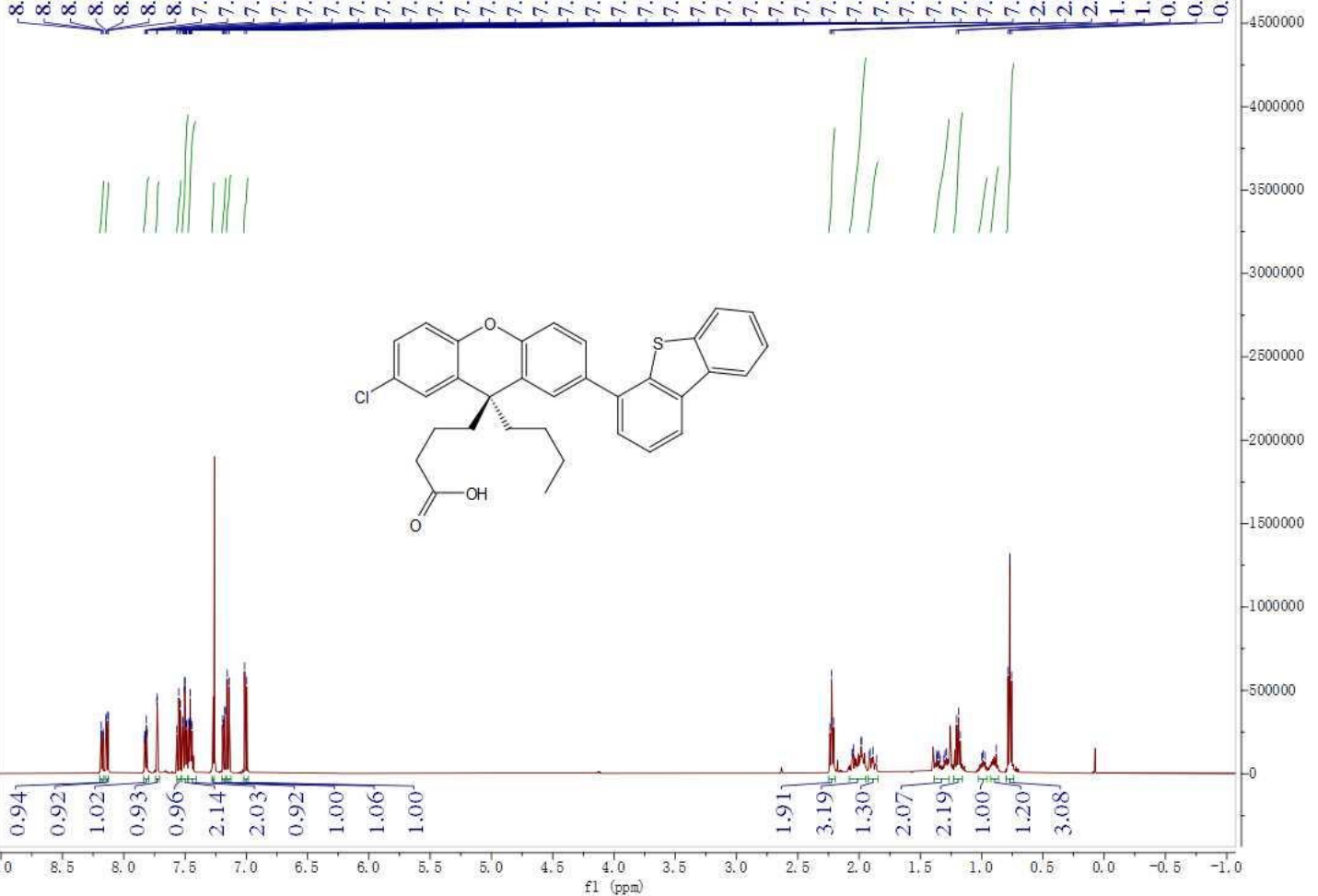

$\left.{ }^{13} \mathrm{C} \mathrm{NMR} \mathrm{(126} \mathrm{MHz,} \mathrm{CDCl}_{3}\right)-44$

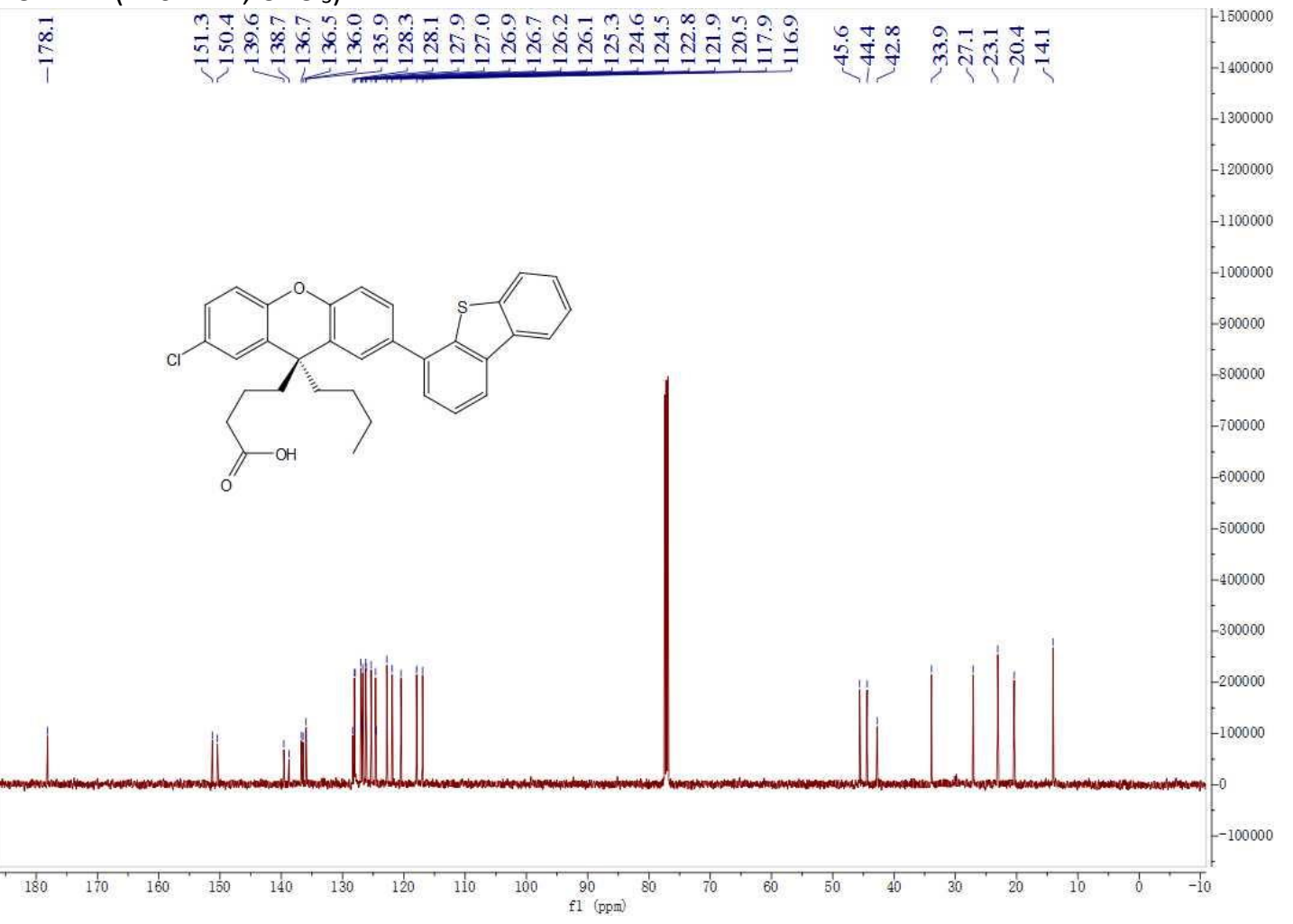


${ }^{1} \mathrm{H}$ NMR $\left(400 \mathrm{MHz}, \mathrm{CDCl}_{3}\right)-45$

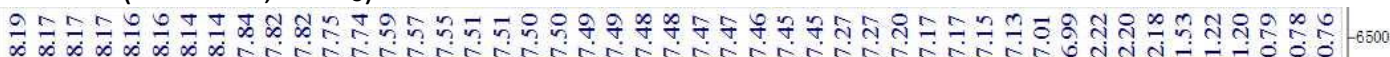

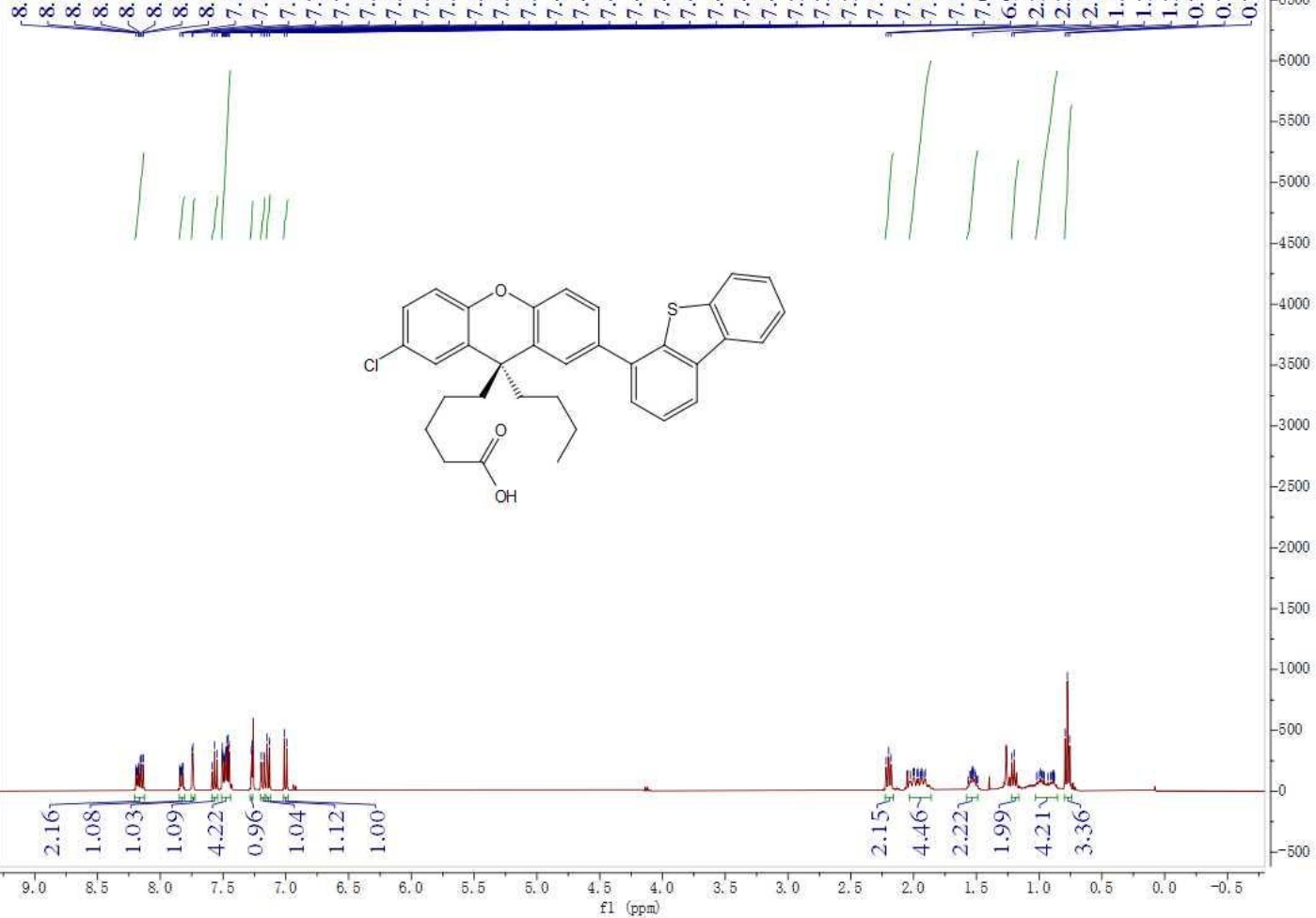

$\left.{ }^{13} \mathrm{C} \mathrm{NMR} \mathrm{(126} \mathrm{MHz,} \mathrm{CDCl}_{3}\right)-45$

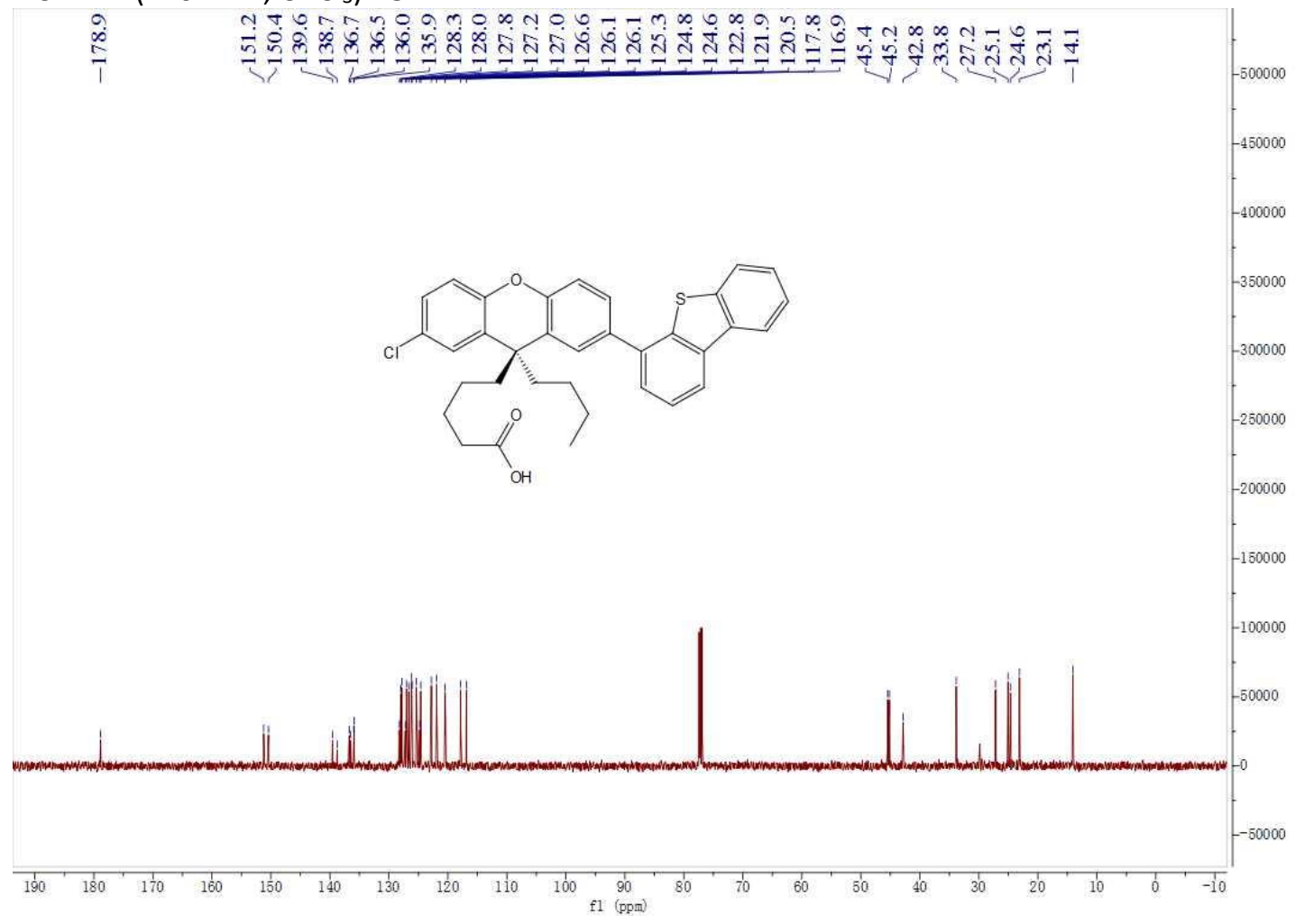




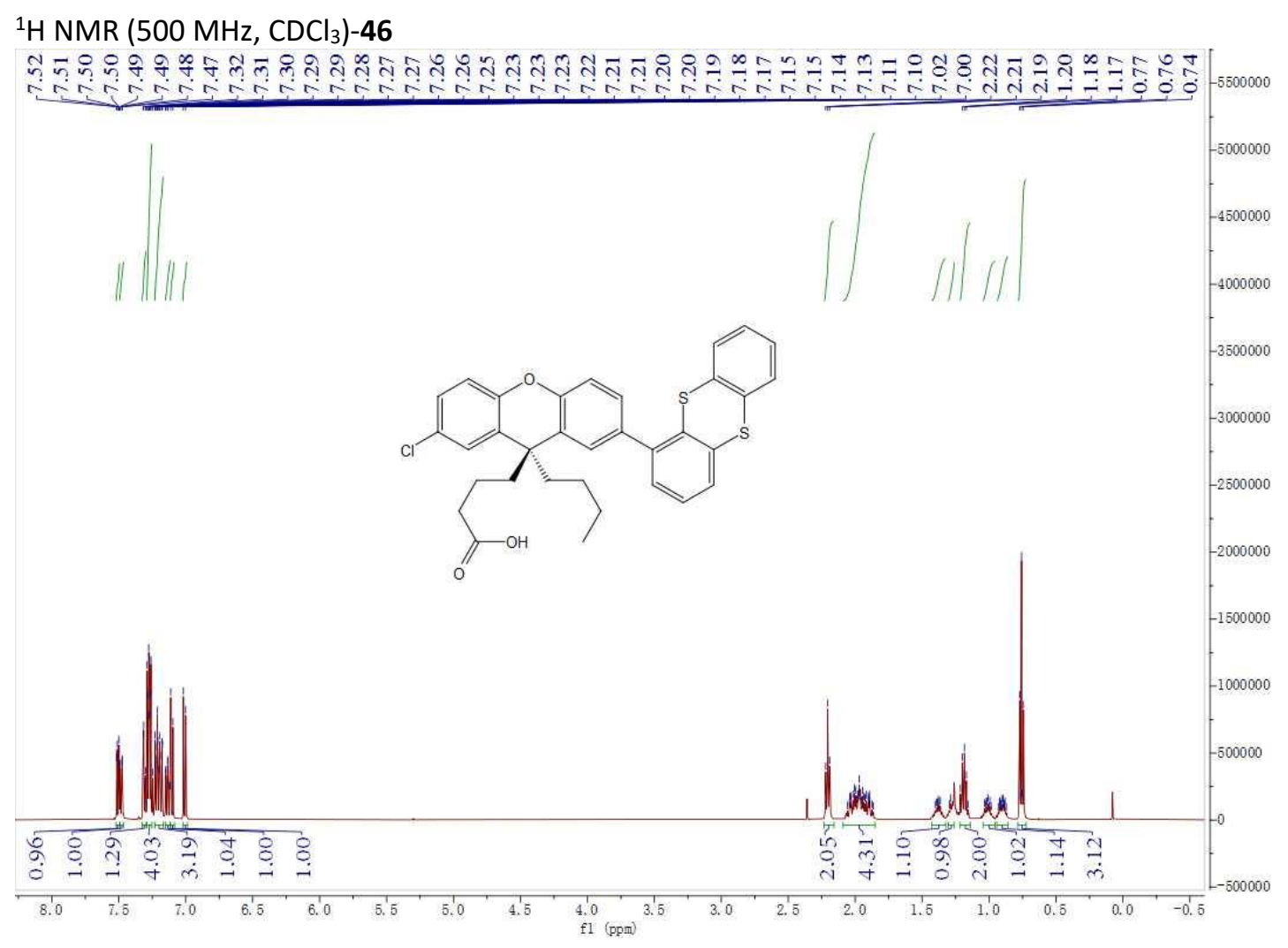

${ }^{13} \mathrm{C}$ NMR (126 MHz, $\left.\mathrm{CDCl}_{3}\right)-46$

0
0
0
0

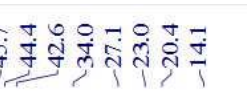

$-2000000$

1900000

1800000

$-1700000$

$-1600000$

1500000<smiles>CCCC[C@@]1(CCCC(=O)O)c2cc(Cl)ccc2Oc2ccc(-c3cccc4c3Sc3ccccc3S4)cc21</smiles>

(2)
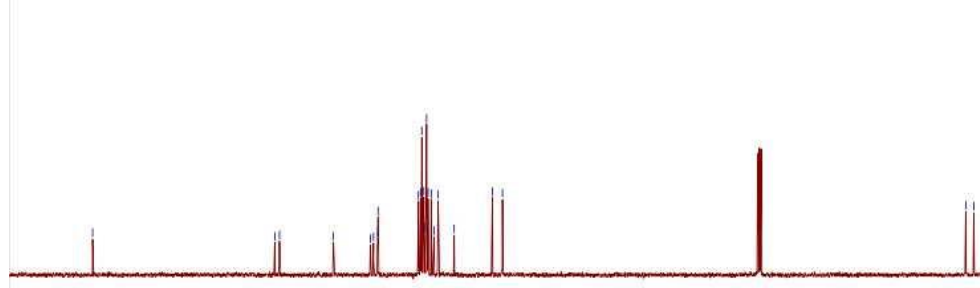

$-1400000$

$-1300000$

$-1200000$

$-1100000$

$-1000000$

$-900000$

800000

700000

$-600000$

$-500000$

$-400000$

300000

200000 
${ }^{1} \mathrm{H}$ NMR $\left(500 \mathrm{MHz}, \mathrm{CDCl}_{3}\right)-47$

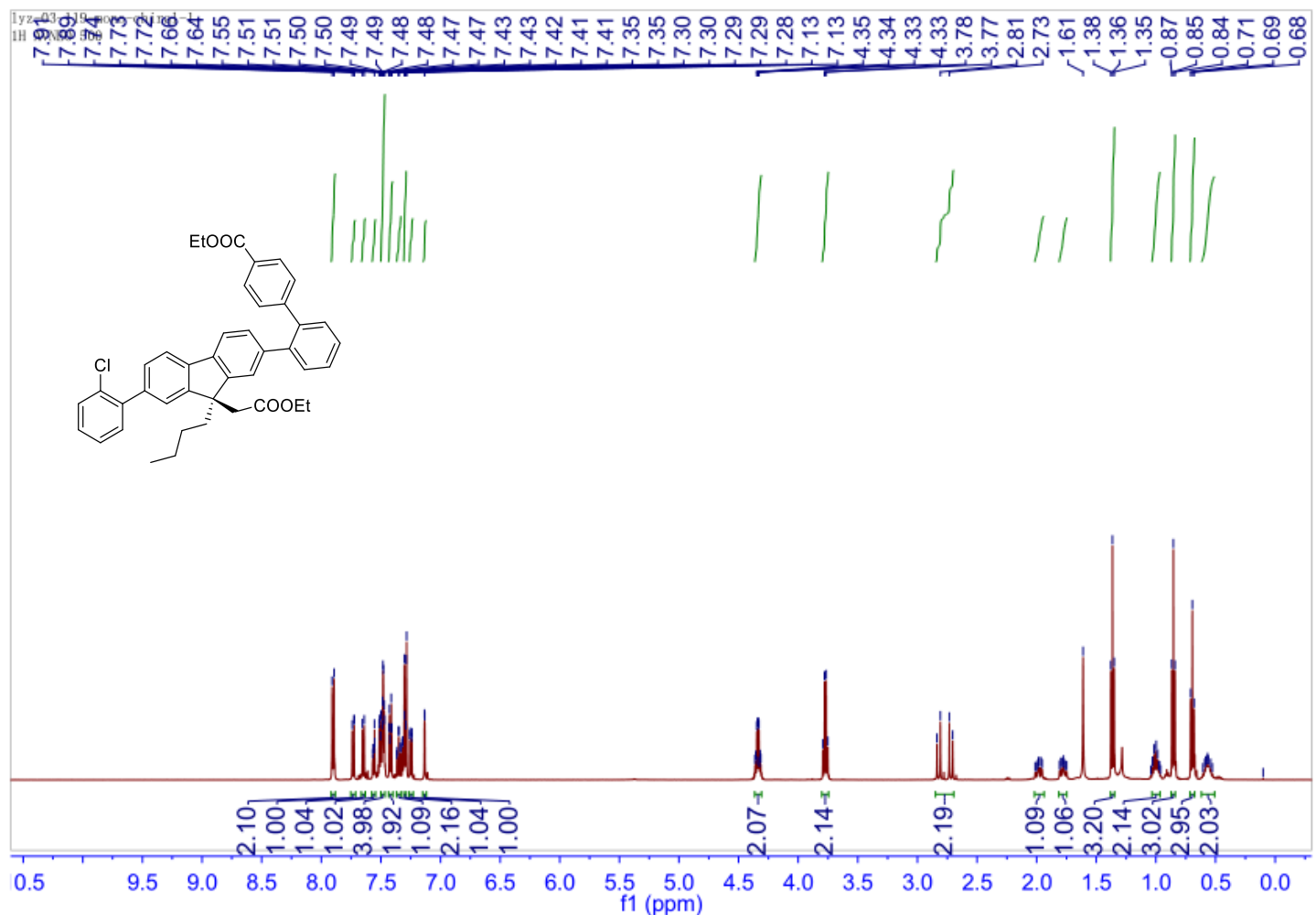

${ }^{13} \mathrm{C}$ NMR $\left(126 \mathrm{MHz}, \mathrm{CDCl}_{3}\right)-47$

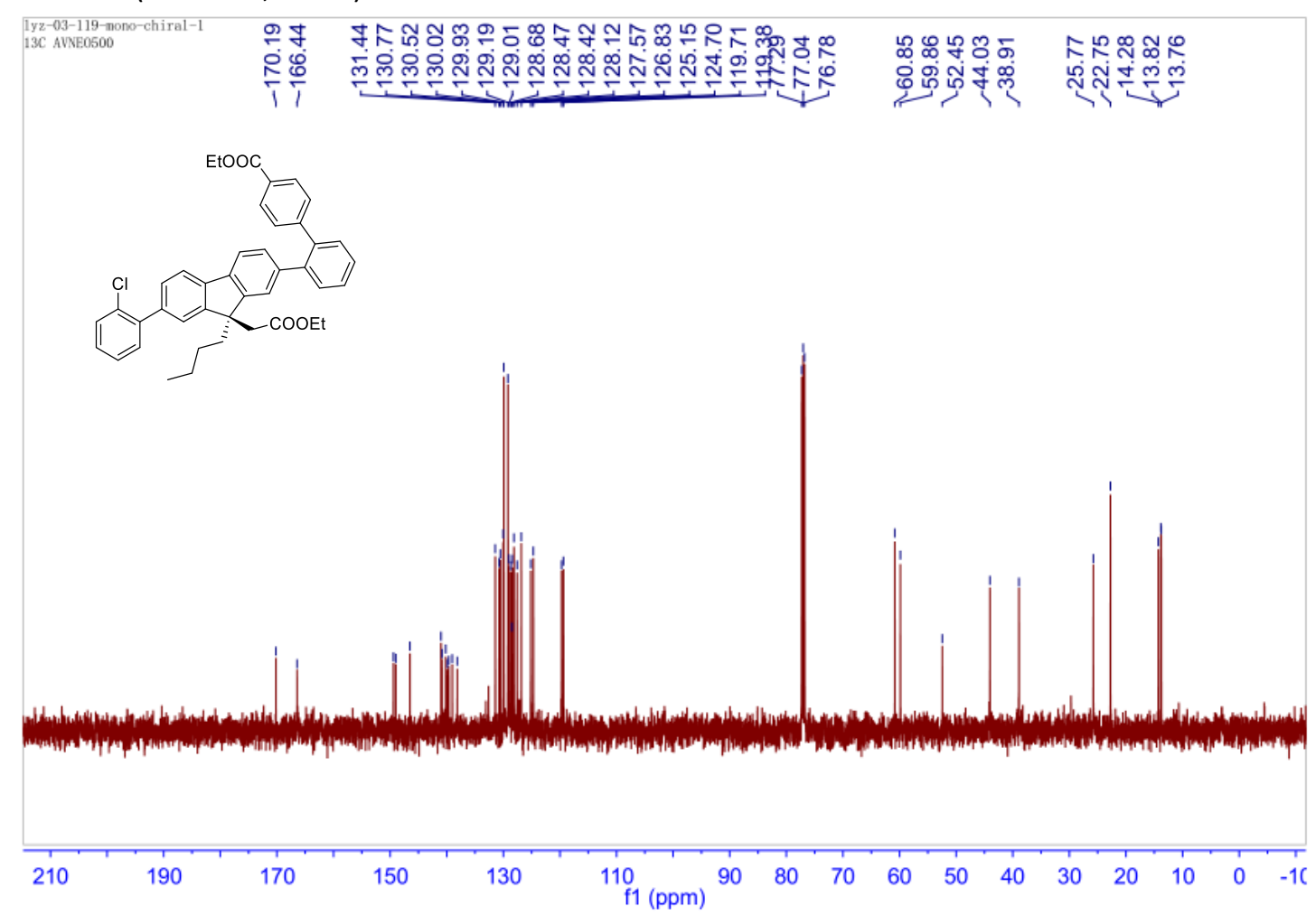


${ }^{1} \mathrm{H}$ NMR $\left(500 \mathrm{MHz}, \mathrm{CDCl}_{3}\right)-48$

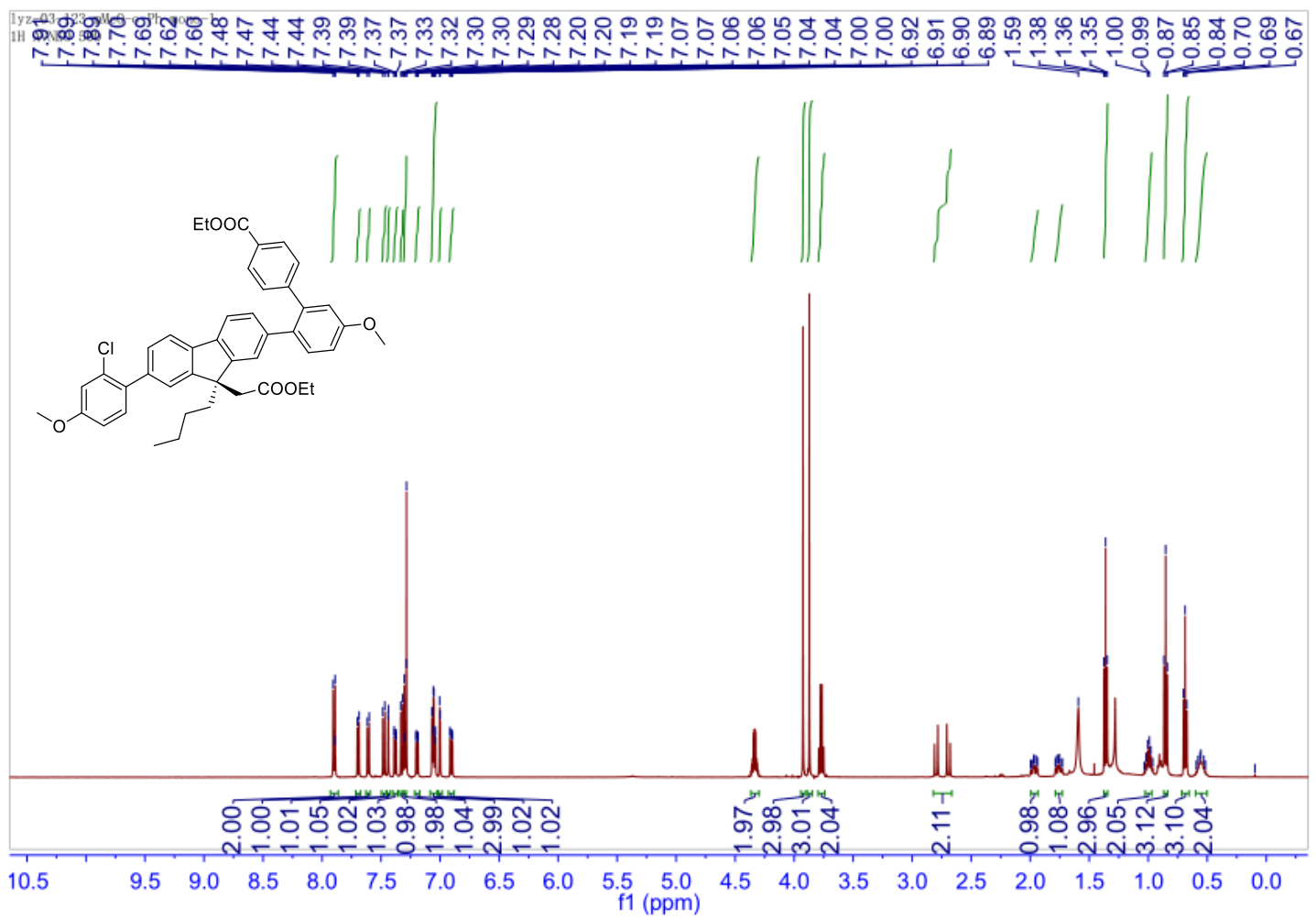

${ }^{13} \mathrm{C} \mathrm{NMR}\left(126 \mathrm{MHz}, \mathrm{CDCl}_{3}\right)-48$

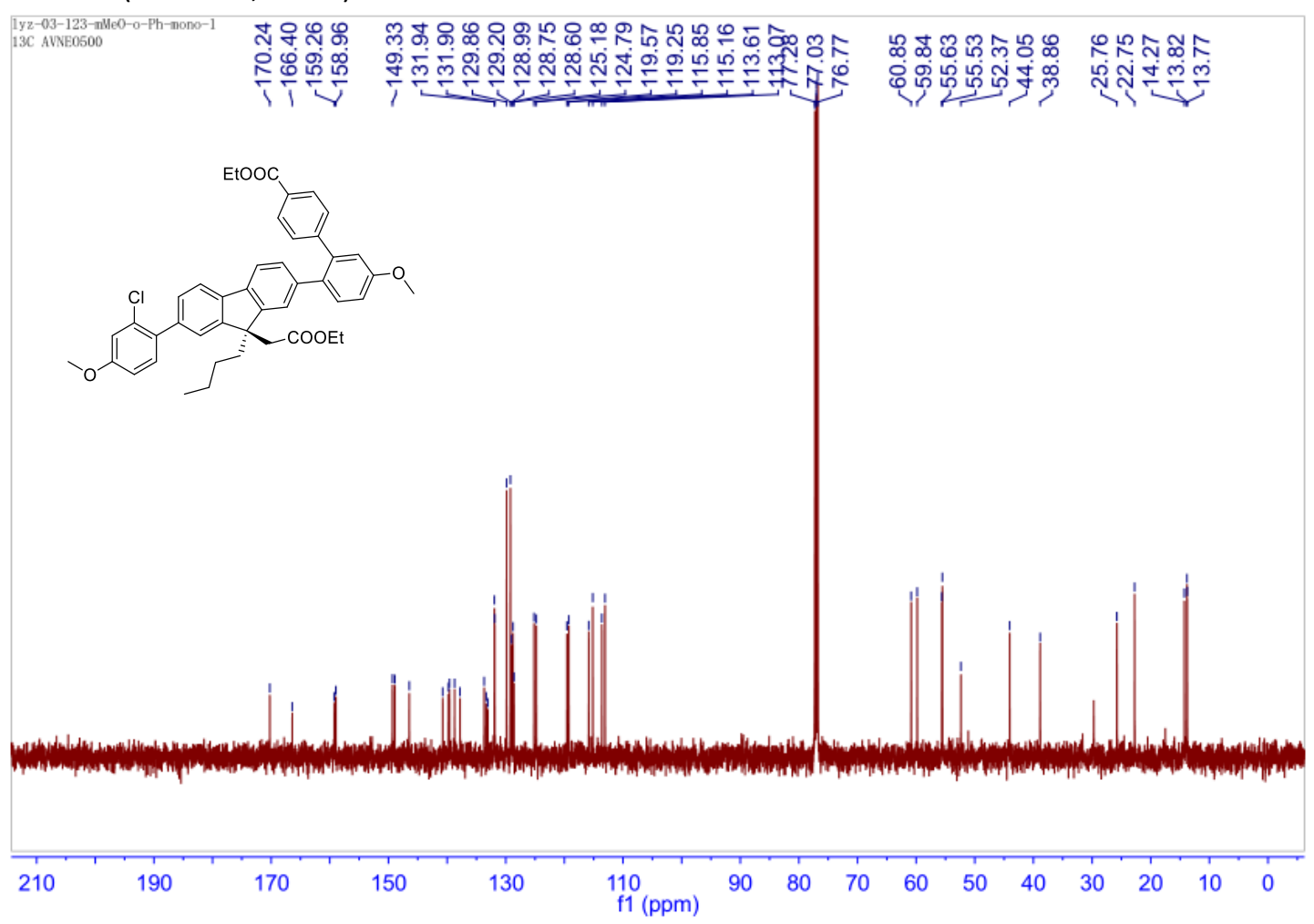


${ }^{1} \mathrm{H} \mathrm{NMR}\left(400 \mathrm{MHz}, \mathrm{CDCl}_{3}\right)-49$

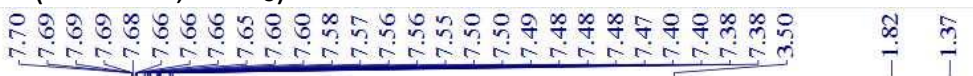

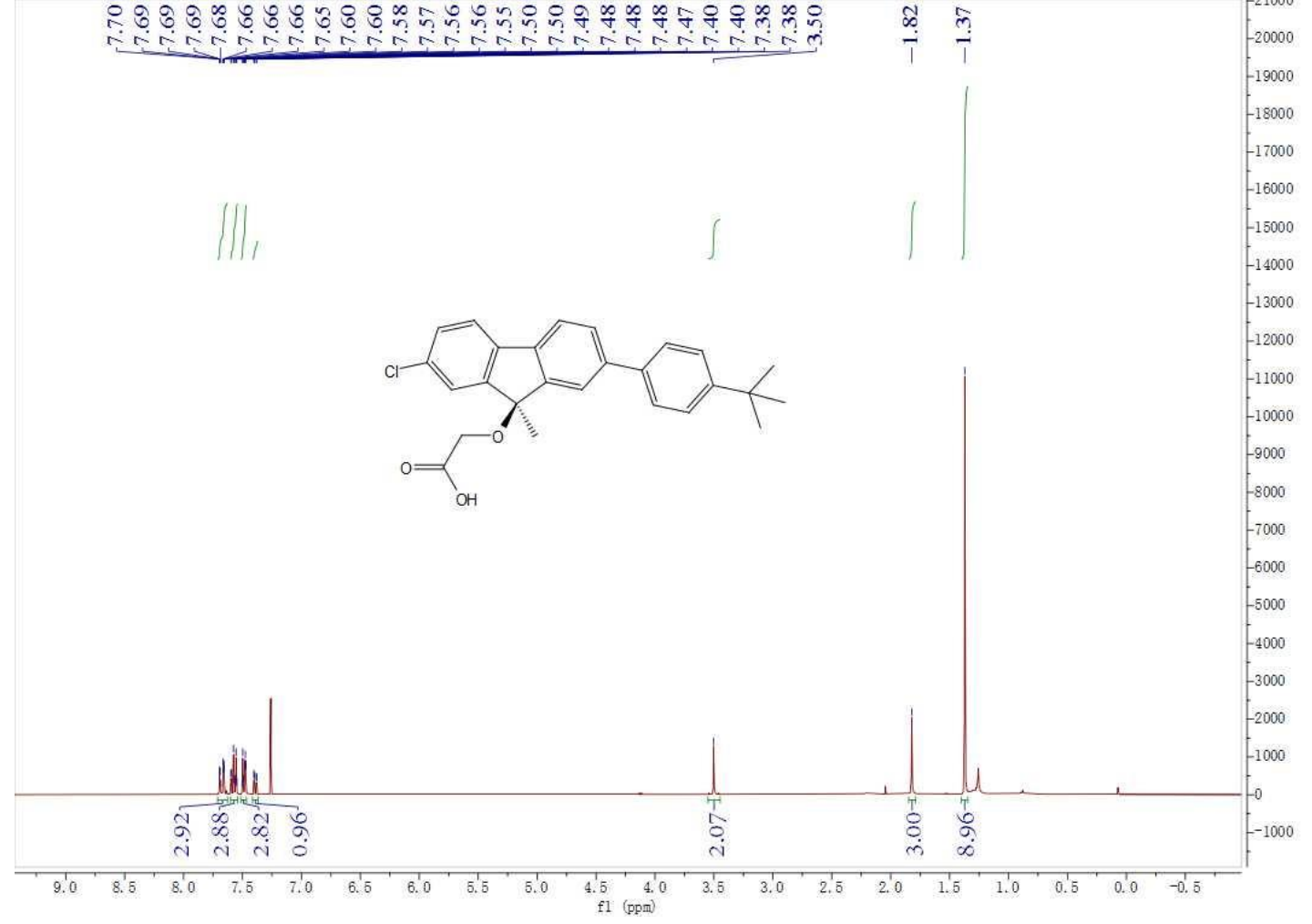

${ }^{13} \mathrm{C}$ NMR (126 MHz, $\left.\mathrm{CDCl}_{3}\right)-49$

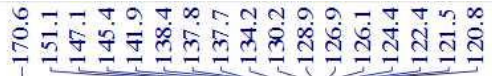

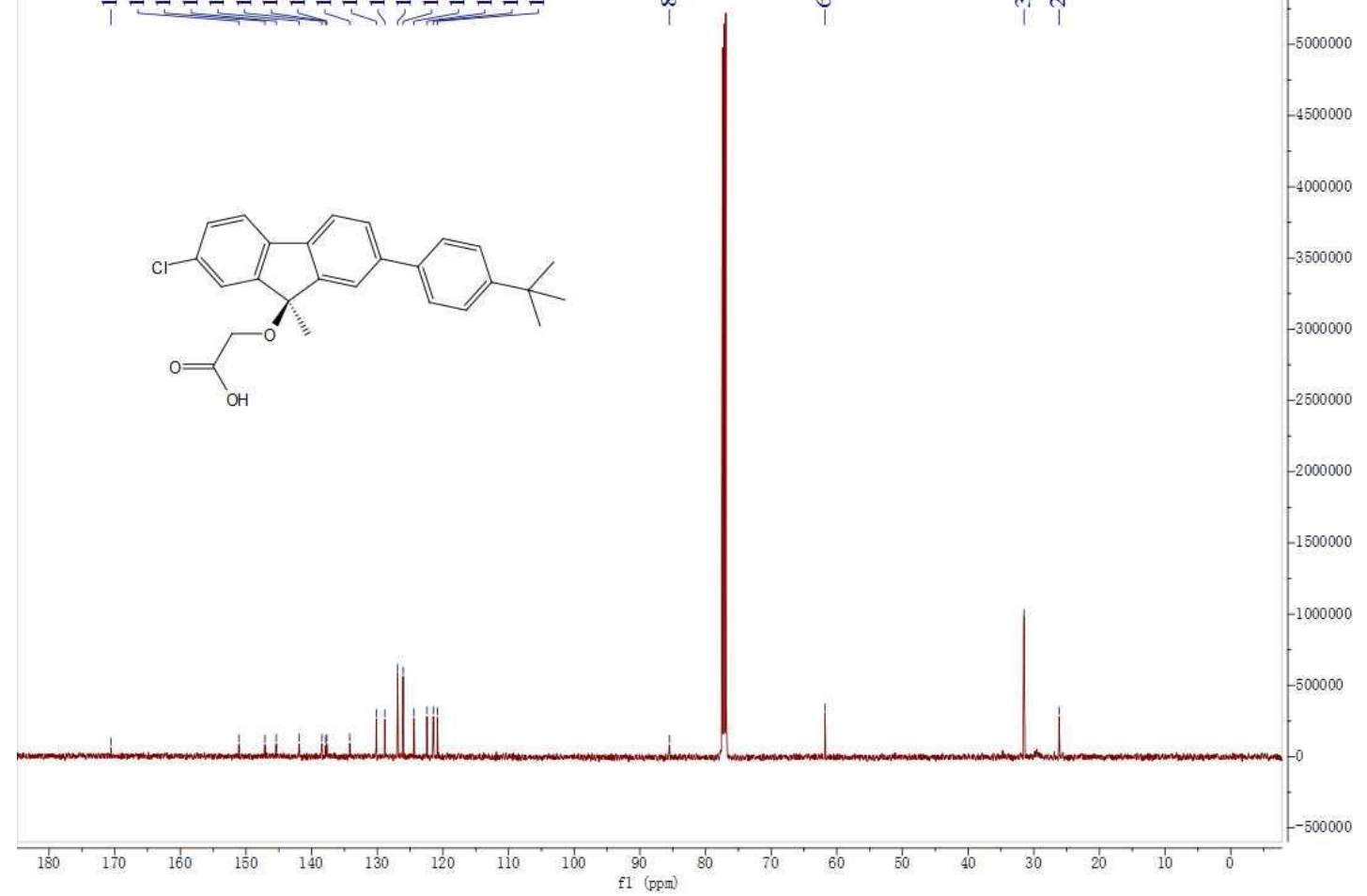




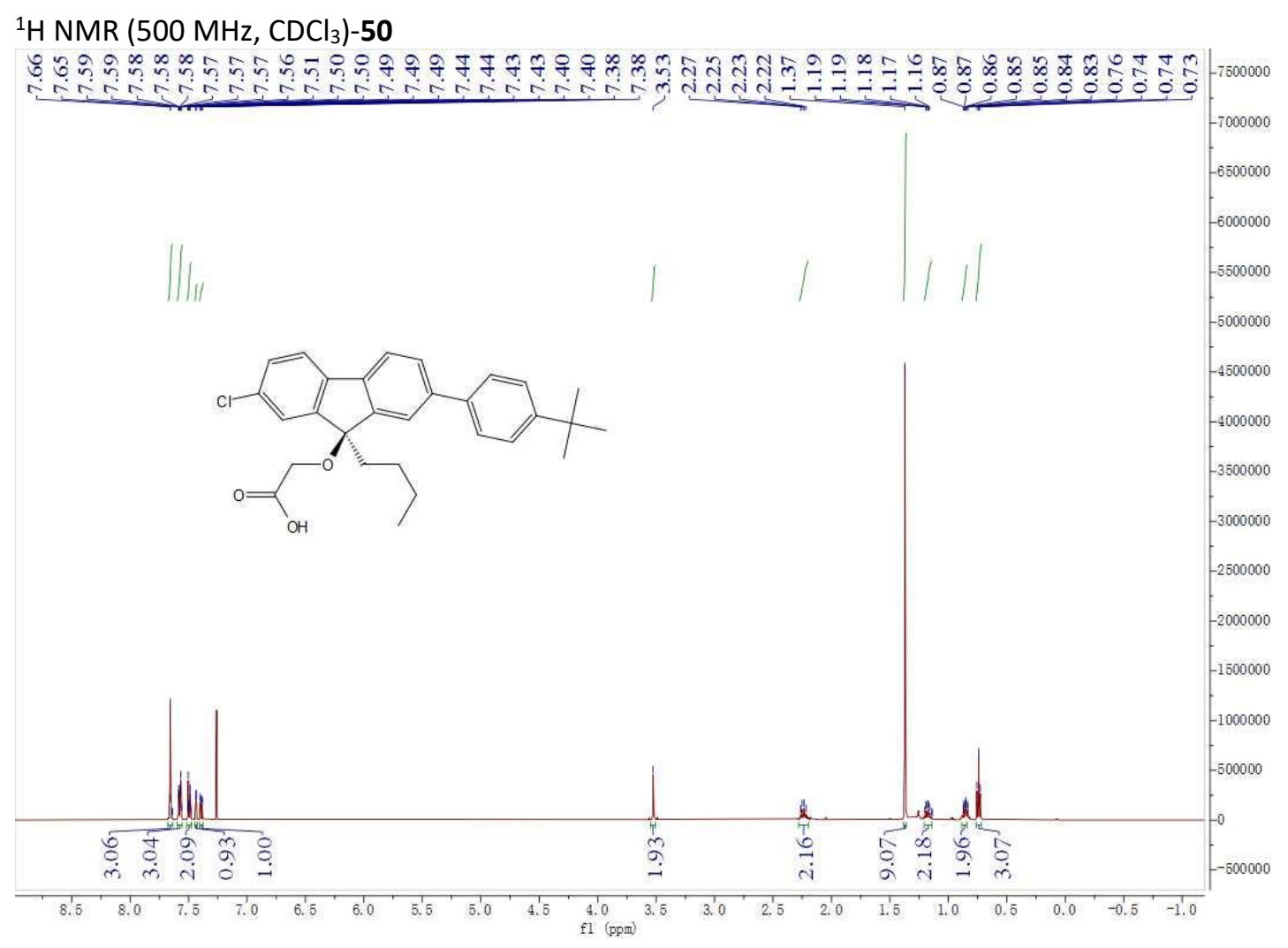

$\left.{ }^{13} \mathrm{C} \mathrm{NMR} \mathrm{(126} \mathrm{MHz,} \mathrm{CDCl}_{3}\right)-50$

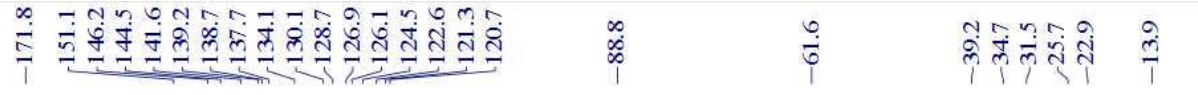
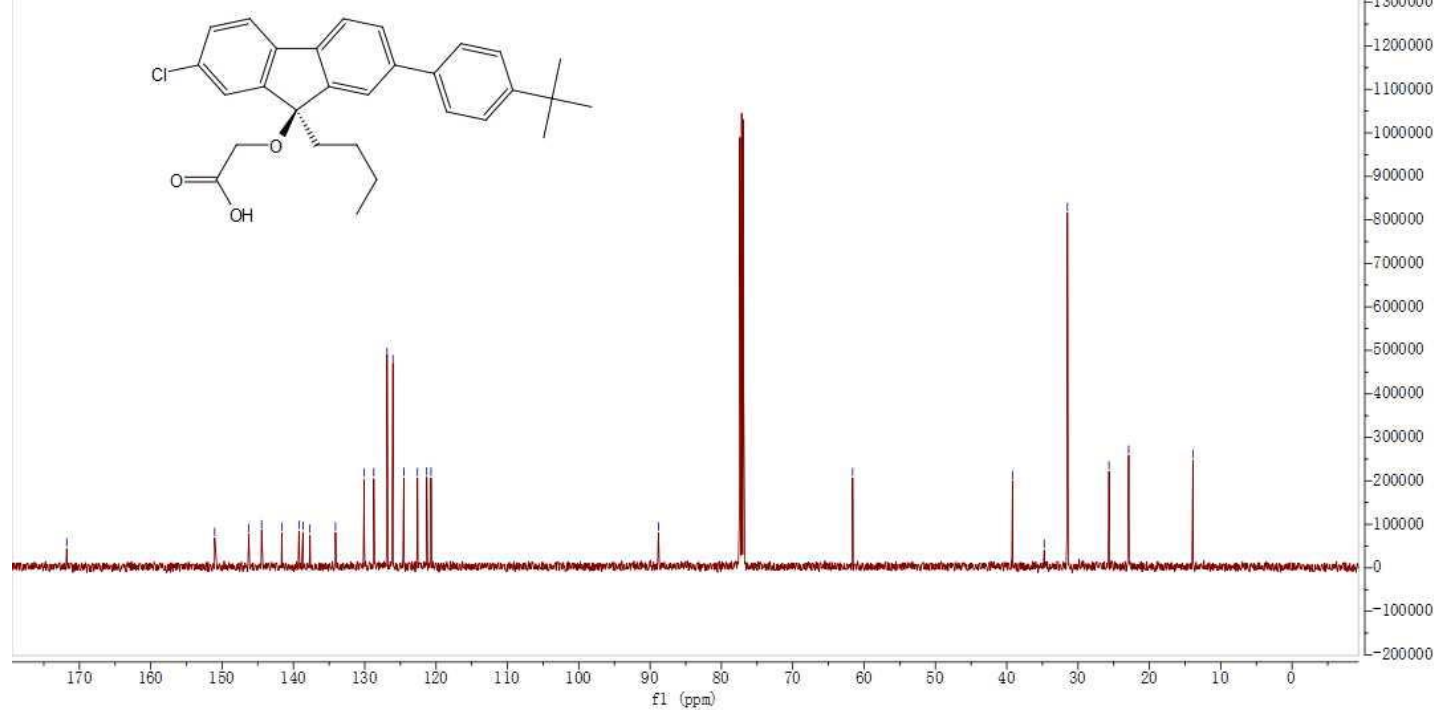
${ }^{1} \mathrm{H}$ NMR (500 MHz, $\mathrm{CDCl}_{3}$ )-53 (coupling product of $\mathbf{5 3}$ from control experiment)

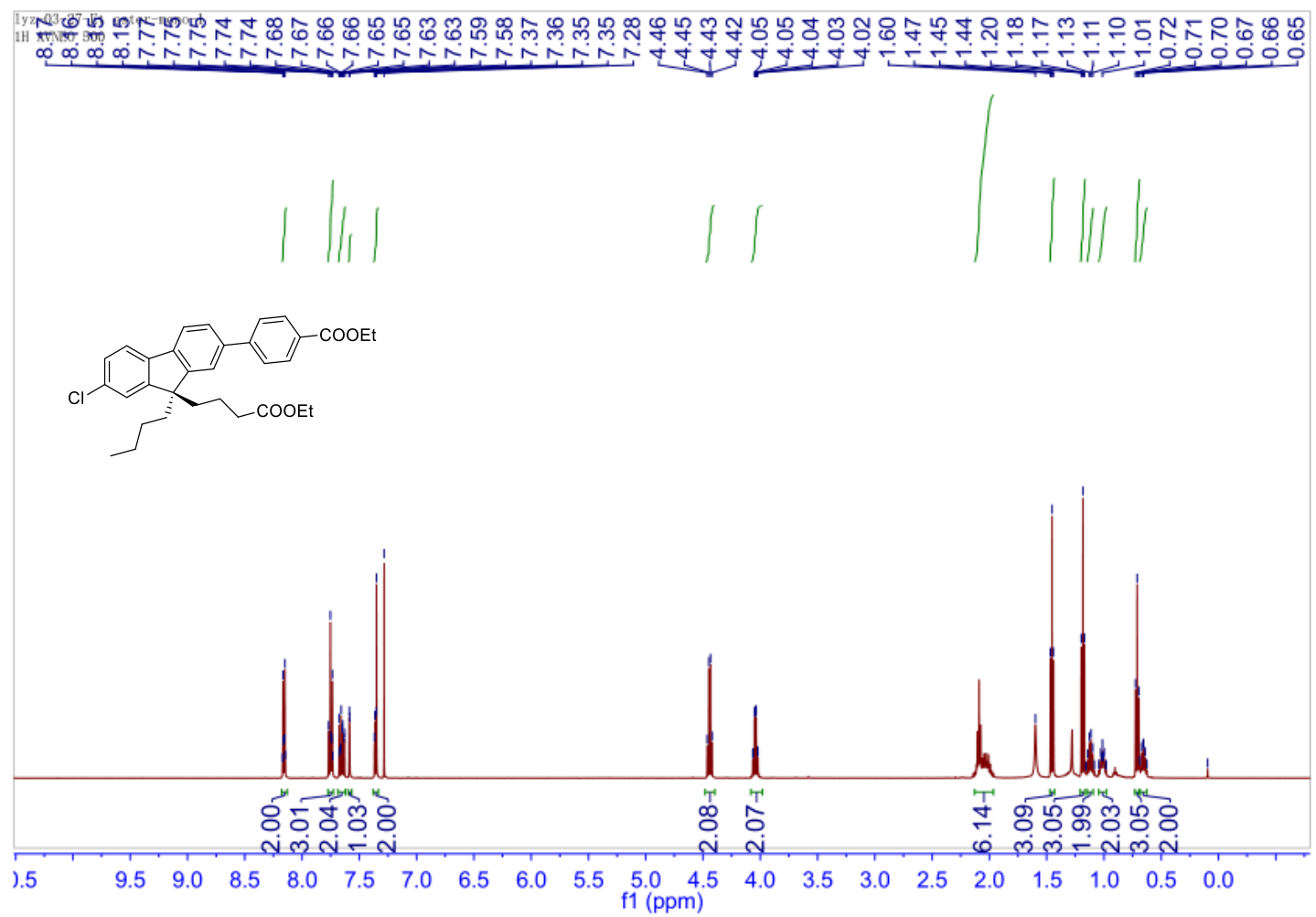

${ }^{13} \mathrm{C} \mathrm{NMR}\left(126 \mathrm{MHz}, \mathrm{CDCl}_{3}\right)-\mathbf{5 3}$ (coupling product of $\mathbf{5 3}$ from control experiment)

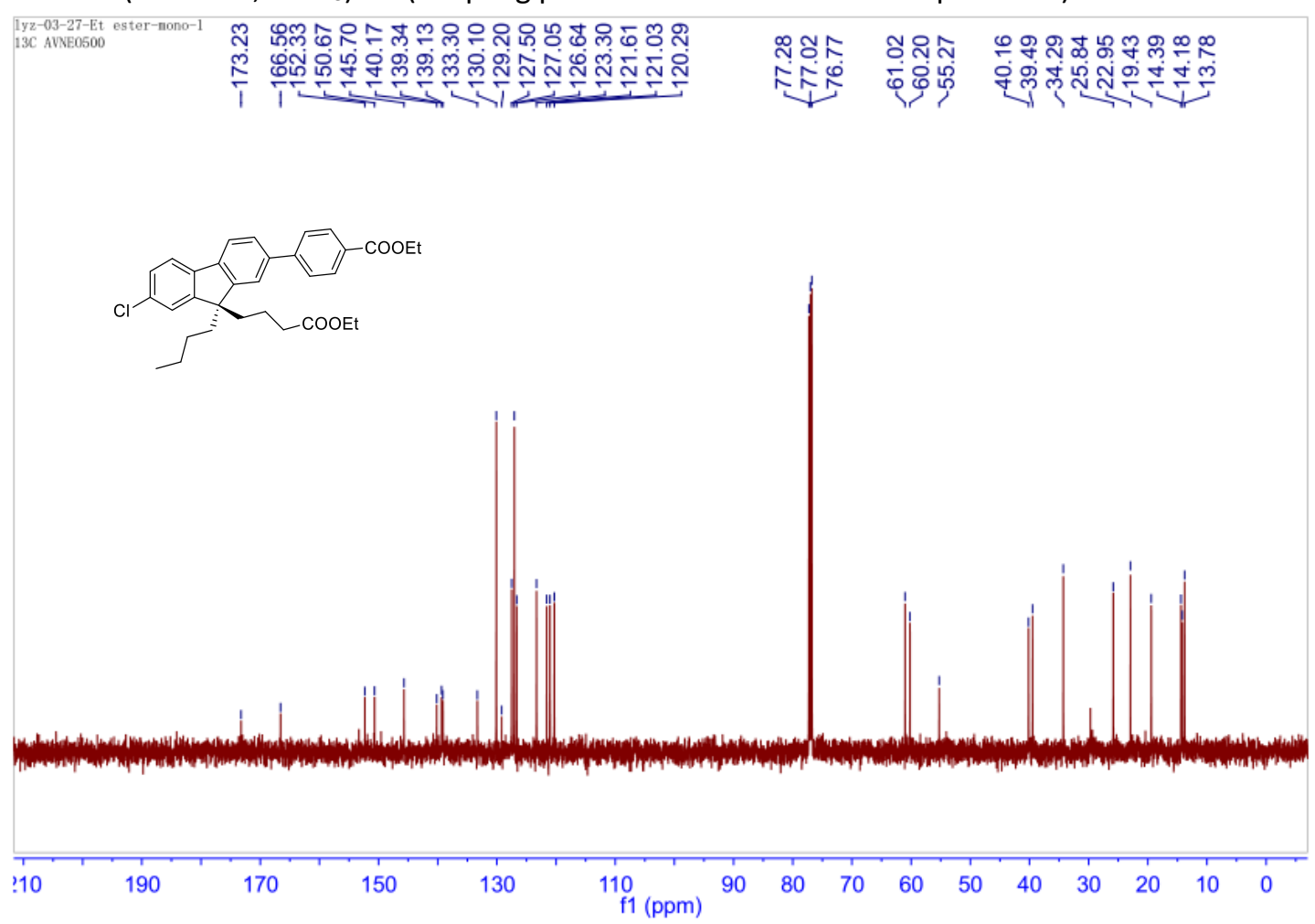



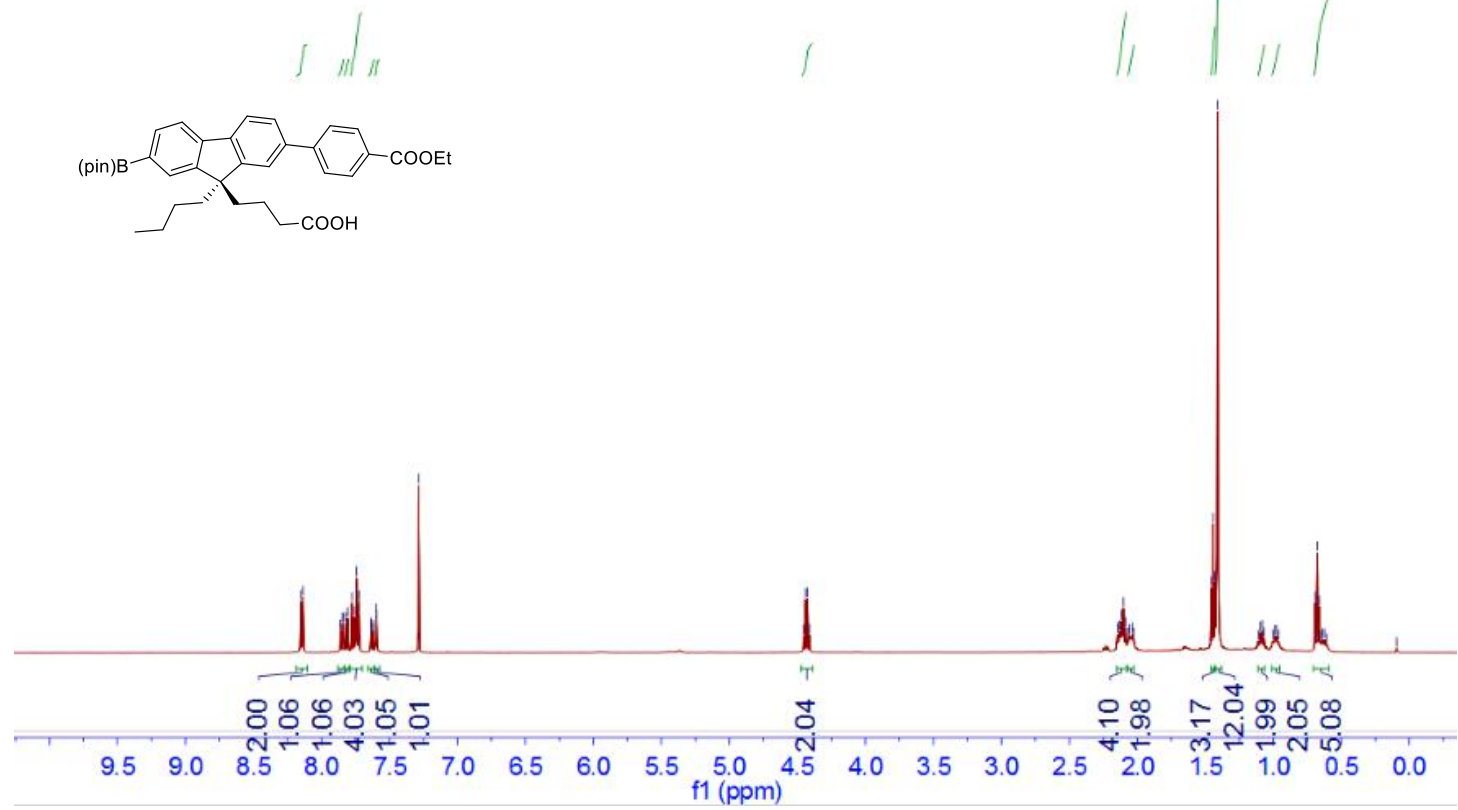

${ }^{13} \mathrm{C} \mathrm{NMR}\left(126 \mathrm{MHz}, \mathrm{CDCl}_{3}\right)-54$

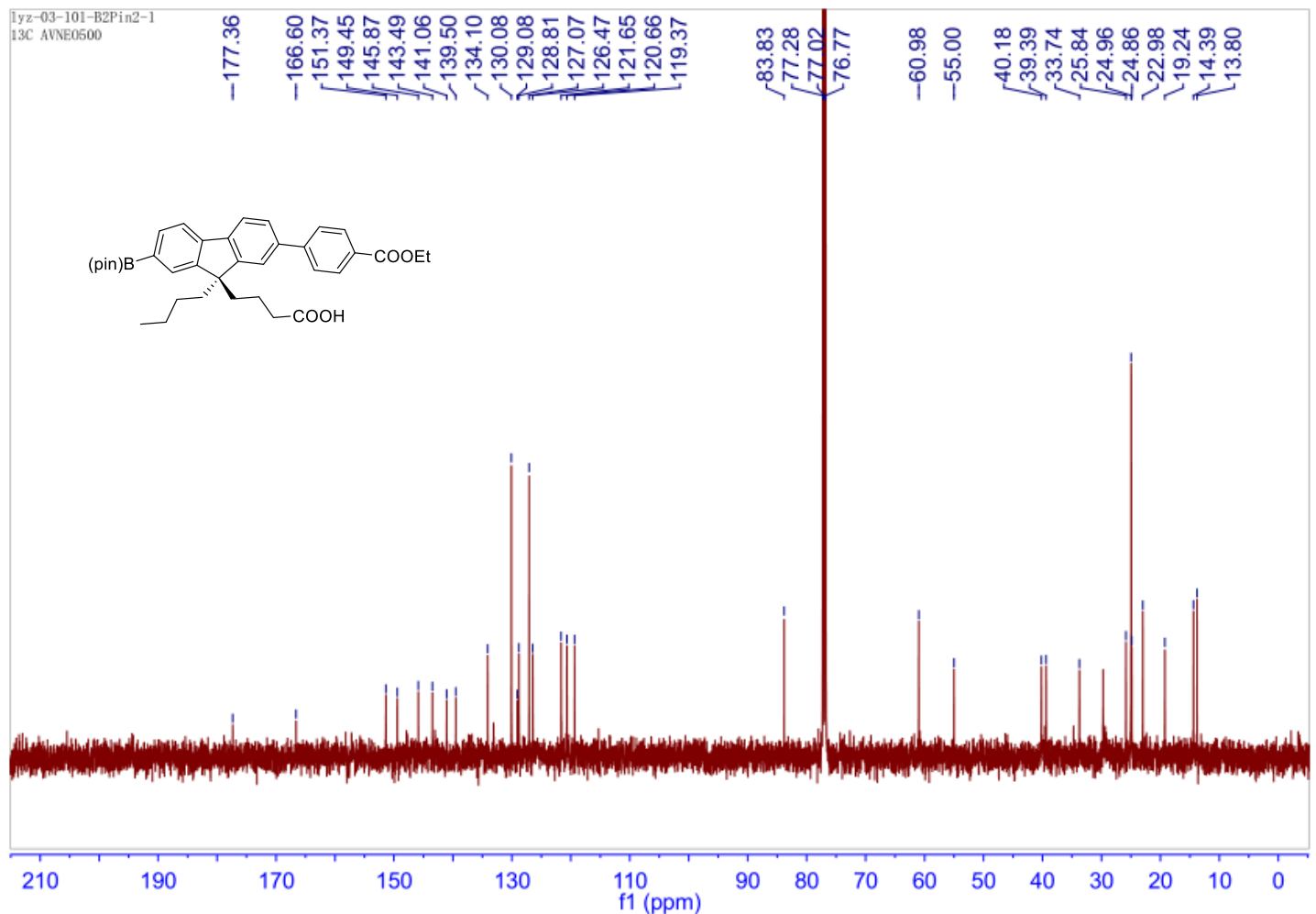


${ }^{1} \mathrm{H}$ NMR $\left.\left(500 \mathrm{MHz}^{\mathrm{CDCl}}\right)_{3}\right)-55$

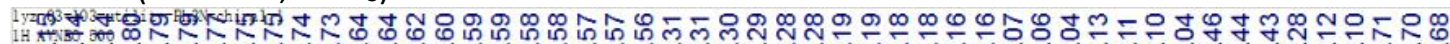

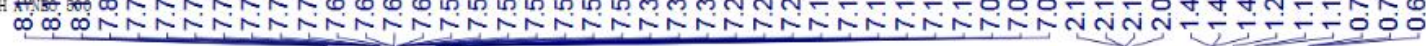
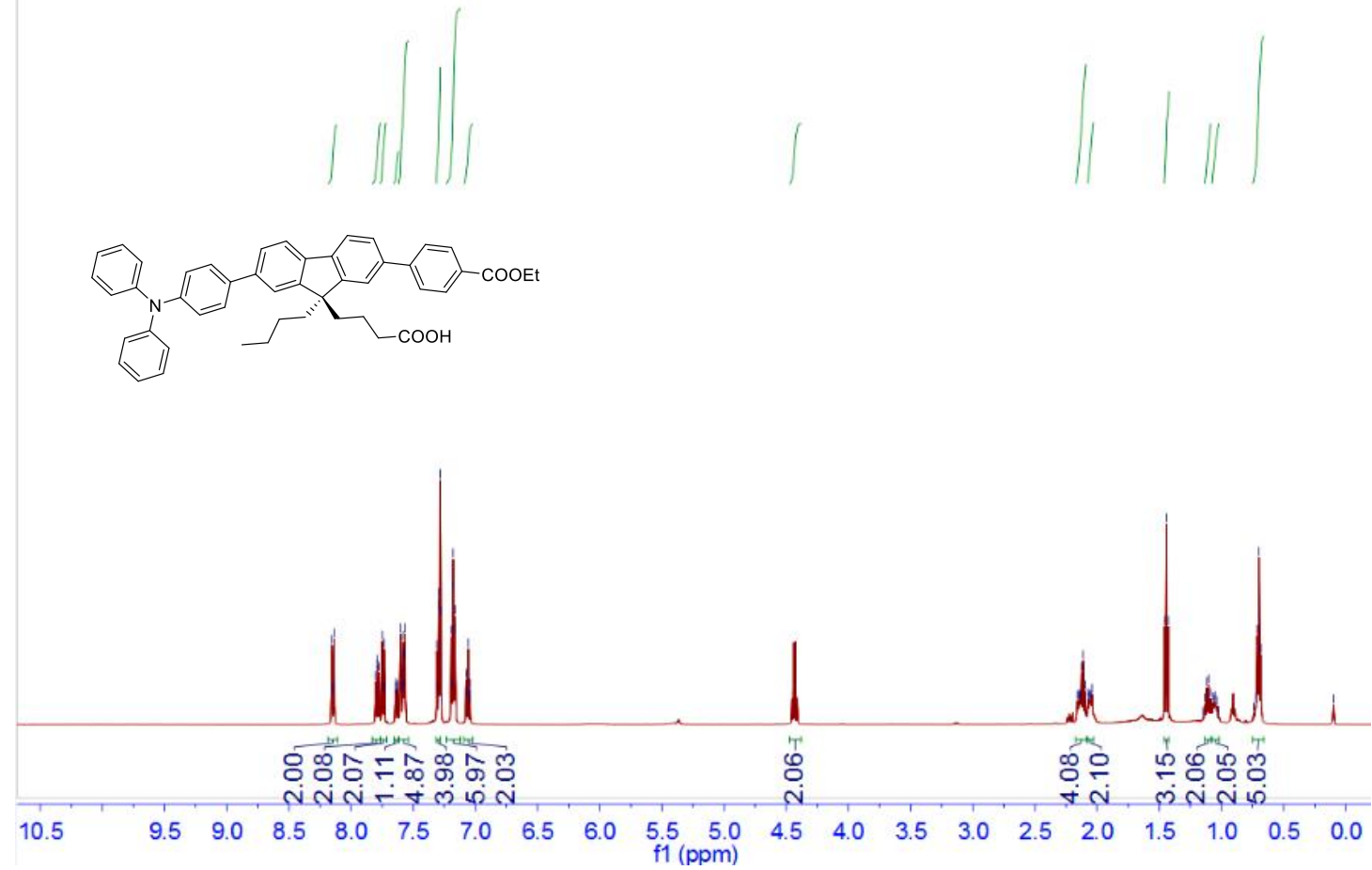

${ }^{13} \mathrm{C} \mathrm{NMR}\left(126 \mathrm{MHz}, \mathrm{CDCl}_{3}\right)-55$

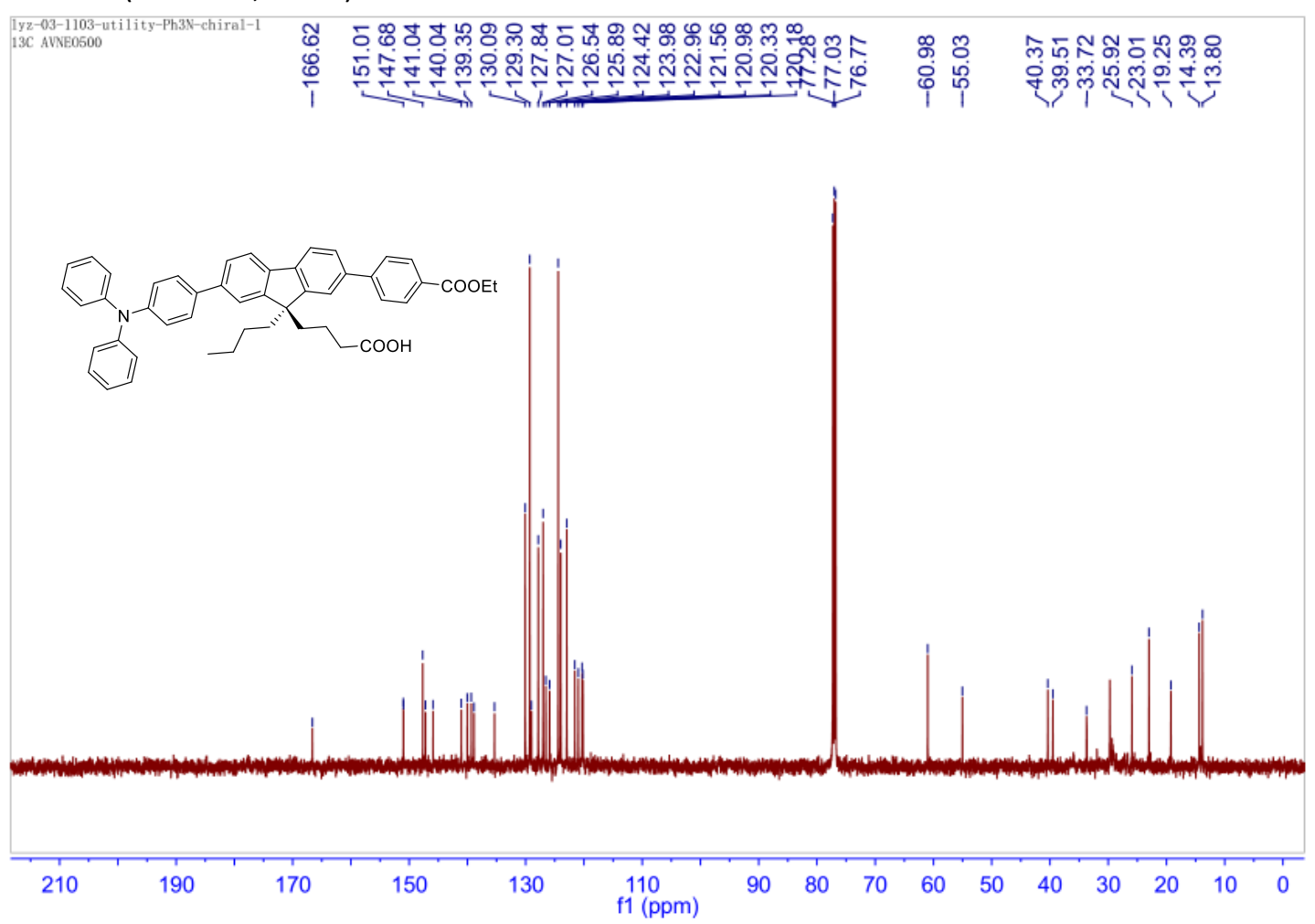


${ }^{1} \mathrm{H}$ NMR $\left(500 \mathrm{MHz}, \mathrm{CDCl}_{3}\right)-56$

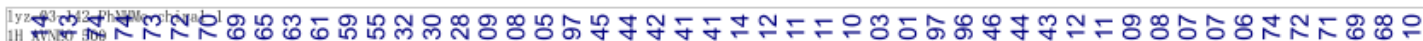

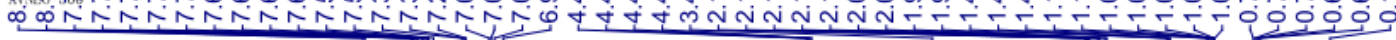
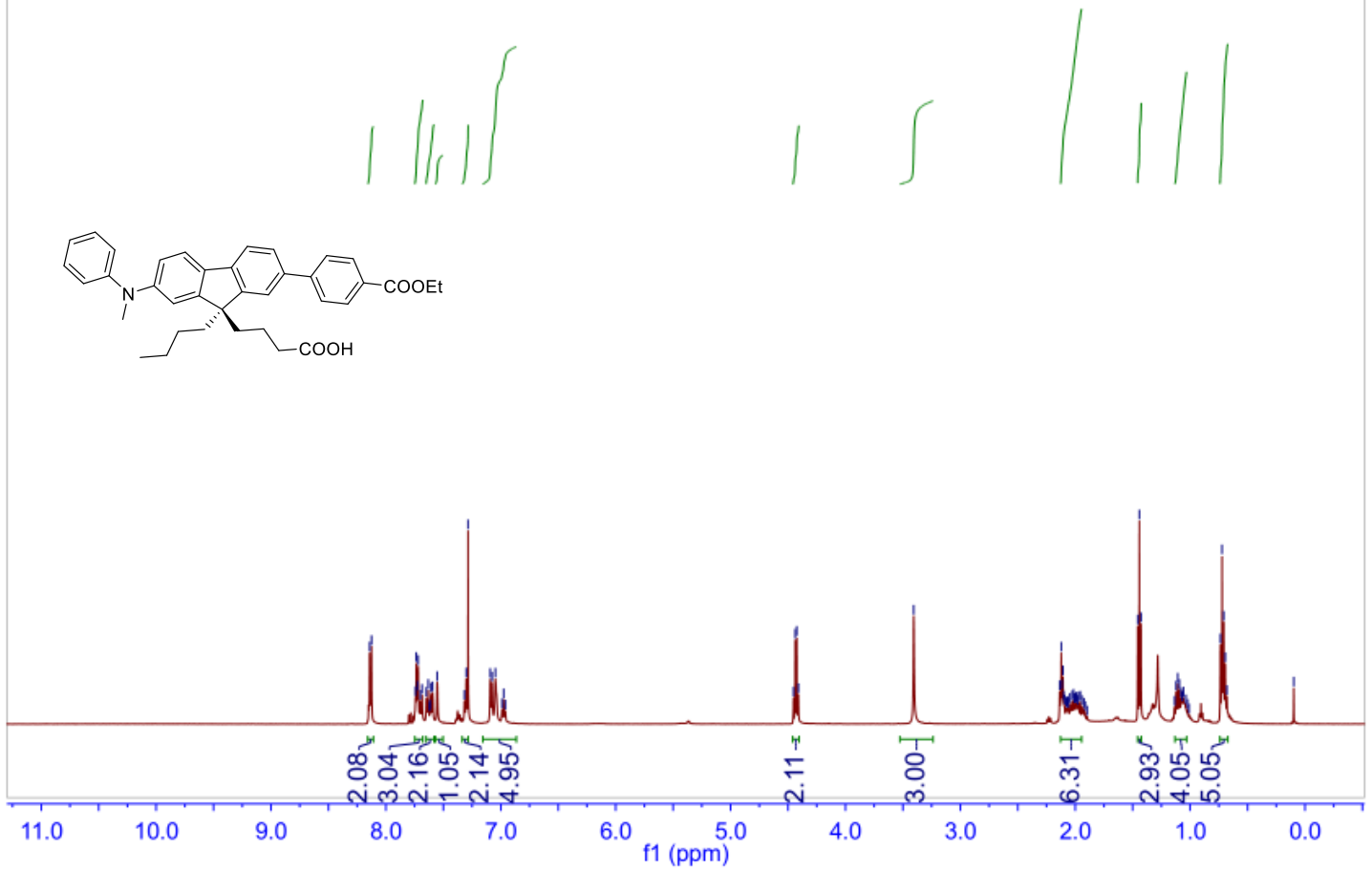

${ }^{13} \mathrm{C} \mathrm{NMR}\left(126 \mathrm{MHz}, \mathrm{CDCl}_{3}\right)-56$

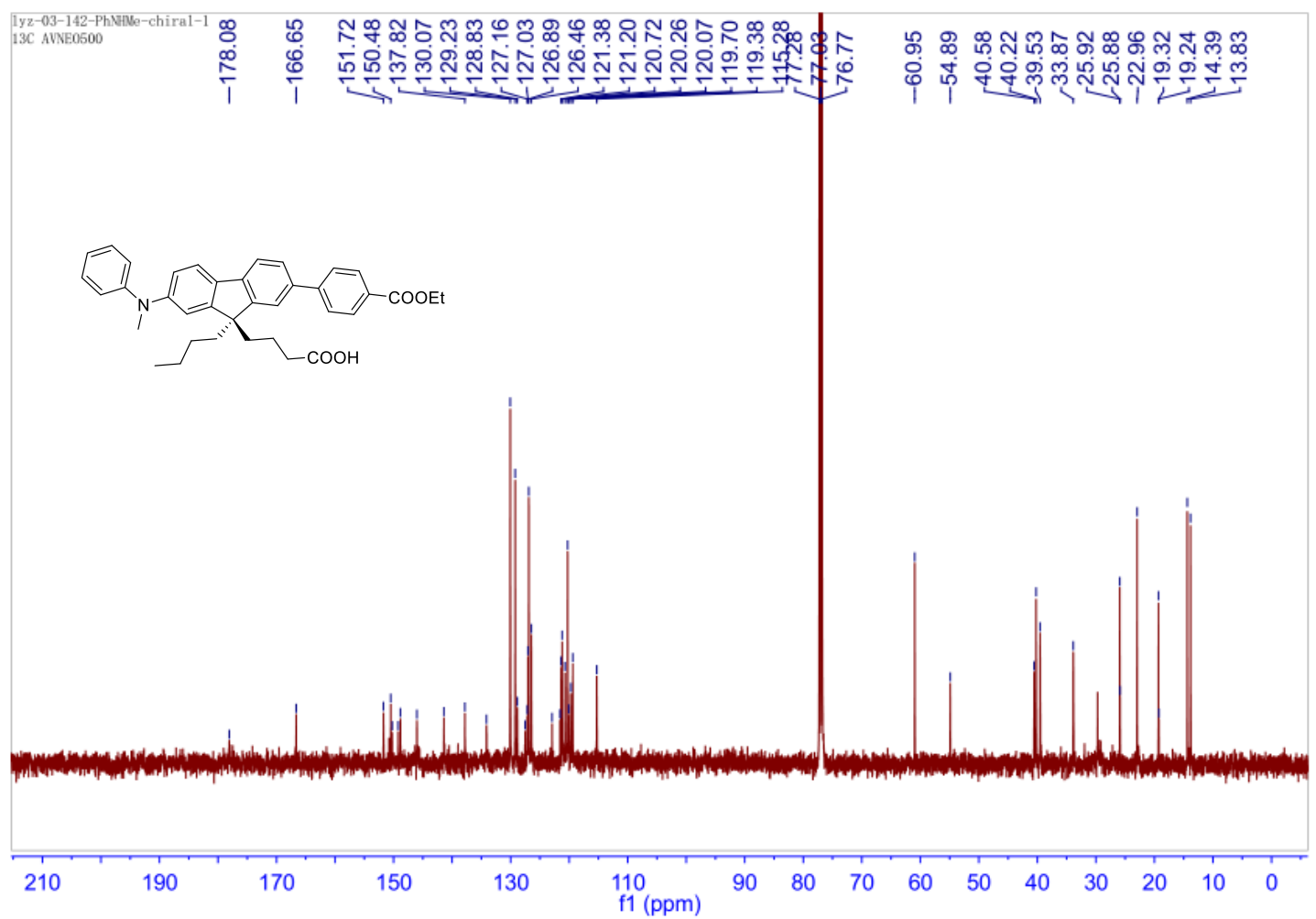




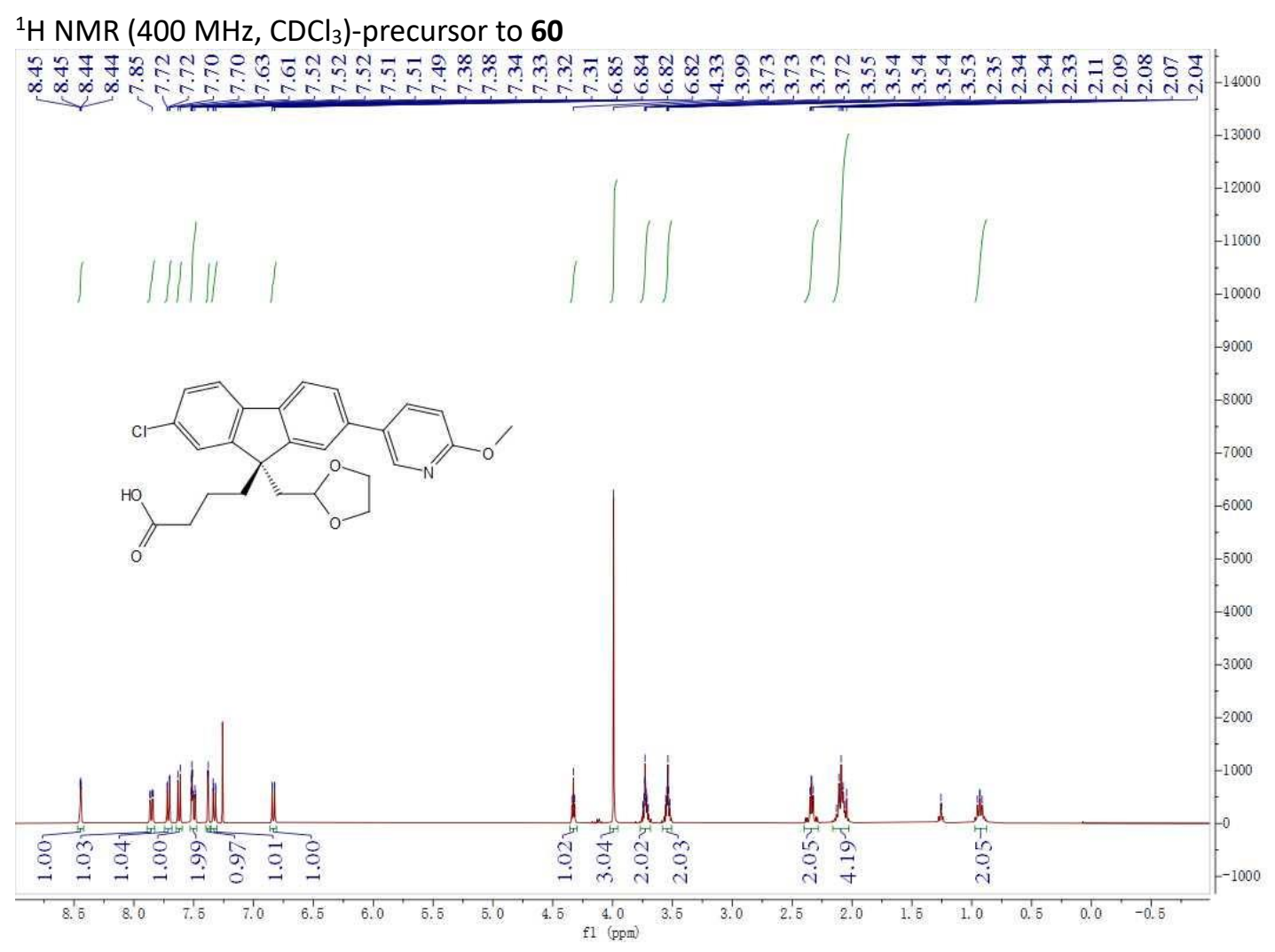

${ }^{13} \mathrm{C}$ NMR (101 MHz, $\mathrm{CDCl}_{3}$ )

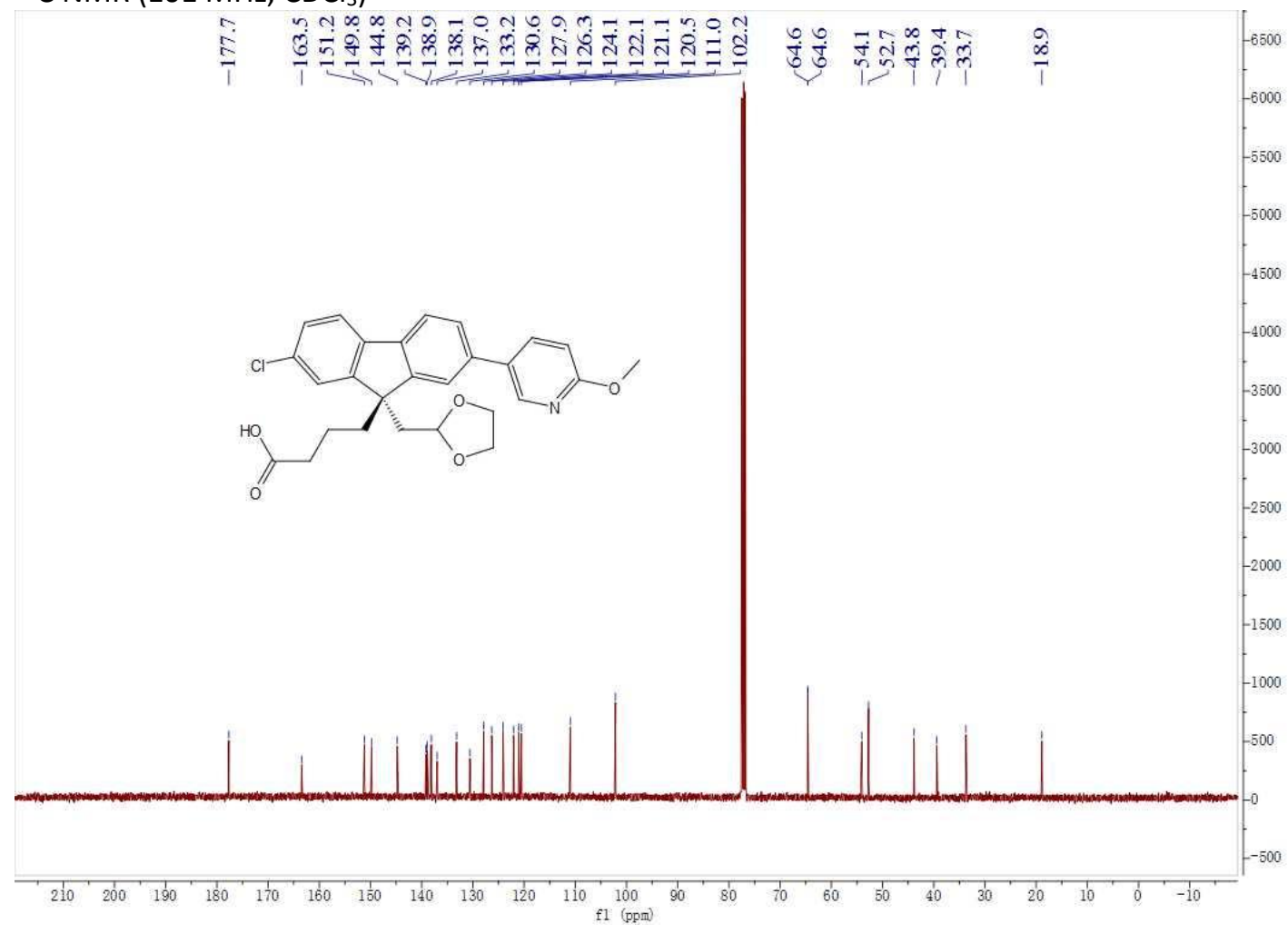


${ }^{1} \mathrm{H}$ NMR $\left(400 \mathrm{MHz}, \mathrm{CDCl}_{3}\right)-60$

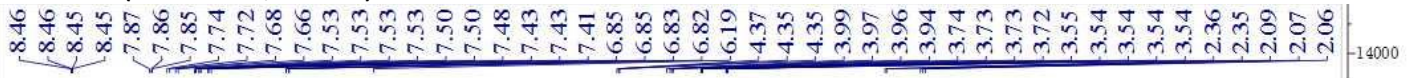

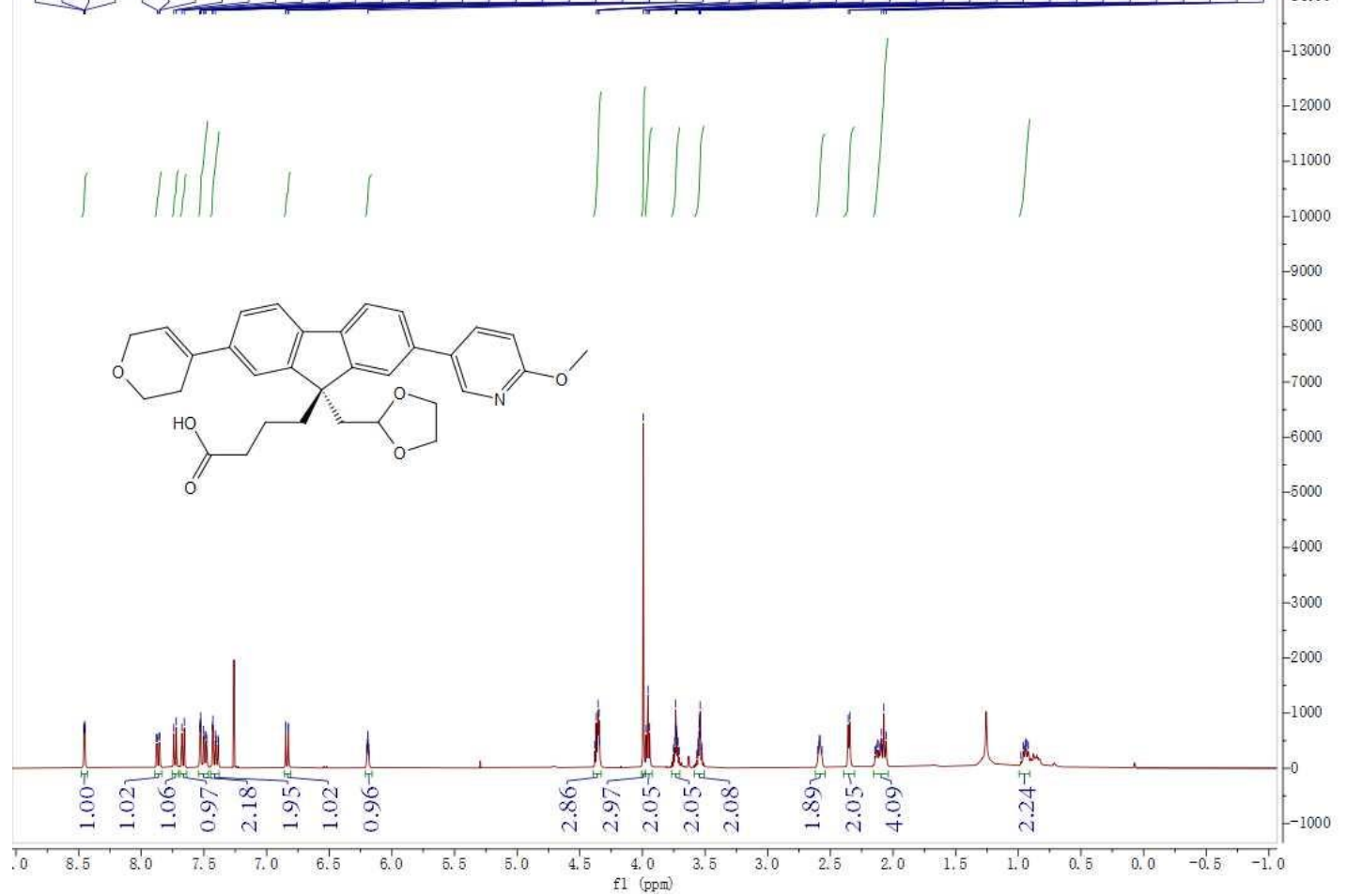

${ }^{13} \mathrm{C}$ NMR (101 MHz, $\left.\mathrm{CDCl}_{3}\right)-60$

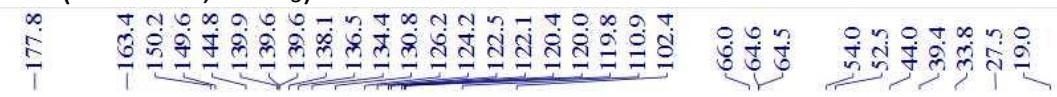

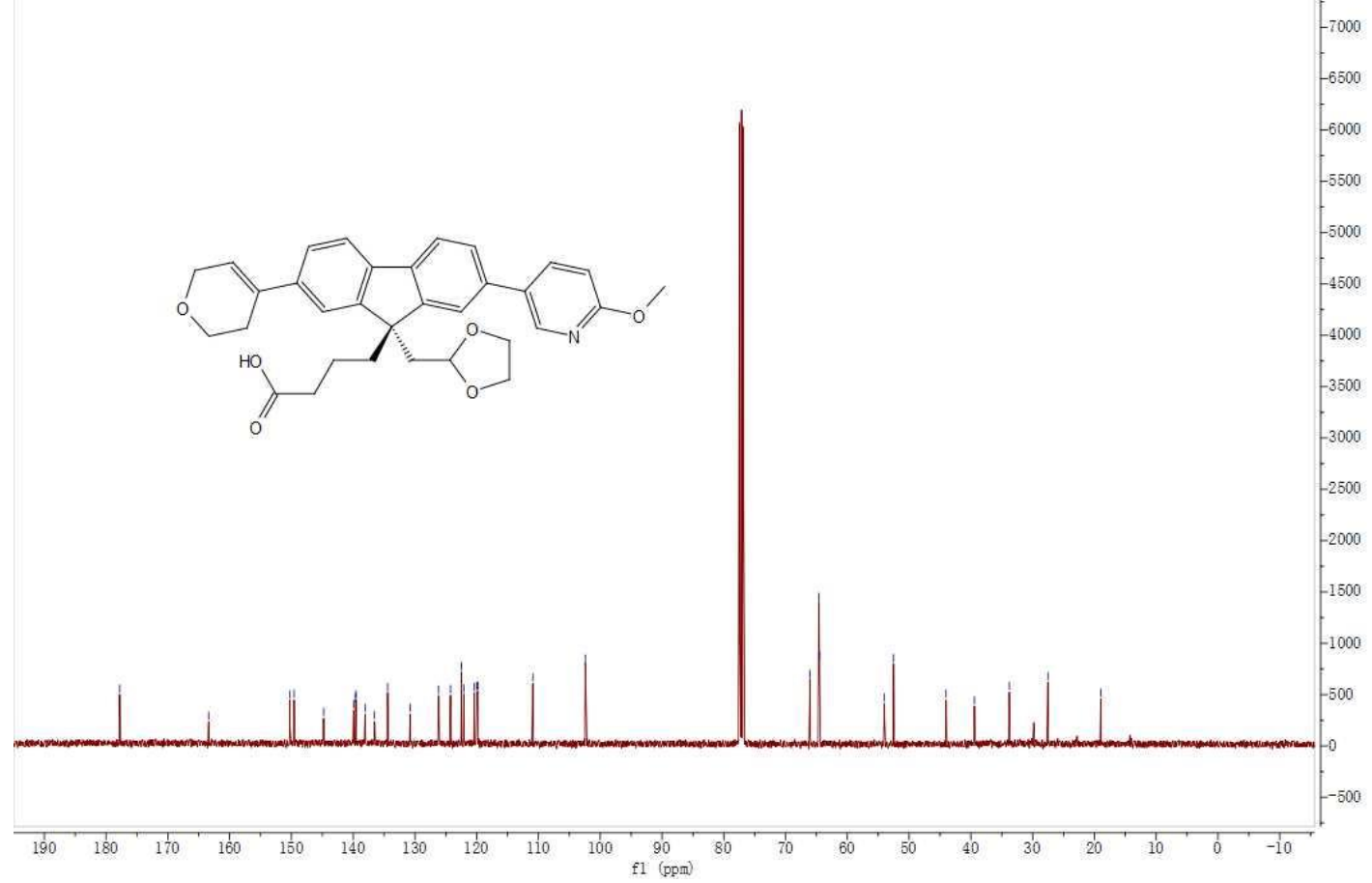


${ }^{1} \mathrm{H}$ NMR (500 MHz, CDCl 3 )-precursor to ent-60

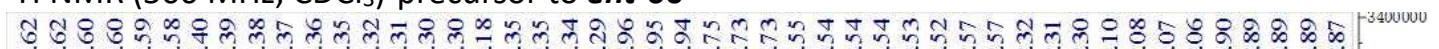

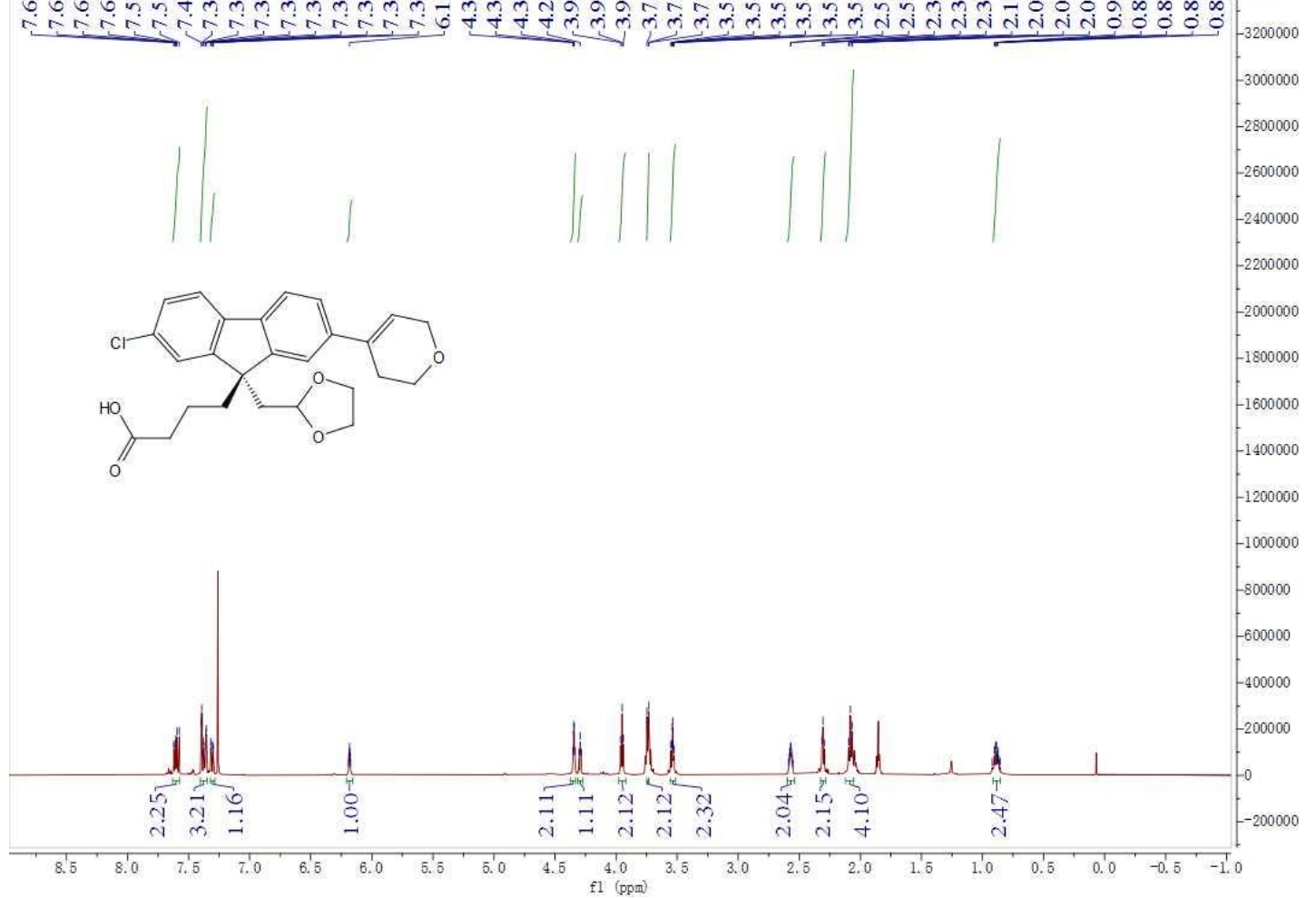

$\left.{ }^{13} \mathrm{C} \mathrm{NMR} \mathrm{(126} \mathrm{MHz,} \mathrm{CDCl}_{3}\right)$

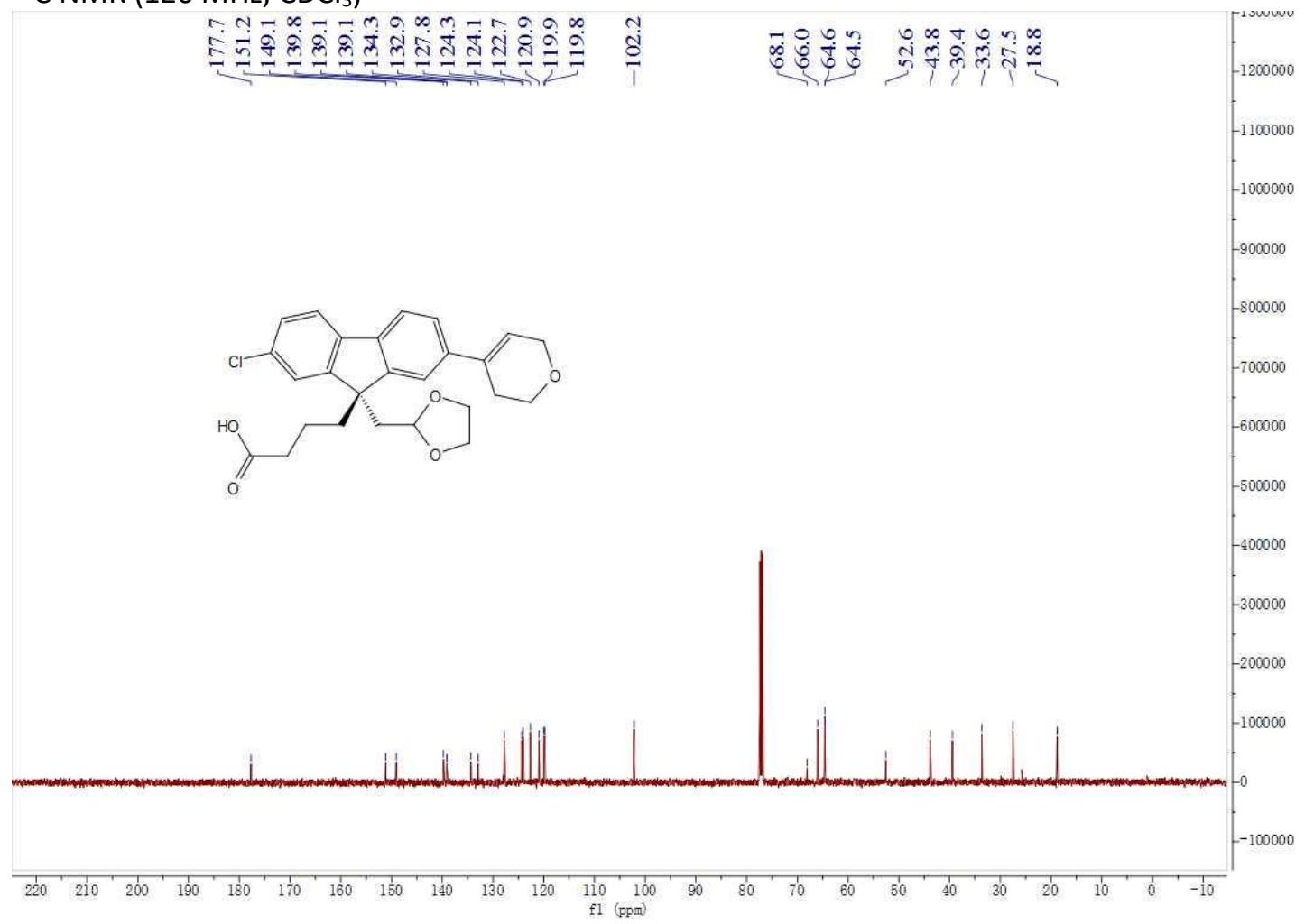




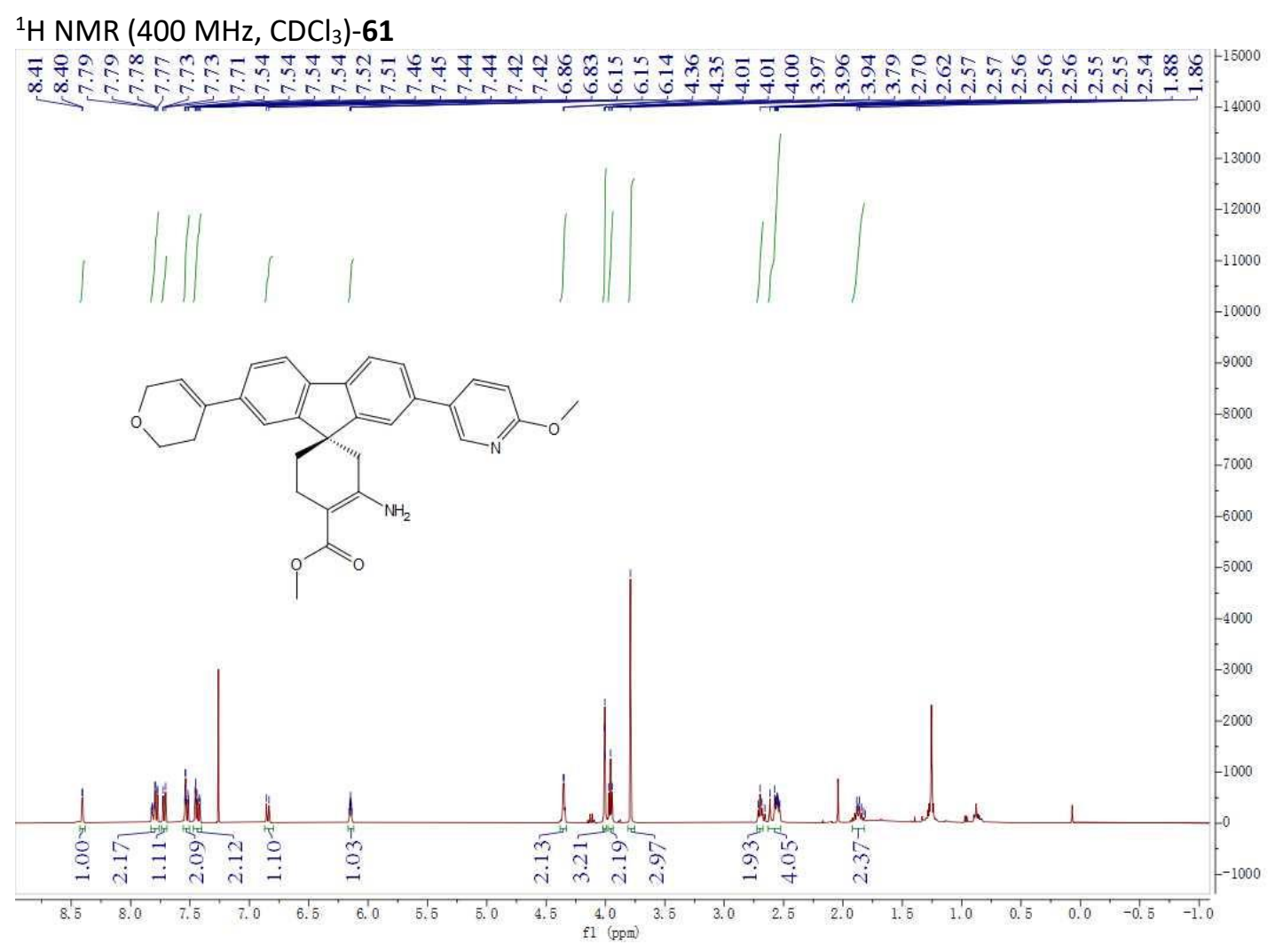

${ }^{13} \mathrm{C}$ NMR (101 MHz, $\left.\mathrm{CDCl}_{3}\right)-61$

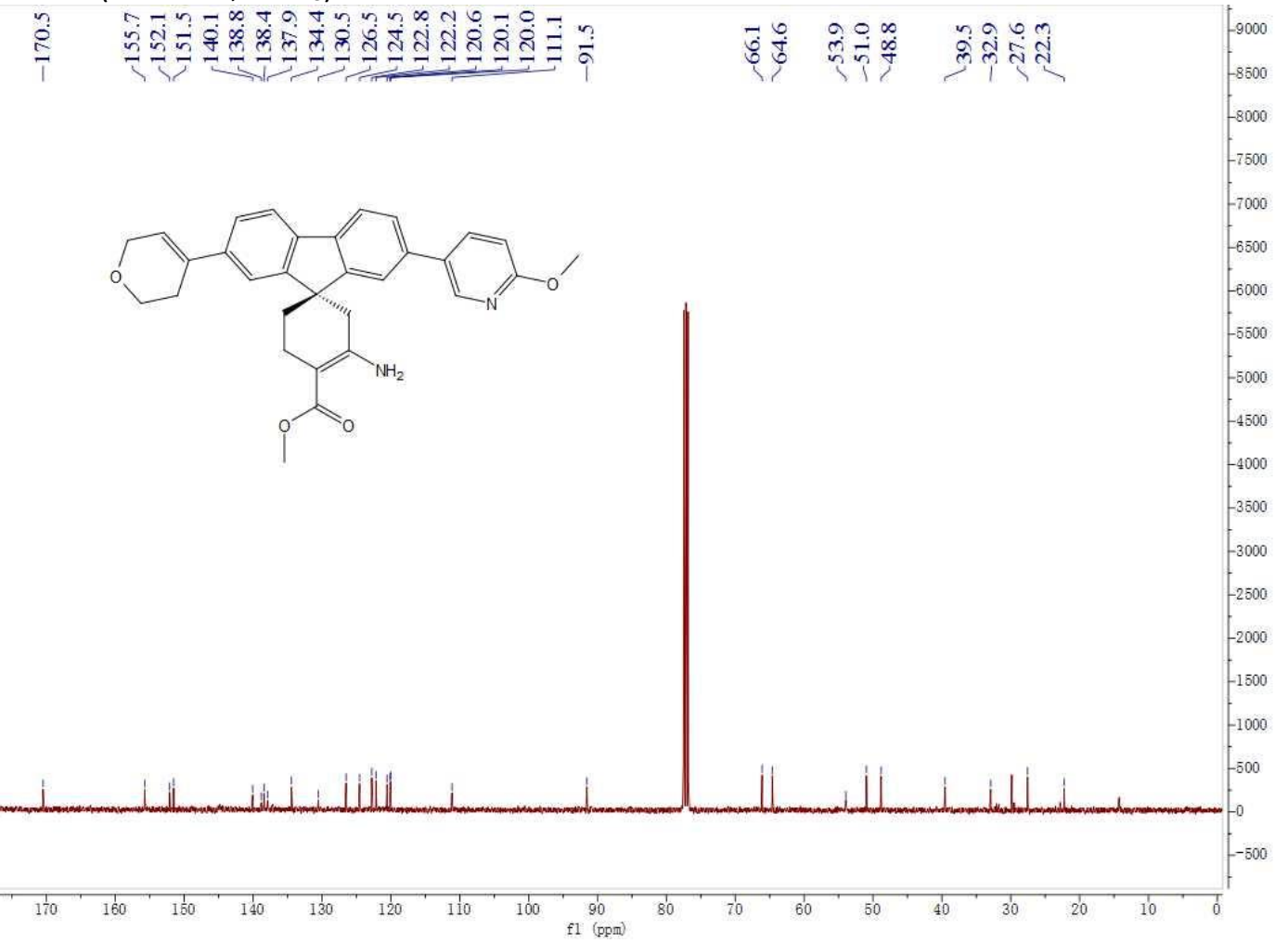


${ }^{1} \mathrm{H}$ NMR $\left(500 \mathrm{MHz}, \mathrm{CDCl}_{3}\right)-62$

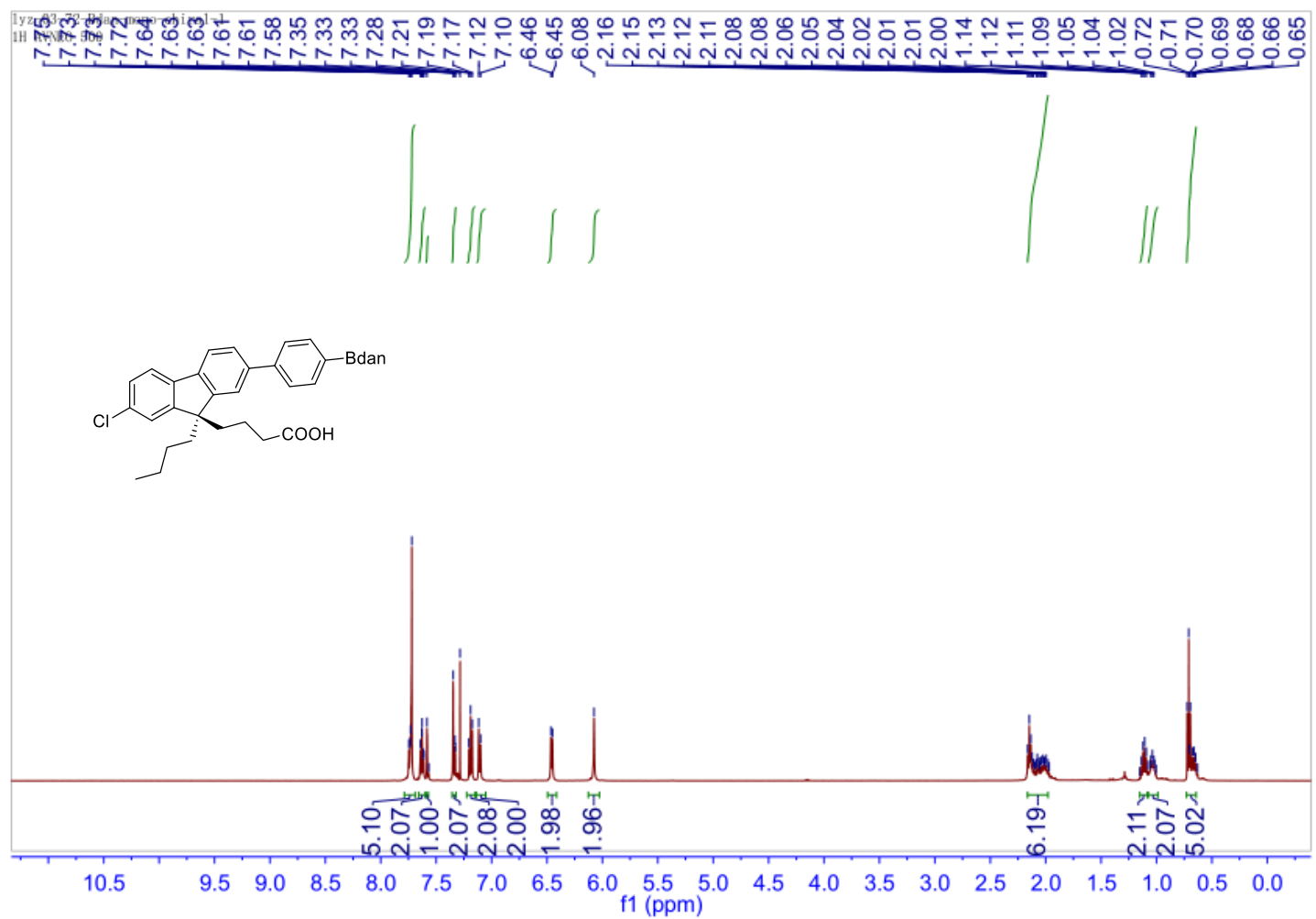

$\left.{ }^{13} \mathrm{C} \mathrm{NMR} \mathrm{(126} \mathrm{MHz,} \mathrm{CDCl}_{3}\right)-62$

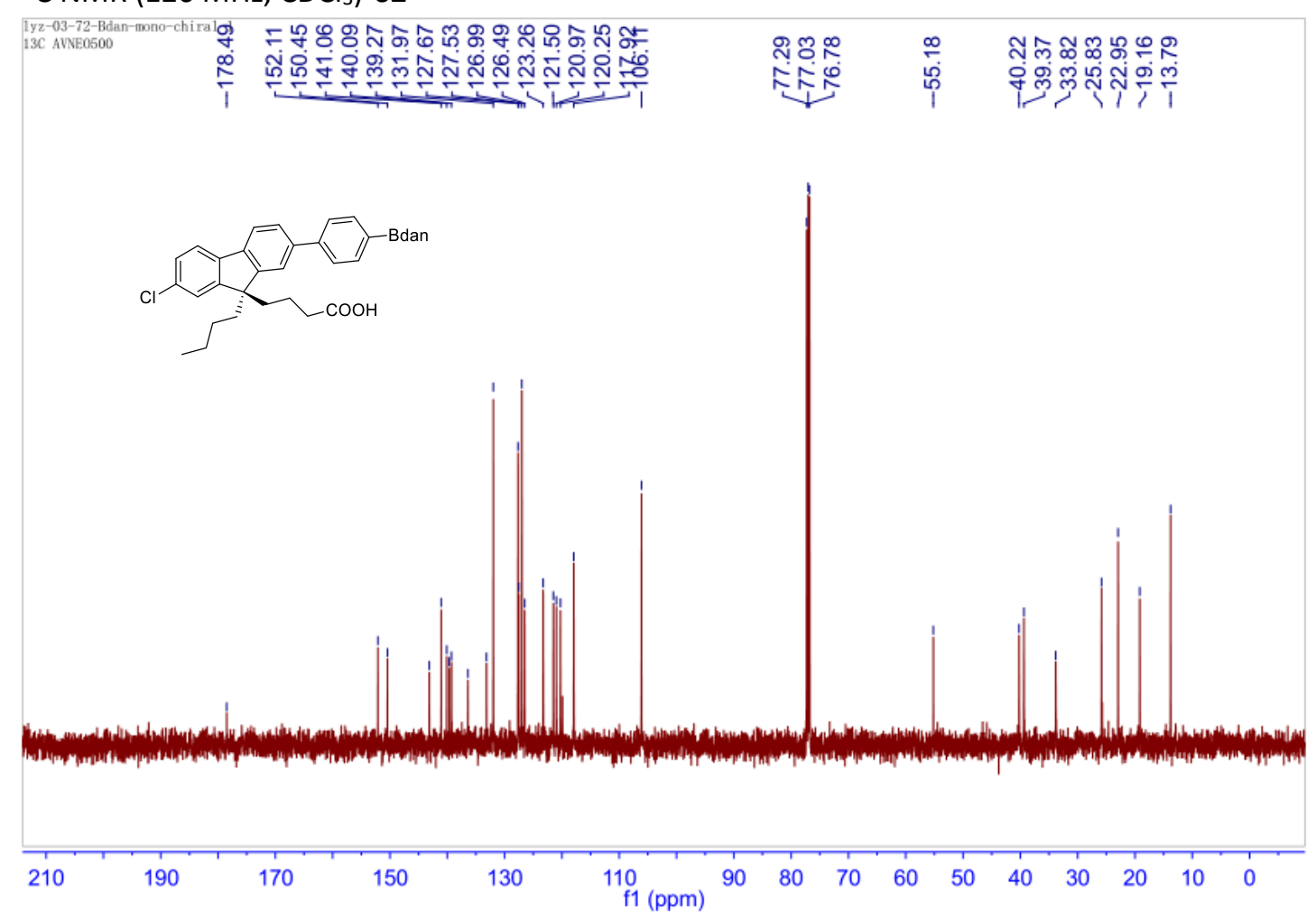


${ }^{1} \mathrm{H}$ NMR (500 MHz, $\mathrm{d}_{6}$-Acetone) -63

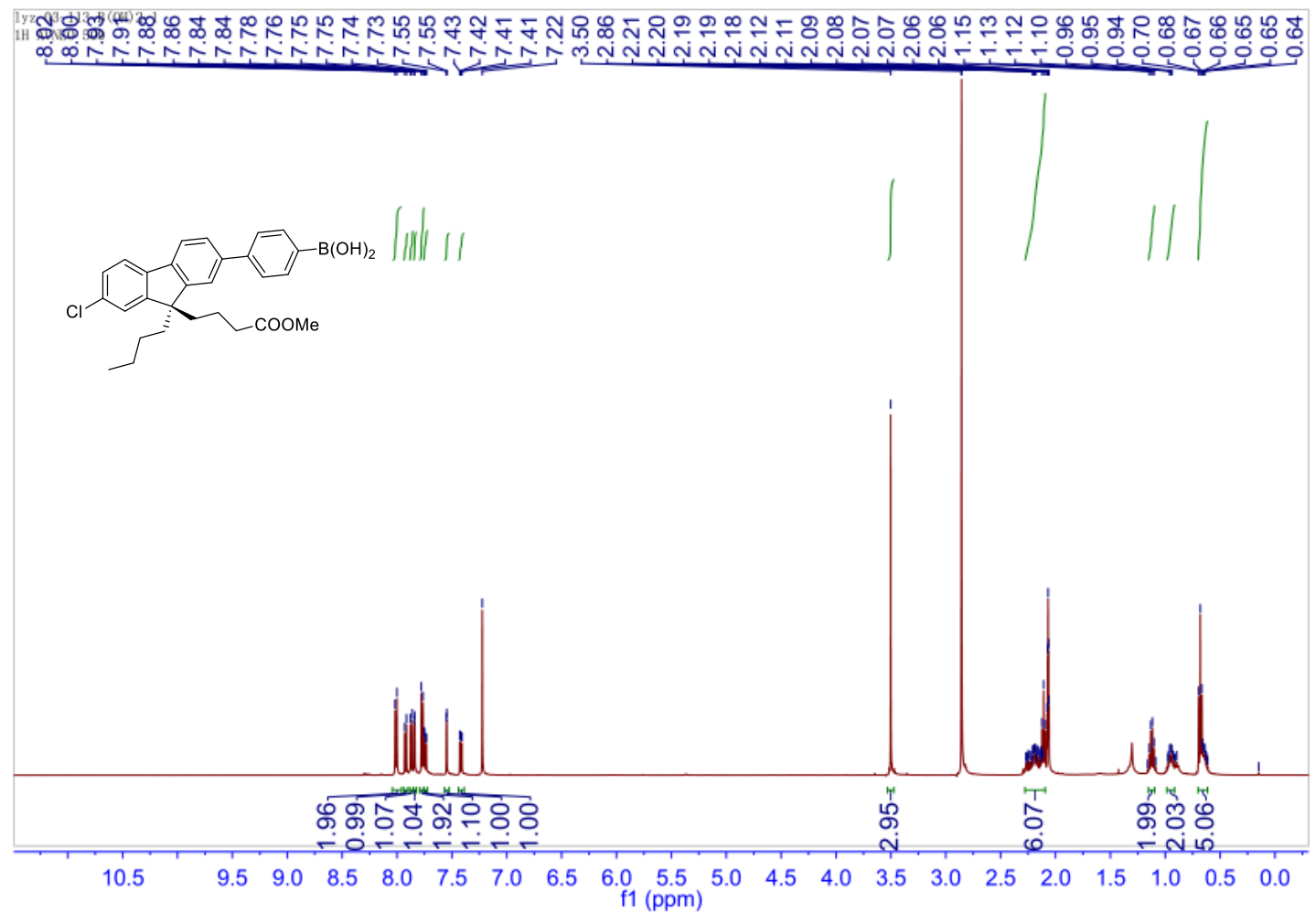

${ }^{13} \mathrm{C}$ NMR (126 MHz, $\mathrm{d}_{6}$-Acetone) -63

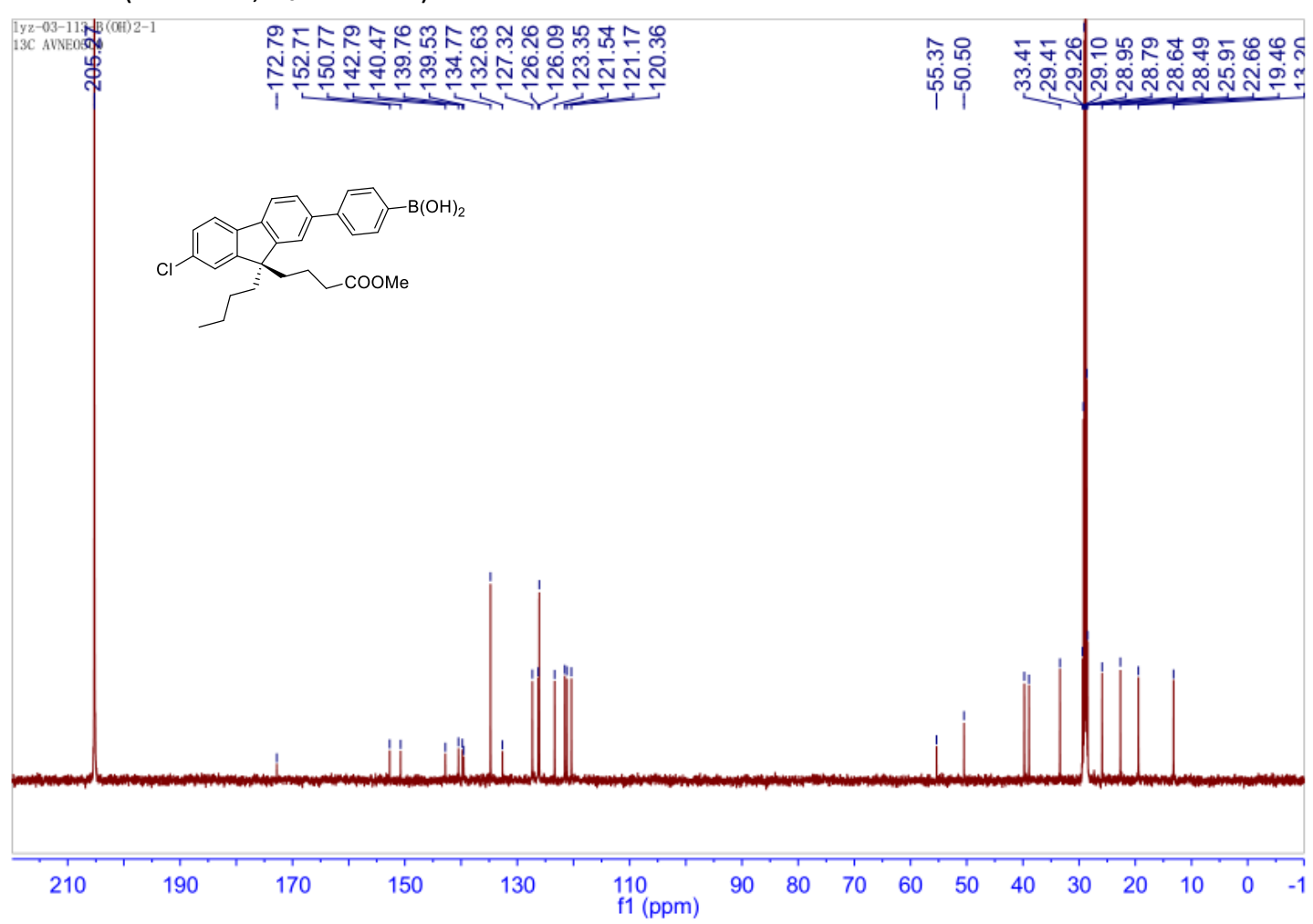


${ }^{1} \mathrm{H}$ NMR (500 MHz, $\mathrm{CDCl}_{3}$ )-64

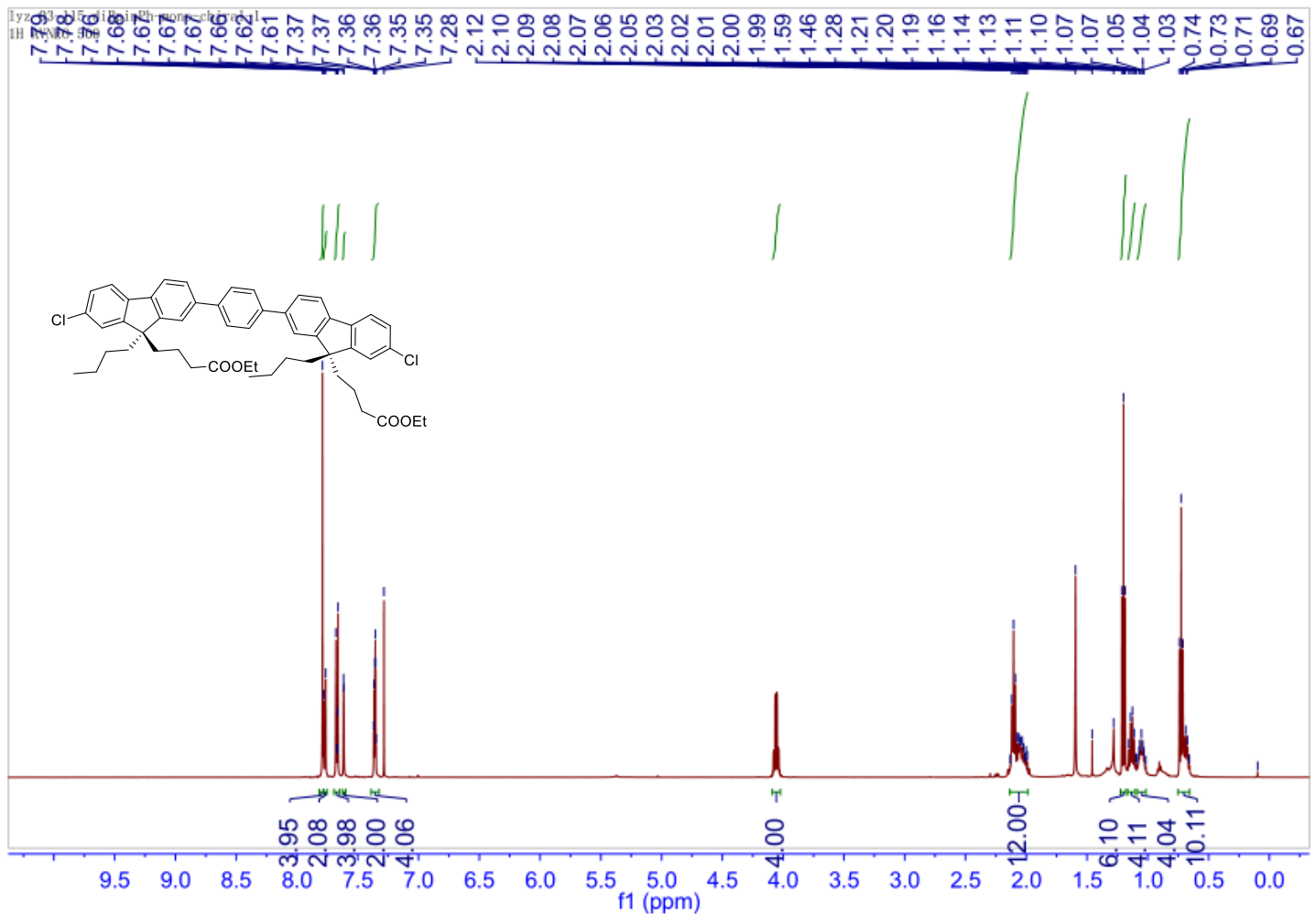

$\left.{ }^{13} \mathrm{C} \mathrm{NMR} \mathrm{(126} \mathrm{MHz,} \mathrm{CDCl}_{3}\right)-64$

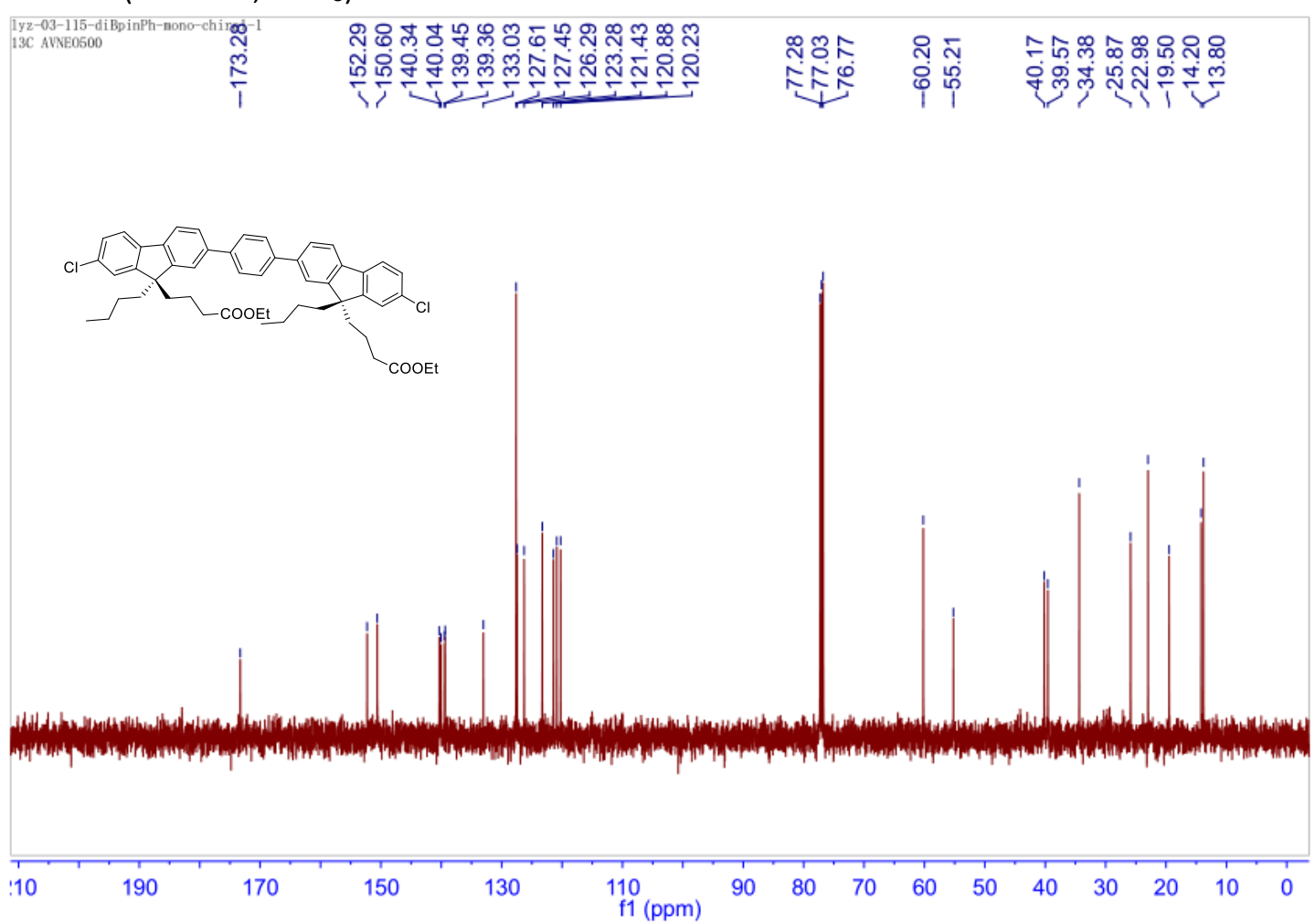


${ }^{1} \mathrm{H}$ NMR $\left(500 \mathrm{MHz}, \mathrm{CDCl}_{3}\right)-$ meso-64

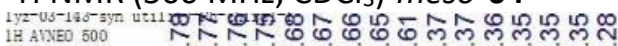

NANANANANTN

ถึ

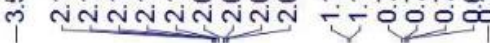

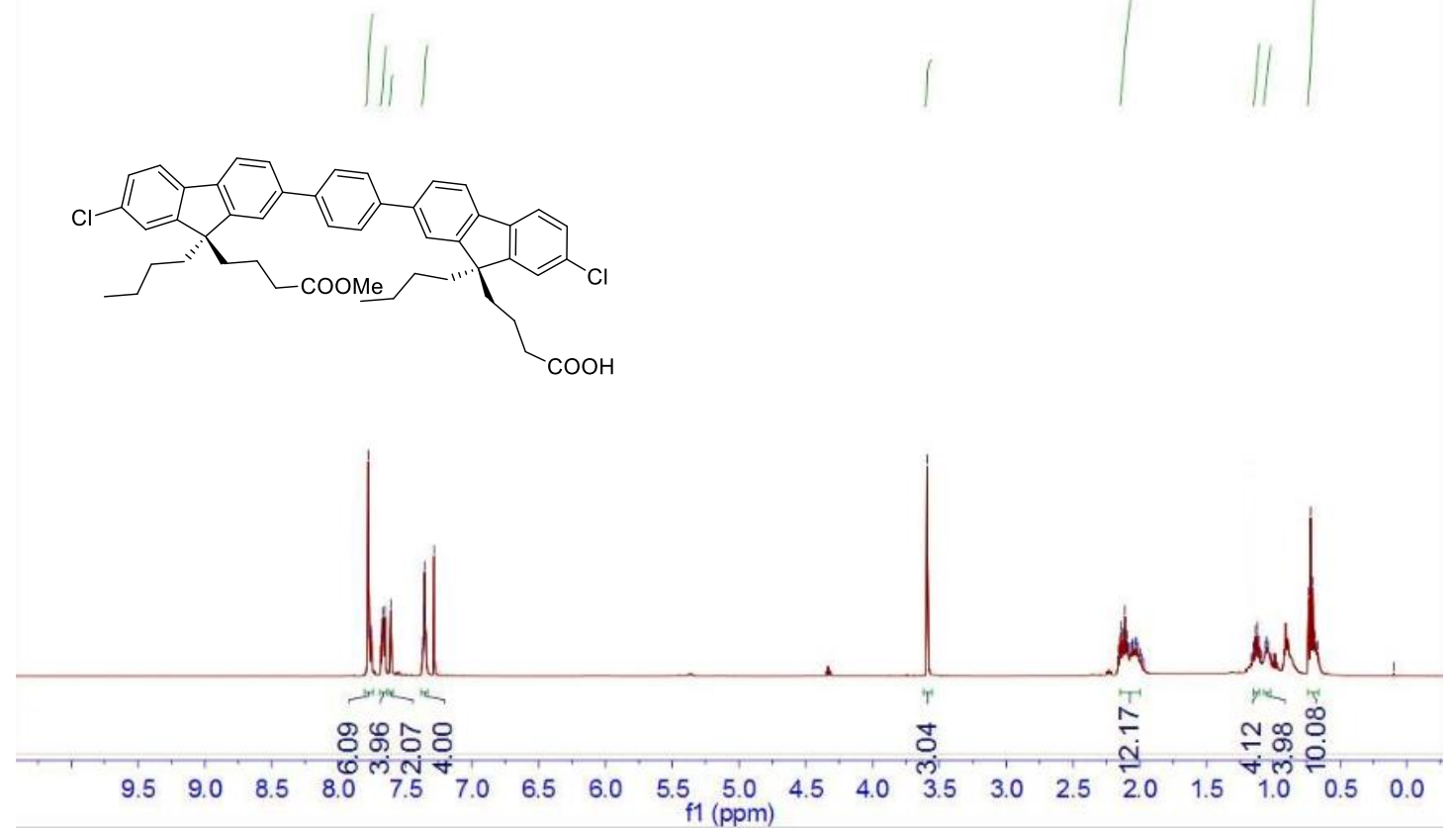

${ }^{13} \mathrm{C} \mathrm{NMR}\left(126 \mathrm{MHz}, \mathrm{CDCl}_{3}\right)-$ meso-64

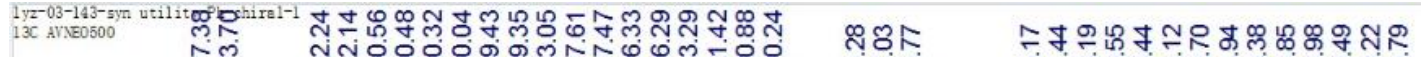

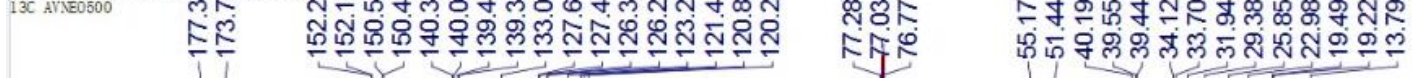
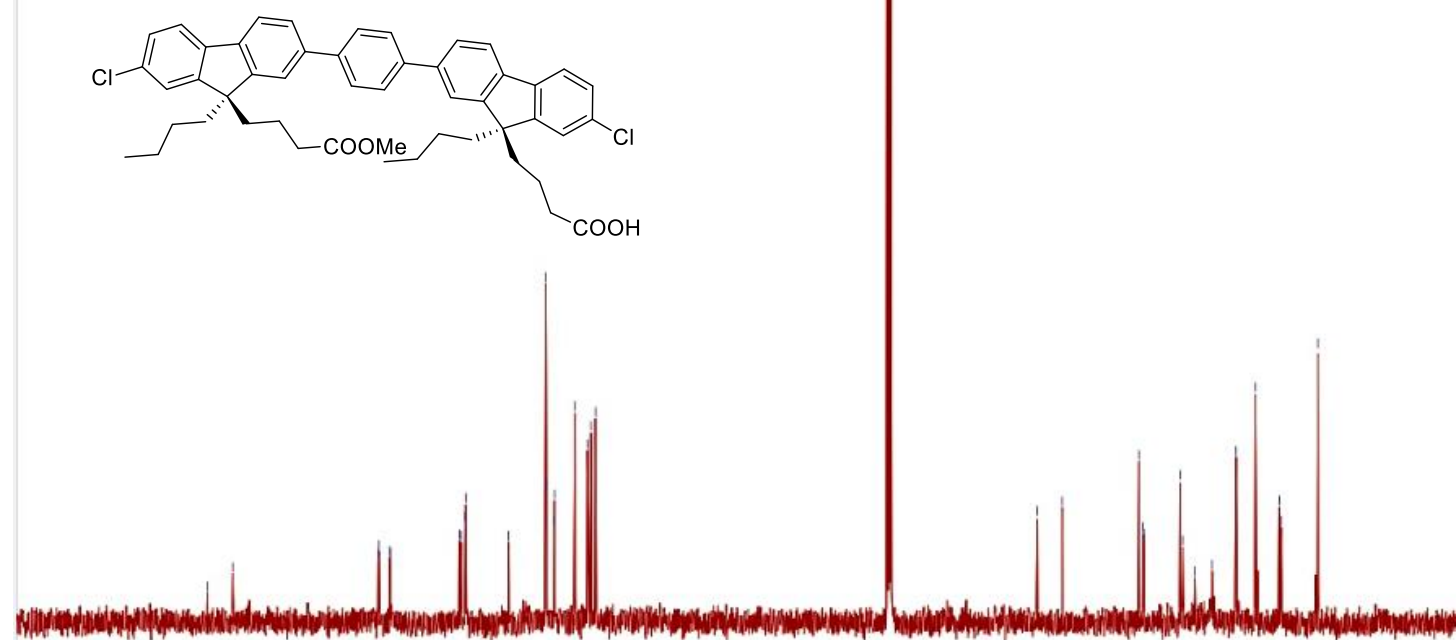

$\begin{array}{lllllllllllllllllllll}200 & 190 & 180 & 170 & 160 & 150 & 140 & 130 & 120 & 110 & \begin{array}{c}100 \\ \mathrm{f} 1(\mathrm{ppm})\end{array} & 90 & 80 & 70 & 60 & 50 & 40 & 30 & 20 & 10 & 0\end{array}$ 


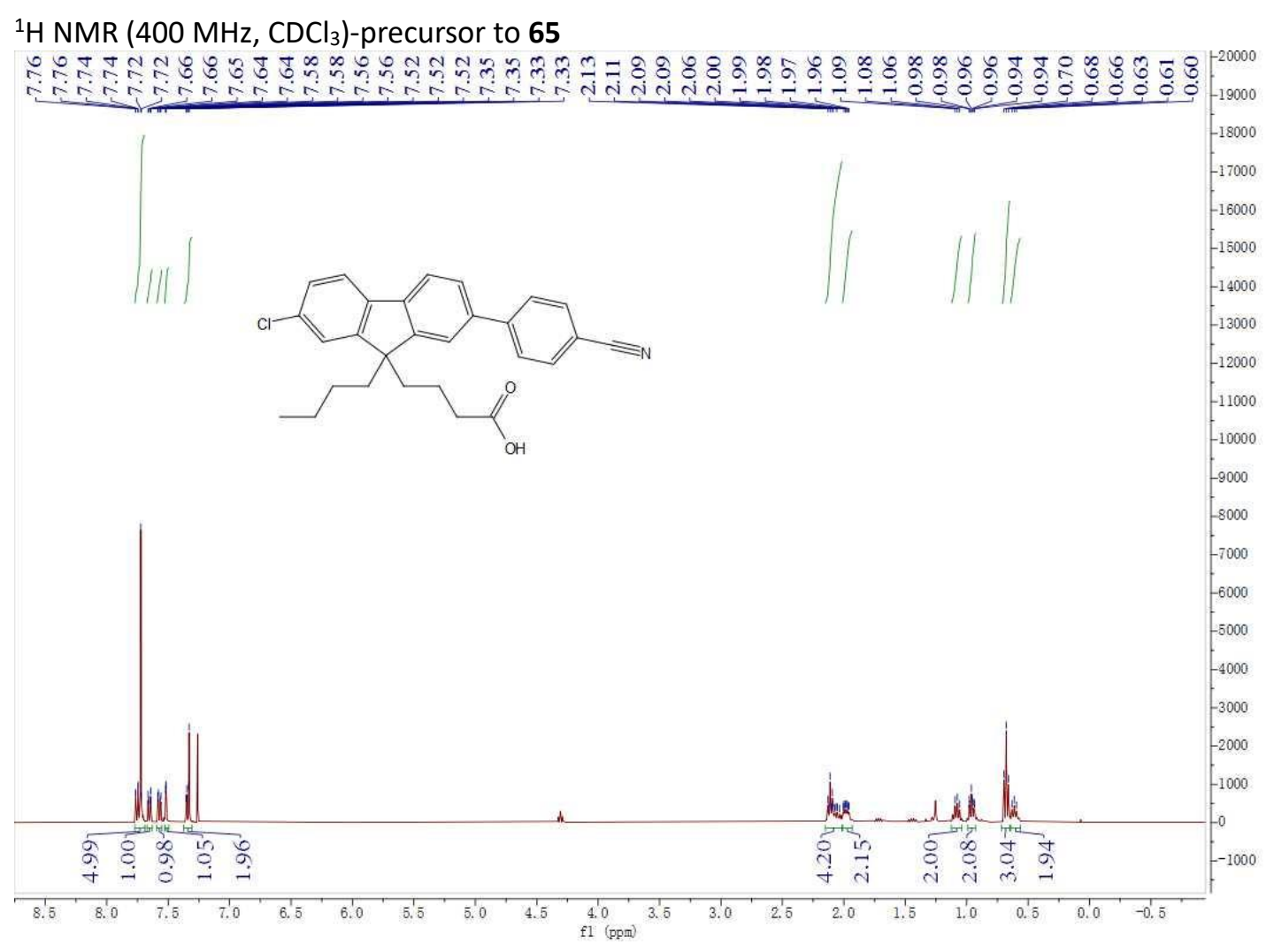

${ }^{13} \mathrm{C}$ NMR (101 MHz, CDCl 3 )

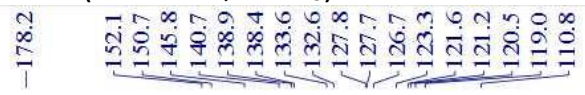

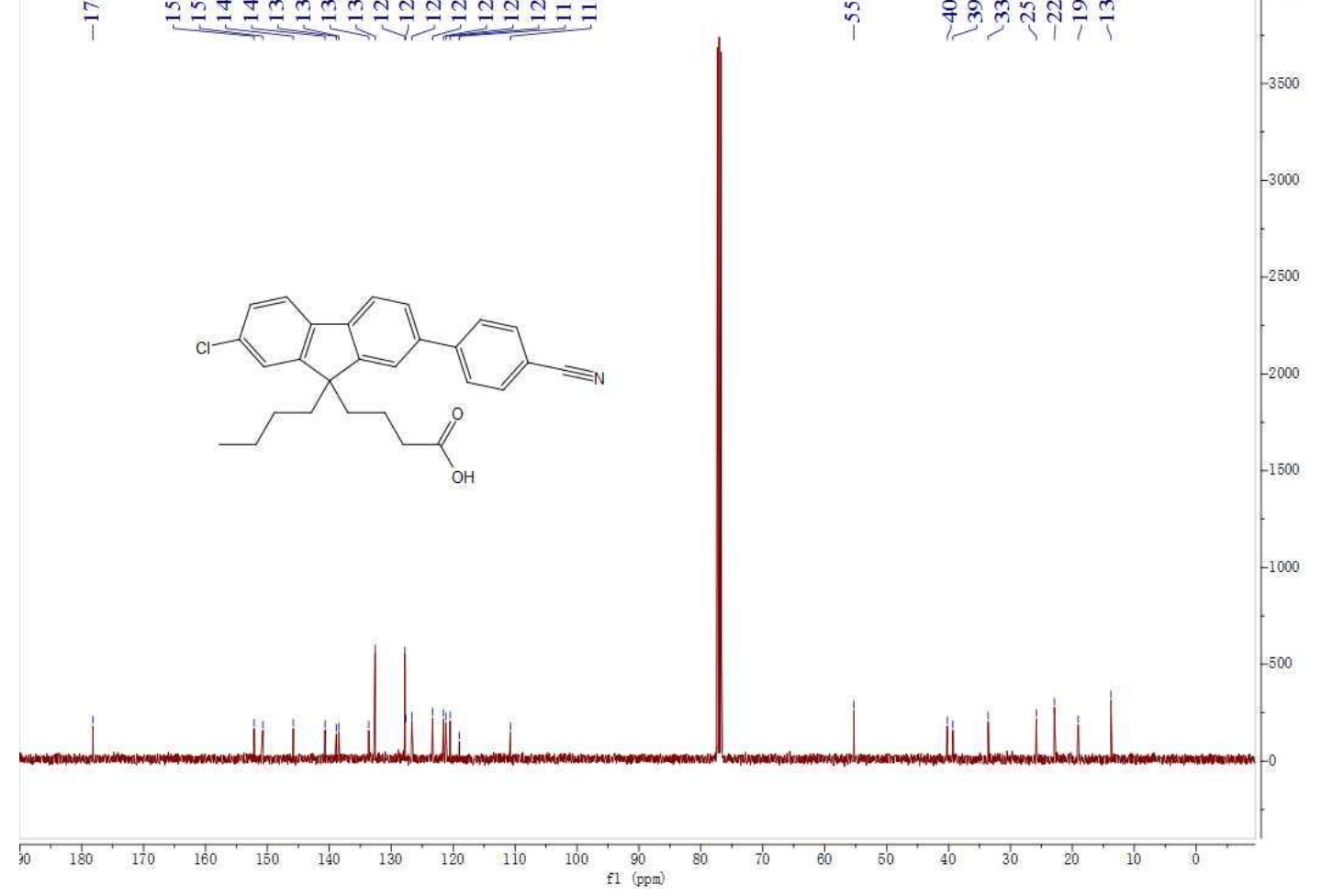


${ }^{1} \mathrm{H}$ NMR (400 MHz, $\mathrm{CDCl}_{3}$ )-precursor to 65

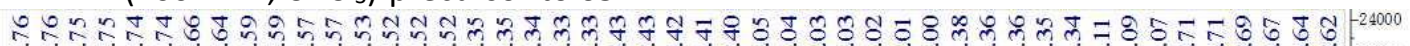

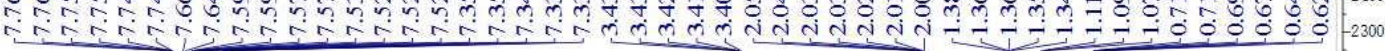

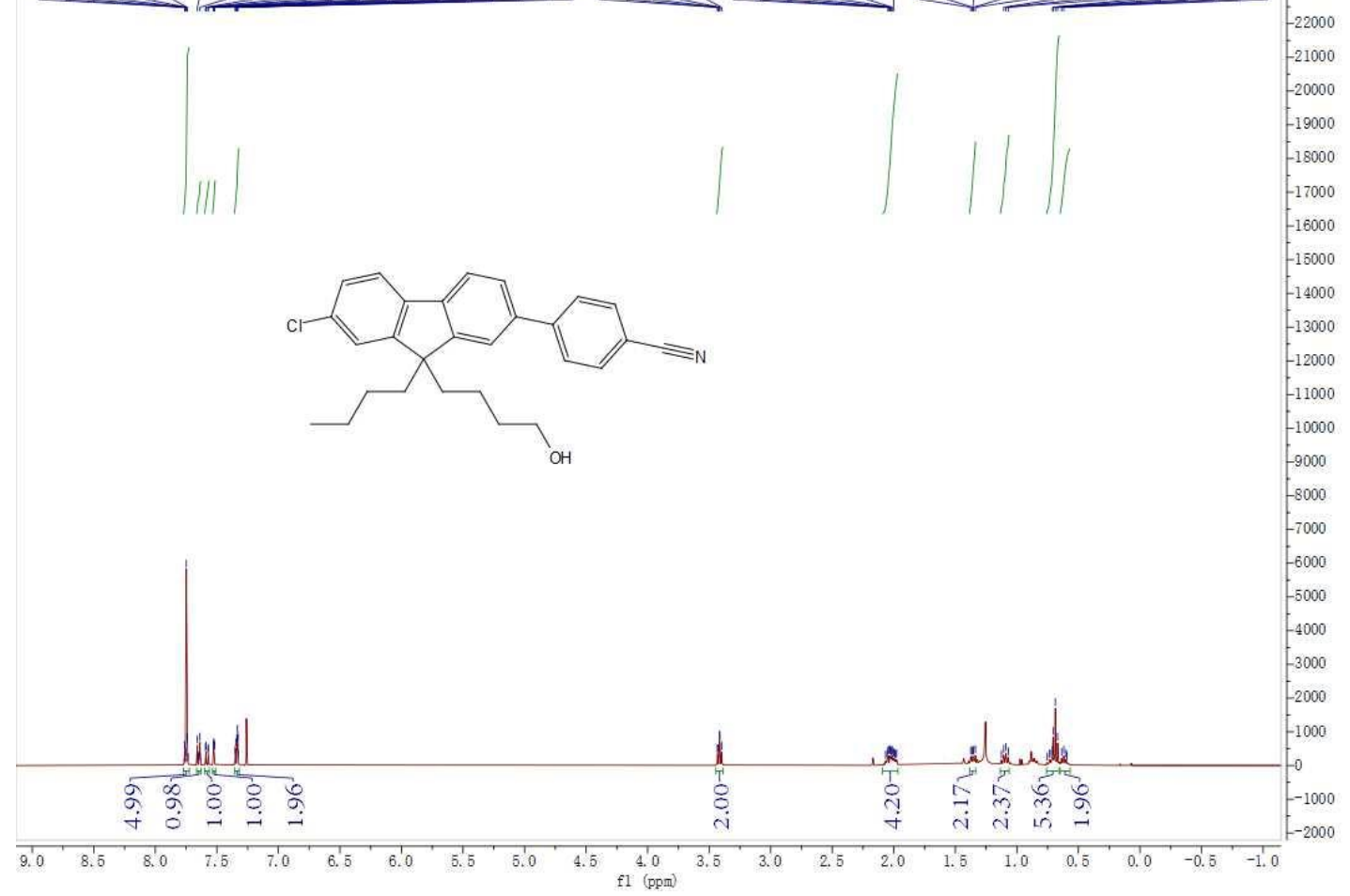

${ }^{13} \mathrm{C} \mathrm{NMR}\left(126 \mathrm{MHz}, \mathrm{CDCl}_{3}\right)$

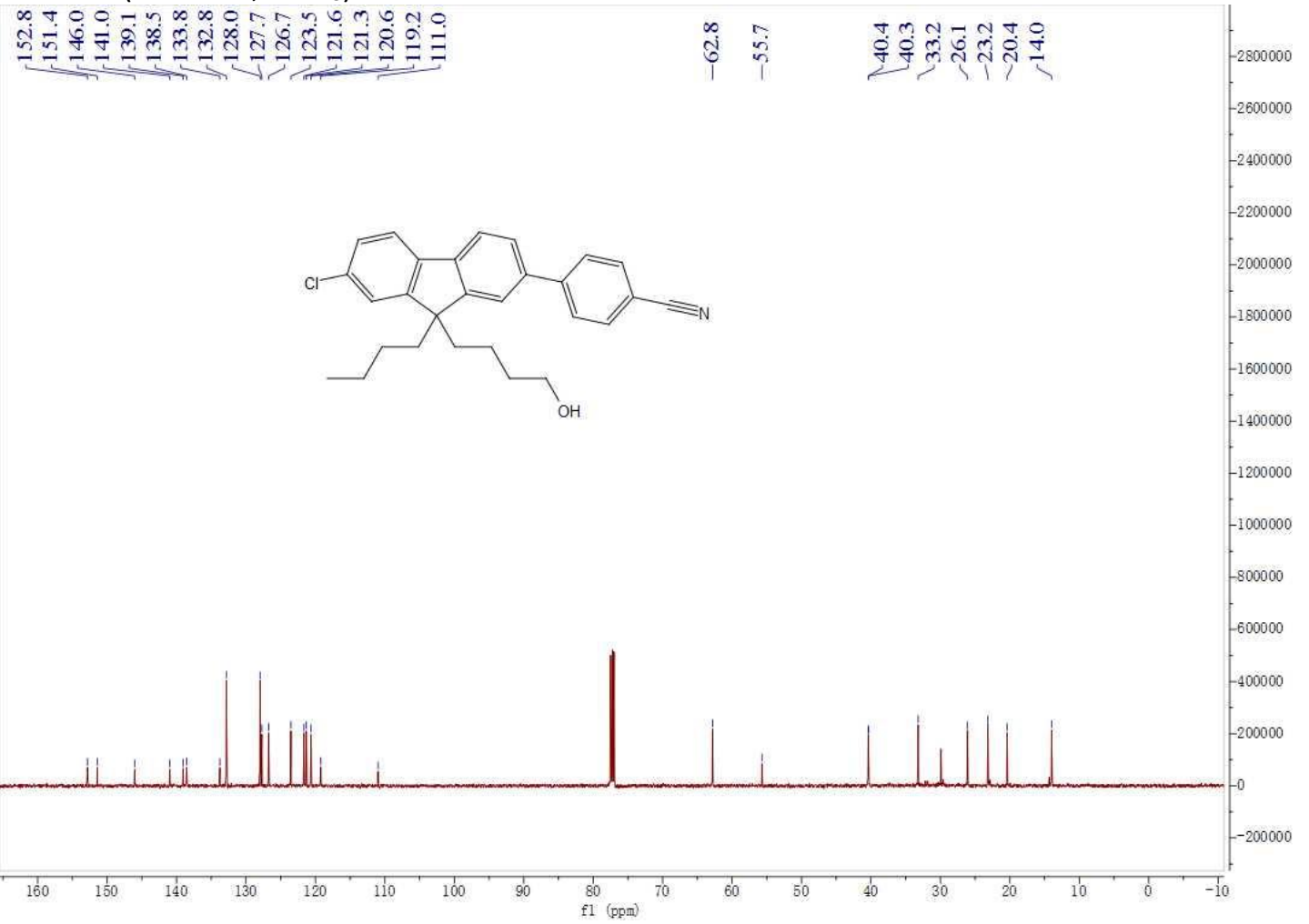




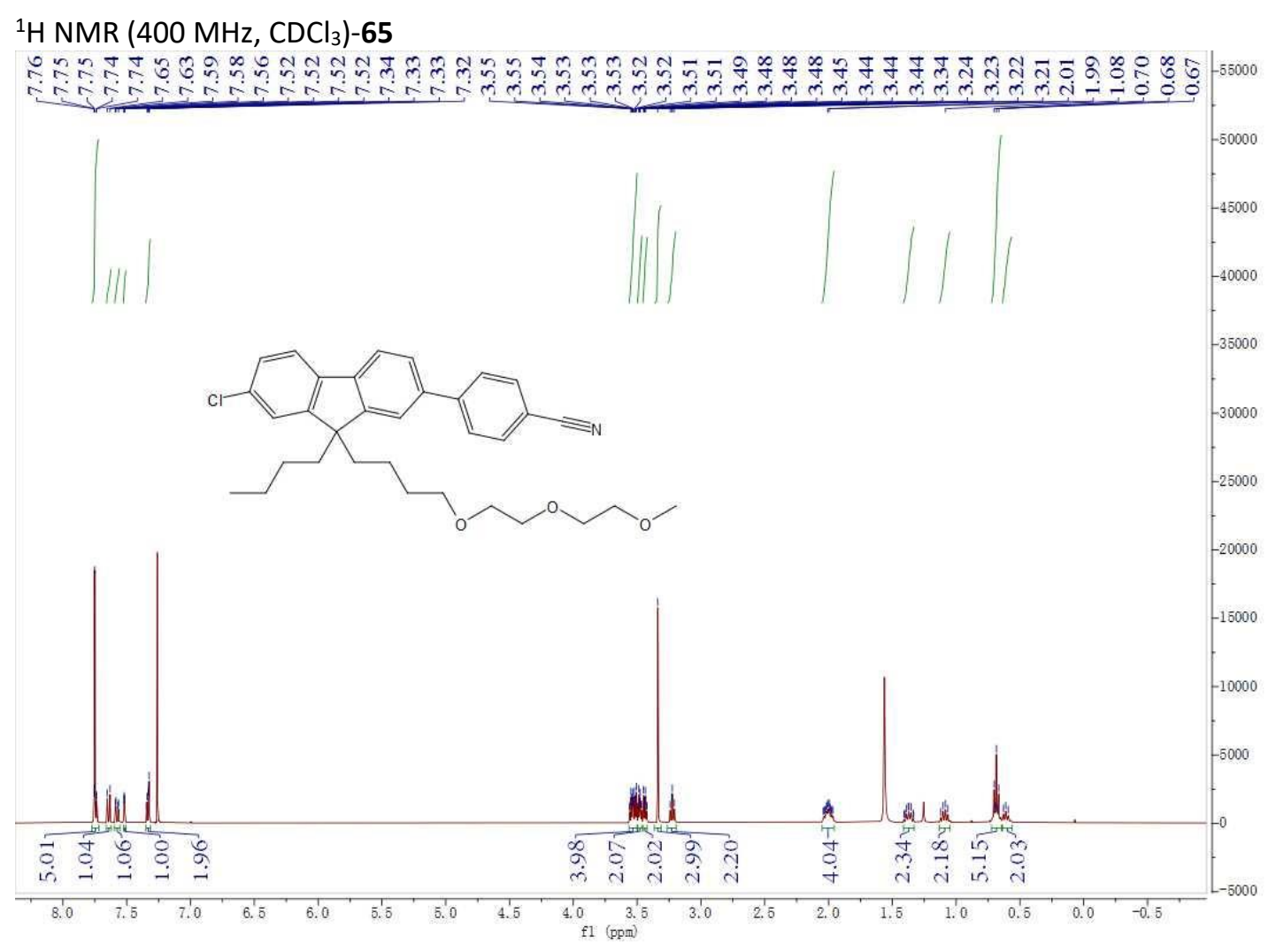

${ }^{13} \mathrm{C}$ NMR $\left(126 \mathrm{MHz}, \mathrm{CDCl}_{3}\right)-65$

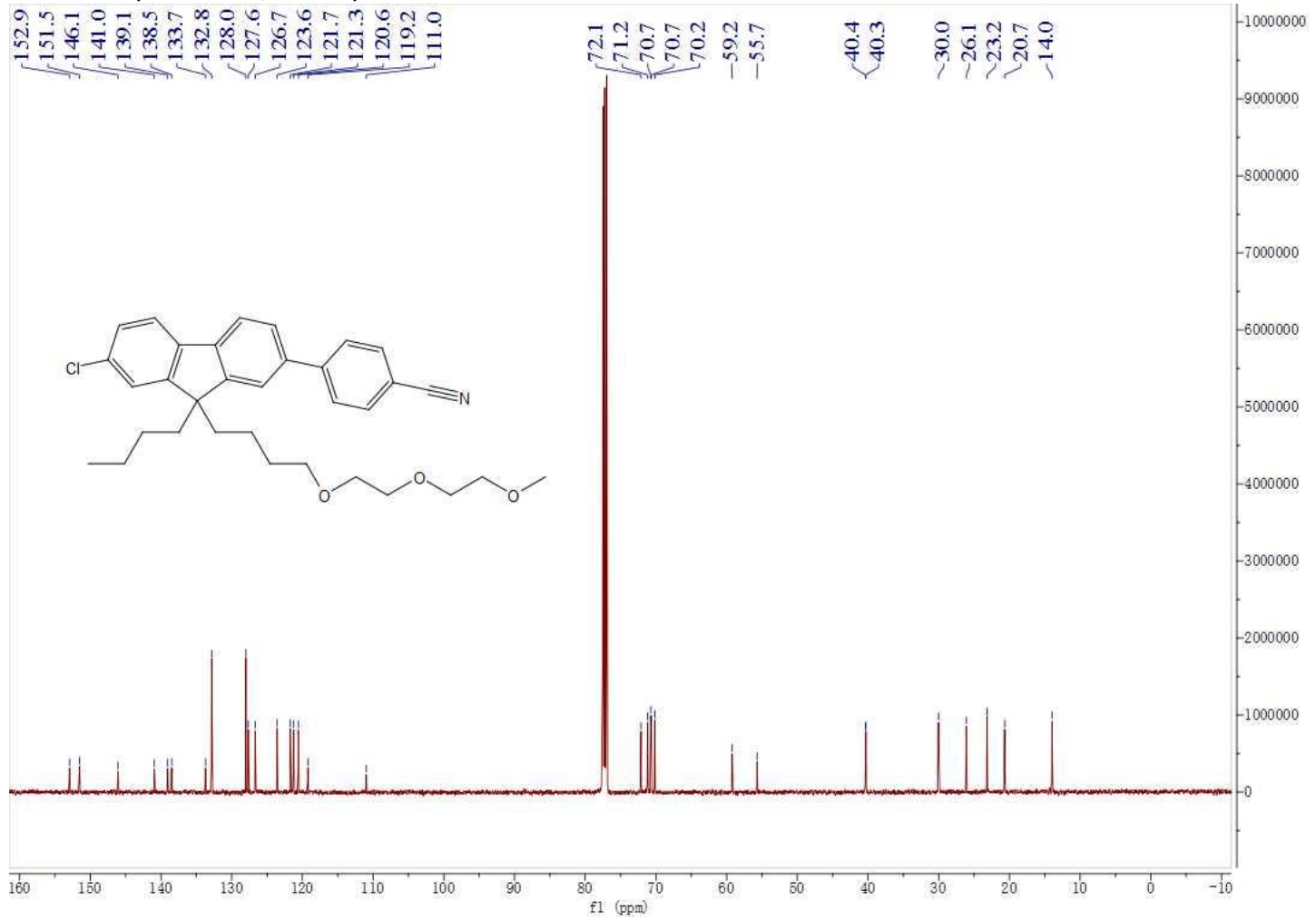




\section{HPLC traces}

L1

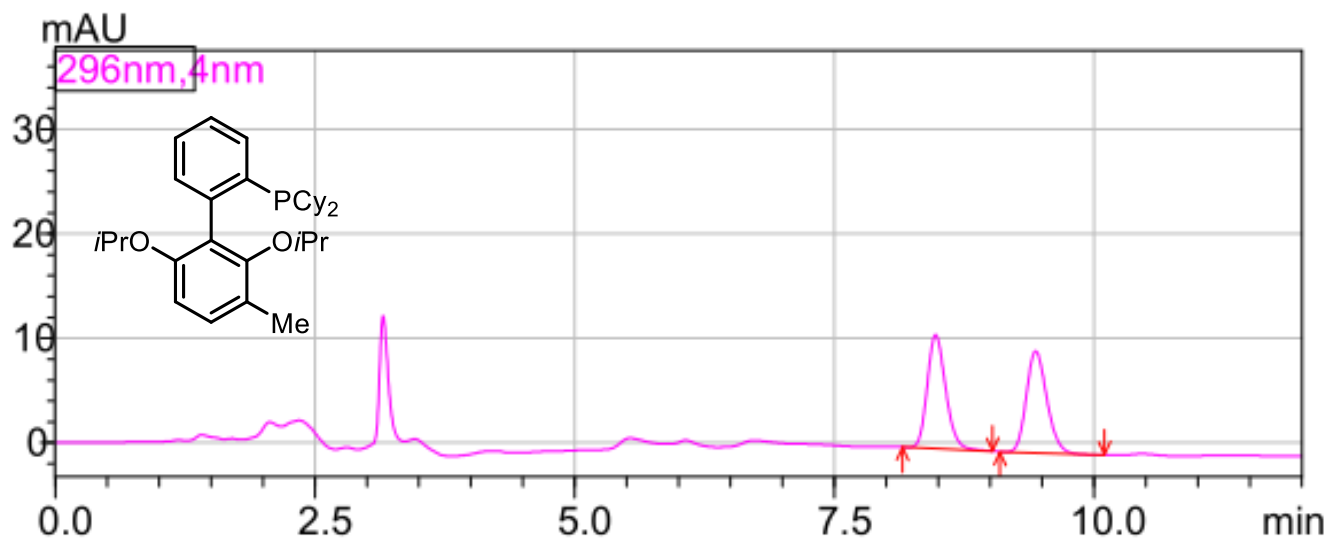

<Peak Table>

PDA Ch1 296nm

\begin{tabular}{|c|c|c|c|c|c|c|c|}
\hline Peak\# & Ret. Time & Area & Height & Mark & Area\% & Peak Start & Peak End \\
\hline 1 & 8.474 & 134690 & 10903 & M & 50.241 & 8.155 & 9.024 \\
\hline 2 & 9.438 & 133396 & 9728 & M & 49.759 & 9.093 & 10.101 \\
\hline Total & & 268086 & 20631 & & 100.000 & & \\
\hline
\end{tabular}

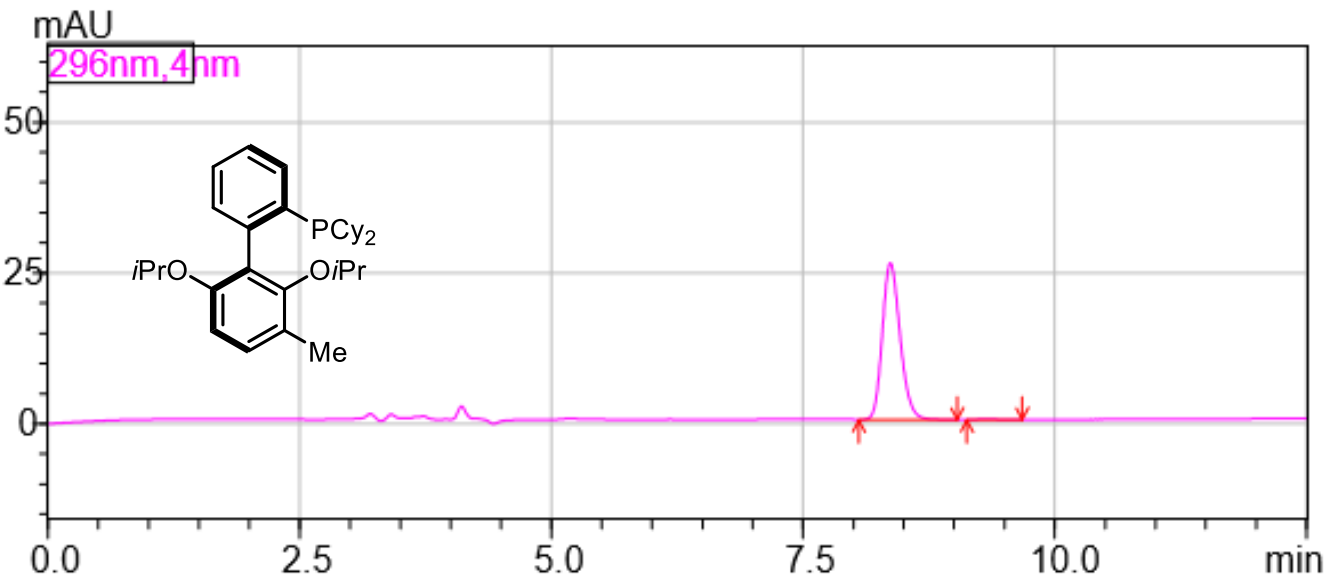

<Peak Table>

PDA Ch1 296nm

\begin{tabular}{|r|r|r|r|c|r|r|r|}
\hline Peak\# & Ret. Time & \multicolumn{1}{c|}{ Area } & \multicolumn{1}{c|}{ Height } & Mark & \multicolumn{1}{c|}{ Area\% } & Peak Start & Peak End \\
\hline 1 & 8.366 & 320654 & 25988 & $\mathrm{M}$ & 99.752 & 8.053 & 9.029 \\
\hline 2 & 9.348 & 797 & 65 & $\mathrm{M}$ & 0.248 & 9.125 & 9.675 \\
\hline Total & & 321451 & 26053 & & 100.000 & & \\
\hline
\end{tabular}




\section{SI-L3}
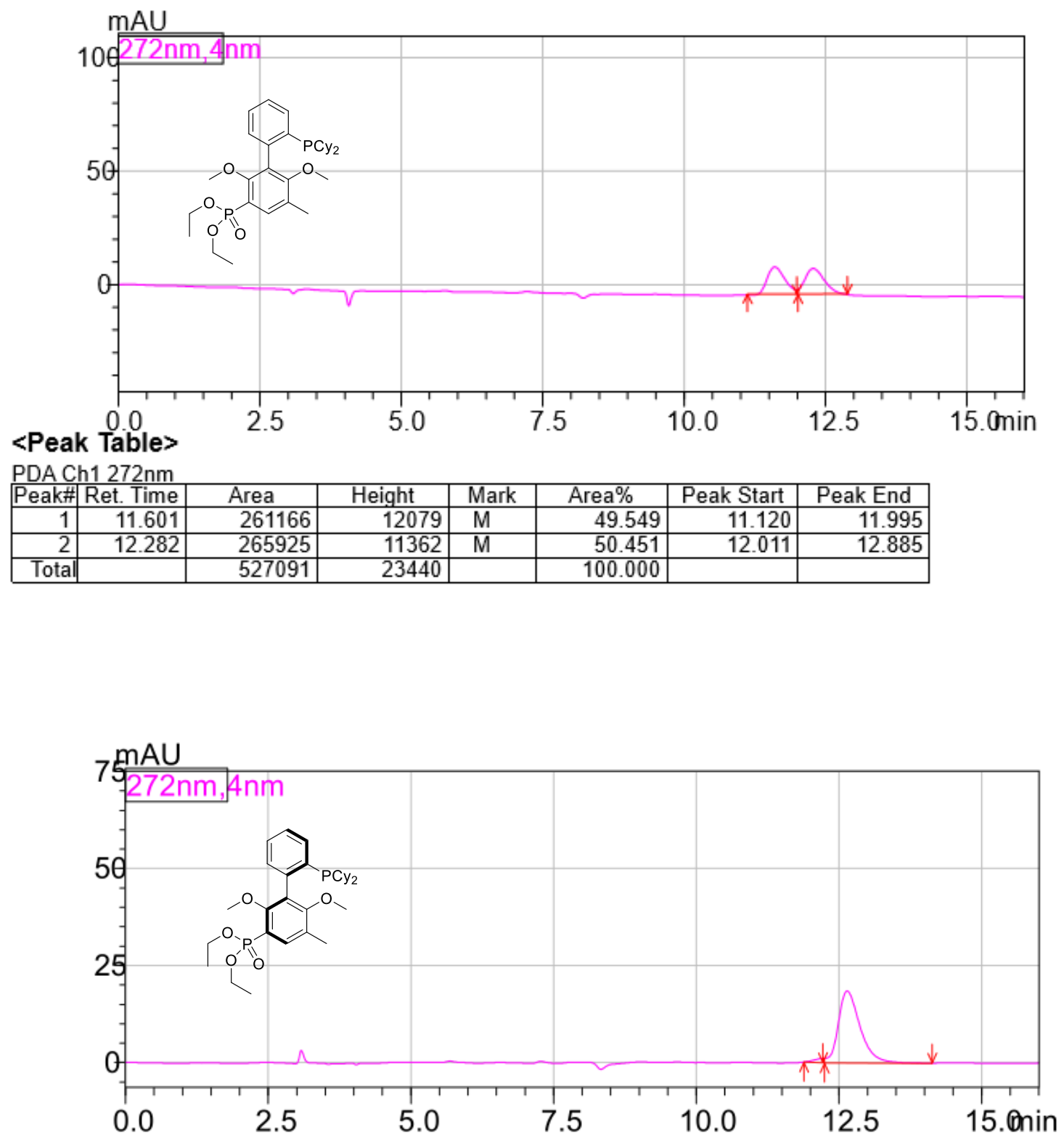

<Peak Table>

PDA Ch1 272nm

Peak\# Ret. Time

\begin{tabular}{|r|r|r|r|l|r|r|r|}
\hline Peak\# & Ret. Time & \multicolumn{1}{|c|}{ Area } & Height & Mark & Area\% & Peak Start & Peak End \\
\hline 1 & 11.885 & 8739 & 1195 & M & 1.392 & 11.685 & 11.888 \\
\hline 2 & 12.265 & 619240 & 24283 & M & 98.608 & 11.931 & 13.696 \\
\hline Total & & 627979 & 25477 & & 100.000 & & \\
\hline
\end{tabular}


1 (Table 1)

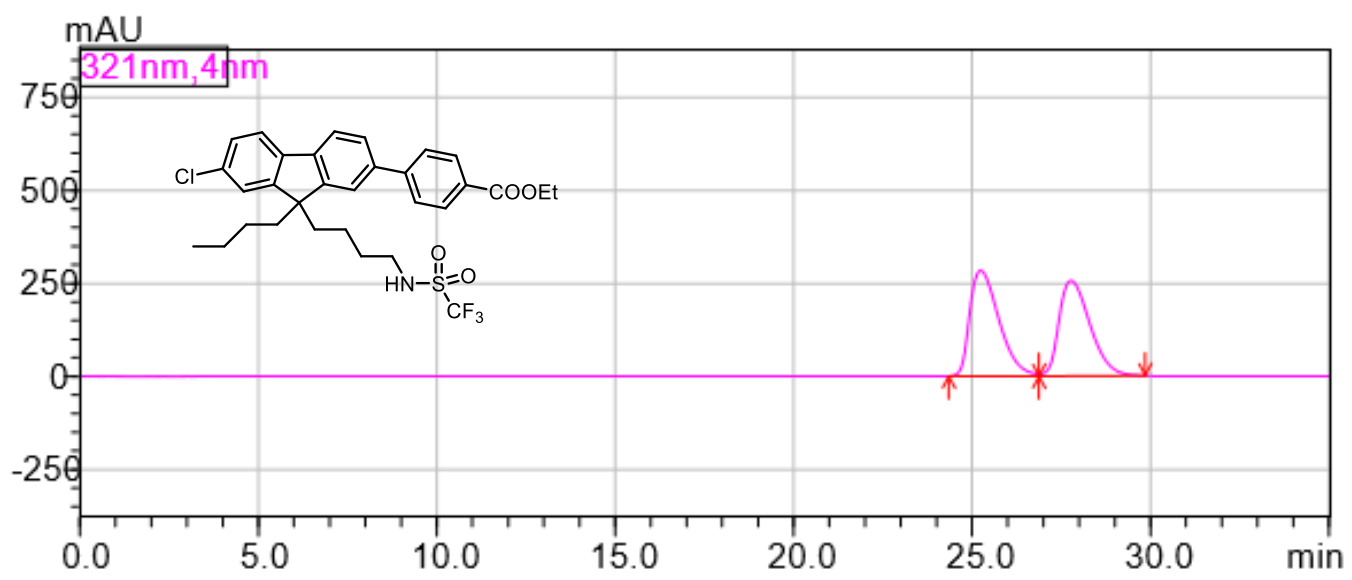

<Peak Table>

PDA Ch1 321nm

Peak\# Ret. Time

\begin{tabular}{|r|r|r|r|r|r|r|}
\hline Peak\# & Ret. Time & \multicolumn{1}{c|}{ Area } & Peak Start & Peak End & Height & \multicolumn{1}{|c|}{ Area\% } \\
\hline 1 & 25.242 & 16069281 & 24.347 & 26.875 & 283170 & 51.049 \\
\hline 2 & 27.774 & 15409018 & 26.875 & 29.856 & 255538 & 48.951 \\
\hline Total & & 31478299 & & & 538707 & 100.000 \\
\hline
\end{tabular}

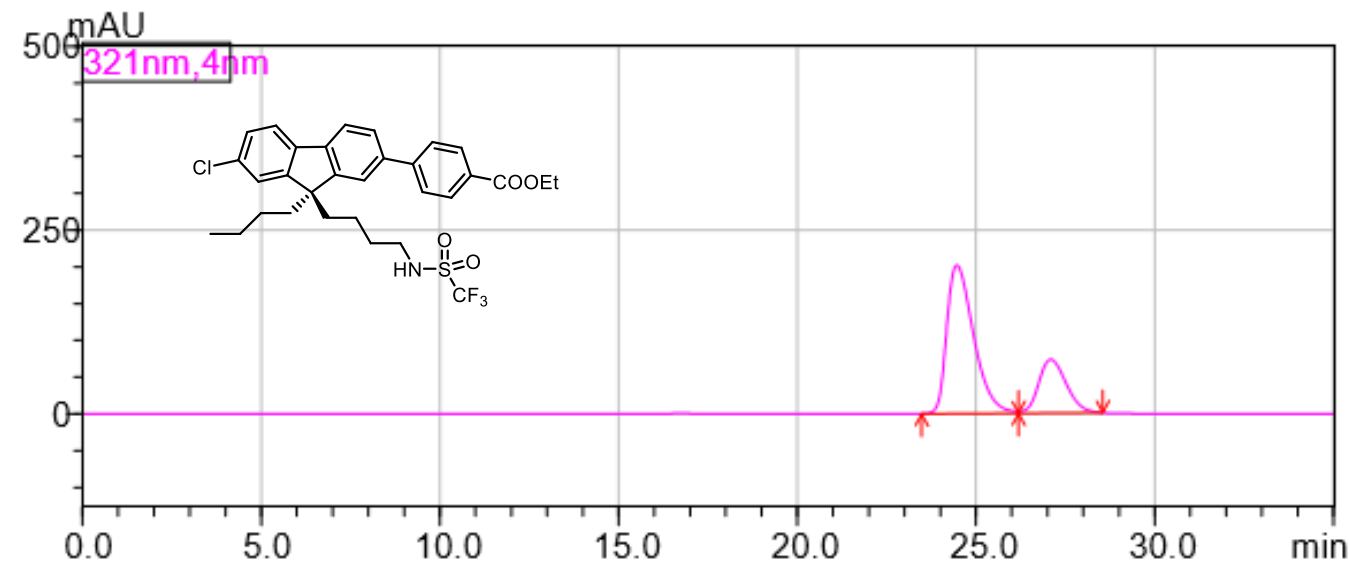

<Peak Table>

PDA Ch1 321nm

\begin{tabular}{|r|r|r|r|r|r|r|}
\hline Peak\# & Ret. Time & \multicolumn{1}{|c|}{ Area } & \multicolumn{1}{c|}{ Height } & Peak Start & Peak End & \multicolumn{1}{c|}{ Area\% } \\
\hline 1 & 24.466 & 10527717 & 201750 & 23.477 & 26.187 & 72.943 \\
\hline 2 & 27.093 & 3905045 & 72832 & 26.187 & 28.549 & 27.057 \\
\hline Total & & 14432761 & 274582 & & & 100.000 \\
\hline
\end{tabular}


4 (Table 1)

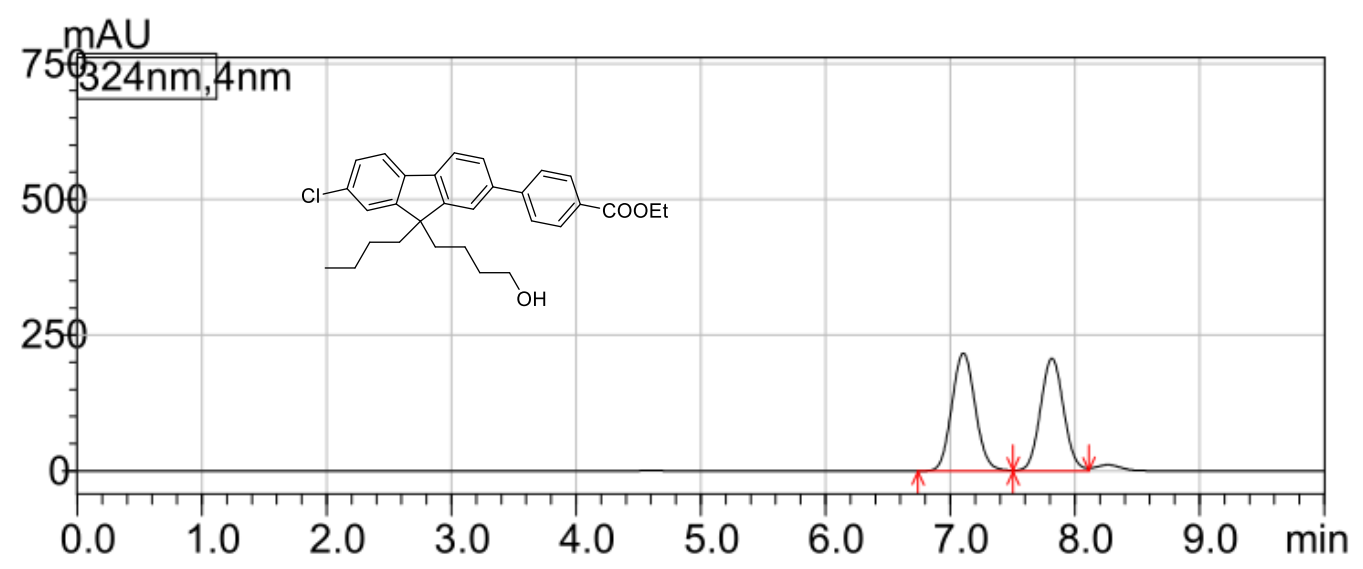

<Peak Table>

\begin{tabular}{|c|c|c|c|c|c|c|c|}
\hline \multicolumn{8}{|c|}{ PDA Ch1 325nm } \\
\hline Peak\# & t. Time & Area & Height & Mark & Area\% & Peak Start & Peak End \\
\hline 1 & 7.105 & 2764300 & 213258 & $\mathrm{M}$ & 50.687 & 6.757 & 7.461 \\
\hline 2 & 7.815 & 2689339 & 205076 & $\mathrm{M}$ & 49.313 & 7.541 & 8.181 \\
\hline Total & & 5453638 & 418334 & & 100.000 & & \\
\hline
\end{tabular}

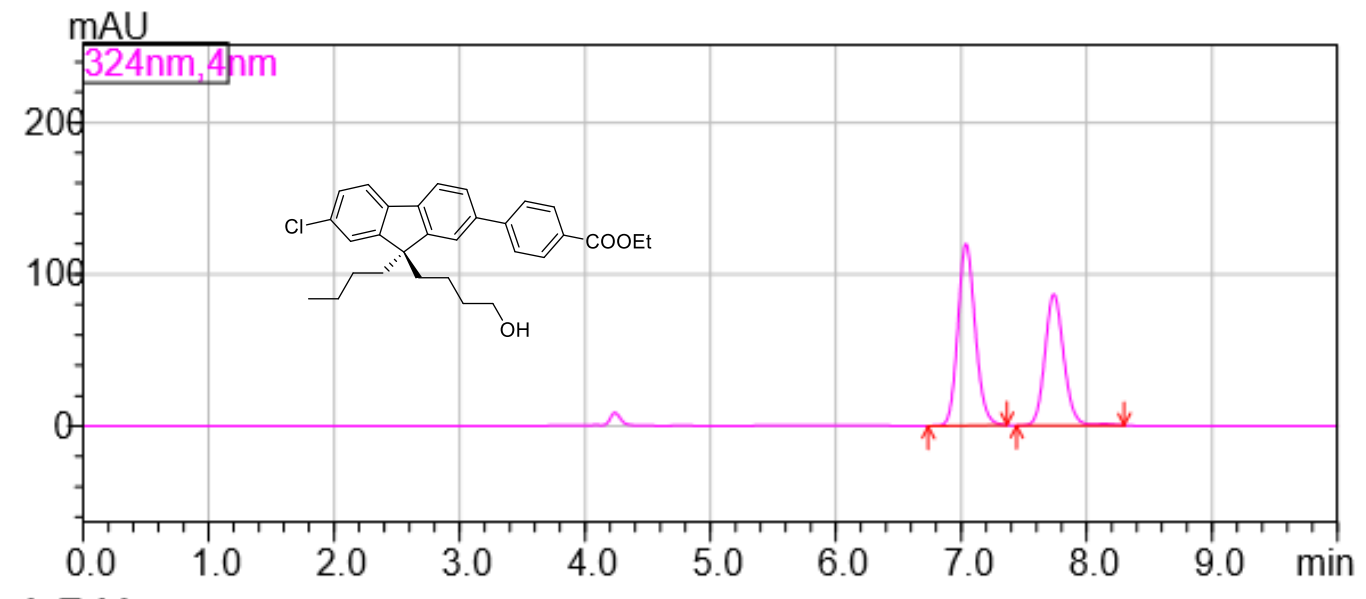

<Peak Table>

\begin{tabular}{|c|c|c|c|c|c|c|c|}
\hline Peak & Ret Timo & $\Delta r e$ & & Mart & $\sin 2$ \& & & Donk Fod \\
\hline 1 & 7.038 & 1158012 & 119562 & $M$ & 55.985 & 6.736 & 7.365 \\
\hline 2 & 7.738 & 910414 & 86520 & $\bar{M}$ & 44.015 & 7.440 & 8.299 \\
\hline Tota & & 2068426 & 206082 & & 100.000 & & \\
\hline
\end{tabular}


5 (Table 1)
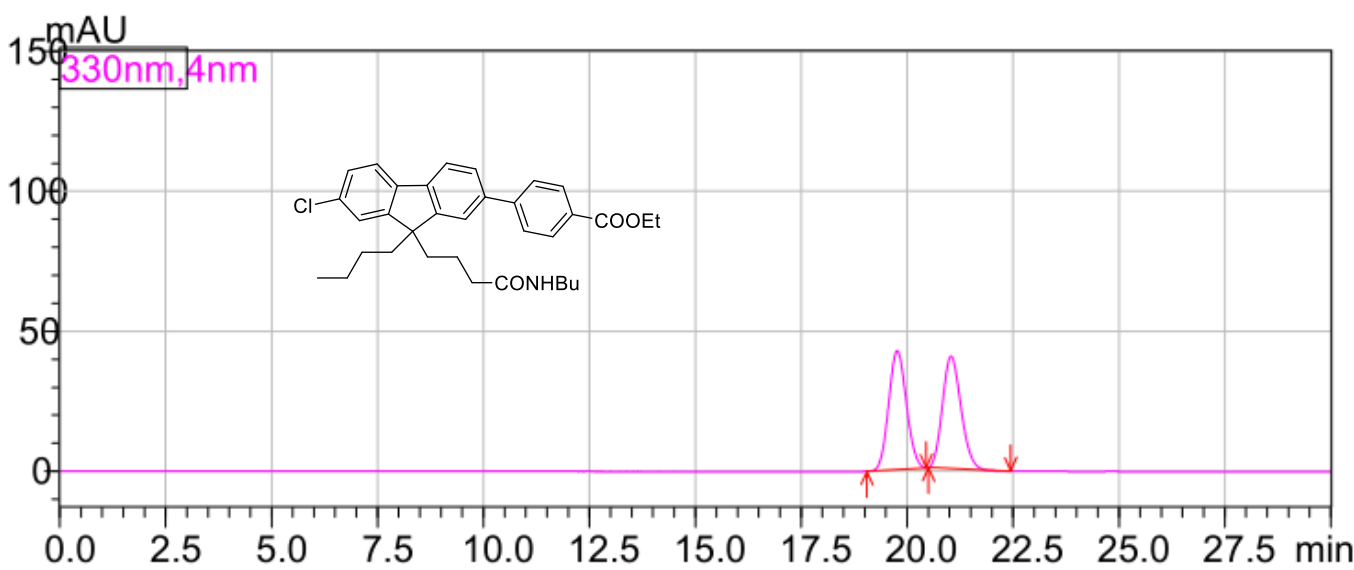

<Peak Table>

PDA Ch1 330nm

\begin{tabular}{|r|r|r|r|r|r|r|r|}
\hline Peak\# Ret. Time & Area & \multicolumn{1}{|c|}{ Height } & Mark & \multicolumn{1}{|c|}{ Area\% } & Peak Start & Peak End \\
\hline 1 & 19.765 & 1240436 & 42511 & M & 50.308 & 19.045 & 20.448 \\
\hline 2 & 21.037 & 1225238 & 40109 & M & 49.692 & 20.507 & 22.443 \\
\hline Total & & 2465673 & 82620 & & 100.000 & & \\
\hline
\end{tabular}

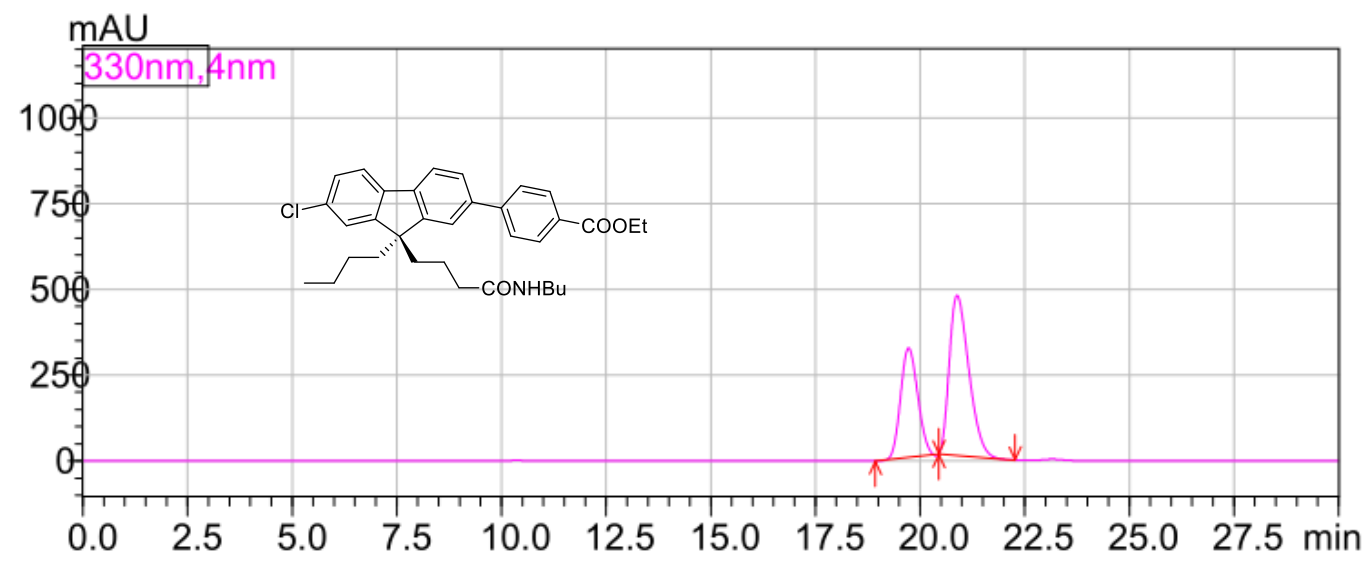

<Peak Table>

PDA Ch1 330nm

\begin{tabular}{|c|c|c|c|c|c|c|c|}
\hline Peak\# & et. Time & Area & Height & Mark & Area\% & Peak Start & Peak End \\
\hline 1 & 19.727 & 8982205 & 318138 & $\mathrm{M}$ & 37.279 & 18.928 & 20.448 \\
\hline 2 & 20.885 & 15112260 & 465848 & M & 62.721 & 20.448 & 22.267 \\
\hline Total & & 24094465 & 783986 & & 100.000 & & \\
\hline
\end{tabular}


6 (Table 1)

mAU

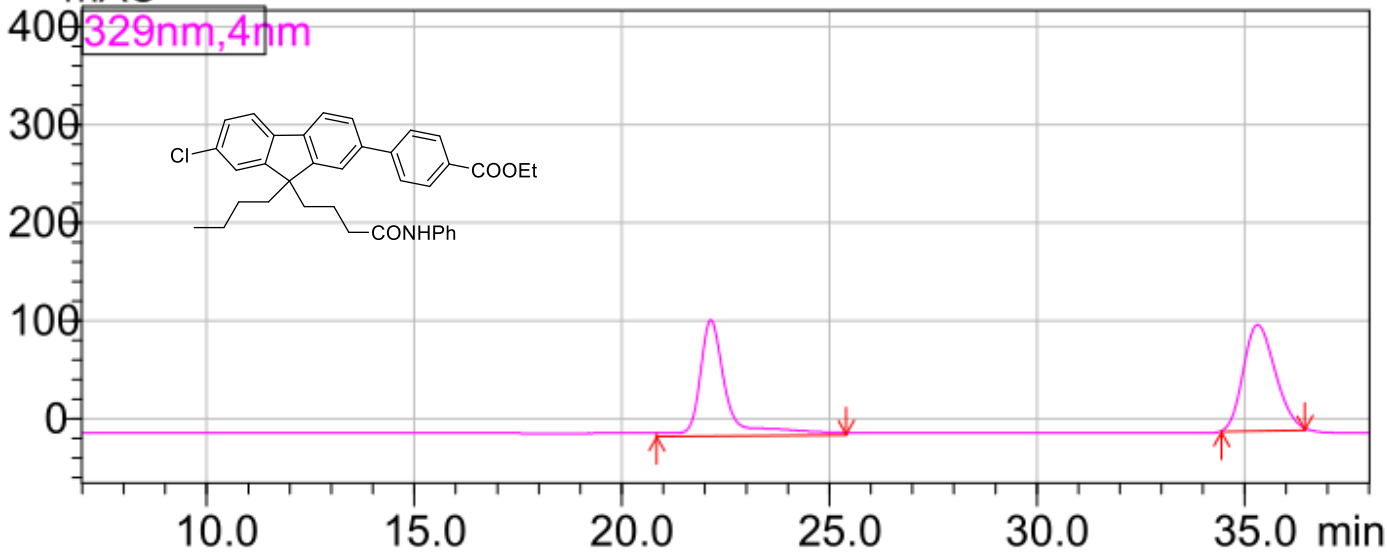

<Peak Table>

PDA Ch1 329nm

Peak\# Ret. Time

\begin{tabular}{|r|r|r|r|r|r|r|}
\hline Peak\# & Ret. Time & Area & Height & \multicolumn{1}{c|}{ Area\% } & Peak Start & Peak End \\
\hline 1 & 22.142 & 5394166 & 118544 & 48.033 & 20.843 & 25.397 \\
\hline 2 & 35.316 & 5835976 & 108458 & 51.967 & 34.443 & 36.453 \\
\hline Total & & 11230142 & 227002 & 100.000 & & \\
\hline
\end{tabular}

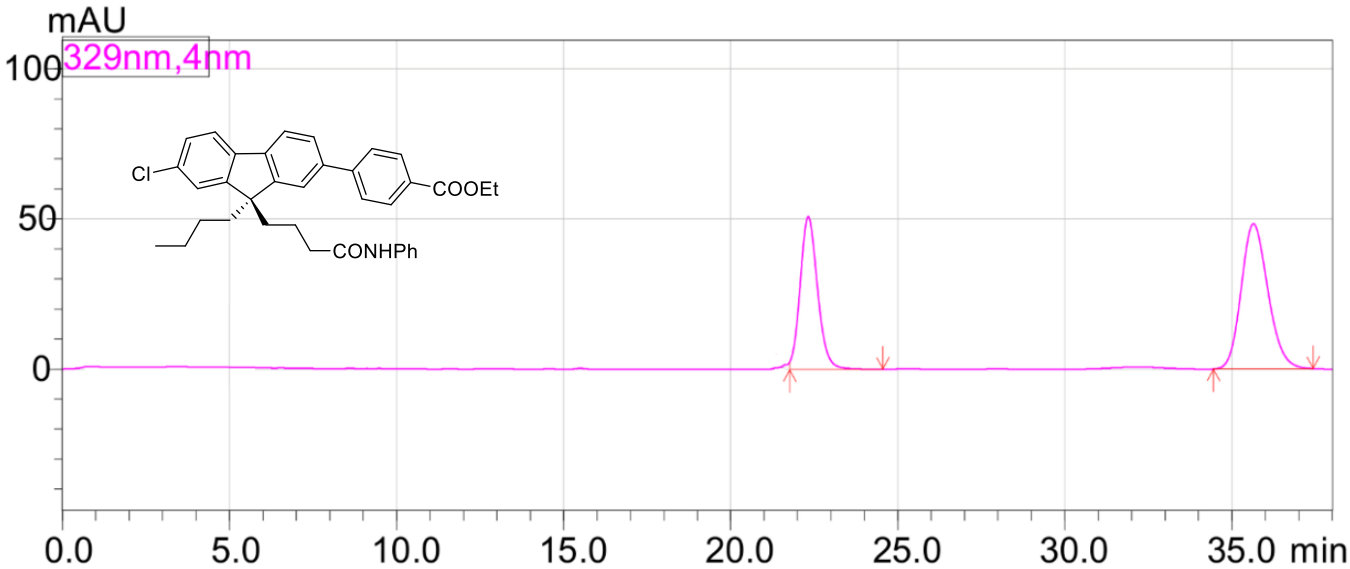

<Peak Table>

PDA Ch1 329nm

\begin{tabular}{|c|c|c|c|c|c|c|}
\hline Peak\# & Ret. Time & Area & Height & Area\% & Peak Start & Peak End \\
\hline 1 & 22.320 & 1832972 & 50802 & 40.480 & 21.765 & 24.549 \\
\hline 2 & 35.646 & 2695129 & 48339 & 59.520 & 34.453 & 37.429 \\
\hline Total & & 4528101 & 99141 & 100.000 & & \\
\hline
\end{tabular}


Substrate scope (Table 2 and Scheme 3)

7
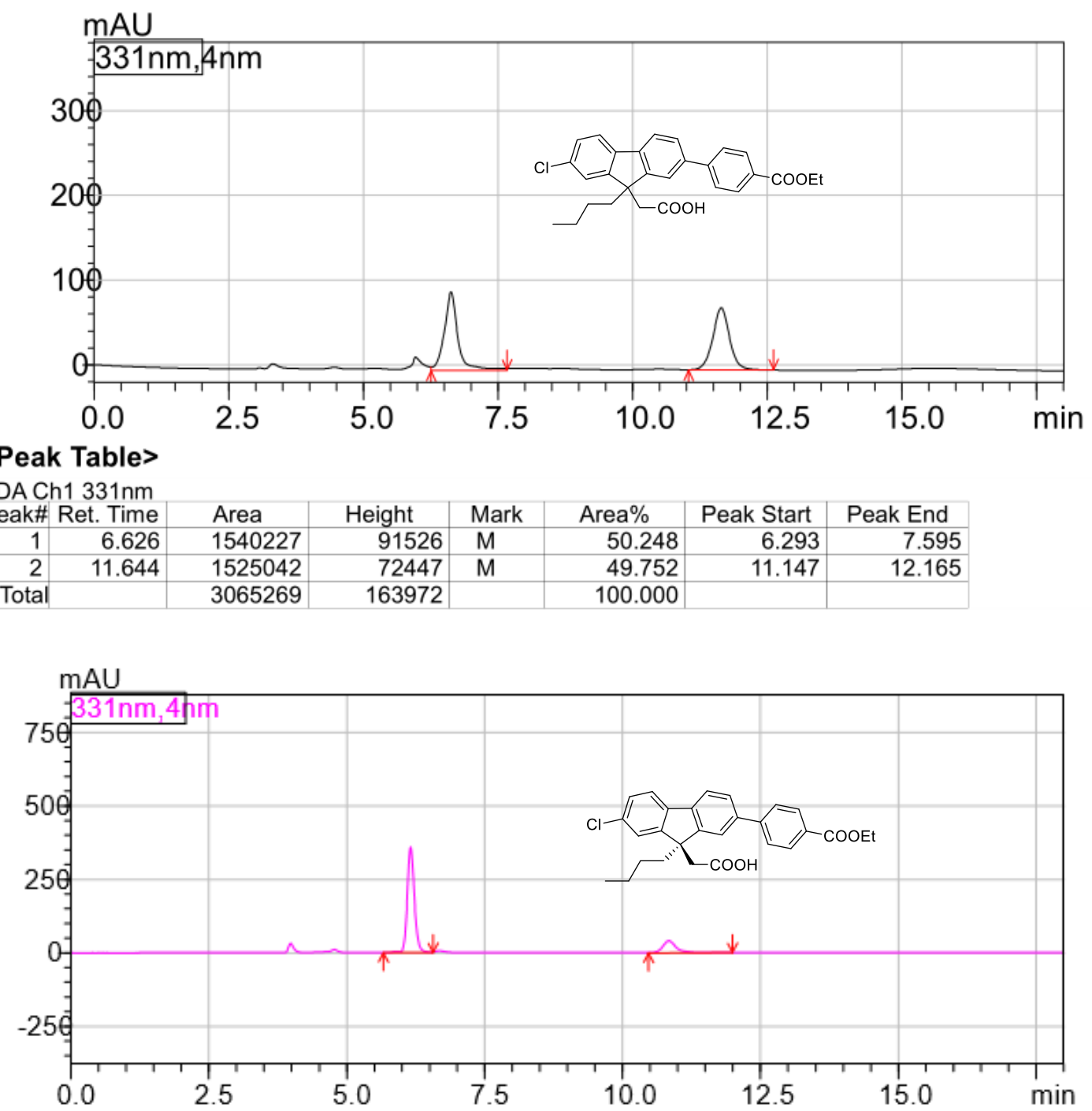

<Peak Table>

PDA Ch1 331nm

\begin{tabular}{|r|r|r|r|r|r|r|r|}
\hline Peak\# & Ret. Time & \multicolumn{1}{c|}{ Area } & \multicolumn{1}{c|}{ Height } & Mark & Area\% & Peak Start & Peak End \\
\hline 1 & 6.157 & 3261362 & 359661 & M & 82.623 & 5.664 & 6.565 \\
\hline 2 & 10.840 & 685925 & 40779 & M & 17.377 & 10.469 & 11.995 \\
\hline Total & & 3947287 & 400441 & & 100.000 & & \\
\hline
\end{tabular}




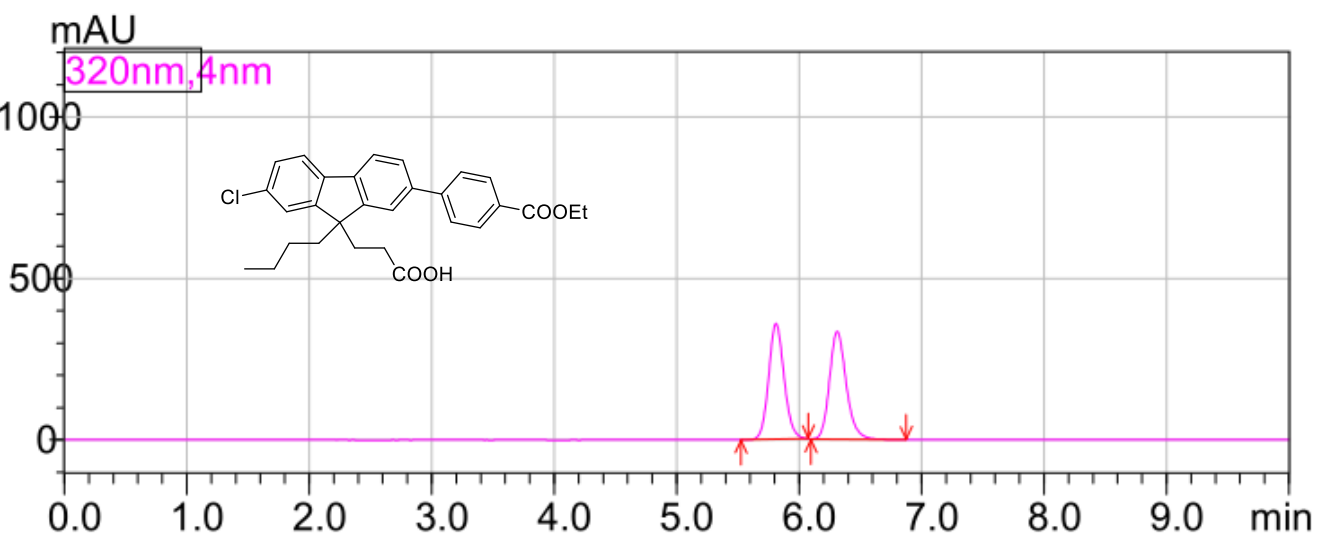

<Peak Table>

PDA Ch1 320nm

\begin{tabular}{|r|r|r|r|l|r|r|r|}
\hline Peak\# Ret. Time & Area & \multicolumn{1}{|c|}{ Height } & Mark & Area\% & Peak Start & Peak End \\
\hline 1 & 5.811 & 3148595 & 358360 & M & 49.862 & 5.525 & 6.075 \\
\hline 2 & 6.310 & 3166084 & 333331 & M & 50.138 & 6.096 & 6.875 \\
\hline Total & & 6314679 & 691691 & & 100.000 & & \\
\hline
\end{tabular}

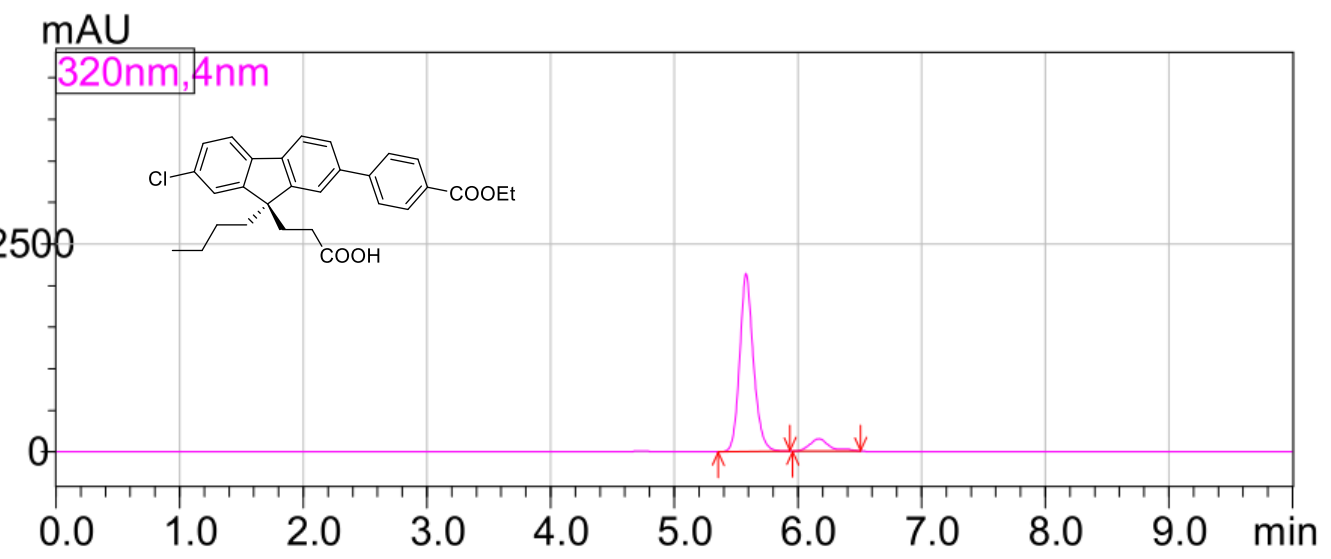

<Peak Table>

PDA Ch1 320nm

\begin{tabular}{|r|r|r|r|l|r|r|r|}
\hline Peak\# Ret. Time & \multicolumn{1}{|c|}{ Area } & \multicolumn{1}{|c|}{ Height } & Mark & Area\% & Peak Start & Peak End \\
\hline 1 & 5.580 & 16589232 & 2145980 & M & 90.595 & 5.355 & 5.936 \\
\hline 2 & 6.169 & 1722235 & 150925 & M & 9.405 & 5.957 & 6.507 \\
\hline Total & & 18311467 & 2296905 & & 100.000 & & \\
\hline
\end{tabular}


3

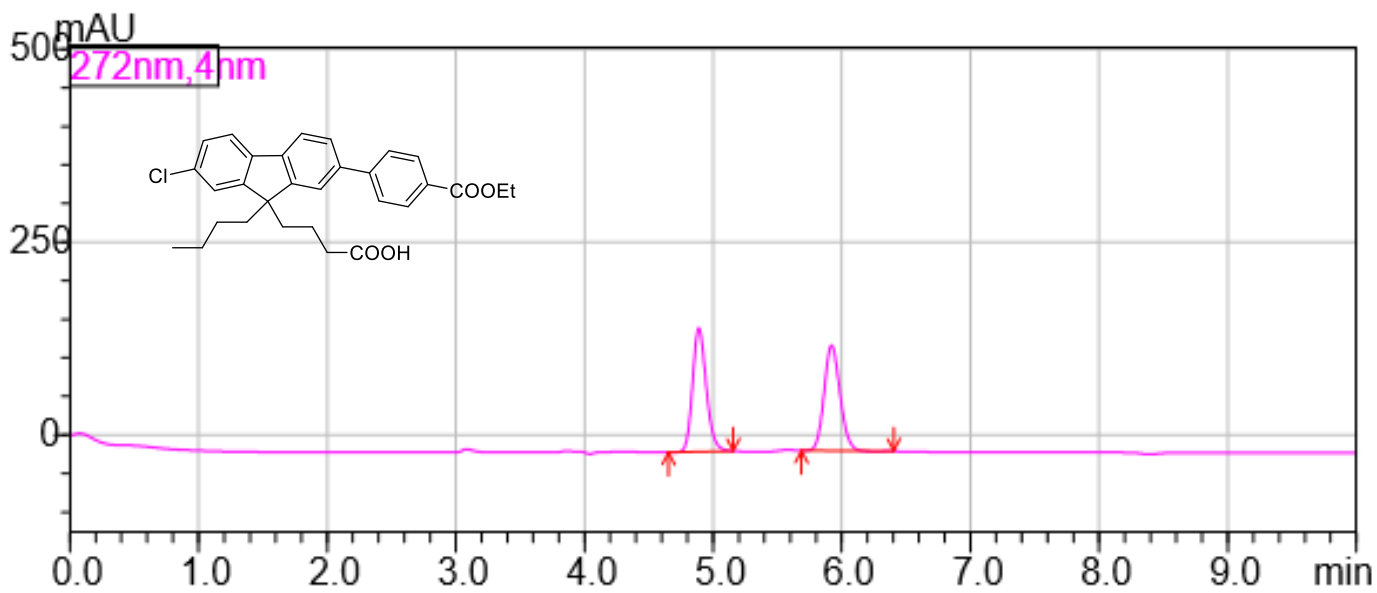

<Peak Table>

PDA Ch1 272nm

\begin{tabular}{|c|c|c|c|c|c|c|c|}
\hline eak\# & Ret. Time & Area & Height & Mark & Area $\%$ & Peak Start & Peak End \\
\hline 1 & 4.889 & 1195937 & 160467 & $\mathrm{M}$ & .337 & 4.651 & 5.157 \\
\hline 2 & 5.921 & 1179907 & 136881 & $\mathrm{M}$ & 49.663 & 5.685 & 6.405 \\
\hline otal & & 2375844 & 297348 & & 100.000 & & \\
\hline
\end{tabular}

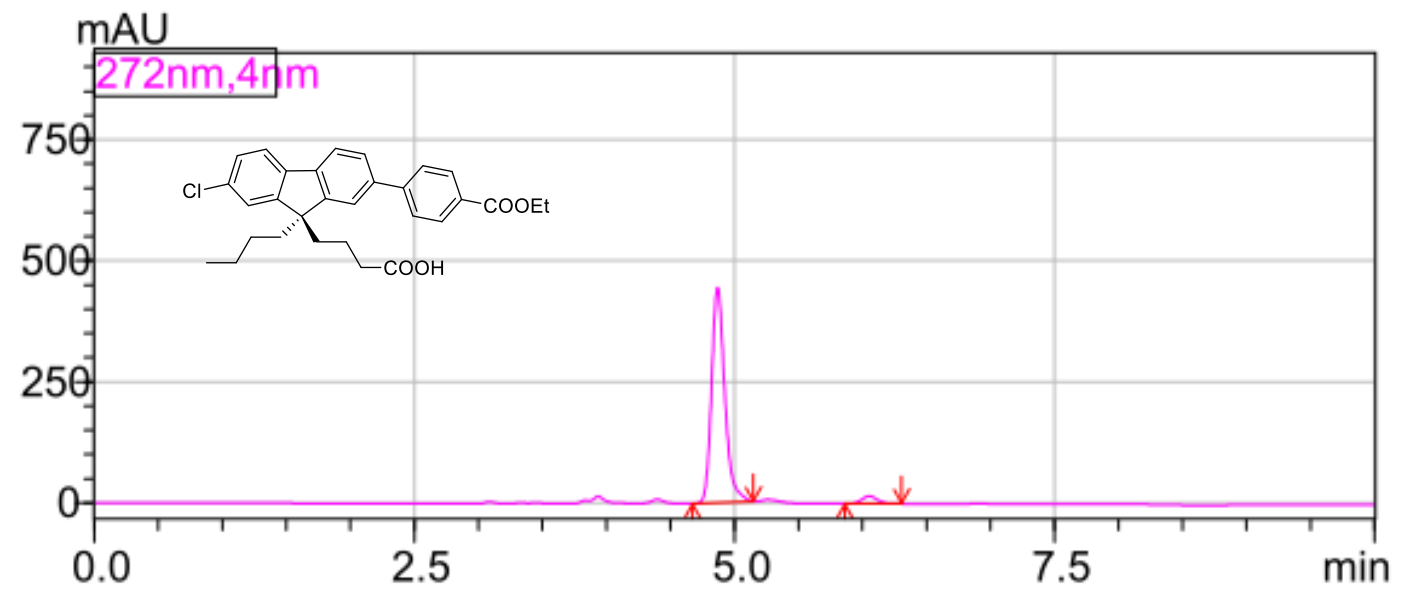

<Peak Table>

PDA Ch1 272nm

Peak\# Ret. Time

\begin{tabular}{|r|r|r|r|r|r|r|}
\hline Peak\# & Ret. Time & \multicolumn{1}{|c|}{ Area } & Height & \multicolumn{1}{c|}{ Area\% } & Peak Start & Peak End \\
\hline 1 & 4.868 & 3205662 & 444990 & 96.019 & 4.672 & 5.147 \\
\hline 2 & 6.053 & 132915 & 15550 & 3.981 & 5.861 & 6.304 \\
\hline Total & & 3338577 & 460540 & 100.000 & & \\
\hline
\end{tabular}




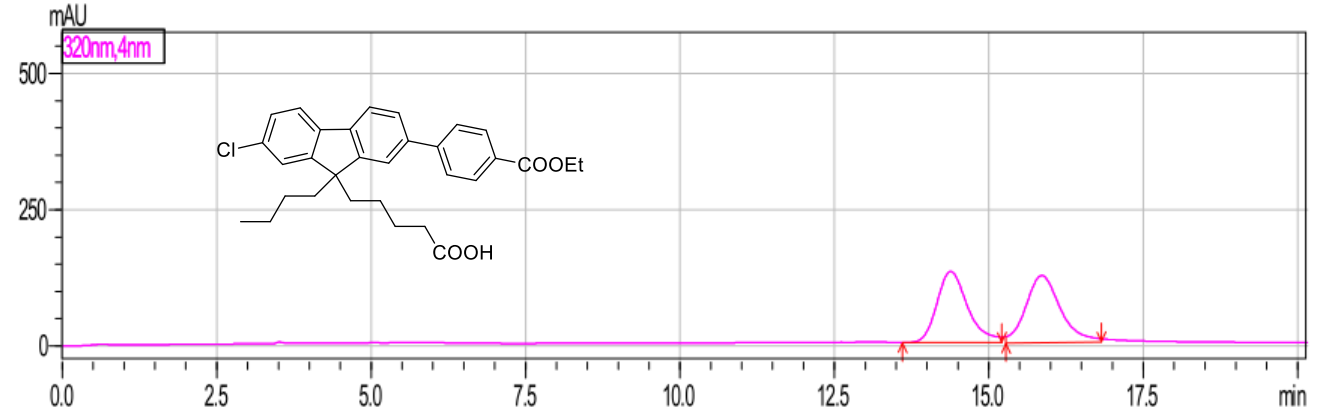

<Peak Table>

PDA Ch1 320nm

Peak\# Ret. Time

\begin{tabular}{|r|r|l|l|r|}
\hline 1 & 14.382 & 4571832 & & Area\% \\
\hline 2 & 15.857 & 4809141 & M & 48.735 \\
\hline Total & & 9380973 & & 100.000 \\
\hline
\end{tabular}

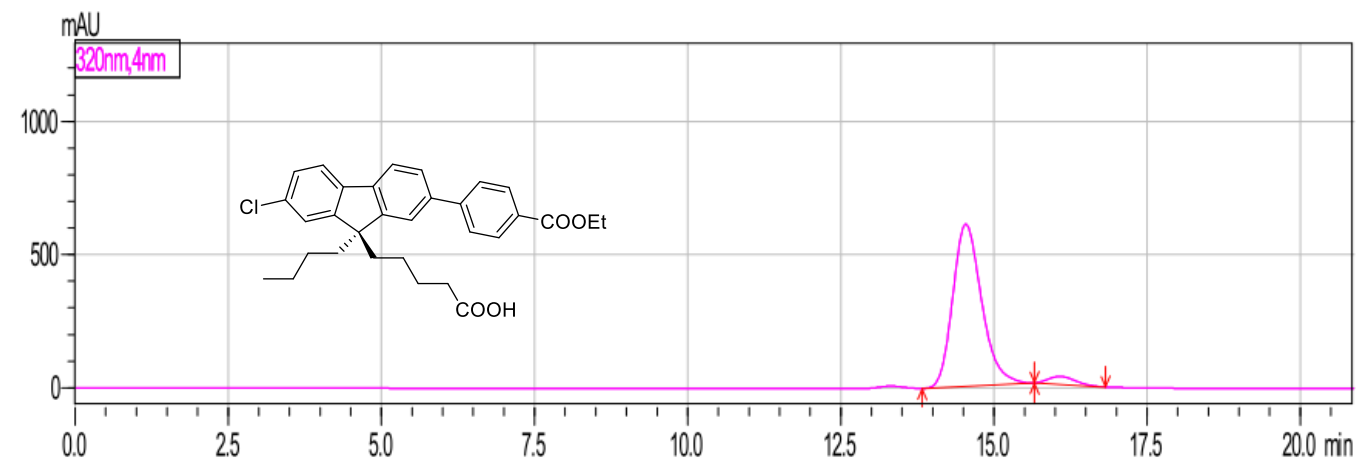

<Peak Table>

PDA Ch1 320nm

\begin{tabular}{|r|r|r|l|r|}
\hline Peak\# Ret. Time & \multicolumn{1}{|c|}{ Area } & Unit & Area\% \\
\hline 1 & 14.540 & 19987622 & & 95.620 \\
\hline 2 & 16.074 & 915481 & & 4.380 \\
\hline Total & & 20903103 & & 100.000 \\
\hline
\end{tabular}




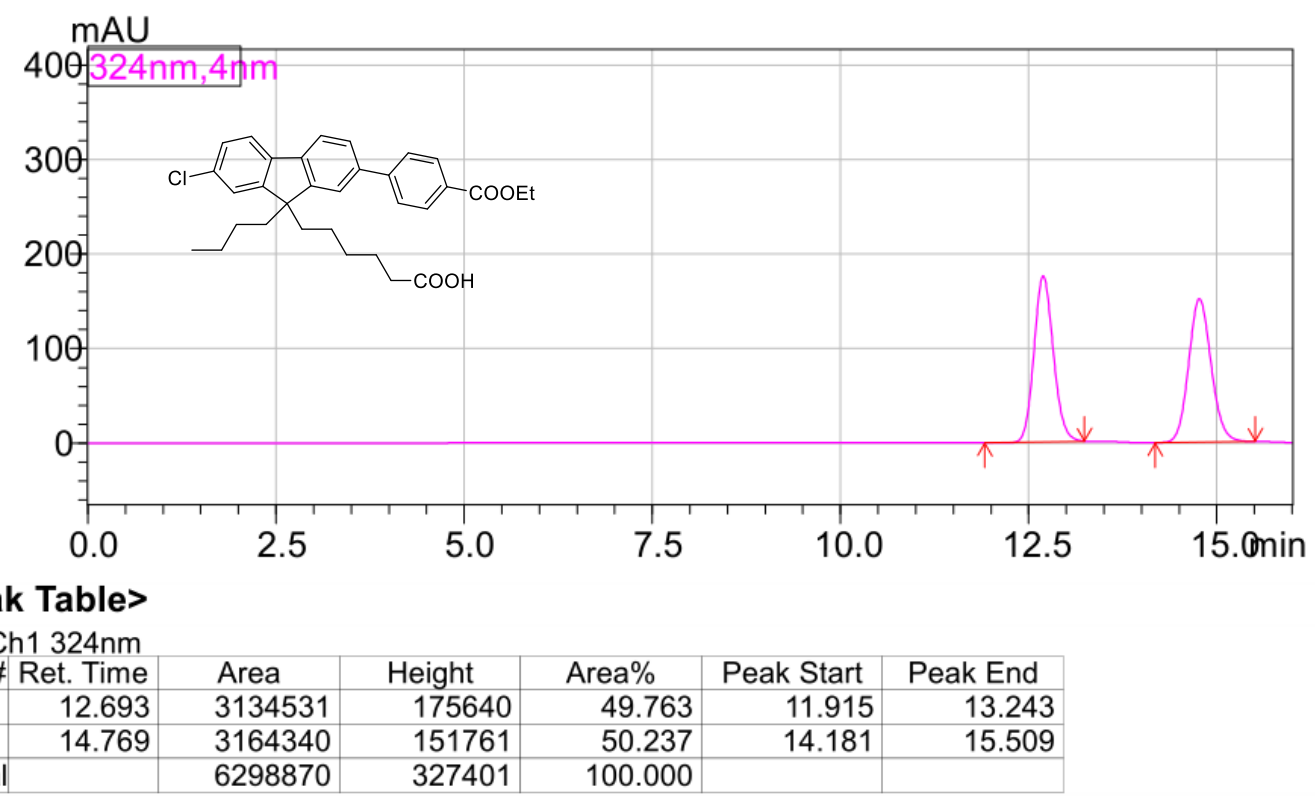

$\mathrm{mAU}$

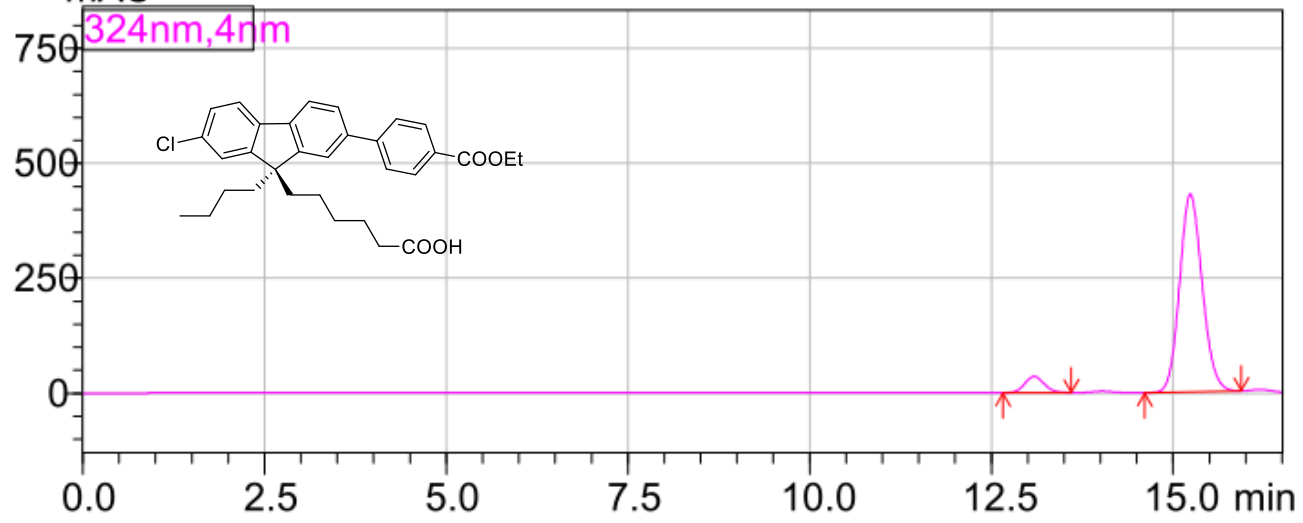

<Peak Table>

PDA Ch1 324nm

Peak\# Ret. Time

\begin{tabular}{|c|c|c|c|c|c|c|}
\hline Peak\# & Ret. Time & Area & Height & Area\% & Peak Start & Peak End \\
\hline 1 & 13.086 & 664913 & 35466 & 6.645 & 12.656 & 13.595 \\
\hline 2 & 15.236 & 9342030 & 430358 & 93.355 & 14.608 & 15.936 \\
\hline Total & & 10006943 & 465823 & 100.000 & & \\
\hline
\end{tabular}




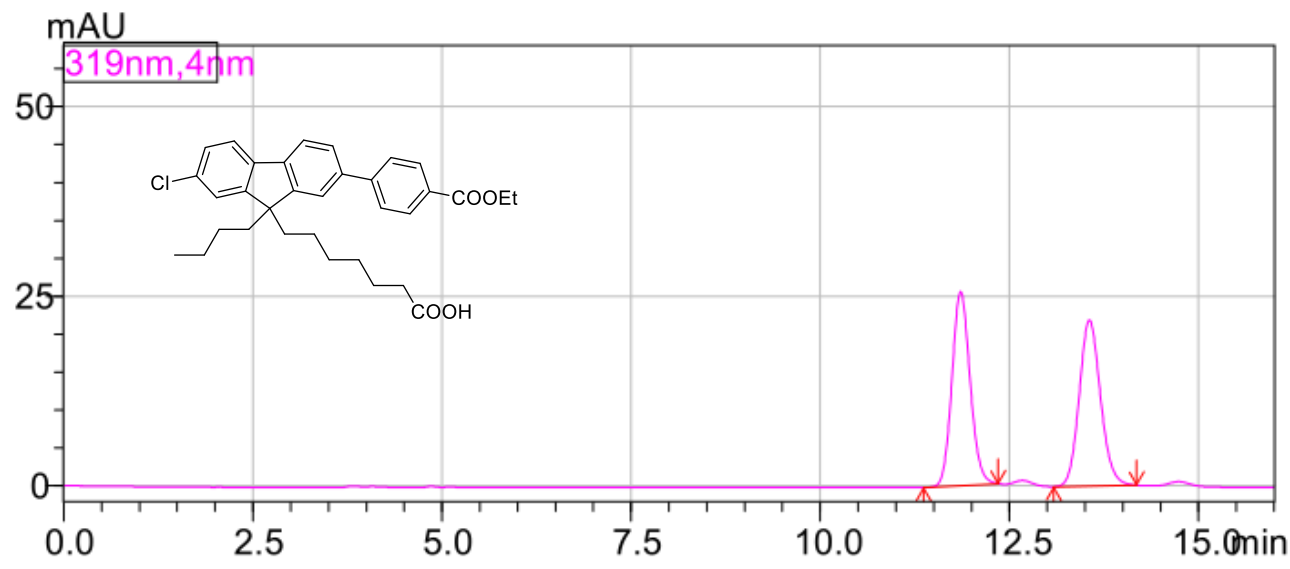

<Peak Table>

PDA Ch1 319nm

\begin{tabular}{|r|r|r|c|r|r|r|r|}
\hline Peak\# Ret. Time & \multicolumn{1}{|c|}{ Height } & Mark & Area\% & Area/Height & Peak Start & Peak End \\
\hline 1 & 11.855 & 25567 & $\mathrm{M}$ & 49.793 & 16.704 & 11.365 & 12.347 \\
\hline 2 & 13.558 & 21908 & $\mathrm{M}$ & 50.207 & 19.655 & 13.088 & 14.181 \\
\hline Total & & 47476 & & 100.000 & & & \\
\hline
\end{tabular}

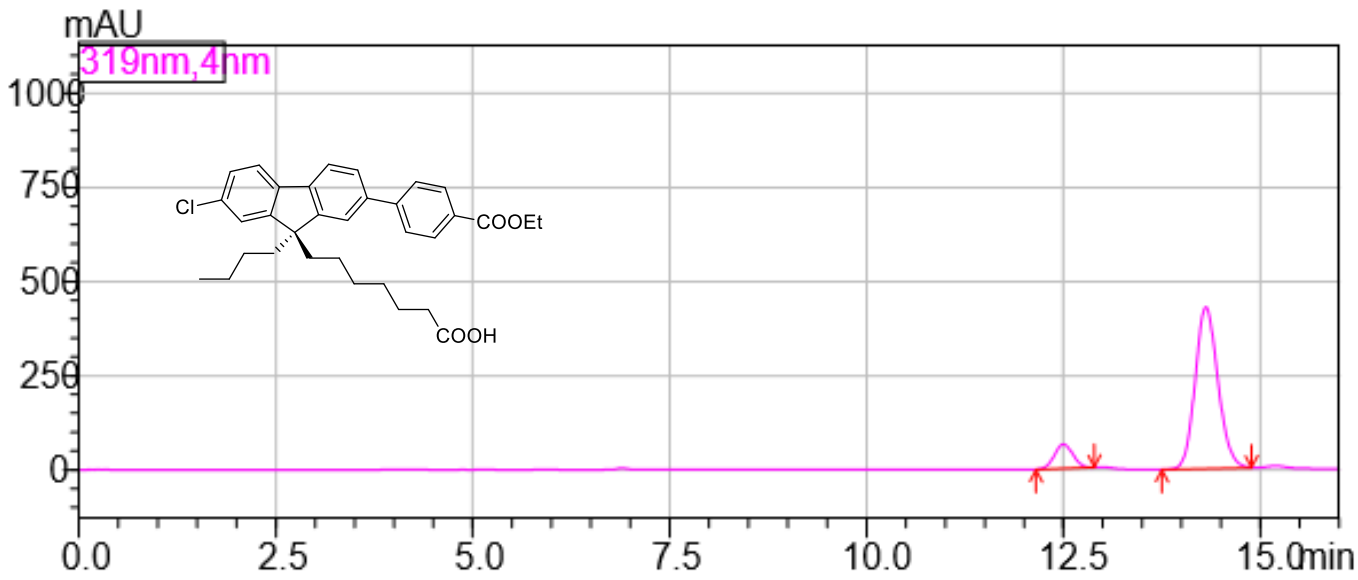

<Peak Table>

PDA Ch1 319nm

\begin{tabular}{|r|r|}
\hline Peak\# & Ret. Time \\
\hline 1 & 12501 \\
\hline
\end{tabular}

\begin{tabular}{|r|r|r|r|r|r|r|}
\hline Peak\# & Ret. Time & Area & \multicolumn{1}{|c|}{ Height } & \multicolumn{1}{|c|}{ Area\% } & Peak Start & Peak End \\
\hline 1 & 12.501 & 1059047 & 64136 & 10.922 & 12.149 & 12.891 \\
\hline 2 & 14.308 & 8637379 & 429041 & 89.078 & 13.749 & 14.885 \\
\hline Total & & 9696426 & 493176 & 100.000 & & \\
\hline
\end{tabular}




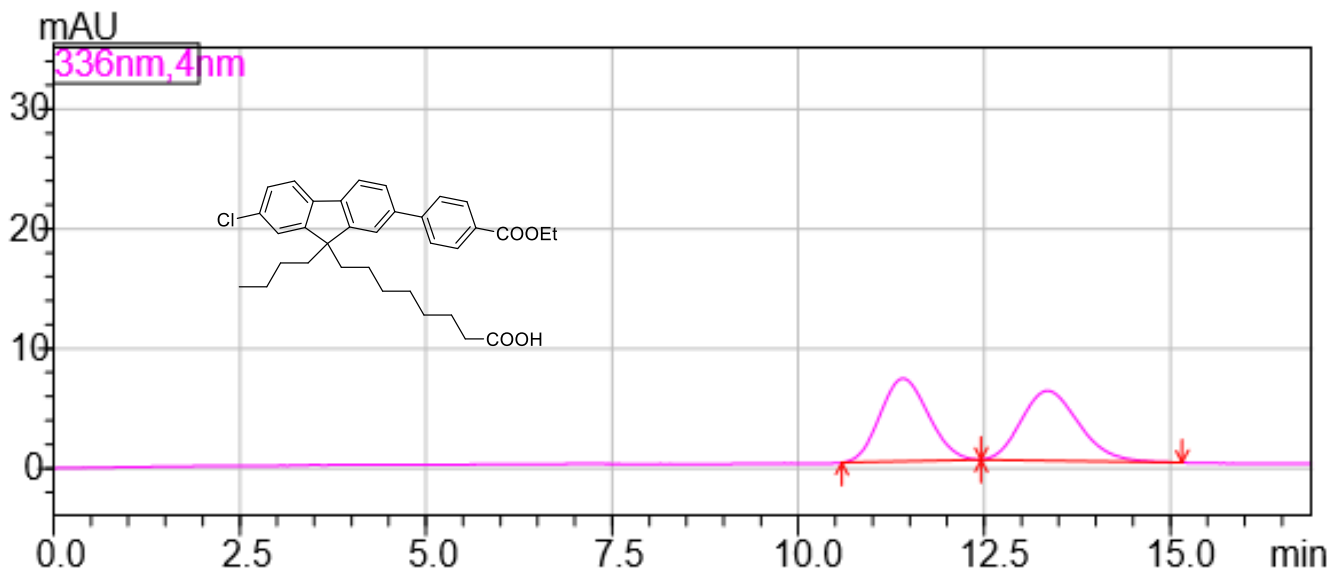

<Peak Table>

PDA Ch1 336nm

\begin{tabular}{|r|r|r|r|r|r|r|r|}
\hline Peak\# & Ret. Time & \multicolumn{1}{c|}{ Area } & \multicolumn{1}{c|}{ Conc. } & \multicolumn{1}{c|}{ Area\% } & \multicolumn{1}{c|}{ Height } & Peak Start & Peak End \\
\hline 1 & 11.409 & 311432 & 0.000 & 49.856 & 6922 & 10.587 & 12.459 \\
\hline 2 & 13.358 & 313228 & 0.000 & 50.144 & 5845 & 12.459 & 15.157 \\
\hline Total & & 624660 & & 100.000 & 12768 & & \\
\hline
\end{tabular}

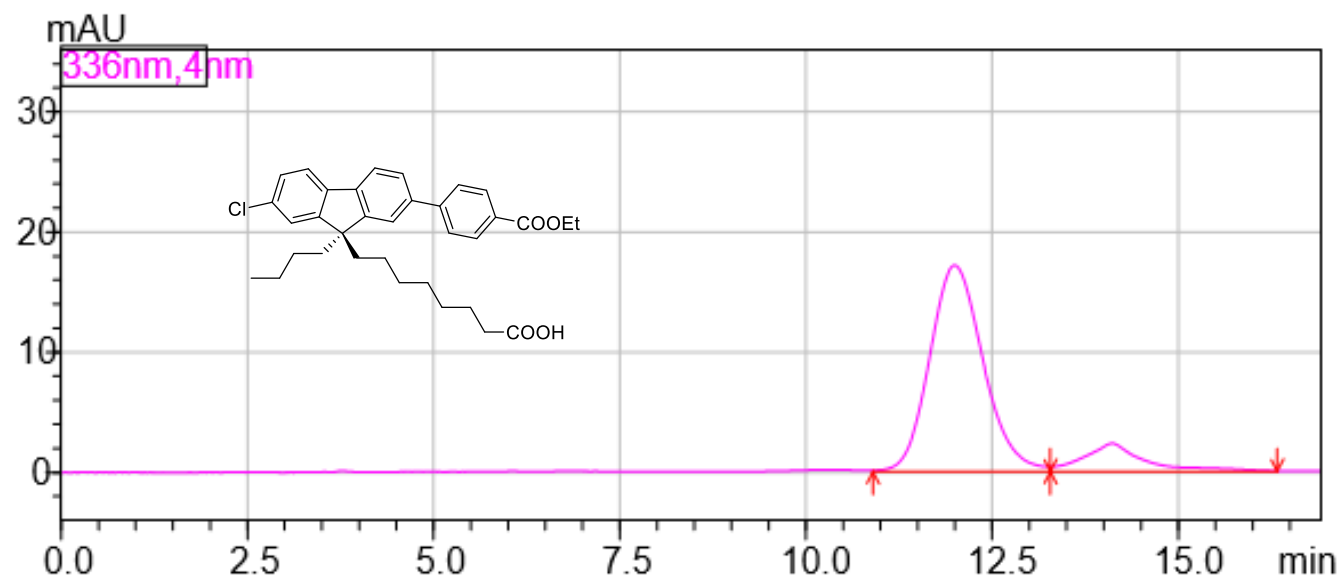

<Peak Table>

PDA Ch1 336nm

\begin{tabular}{r|r|r|r|r|r|r|r|}
\hline Peak\# & Ret. Time & Unit & Mark & \multicolumn{1}{|c|}{ Area\% } & \multicolumn{1}{c|}{ Height } & Peak Start & Peak End \\
\hline 1 & 11.996 & & $\mathrm{M}$ & 86.394 & 17179 & 10.896 & 13.280 \\
\hline 2 & 14.115 & & $\mathrm{M}$ & 13.606 & 2343 & 13.280 & 16.331 \\
\hline Total & & & & 100.000 & 19522 & & \\
\hline
\end{tabular}


$\mathrm{mAU}$

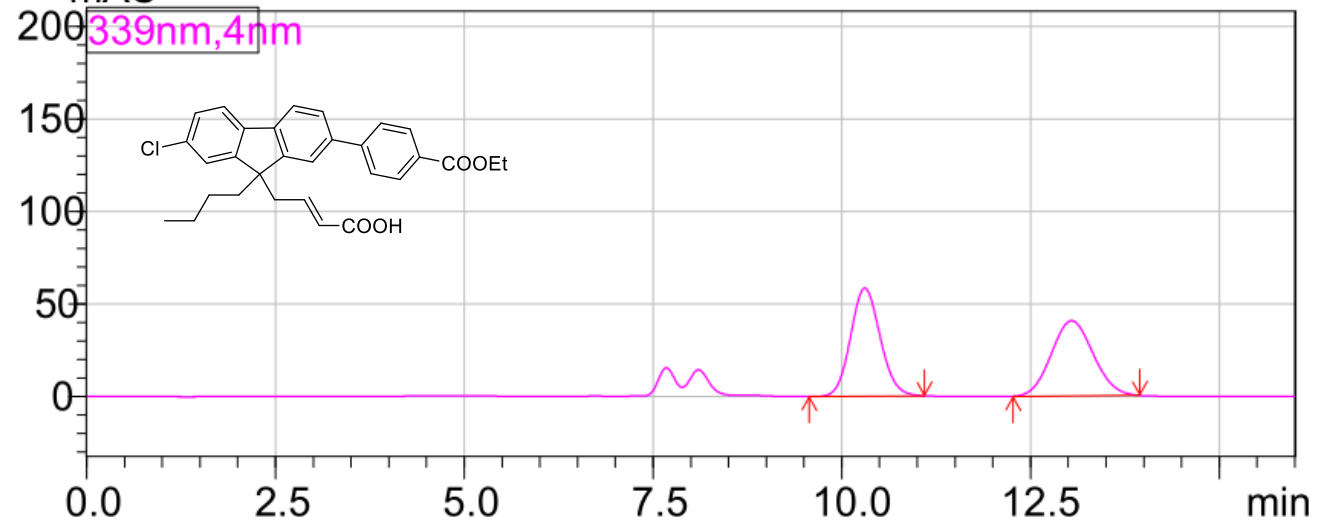

<Peak Table>

PDA Ch1 339nm

\begin{tabular}{|r|r|l|r|r|r|r|}
\hline Peak\# Ret. Time & Area & \multicolumn{1}{|c|}{ Height } & \multicolumn{1}{|c|}{ Area\% } & Peak Start & Peak End \\
\hline 1 & 10.304 & 1600515 & 58524 & 50.493 & 9.568 & 11.093 \\
\hline 2 & 13.043 & 1569239 & 40609 & 49.507 & 12.267 & 13.947 \\
\hline Total & & 3169754 & 99133 & 100.000 & & \\
\hline
\end{tabular}

$\mathrm{mAU}$

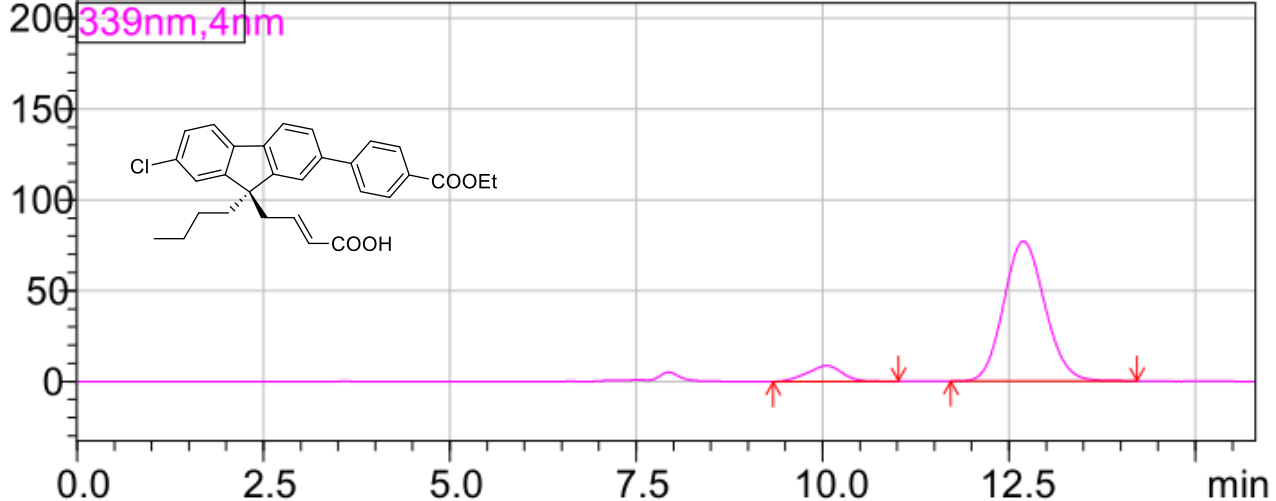

<Peak Table>

PDA Ch1 339nm

\begin{tabular}{|r|r|r|c|r|r|r|}
\hline Peak\# Ret. Time & Height & Mark & Area\% & Peak Start & Peak End \\
\hline 1 & 10.052 & 8652 & $\mathrm{M}$ & 8.638 & 9.339 & 11.019 \\
\hline 2 & 12.692 & 77050 & $\mathrm{M}$ & 91.362 & 11.717 & 14.219 \\
\hline Total & & 85702 & & 100.000 & & \\
\hline
\end{tabular}




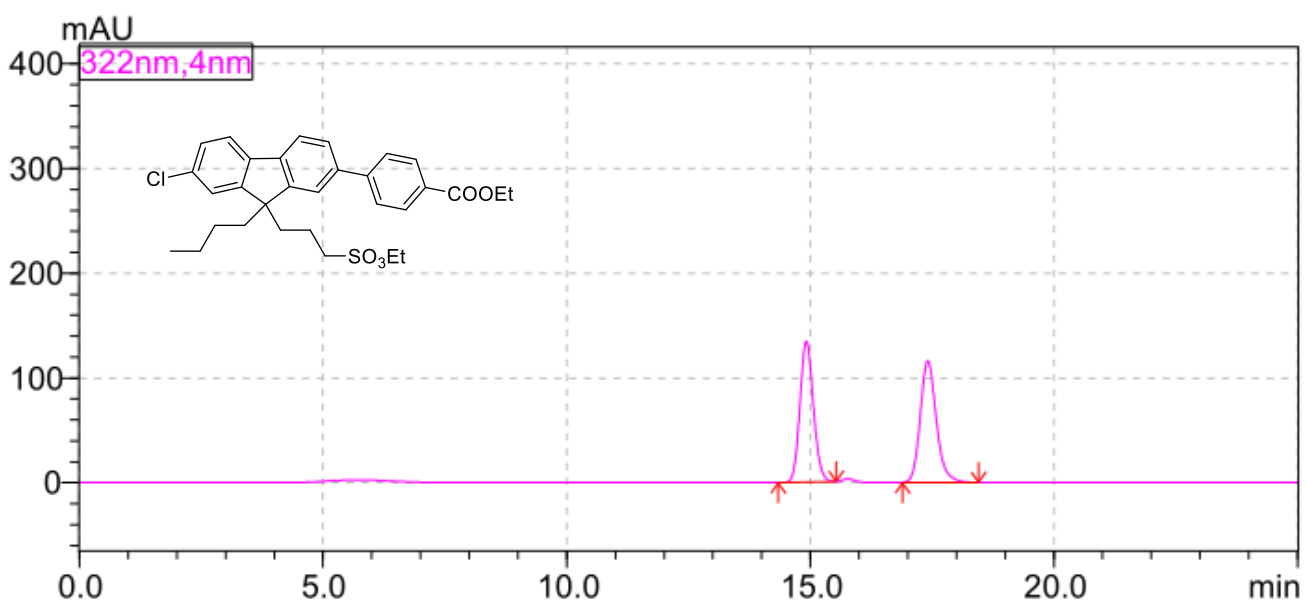

<Peak Table>

PDA Ch1 322nm

\begin{tabular}{|r|r|r|r|r|r|r|}
\hline Peak\# Ret. Time & \multicolumn{1}{|c|}{ Area } & Height & \multicolumn{1}{|c|}{ Area\% } & Peak Start & Peak End \\
\hline 1 & 14.918 & 2587026 & 134541 & 49.193 & 14.341 & 15.525 \\
\hline 2 & 17.411 & 2671887 & 116080 & 50.807 & 16.896 & 18.453 \\
\hline Total & & 5258912 & 250621 & 100.000 & & \\
\hline
\end{tabular}

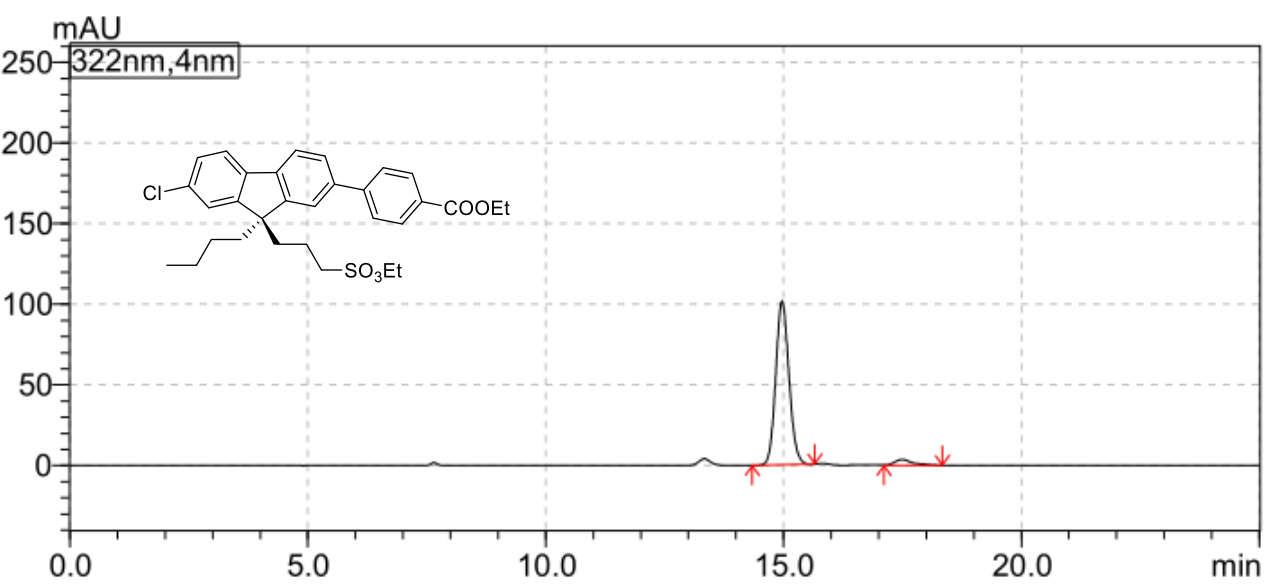

<Peak Table>

PDA Ch1 322nm

\begin{tabular}{|r|r|r|r|l|r|r|r|}
\hline Peak\# Ret. Time & \multicolumn{1}{|c|}{ Area } & \multicolumn{1}{|c|}{ Height } & Mark & Area\% & Peak Start & Peak End \\
\hline 1 & 14.968 & 1973528 & 101586 & M & 95.081 & 14.400 & 15.541 \\
\hline 2 & 17.492 & 102100 & 3717 & M & 4.919 & 17.045 & 18.480 \\
\hline Total & & 2075628 & 105303 & & 100.000 & & \\
\hline
\end{tabular}


14

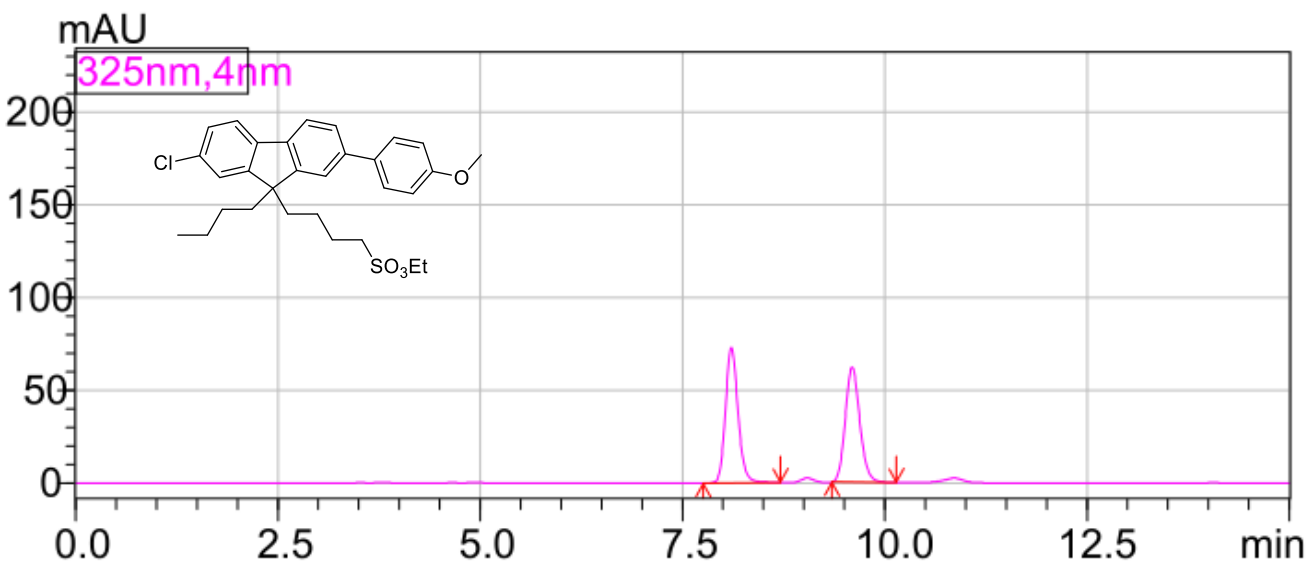

<Peak Table>

PDA Ch1 325nm

Peak\# Ret. Time

\begin{tabular}{|r|r|r|r|r|r|r|}
\hline Peak\# Ret. Time & \multicolumn{1}{|c|}{ Area } & Height & \multicolumn{1}{c|}{ Area\% } & Peak Start & Peak End \\
\hline 1 & 8.104 & 788190 & 73055 & 50.293 & 7.755 & 8.709 \\
\hline 2 & 9.596 & 779019 & 62088 & 49.707 & 9.344 & 10.144 \\
\hline Total & & 1567209 & 135143 & 100.000 & & \\
\hline
\end{tabular}

$\mathrm{mAU}$

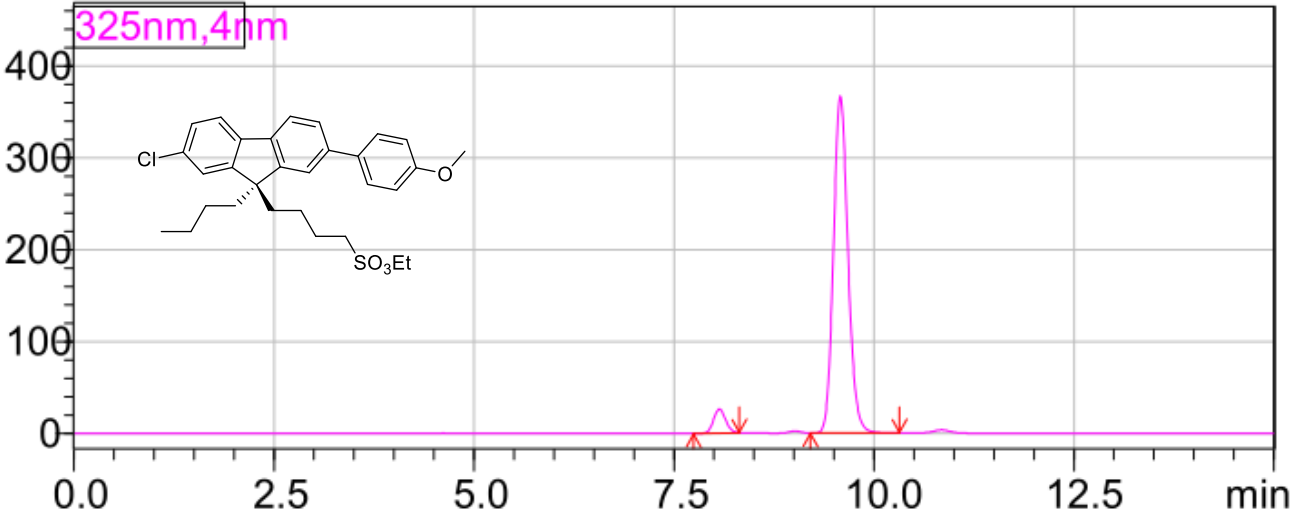

<Peak Table>

PDA Ch1 325nm

\begin{tabular}{|r|r|r|r|r|r|r|r|}
\hline Peak\# Ret. Time & \multicolumn{1}{|c|}{ Area } & Height & \multicolumn{1}{c|}{ Conc. } & Area\% & Peak Start & Peak End \\
\hline 1 & 8.067 & 273386 & 26155 & 0.000 & 5.551 & 7.744 & 8.315 \\
\hline 2 & 9.579 & 4651933 & 367155 & 0.000 & 94.449 & 9.205 & 10.320 \\
\hline Total & & 4925319 & 393310 & & 100.000 & & \\
\hline
\end{tabular}




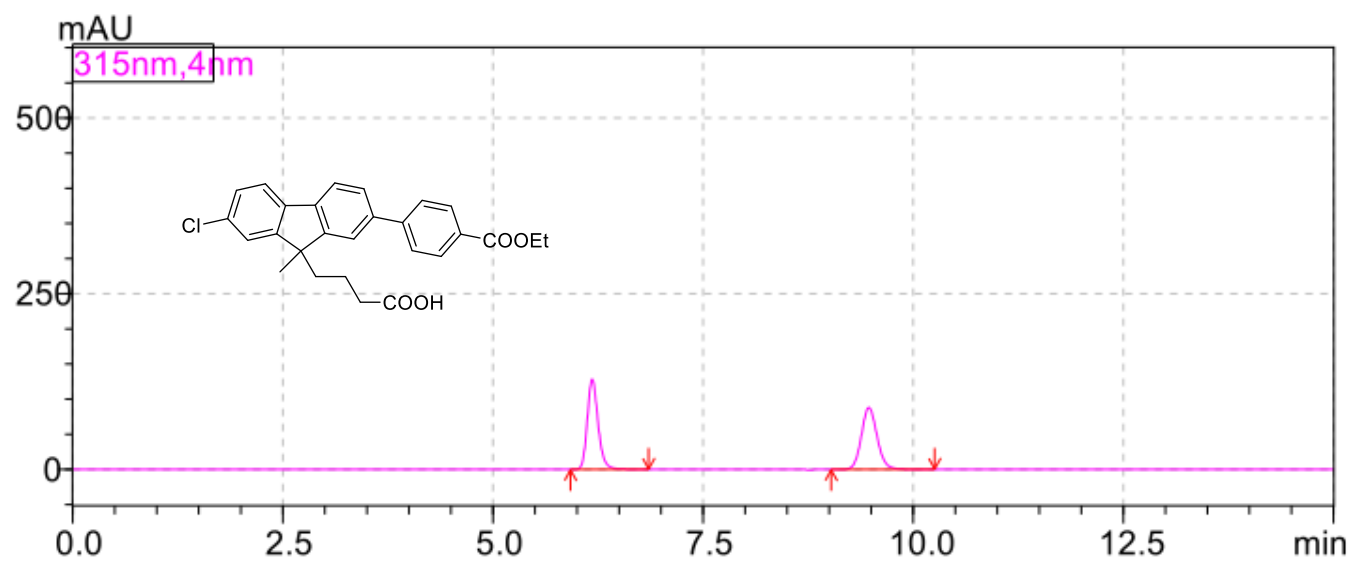

<Peak Table>

PDA Ch1 315nm

\begin{tabular}{|r|r|r|r|l|r|r|r|}
\hline Peak\# Ret. Time & Area & \multicolumn{1}{|c|}{ Height } & Mark & Area\% & Peak Start & Peak End \\
\hline 1 & 6.180 & 1155997 & 128659 & M & 50.045 & 5.920 & 6.853 \\
\hline 2 & 9.473 & 1153906 & 88102 & M & 49.955 & 9.024 & 10.256 \\
\hline Total & & 2309903 & 216761 & & 100.000 & & \\
\hline
\end{tabular}

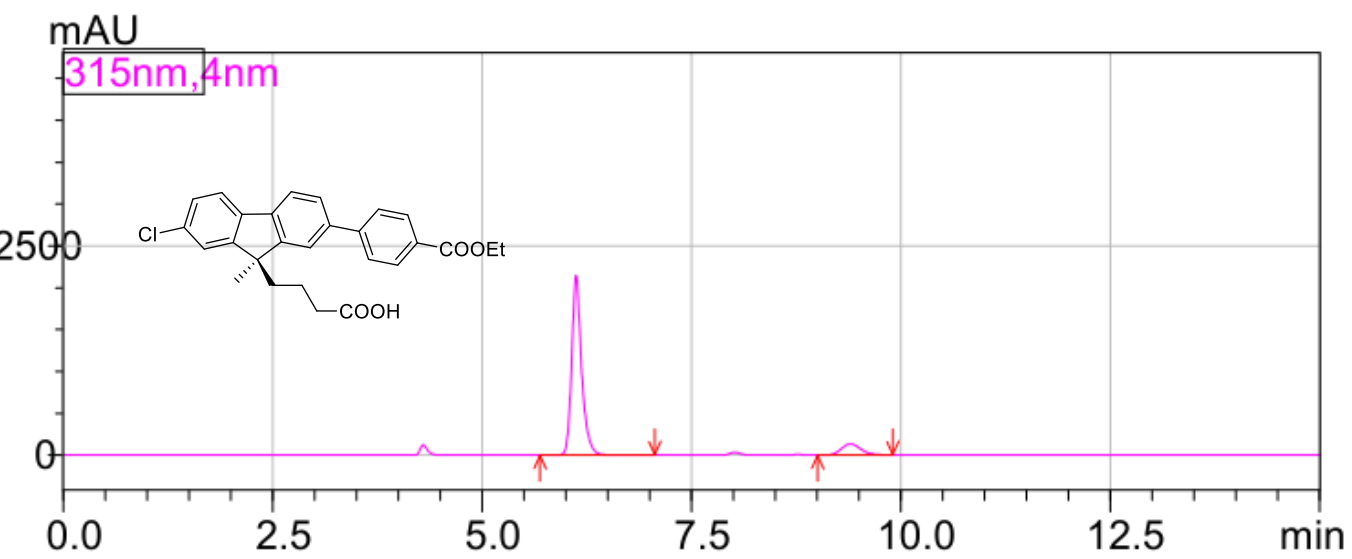

<Peak Table>

PDA Ch1 315nm

\begin{tabular}{|c|c|c|c|c|c|c|c|}
\hline Peak\# & t. Time & Area & Height & Mark & Area\% & Peak Start & Peak End \\
\hline $\begin{array}{l}1 \\
\end{array}$ & 6.121 & 17799005 & 2150253 & $\mathrm{M}$ & 88.882 & 5.691 & 7.061 \\
\hline 2 & 9.397 & 2226353 & 133405 & M & 11.118 & 9.003 & 9.904 \\
\hline Total & & 20025357 & 2283658 & & 100.000 & & \\
\hline
\end{tabular}




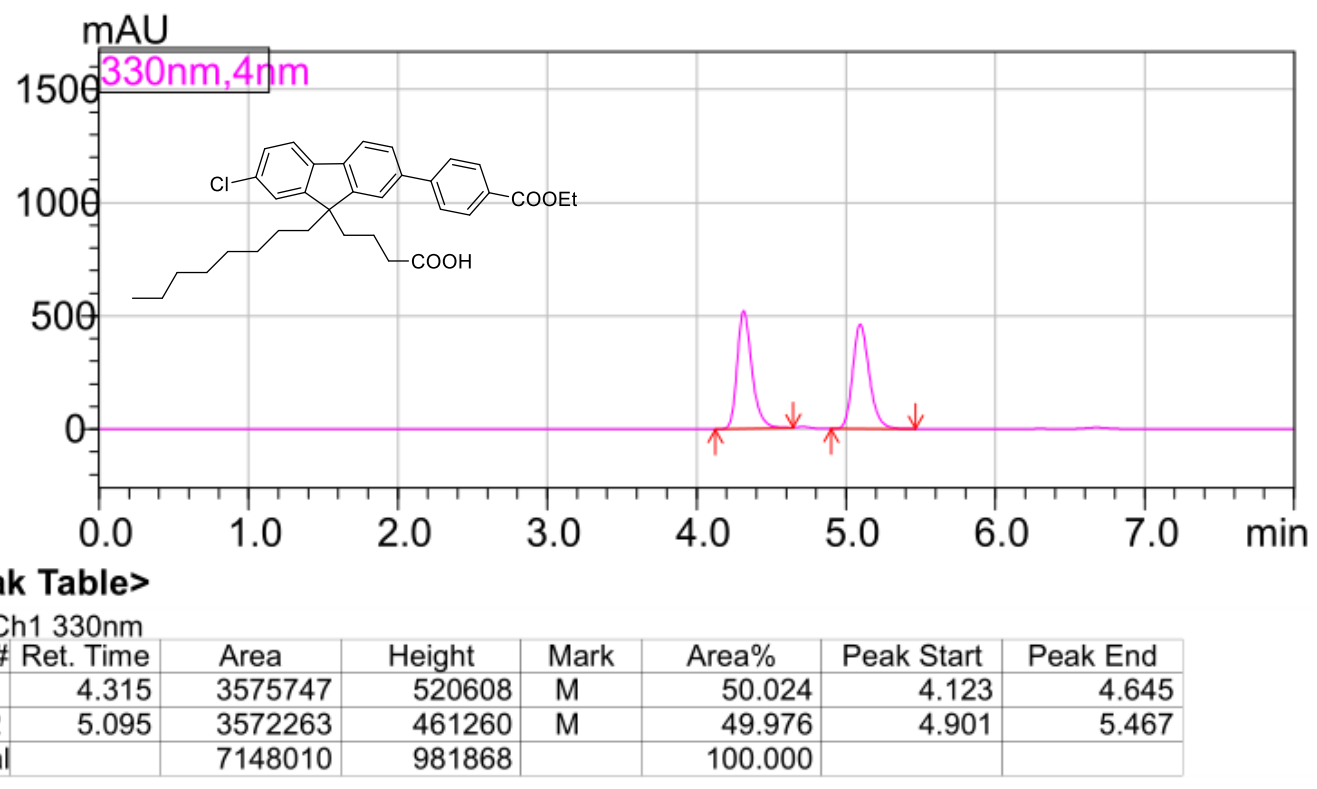

<Peak Table>

PDA Ch1 330nm

\begin{tabular}{|r|r|r|r|l|r|r|r|}
\hline Peak\# Ret. Time & Area & Height & Mark & \multicolumn{1}{|c|}{ Area\% } & Peak Start & Peak End \\
\hline 1 & 4.315 & 3575747 & 520608 & M & 50.024 & 4.123 & 4.645 \\
\hline 2 & 5.095 & 3572263 & 461260 & M & 49.976 & 4.901 & 5.467 \\
\hline Total & & 7148010 & 981868 & & 100.000 & & \\
\hline
\end{tabular}

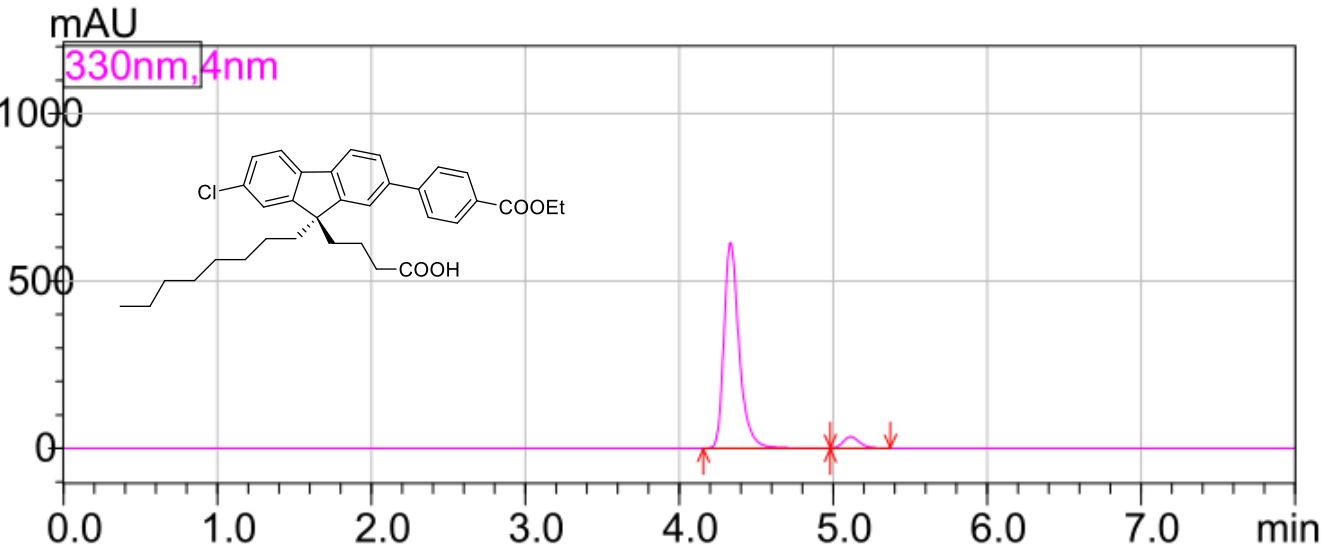

<Peak Table>

PDA Ch1 330nm

\begin{tabular}{|r|r|r|r|l|r|r|r|}
\hline Peak\# Ret. Time & \multicolumn{1}{|c|}{ Area } & Height & Mark & Area\% & Peak Start & Peak End \\
\hline 1 & 4.332 & 4218194 & 615272 & $\mathrm{M}$ & 94.294 & 4.155 & 4.981 \\
\hline 2 & 5.112 & 255263 & 33944 & $\mathrm{M}$ & 5.706 & 4.981 & 5.371 \\
\hline Total & & 4473457 & 649216 & & 100.000 & & \\
\hline
\end{tabular}


$m A U$

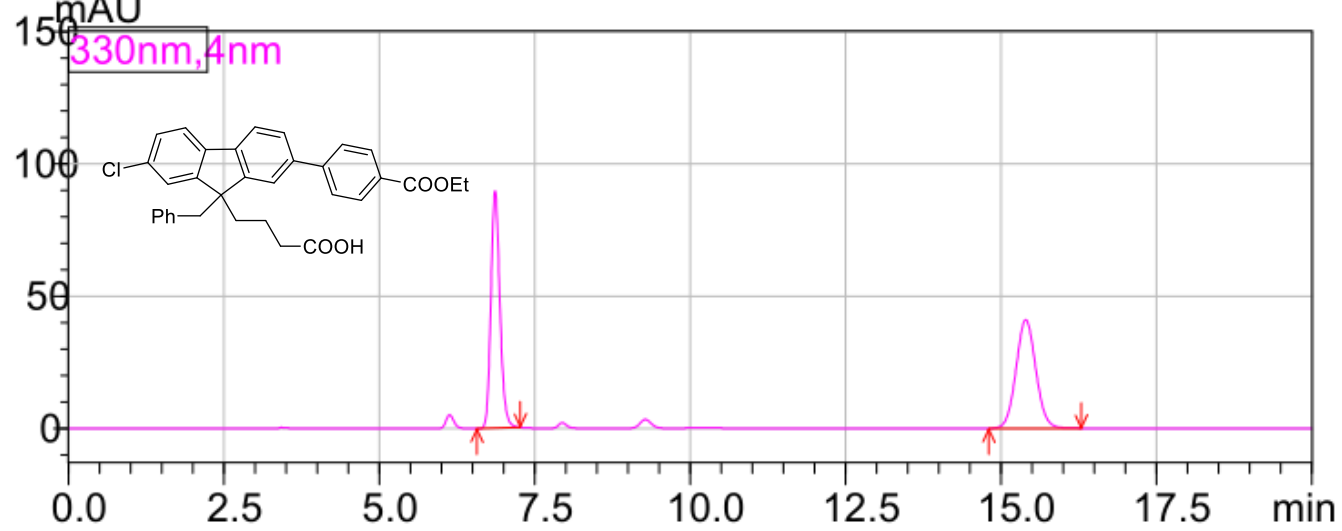

<Peak Table>

PDA Ch1 330nm

\begin{tabular}{|r|r|r|r|r|r|r|r|}
\hline Peak\# Ret. Time & \multicolumn{1}{|c|}{ Area } & \multicolumn{1}{|c|}{ Height } & Mark & Area\% & Peak Start & Peak End \\
\hline 1 & 6.861 & 903151 & 89618 & M & 49.791 & 6.565 & 7.264 \\
\hline 2 & 15.397 & 910737 & 40979 & M & 50.209 & 14.805 & 16.288 \\
\hline Total & & 1813888 & 130597 & & 100.000 & & \\
\hline
\end{tabular}

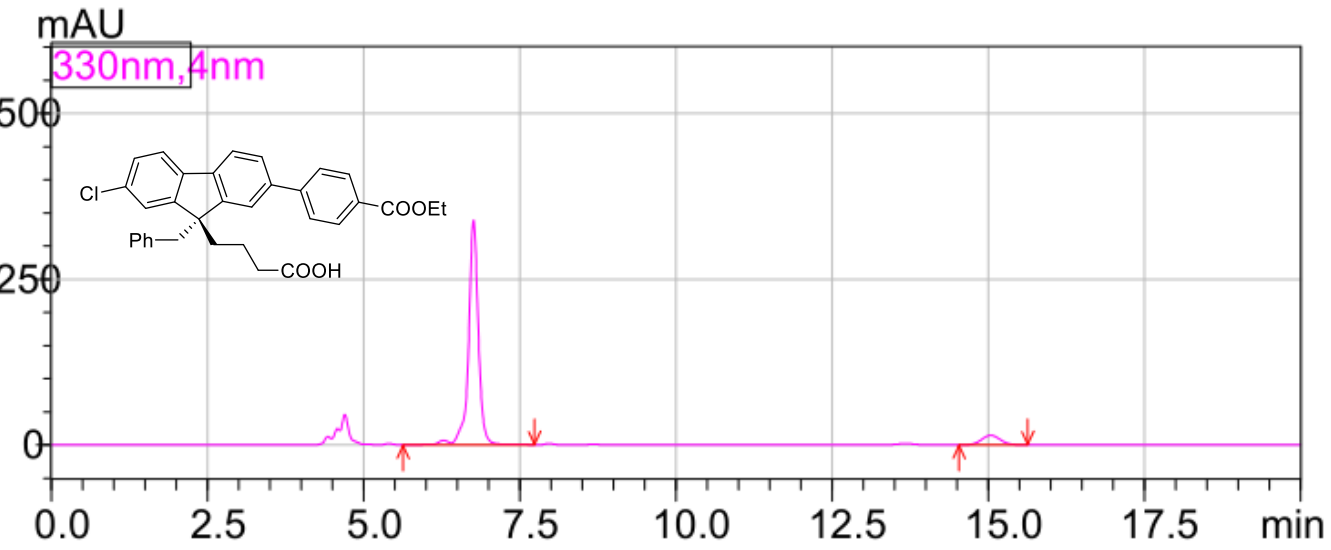

<Peak Table>

PDA Ch1 330nm Peak\# Ret. Time

\begin{tabular}{|r|r|} 
Peak\# Ret. Time \\
\hline 1 & 6.758 \\
\hline 2 & 15.039 \\
\hline
\end{tabular}

\begin{tabular}{|r|r|l|r|r|r|}
\hline \multicolumn{1}{|c|}{ Area } & \multicolumn{1}{|c|}{ Height } & Mark & \multicolumn{1}{|c|}{ Area\% } & Peak Start & Peak End \\
\hline 3816870 & 339173 & M & 92.527 & 5.627 & 7.733 \\
\hline 308283 & 14555 & M & 7.473 & 14.533 & 15.627 \\
\hline 4125154 & 353727 & & 100.000 & & \\
\hline
\end{tabular}




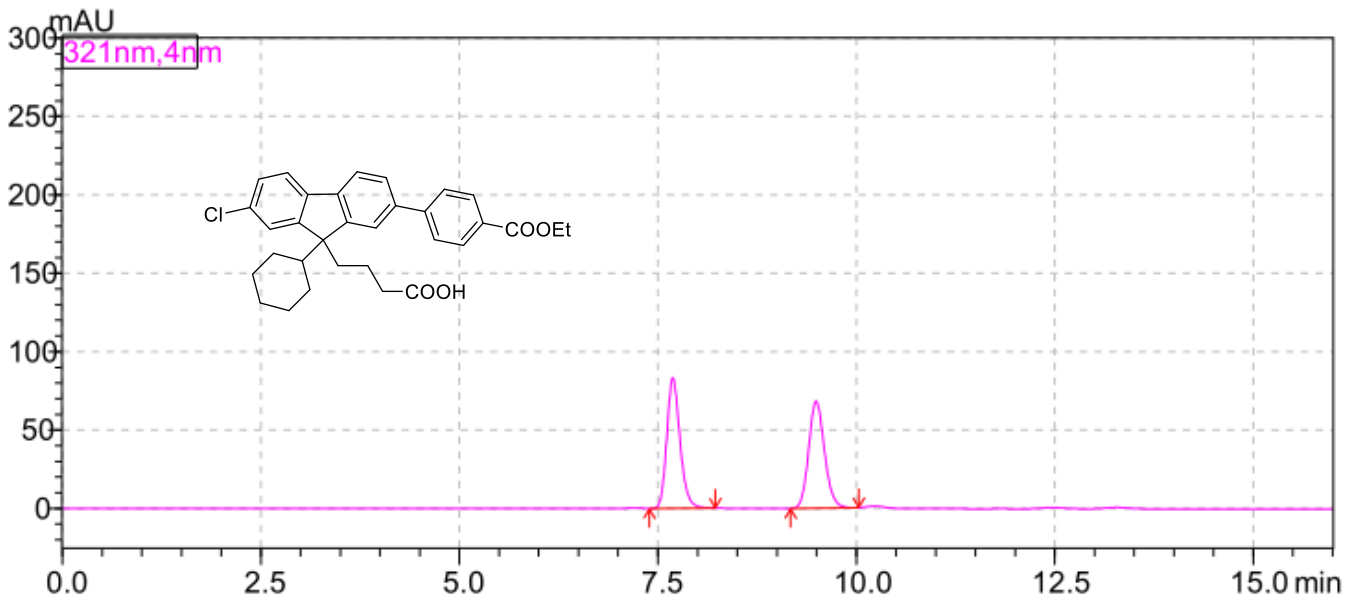

<Peak Table>

PDA Ch1 321nm

\begin{tabular}{|r|r|r|r|r|r|r|}
\hline Peak\# Ret. Time & \multicolumn{1}{|c|}{ Area } & \multicolumn{1}{|c|}{ Height } & Area\% & Peak Start & Peak End \\
\hline 1 & 7.688 & 967048 & 83194 & 50.450 & 7.392 & 8.224 \\
\hline 2 & 9.493 & 949801 & 68318 & 49.550 & 9.173 & 10.032 \\
\hline Total & & 1916849 & 151512 & 100.000 & & \\
\hline
\end{tabular}

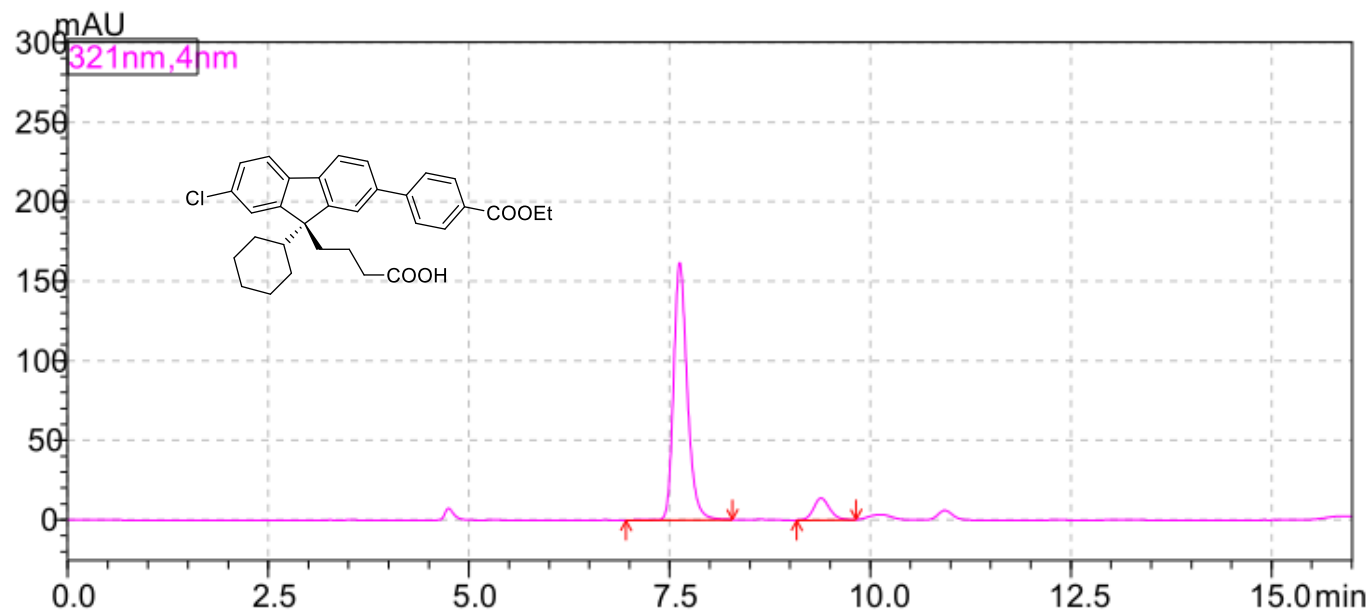

<Peak Table>

PDA Ch1 321nm

Peak\# Ret. Time

\begin{tabular}{|r|r|r|r|r|r|r|}
\hline Peak\# Ret. Time & \multicolumn{1}{|c|}{ Area } & \multicolumn{1}{|c|}{ Height } & \multicolumn{1}{c|}{ Area\% } & Peak Start & \multicolumn{1}{c|}{ Peak End } \\
\hline 1 & 7.626 & 1916651 & 161845 & 91.108 & 6.955 & 8.283 \\
\hline 2 & 9.388 & 187064 & 13659 & 8.892 & 9.083 & 9.824 \\
\hline Total & & 2103715 & 175504 & 100.000 & & \\
\hline
\end{tabular}



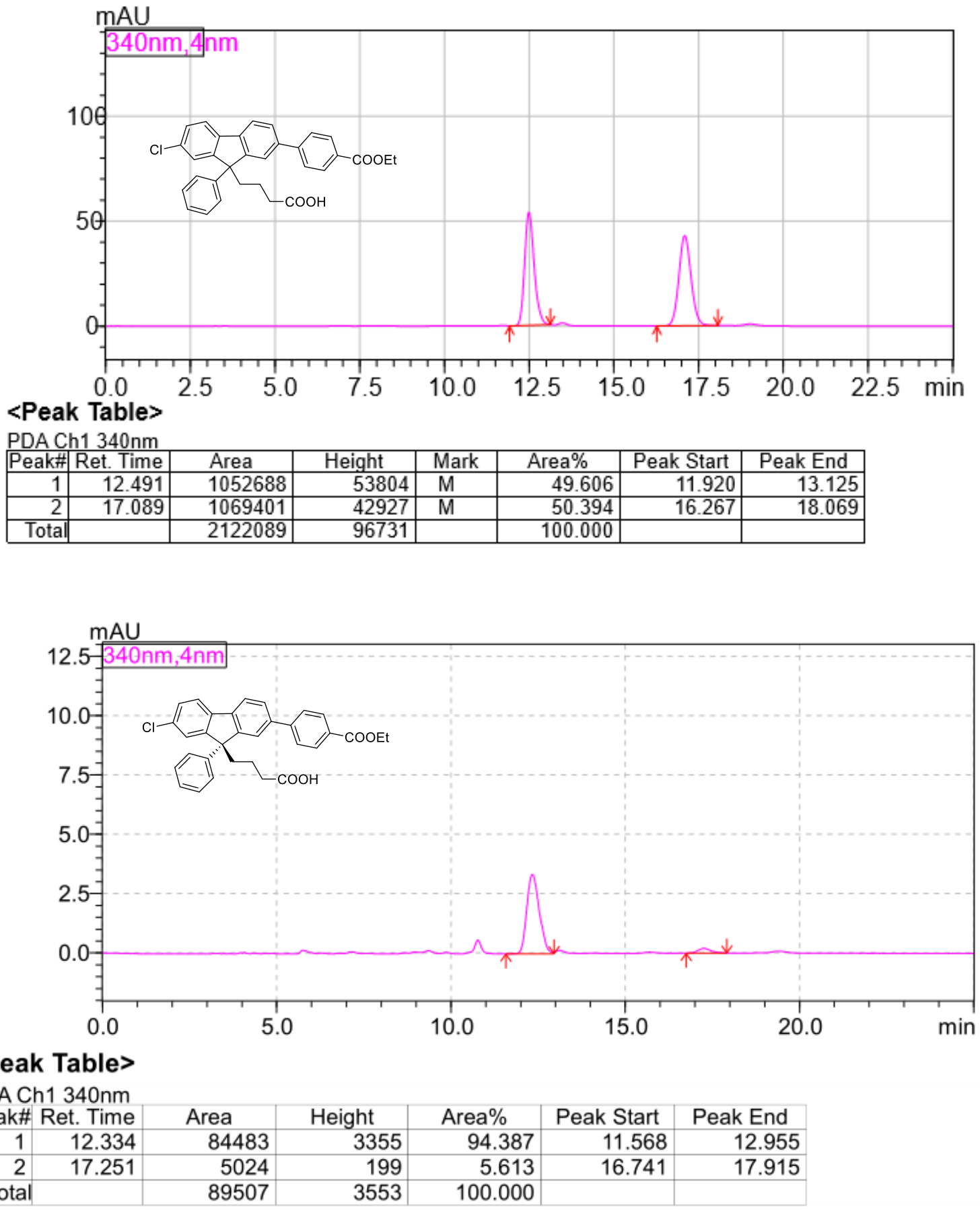


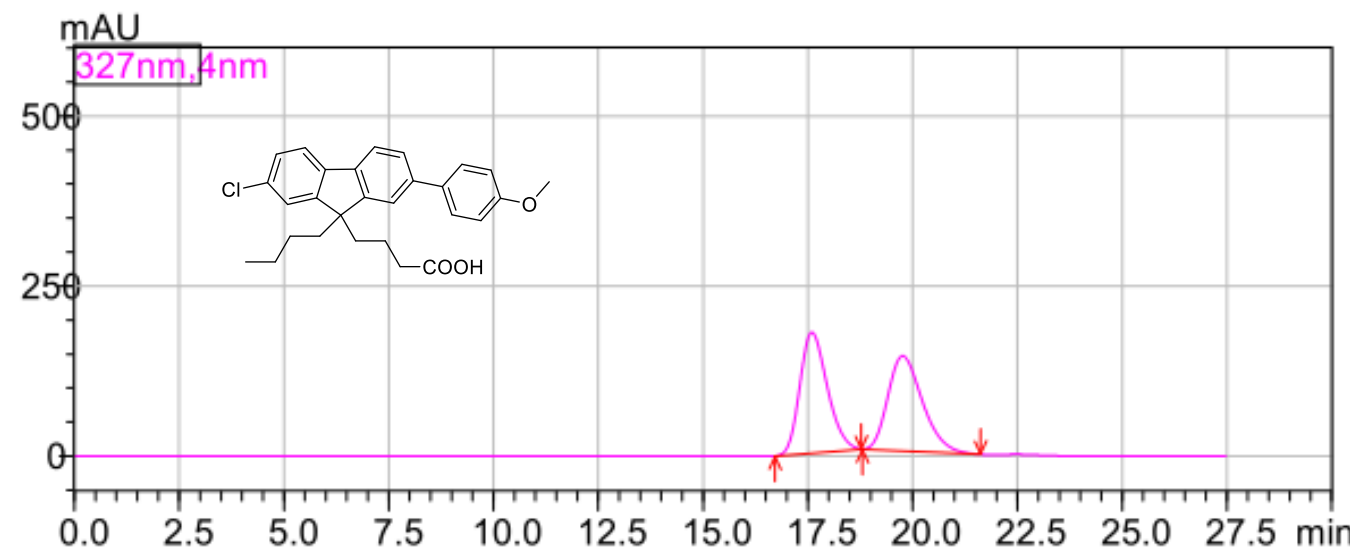

<Peak Table>

PDA Ch1 327nm

\begin{tabular}{|r|r|r|r|r|r|r|r|}
\hline Peak\# & Ret. Time & \multicolumn{1}{|c|}{ Area } & Height & Mark & Area\% & Peak Start & Peak End \\
\hline 1 & 17.597 & 8088778 & 177472 & M & 50.255 & 16.715 & 18.773 \\
\hline 2 & 19.763 & 8006616 & 139737 & M & 49.745 & 18.805 & 21.621 \\
\hline Total & & 16095394 & 317209 & & 100.000 & & \\
\hline
\end{tabular}

$\mathrm{mAU}$

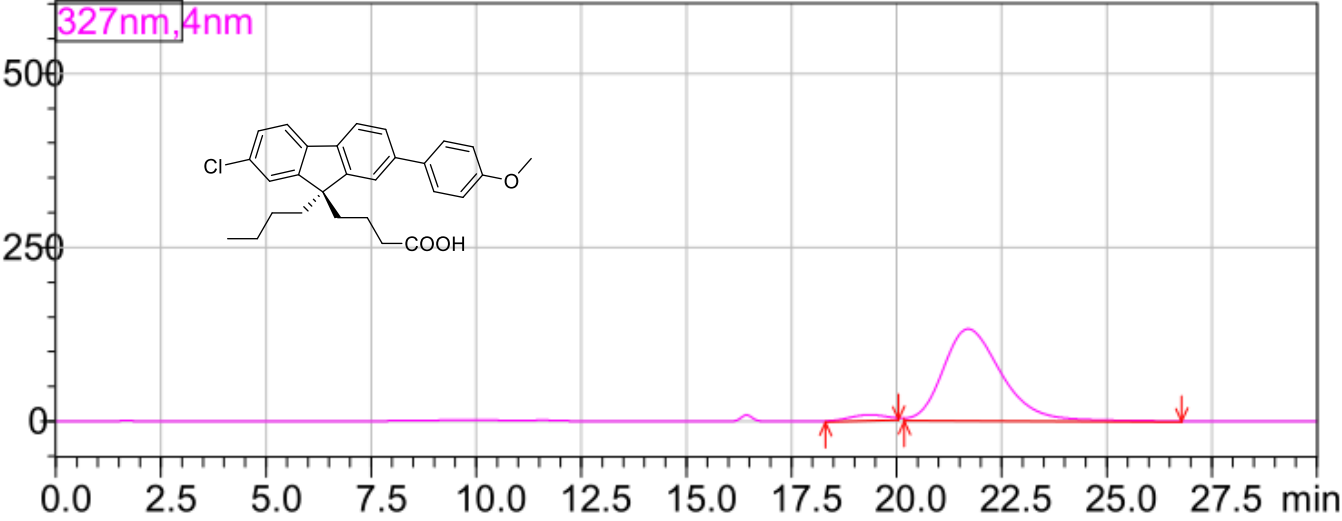

<Peak Table>

PDA Ch1 327nm

\begin{tabular}{|r|r|r|r|c|r|r|r|}
\hline Peak\# Ret. Time & \multicolumn{1}{|c|}{ Area } & \multicolumn{1}{|c|}{ Height } & Mark & Area\% & Peak Start & Peak End \\
\hline 1 & 19.363 & 553959 & 8652 & M & 4.073 & 18.309 & 20.048 \\
\hline 2 & 21.704 & 13045579 & 132482 & M & 95.927 & 20.181 & 26.779 \\
\hline Total & & 13599537 & 141133 & & 100.000 & & \\
\hline
\end{tabular}




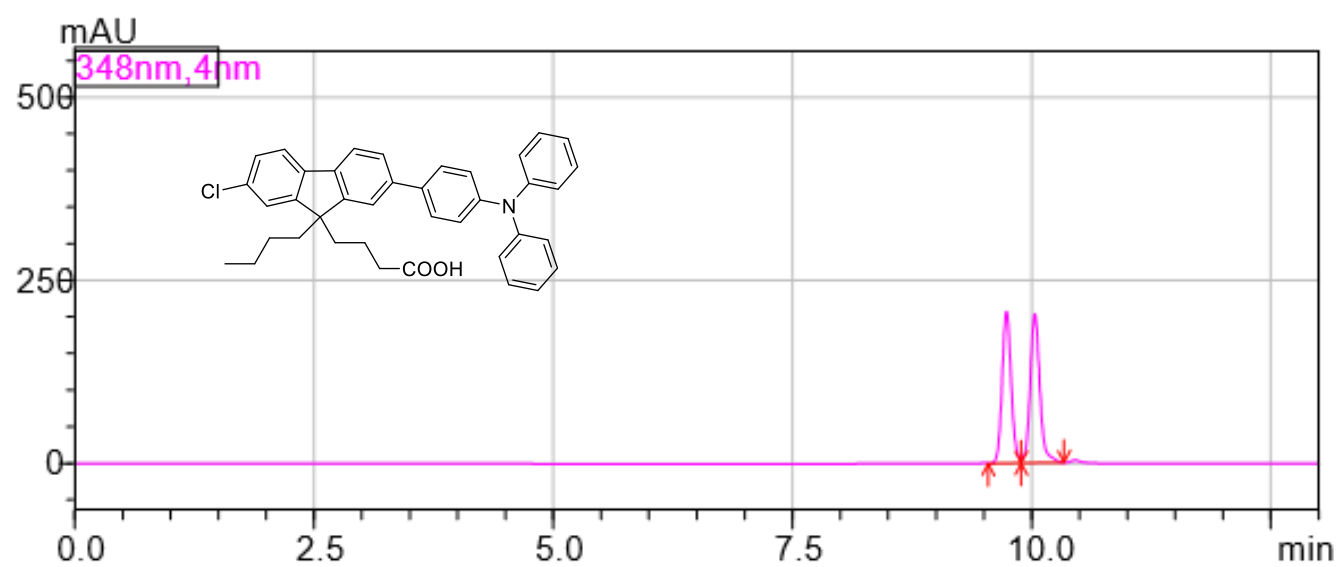

<Peak Table>

PDA Ch1 348nm

\begin{tabular}{|r|r|r|r|r|r|r|}
\hline Peak\# & Ret. Time & Area & \multicolumn{1}{c|}{ Height } & Peak Start & Peak End & \multicolumn{1}{c|}{ Area\% } \\
\hline 1 & 9.736 & 1361527 & 206723 & 9.547 & 9.888 & 48.654 \\
\hline 2 & 10.032 & 1436878 & 203282 & 9.888 & 10.336 & 51.346 \\
\hline Total & & 2798406 & 410005 & & & 100.000 \\
\hline
\end{tabular}

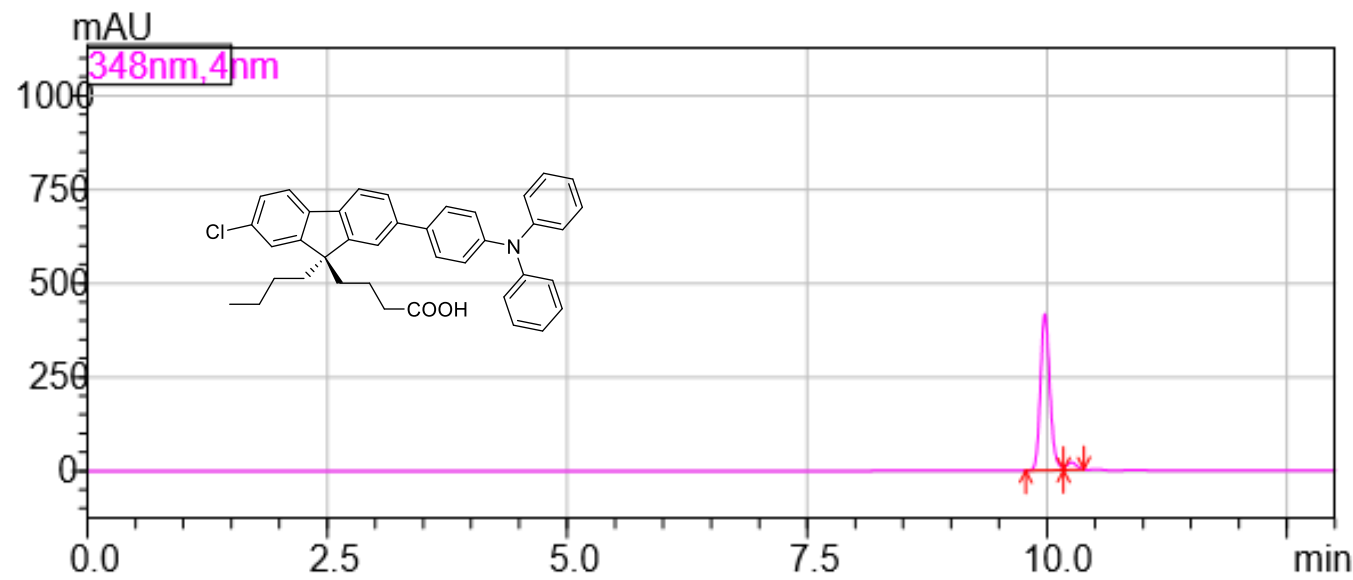

<Peak Table>

PDA Ch1 348nm

\begin{tabular}{|r|r|r|r|r|r|r|}
\hline Peak\# & Ret. Time & \multicolumn{1}{|c|}{ Area } & \multicolumn{1}{c|}{ Height } & Peak Start & Peak End & \multicolumn{1}{c|}{ Area\% } \\
\hline 1 & 9.977 & 2863714 & 416396 & 9.776 & 10.171 & 95.420 \\
\hline 2 & 10.257 & 137452 & 19619 & 10.171 & 10.384 & 4.580 \\
\hline Total & & 3001166 & 436015 & & & 100.000 \\
\hline
\end{tabular}




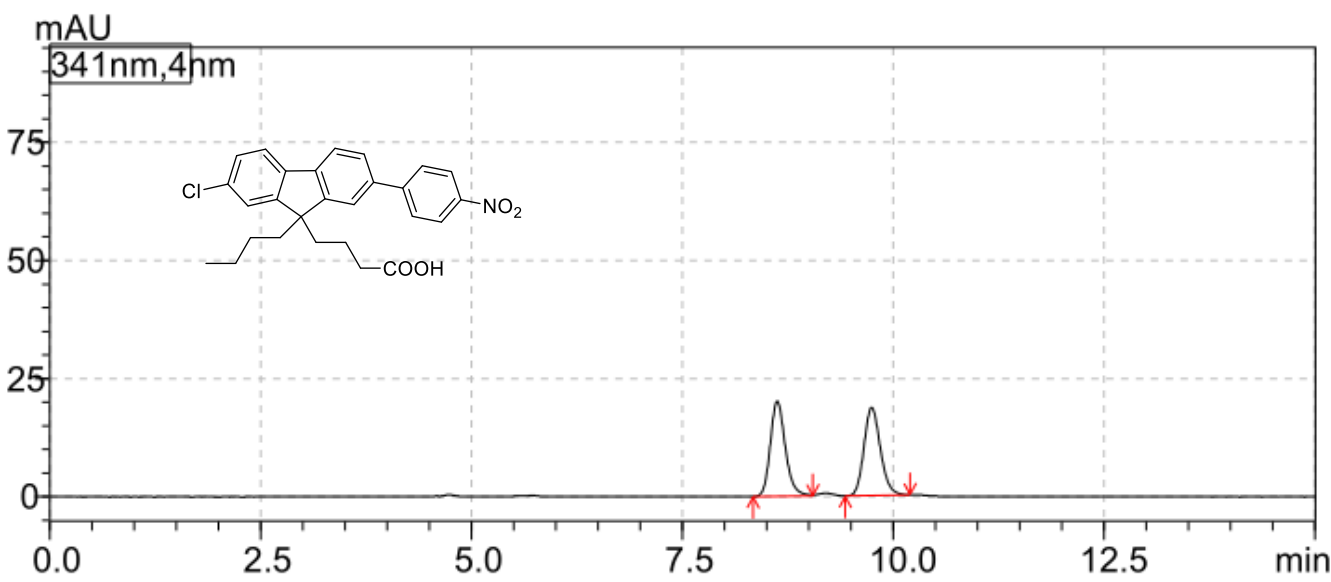

<Peak Table>

PDA Ch1 340nm

\begin{tabular}{|c|c|c|c|c|c|c|c|}
\hline Peak\# & t. Time & Area & Height & Mark & Area\% & Peak Start & Peak End \\
\hline 1 & 8.624 & 247208 & 20228 & $\mathrm{M}$ & 49.076 & 8.352 & 8.992 \\
\hline 2 & 9.744 & 256514 & 18866 & $M$ & 50.924 & 9.435 & 10.224 \\
\hline Total & & 503722 & 39094 & & 100.000 & & \\
\hline
\end{tabular}

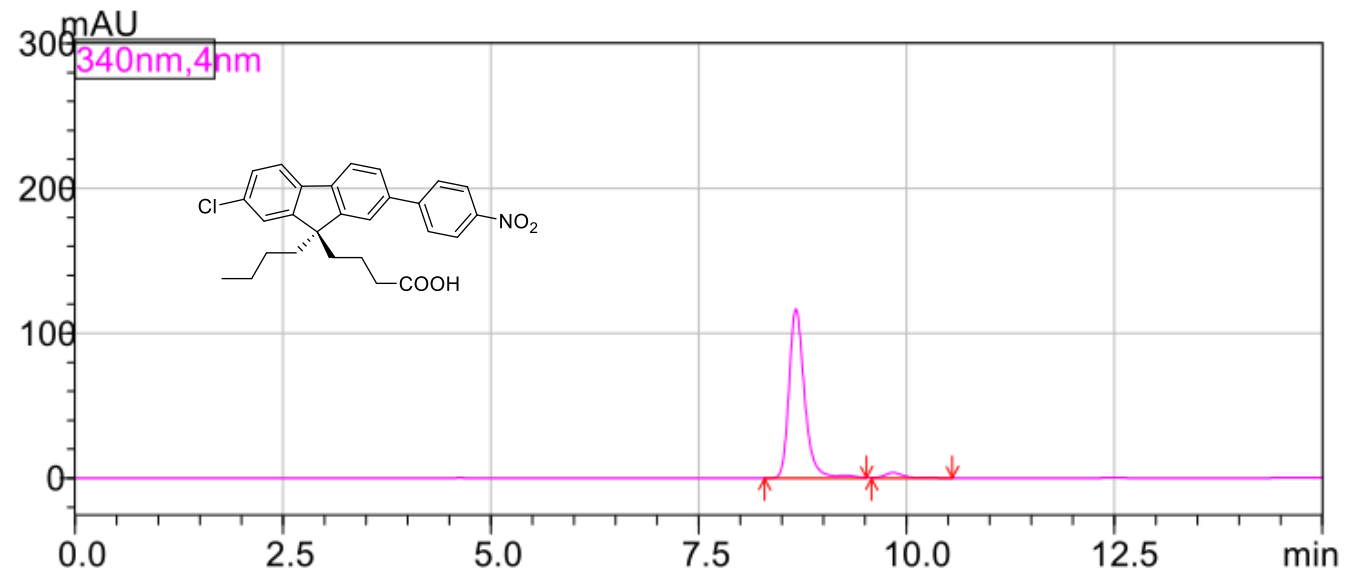

<Peak Table>

PDA Ch1 340nm

Peak\# Ret. Time

\begin{tabular}{|c|c|c|c|c|c|c|c|}
\hline Peak\# & Ret. Time & Area & Height & Mark & Area\% & Peak Start & Peak End \\
\hline 1 & 8.669 & 1536823 & 117003 & M & 96.023 & 8.293 & 9.520 \\
\hline 2 & 9.837 & 63650 & 3811 & M & 3.977 & 9.579 & 10.549 \\
\hline Total & & 1600473 & 120814 & & 100.000 & & \\
\hline
\end{tabular}




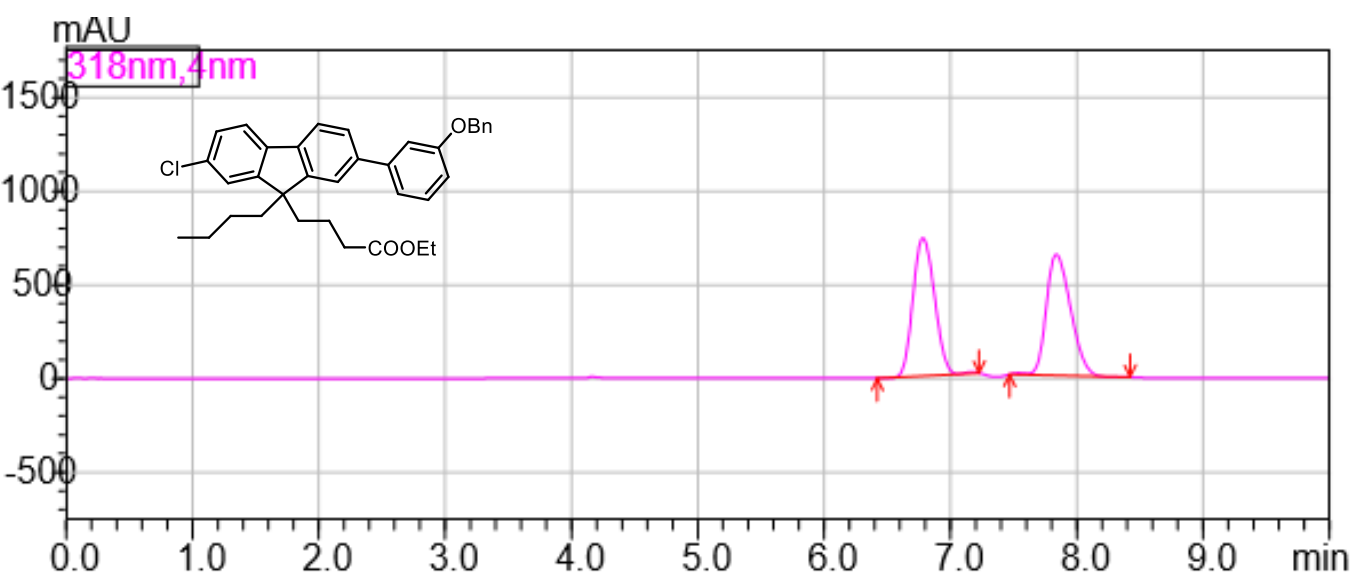

<Peak Table>

PDA Ch1 318nm

\begin{tabular}{|r|r|r|r|r|r|r|}
\hline Peak\# & Ret. Time & \multicolumn{1}{c|}{ Area } & \multicolumn{1}{c|}{ Height } & Peak Start & Peak End & \multicolumn{1}{c|}{ Area\% } \\
\hline 1 & 6.782 & 8763723 & 735594 & 6.421 & 7.227 & 50.226 \\
\hline 2 & 7.838 & 8684872 & 645717 & 7.467 & 8.421 & 49.774 \\
\hline Total & & 17448595 & 1381311 & & & 100.000 \\
\hline
\end{tabular}

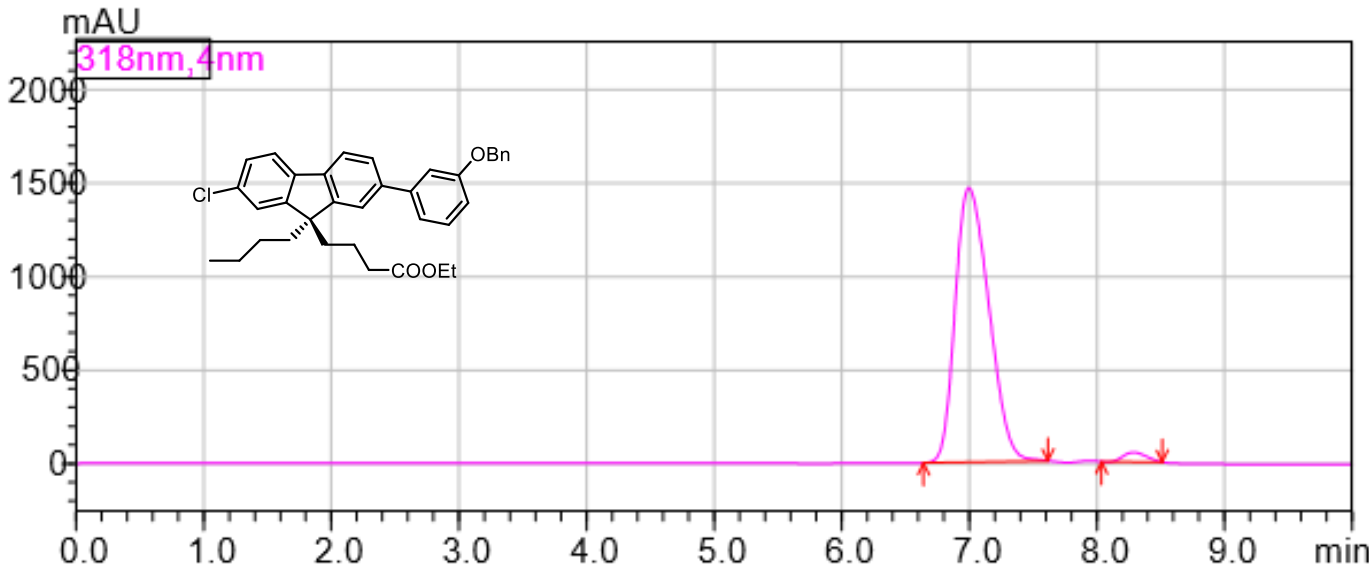

<Peak Table>

PDA Ch1 318nm

\begin{tabular}{|r|r|r|r|r|r|r|}
\hline Peak\# & Ret. Time & \multicolumn{1}{c|}{ Area } & Height & Peak Start & Peak End & \multicolumn{1}{c|}{ Area\% } \\
\hline 1 & 6.997 & 26933869 & 1467169 & 6.640 & 7.616 & 97.678 \\
\hline 2 & 8.287 & 640297 & 51242 & 8.037 & 8.512 & 2.322 \\
\hline Total & & 27574165 & 1518411 & & & 100.000 \\
\hline
\end{tabular}




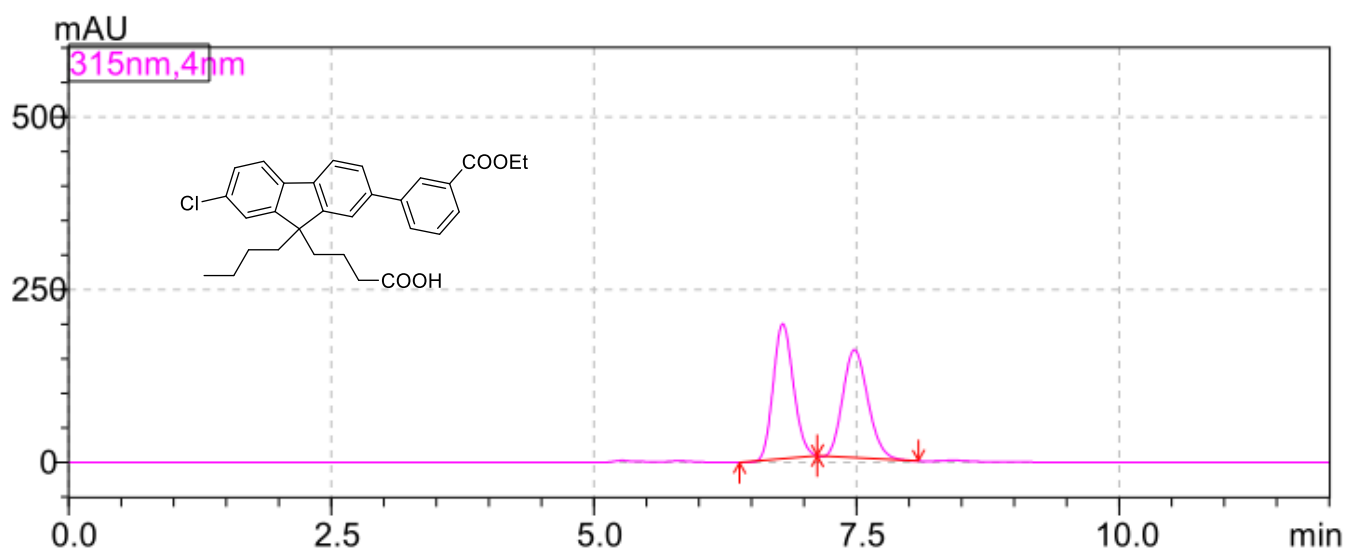

<Peak Table>

PDA Ch1 315nm

Peak\# Ret. Time

\begin{tabular}{|r|r|r|r|l|r|r|r|}
\hline Peak\# & Ret. Time & Area & Height & Mark & Area\% & Peak Start & Peak End \\
\hline 1 & 6.797 & 2611192 & 195302 & M & 50.069 & 6.384 & 7.125 \\
\hline 2 & 7.479 & 2604031 & 156414 & M & 49.931 & 7.125 & 8.091 \\
\hline Total & & 5215224 & 351716 & & 100.000 & & \\
\hline
\end{tabular}

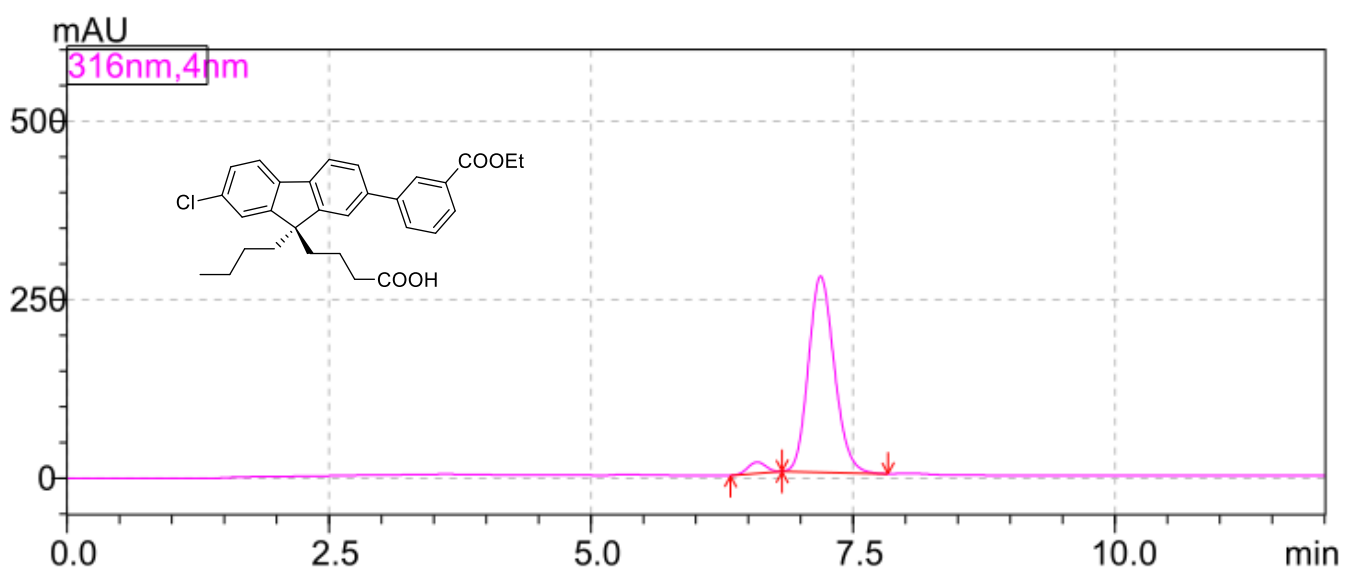

<Peak Table>

PDA Ch1 316nm

Peak\# Ret. Time

\begin{tabular}{|r|r|r|r|l|r|r|r|}
\hline Peak\# & Ret. Time & \multicolumn{1}{|c|}{ Area } & Height & Mark & Area\% & Peak Start & Peak End \\
\hline 1 & 6.585 & 186956 & 15590 & $\mathrm{M}$ & 3.800 & 6.331 & 6.821 \\
\hline 2 & 7.190 & 4732592 & 274482 & $\mathrm{M}$ & 96.200 & 6.821 & 7.835 \\
\hline Total & & 4919548 & 290072 & & 100.000 & & \\
\hline
\end{tabular}




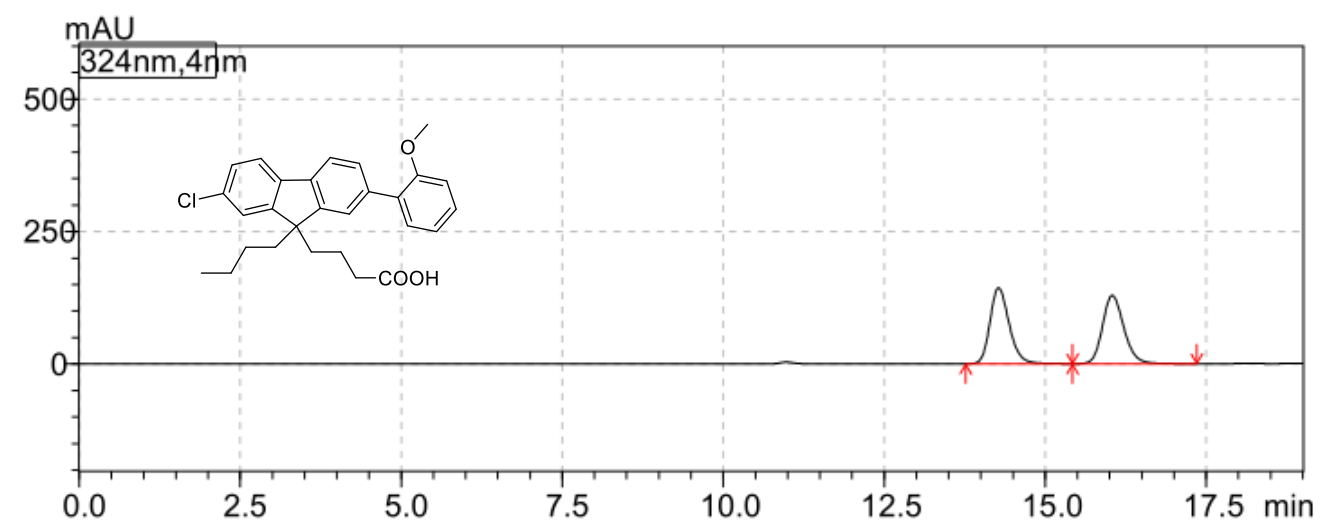

<Peak Table>

PDA Ch1 324nm

\begin{tabular}{|r|r|l|r|l|r|r|r|}
\hline Peak\# Ret. Time & \multicolumn{1}{|c|}{ Area } & \multicolumn{1}{|c|}{ Height } & Mark & Area\% & Peak Start & Peak End \\
\hline 1 & 14.275 & 2982301 & 143459 & M & 49.944 & 13.691 & 15.328 \\
\hline 2 & 16.040 & 2988939 & 128994 & M & 50.056 & 15.365 & 17.259 \\
\hline Total & & 5971240 & 272453 & & 100.000 & & \\
\hline
\end{tabular}

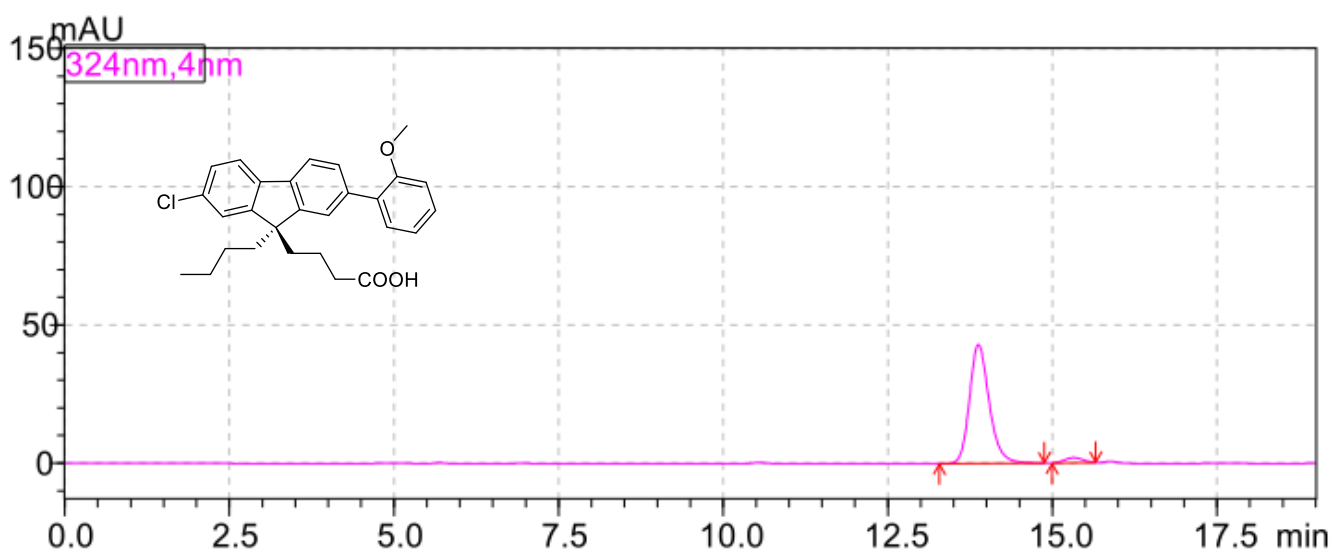

<Peak Table>

PDA Ch1 324nm

\begin{tabular}{|r|r|r|r|r|r|r|r|}
\hline Peak\# Ret. Time & \multicolumn{1}{|c|}{ Area } & \multicolumn{1}{|c|}{ Height } & Mark & \multicolumn{1}{c|}{ Area\% } & Peak Start & Peak End \\
\hline 1 & 13.876 & 896974 & 43068 & $\mathrm{M}$ & 96.278 & 13.285 & 14.880 \\
\hline 2 & 15.321 & 34680 & 1824 & $\mathrm{M}$ & 3.722 & 14.992 & 15.659 \\
\hline Total & & 931655 & 44892 & & 100.000 & & \\
\hline
\end{tabular}




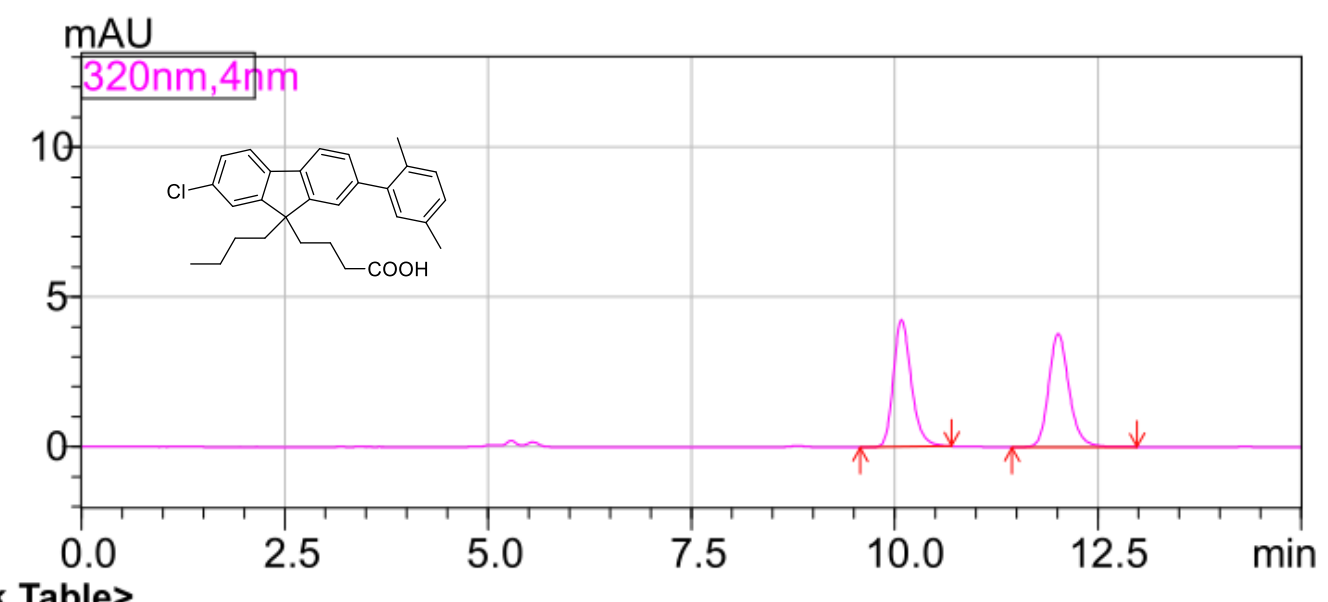

<Peak Table>

PDA Ch1 320nm

\begin{tabular}{|r|r|r|r|r|r|r|}
\hline Peak\# Ret. Time & \multicolumn{1}{|c|}{ Area } & Height & Area\% & Peak Start & Peak End \\
\hline 1 & 10.085 & 65184 & 4242 & 49.705 & 9.579 & 10.699 \\
\hline 2 & 12.011 & 65957 & 3790 & 50.295 & 11.445 & 12.981 \\
\hline Total & & 131141 & 8032 & 100.000 & & \\
\hline
\end{tabular}

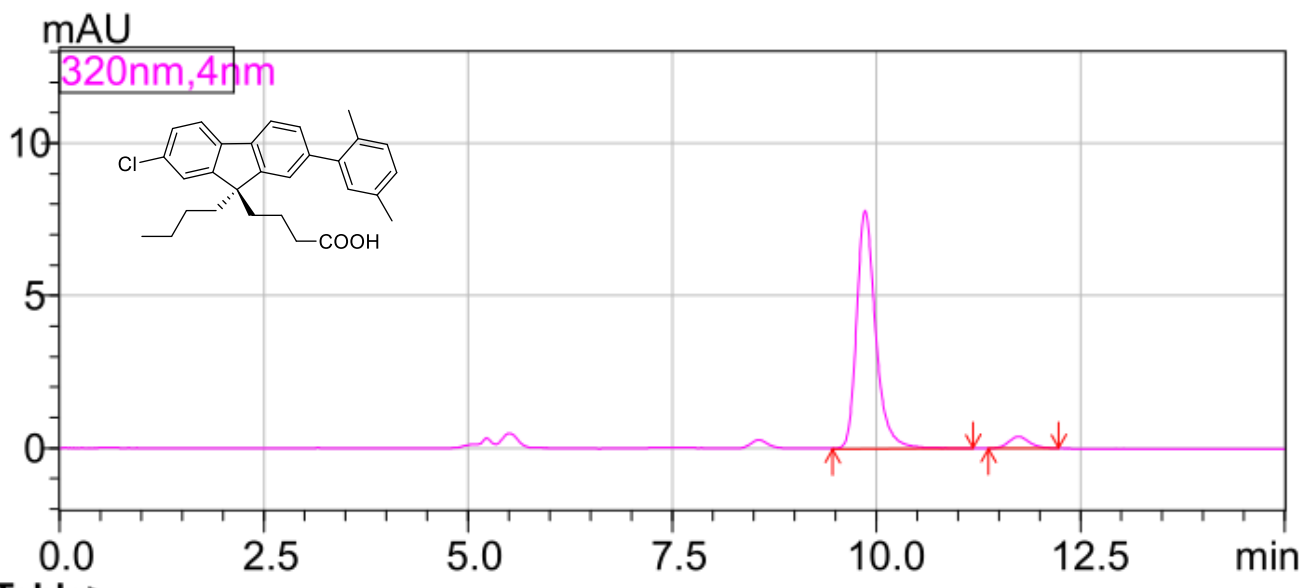

<Peak Table>

PDA Ch1 320nm

\begin{tabular}{|r|r|r|r|r|r|r|}
\hline Peak\# Ret. Time & \multicolumn{1}{|c|}{ Area } & Height & Area\% & Peak Start & Peak End \\
\hline 1 & 9.862 & 126730 & 7809 & 94.842 & 9.467 & 11.184 \\
\hline 2 & 11.746 & 6892 & 388 & 5.158 & 11.371 & 12.235 \\
\hline Total & & 133621 & 8197 & 100.000 & & \\
\hline
\end{tabular}




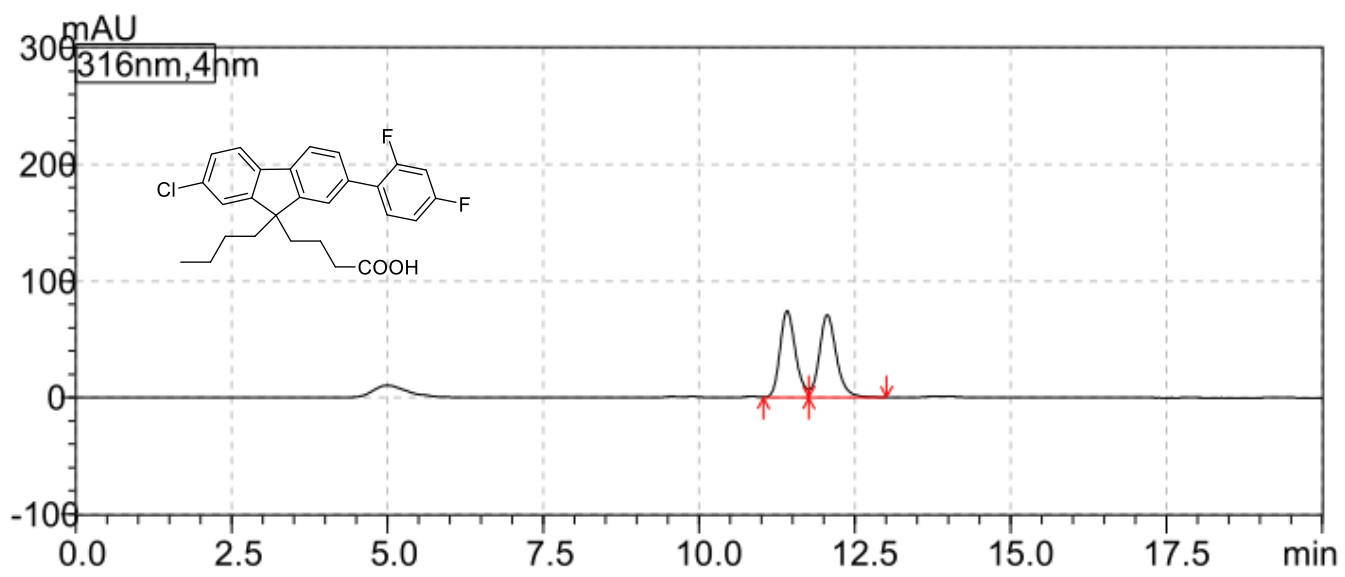

<Peak Table>

PDA Ch1 316nm

\begin{tabular}{|c|c|c|c|c|c|c|c|}
\hline eak\# & Ret. Time & Area & Height & Mark & Area\% & Peak Start & Peak End \\
\hline 1 & 11.4 & 1135981 & 70656 & M & 50.071 & 11.019 & 11.760 \\
\hline 2 & 12.056 & 1132760 & 66036 & M & 49.929 & 11.760 & 12.811 \\
\hline Total & & 2268742 & 136692 & & 100.000 & & \\
\hline
\end{tabular}

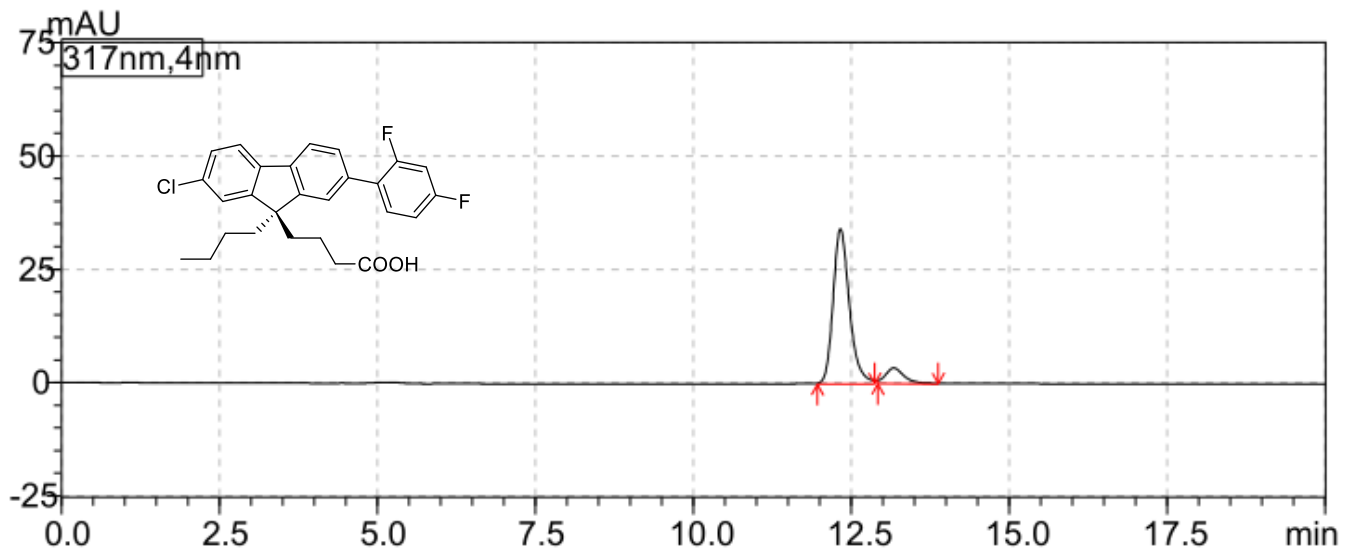

<Peak Table>

PDA Ch1 316nm

Peak\# Ret. Time

\begin{tabular}{|r|r|r|r|l|r|r|r|}
\hline Peak\# & Ret. Time & \multicolumn{1}{|c|}{ Area } & Height & Mark & Area\% & Peak Start & Peak End \\
\hline 1 & 12.328 & 606322 & 34836 & $\mathrm{M}$ & 92.982 & 11.680 & 12.928 \\
\hline 2 & 13.174 & 45762 & 2794 & $\mathrm{M}$ & 7.018 & 12.971 & 13.632 \\
\hline Total & & 652085 & 37630 & & 100.000 & & \\
\hline
\end{tabular}




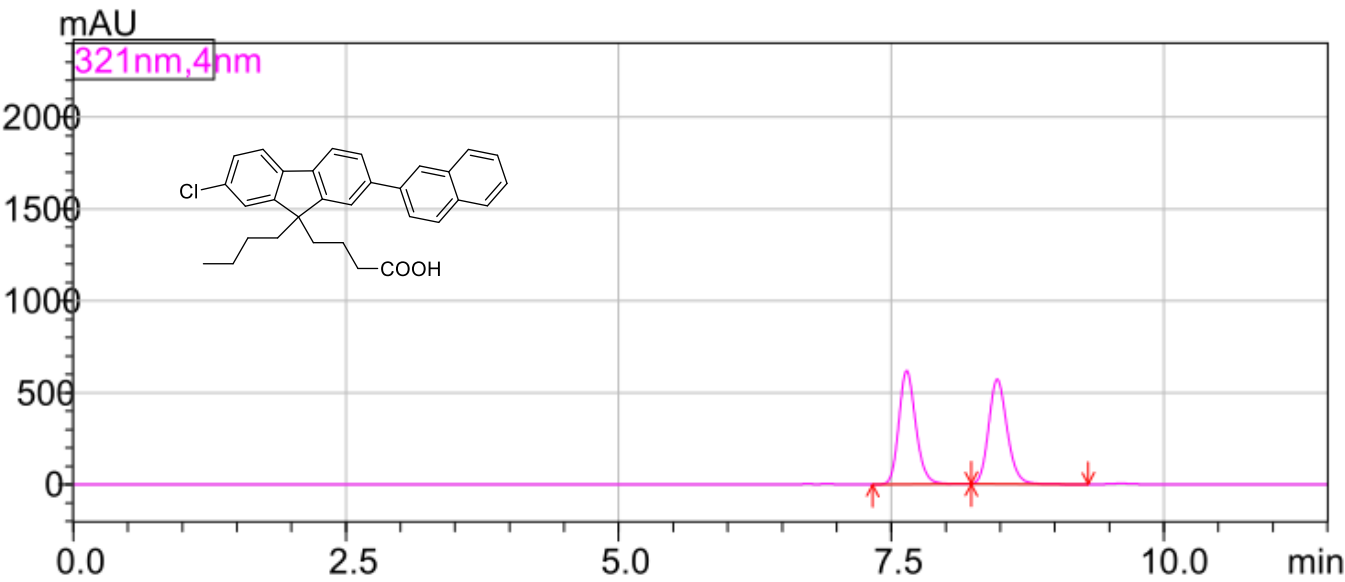

<Peak Table>

PDA Ch1 321nm

\begin{tabular}{|r|r|r|r|l|r|r|r|}
\hline Peak\# Ret. Time & \multicolumn{1}{|c|}{ Area } & \multicolumn{1}{|c|}{ Height } & Mark & Area\% & Peak Start & Peak End \\
\hline 1 & 7.641 & 6650777 & 618104 & M & 49.850 & 7.328 & 8.235 \\
\hline 2 & 8.472 & 6690701 & 569043 & M & 50.150 & 8.235 & 9.307 \\
\hline Total & & 13341478 & 1187148 & & 100.000 & & \\
\hline
\end{tabular}

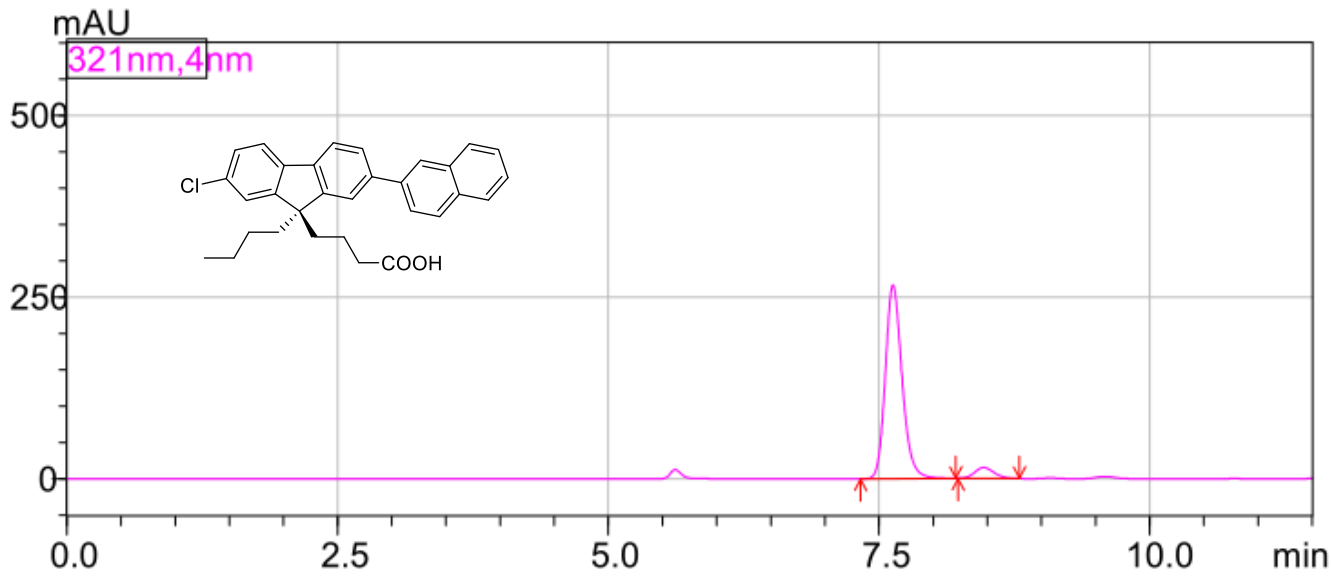

<Peak Table>

PDA Ch1 321nm

\begin{tabular}{|r|r|r|r|r|r|r|r|}
\hline Peak\# Ret. Time & \multicolumn{1}{|c|}{ Area } & \multicolumn{1}{|c|}{ Height } & Mark & \multicolumn{1}{|c|}{ Area\% } & Peak Start & Peak End \\
\hline 1 & 7.629 & 2860174 & 266404 & M & 94.007 & 7.328 & 8.208 \\
\hline 2 & 8.467 & 182351 & 14803 & M & 5.993 & 8.229 & 8.795 \\
\hline Total & & 3042525 & 281207 & & 100.000 & & \\
\hline
\end{tabular}




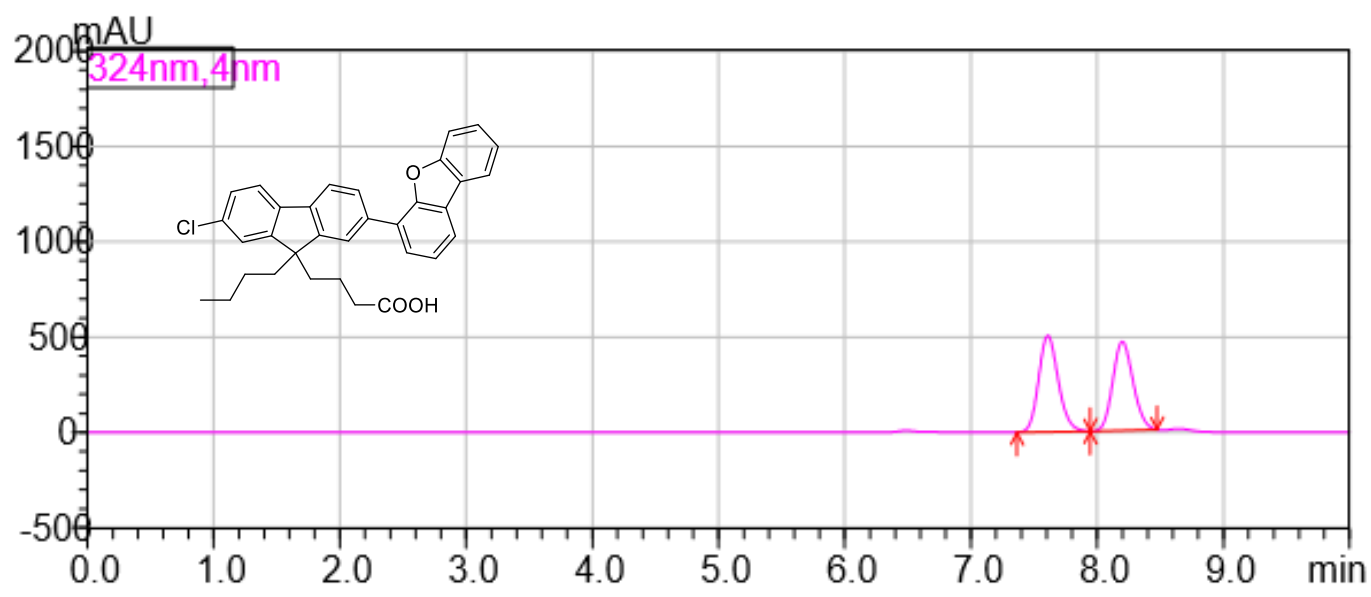

<Peak Table>

PDA Ch1 324nm

\begin{tabular}{|r|r|r|r|r|r|r|r|}
\hline Peak\# & Ret. Time & Area & Height & Mark & Area\% & Peak Start & Peak End \\
\hline 1 & 7.608 & 5462436 & 504995 & M & 50.922 & 7.365 & 7.947 \\
\hline 2 & 8.199 & 5264578 & 467558 & M & 49.078 & 7.947 & 8.475 \\
\hline Total & & 10727014 & 972553 & & 100.000 & & \\
\hline
\end{tabular}

$30 \mathrm{mAL}$

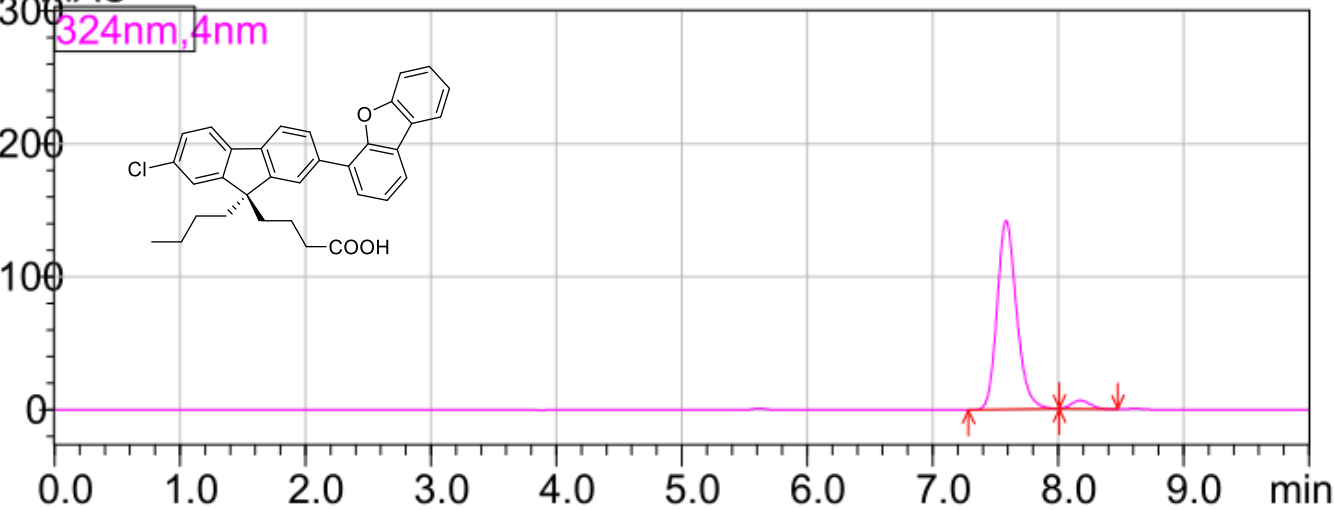

<Peak Table>

PDA Ch1 324nm

\begin{tabular}{|r|r|r|r|r|r|r|r|}
\hline Peak\# Ret. Time & \multicolumn{1}{|c|}{ Area } & \multicolumn{1}{|c|}{ Height } & Mark & Area\% & Peak Start & Peak End \\
\hline 1 & 7.585 & 1528908 & 141835 & M & 95.937 & 7.285 & 8.011 \\
\hline 2 & 8.176 & 64744 & 6234 & M & 4.063 & 8.011 & 8.475 \\
\hline Total & & 1593652 & 148069 & & 100.000 & & \\
\hline
\end{tabular}


30

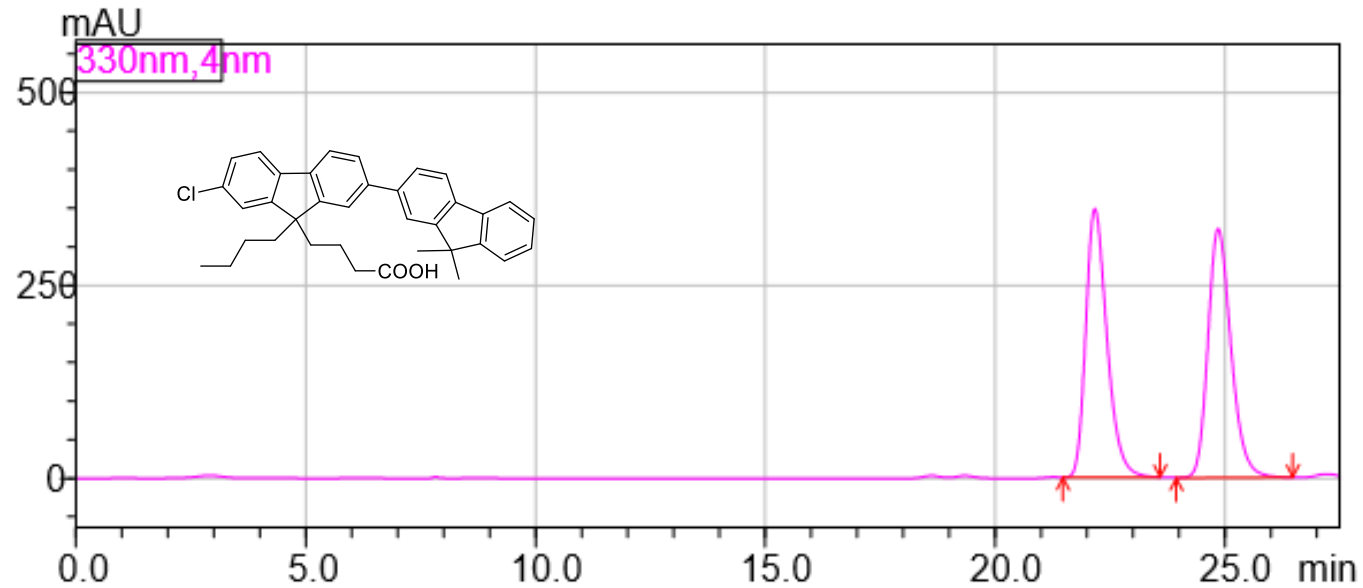

<Peak Table>

PDA Ch1 330nm

\begin{tabular}{r|r|r}
\hline Peak\# & Ret. Time \\
\hline 1 & 22.168 \\
\hline
\end{tabular}

\begin{tabular}{|r|r|r|r|r|r|r|r|}
\hline Peak\# & Ret. Time & \multicolumn{1}{c|}{ Area } & Height & Mark & \multicolumn{1}{c|}{ Area\% } & Peak Start & Peak End \\
\hline 1 & 22.168 & 11172774 & 347968 & $\mathrm{M}$ & 49.811 & 21.472 & 23.589 \\
\hline 2 & 24.851 & 11257343 & 322486 & $\mathrm{M}$ & 50.189 & 23.941 & 26.469 \\
\hline Total & & 22430117 & 670454 & & 100.000 & & \\
\hline
\end{tabular}

mAU

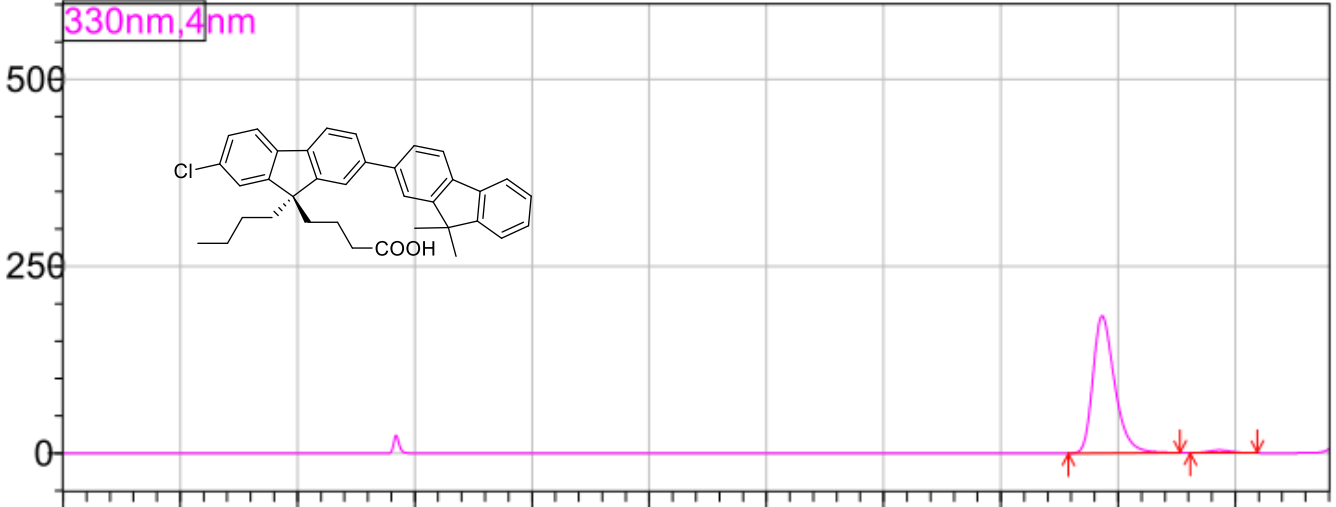

<Peak Table>

PDA Ch1 330nm

Peak\# Ret. Time

\begin{tabular}{|r|r|}
\hline 1 & 22.160 \\
\hline 2 & 24.657 \\
\hline
\end{tabular}

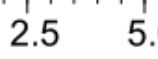

5.07 .5

$10.0 \quad 12.5$

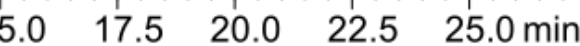

Total

24.657

\begin{tabular}{r|r|r|}
\multicolumn{1}{|c|}{ Area } & Height & 183594 \\
\hline 5934338 & 1835 \\
\hline 121821 & 3695 & \\
\hline 6056159 & 187289 &
\end{tabular}

\begin{tabular}{|c|r|r|r|}
\hline Mark & \multicolumn{1}{|c|}{ Area\% } & Peak Start & Peak End \\
\hline M & 97.988 & 21.440 & 23.824 \\
\hline M & 2.012 & 24.037 & 25.467 \\
\hline & 100.000 & & \\
\hline
\end{tabular}




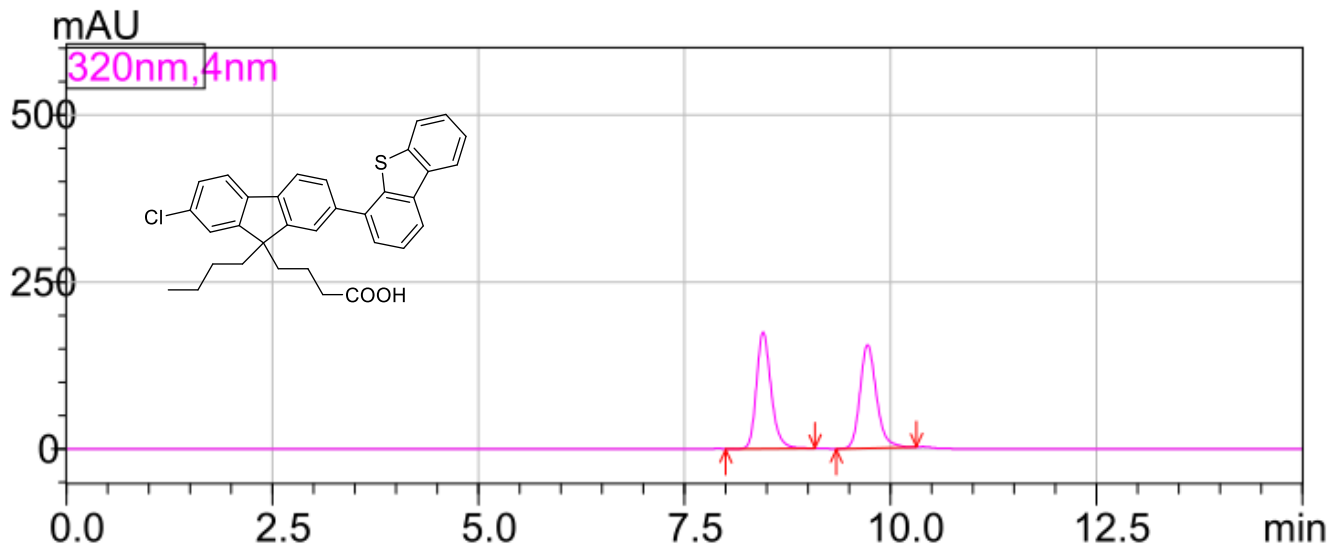

<Peak Table>

PDA Ch1 320nm

\begin{tabular}{|r|r|r|r|l|r|r|r|}
\hline Peak\# Ret. Time & Area & Height & Mark & Area\% & Peak Start & Peak End \\
\hline 1 & 8.454 & 2150008 & 174394 & M & 50.057 & 8.000 & 9.083 \\
\hline 2 & 9.722 & 2145135 & 154725 & M & 49.943 & 9.344 & 10.315 \\
\hline Total & & 4295142 & 329119 & & 100.000 & & \\
\hline
\end{tabular}

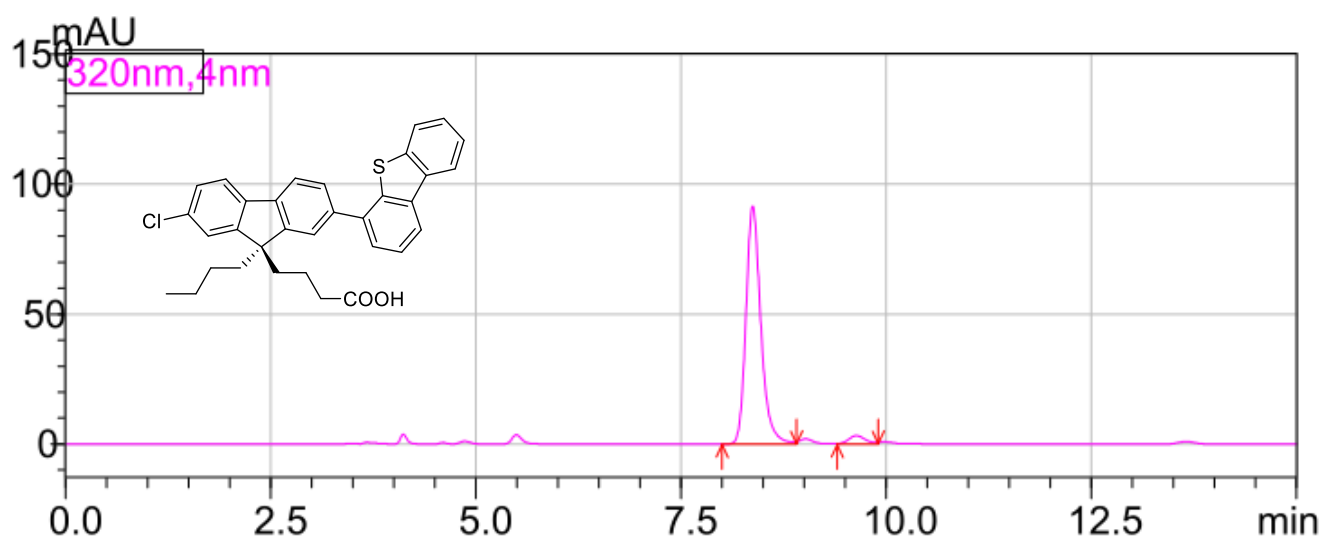

<Peak Table>

PDA Ch1 320nm

\begin{tabular}{|r|r|r|r|c|r|r|r|}
\hline Peak\# Ret. Time & \multicolumn{1}{|c|}{ Area } & \multicolumn{1}{|c|}{ Height } & Mark & Area\% & Peak Start & Peak End \\
\hline 1 & 8.373 & 1161882 & 91670 & M & 96.007 & 8.000 & 8.907 \\
\hline 2 & 9.638 & 48320 & 3275 & M & 3.993 & 9.403 & 9.904 \\
\hline Total & & 1210202 & 94945 & & 100.000 & & \\
\hline
\end{tabular}




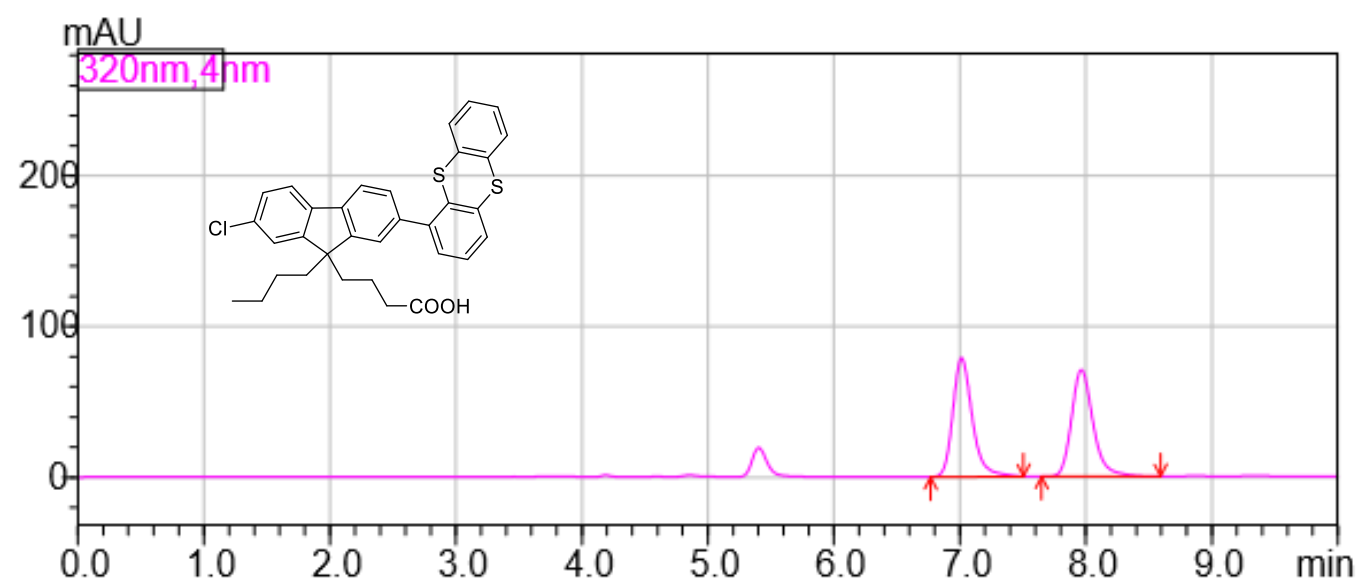

<Peak Table>

PDA Ch1 320nm

\begin{tabular}{|r|r|r|r|r|r|r|}
\hline Peak\# & Ret. Time & \multicolumn{1}{c|}{ Area } & \multicolumn{1}{c|}{ Height } & Peak Start & Peak End & \multicolumn{1}{c|}{ Area \% } \\
\hline 1 & 7.013 & 852808 & 79008 & 6.768 & 7.504 & 50.289 \\
\hline 2 & 7.964 & 843016 & 70841 & 7.648 & 8.592 & 49.711 \\
\hline Total & & 1695824 & 149849 & & & 100.000 \\
\hline
\end{tabular}

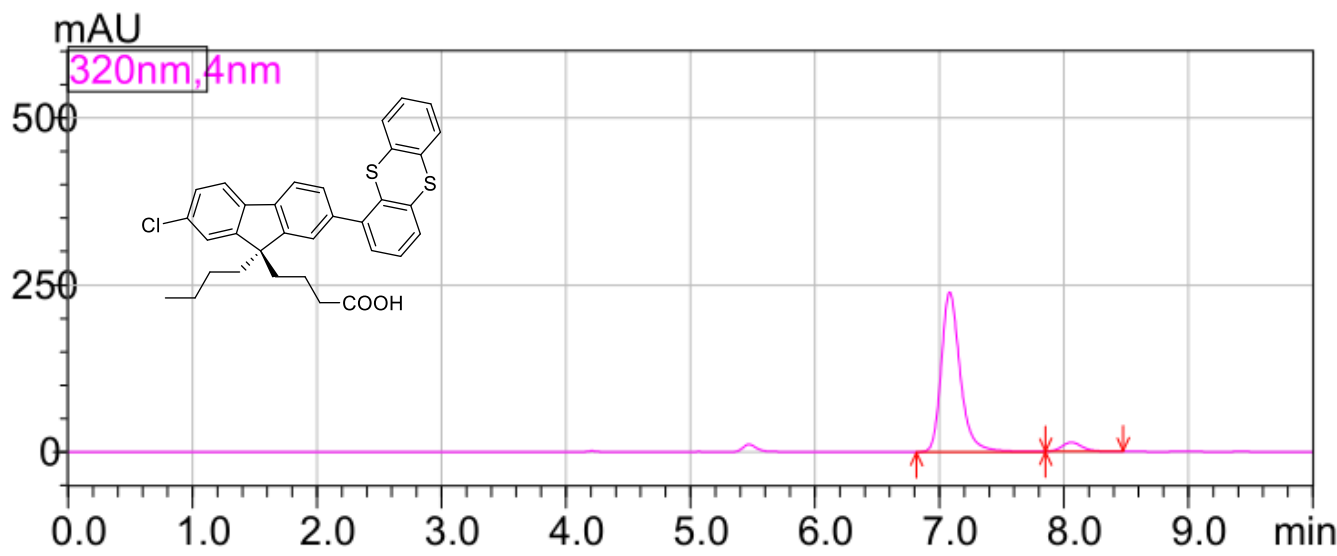

<Peak Table>

PDA Ch1 320nm

\begin{tabular}{|c|c|c|c|c|c|c|c|}
\hline Peak\# & t. Time & Area & Height & Mark & Area\% & Peak Start & Peak End \\
\hline 1 & 7.082 & 2550028 & 239479 & $\mathrm{M}$ & 94.269 & 6.816 & 7.851 \\
\hline 2 & 8.060 & 155032 & 13654 & M & 5.731 & 7.851 & 8.475 \\
\hline Total & & 2705060 & 253133 & & 100.000 & & \\
\hline
\end{tabular}


mAU

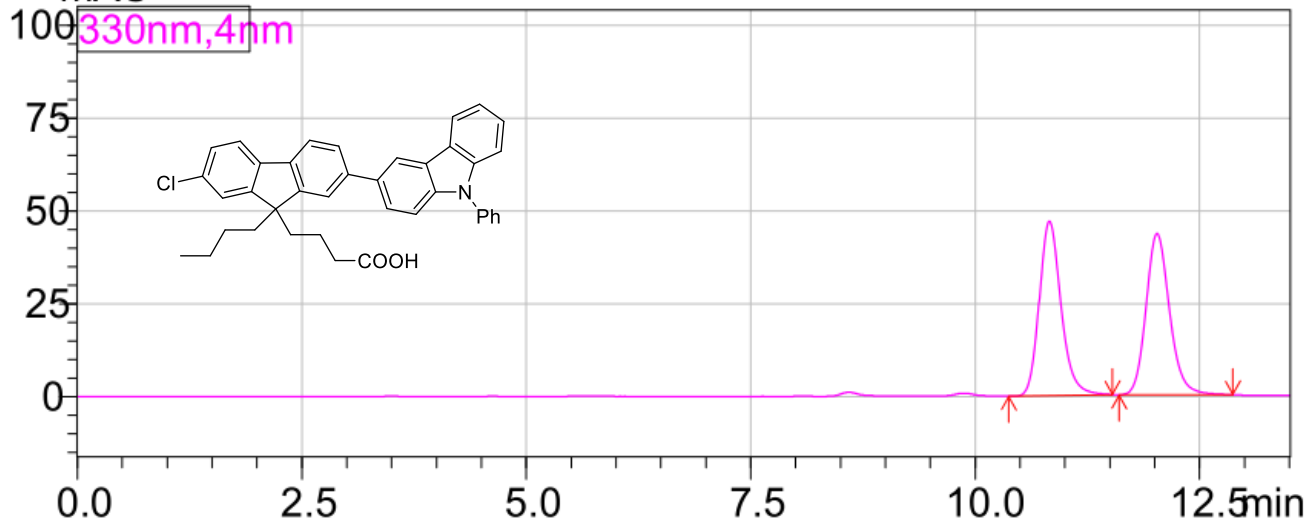

<Peak Table>

PDA Ch1 330nm

\begin{tabular}{|r|r|r|r|r|r|r|r|}
\hline Peak\# Ret. Time & \multicolumn{1}{|c|}{ Area } & Height & Mark & Area\% & Peak Start & Peak End \\
\hline 1 & 10.824 & 792380 & 47029 & $\mathrm{M}$ & 49.866 & 10.373 & 11.531 \\
\hline 2 & 12.026 & 796636 & 43460 & $\mathrm{M}$ & 50.134 & 11.600 & 12.869 \\
\hline Total & & 1589016 & 90489 & & 100.000 & & \\
\hline
\end{tabular}

mAU

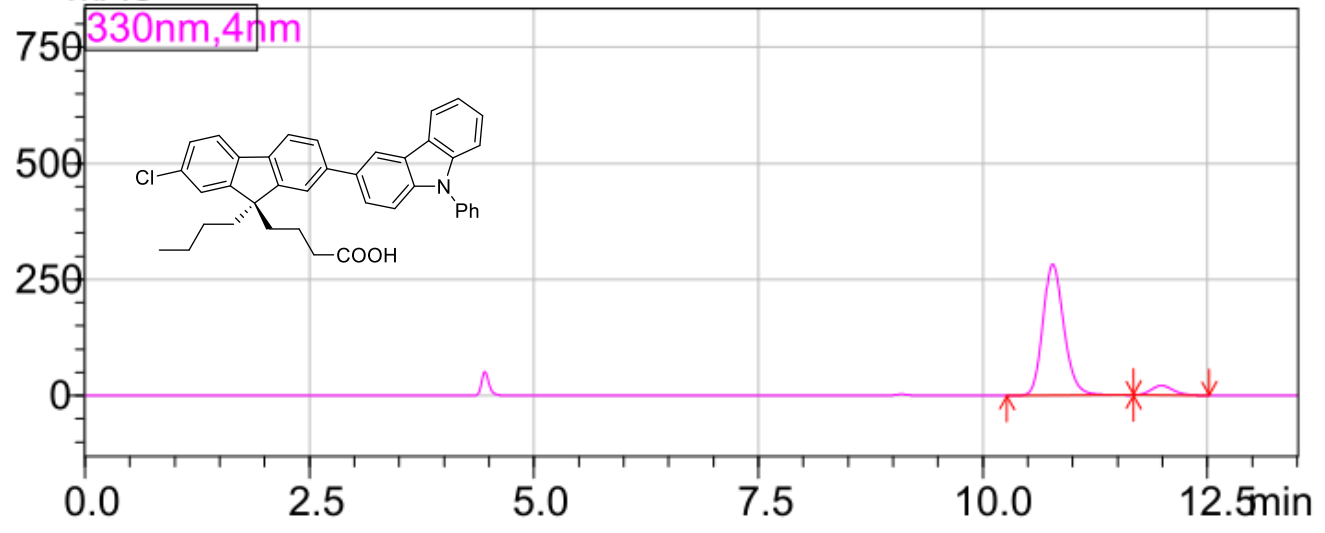

<Peak Table>

PDA Ch1 330nm

\begin{tabular}{|r|r|r|r|r|r|r|r|}
\hline Peak\# Ret. Time & \multicolumn{1}{|c|}{ Area } & \multicolumn{1}{|c|}{ Height } & Mark & Area\% & Peak Start & Peak End \\
\hline 1 & 10.775 & 4590288 & 282862 & M & 92.689 & 10.267 & 11.675 \\
\hline 2 & 11.988 & 362090 & 20630 & M & 7.311 & 11.675 & 12.517 \\
\hline Total & & 4952378 & 303492 & & 100.000 & & \\
\hline
\end{tabular}




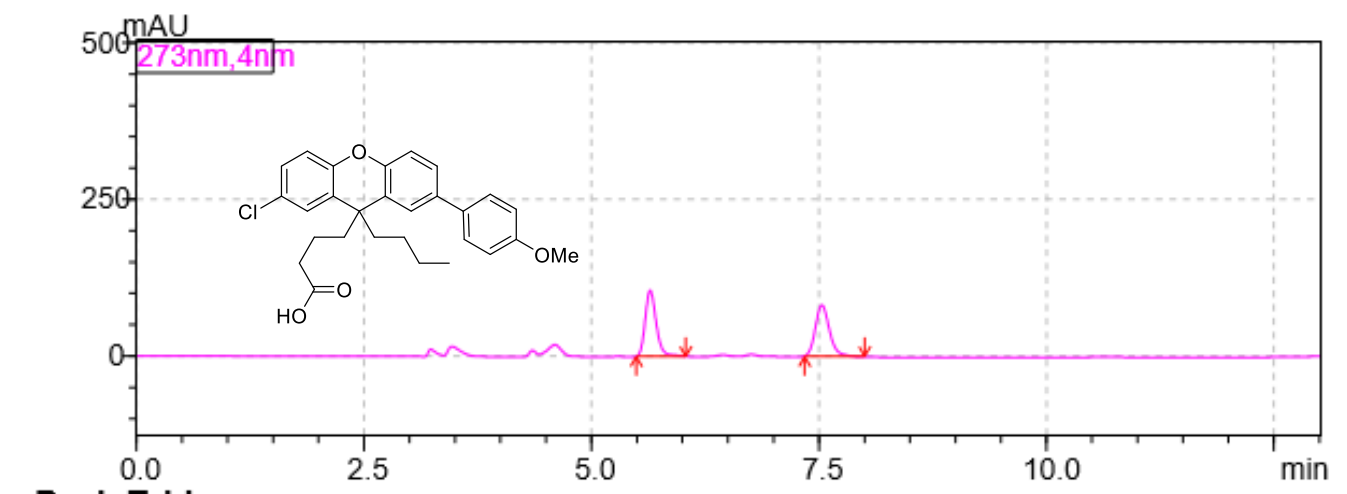

<Peak Table>

PDA Ch1 273nm

\begin{tabular}{|r|r|r|r|r|r|r|}
\hline Peak\# Ret. Time & \multicolumn{1}{|c|}{ Area } & \multicolumn{1}{c|}{ Height } & \multicolumn{1}{c|}{ Area\% } & Peak Start & Peak End \\
\hline 1 & 5.645 & 906091 & 105748 & 50.371 & 5.493 & 6.037 \\
\hline 2 & 7.532 & 892761 & 82420 & 49.629 & 7.344 & 8.000 \\
\hline Total & & 1798853 & 188167 & 100.000 & & \\
\hline
\end{tabular}

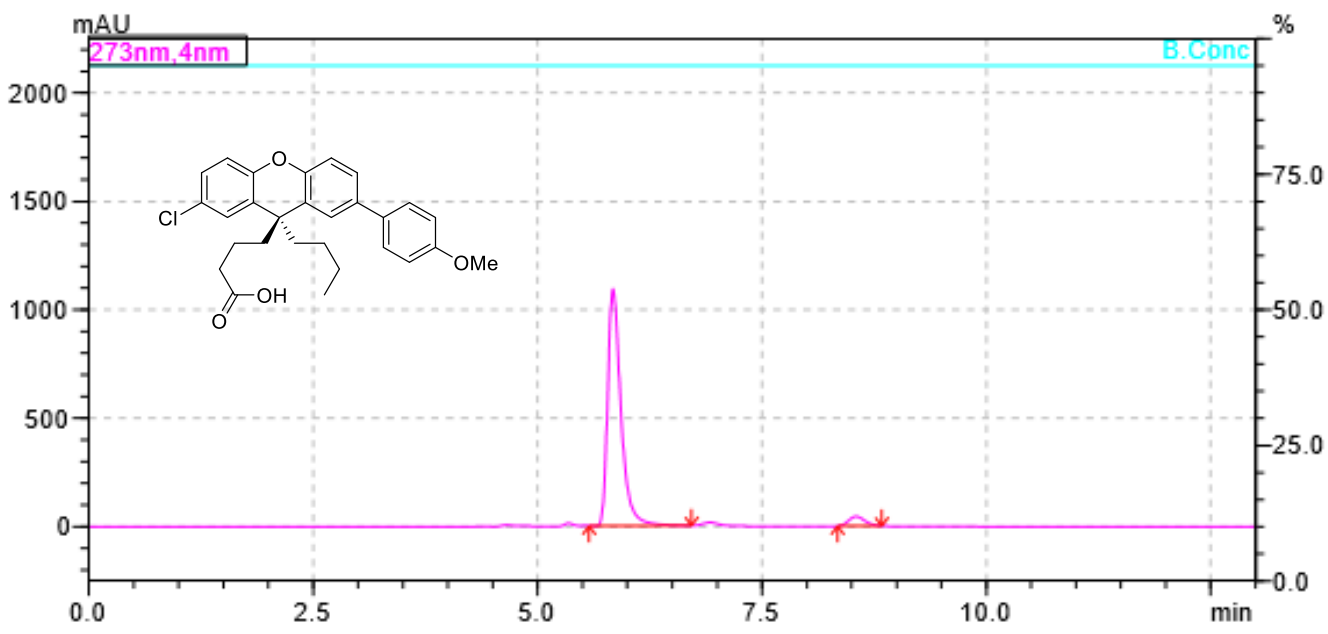

<Peak Table>

PDA Ch1 273nm

\begin{tabular}{|r|r|r|r|r|r|r|}
\hline Peak\# & Ret. Time & \multicolumn{1}{c|}{ Area } & \multicolumn{1}{c|}{ Height } & \multicolumn{1}{c|}{ Area\% } & Peak Start & Peak End \\
\hline 1 & 5.840 & 11259551 & 1092140 & 95.297 & 5.573 & 6.715 \\
\hline 2 & 8.549 & 555671 & 43655 & 4.703 & 8.341 & 8.832 \\
\hline Total & & 11815222 & 1135795 & 100.000 & & \\
\hline
\end{tabular}




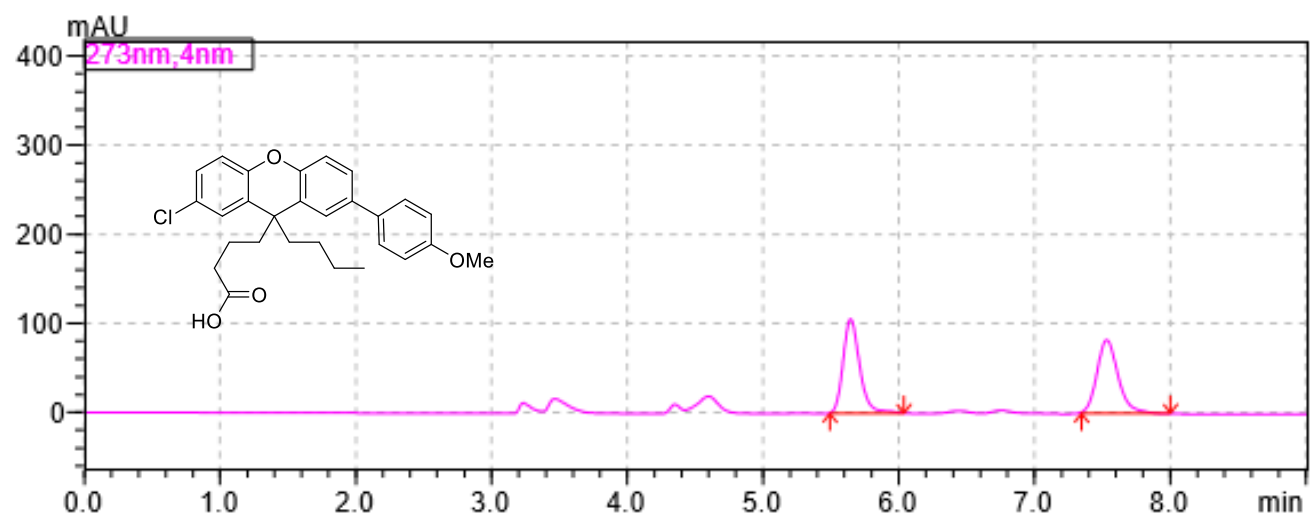

<Peak Table>

PDA Ch1 273nm

\begin{tabular}{|c|c|c|c|c|c|c|}
\hline Peak\# & Ret. Time & Area & Height & Area $\%$ & Peak Start & Peak End \\
\hline 1 & 5.645 & 906091 & 105748 & 50.371 & 5.493 & 6.03 \\
\hline 2 & 7.532 & 892761 & 82420 & 49.629 & 7.344 & 8.00 \\
\hline Total & & 1798853 & 188167 & 100.000 & & \\
\hline
\end{tabular}

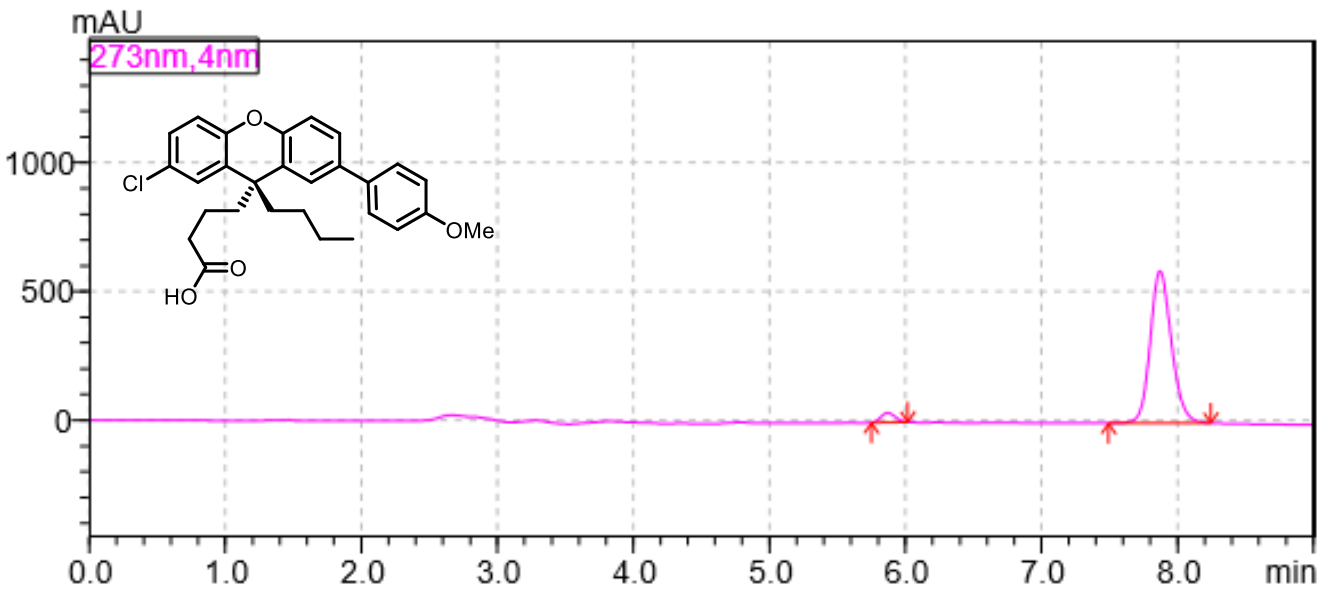

<Peak Table>

PDA Ch1 273nm

\begin{tabular}{|r|r|r|r|r|r|r|}
\hline Peak\# & Ret. Time & \multicolumn{1}{c|}{ Area } & \multicolumn{1}{c|}{ Height } & \multicolumn{1}{c|}{ Area\% } & Peak Start & Peak End \\
\hline 1 & 5.870 & 269620 & 37049 & 4.025 & 5.749 & 6.016 \\
\hline 2 & 7.871 & 6428568 & 590825 & 95.975 & 7.493 & 8.245 \\
\hline Total & & 6698188 & 627874 & 100.000 & & \\
\hline
\end{tabular}




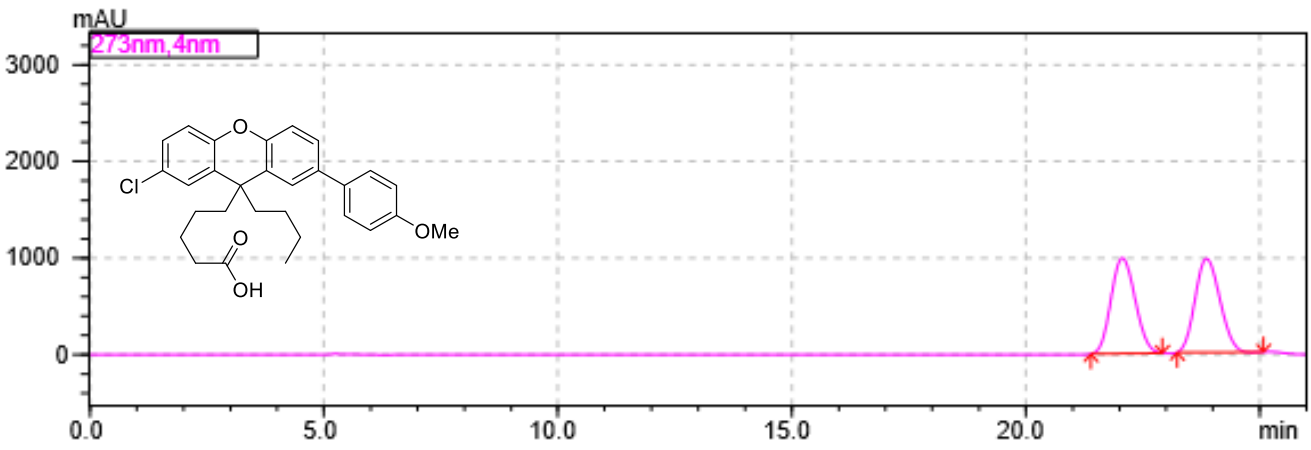

<Peak Table>

PDA Ch1 273nm

\begin{tabular}{|r|r|r|r|c|r|r|r|}
\hline Peak\# & Ret. Time & \multicolumn{1}{c|}{ Area } & \multicolumn{1}{c|}{ Height } & Mark & Peak Start & Peak End & \multicolumn{1}{c|}{ Area\% } \\
\hline 1 & 22.063 & 35610635 & 988909 & $\mathrm{M}$ & 21.387 & 22.912 & 49.999 \\
\hline 2 & 23.862 & 35612259 & 968627 & $\mathrm{M}$ & 23.232 & 25.077 & 50.001 \\
\hline Total & & 71222894 & 1957536 & & & & 100.000 \\
\hline
\end{tabular}

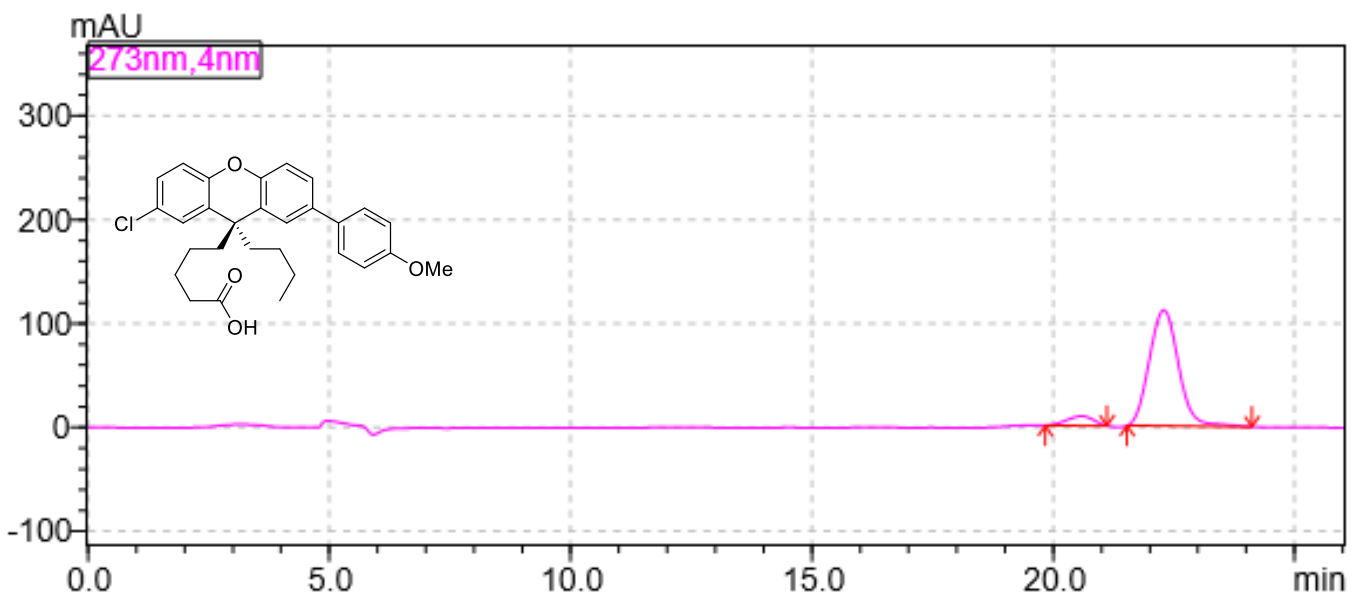

<Peak Table>

PDA Ch1 273nm

\begin{tabular}{|r|r|r|r|r|r|r|r|}
\hline Peak\# & Ret. Time & \multicolumn{1}{c|}{ Area } & Height & Mark & Peak Start & Peak End & \multicolumn{1}{c|}{ Area\% } \\
\hline 1 & 20.587 & 344742 & 8950 & M & 19.829 & 21.115 & 6.826 \\
\hline 2 & 22.291 & 4705984 & 111632 & M & 21.531 & 24.112 & 93.174 \\
\hline Total & & 5050725 & 120583 & & & & 100.000 \\
\hline
\end{tabular}




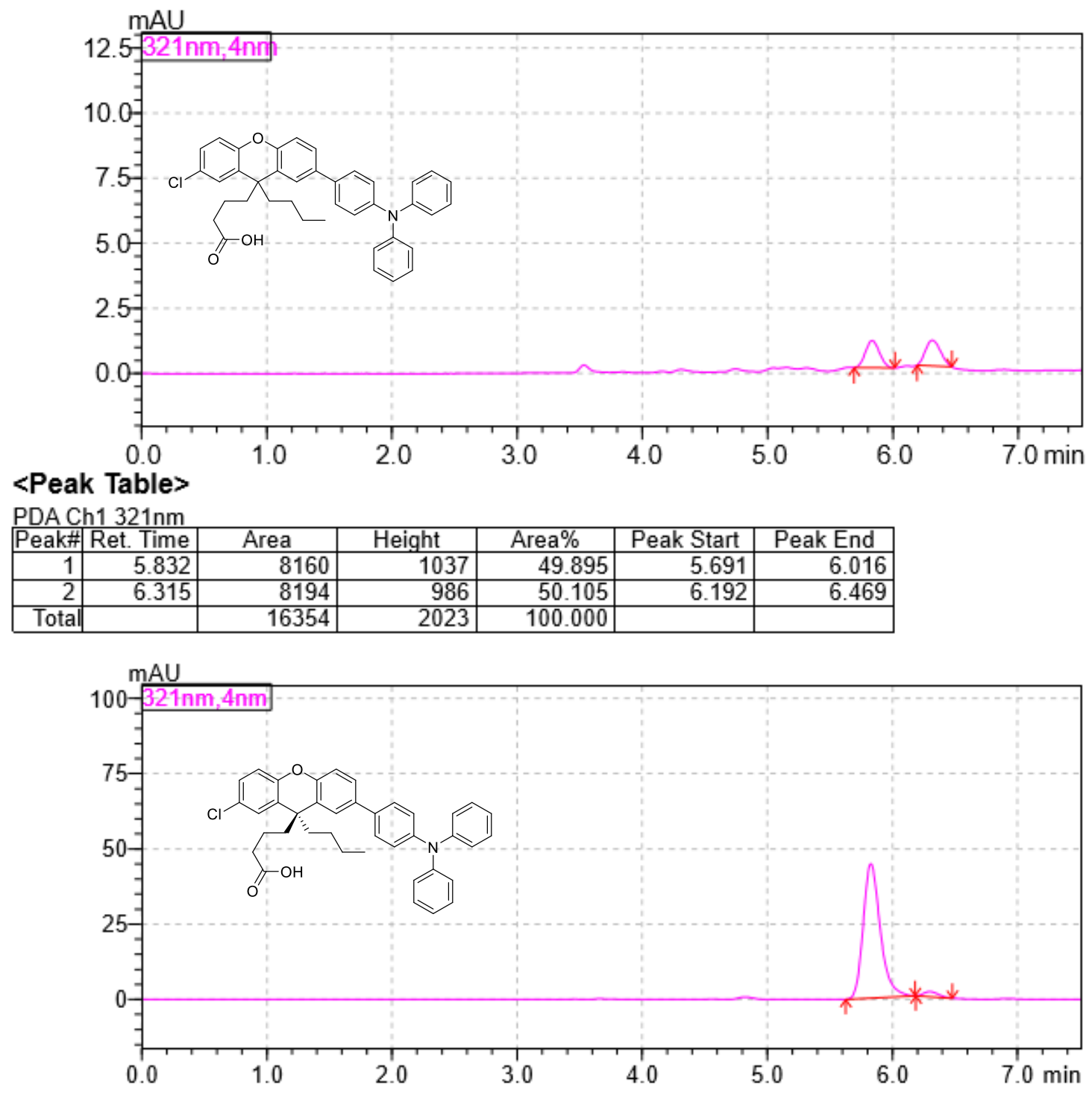

<Peak Table>

PDA Ch1 321nm

\begin{tabular}{|r|r|r|r|r|r|r|}
\hline Peak\# & Ret. Time & \multicolumn{1}{|c|}{ Area } & Height & \multicolumn{1}{c|}{ Area\% } & Peak Start & Peak End \\
\hline 1 & 5.827 & 425862 & 44585 & 96.856 & 5.627 & 6.181 \\
\hline 2 & 6.301 & 13826 & 1698 & 3.144 & 6.187 & 6.480 \\
\hline Total & & 439688 & 46283 & 100.000 & & \\
\hline
\end{tabular}




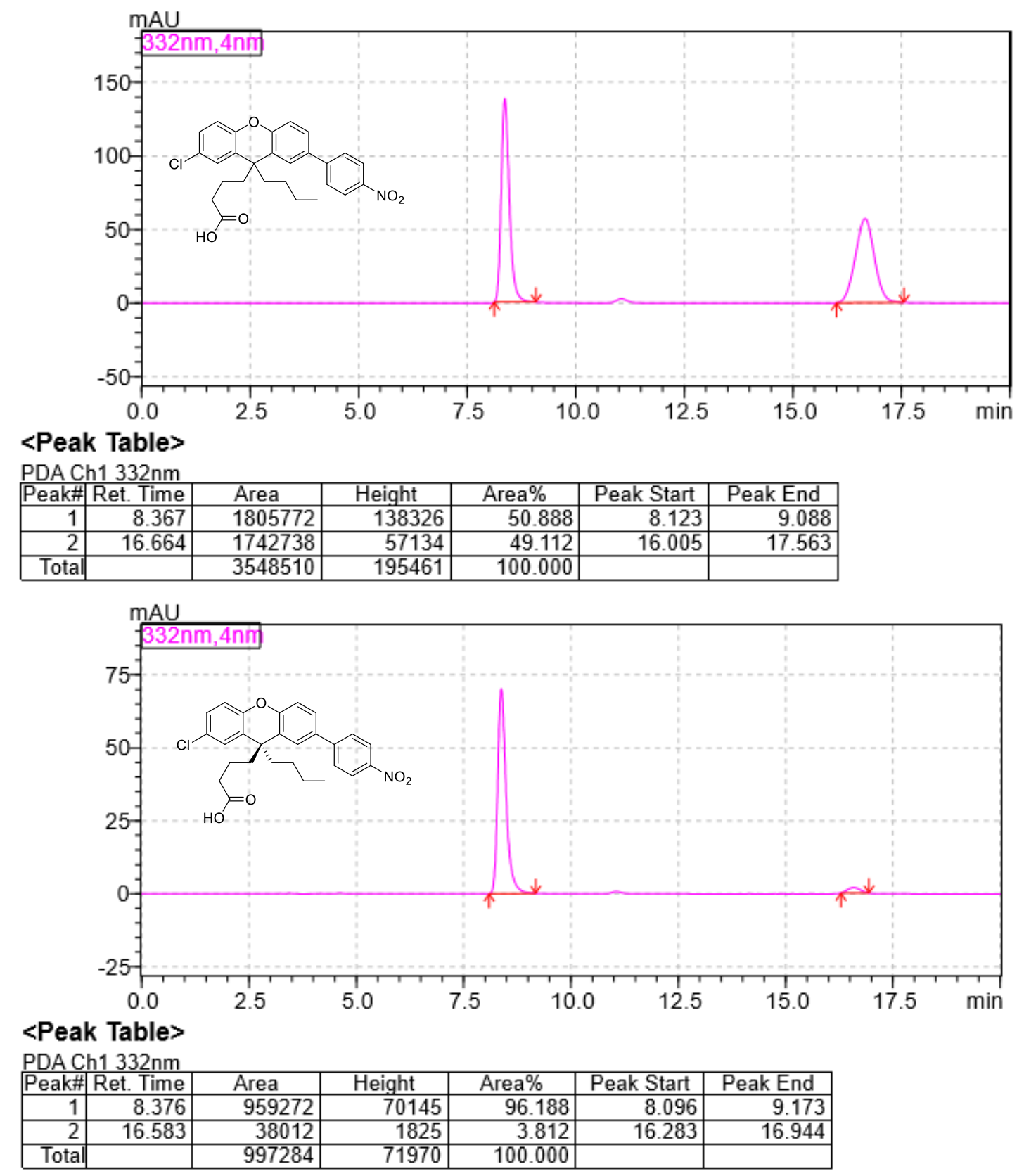




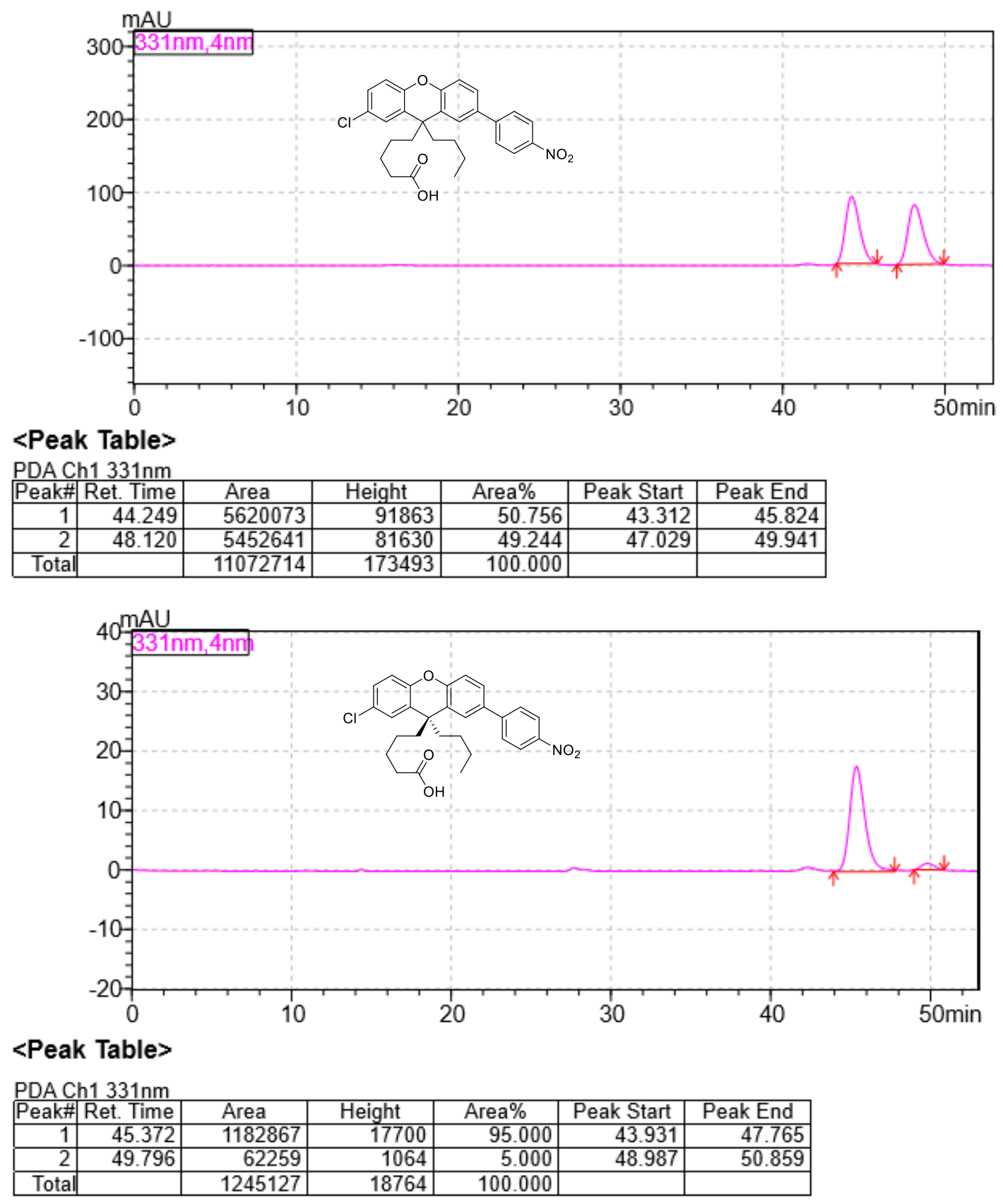




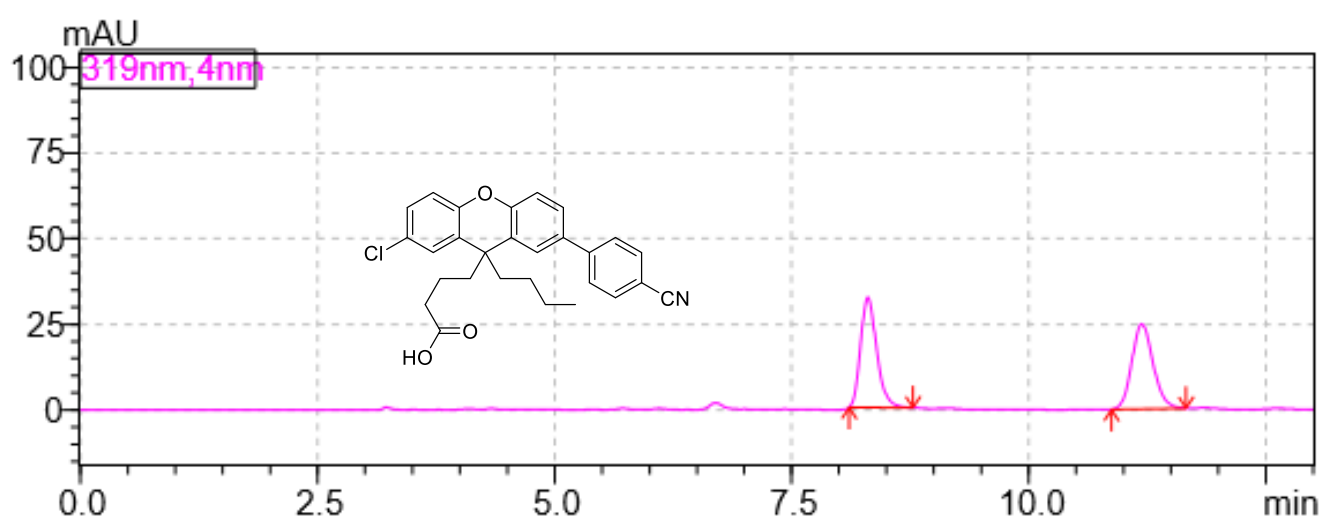

<Peak Table>

PDA Ch1 319nm

\begin{tabular}{|r|r|r|r|c|r|r|r|}
\hline Peak\# & Ret. Time & Area & \multicolumn{1}{c|}{ Height } & Mark & \multicolumn{1}{c|}{ Area\% } & Peak Start & Peak End \\
\hline 1 & 8.303 & 383604 & 32162 & $\mathrm{M}$ & 49.719 & 8.107 & 8.779 \\
\hline 2 & 11.192 & 387939 & 24761 & $\mathrm{M}$ & 50.281 & 10.869 & 11.659 \\
\hline Total & & 771543 & 56923 & & 100.000 & & \\
\hline
\end{tabular}

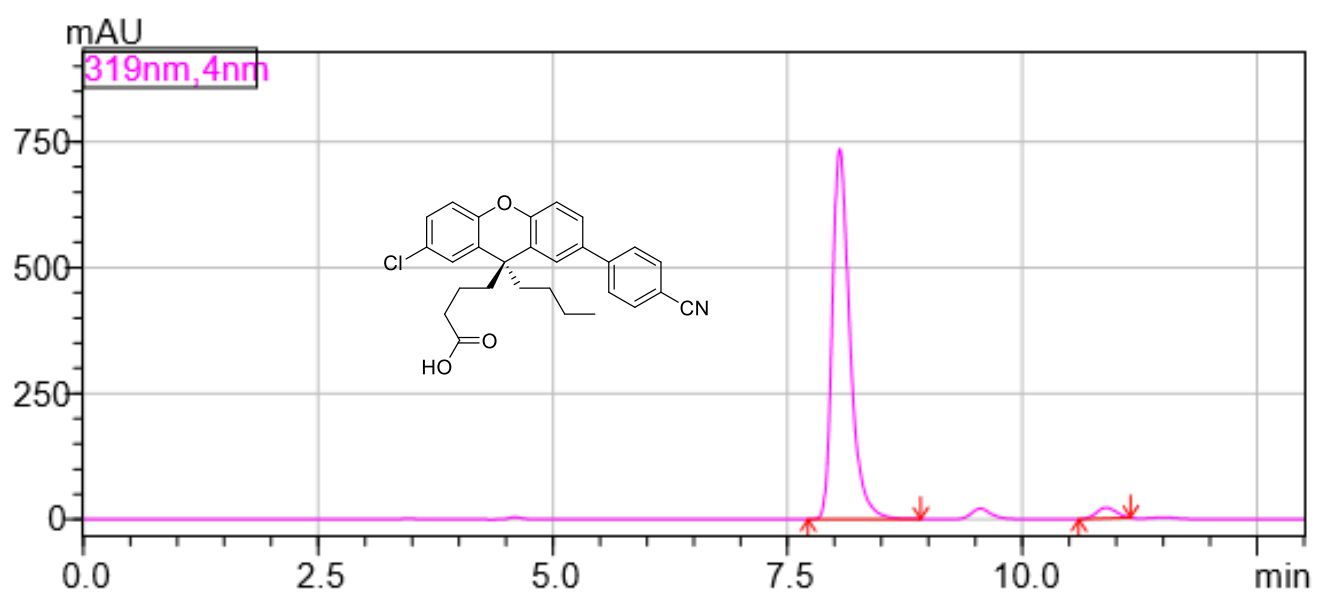

<Peak Table>

PDA Ch1 319nm

\begin{tabular}{|r|r|r|r|r|r|r|}
\hline Peak\# & Ret. Time & \multicolumn{1}{|c|}{ Area } & Height & Area\% & Peak Start & Peak End \\
\hline 1 & 8.054 & 9594456 & 736404 & 96.844 & 7.717 & 8.917 \\
\hline 2 & 10.893 & 312700 & 21266 & 3.156 & 10.603 & 11.152 \\
\hline Total & & 9907156 & 757670 & 100.000 & & \\
\hline
\end{tabular}


MAU

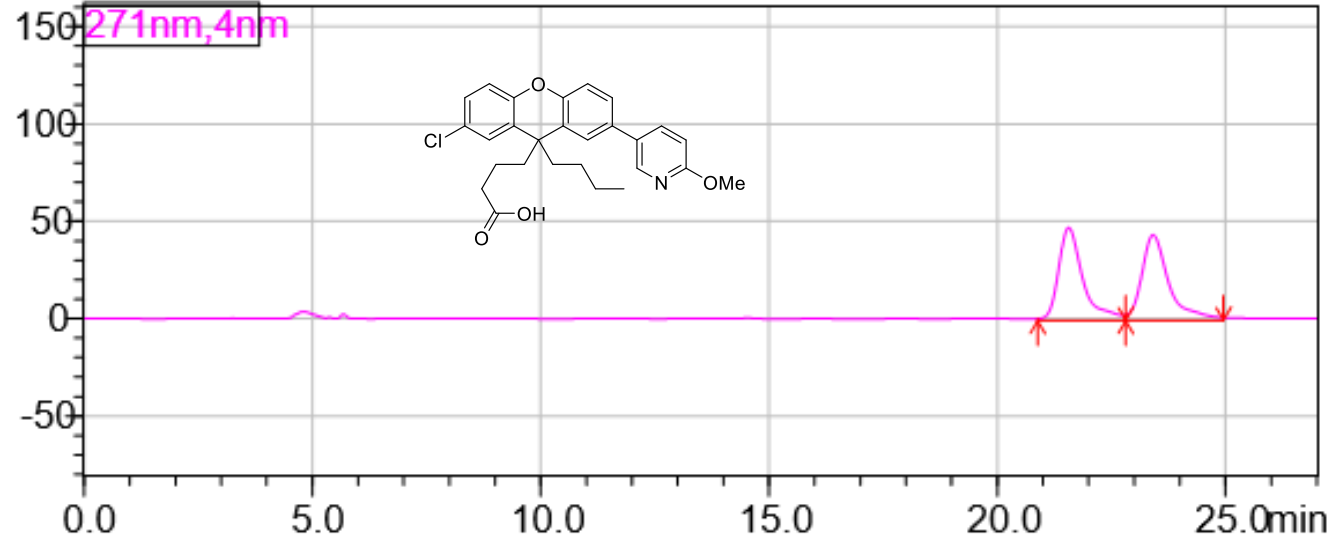

<Peak Table>

PDA Ch1 271nm

\begin{tabular}{|r|r|r|r|r|r|r|r|}
\hline Peak\# & Ret. Time & \multicolumn{1}{|c|}{ Area } & \multicolumn{1}{c|}{ Height } & Mark & Peak Start & Peak End & \multicolumn{1}{c|}{ Area\% } \\
\hline 1 & 21.563 & 1792694 & 47725 & $\mathrm{M}$ & 20.885 & 22.811 & 49.368 \\
\hline 2 & 23.413 & 1838617 & 44044 & $\mathrm{M}$ & 22.811 & 24.955 & 50.632 \\
\hline Total & & 3631312 & 91769 & & & & 100.000 \\
\hline
\end{tabular}

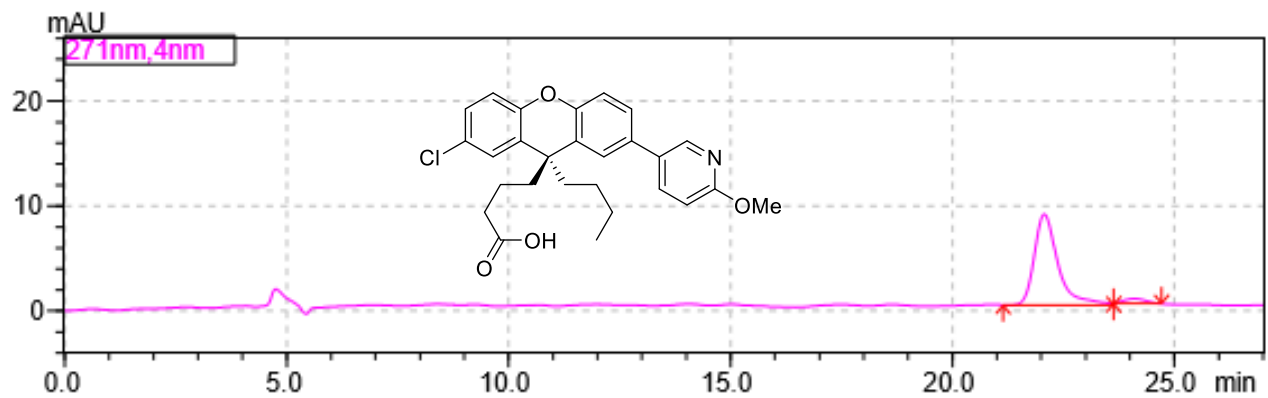

<Peak Table>

PDA Ch1271nm

\begin{tabular}{|c|c|c|c|c|c|c|c|}
\hline Peak\# & Ret. Time & Area & Area \% & Mark & Peak Start & Peak End & Height \\
\hline 1 & 22.070 & 323283 & 95.226 & $M$ & 21.141 & 23.632 & 8669 \\
\hline 2 & 24.070 & 16207 & 4.774 & M & 23.632 & 24.709 & 468 \\
\hline Total & & 339490 & 100.000 & & & & 9138 \\
\hline
\end{tabular}




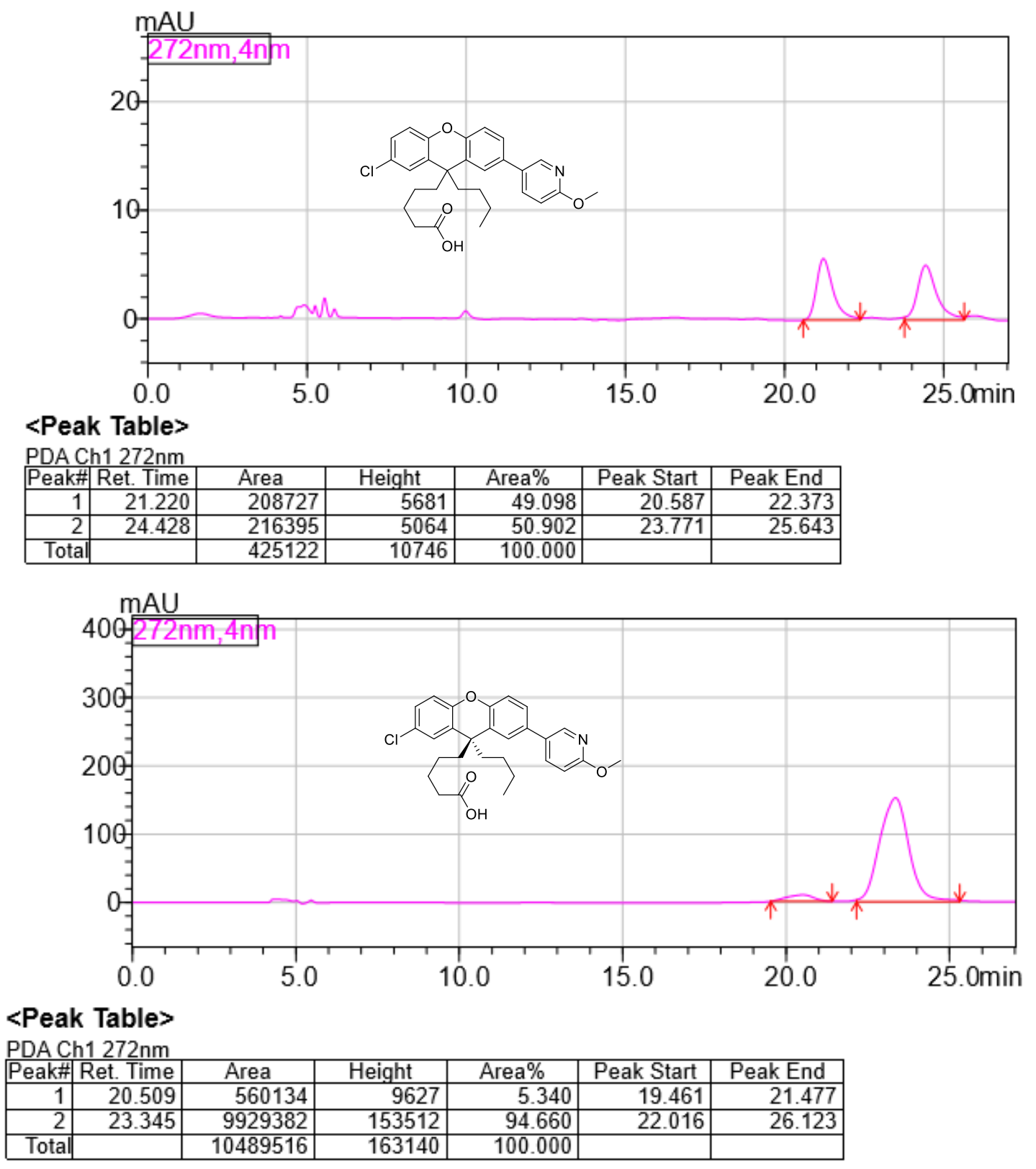




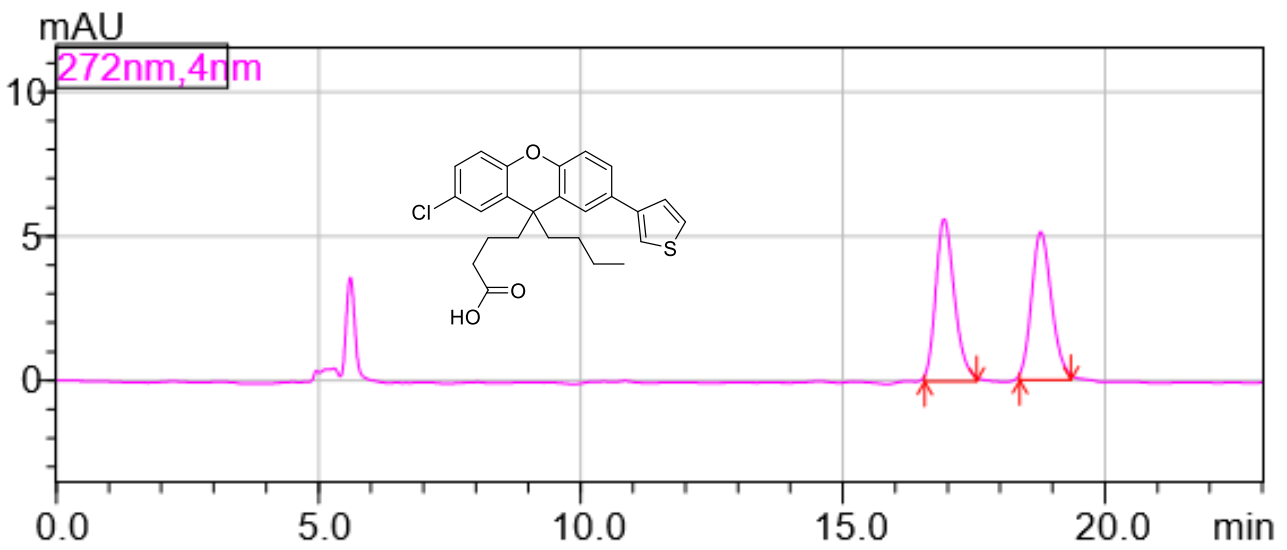

<Peak Table>

PDA Ch1 272nm

\begin{tabular}{|c|c|c|c|c|c|c|}
\hline Peak\# & Ret. Time & Area & Height & Area $\%$ & Peak Start & Peak End \\
\hline 1 & 16.934 & 141070 & 5629 & 50.695 & 16.565 & 17.552 \\
\hline 2 & 18.775 & 137203 & 5147 & 49.305 & 18.379 & 19.349 \\
\hline Total & & 278273 & 10776 & 100.000 & & \\
\hline
\end{tabular}

$\mathrm{mAU}$

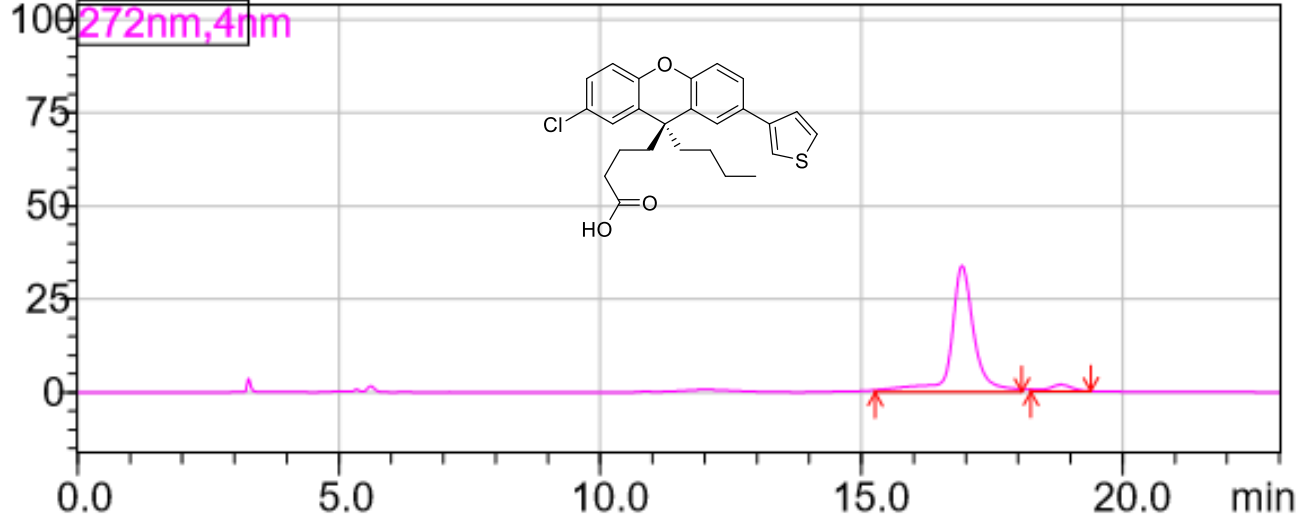

<Peak Table>

PDA Ch1 272nm

\begin{tabular}{|c|c|c|c|c|c|c|}
\hline Peak\# & Ret. Time & Area & Height & Area $\%$ & Peak Start & Peak End \\
\hline 1 & 16.925 & 1029787 & 33772 & 95.132 & 15.259 & 18.069 \\
\hline 2 & 18.822 & 52697 & 1732 & 4.868 & 18.240 & 19.387 \\
\hline Total & & 1082484 & 35503 & 100.000 & & \\
\hline
\end{tabular}




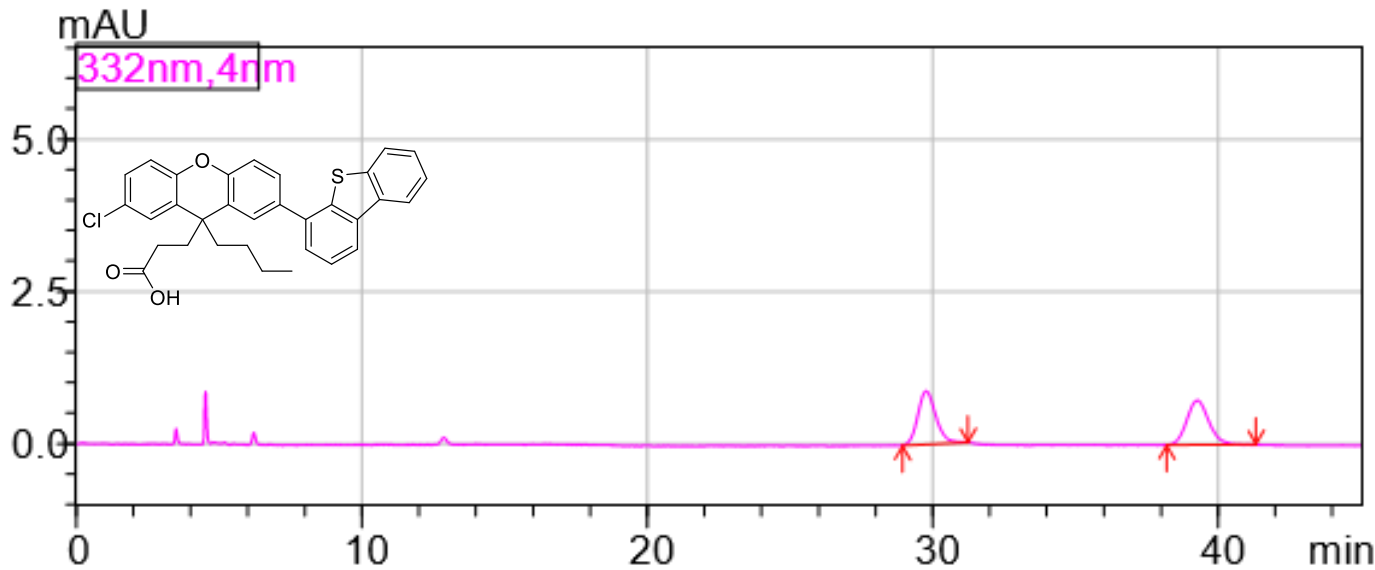

\section{<Peak Table>}

PDA Ch1 332nm

\begin{tabular}{|r|r|r|r|r|r|r|}
\hline Peak\# & Ret. Time & \multicolumn{1}{c|}{ Area } & \multicolumn{1}{c|}{ Height } & Area\% & Peak Start & Peak End \\
\hline 1 & 29.779 & 38675 & 877 & 49.664 & 28.939 & 31.243 \\
\hline 2 & 39.274 & 39198 & 733 & 50.336 & 38.197 & 41.323 \\
\hline Total & & 77873 & 1610 & 100.000 & & \\
\hline
\end{tabular}

\section{mAU}

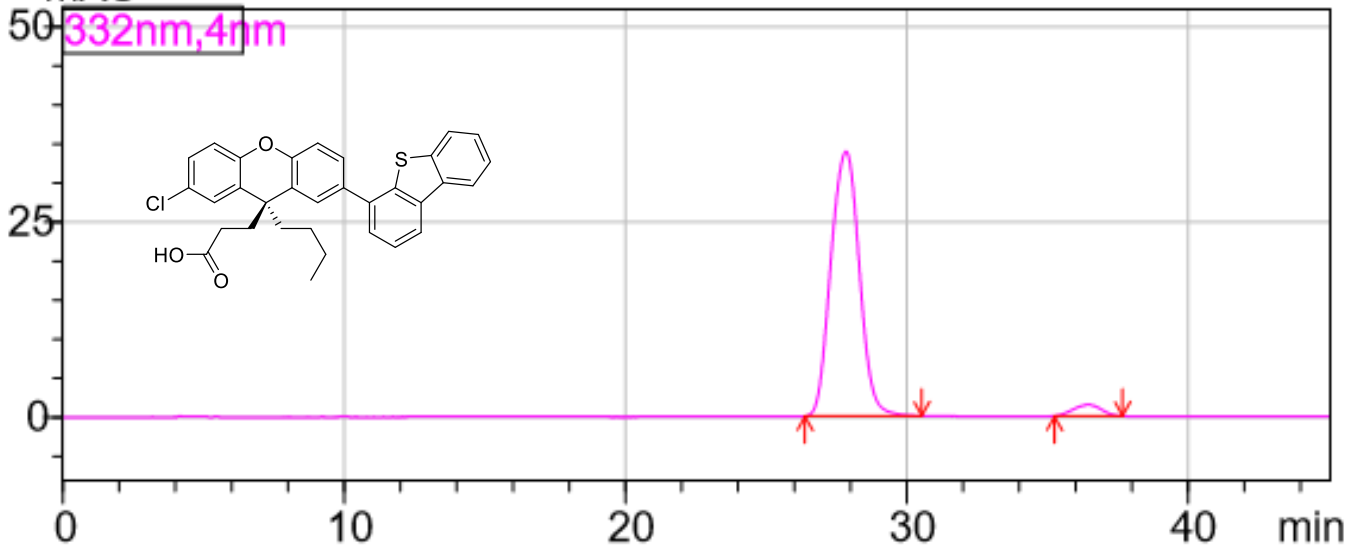

<Peak Table>

PDA Ch1 332nm

\begin{tabular}{|r|r|r|r|r|r|r|}
\hline Peak\# & Ret. Time & \multicolumn{1}{c|}{ Area } & \multicolumn{1}{c|}{ Height } & \multicolumn{1}{c|}{ Area\% } & Peak Start & Peak End \\
\hline 1 & 27.837 & 2384424 & 33873 & 95.965 & 26.373 & 30.523 \\
\hline 2 & 36.433 & 100261 & 1439 & 4.035 & 35.253 & 37.675 \\
\hline Total & & 2484685 & 35312 & 100.000 & & \\
\hline
\end{tabular}




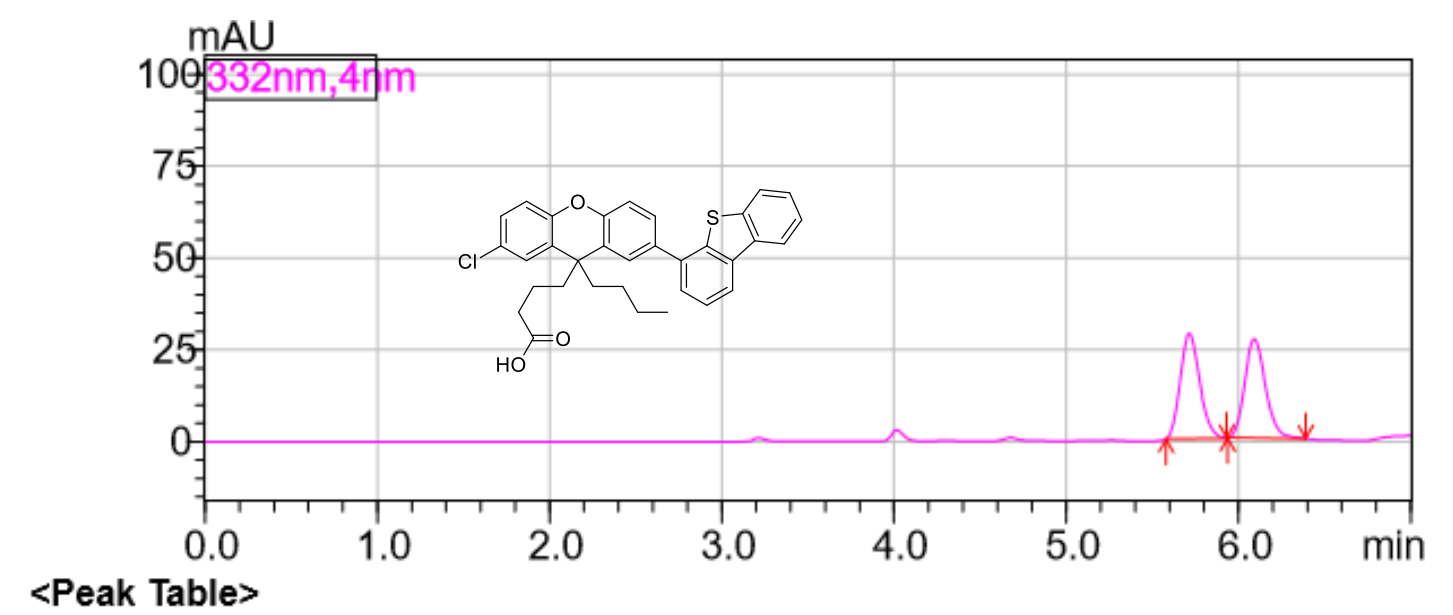

PDA Ch1 332nm

\begin{tabular}{|c|c|c|c|c|c|c|c|}
\hline Peak\# & Ret. Time & Area & Height & Mark & Area \% & Peak Start & Peak End \\
\hline 1 & 5.714 & 223782 & 28565 & $\mathrm{M}$ & 49.626 & 5.579 & 5.931 \\
\hline 2 & 6.091 & 227152 & 26973 & $M$ & 50.374 & 5.936 & 6.389 \\
\hline Total & & 450934 & 55538 & & 100.000 & & \\
\hline
\end{tabular}

mAU

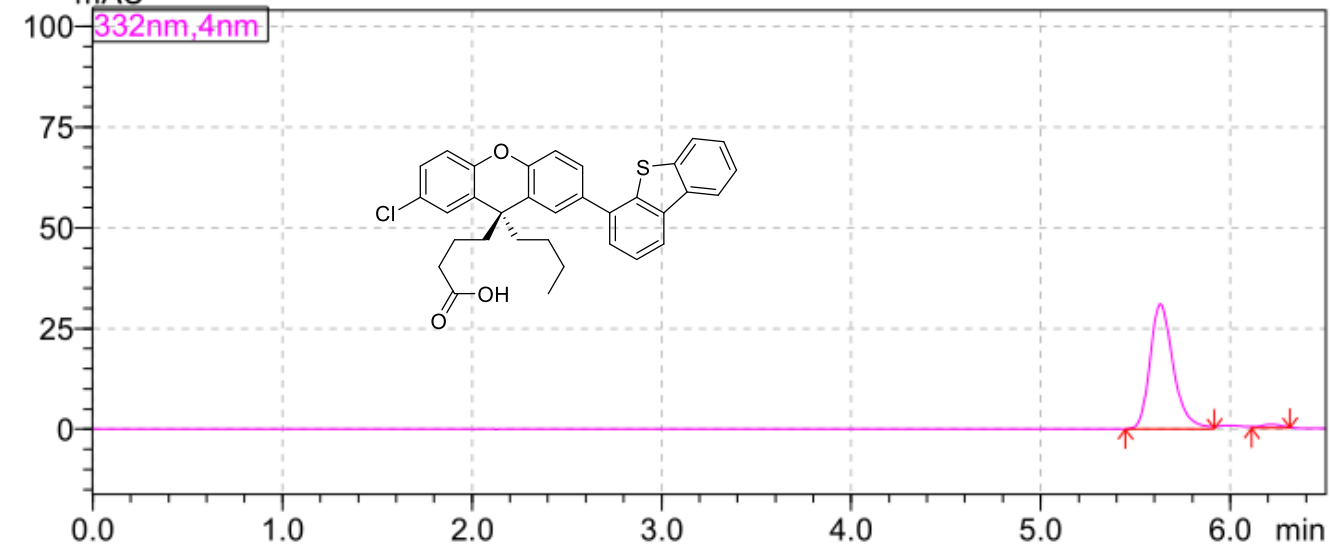

<Peak Table>

PDA Ch1 332nm

\begin{tabular}{|r|r|r|r|r|r|r|}
\hline Peak\# Ret. Time & \multicolumn{1}{|c|}{ Area } & \multicolumn{1}{|c|}{ Height } & Area\% & Peak Start & Peak End \\
\hline 1 & 5.631 & 267341 & 31103 & 97.628 & 5.445 & 5.915 \\
\hline 2 & 6.217 & 6494 & 828 & 2.372 & 6.112 & 6.315 \\
\hline Total & & 273836 & 31932 & 100.000 & & \\
\hline
\end{tabular}




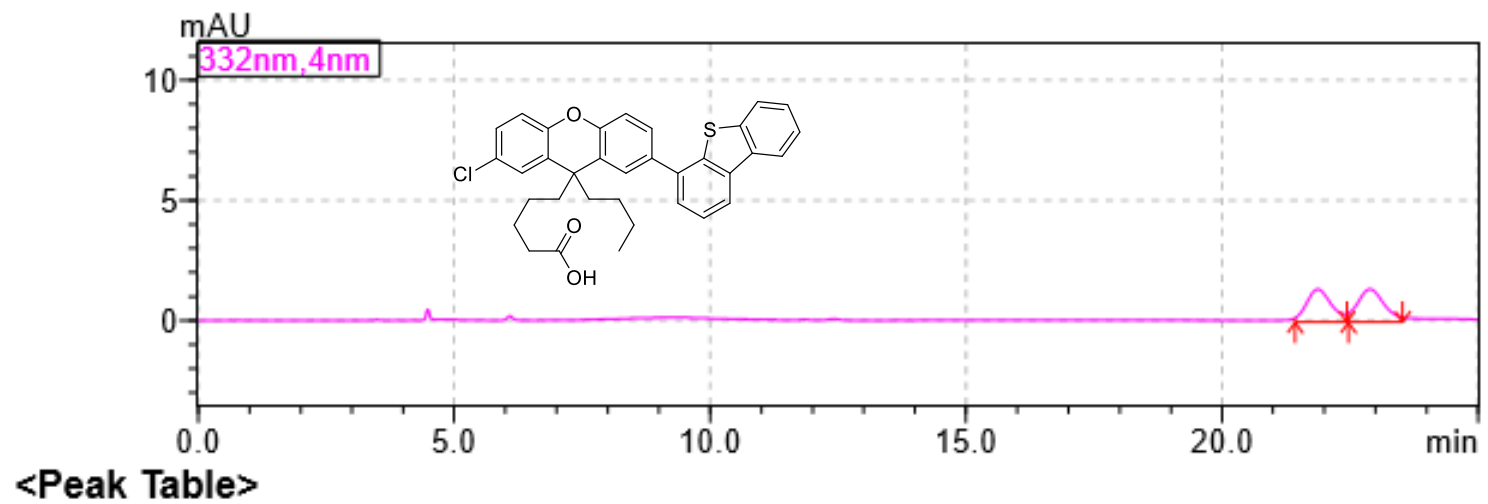

PDA Ch1 332nm

\begin{tabular}{|r|r|r|r|r|r|r|}
\hline Peak\# & Ret. Time & \multicolumn{1}{c|}{ Area } & \multicolumn{1}{c|}{ Height } & \multicolumn{1}{c|}{ Area\% } & Peak Start & Peak End \\
\hline 1 & 21.871 & 47644 & 1380 & 48.400 & 21.424 & 22.443 \\
\hline 2 & 22.883 & 50795 & 1383 & 51.600 & 22.464 & 23.531 \\
\hline Total & & 98439 & 2764 & 100.000 & & \\
\hline
\end{tabular}

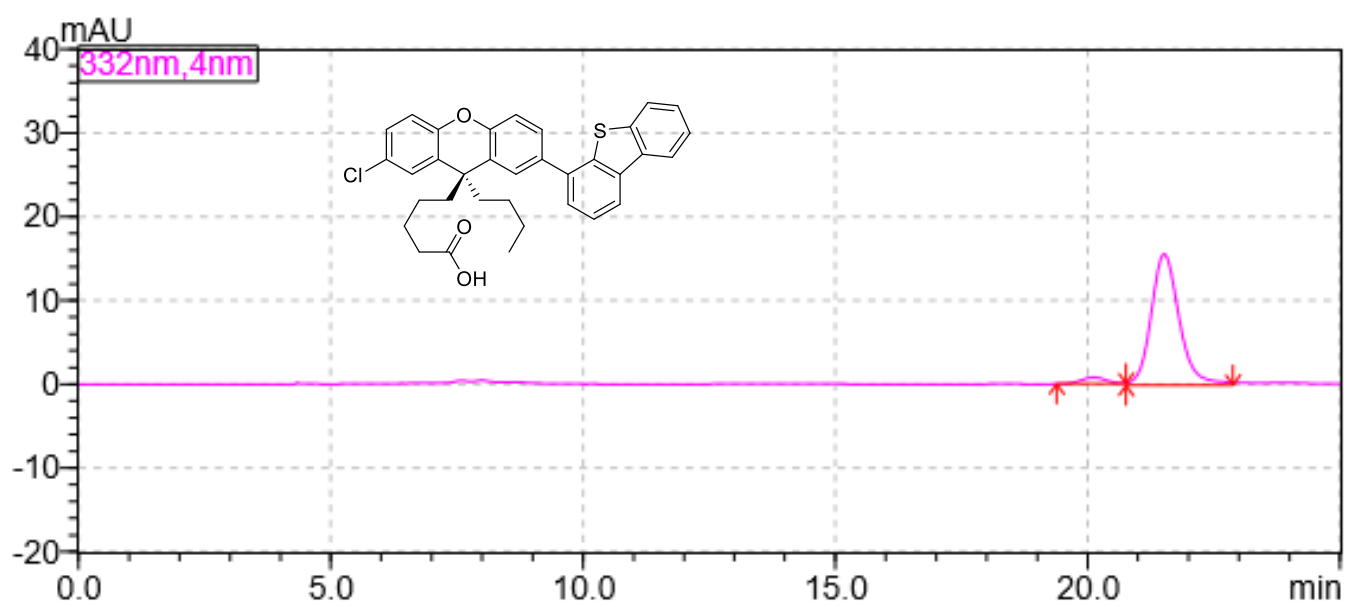

<Peak Table>

PDA Ch1 332nm Peak\# Ret. Time

\begin{tabular}{|r|r|r|r|r|r|r|}
\hline Peak\# & Ret. Time & \multicolumn{1}{c|}{ Area } & \multicolumn{1}{c|}{ Height } & \multicolumn{1}{|c|}{ Area\% } & Peak Start & Peak End \\
\hline 1 & 20.118 & 28206 & 784 & 4.413 & 19.387 & 20.763 \\
\hline 2 & 21.517 & 610994 & 15668 & 95.587 & 20.763 & 22.880 \\
\hline Total & & 639200 & 16452 & 100.000 & & \\
\hline
\end{tabular}


mAU

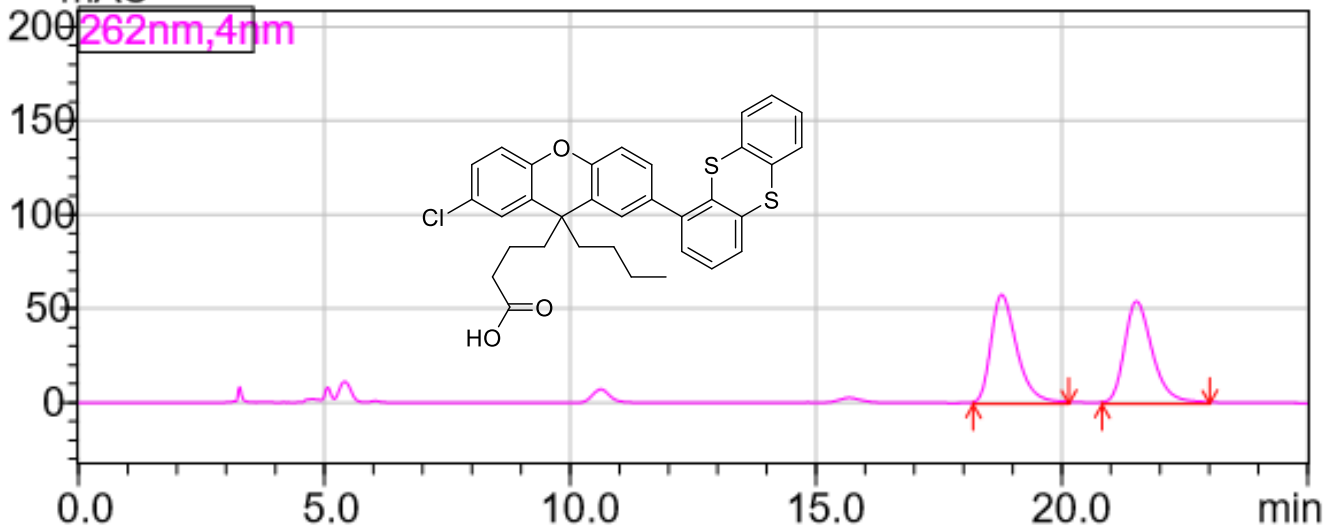

<Peak Table>

PDA Ch1 262nm

\begin{tabular}{|r|r|r|r|r|r|r|}
\hline Peak\# & Ret. Time & Area & \multicolumn{1}{c|}{ Height } & Area\% & Peak Start & Peak End \\
\hline 1 & 18.778 & 2161985 & 58068 & 49.635 & 18.203 & 20.139 \\
\hline 2 & 21.519 & 2193740 & 54571 & 50.365 & 20.821 & 23.003 \\
\hline Total & & 4355725 & 112639 & 100.000 & & \\
\hline
\end{tabular}

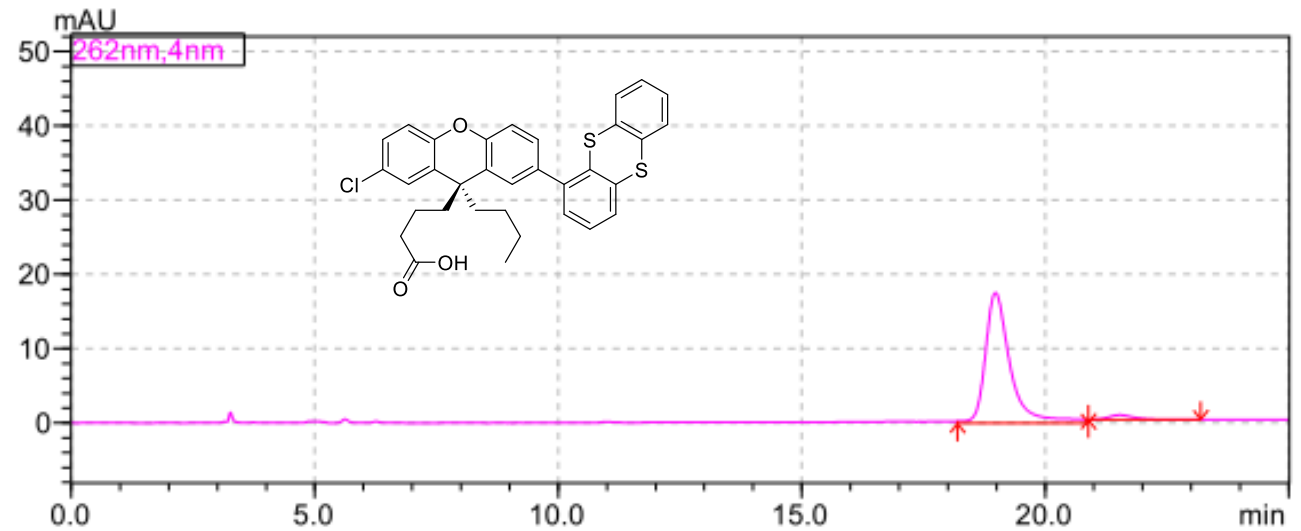

<Peak Table>

PDA Ch1 262nm

Peak\# Ret. Time

\begin{tabular}{|r|r|r|r|r|r|r|}
\hline Peak\# Ret. Time & \multicolumn{1}{|c|}{ Area } & \multicolumn{1}{c|}{ Height } & \multicolumn{1}{c|}{ Area\% } & Peak Start & Peak End \\
\hline 1 & 18.978 & 626442 & 17572 & 96.934 & 18.203 & 20.885 \\
\hline 2 & 21.523 & 19814 & 622 & 3.066 & 20.885 & 23.195 \\
\hline Total & & 646256 & 18194 & 100.000 & & \\
\hline
\end{tabular}



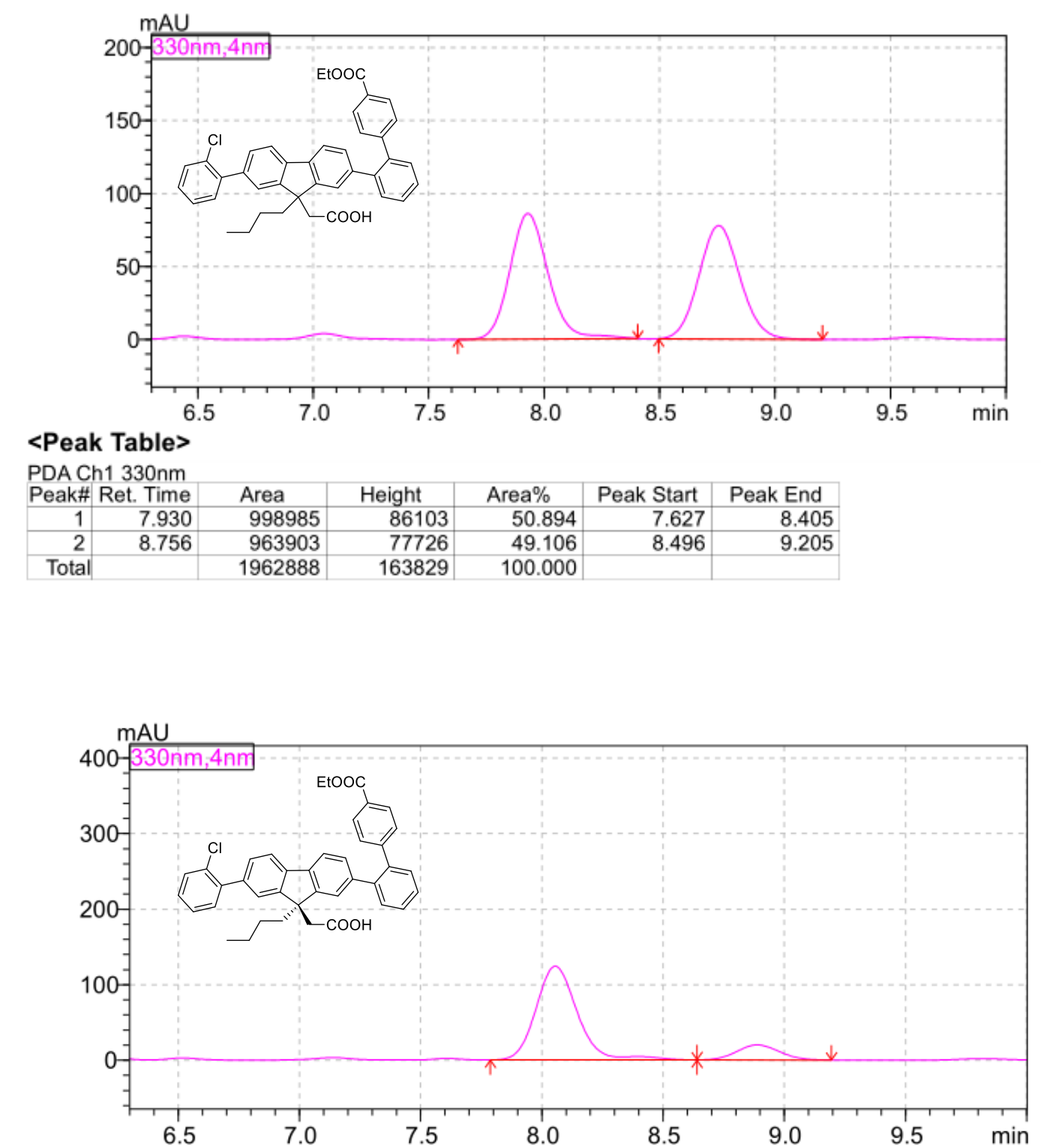

<Peak Table>

PDA Ch1 330nm

\begin{tabular}{|r|r|r|r|r|r|r|}
\hline Peak\# Ret. Time & \multicolumn{1}{|c|}{ Area } & \multicolumn{1}{|c|}{ Height } & Area\% & Peak Start & Peak End \\
\hline 1 & 8.055 & 1471903 & 124104 & 85.718 & 7.787 & 8.640 \\
\hline 2 & 8.889 & 245242 & 19903 & 14.282 & 8.640 & 9.195 \\
\hline Total & & 1717145 & 144007 & 100.000 & & \\
\hline
\end{tabular}




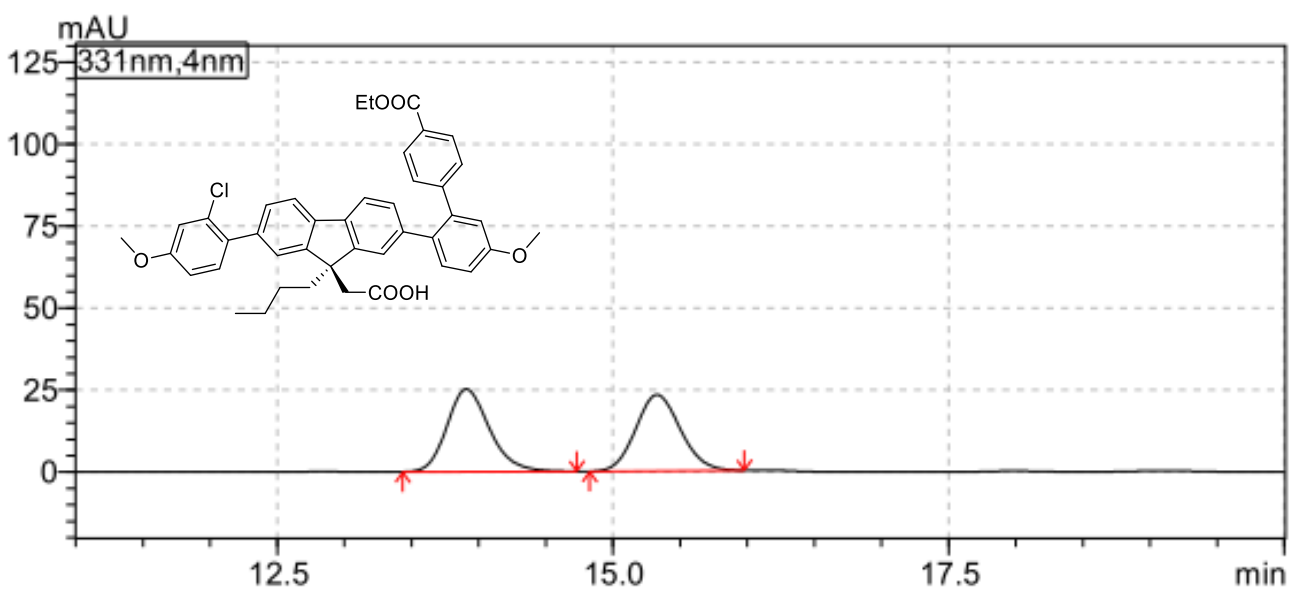

<Peak Table>

PDA Ch1 350nm

\begin{tabular}{|r|r|r|r|r|r|r|}
\hline Peak\# & Ret. Time & \multicolumn{1}{|c|}{ Area } & \multicolumn{1}{|c|}{ Height } & Peak Start & Peak End & \multicolumn{1}{c|}{ Area\% } \\
\hline 1 & 13.909 & 118715 & 5406 & 13.403 & 14.688 & 50.200 \\
\hline 2 & 15.331 & 117769 & 5019 & 14.731 & 16.315 & 49.800 \\
\hline Total & & 236484 & 10424 & & & 100.000 \\
\hline
\end{tabular}

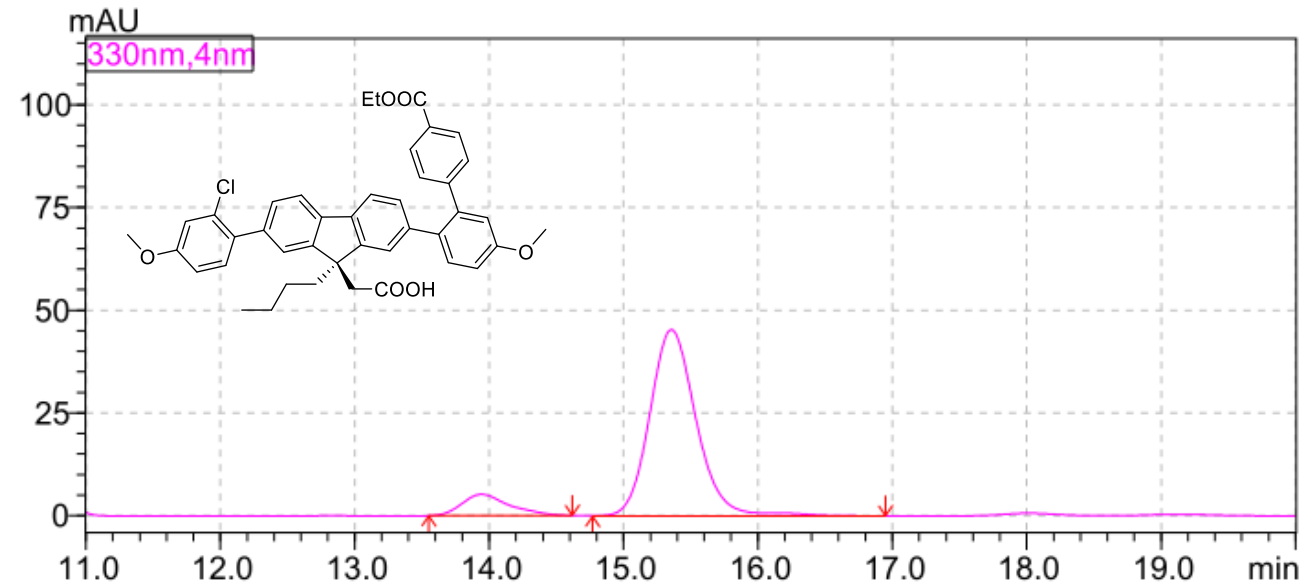

<Peak Table>

PDA Ch1 330nm

\begin{tabular}{|r|r|r|r|r|r|r|}
\hline Peak\# Ret. Time & \multicolumn{1}{|c|}{ Area } & \multicolumn{1}{|c|}{ Height } & \multicolumn{1}{|c|}{ Area\% } & Peak Start & Peak End \\
\hline 1 & 13.942 & 128004 & 5203 & 10.457 & 13.552 & 14.619 \\
\hline 2 & 15.356 & 1096155 & 45395 & 89.543 & 14.768 & 16.949 \\
\hline Total & & 1224159 & 50598 & 100.000 & & \\
\hline
\end{tabular}




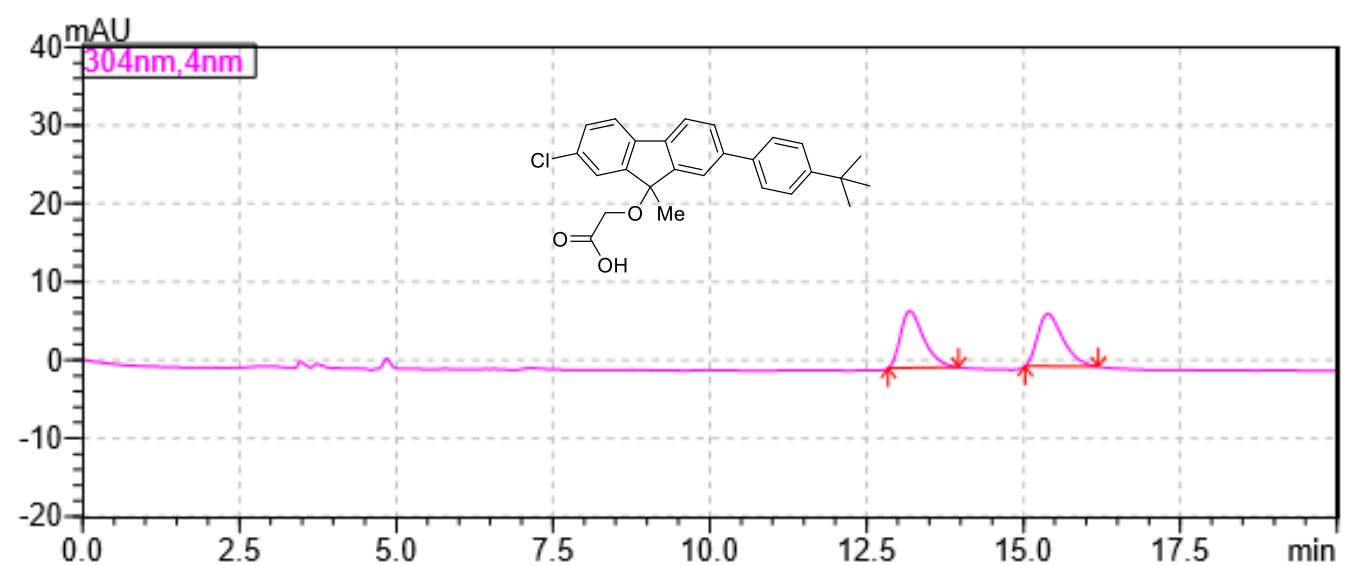

<Peak Table>

PDA Ch1 304nm

\begin{tabular}{|r|r|r|r|r|r|r|}
\hline Peak\# & Ret. Time & \multicolumn{1}{c|}{ Area } & Height & \multicolumn{1}{c|}{ Area\% } & Peak Start & Peak End \\
\hline 1 & 13.189 & 188773 & 7292 & 49.783 & 12.848 & 13.963 \\
\hline 2 & 15.390 & 190421 & 6739 & 50.217 & 15.029 & 16.197 \\
\hline Total & & 379194 & 14031 & 100.000 & & \\
\hline
\end{tabular}

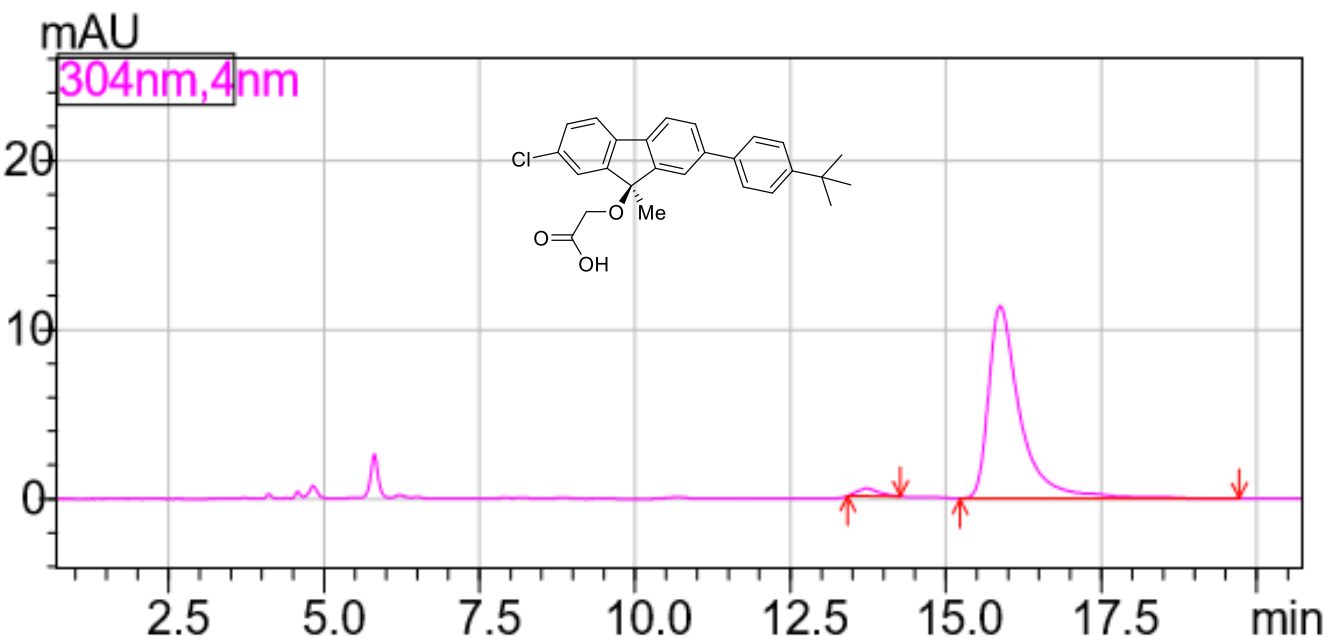

<Peak Table>

PDA Ch1 304nm

\begin{tabular}{|r|r|r|r|r|r|r|}
\hline Peak\# & Ret. Time & \multicolumn{1}{c|}{ Area } & \multicolumn{1}{c|}{ Height } & Area\% & Peak Start & Peak End \\
\hline 1 & 13.728 & 7209 & 362 & 1.734 & 13.483 & 14.133 \\
\hline 2 & 15.875 & 408642 & 11397 & 98.266 & 15.227 & 19.717 \\
\hline Total & & 415851 & 11758 & 100.000 & & \\
\hline
\end{tabular}




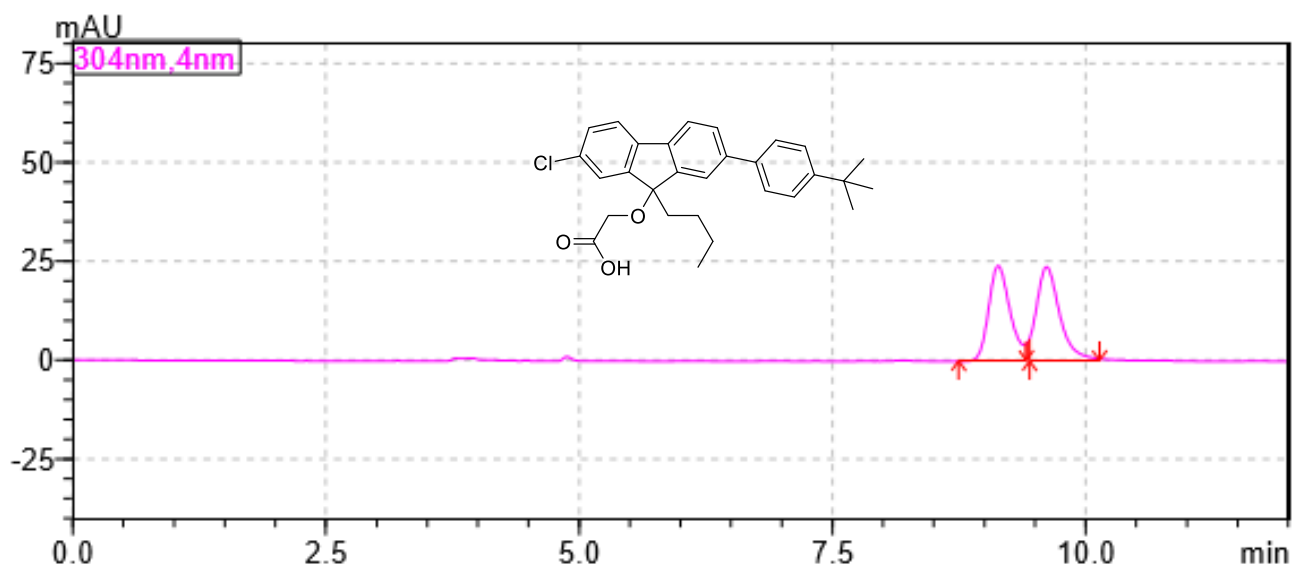

<Peak Table>

PDA Ch1 304nm

\begin{tabular}{|c|c|c|c|c|c|c|}
\hline Peak\# & Ret. Time & Area & Height & Area $\%$ & Peak Start & Peak End \\
\hline 1 & 9.137 & 353184 & 24037 & 48.369 & 8.747 & 9.413 \\
\hline 2 & 9.615 & 377004 & 23760 & 51.631 & 9.445 & 10.139 \\
\hline Total & & 730188 & 47797 & 100.000 & & \\
\hline
\end{tabular}

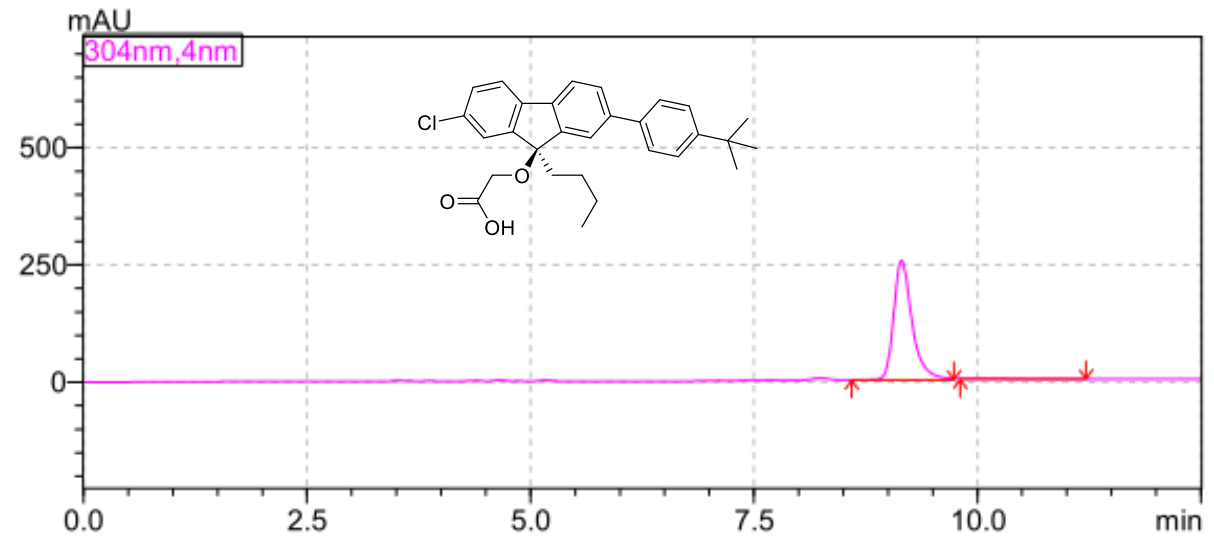

<Peak Table>

PDA Ch1 304nm

\begin{tabular}{|r|r|r|r|r|r|r|}
\hline Peak\# & Ret. Time & \multicolumn{1}{|c|}{ Area } & \multicolumn{1}{|c|}{ Height } & \multicolumn{1}{|c|}{ Area\% } & Peak Start & Peak End \\
\hline 1 & 9.150 & 3682060 & 254991 & 99.201 & 8.592 & 9.739 \\
\hline 2 & 10.175 & 29653 & 1196 & 0.799 & 9.808 & 11.216 \\
\hline Total & & 3711713 & 256188 & 100.000 & & \\
\hline
\end{tabular}


Control experiment (product of reaction using 53)

Racemic marker

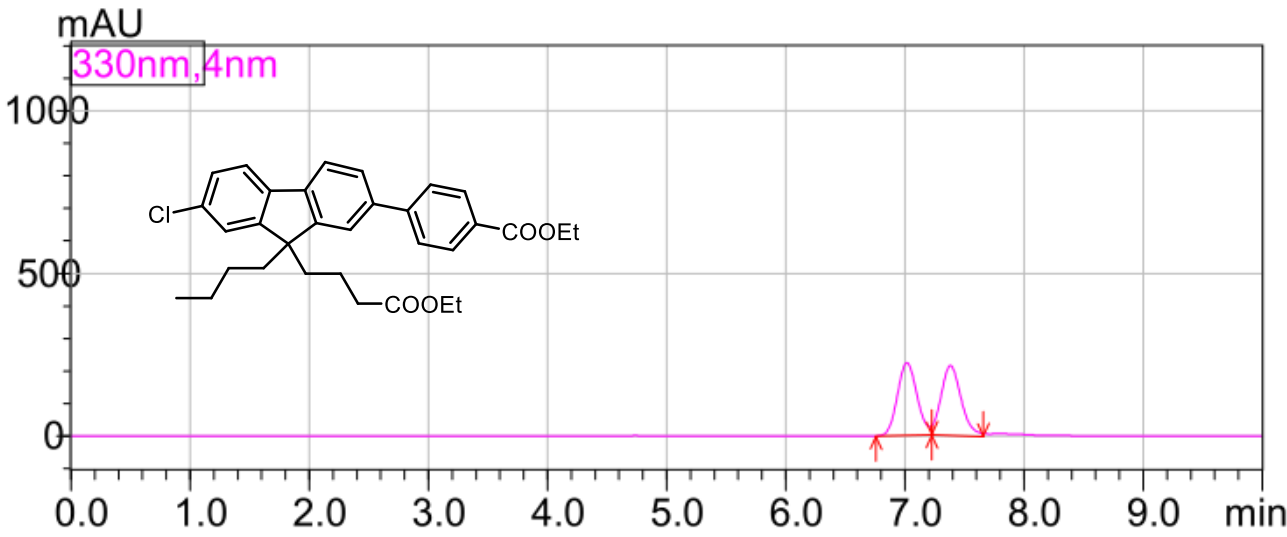

<Peak Table>

PDA Ch1 330nm

\begin{tabular}{|r|r|r|r|l|r|r|r|}
\hline Peak\# Ret. Time & Area & Height & Mark & Area\% & Peak Start & Peak End \\
\hline 1 & 7.016 & 2551333 & 222426 & M & 50.105 & 6.757 & 7.227 \\
\hline 2 & 7.382 & 2540657 & 215334 & M & 49.895 & 7.227 & 7.659 \\
\hline Total & & 5091990 & 437761 & & 100.000 & & \\
\hline
\end{tabular}

HPLC trace of the coupling product of ethyl ester $\mathbf{5 3}$ under the optimized reaction conditions. It was found that the product was racemic.

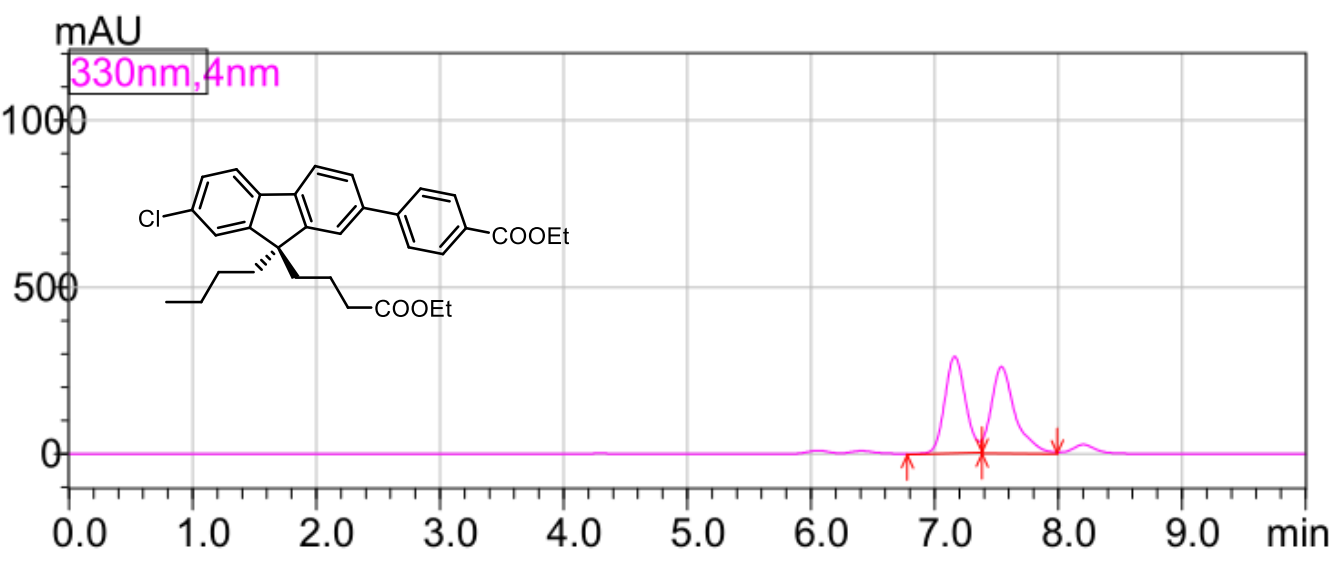

<Peak Table>

PDA Ch1 330nm

\begin{tabular}{|r|r|r|r|r|r|r|r|}
\hline Peak\# Ret. Time & Area & \multicolumn{1}{|c|}{ Height } & Mark & Area\% & Peak Start & Peak End \\
\hline 1 & 7.162 & 3434882 & 290805 & M & 49.686 & 6.779 & 7.381 \\
\hline 2 & 7.540 & 3478269 & 259984 & M & 50.314 & 7.381 & 7.989 \\
\hline Total & & 6913151 & 550788 & & 100.000 & & \\
\hline
\end{tabular}


Synthetic applications (Scheme 4)

54

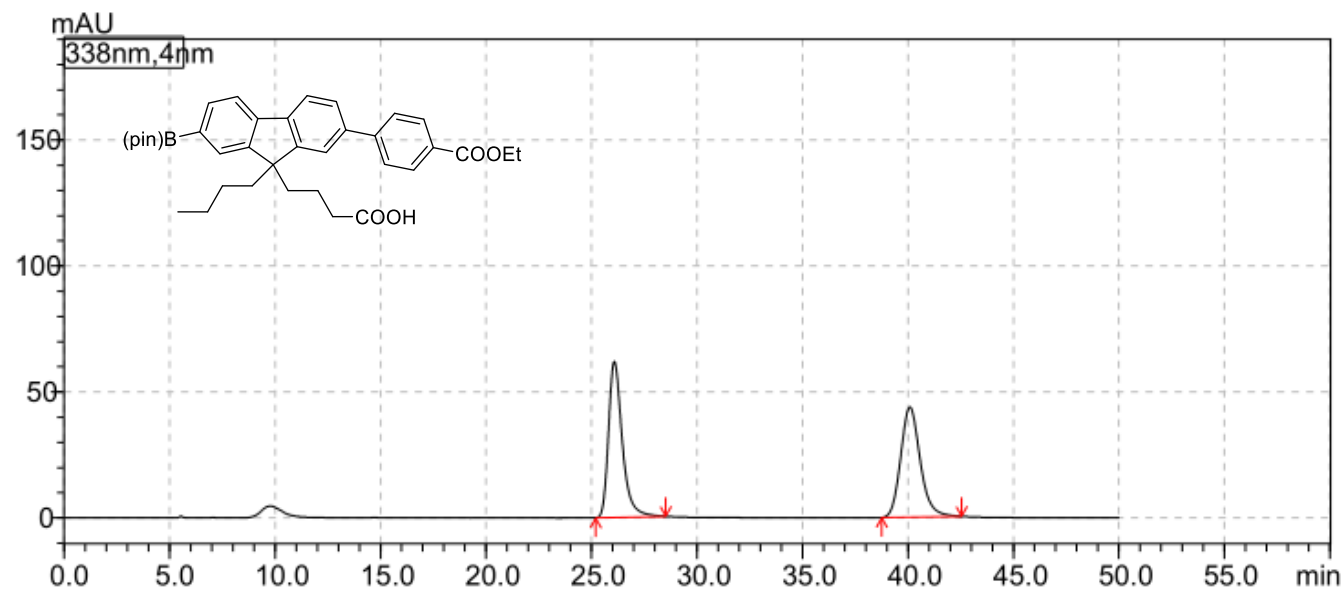

<Peak Table>

PDA Ch1 338nm

\begin{tabular}{|r|r|r|r|r|r|r|}
\hline Peak\# Ret. Time & Area & Height & Area\% & Peak Start & Peak End \\
\hline 1 & 26.081 & 2953745 & 62178 & 50.553 & 24.635 & 33.344 \\
\hline 2 & 40.091 & 2889143 & 43921 & 49.447 & 37.915 & 43.856 \\
\hline Total & & 5842887 & 106100 & 100.000 & & \\
\hline
\end{tabular}

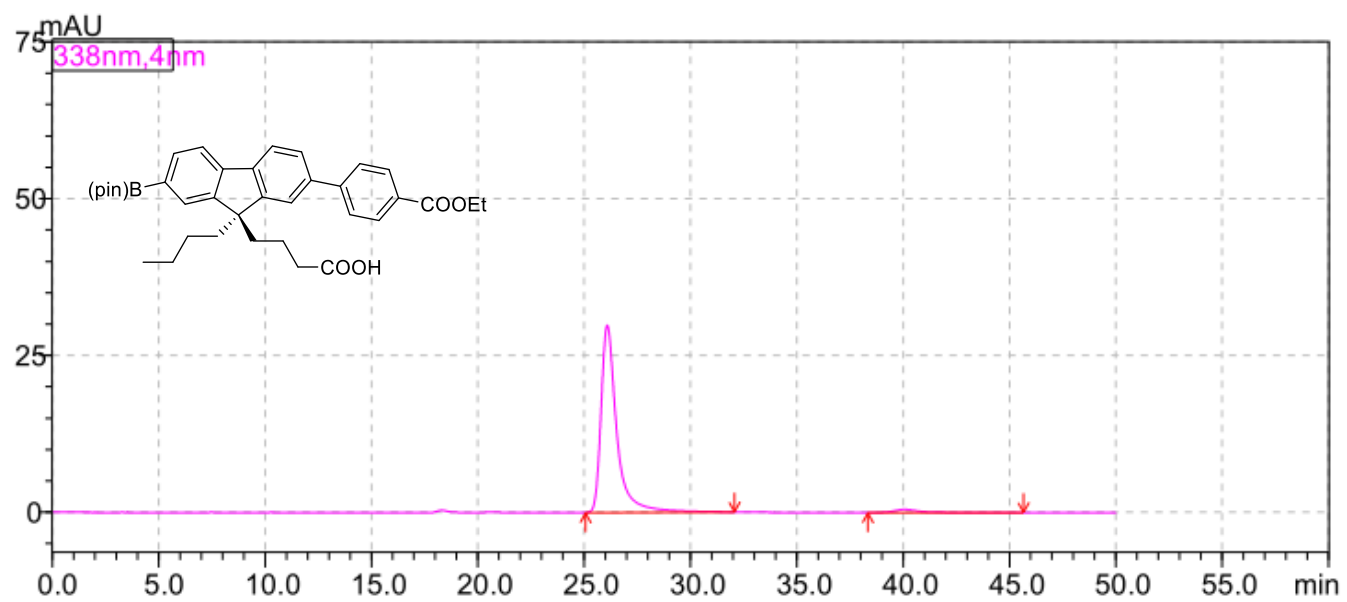

<Peak Table>

PDA Ch1 338nm

\begin{tabular}{|r|r|r|r|r|r|r|}
\hline Peak\# Ret. Time & \multicolumn{1}{|c|}{ Area } & \multicolumn{1}{c|}{ Height } & \multicolumn{1}{c|}{ Area\% } & Peak Start & Peak End \\
\hline 1 & 26.090 & 1545538 & 29868 & 97.144 & 25.061 & 32.069 \\
\hline 2 & 40.057 & 45439 & 521 & 2.856 & 38.336 & 45.664 \\
\hline Total & & 1590977 & 30389 & 100.000 & & \\
\hline
\end{tabular}




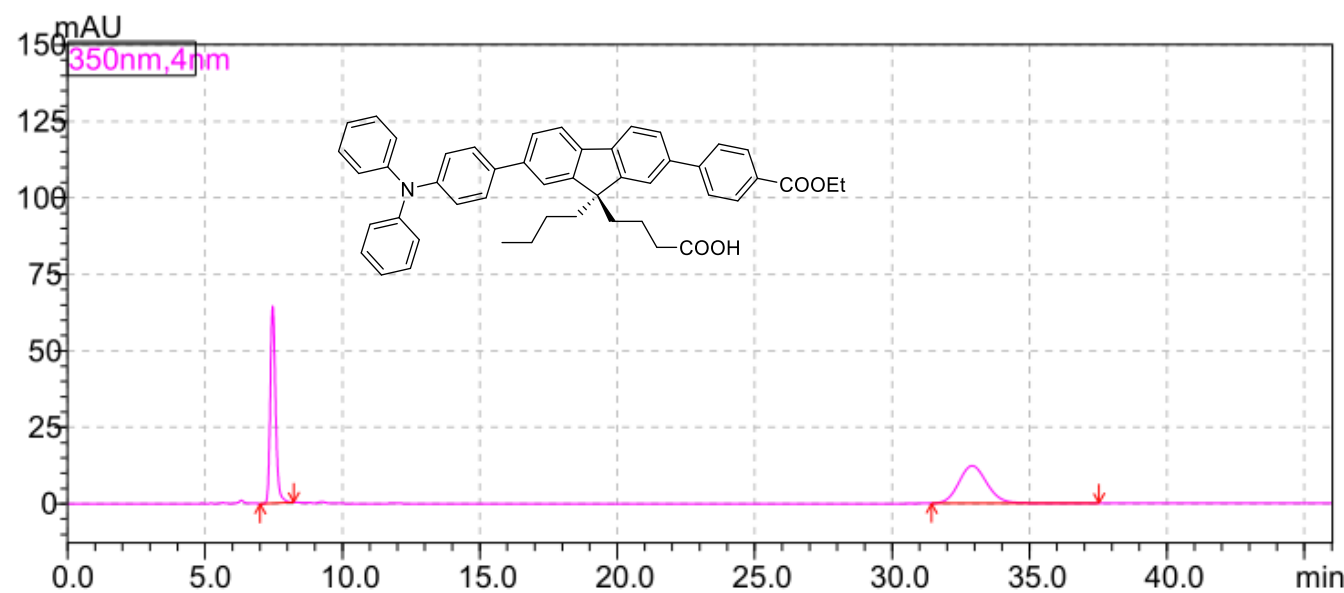

<Peak Table>

\begin{tabular}{|c|c|c|c|c|c|c|}
\hline \multicolumn{7}{|c|}{ PDA Ch1 350nm } \\
\hline Peak\# & Ret. Time & Area & Height & Area\% & Peak Start & Peak End \\
\hline 1 & 7.463 & 865327 & 64355 & 49.596 & 7.003 & 8.224 \\
\hline 2 & 32.907 & 879408 & 12317 & 50.404 & 31.429 & 37.531 \\
\hline Total & & 1744735 & 76673 & 100.000 & & \\
\hline
\end{tabular}

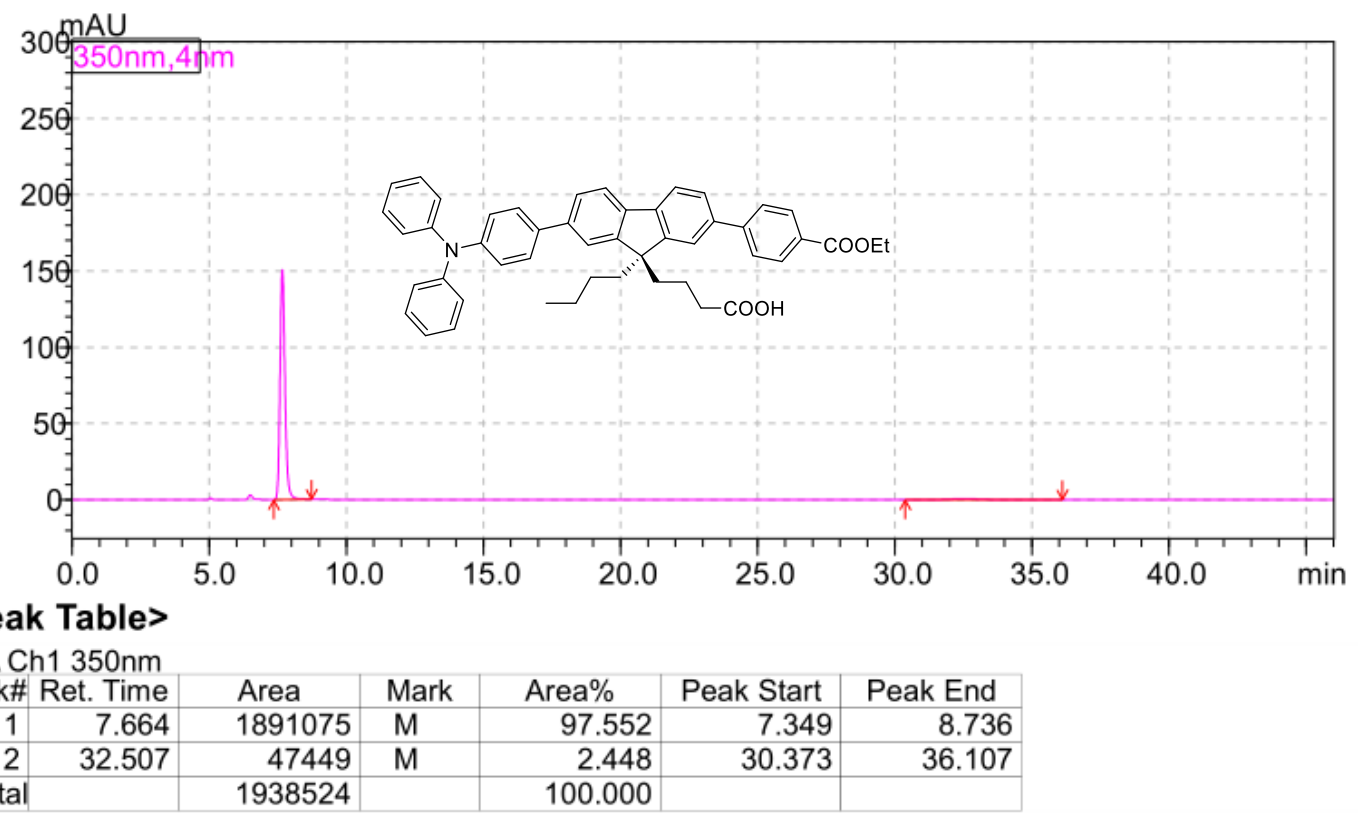




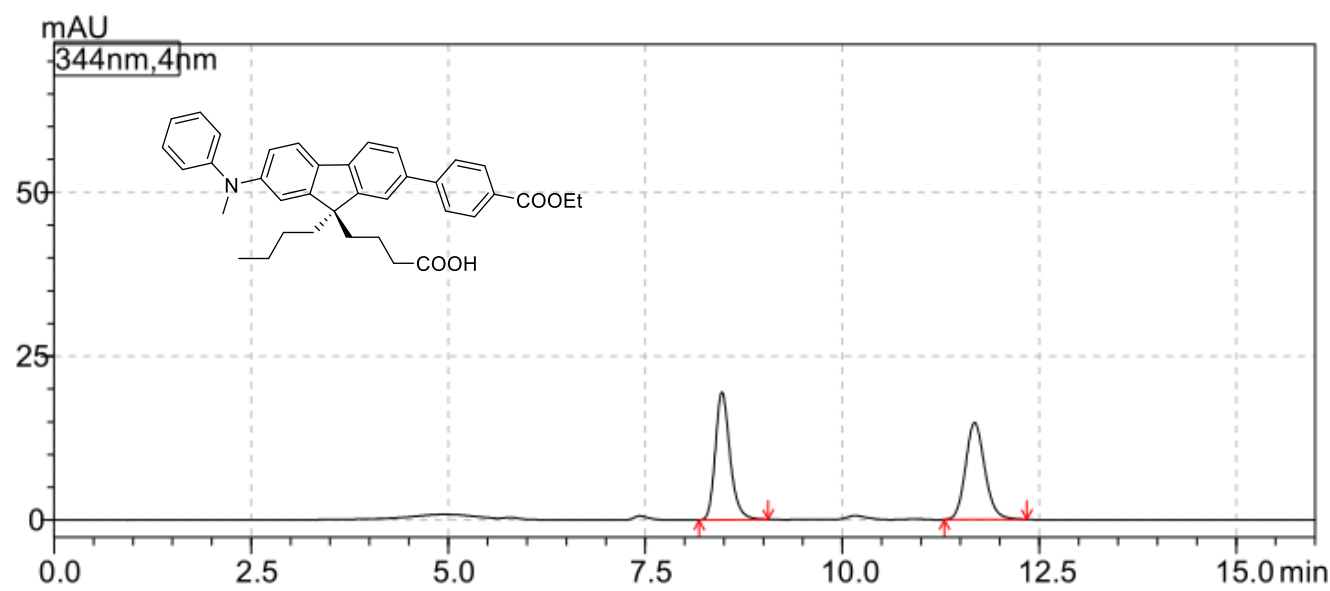

<Peak Table>

PDA Ch1 344nm

\begin{tabular}{|r|r|r|r|r|r|r|}
\hline Peak\# Ret. Time & \multicolumn{1}{|c|}{ Area } & Height & Peak Start & Peak End & \multicolumn{1}{c|}{ Area\% } \\
\hline 1 & 8.474 & 255586 & 19511 & 8.027 & 9.195 & 49.952 \\
\hline 2 & 11.680 & 256074 & 14844 & 11.168 & 12.528 & 50.048 \\
\hline Total & & 511659 & 34354 & & & 100.000 \\
\hline
\end{tabular}

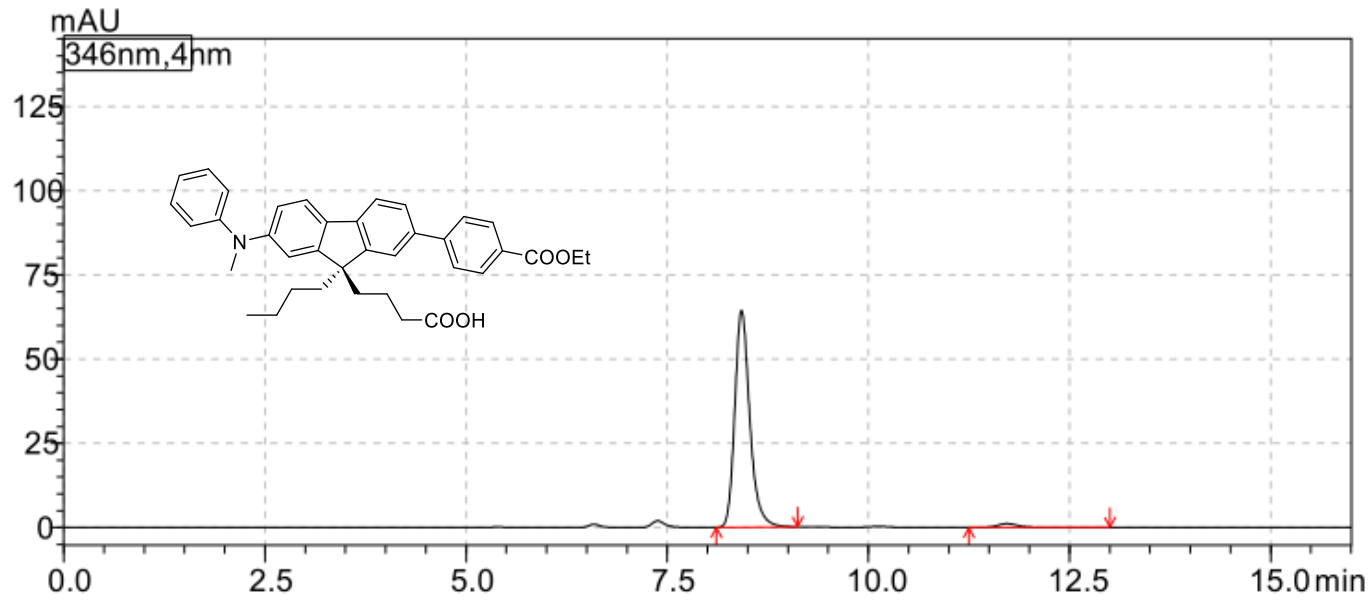

<Peak Table>

PDA Ch1 346nm

\begin{tabular}{|r|r|r|r|r|r|r|}
\hline Peak\# Ret. Time & \multicolumn{1}{|c|}{ Area } & Height & Peak Start & Peak End & \multicolumn{1}{|c|}{ Area\% } \\
\hline 1 & 8.423 & 840279 & 64403 & 8.085 & 9.307 & 97.332 \\
\hline 2 & 11.724 & 23032 & 1158 & 11.221 & 12.971 & 2.668 \\
\hline Total & & 863311 & 65561 & & & 100.000 \\
\hline
\end{tabular}




\section{Precursor to 60}

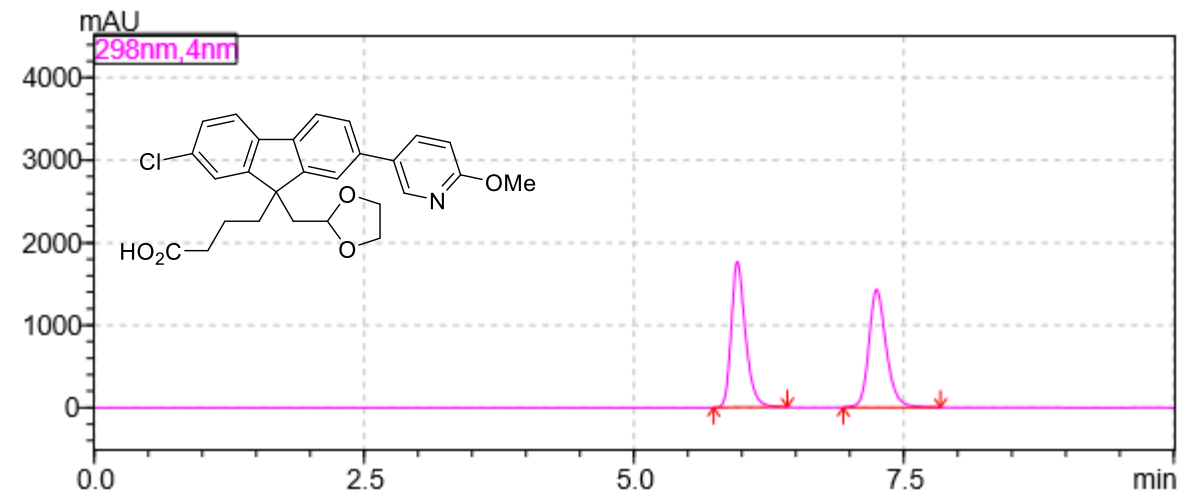

<Peak Table>

PDA Ch1 298nm

\begin{tabular}{|r|r|r|r|r|r|r|}
\hline Peak\# & Ret. Time & Area & Height & Peak Start & Peak End & \multicolumn{1}{c|}{ Area\% } \\
\hline 1 & 5.957 & 16224547 & 1768166 & 5.739 & 6.421 & 50.350 \\
\hline 2 & 7.247 & 15998750 & 1428746 & 6.939 & 7.840 & 49.650 \\
\hline Total & & 32223297 & 3196912 & & & 100.000 \\
\hline
\end{tabular}

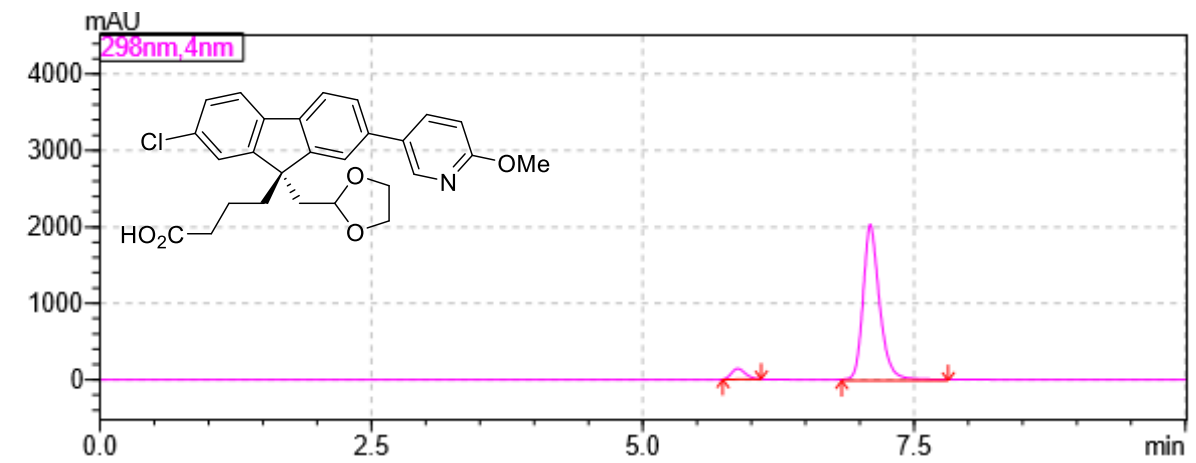

<Peak Table>

PDA Ch1 298nm

\begin{tabular}{|c|c|c|c|c|c|c|}
\hline Peak\# & Ret. Time & Area & Height & Peak Start & Peak End & Area $\%$ \\
\hline 1 & 5.875 & 1245832 & 139877 & 5.739 & 6.091 & 5.328 \\
\hline 2 & 7.096 & 22137992 & 2046523 & 6.832 & 7.813 & 94.672 \\
\hline Total & & 23383823 & 2186400 & & & 100.000 \\
\hline
\end{tabular}




\section{Precursor to ent-60}

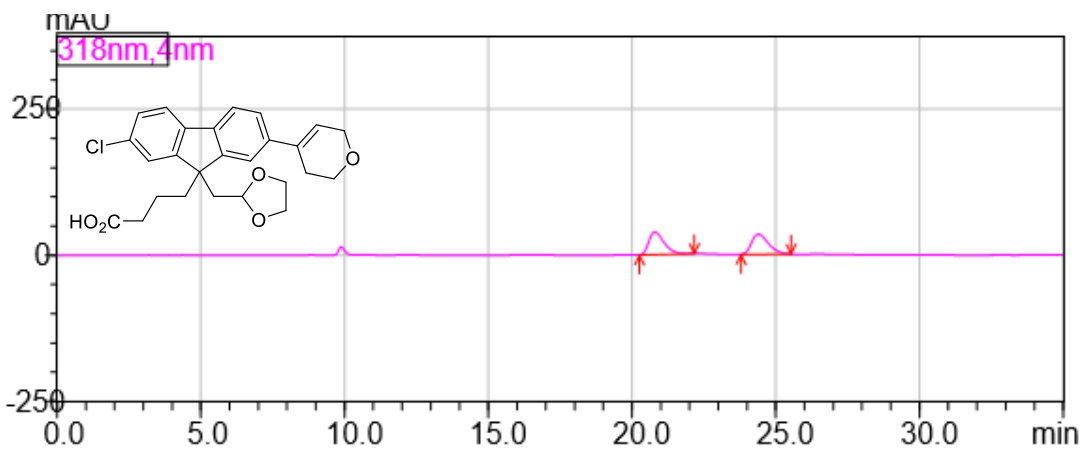

<Peak Table>

PDA Ch1 318nm

\begin{tabular}{|r|r|r|r|r|r|r|}
\hline Peak\# & Ret. Time & \multicolumn{1}{c|}{ Area } & Peak Start & Peak End & Area\% & Height \\
\hline 1 & 20.792 & 1409390 & 20.251 & 22.165 & 49.818 & 38788 \\
\hline 2 & 24.398 & 1419696 & 23.792 & 25.531 & 50.182 & 34637 \\
\hline Total & & 2829086 & & & 100.000 & 73425 \\
\hline
\end{tabular}

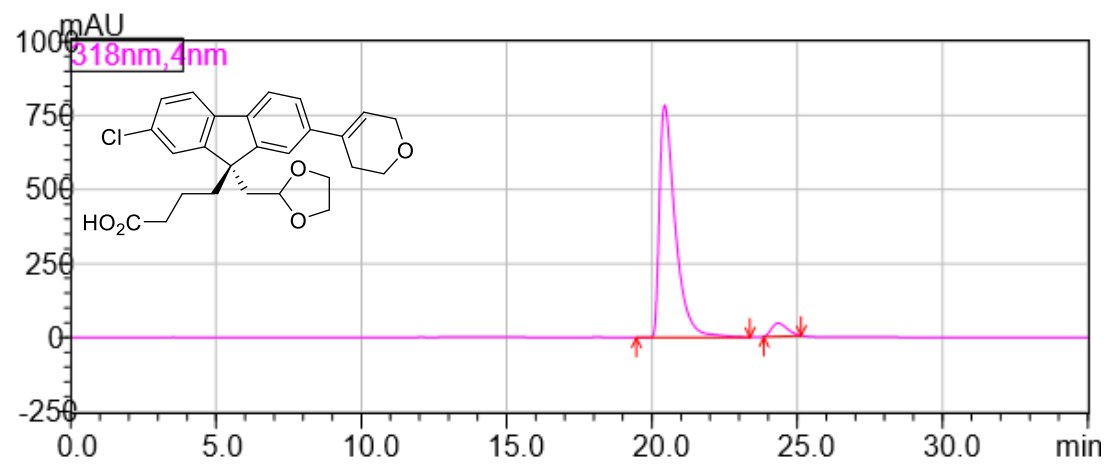

<Peak Table>

PDA Ch1 318nm
Peak\# Ret. Time

\begin{tabular}{|c|c|c|c|c|c|c|}
\hline Peak\# & Ret. Time & Area & Height & Peak Start & Peak End & Area \% \\
\hline 1 & 20.438 & 29970030 & 786189 & 19.461 & 23.376 & 94.8 \\
\hline 2 & 24.344 & 1633227 & 43861 & 23.851 & 25.136 & 0 \\
\hline
\end{tabular}


60

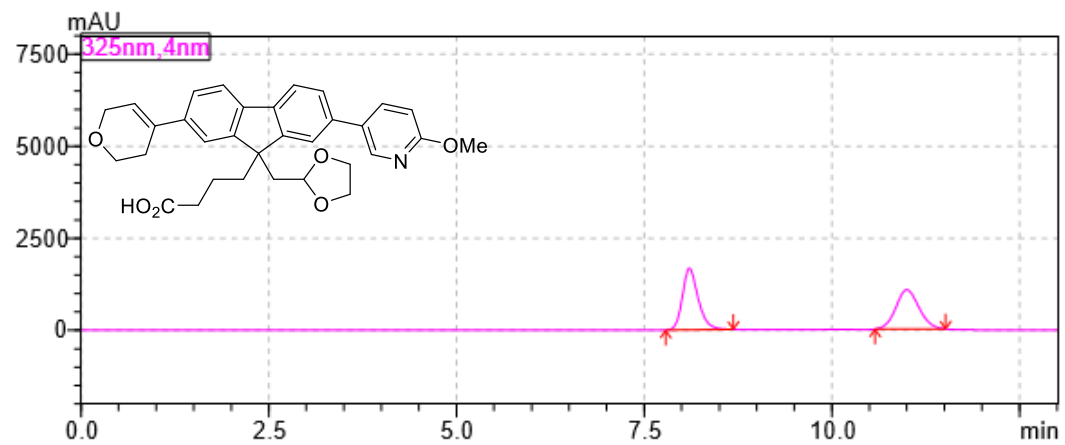

<Peak Table>

PDA Ch1 325nm
\begin{tabular}{r|r|r|r|r|r|r|}
\hline Peak\#\# & Ret. Time & Area & Height & Peak Start & Peak End & \multicolumn{1}{|c|}{ Area $\%$} \\
\hline 1 & 8.102 & 24589901 & 1674022 & 7.787 & 8.688 & 51.668 \\
\hline 2 & 10.999 & 23002148 & 1067279 & 10.576 & 11.515 & 48.332 \\
\hline Total & & 47592049 & 2741301 & & & 100.000 \\
\hline
\end{tabular}

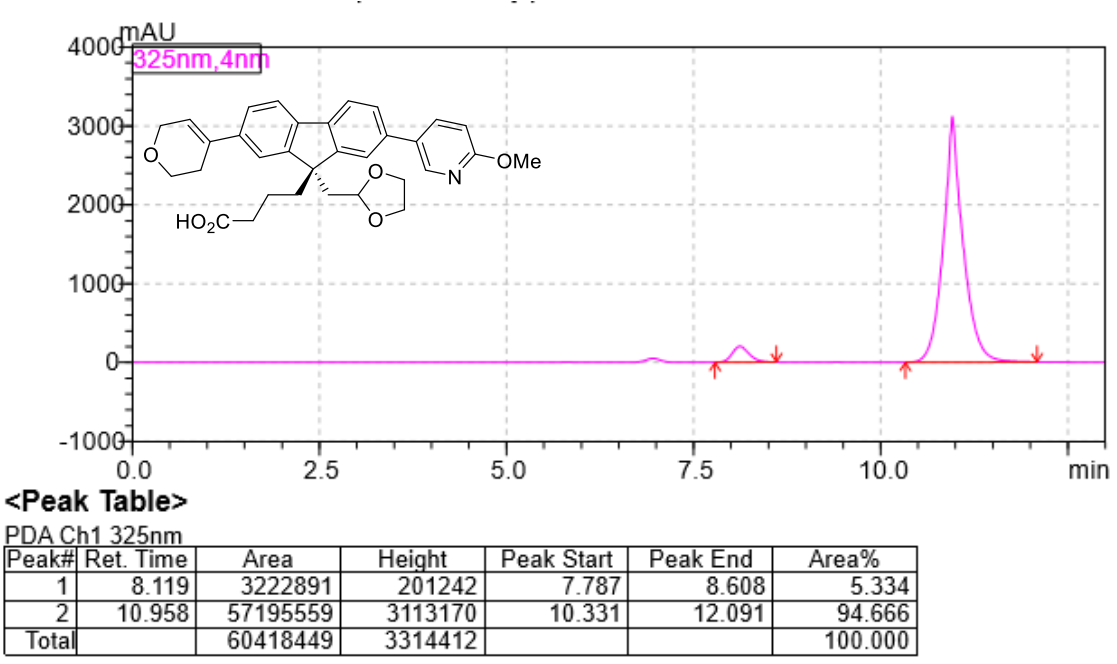


ent-60

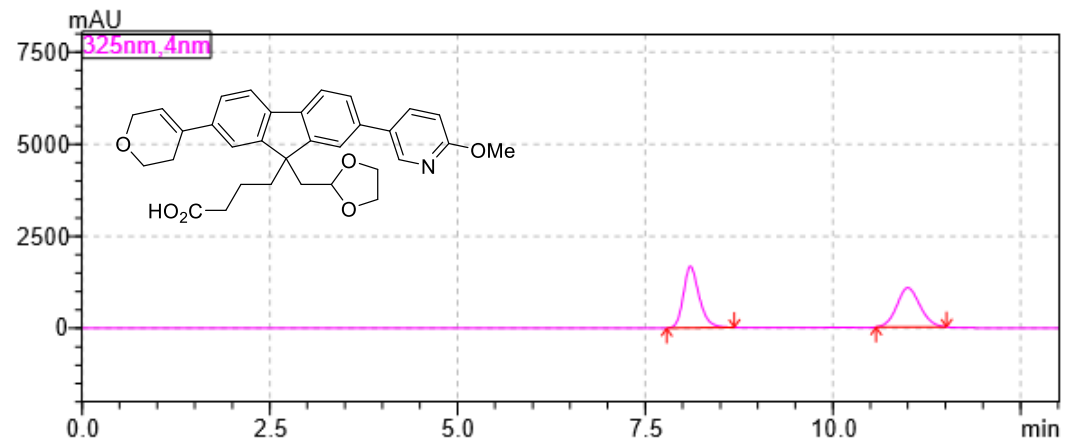

<Peak Table>

PDA Ch1 325nm
\begin{tabular}{r|r|c|c|r|r|r|}
\hline Peak\# & Ret. Time & Area & Height & Peak Start & Peak End & \multicolumn{1}{|c|}{ Area $\%$} \\
\hline 1 & 8.102 & 24589901 & 1674022 & 7.787 & 8.688 & 51.668 \\
\hline 2 & 10.999 & 23002148 & 1067279 & 10.576 & 11.515 & 48.332 \\
\hline Total & & 47592049 & 2741301 & & & 100.000 \\
\hline
\end{tabular}

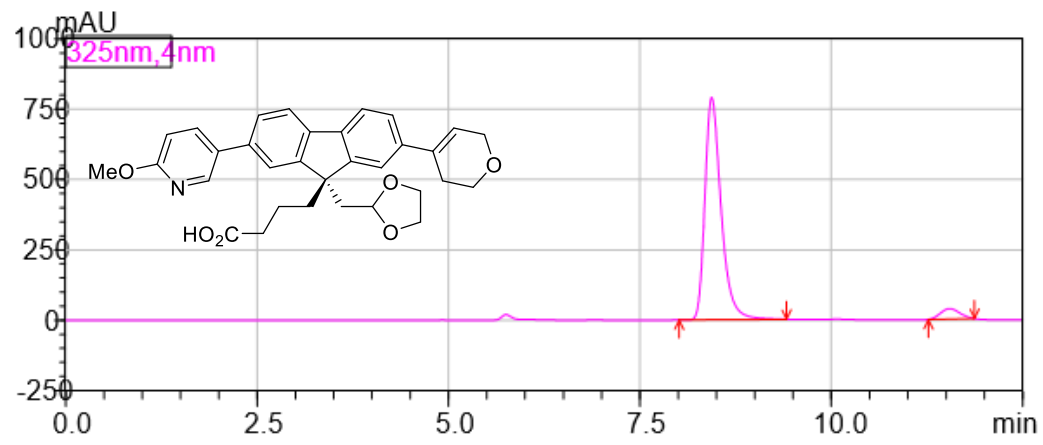

$<$ Peak Table>

PDA Ch1 325nm

\begin{tabular}{|c|c|c|c|c|c|}
\hline Peak\# Ret. Time & Area & Height & Peak Start & Peak End & Area\% \\
\hline
\end{tabular}

\begin{tabular}{|r|r|r|r|r|r|r|}
\hline Peak\# & Ret. Time & Area & Height & Peak Start & Peak End & \multicolumn{1}{|c|}{ Area\% } \\
\hline 1 & 8.438 & 11654532 & 790526 & 8.011 & 9.413 & 94.759 \\
\hline 2 & 11.546 & 644599 & 36927 & 11.269 & 11.872 & 5.241 \\
\hline Total & 12299131 & 827453 & & & 100.000 \\
\hline
\end{tabular}




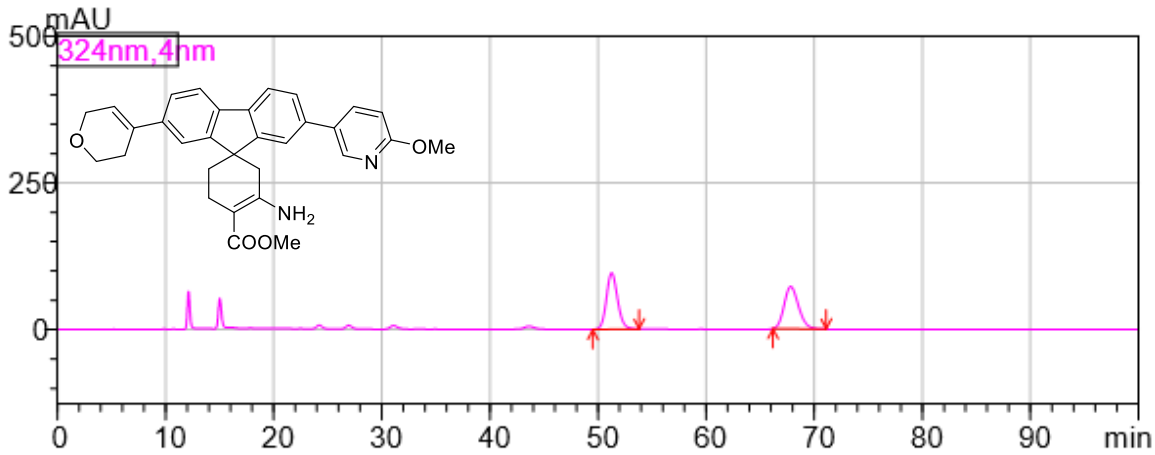

<Peak Table>

PDA Ch1 324nm

\begin{tabular}{|r|r|r|r|r|r|r|}
\hline Peak\# & Ret. Time & \multicolumn{1}{|c|}{ Area } & \multicolumn{1}{c|}{ Height } & Peak Start & Peak End & \multicolumn{1}{c|}{ Area\% } \\
\hline 1 & 51.267 & 6882299 & 96063 & 49.515 & 53.803 & 50.667 \\
\hline 2 & 67.828 & 6701199 & 72095 & 66.197 & 71.109 & 49.333 \\
\hline Total & & 13583498 & 168157 & & & 100.000 \\
\hline
\end{tabular}

$\mathrm{mAU}$

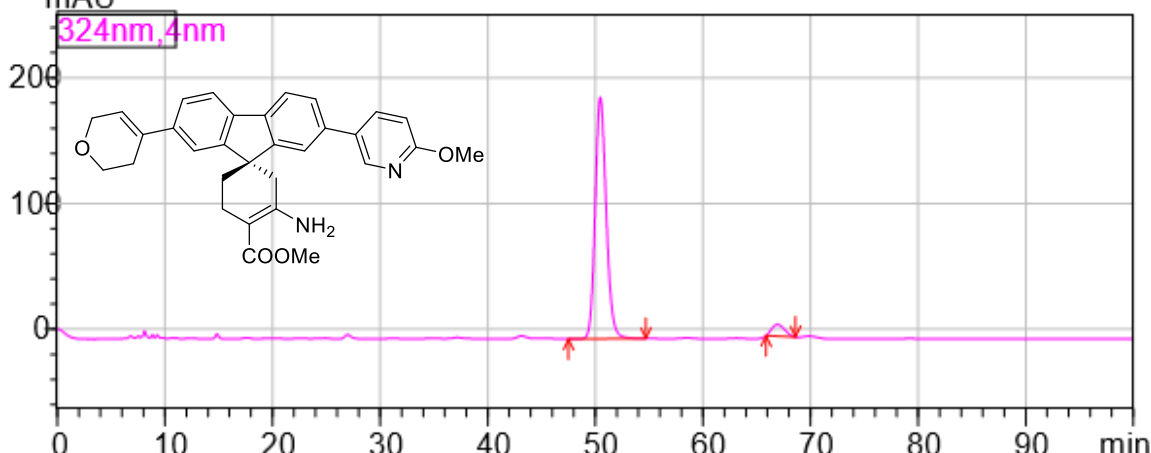

<Peak Table>

PDA Ch1 324nm

\begin{tabular}{|r|r|r|r|r|r|}
\hline Peak\#住 & Ret. Time & \multicolumn{1}{c|}{ Area } & Peak Start & Peak End & \multicolumn{1}{c|}{ Area\% } \\
\hline 1 & 50.460 & 13723428 & 47.504 & 54.683 & 94.581 \\
\hline 2 & 66.924 & 786333 & 65.872 & 68.619 & 5.419 \\
\hline Total & & 14509761 & & & 100.000 \\
\hline
\end{tabular}




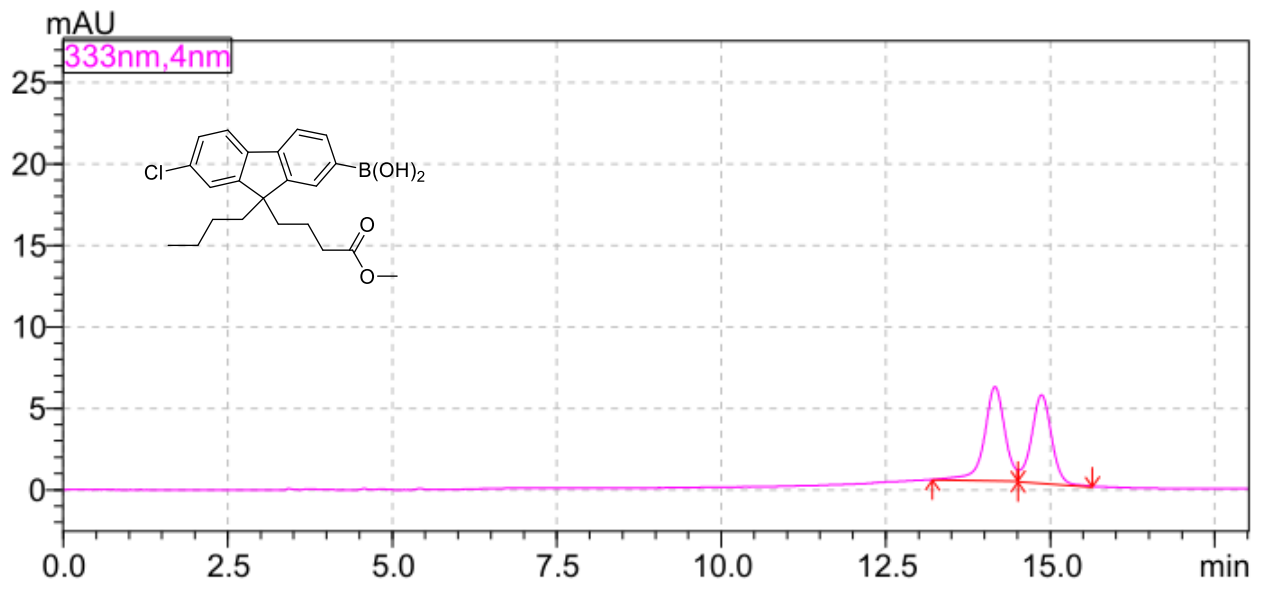

<Peak Table>

PDA Ch1 333nm

\begin{tabular}{|r|r|r|l|r|r|r|}
\hline Peak\# Ret. Time & \multicolumn{1}{|c|}{ Area } & Mark & \multicolumn{1}{|c|}{ Area\% } & Peak Start & Peak End \\
\hline 1 & 14.160 & 125568 & $\mathrm{M}$ & 51.789 & 13.211 & 14.512 \\
\hline 2 & 14.869 & 116893 & $\mathrm{M}$ & 48.211 & 14.512 & 15.643 \\
\hline Total & & 242461 & & 100.000 & & \\
\hline
\end{tabular}

mAU

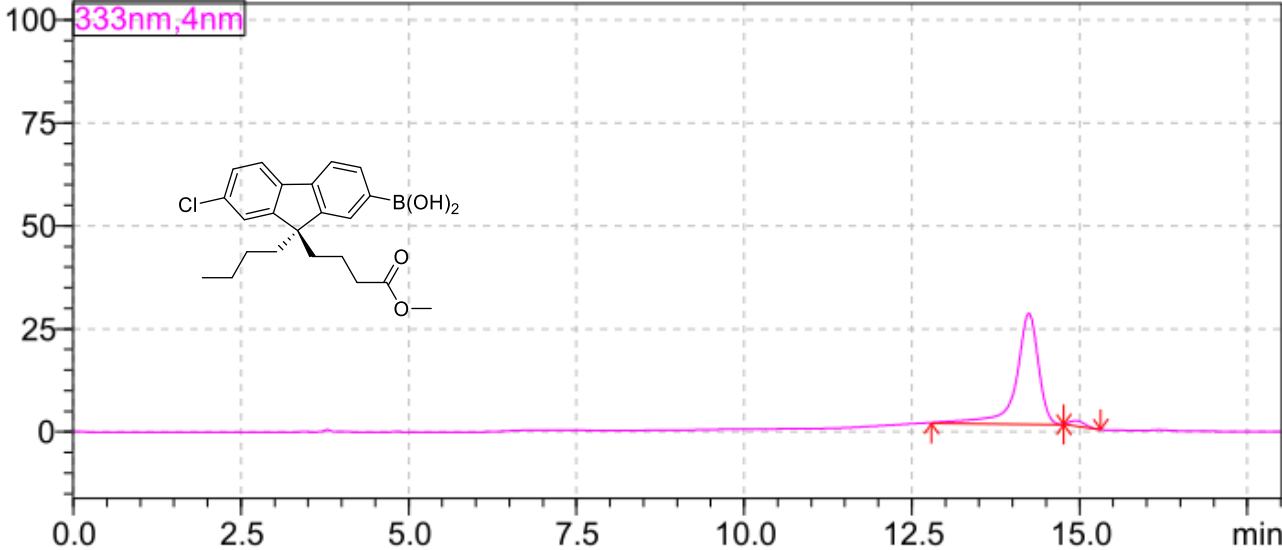

<Peak Table>

PDA Ch1 333nm

Peak\# Ret. Time

\begin{tabular}{|r|r|r|r|c|r|r|r|}
\hline Peak\# & Ret. Time & \multicolumn{1}{|c|}{ Area } & \multicolumn{1}{|c|}{ Height } & Mark & Area\% & Peak Start & Peak End \\
\hline 1 & 14.244 & 621650 & 26912 & M & 97.002 & 12.789 & 14.763 \\
\hline 2 & 14.943 & 19211 & 1263 & M & 2.998 & 14.763 & 15.312 \\
\hline Total & & 640861 & 28175 & & 100.000 & & \\
\hline
\end{tabular}




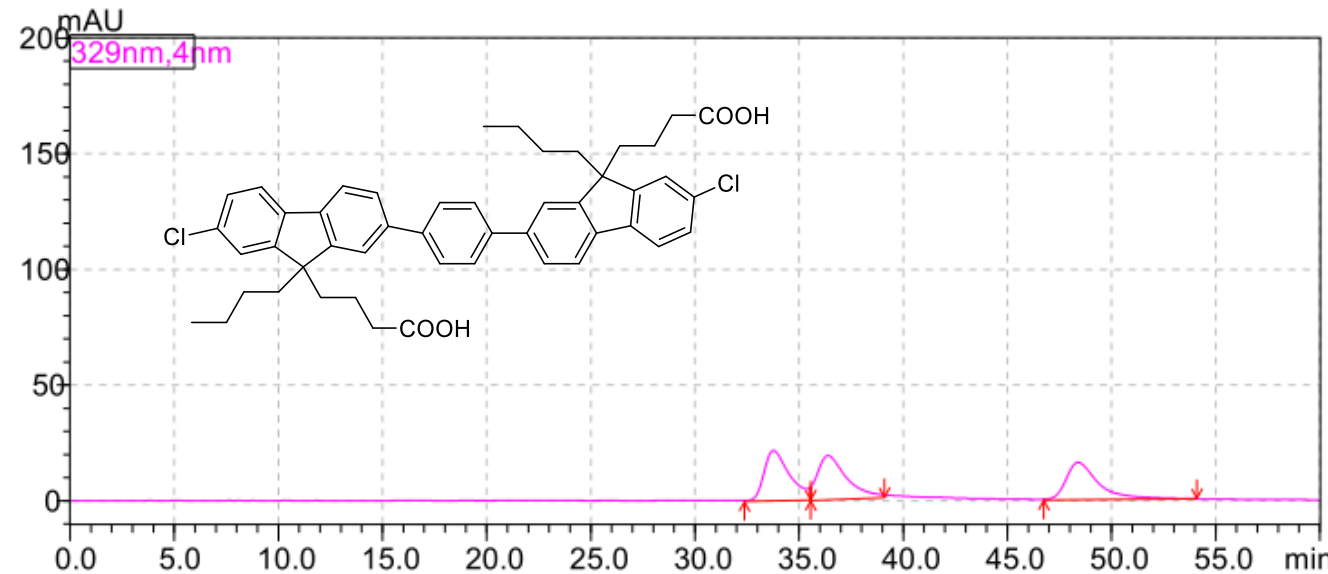

<Peak Table>

PDA Ch1 329nm

Peak\# Ret. Time

\begin{tabular}{|r|r} 
Peak\# Ret. Time \\
\hline 1 & 33.770
\end{tabular}

\begin{tabular}{|r|r|r|r|r|r|r|}
\hline 1 & 33.770 & 1874907 & 21692 & 32.379 & 35.552 & Area\% \\
\hline 2 & 36.389 & 1866352 & 19072 & 35.552 & 39.099 & 33.997 \\
\hline 3 & 48.390 & 1748519 & 16102 & 46.747 & 54.091 & 31.850 \\
\hline Total & & 5489778 & 56866 & & & 100.000 \\
\hline
\end{tabular}

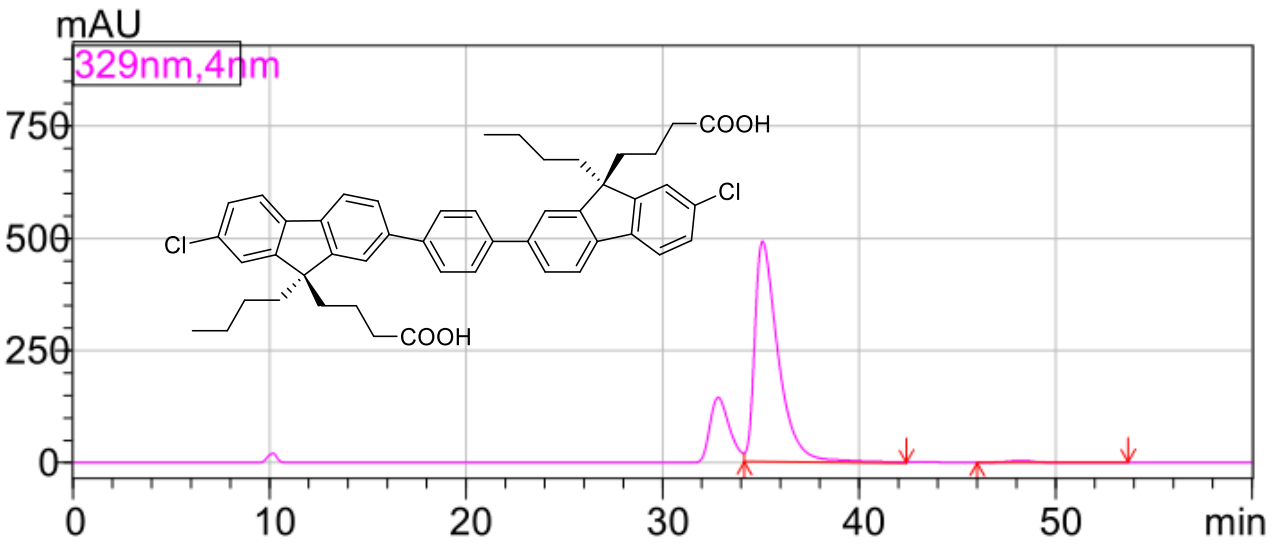

<Peak Table>

PDA Ch1 329nm

\begin{tabular}{|r|r|r|r|r|r|r|}
\hline Peak\# Ret. Time & \multicolumn{1}{|c|}{ Area } & \multicolumn{1}{c|}{ Height } & Peak Start & Peak End & \multicolumn{1}{c|}{ Area\% } \\
\hline 1 & 35.092 & 40983994 & 490819 & 34.165 & 42.395 & 99.048 \\
\hline 2 & 48.223 & 393805 & 4241 & 46.016 & 53.685 & 0.952 \\
\hline Total & & 41377798 & 495060 & & & 100.000 \\
\hline
\end{tabular}


meso-64

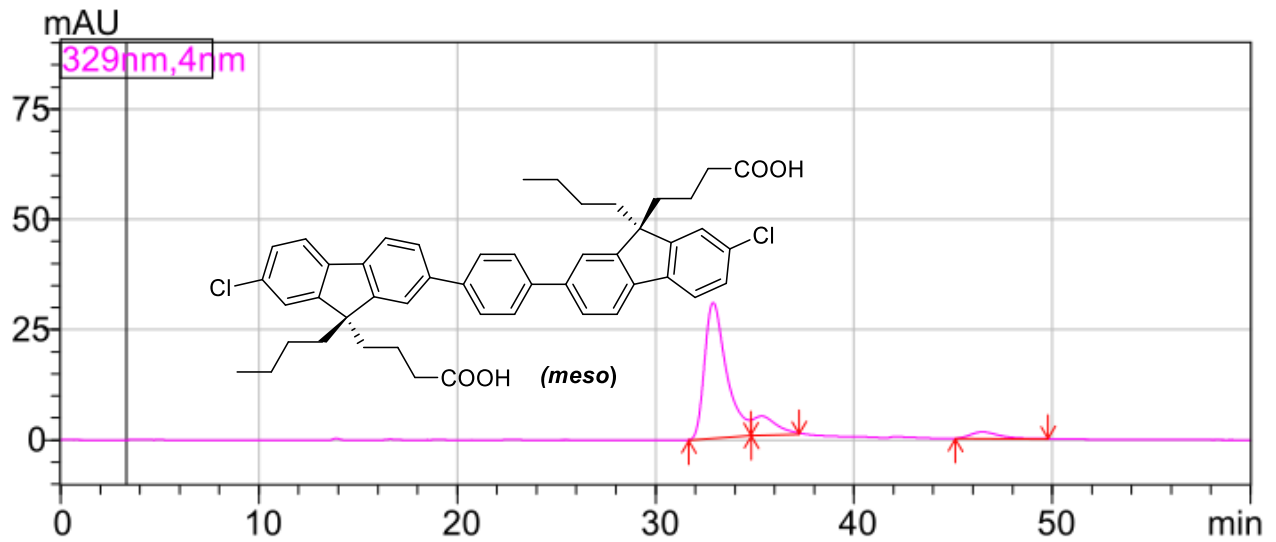

<Peak Table>

PDA Ch1 329nm

\begin{tabular}{|r|r|r|r|r|r|}
\hline Peak\# Ret. Time & \multicolumn{1}{|c|}{ Area } & Peak Start & Peak End & Area\% \\
\hline 1 & 32.906 & 2387781 & 31.669 & 34.795 & 82.632 \\
\hline 2 & 35.330 & 351513 & 34.837 & 37.232 & 12.165 \\
\hline 3 & 46.473 & 150356 & 45.104 & 49.792 & 5.203 \\
\hline Total & & 2889650 & & & 100.000 \\
\hline
\end{tabular}




\section{Precursor to 65}

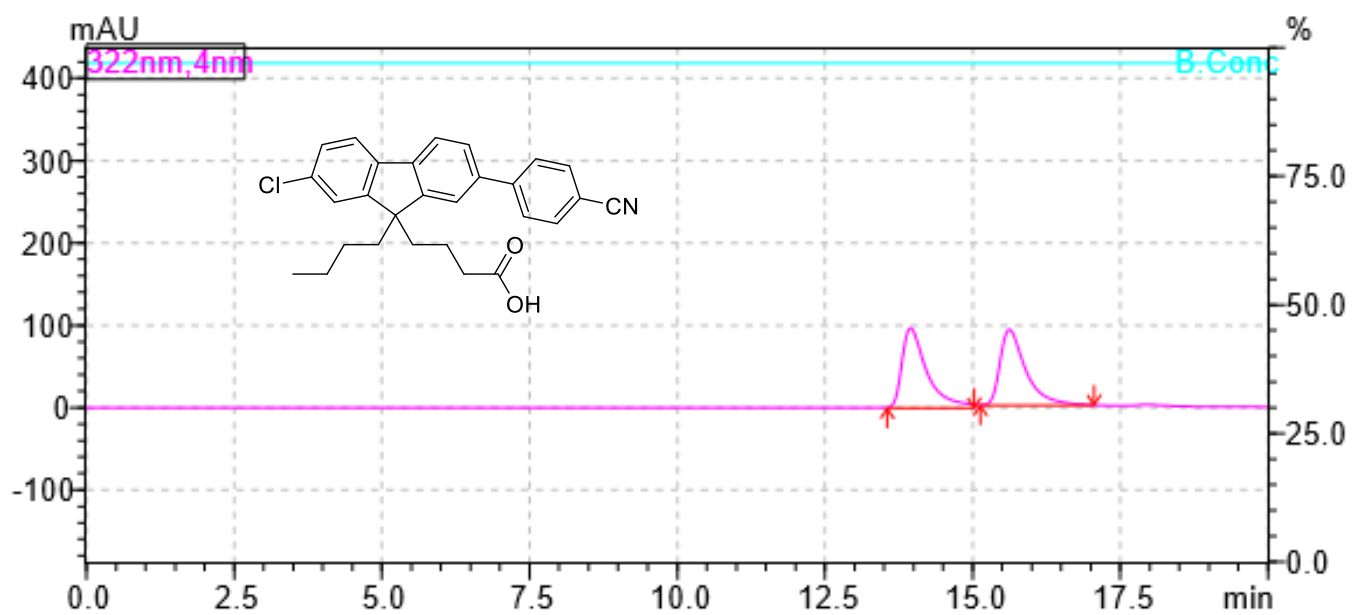

<Peak Table>

PDA Ch1 322nm

\begin{tabular}{|c|c|c|c|c|c|c|}
\hline Peak\# & Ret. Time & Area & Height & Area $\%$ & Peak Start & Peak End \\
\hline 1 & 13.947 & 2889213 & 97370 & 50.854 & 13.552 & 15.024 \\
\hline 2 & 15.620 & 2792205 & 91985 & 49.146 & 15.125 & 17.056 \\
\hline Total & & 5681418 & 189354 & 100.000 & & \\
\hline
\end{tabular}

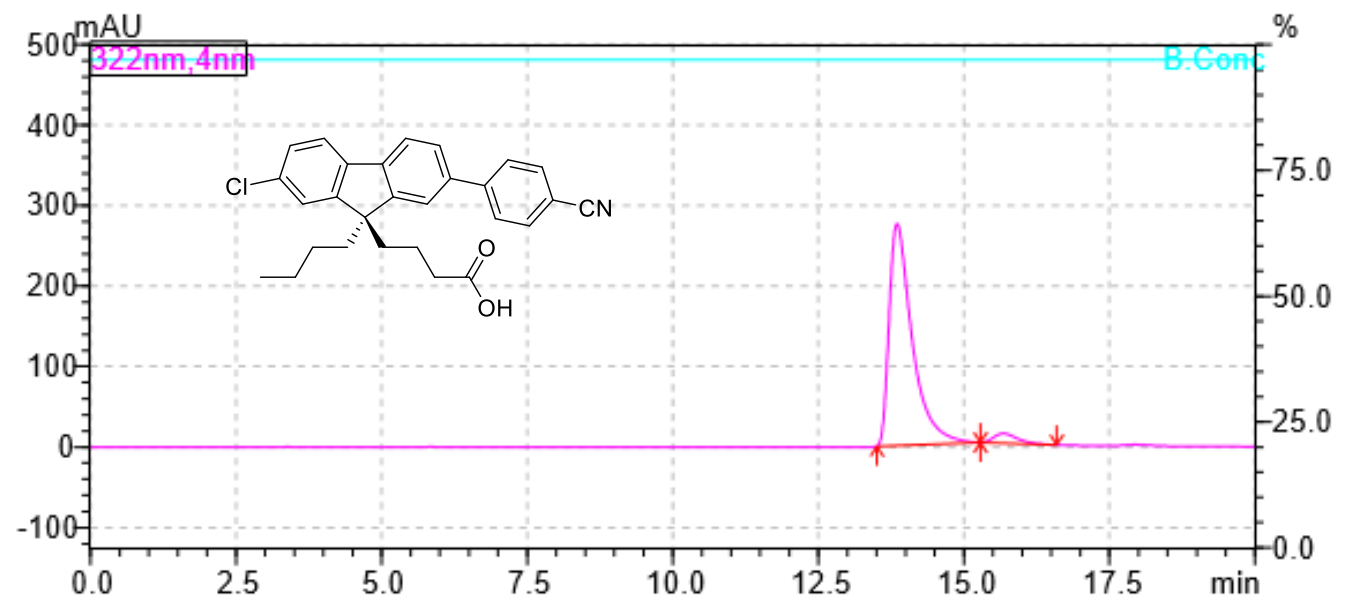

<Peak Table>

PDA Ch1 322nm

Peak\# Ret. Time

\begin{tabular}{|r|r|r|r|r|r|r|}
\hline Peak\# & Ret. Time & \multicolumn{1}{|c|}{ Area } & \multicolumn{1}{|c|}{ Height } & \multicolumn{1}{c|}{ Area\% } & Peak Start & Peak End \\
\hline 1 & 13.853 & 7587079 & 275446 & 95.639 & 13.504 & 15.280 \\
\hline 2 & 15.676 & 345967 & 12101 & 4.361 & 15.280 & 16.597 \\
\hline Total & & 7933046 & 287546 & 100.000 & & \\
\hline
\end{tabular}


65

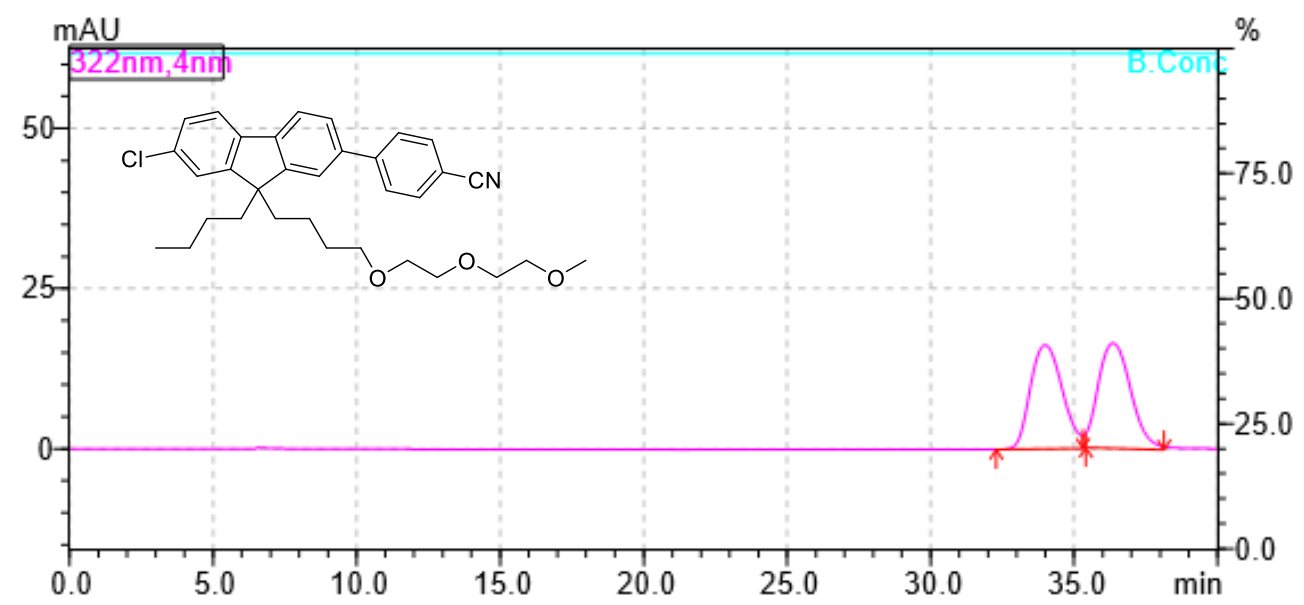

<Peak Table>

PDA Ch1 322nm

\begin{tabular}{|r|r|r|r|r|r|r|}
\hline Peak\# & Ret. Time & \multicolumn{1}{c|}{ Area } & \multicolumn{1}{c|}{ Height } & \multicolumn{1}{c|}{ Area\% } & Peak Start & Peak End \\
\hline 1 & 33.994 & 1251976 & 16176 & 49.189 & 32.288 & 35.312 \\
\hline 2 & 36.363 & 1293281 & 16395 & 50.811 & 35.413 & 38.139 \\
\hline Total & & 2545257 & 32572 & 100.000 & & \\
\hline
\end{tabular}

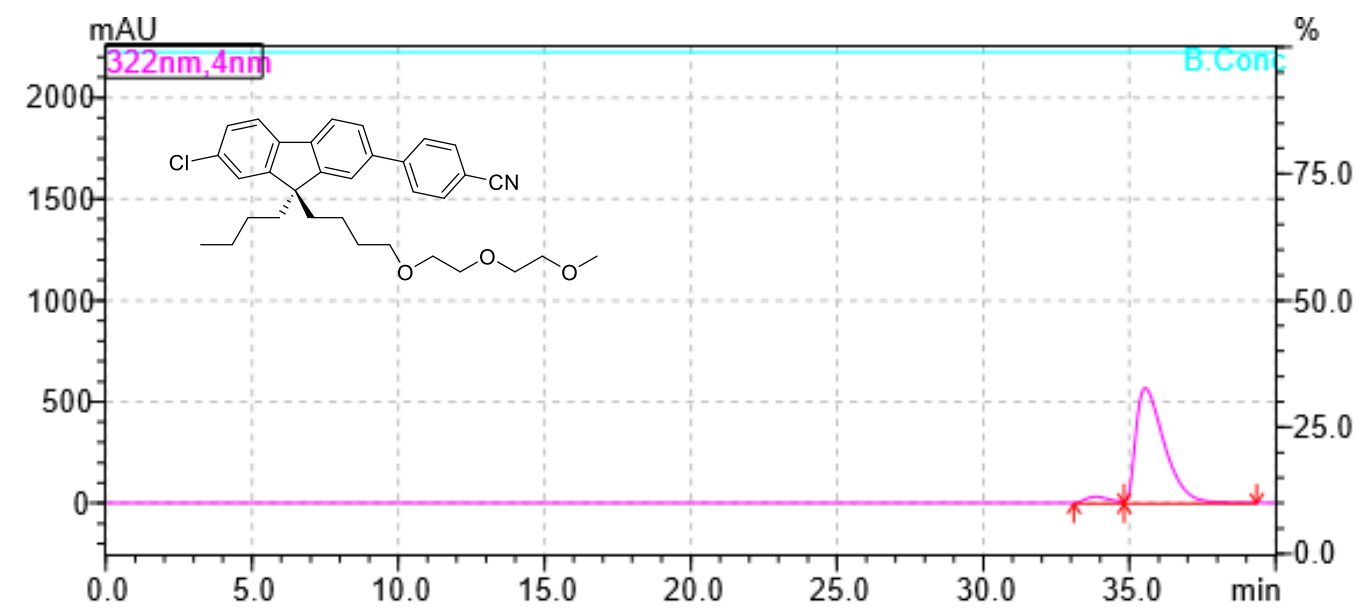

<Peak Table>

PDA Ch1 322nm

\begin{tabular}{|r|r|r|r|r|r|r|}
\hline Peak\# & Ret. Time & \multicolumn{1}{c|}{ Area } & Height & \multicolumn{1}{c|}{ Area\% } & Peak Start & Peak End \\
\hline 1 & 33.844 & 2018165 & 33524 & 4.906 & 33.093 & 34.811 \\
\hline 2 & 35.541 & 39122132 & 569940 & 95.094 & 34.811 & 39.349 \\
\hline Total & & 41140297 & 603464 & 100.000 & & \\
\hline
\end{tabular}




\section{X-ray crystallography data}

Compound $\left(\mathrm{S}_{S}, \mathrm{~S}\right)-\mathrm{L} 2$

The .cif data file is attached as a separate document. CCDC Deposition Number 2054849.

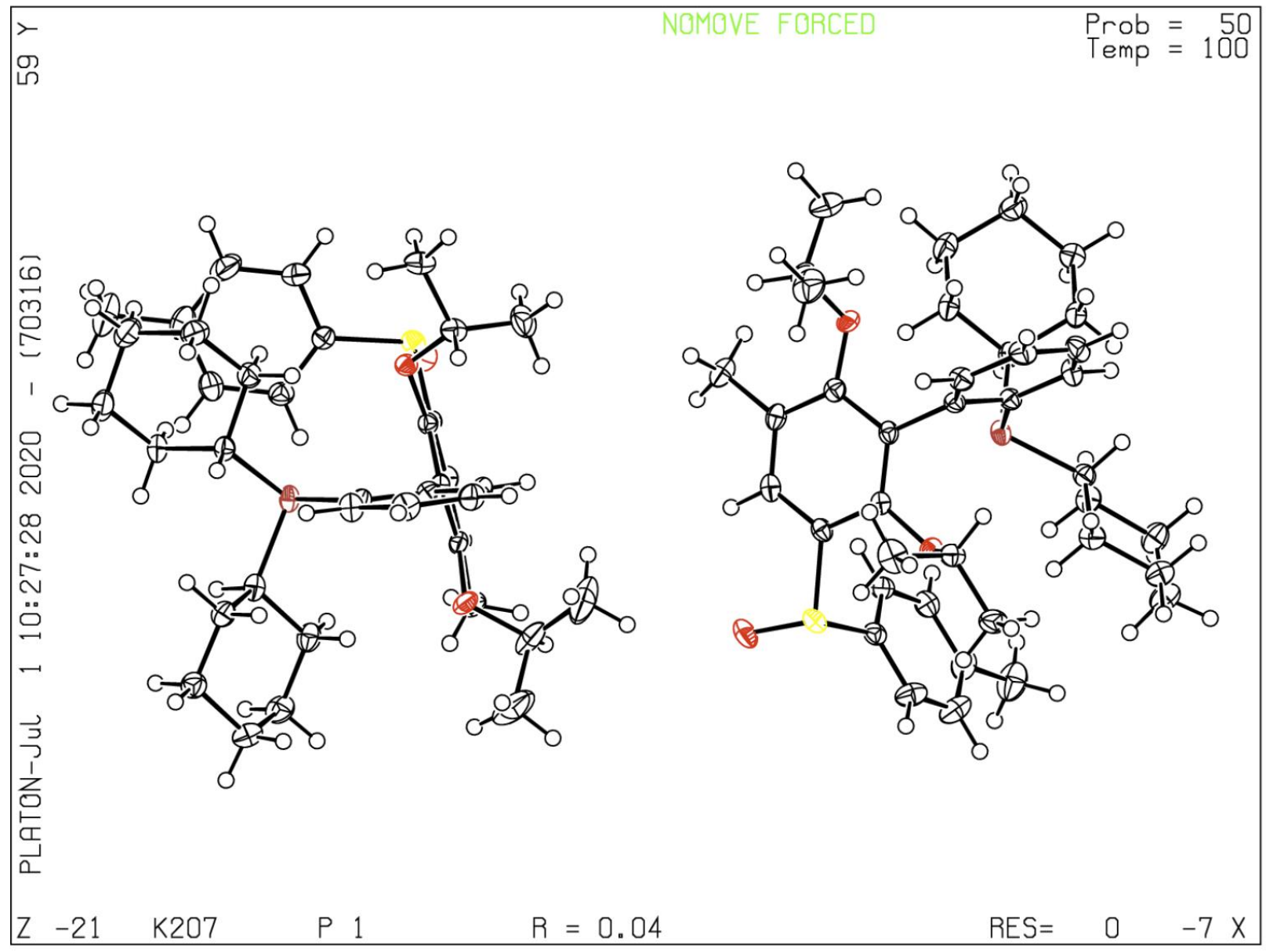




\section{Compound 37}

The .cif data file is attached as a separate document. CCDC Deposition Number 2054848.

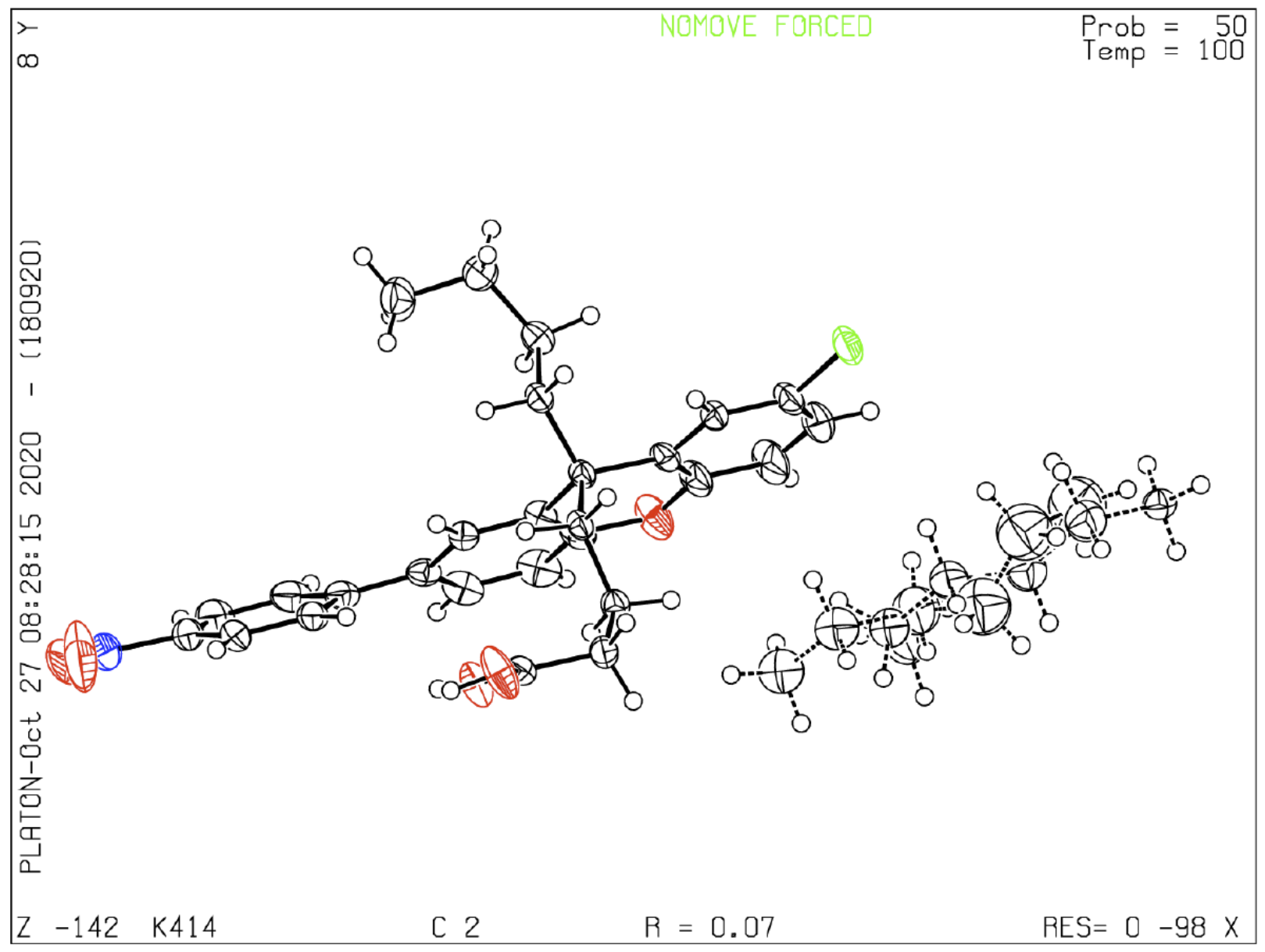

